\title{
Characterization of zampanolide as a microtubule-stabilizing agent
}

\author{
By \\ Jessica J. Field
}

\begin{abstract}
A thesis
submitted to the Victoria University of Wellington

in fulfillment of the requirements for the degree of

Doctor of Philosophy in Biomedical Science
\end{abstract}

Victoria University of Wellington

2013 



\section{Abstract}

Microtubule-stabilizing agents (MSAs) are extremely important chemotherapeutic drugs since microtubules (MTs) are one of the most successful cancer drug targets. Currently there are four MSAs that are clinically used for the treatment of cancer. Cancer cells, however, can develop resistance towards these drugs, the most common being overexpression of the P-glycoprotein drug efflux pump. Zampanolide (ZMP), a novel secondary metabolite isolated from a marine sponge consists of a 20-membered macrolide ring with an unusual $N$-acyl-hemiaminal side chain. It is a potent MSA with similar cellular effects to the clinically relevant MSAs, Taxol ${ }^{\circledR}$, Taxotere ${ }^{\circledR}$ and Ixempra ${ }^{\circledR}$. ZMP has a small number of stereogenic centers and therefore is relatively easier to synthesize than other macrolide natural products.

Using established cancer cell lines and isolated bovine brain tubulin ZMP in the present study was further characterized as a potential anti-cancer compound and was shown to have significant advantages over currently used MSAs. These studies provided insight into how this important drug class induces MT assembly, suggesting strategies for the development of new generation MSAs for use in the clinic.

ZMP and its less active analog dactylolide competed with paclitaxel for binding to MTs and represented a novel MSA chemotype. Unlike traditional taxoid site ligands, ZMP remained significantly more cytotoxic in cell lines with mutations in the taxoid binding site, and behaved in an unusual manner in vitro. This was later found to be due to its mechanism of binding which involved covalent modification of two amino acids in the taxoid binding site, histidine 229 as the major product and asparagine 228 as the minor product. Alkylation of both these luminal site residues was also detected in unassembled tubulin, providing the first direct evidence that the taxoid binding site exists in unassembled tubulin and suggesting that the induction of MT nucleation by MSAs may proceed through an allosteric mechanism. X-ray crystallography data confirmed the presence of this binding site in unassembled tubulin and indicated that covalent modification occurs at $\mathrm{C} 9$ of ZMP with the NE2 of the histidine side chain. The potent stabilization of MTs observed with ZMP occurred due to its side chain interaction with the stabilizing M-loop of $\beta$-tubulin. In unassembled tubulin the M-loop is unordered. Upon ZMP binding, it is restructured into a short, well-defined helix. It is this restructuring that leads to the potent stabilization by ZMP and most likely other MSAs, including those currently used in the clinic. This information provides a basis for 
structure-guided drug engineering to design and develop new generation MSAs with potent stabilizing activity. In addition, covalent binding of ZMP means that it is able to avoid drug efflux pumps and thus evade the main mechanism of resistance presented to MSAs in the clinic. It was shown by studying structure-activity relationships that there are a number of key chemical motifs in ZMP responsible for its potent activity. Simpler analog structures that retain significant stabilizing activity could be used as lead compounds for further drug development. Moreover, MSAs have clinically relevant antiangiogenic and vascular-disrupting properties, and ZMP was also shown to potently inhibit cell migration and thus have possible benefits as a vasculature-targeting compound.

It was concluded that ZMP is a potent covalently-binding MSA in both cells and in vitro. Given these promising results, further preclinical development of the compound is warranted. 
Some of the work presented in this thesis was published prior to submission of the thesis for examination.

Field, J. J., Pera, B., Calvo, E., Canales, A., Zurwerra, D., Trigili, C., Rodríguez-Salarichs, J., Matesanz, R., Kanakkanthara, A., Wakefield, S., Singh, A. J., Jiménez-Barbero, J., Northcote, P., Miller, J. H., López, J. A., Hamel, E., Barasoain, I., Altmann, K. H. \& Díaz, J. F. (2012). Zampanolide, a potent new microtubule-stabilizing agent, covalently reacts with the taxane luminal site in tubulin $\alpha, \beta$-heterodimers and microtubules. Chem. Biol. 19, 686-698.

Prota, A. E., Bargsten, K., Zurwerra, D., Field, J. J., Diaz, J. F., Altmann, K. H. \& Steinmetz, M. O. (2013). Molecular mechanism of action of microtubule-stabilizing anticancer agents. Science 339, 587-590.

Field J. J., Calvo, E., Northcote, P., Miller, J. H., Altmann, K. H. \& Díaz, J. F. (2013). Methods for studying microtubule binding site interactions: Zampanolide as a covalent binding agent. In Methods in Cell Biology: Microtubules in vitro II. (L. Wilson \& J. J. Correia, Eds.), Chapter 25. Elsevier Academic Press. In press.

Field J. J., Díaz, J. F., Miller, J. H. (2013). The binding sites of microtubule-stabilizing agents. Chem. Biol. 20, 301-315. 


\section{Acknowledgements}

First and foremost I would like to thank my amazing supervisor Professor John H. Miller. Without you I wouldn't be where I am today. Your constant guidance, support, encouragement and words of wisdom have made this project possible. I couldn't have asked for a better supervisor and I will always be grateful that I had the opportunity to be your PhD student - I learnt from the best. Thank you for always making us smile and laugh. And of course, I must thank you for also teaching me how to write and to stop "vomiting words onto a piece of paper". Thank you also to Julie for her amazing baking and always making us feel welcome.

I would like to thank my Co-supervisor Professor. J. Fernando Díaz at CIB, Madrid. Fer - I will be forever grateful, I have learnt so much from you and have developed a love for biochemistry. This thesis wouldn't exist without your expertise, guidance and support, and of course the use of your lab! Again I have learnt from the best. ¡Muchas Gracias!

This thesis would not have been possible without the expertise, support, and guidance of many different people. It is with immense gratitude that I acknowledge and thank the following people:

My secondary supervisor Assoc. Professor Peter Northcote for providing the zampanolide and also for your excitement and support throughout this research.

Dr. Benet Pera for teaching me everything I know about biochemistry and for your help in the lab. Again, thank you to Benet and his wife Eli for taking me in when I was in Spain for the first time, looking after me like I was family and showing me your beautiful country. Without you guys, my time in Spain wouldn't have been as amazing as it was. I am really grateful for everything you guys did for me. Again I would like to thank both of you for having Ryan and I stay the second time I came to Spain.

Dr. José Manuel Andreu for the use of lab equipment and materials and for his help with the project. I would also like to thank labs 308 and 309 at the CIB in Madrid for having me stay, for their help in lab and patience with stupid questions and trying to understand my kiwi accent and also for putting up with bad Spanish! Especially Mariano Redondo Horcajo.

Dr. Jonathan Singh for isolating zampanolide and for all his help with chemistry that he once taught me in undergrad that I forgot. Assoc. Professor Paul Teesdale-Spittle - For always being interested in my project, helping me with SAR and other chemistry related matters. Professor Karl-Heinz Altmann (ETH, Zürich, Switzerland), Dr. Michel Steinmetz and colleagues (Paul Scherrer Institut, Switzerland), Dr. Enrique Calvo (Unidad de Proteómica, Centro Nacional de Investigaciones Cardiovasculares, Madrid), AUC staff at CIB, Dr. Aurora Nogales (Instituto de Estructura de la Materia, Madrid). And of course all the SBS VUW admin staff, who make life so much easier!

Team JHM! Drs Arun Kanakkanthara, Ariane Chan, Vishy Das for all your help and for being great lab mates! The old crew from KK 601 especially Peter Bosch who has been on this journey with me and of course Miles Benton for help with computerish stuff which has proved invaluable. AM304 and everyone who I have shared the past 3 years with, there are too many people to name. Thank you.

Thank you to all my friends who have been supportive and put up with me being married to my $\mathrm{PhD}$ for the past few years, sorry I can't name you all but you know who you are. Special thanks to Elizabeth Stichbury my best bud and fantastic chief science editor! To Keelan Law for his enthusiasm and support! And lastly Lucía García -we can party now gatito!

I would like to thank my family. My mother, grandmother, brother and also Mike, without all your support and guidance I wouldn't be where I am today. Also thank you to the Steel family.

Finally and most importantly thank you to Ryan Steel - For your understanding, unconditional love and support throughout. I am so grateful. Love you ${ }^{\wedge} \boldsymbol{e}^{\wedge}$ 
This $\mathrm{PhD}$ was supported by a Victoria University of Wellington Postgraduate Scholarship to Jessica Field. Research in Wellington was supported by grants from the Cancer Society of New Zealand (Wellington Division), the Wellington Medical Research Foundation, and Victoria University of Wellington. Research in Madrid was supported by grants awarded to Dr. J. Fernando Díaz of the Centro de Investigaciones Biológicas from the Ministerio de Economia y Competitividad (BIO2010-16351) and the Comunidad Autónoma de Madrid (S2010/BMD-2457 BIPEDD2). Travel to and from Madrid and to conferences was funded by a Genesis Oncology Trust Professional Development award, a Wellington Medical Research Foundation travel grant, a European Molecular Biology Short-Term Fellowship award, a Victoria University Postgraduate Student Association travel grant, a Cancer Society of New Zealand (Wellington Division) travel grant, a New Zealand Society of Biochemistry and Molecular Biology student travel grant, a European Molecular Biology Laboratory Corporate Partnership travel grant, a Victoria University Faculty Strategic Research Grant, a Centre for Biodiscovery, Victoria University of Wellington grant, and a Maurice and Phyllis Paykel Trust travel grant. 
Table of contents

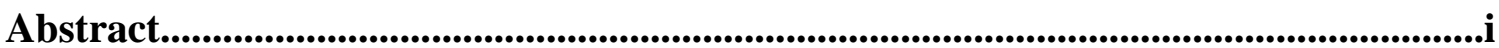

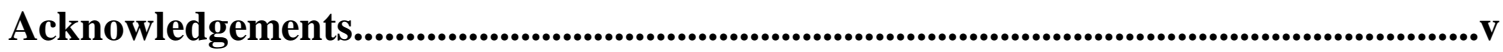

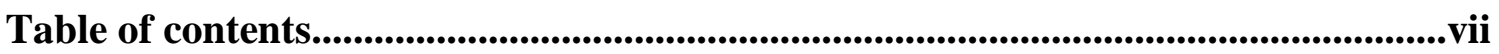

List of figures...............................................................................................................................................xiv

List of tables...........................................................................................................................xvii

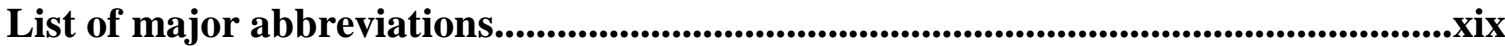

Chapter 1 General introduction........................................................................1

1.1 Drug discovery and natural products...........................................................

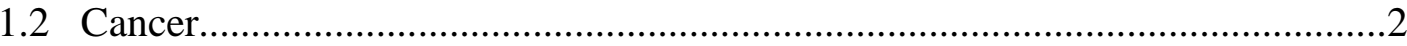

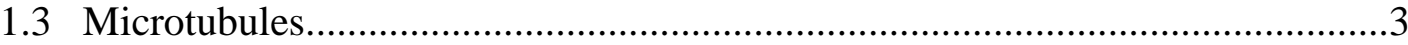

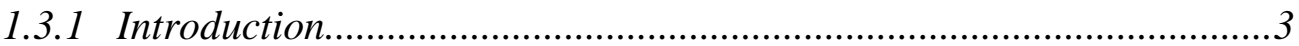

1.3.2 Microtubule dynamics.........................................................................4

1.3.3 Microtubules in mitosis and chemotherapy........................................5

1.4 Microtubule-destabilizing agents............................................................

1.4.1 Vinca domain agents....................................................................

1.4.2 Colchicine-like compounds..............................................................10

1.4.3 Covalent microtubule-destabilizers................................................13

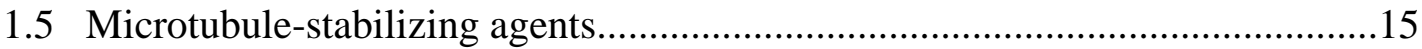

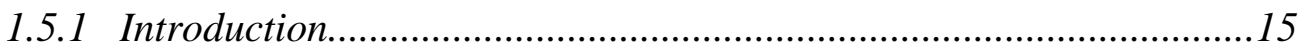

1.5.2 Compounds that stabilize microtubules..........................................15

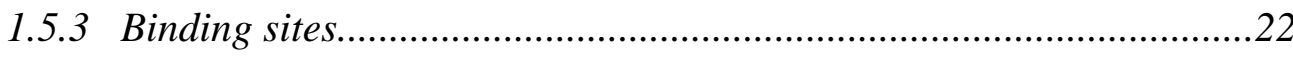

1.5.4 Clinical limitations of the taxanes....................................................23

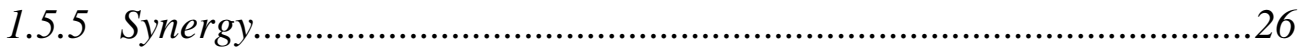

1.5.6 Microtubule-stabilizing agents in other diseases..............................27

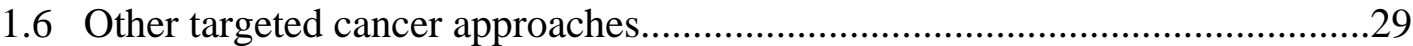

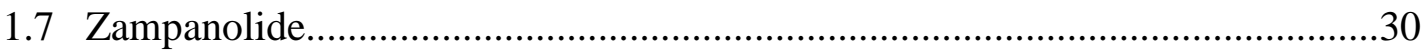

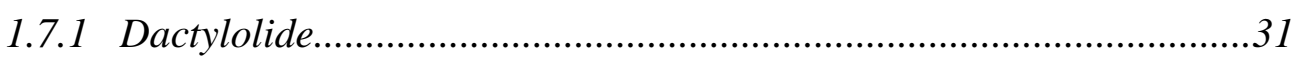

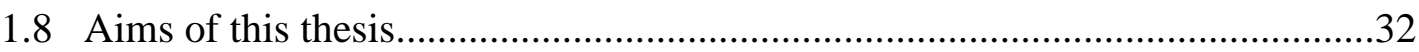


Chapter 2 General methods............................................................................33

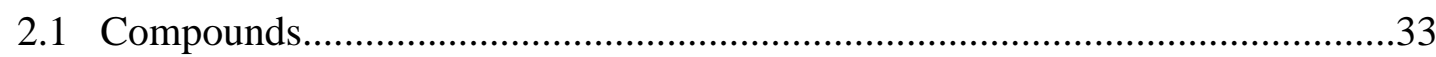

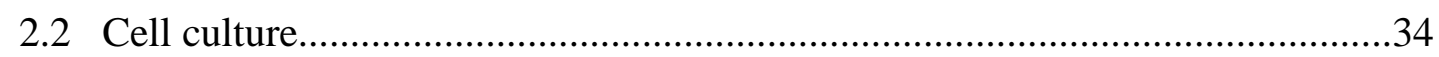

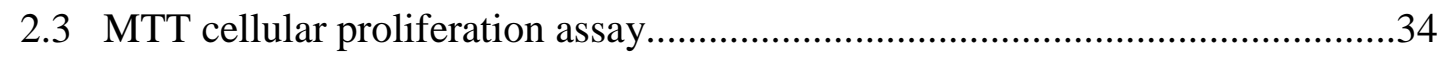

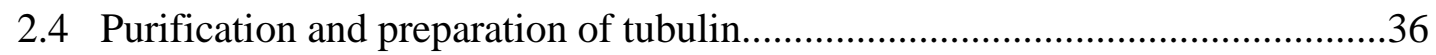

2.5 Preparation of stabilized taxoid binding sites.................................................

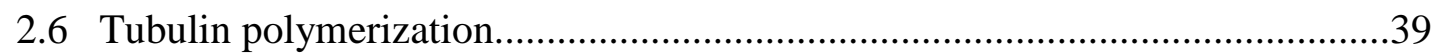

2.6.1 Microtubule assembly in glycerol buffer...........................................39

2.6.2 Induction of microtubule assembly in unfavorable conditions............40

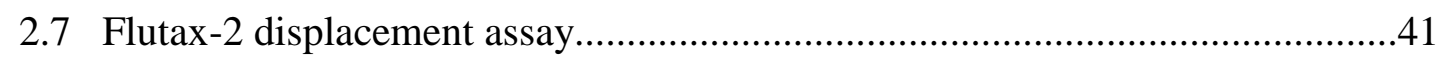

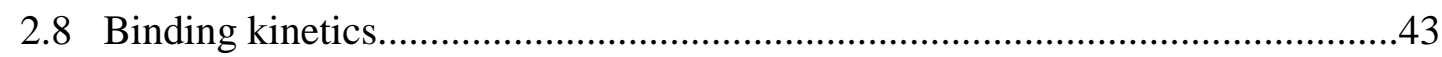

2.9 High performance liquid chromatography..................................................4

2.10 Nano-liquid chromatography and tandem mass spectrometry........................44

2.10.1 Assembled microtubules..................................................................45

2.10.2 Stabilized cross-linked microtubules............................................45

2.10.3 Oligomeric unassembled tubulin...................................................45

2.10.4 Dimeric unassembled tubulin.........................................................46

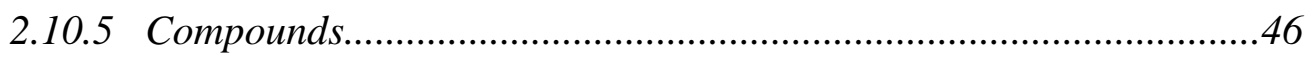

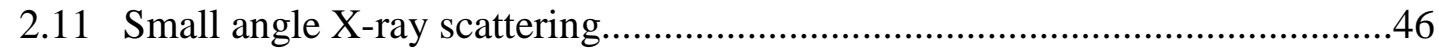

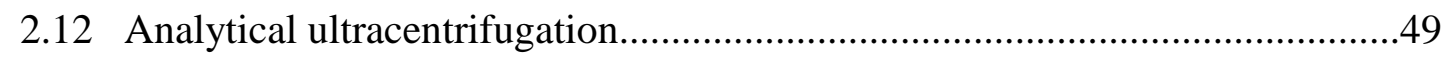

Chapter 3 Cellular effects of zampanolide...................................................53

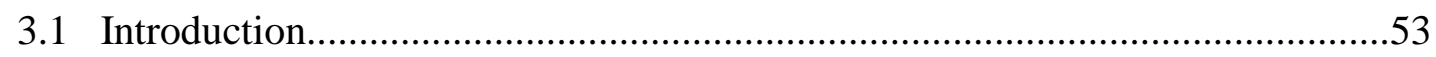

3.1.1 Resistance to microtubule-stabilizing agents....................................53

3.1.2 Resistant cell lines................................................................................53

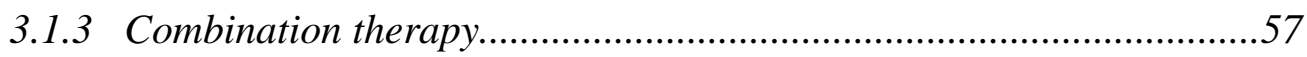

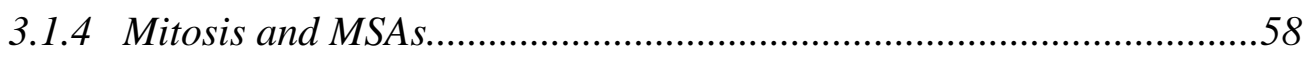

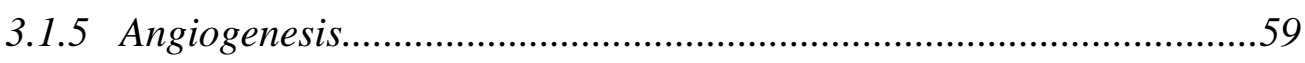

3.1.6 Aims of this chapter...................................................................61

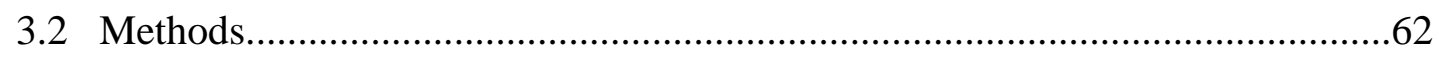

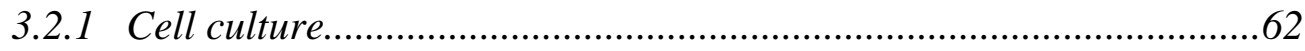

3.2.2 MTT cellular proliferation assay.................................................62

3.2.3 Wound scratch healing assay........................................................64 
3.2.4 Generation of a zampanolide-resistant cell line......

3.3 Results .66

3.3.1 Cytotoxicity of ZMP in different cell lines..... 66

3.3.2 Profile of zampanolide in resistant cell lines....................................67

3.3.3 Zampanolide does not synergize with other MSAs............................70

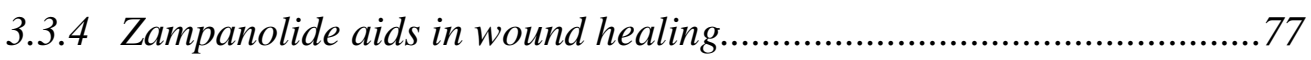

3.3.5 Generating a zampanolide-resistant cell line....................................82

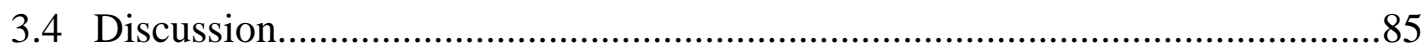

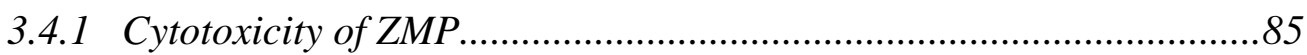

3.4.2 Investigation into the binding site of ZMP using resistant cells..........85

3.4.3 Lack of synergistic interaction of ZMP with MSAs............................89

3.4.4 ZMP inhibits cell migration...............................................................90

3.4.5 Development of a ZMP-resistant cell line........................................92

3.4.6 Conclusions and future directions...................................................94

Chapter 4 Determination of the zampanolide binding site..................................97

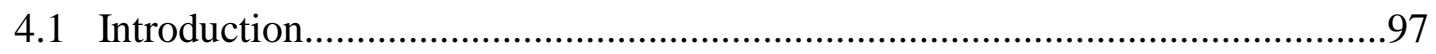

4.1.1 The taxoid binding site..................................................................97

4.1.2 The two-site binding hypothesis.....................................................105

4.1.3 Other important taxoid site MSAs.................................................111

4.1.4 The laulimalide/peloruside binding site...........................................112

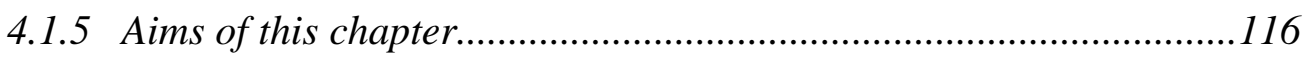

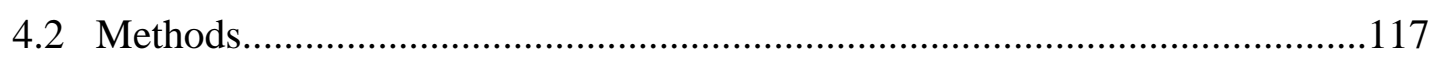

4.2.1 Preparation of zampanolide ........................................................ 117

4.2.2 Ligand induced microtubule assembly..........................................117

4.2.3 Binding site determination studies..................................................119

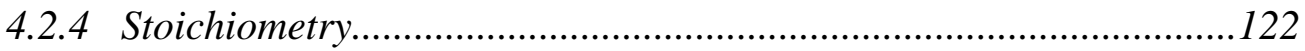

4.2.5 Probing the pore type I site ......................................................... 122

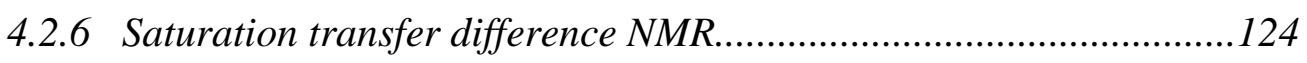

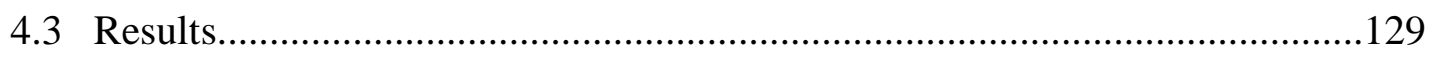

4.3.1 Biochemical properties of zampanolide...........................................129

4.3.2 Zampanolide is a potent MSA..........................................................130

4.3.3 Zampanolide and dactylolide are ligands of the taxoid site..............131 
4.3.4 Zampanolide binds to the taxoid site with 1:1 stoichiometry............133

4.3.5 Zampanolide binding blocks binding at the pore site........................134

4.3.6 Bioactive conformation of DAC bound to microtubules....................135

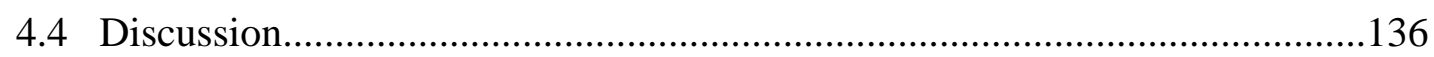

4.4.1 Zampanolide is a potent inducer of MT assembly...........................136

4.4.2 Zampanolide is a taxoid site ligand.................................................136

4.4.3 Zampanolide binds with 1:1 stoichiometry.....................................138

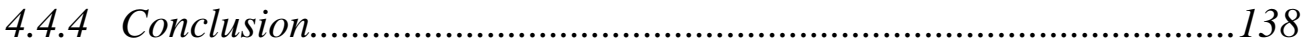

Chapter 5 Zampanolide covalently modifies tubulin....................................141

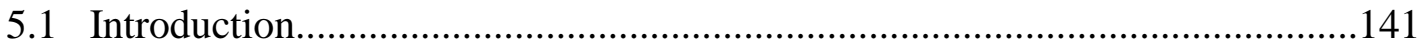

5.1.1 Luminal binding site in dimeric tubulin..........................................141

5.1.2 Mechanisms of MSA-induced assembly.........................................141

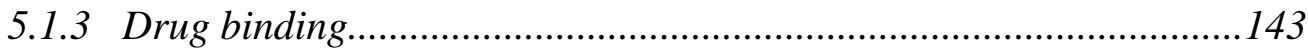

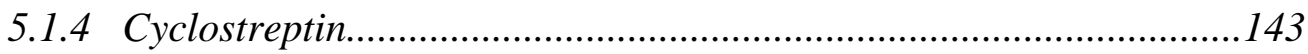

5.1.5 Characteristics of covalent binding in pharmacology.....................144

5.1.6 Covalent binding and resistance..................................................145

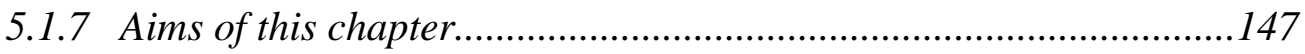

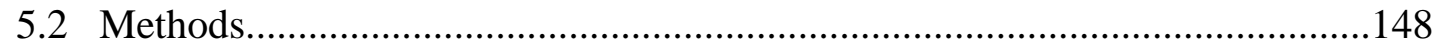

5.2.1 Detection of irreversible binding...................................................148

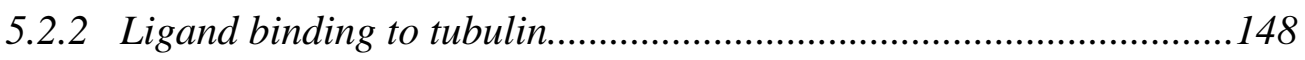

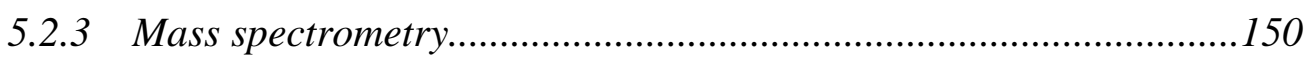

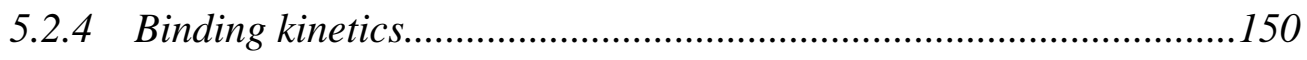

5.2.5 Immunofluorescence and confocal microscopy...............................150

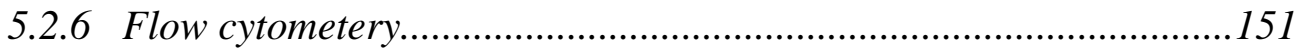

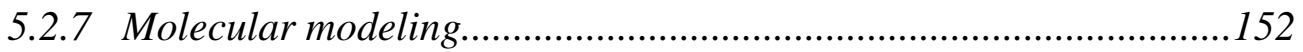

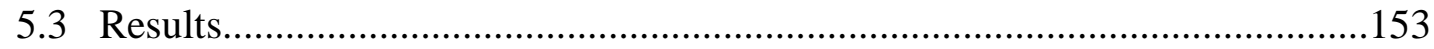

5.3.1 Irreversible binding of zampanolide and dactylolide to tubulin.......153

5.3.2 Mass spectrometry of ligand-modified tubulin................................... 153

5.3.3 Binding kinetics of zampanolide and dactylolide ..............................159

5.3.4 Covalent modification of cellular tubulin.........................................161

5.3.5 Cell recovery after MSA treatment................................................164

5.3.6 Molecular modeling of ZMP bound to the taxoid site.......................167 
5.4 Discussion 172

5.4.1 Zampanolide binds covalently to the MT. 172

5.4.2 Labeling of residue 288. 173

5.4.3 Taxoid site and the pore type I site are mutually exclusive. 175

5.4.4 The luminal binding site exists in dimeric tubulin. 176

5.4.5 Binding kinetics 177

5.4.6 Structural basis for the ZMP MT-stabilizing effect. 178

5.4.7 Zampanolide overcomes resistance. 178

5.4.8 Conclusions. 179

Chapter 6 Biochemistry of the zampanolide adduct. .181

6.1 Introduction 181

6.1.1 Microtubule formation and tubulin aggregation. 181

6.1.2 GTP-bound dimers versus GDP-bound dimmers. 185

6.1.3 Allosteric or lattice model of tubulin straightening. 185

6.1.4 Colchicine site. 187

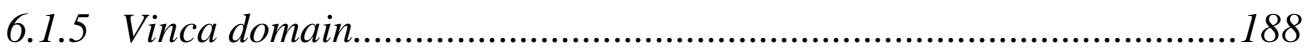

6.1.6 MSA binding determines MT size................................................192

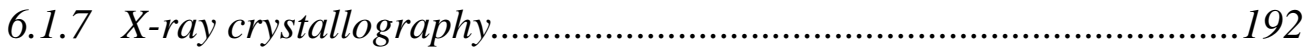

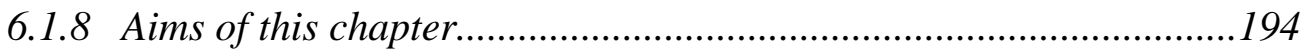

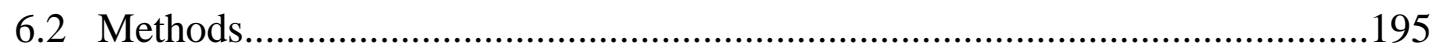

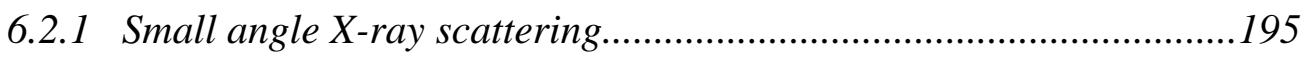

6.2.2 Analytical ultracentrifugation........................................................195

6.2.3 Effect of zampanolide on laulimalide/peloruside binding site..........196

6.2.4 Effect of zampanolide on colchicine binding site.............................196

6.2.5 Effect of zampanolide on the Vinca domain.....................................197

6.2.6 Effect of zampanolide on the E-site...............................................198

6.2.7 Preparation of the zampanolide-tubulin adduct for crystallization..199

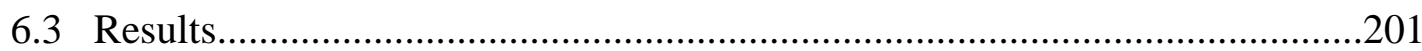

6.3.1 Microtubule diameter and number of protofilaments.......................201

6.3.2 Tubulin aggregation..................................................................203

6.3.3 Effect of zampanolide on the laulimalide/peloruside A site..............206

6.3.4 Effect of zampanolide on colchicine binding site. 207 
6.3.5 Effect of zampanolide on nucleotide E-site...................................209

6.3.6 Crystallization of zampanolide bound to unassembled tubulin.........211

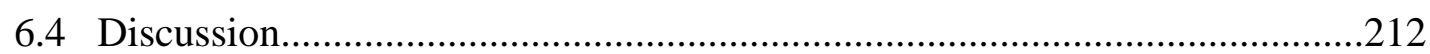

6.4.1 Crystal structure of zampanolide bound to unassembled tubulin .....212

6.4.2 Molecular mechanism of action of MSAs.......................................215

6.4.3 Thermodynamics of MSA binding.................................................214

6.4.4 Zampanolide influences MT size..................................................220

6.4.5 Effect of adduct formation on tubulin aggregation..........................220

6.4.6 ZMP binding results in loss of the E-site affinity for nucleotide.......221

6.4.7 Effect of adduct formation on other drug binding sites....................222

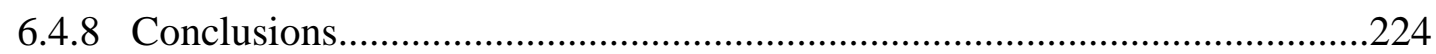

Chapter 7 Zampanolide analogs and SAR ........................................................225

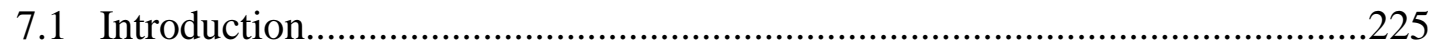

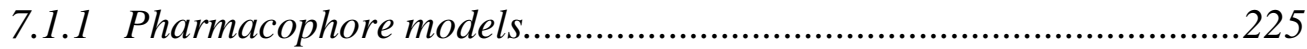

7.1.2 The zampanolide chemotype .......................................................229

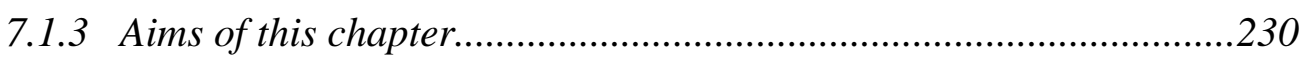

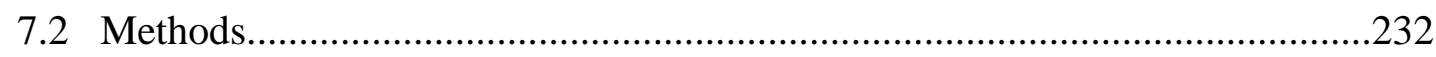

7.2.1 Effects on microtubule assembly..................................................232

7.2.2 Binding at the taxoid binding site................................................232

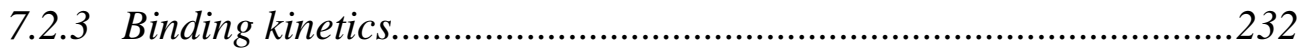

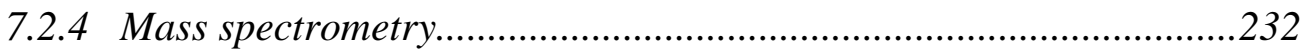

7.2.5 Small angle $X$-ray scattering....................................................232

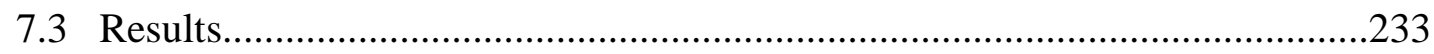

7.3.1 Effects on microtubule assembly ................................................233

7.3.2 Binding at the taxoid binding site and binding kinetics...................234

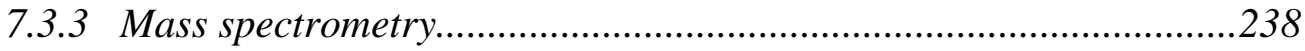

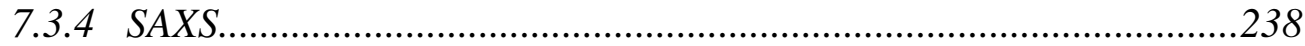

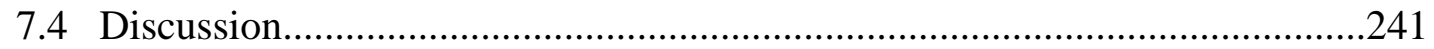

7.4.1 Structure-activity relationships of the analogs................................241

7.4.2 Microtubule size........................................................................248

7.4.3 Conclusions and future directions...............................................248 
Chapter 8 General discussion, conclusions and future direction..

8.1 Overall findings and conclusions

8.1.1 Zampanolide binds covalently to the taxoid site and potently

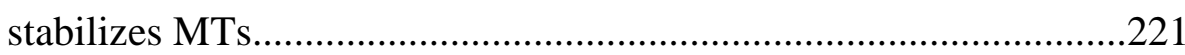

8.1.2 The luminal binding site exists in dimeric tubulin.........................221

8.1.3 Zampanolide avoids the main mechanisms of resistance.................252

8.1.4 Zampanolide activity in A8 EPOA-resistant mutant cells.................253

8.1.5 Cells grown in high concentrations of zampanolide.......................256

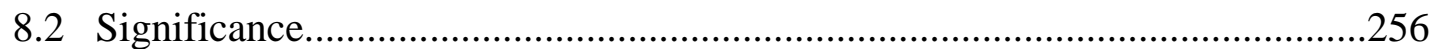

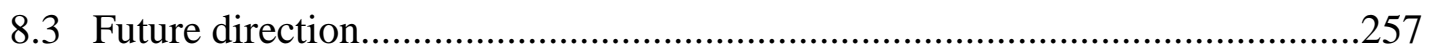

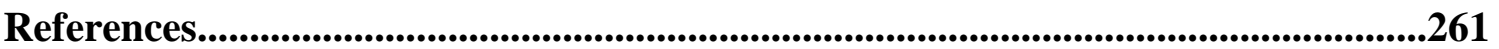

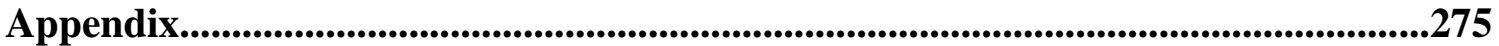




\section{Chapter 1}

Figure 1.1 Structure of microtubules and their dynamic instability...........................6

Figure 1.2 Structure of ligands that bind to the Vinca binding domain.......................

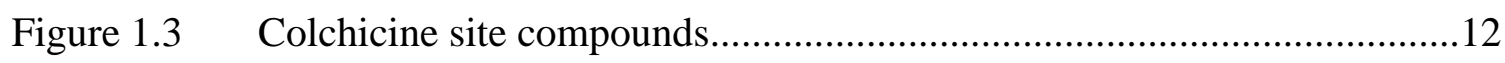

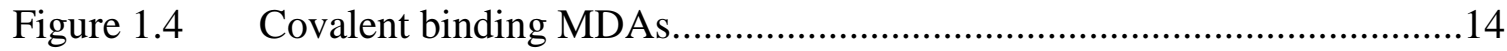

Figure 1.5 Structures of selected compounds that bind and stabilize the MT............21

Figure 1.6 Microtubule-stabilizing agent binding sites.........................................22

\section{Chapter 3}

Figure 3.1 Resistance ratios of MSAs in mutant cell lines.....................................69

Figure 3.2 Synergy interactions of selected MSAs with one another........................75

Figure 3.3 Synergy interactions of ZMP with selected MSAs.................................76

Figure 3.4 ZMP and DTX inhibition of HUVEC cell migration..............................79

Figure 3.5 ZMP and DTX inhibition of D551 cell migration...................................81

Figure 3.6 Changes in morphology of long-term ZMP-treated cells.........................83

\section{Chapter 4}

Figure 4.1 Sequence and secondary structure of $\beta$-tubulin......................................99

Figure 4.2 The luminal taxoid binding site with bound paclitaxel...........................103

Figure 4.3 Pore type I site interacting structures................................................... 110

Figure 4.4 Models of the proposed LAU/PEL sites...............................................115

Figure 4.5 Structure of Flutax compounds.......................................................... 121

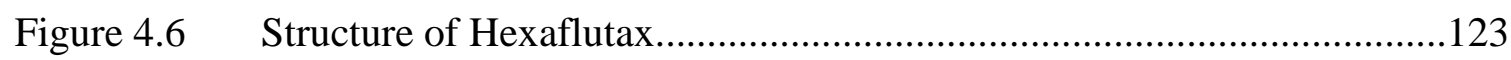

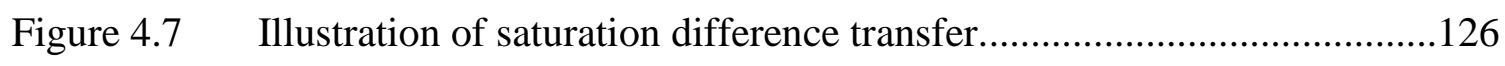

Figure 4.8 Spectra and extinction coefficients.......................................................129

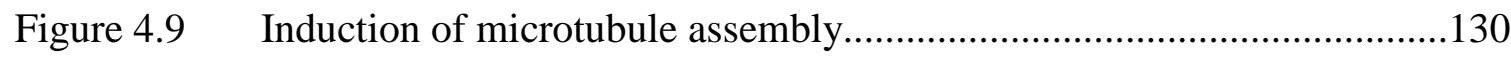

Figure 4.10 Flutax-2 displacement by ZMP and DAC ..........................................131

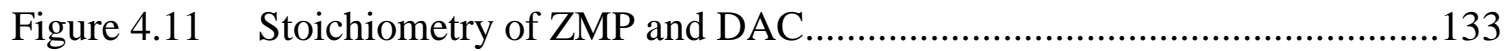

Figure 4.12 Hexaflutax binding to microtubules in the presence of ZMP.................134

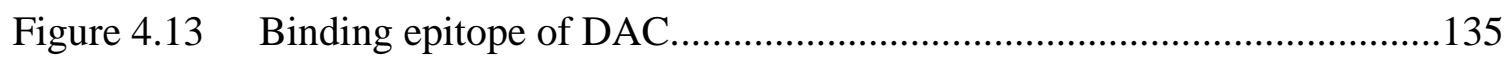




\section{Chapter 5}

Figure 5.1 Possible mechanisms for MSA-induced elongation of MTs 142

Figure 5.2 MS analysis of ZMP binding to MTs....................................................

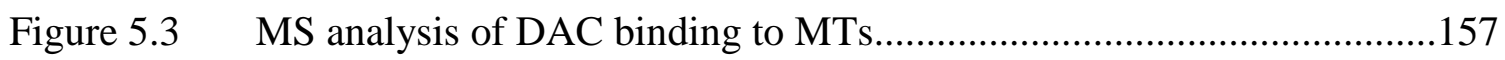

Figure 5.4 ZMP and DAC interaction with tubulin and MTs................................158

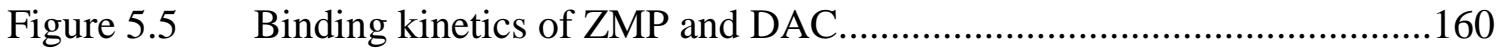

Figure 5.6 FTX-2 staining of cellular MTs with different MSAs.............................162

Figure 5.7 Percentage of cells in the $\mathrm{G}_{2} \mathrm{M}$ phase of the cell cycle...........................166

Figure 5.8 Molecular model of the ZMP-MT adduct.............................................169

Figure 5.9 Molecular model of the DAC-MT adduct.............................................171

\section{Chapter 6}

Figure 6.1 MSA induced MT-assembly from GDP bound tubulin..... 183

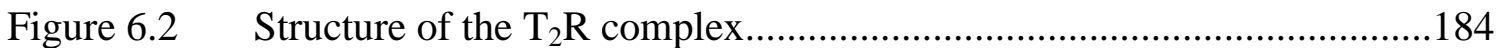

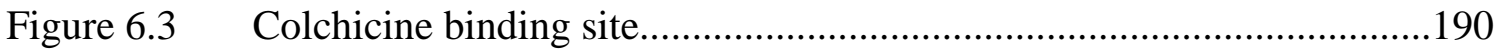

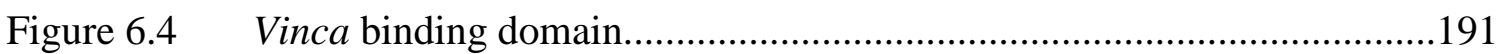

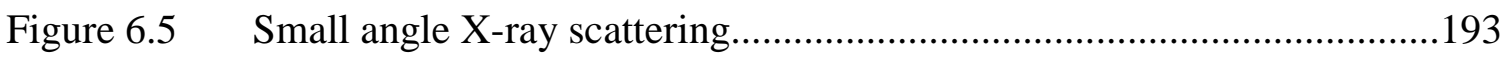

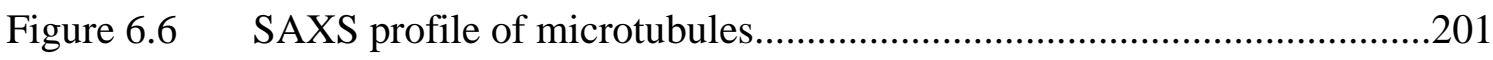

Figure 6.7 Tubulin aggregation of unligated tubulin and the ZMP-tubulin adduct..204

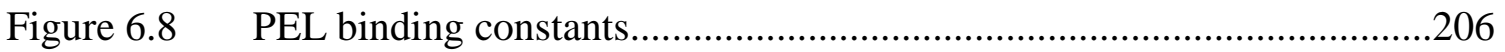

Figure 6.9 Effect of adduct formation on the affinity of the COL binding site........208

Figure 6.10 Michael addition of ZMP and histidine..........................................213

Figure 6.11 Crystal structure of ZMP bound to unassembled tubulin.......................214

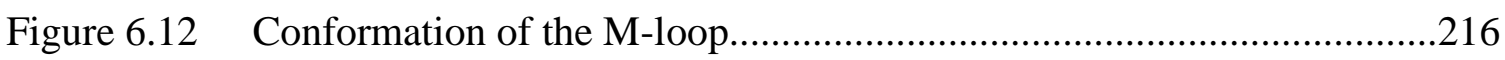

Figure 6.13 Molecular mechanism of action of MSAs.........................................219

\section{Chapter 7}

Figure 7.1 ZMP, EPOA and PTX in the taxoid binding site..............................228

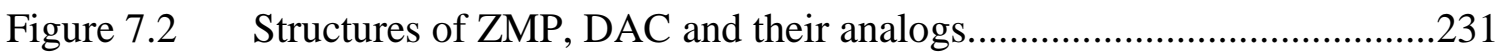

Figure 7.3 Enhancement of MT assembly by ZMP and analogs...........................233

Figure 7.4 Flutax-2 displacement by the analog compounds.................................235

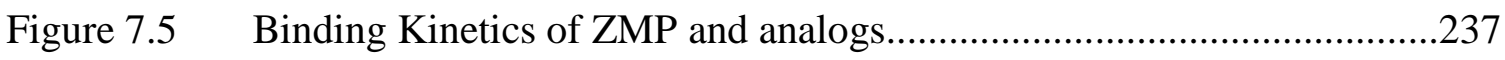

Figure 7.6 Mass spectrometry ion chromatograms of the analogs..........................238 


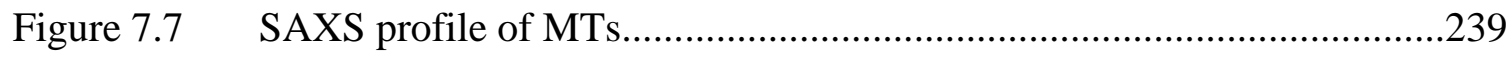

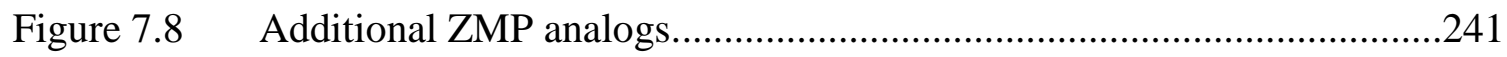

Figure 7.9 Molecular modelling of the ZMP crystal structure................................247

\section{Chapter 8}

Figure 8.1 ZMP and EPOA binding in A8 cells. .255 


\section{List of tables}

\section{Chapter 2}

Table 2.1 Fluorescence emission anisotropy of FTX-2

Table 2.2 Equilibrium constants of FTX-2 binding.....

\section{Chapter 3}

Table 3.1 Mutations in $\beta$-tubulin that confer resistance to MSAs................................56

Table 3.2 Cytotoxicity of ZMP in different cell lines................................................66

Table 3.3 IC $\mathrm{IC}_{50}$ values of MSAs in 1A9 parental cells and mutant cell lines..................67

Table 3.4 Resistant ratios for MSAs in mutant cells compared to 1A9 parental cells...68

Table $3.548 \mathrm{~h} \mathrm{IC}_{50}$ values of individual compounds.................................................

Table 3.6 CI values for different MSA combinations in 1A9 cells..............................72

Table 3.7 CI values for ZMP + MSA combinations in 1A9 cells................................73

Table $3.8 \%$ of wound recovery after $18 \mathrm{~h}$ in HUVEC cells........................................78

Table $3.9 \%$ of wound recovery after $18 \mathrm{~h}$ in D551 cells............................................80

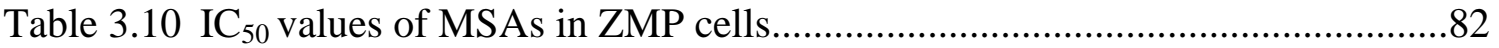

\section{Chapter 4}

Table 4.1 Tubulin regions and amino acid residues involved in MSA stabilization....100

Table 4.2 Binding constants of ligands for the taxoid site. .132

\section{Chapter 5}

Table 5.1 Cell cycle distribution (\%) of 1A9 cells treated with MSA 162

\section{Chapter 6}

Table 6.1 Structural data of ligand induced MTs.....................................................202

Table 6.2 Binding affinity of PEL for unmodified and modified MTs.......................206

Table 6.3 Binding constants of MTC binding to unmodified and modified tubulin....208

Table 6.4 Effect of adduct formation on the E-site. .210

\section{Chapter 7}

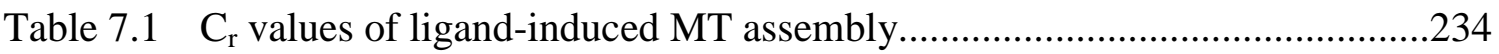

Table 7.2 Binding constants of the compounds to the taxoid site..............................236 
Table 7.3 \% of taxoid binding sites available for FTX-2 exchange...........................237

Table 7.4 Structural data of the ligand-induced MTs................................................240

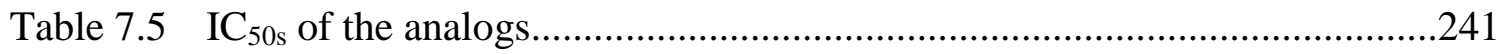


ACN Acetonitrile

AUC Analytical ultracentrifugation

BBT Bovine brain tubulin

$\mathrm{C}_{\mathrm{r}} \quad$ Critical concentration of tubulin assembly

DAC Dactylolide

DCBT Dichlorobenzyl thiocyanate

DSC Discodermolide

DTX Docetaxel (Taxotere ${ }^{\circledR}$ )

E-site Exchangeable nucleotide site

EPO Epothilone

FPLC Fast protein liquid chromatography

FTX-2 Flutax-2

GAB Glycerol EGTA buffer

GDP Guanosine 5'-diphosphate

GMP-PCP Guanylyl 5'-( $\beta, \gamma$-methylenediphosphonate

HDX-MS Hydrogen deuterium exchange mass spectrometry

HPLC High performance liquid chromatography

HXF Hexaflutax

IXA Ixabepilone (Ixempra ${ }^{\circledR}$ )

LAU Laulimalide

MAP Microtubule-associated protein

MDA Microtubule-destabilizing agent

MSA Microtubule-stabilizing agent

MT Microtubule

MTA Microtubule-targeting agent

MTC 2-methoxy-5-(2,3,4-trimethoxyphenyl)-2,4,6-cycloheptatrien-1-one

MTT 2-(4,5-dimethyl-2thiazolyl)-3,5-diphenyl-2H-tetrazolium bromide

$\mathrm{N}$-site Non-exchangeable nucleotide site

$\mathrm{NaPi} \quad$ Sodium phosphate

NOE Nuclear Overhauser Effect

PDP Podophyllotoxin

PEDTA Phosphate EDTA buffer 
PEL Peloruside A

P-gp P-glycoprotein drug efflux pump

PI Propidium iodide

PTX $\quad$ Paclitaxel $\left(\right.$ Taxol $\left.^{\circledR}\right)$

RB3 Bacterial protein with a SLD

SAR Structure-activity relationships

SAXS Small angle $x$-ray scattering

SLD Stathmin-like domain

STD Saturation transfer difference

$\mathrm{T}_{2} \mathrm{R} \quad$ Two tubulin heterodimers in complex with a SLD

TTL Tubulin tyrosine ligase

trNOESY Transferred nuclear Overhauser spectroscopy

VBL Vinblastine $\left(\right.$ Velban $\left.^{\circledR}\right)$

ZMP Zampanolide 


\subsection{DRUG DISCOVERY AND NATURAL PRODUCTS}

Traditionally the majority of new drugs translated into the clinic are natural products or compounds derived from them, although recently research into natural products has decreased (Harvey et al., 2010). This decrease is partly due to the increase in highthroughput screening technologies which are useful tools for screening large synthetic libraries for active compounds (Li \& Vederas, 2009). An advantage natural products have over purely synthetic compounds is that they have been evolutionarily selected to bind biological macromolecules and therefore serve as excellent potential drug compounds or advanced structure templates for the synthesis of novel biologically active analogs. Based on natural product scaffolds, synthetic chemists can create libraries of analogs and screen them using high-throughput screening to develop 'ideal' molecules based on structure-activity relationships (SARs). De novo design and synthesis of bioactive molecules; however, is unlikely to replace the millions of years of evolution and natural selection available to living organisms, thus even though natural products research has decreased, they still remain at the forefront of drug discovery.

Given their broad set of natural functions, secondary metabolites have a wide array of useful pharmacological properties, including antibacterial, anticoagulant, antimalarial and antiviral activity (Mayer \& Gustafson, 2008). Bacteria often have a symbiotic relationship with their host and are generally the main source of the active secondary metabolites, not the host eukaryotic organism (Belarbi et al., 2003). Secondary metabolites are used by their producers as powerful mechanisms of defense and often have eukaryotic targets such as tubulin and actin, as the microbes lack these proteins, and the marine organism's proteins have significant differences in homology to other eukaryotes or have defense mechanisms for preventing access to self-targets (Newman \& Cragg, 2004). Currently, $47 \%$ of compounds used to treat cancer are natural products or have been directly derived from natural products (Newman \& Cragg, 2012). Marine organisms exhibit extreme biodiversity and provide a wealthy source of natural products with unusual structure and significant biological activities. This makes them an exceptionally valuable resource for anticancer compounds. Many of these compounds have potent anticancer activity, and they often have a macrolide structure (Qi \& Ma, 2011). A number of compounds isolated from marine organisms also have potent 
microtubule-stabilizing activity, including discodermolide (DSC) (ter Haar et al., 1996), the sarcodictyins (Ciomei et al., 1997), eleutherobin (Long et al., 1998), the pelorusides (Hood et al., 2002; Singh et al., 2010), the laulimalides (Mooberry et al., 1999), dictyostatin (Isbrucker et al., 2003), ceratamines (Karjala et al., 2005), and zampanolide (ZMP) (Field et al., 2009) and dactylolide (DAC) (Zurwerra et al., 2010).

\subsection{CANCER}

Despite many recent medical advances in the past 50 years, cancer remains the second most common cause of death, after heart disease, in most countries (Ma \& Yu, 2006). In 2008, it was estimated that 12.7 million people were diagnosed with cancer, and 7.6 million people died worldwide. The number of global cancer mortalities is expected to rise to over 11 million in 2030 (Jemal et al., 2011). In New Zealand, cancer is the leading cause of death, accounting for $29 \%$ of all deaths with 20,317 people diagnosed and 8,566 deaths in 2008 (NZ Ministry of Health, 2011).

Cancer stems from the accumulation of gene mutations that control either cell proliferation or cell death. The majority of cancers therefore are genetically unstable (Lengauer et al., 1998). There are many different types of cancer, all characterized by uncontrolled cellular proliferation (Jordan et al., 1998). Cancer cells can often dislodge from their primary site and move to other organs in the body. This process is termed metastasis, and under these conditions, chemotherapy is needed to target all the cancer cells as it is no longer localized and the tumors cannot be removed by surgery or radiation therapy (Jordan et al., 1998). In metastatic conditions, cancer is a systemic disease, and a systemic approach is therefore required to treat the disease. Chemotherapy has now become the main focus in treatment of metastasized cancers, and is also used to treat primary tumors (DeVita \& Chu 2008)

Medical advances and improved understanding has increased knowledge as to how cancer behaves and progresses (Yap \& Workman 2012). Cancer cells are extremely well adapted for survival. The main issue in cancer chemotherapy therefore is to target the malignant cancer cells without affecting normal cells of the body (Jordan et al., 1998). It is relatively easy to chemically distinguish between a bacterial and a eukaryotic cell, simply because of the large evolutionary differences between their proteins. The task is more difficult, for example, with eukaryotic parasites because of the closer genetic similarity between the parasite proteins and those of humans. The most difficult case, 
however, occurs when the proteins of the target and the host cells are near identical, as is the case for neoplastic diseases. Drugs that can distinguish between these two cell types need to be designed and developed to treat cancer (Yap \& Workman 2012). In some cases, drugs are able to target cancer specific proteins, which often consist of embryonic proteins that are expressed only in cancer cells, or mutant oncogene proteins. One of the main functional distinctions, however, between cancer cells and most healthy cells is that most cancerous cells rapidly proliferate (Jordan \& Wilson 2004). Many cell cycle checkpoints exist that regulate cell division and apoptosis. Thus, if a chemical agent blocks any of the cell division components, the failure to undergo cell division will activate these checkpoints and result in cell death. Cancer cells are therefore sensitized to antimitotic drugs that block components of the cell division machinery (Jordan \& Wilson, 2004). Despite the new focus on molecular targeted therapy, such as the use of antibodies, chemotherapeutics have a more general cytotoxic effect and can thus target a broad range of cancer types, meaning they are still at the forefront of drug research for cancer chemotherapy.

\subsection{MicROTUBULES}

\subsubsection{Introduction}

Microtubules (MTs) are ubiquitous proteins, essential in all eukaryotic cells as key components of the cytoskeleton. They are critical in a number of cellular processes, most importantly, cell division and mitosis where they are involved in correctly assembling the mitotic spindle and consequent separation of the chromosomes to the spindle poles. MTs are characterized by their highly dynamic (non-equilibrium) behavior, constantly switching between periods of elongation and shortening, driven by the energy obtained from GTP hydrolysis. Hydrolysis of GTP leads to polymer formation in which tubulin assumes a straight conformation rather than a curved one, and potential energy is stored within the MT lattice. Depolymerization releases this energy, and, the dimers that are released have a curved conformation (Nogales et al., 2003). The tubulin dimer is made up of an $\alpha$ - and a $\beta$-subunit, arranged in a polar head-to-tail fashion, and these dimers assemble into protofilaments, with thirteen protofilaments arranging in a parallel manner to form a MT (Figure 1.1). In vivo MTs are made up of approximately 13 protofilaments, but this number varies in purified tubulin in vitro depending on the experimental conditions in which the MTs are formed (Chrétien et al., 1992; Matesanz et al., 2011a). 
Each tubulin monomer is made up of three distinct domains, the $\mathrm{N}$-terminal domain, the intermediate domain and the C-terminal domain, and each monomer also contains a nucleotide binding site. The $\alpha$-subunit has a stable non-exchangeable nucleotide binding site (N-site) that only binds GTP. The $\beta$-subunit contains an exchangeable nucleotide site (E-site) that also binds GTP, which is hydrolyzed to GDP soon after assembly of the MT. Assembly is favored when GTP is bound to the E-site; whereas, when this nucleotide is GDP, disassembly is favored. A polymerizing MT therefore has a 'GTP cap' on its growing end, which promotes stability of the straight MT conformation (Akhmanova \& Steinmetz, 2008). MTs are present either in an elongation state or a rapidly shortening state, with rapid transition between the two. This is termed the dynamic instability of MTs and involves the addition and loss of tubulin at both ends of the polymer (treadmilling) (Mitchison \& Kirschner, 1984). Upon depolymerization the protofilaments peel away from the lattice and curl at the ends due to loss of lateral contacts, rather than loss of subunits (Nogales \& Wang, 2006).

\subsubsection{Microtubule dynamics}

Tubulin dimers arrange into protofilaments through a mechanism called nucleationelongation, in which a small MT center is slowly formed, and this is followed by rapid growth at both ends by the reversible addition of tubulin dimers (Jordan \& Wilson, 2004). These dimers are then arranged into the 'long hollow tubes' (the MTs) that are made up of parallel protofilaments (Akhmanova \& Steinmetz, 2008). The final structure is organized so that $\alpha$-subunits are at one end (minus end) and $\beta$-subunits are at the other end (plus end). Dynamic instability of MT formation involves the addition and loss of tubulin dimers at both polymer ends (Mitchison \& Kirschner, 1984). The addition or loss of dimers occurs more readily at the plus end of the MT (Akhmanova \& Steinmetz, 2008).

Dynamic instability is characterized by four processes: growth, shortening, and the transition from the growth phase to the shorting phase (catastrophe) or from the shortening to growth phase (rescue) (Jordan \& Wilson, 2004). Hydrolysis of GTP is required to switch between catastrophe and rescue (Akhmanova \& Steinmetz, 2008) (Figure 1.1). MTs undergo two distinct dynamic behaviors neither of which occurs in equilibrium. First, if the $\beta$-tubulin GTP is hydrolyzed before more GTP bound tubulin is added to the MT, the exposed GDP causes a conformational change resulting in fast 
depolymerization of the MT (catastrophe). Rescue is the opposite of this process and involves the transition between a rapidly shortening MT to a growing MT or a stable MT (Jordan \& Wilson, 2004). In addition to dynamic instability, more controlled loss of subunits from the minus end can occur while subunits are added to the plus end, resulting in no overall change in MT mass. This is referred to as treadmilling (Risinger et al., 2009). This process involves flow of subunits from the shortening minus end to the growing plus end. MT-associated proteins (MAPs) can advance or hamper these dynamic behaviors. Treadmilling or dynamic instability can be occurring at the same time or individually in a population of MTs. Populations of MTs are generally arranged in "an intracellular lattice-like structure", except when a cell is entering in mitosis when MTs rearrange to form the mitotic spindle and asters. The mitotic spindle is much more dynamic, polymerizing and depolymerizing at greater rates in order to rapidly attach and separate the chromosomes (Risinger et al., 2009). Therefore, compounds that disrupt normal dynamics of the MTs prevent these mitotic processes and cause inhibition of mitosis and consequent cellular death by apoptosis (Jordan \& Wilson, 2004).

\subsubsection{Microtubules in mitosis and chemotherapy}

MTs are involved in a number of important cellular processes, including for example cell division, maintenance of cell shape, intracellular transport and cell signalling. Tumour cell division, invasive metastasis, and angiogenesis all involve MTs and are important targets in chemotherapy. Metastasis and angiogenesis will be discussed in Chapter 31.

The dynamicity of MTs is an excellent drug target for chemotherapy and one of the most successful approaches in cancer treatment is to inhibit MT function. There are two classes of drugs that inhibit MT function, MT-destabilizing agents (MDAs) and MTstabilizing agents (MSAs). Each group of compounds is extremely structurally diverse, and all act as potent antimitotics. At high concentrations, these two classes have opposing effects on tubulin (depolymerisation versus polymerization). At low concentrations, however, these drugs share the same effect of inhibiting the dynamic instability of MTs that leads to mitotic arrest but causes no major change in the total polymer mass (Jordan et al., 1993). MT-targeting agents (MTAs) generally consist of natural products. Over several hundred compounds have been reported to arrest mitosis via interaction with MTs, and both MDAs and MSAs have proven successful in the clinic in treating a number of different cancers (Sui \& Downing, 2010). 


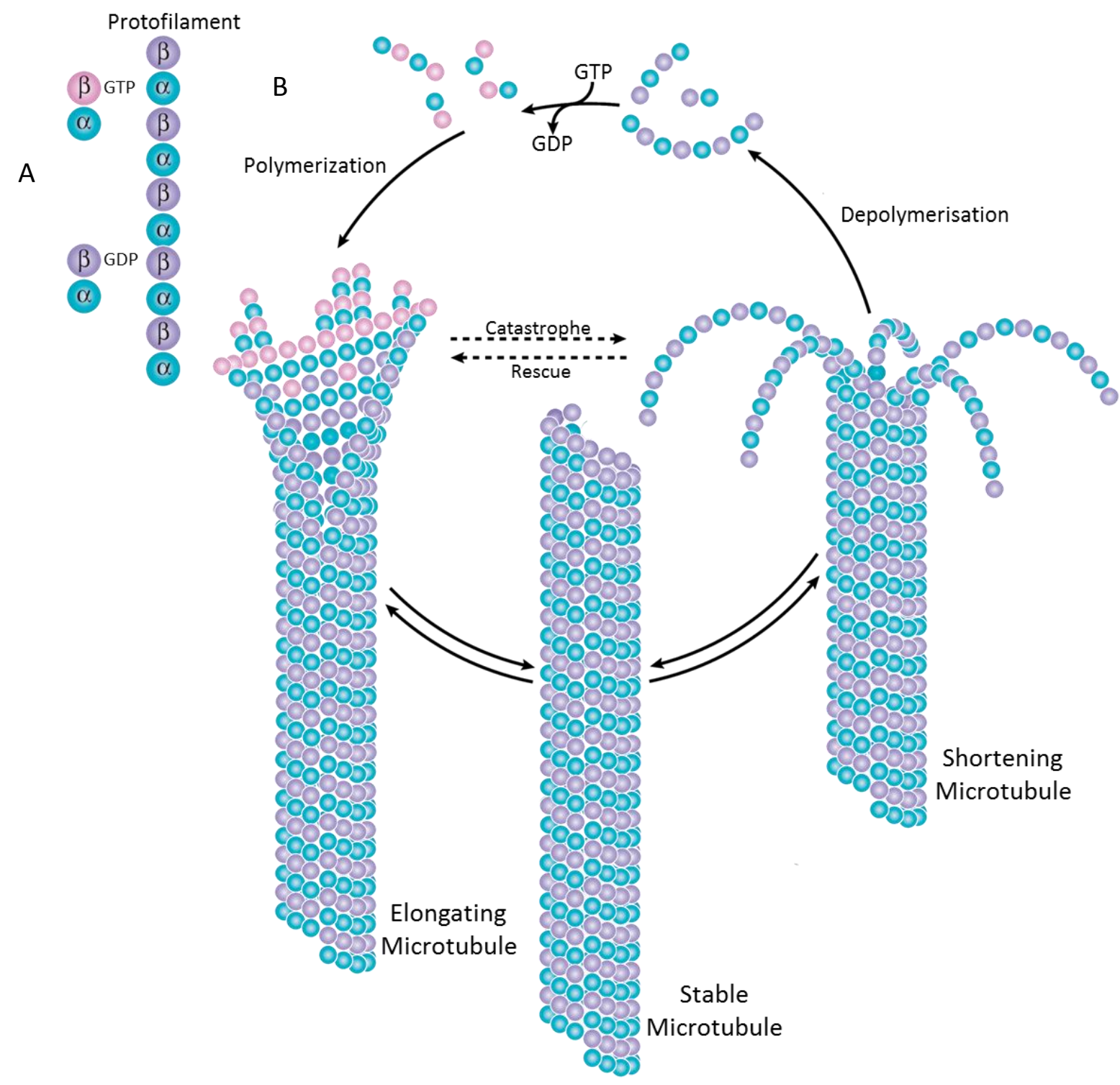

Figure 1.1 Structure of microtubules and their dynamic instability

A. Tubulin $\alpha$ - and $\beta$-subunits form heterodimers, which then arrange in a head to tail fashion to form a protofilament. A typical MT is made up of 13 protofilaments arranged in a parallel manner. B. The binding, hydrolysis and exchange of the guanine nucleotide on the $\beta$-subunit drives MT-assembly (polymerization) and disassembly (depolymerization). Polymerization of MTs occurs from a pool of heterodimers that have GTP bound to the $\beta$-subunit (pink), and this guanine nucleotide is hydrolyzed soon after assembly (purple). Thus, the MT is made up of GDP-bound $\beta$-subunits (purple) with a 'cap' of GTP-bound $\beta$-subunits (pink) that stabilizes the straight conformation of tubulin (due to the nucleotide-dependent change that occurs upon hydrolysis). This open-ended structure can close to form a "metastable, blunt-ended" MT that is considered an intermediate between a growing and a shortening MT. To switch between phases of rescue and catastrophe, GTP hydrolysis is essential.

Figure adapted from Akhmanova \& Steinmetz (2008) with permission from the author. 


\subsection{MICROTUBULE-DESTABILIZING AGENTS}

MDAs bind to and destabilize tubulin, inhibiting MT assembly. Cells treated with an MDA show abnormal spindles or complete loss of MTs at high concentrations (Jordan \& Wilson, 2004). These compounds bind to tubulin dimers and prevent their assembly into MTs and/or bind to the MT and induce depolymerization, both mechanisms resulting in loss of MTs. MDAs can bind to the Vinca domain or the colchicine (COL) binding site, or they can covalently modify tubulin at specific residues.

\subsubsection{Vinca domain agents}

The Vinca alkaloids are dimeric asymmetrical compounds with two multi-ringed motifs linked by a carbon-carbon linkage. The Vinca alkaloids have had an important role in cancer chemotherapy for over 50 years. They were originally isolated from the leaves of the periwinkle plant Catharanthus rosea, which have been used medicinally since the 1600s; however, their anti-proliferative activity was not discovered until the late 1950s (Noble et al., 1958; Johnson et al., 1959). Vinca drugs bind to the Vinca domain on a tubulin tetramer (Gigant et al., 2005). These drugs include vinblastine (Velban ${ }^{\circledR}$ ) (VBL), vincristine $\left(\right.$ Oncovin $\left.^{\circledR}\right)$, vindesine $\left(\right.$ Eldisine $\left.^{\circledR}\right)$, vinflunine $\left(\right.$ Javlor $\left.^{\circledR}\right)$ and vinorelbine $\left(\right.$ Navelbine $\left.^{\circledR}\right)$. VBL and vincristine were approved for clinical use in 1961 and 1963, respectively (Yue et al., 2010). All the Vinca alkaloids listed have now been approved for clinical use in various cancer treatment applications, including acute lymphoblastic leukaemia, lymphomas, breast cancer, bladder cancer and various other solid tumors (Dumontet \& Jordan, 2010). Vinca alkaloids are also able to synergize with a number of other chemotherapeutic agents in combination therapy; for example, VBL is able to synergize with the MSA paclitaxel (PTX) (Giannakakou et al., 1998). Although these drugs have been and still are hugely successful in the clinic they have associated neurotoxicity, often causing myelosupression (decreases in bone marrow haematopoiesis), and they are multiple drug resistant (MDR) efflux pump substrates, being pumped out of the cell before they can reach their threshold concentration to have a biological effect. Therefore, there is on-going investigation into improving this important class of drugs. Other compounds which bind the site include the cryptophycins, the dolastatins, the halichondrins, hemiasterlin and its analogs and a number of others (Dumontet \& Jordan, 2010) (Figure 1.2). There are three dolastatin analogs in clinical trials, one of which, romidepsin $\left(\right.$ Istodaxi $\left.^{\circledR}\right)$, is currently used in the treatment of 
cutaneous T-cell lymphoma. Cryptophycin-52 was withdrawn from clinical trials due to toxicity issues (Dumontet \& Jordan, 2010). Eribulin (Halaven $^{\mathrm{TM}}$ ), a macrocyclic ketone analog of halichondrin B, was FDA approved in 2010 for the treatment of breast cancer (www.fda.gov/). Eribulin is a "global inhibitor' of tubulin polymerization, as it is able to bind at MT plus ends to disrupt polymer growth, inhibit the formation of the tubulinstathmin complex and inhibit VBL-induced tubulin spirals (Alday \& Correia, 2009). Vinca agents bind between two heterodimers; thus, one heterodimer on its own does not have a binding site. Completion of the site occurs when two heterodimers interact. Interestingly Eribulin, although classed as a vinca domain compound, is able to bind on the subunit at a Vinca 'half site' (John Correia personal communication). The Vinca binding domain will be discussed in more detail in Chapter 6.1. 


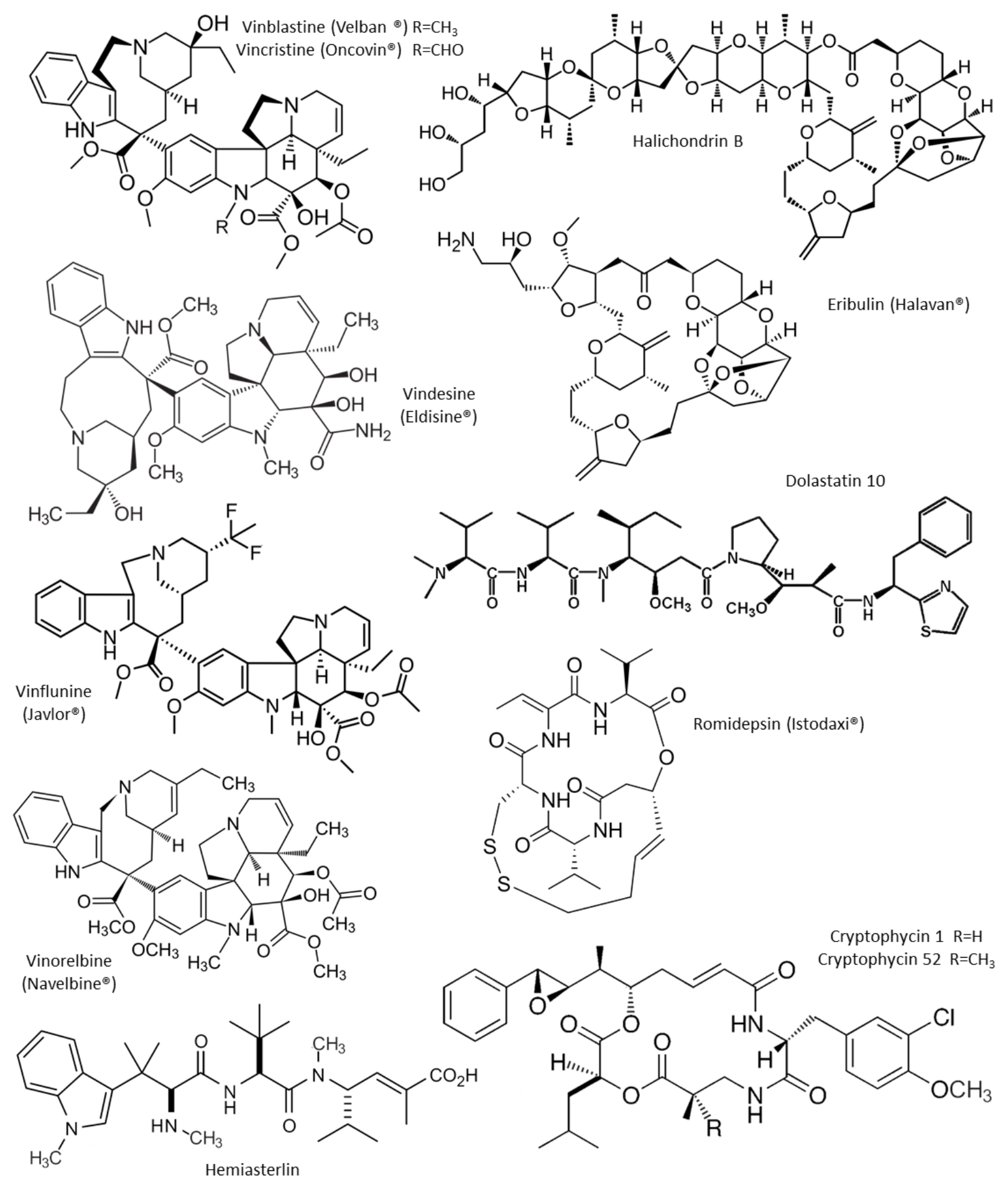

Figure 1.2 Structure of ligands that bind to the Vinca binding domain

Compounds that bind the Vinca domain are identified by their ability to displace VBL from its binding site. The Vinca alkaloids (VBL, vincristine, vindesine, vinflunine and vinorelbine) are all used in the clinic in treating various cancer types. Eribulin and romidepsin are also used clinically. 


\subsubsection{Colchicine-like compounds}

COL (Fig 1.3), a potent MDA, is a plant natural product isolated from the leaves of meadow saffron and has been used to treat gout since the 1700s (Massarotti et al., 2012). The biological target of COL was not known until 1968 when it was used in a specific assay to detect the presence of MTs, COL was therefore involved in the discovery of tubulin itself (Weisenberg et al., 1968). Binding to the COL site prevents curved tubulin from adopting a straight structure, resulting in inhibition of assembly into MTs (Ravelli et al., 2004). A more detailed discussion of the COL binding site is presented in Chapter 6.1. COL binds tightly to tubulin in a two-step process. The first step is slow, occurs with low affinity and forms a reversible complex. This is followed by a slow conformational change that results in high affinity binding and produces a "pseudo-irreversible' complex (Skoufias \& Wilson, 1992; Engelborghs, 1998). In addition to inhibition of tubulin polymerization, COL is also able to increase GTP hydrolysis of tubulin (David-Pfeuty et al., 1979).

COL has been under investigation as a vascular-targeting agent in treating cancer (related to tumor angiogenesis and the involvement of MTs in this process (see Chapter 3.1); however, it has failed in clinical trials due to its high toxicity (Jordan \& Wilson, 2004). Although a number of COL-like compounds have been reported, for example, those shown in Figure 1.3, there is no such agent yet used in cancer chemotherapy (Massarotti et al., 2012). Combretastatin A-4, isolated from the bark of the Combretum caffrum tree, is a vascular disrupting agent that targets existing tumor neovasculature and binds to the COL site (Sackett, 1993; Dumontet \& Jordan, 2010). The phosphorylated prodrug, fosbretabulin (Zybrestat), which binds to the COL site reversibly, is currently in phase II/III trials on its own or in combination with other anticancer agents or radiotherapy for the treatment of various solid tumors (Mooney et al., 2009), including anaplastic thyroid cancer. In addition, a number of other COL site agents are in clinical trials (Dumontet \& Jordan 2010). Ombrabulin, for example, a combretastatin derivative, is in phase III for soft tissue sarcoma as a vascular disrupting agent, and indibulin $\left(\right.$ Zybulin $^{\mathrm{TM}}$ ) is in phase I/II trails for metastatic breast cancer (Dumontet \& Jordan 2010). Interestingly, indibulin is able to distinguish between mature neuronal (highly posttranslationally modified tubulin) and immature or non-neuronal, (less-modified) tubulin and only binds the latter (Wienecke \& Bacher, 2009); hence, indibulin has no neurotoxicity effects in preclinical adult animal studies (Bacher et al., 2001). 
Podophyllotoxin (PDP), a lignan, was first isolated in the 1800s and competes with COL for binding to tubulin, binding more rapidly than COL and in a reversible manner (Sackett, 1993). Despite differences in binding mechanisms, COL and PDP share the same binding site and do not just show minor overlap in their binding (Ravelli et al., 2004). In contrast to COL, PDP does not affect the association-dissociation equilibrium of tubulin dimers (Detrich et al., 1982) nor does it enhance GTP hydrolysis but actually inhibits it similar to VBL (David-Pfeuty et al., 1979). PDP is currently under investigation as an anticancer agent, but it has been used in the clinic since 1942 as a topical treatment for genital warts (www.fda.gov/; Sackett, 1993). A number of PDP derivatives, such as etoposide, do not target tubulin and are used clinically to treat various types of cancer. These compounds target topoisomerases, inhibiting DNA replication by promoting topoisomerase dependent DNA strand breaks (www.fda.gov/; Sackett, 1993). Although PDP does not target DNA, some of its derivatives can target both tubulin and DNA depending on what concentration they are used at (Sackett, 1993). 

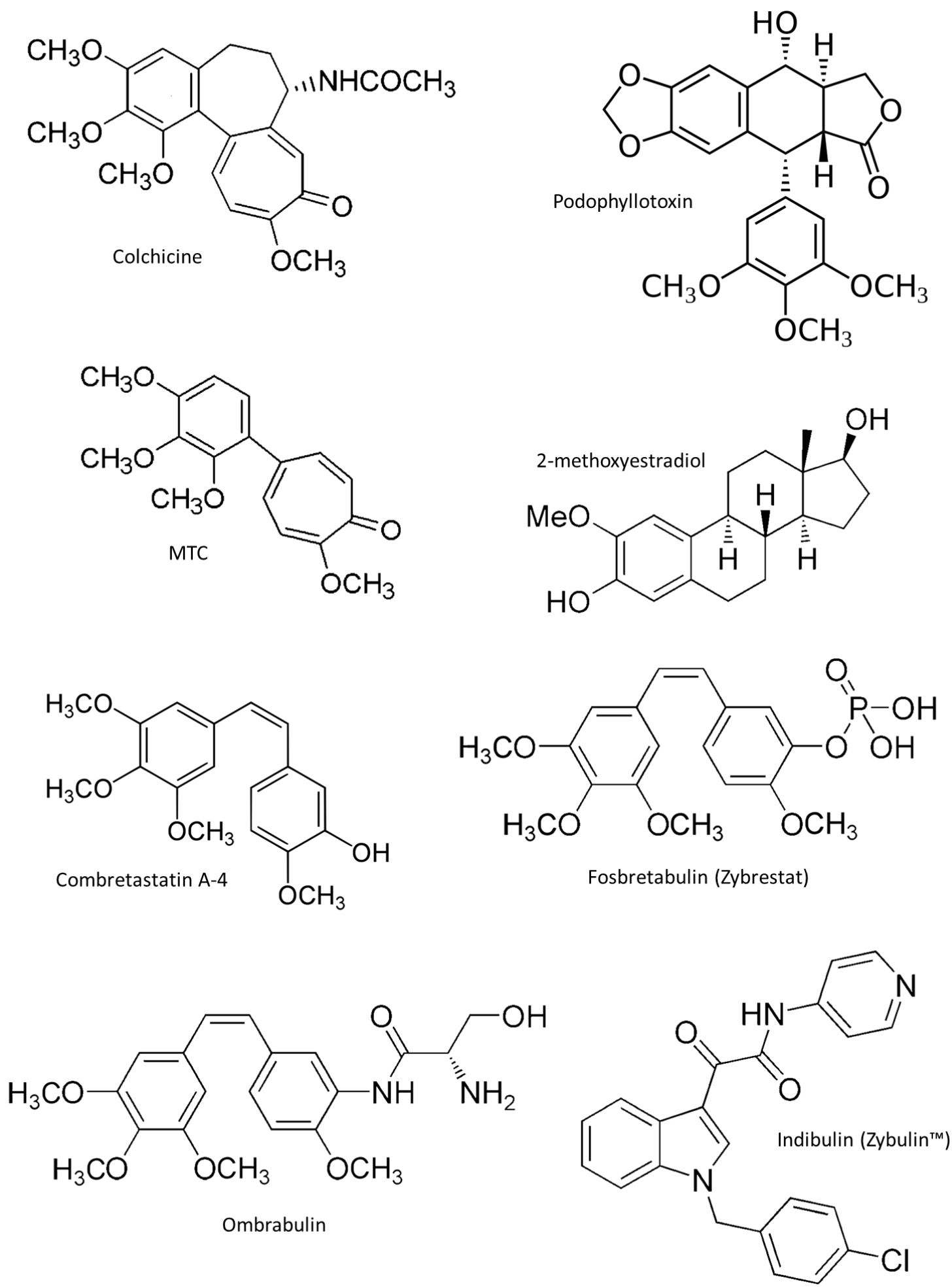

Figure 1.3 Colchicine site compounds

Structure of compounds that bind the colchicine site. 


\subsubsection{Covalent microtubule-destabilizers}

The majority of MTAs bind to tubulin in a reversible manner. However there is a diverse set of MDAs that are able to alkylate tubulin at nucleophilic residues via formation of a permanent bond. Covalent modification by MDAs generally occurs at the sulfhydryl moiety of cysteine residues, given the residues ability to act as a nucleophile, and this generally occurs in the $\beta$-tubulin subunit (Sackett, 2008). Cys239 is the most reactive cysteine residue in tubulin, and a number of compounds can alkylate tubulin at this site. Covalent attachment at this residue results in inhibition of the ability of tubulin to polymerize. Cys 239 is located near the $\alpha, \beta$-intradimer interface and is not exposed to the surface of the protein. Given its location, modification of this residue inhibits COL binding (Hamel, 2008; Sackett, 2008). Covalent MTAs act as electrophiles, and therefore it is likely that they will also react with other proteins and not just tubulin. Some MTAs, however, are tubulin specific. For example, T138067 (Fig 1.4), an arylpentafluorosulfonamide, reacts exclusively with tubulin at residue 239 (Shan et al., 1999) and is unable to interact with the $\beta I I I$ isotype of tubulin, in which residue 239 is a serine. T138067 has structural similarity to COL site binding agents and inhibits the binding of COL, and COL is also able to inhibit attachment of T138067. This compound underwent phase II clinical trials for refractory colorectal carcinoma; however, there was no evidence for clinical activity of the compound in this setting (Berlin et al., 2008). 2,4Dichlorobenzyl thiocyanate (DCBT) (Figure 1.4) reacts specifically with Cys239, although there is evidence it may alkylate other proteins, including $\alpha$-tubulin. Interestingly, pre-incubation of tubulin with COL inhibits binding; whereas, DCBT cannot inhibit COL binding, indicating that the cysteine residue is not essential in COL binding (Bai et al., 1989). The natural product ottelione A (Fig 1.4), an analog of COL site drugs, selectively alkylates an unidentified cysteine residue and is currently the most potent drug in this class with an $\mathrm{IC}_{50}$ value of $20 \mathrm{pM}$ (Hamel, 2008). Other residues such as lysine can also be targeted by covalent MDAs. Covalent MTAs have advantages and disadvantages over 'normal' reversible agents, and this will be addressed in Chapter 5.1. 
<smiles>COc1ccc(NS(=O)(=O)c2c(F)c(F)c(F)c(F)c2F)cc1F</smiles><smiles>C=C[C@H]1C[C@H](Cc2ccc(OC)c(O)c2)[C@@H]2C(=O)C=CC(=C)[C@@]21C</smiles>

Figure 1.4 Covalent binding MDAs

Structures of compounds that bind to the $\beta$ tubulin subunit covalently. These compounds all modify the tubulin structure by electrophilic attack at cysteine residues. 


\subsection{MiCROTUBULE-STABILIZING AGENTS}

\subsubsection{Introduction}

MSAs block disassembly of the GDP-bound form of tubulin resulting in impaired dynamics. These compounds also form stable tubulin polymers even when GDP is bound in the E-site; whereas, normally under these conditions tubulin does not assemble into MTs. MSAs are thought to take advantage of sites in which natural MT associated proteins (MAPs) bind to and regulate assembly. However, the drug-like MSAs bind more tightly, inhibiting the natural activity of tubulin and promoting near irreversible assembly (Amos, 2011). Interestingly, the majority of the known potent MSAs are natural products or synthetic derivatives of natural products. These compounds have most likely evolved and been produced as broad-spectrum toxins to target eukaryotic tubulin in predators and/or prey (Altmann \& Gertsch, 2007). In addition to cancer, some MSAs have also shown promise as potential therapeutics in the treatment of multiple sclerosis (Crume et al., 2009) and other neurodegenerative diseases (Lee et al., 1994; Brunden et al., 2009). Currently there are four MSAs used in the clinic, in various formulations, to treat a number of different cancer types. In addition, there are many identified MSAs and numerous analogs at various stages of preclinical and clinical development.

\subsubsection{Compounds that stabilize microtubules}

MSAs are considered a structurally diverse class of compounds (Figure 1.5). Some structures do however the share a common largely polyoxygenated macrolide ring with a substituted hydrophobic side chain. MSAs may mimic endogenous, less potent cellular molecules in their target cells (Amos \& Löwe, 1999); therefore, it is not surprising there is such a large variety of compounds that can bind to MTs. MSAs bind tightly to the assembled form of tubulin; however, they generally do not bind the unassembled form with measurable affinity (Díaz et al., 1993). The exact mechanism of mitotic arrest and consequent apoptosis induced by MSAs are still not fully understood. It is, however, widely accepted that their antimitotic action occurs through interference with the dynamics of spindle MTs within the cell. Affected cells fail to pass mitotic checkpoints and are arrested at the $G_{2} / M$ phase of the cell cycle. $A G_{2} / M$ block is the hallmark of MTAs, and cells blocked in $\mathrm{G}_{2} / \mathrm{M}$ consequently undergo cell death (Zhao et al., 2009). 


\section{The taxanes}

The first agent to be identified as an MSA by Susan Horwitz in 1979 (Schiff et al., 1979) was paclitaxel $\left(\right.$ Taxol $\left.^{\circledR}\right)($ PTX), a plant compound isolated from Yew tree bark (Wani et $a l .$, 1971). PTX is a complex polyoxygenated diterpene with a taxane ring structure fused with a four-membered oxetane ring. PTX received FDA approval in 1992 and has been clinically available since 1993, along with one of its semi-synthetic analogs docetaxel (taxotere $^{\circledR}$ ) (DTX), which has been clinically available since 1996 (Altmann \& Gertsch, 2007). These drugs are used to treat solid tumor malignancies such as breast cancer, ovarian cancer, non-small cell lung cancer, gastroesophageal cancer, germ cell tumors, and cancers of the head and neck. The taxanes can be used in the neoadjuvant, adjuvant and metastatic settings or in synergistic combinations with other drugs (Morris \& Fornier, 2008). More recently, cabazitaxel (Jevtana ${ }^{\circledR}$ ), another semi-synthetic PTX analogue, has been FDA approved. Cabazitaxel has poor affinity for the P-glycoprotein (P-gp) drug efflux pump that is involved in MDR and is used in the clinic along with other anticancer drugs to treat hormone-refractory metastatic prostate cancer (Galsky et $a l ., 2010)$. A number of other taxane analogs and taxanes in different formulations are currently in clinical trials (Dumontet and Jordan, 2010); one example being Abraxane ${ }^{\circledR}$ which is PTX bound to human albumin protein particles (www.fda.gov/).

\section{The epothilones}

The epothilones (EPOs) are macrolide antibiotics, first discovered as antifungal agents in 1986. EPOA and EPOB were isolated from myxobacterium Sorangium cellulosum. They are 16-membered polyketide macrolactones with an aromatic methylthiazole group connected via an unsaturated side chain. Like the taxanes, they bind to and stabilize MTs (Bollag et al., 1995). Preclinical and early clinical studies of the EPOs were hindered by difficulties in pharmacokinetics and metabolic instability. But if the taxanes were anything to go by, these drugs still had a promising future in treating cancer, and thus a number of synthetic and semisynthetic analogs were made (Morris \& Fornier, 2008). One synthetic derivative, the lactam analogue of EPOB (aza-EPOB (ixabepilone, Ixempra ${ }^{\circledR}$ ) (IXA) was given FDA approval in 2007 and is currently used in the clinic to treat metastatic breast cancer (Lechleider et al., 2008), and is in a number of clinical trials for treatment of solid tumors (Dumontet \& Jordan 2010). The EPOs are an important class of compounds as they are active in some taxane-resistant tumors, have better water 
solubility and are not sensitive to $\beta$-tubulin content as are the taxanes (Chou et al., 2008; Dumontet \& Jordan 2010). Some are able to penetrate the blood brain barrier (BBB), making them candidates for treatment of brain metastases (Hoffmann et al., 2009).

There are several EPOs being clinically evaluated in a number of solid tumors. For example, natural EPOB (patupilone) is able to penetrate the BBB and has effects in the central nervous system in animal models. It is currently in clinical trials for a number of different solid tumors, including brain metastases in breast and ovarian cancer, melanoma and other solid tumors (Dumontet \& Jordan, 2010) . It has shown promising effects in a phase II study in refractory brain metastases in non-small cell lung cancer (Abrey et al., 2007). Compared to PTX, patupilone is up to 20 times more potent and is effective in taxane-resistant cells. Patupilone's main side effect is diarrhea which limits its dose, but this is a relatively minor side effect and gives it potential for use in targeting tumors in which the patient has developed cumulative neurotoxicity from taxane use (Morris \& Fornier, 2008). Sagopilone is a fully synthetic analog that also penetrates the $\mathrm{BBB}$ and is in trials for glioblastoma, prostate and lung cancers (Dumontet \& Jordan, 2010). EPO compounds are extremely similar to one another in chemical structure; however, they have very different side effects. These differences are most likely related to their pharmacodynamics and pharmacokinetics (Goodin et al., 2004).

\section{Discodermolide and dictyostatin}

DSC, a polyhydroxylated alkatetraene lactone, was originally isolated from the marine sponge Discodermia dissoluta (ter Haar et al., 1996). It has been shown to compete for binding with PTX (Hung et al., 1996); however, these two compounds are also known to synergize with one another (Martello et al., 2000), which led to the finding that instead of sharing a binding site they establish distinct poses within the same binding site region (Khrapunovich-Baine et al., 2009). A large-scale synthetic route was developed for DSC, and it entered into a phase I clinical trial; however, it was discontinued due to its pulmonary toxicity (Dumontet \& Jordan, 2010). Regardless, there has been continuous work done on synthesizing analogues of DSC, and it is possible that a synthetic derivative may become a clinical contender in the future. Dictyostatin, a polyketidederived macrolide, is a close structural congener of DSC isolated from the same Spongia species (Isbrucker et al., 2003). Like DSC, dictyostatin is active in PTX-resistant cell lines and competes for the taxoid binding site (Madiraju et al., 2005). It is thought that 
the structure of dictyostatin represents the template for the bioactive conformation of DSC, which in general is a linear compound (Figure 1.5).

\section{Eleutherobin and sarcodictyins}

Eleutherobin is a tricyclic diterpene, isolated from marine soft coral (Long et al., 1998). It has also had its total synthesis described a number of times, and a number of analogs have been described. Eleutherobin has had little clinical interest since it is highly susceptible to the P-gp drug efflux pump (Altmann \& Gertsch, 2007). The sarcodictyins from the Mediterranean stoloniferan coral Sarcodictyon roseum (Ciomei et al., 1997) have a core structure identical to that of eleutherobin (oxa-bridged tricyclic skeleton). Although significantly less potent compared to the taxanes, the sarcodictyins, unlike eleutherobin, are not susceptible to the P-gp drug efflux pump (Ciomei et al., 1997) and have been found to be active in two taxane-resistant cell lines (Hamel et al., 1999).

\section{The ceratamines}

The ceratamines, isolated from the marine sponge Pseudoceratina sp., are heterocyclic alkaloids, the first of their type shown to stabilize MTs, and introducing a novel pharmacophore into this area of research. Their simple structure has no chiral centers, making them an attractive compound from a synthetic perspective (Coleman et al., 2009). These compounds appear to be distinctly different from known MSAs as they do not compete with PTX for binding to the MT and have unusual cellular effects, such as inducing the formation of pillar-like tubulin structures. They are not as strong as PTX in causing tubulin polymerization, and therefore their observed inability to displacing PTX may be the result of concentrations not reaching the threshold to compete with PTX (Karjala et al., 2005).

\section{The taccalonolides}

The taccalonolides, highly acetylated steroids, isolated from the roots of the Taccachantrieri tropical plant, have been shown to have an MSA-like phenotype in cells (Tinley et al., 2003). However, they are unable to modulate tubulin assembly in vitro or bind to stabilized MTs with measureable affinity, indicating they may have a different mechanism of action. The lack of ability of these compounds to displace taxoid-site ligands suggests they do not bind the taxoid binding site (Buey et al., 2005). 
Interestingly, a number of these compounds have been shown to be effective in cell lines that over express drug efflux pumps, and in cells that overexpress the $\beta$ III-tubulin isotype, indicating they have superior advantages over other MSAs (Risinger et al., 2008).

\section{The laulimalides}

The laulimalides are 20-membered macrolide lactones isolated from the marine sponge Cacospongia mycofijiensis. Laulimalide (LAU) contains a three-membered epoxide ring; whereas, its natural congener isolaulimalide contains a five-membered tetrahydrofuran ring, with both compounds stabilizing MTs (Mooberry et al., 1999). Neolaulimalide, a ring-enlarged natural isomer of LAU with an intact epoxide moiety has also been identified in a different sponge, Fasciospongia rimosa, collected in Japan (Tanaka et al., 1996). Neolaulimalide possesses high cytotoxicity in the same range as LAU and is a far more stable compound than LAU. LAU easily rearranges to form isolaulimilde (Qi \& Ma, 2011). All compounds and other analogs have been synthesized a number of times. Although potent at inducing MT assembly, the laulimalides are lipophilic which limits their solubility (Mooberry et al., 1999). LAU binds to a distinct site from PTX (Pryor et $a l ., 2002$ ) and is known to synergize with compounds with bind the taxoid site (Clark et $a l .$, 2006). In preclinical tests in mice, despite showing promising results in cell-based and pharmacokinetic studies, LAU was shown to be ineffective at preventing xenografted cancer growth and also presented with significant systemic toxicity and mortality (Liu et al., 2007).

\section{The pelorusides}

The pelorusides are polyoxygenated 16-membered macrolides isolated from the New Zealand marine sponge Mycale hentscheli using spectroscopy-guided isolation techniques. Peloruside A (PEL) was isolated in 2000 (West et al., 2000) and shown to stabilize microtubles in a PTX like manner (Hood et al., 2002). Peloruside B, a natural congener of PEL, was isolated in 2010 and shown to have activity similar to PEL (Singh et al., 2010). PEL shares a binding site with LAU (Gaitanos et al., 2004) and is a poor substrate of the P-gp. It is known to act synergistically with taxoid site compounds (Wilmes et al., 2007). PEL has shown good efficacy in preclinical trials in mice. Three xenograft studies were done and PEL compared to standard MSAs, PTX and DTX. 
Overall PEL caused sustained tumor regression, showing superior action compared to the taxanes and was better tolerated (Meyer et al., 2006). Given these results preclinical development of PEL was started but because a large-scale synthetic program has not been possible, no clinical trials have been entered into.

\section{Cyclostreptin}

The first MSA to be identified as a covalent modifier of tubulin was cyclostreptin (CYC). CYC has an interesting structure with an unusual ring system and a highly strained $\alpha-\beta$ unsaturated lactone that is susceptible to nucleophilic attack (Sato et al., 2000; Edler et al., 2005; Buey et al., 2007). Although an interesting compound, CYC is a weak MSA and would not be suitable for clinical development but makes a good probe for studying MSA interactions with MTs.

\section{Zampanolide and dactylolide}

ZMP is a 20-membered macrolide isolated from the Tongan marine sponge Cacospongia mycofijiensis in a similar manner to the isolation of the pelorusides. It was originally isolated by Tanaka and Higa (1996) from the marine sponge Fasciospongia rimosa (collected from Capa Zampa, Okinawa, Japan), but its mode of action at that time was unknown. It is a potent inducer of MT assembly and is not susceptible to overexpression of the P-gp drug efflux pump (Field et al., 2009). An enantiomer of ZMP, DAC was isolated from a different marine sponge but has less potent MSA activity (Cutignano et al., 2001). Neither ZMP nor DAC have progressed into preclinical trials as they are still in the very early stages of development. 


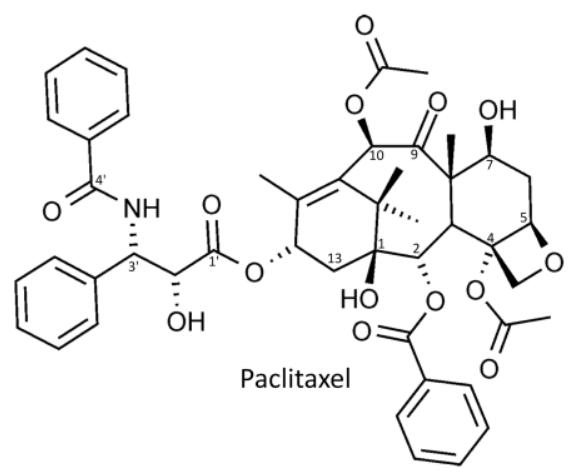

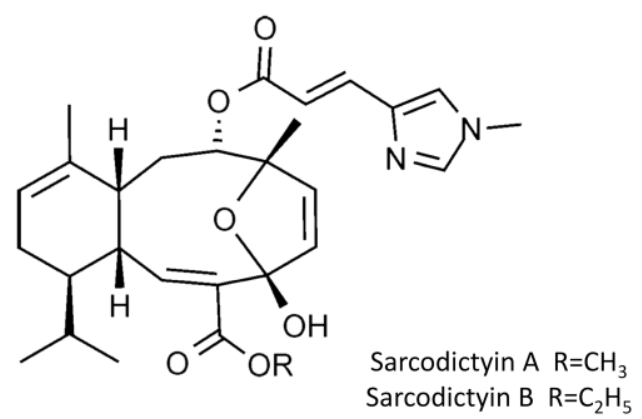<smiles>C=C/C=C\[C@H](C)[C@H](OC(N)=O)[C@@H](C)[C@H](O)[C@@H](C)C/C(C)=C\[C@@H](C)[C@H](O)[C@@H](C)/C=C\[C@H](O)C[C@H]1OC(=O)[C@H](C)[C@@H](O)[C@H]1C</smiles>

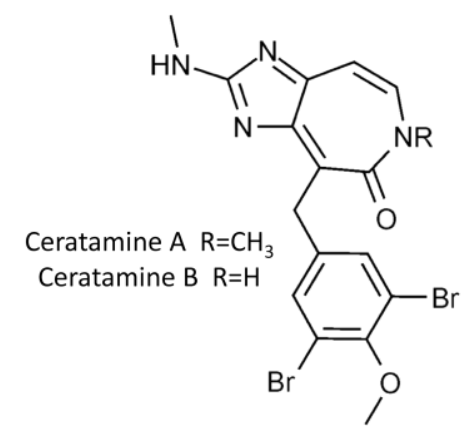

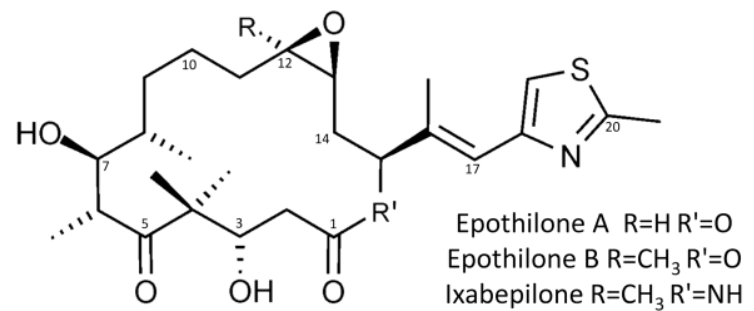

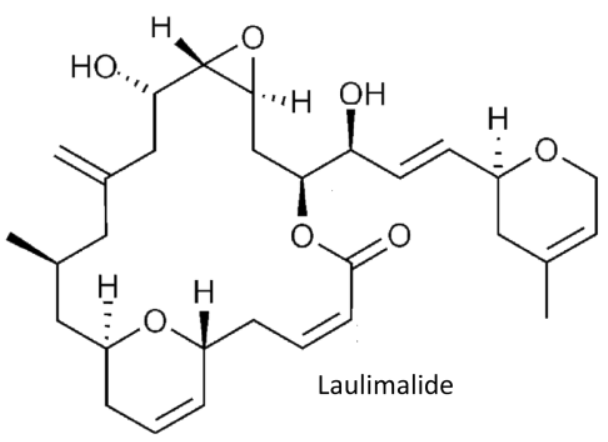

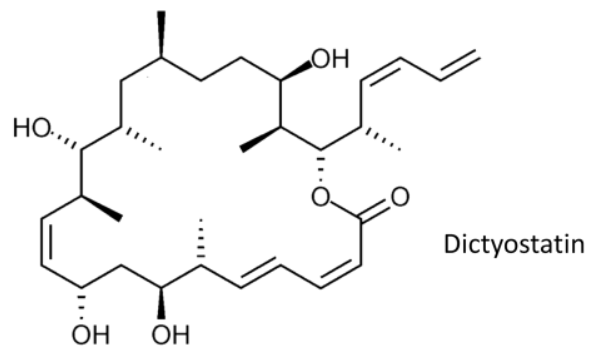

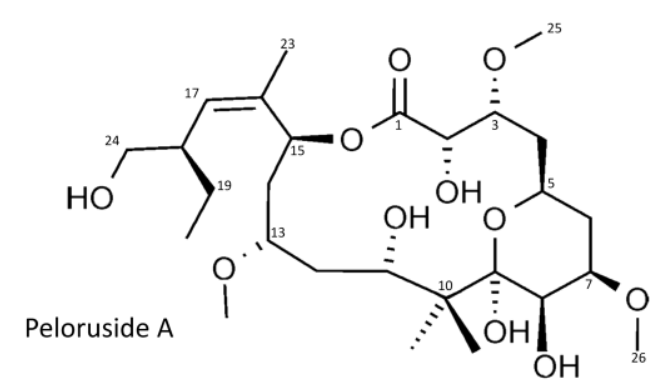

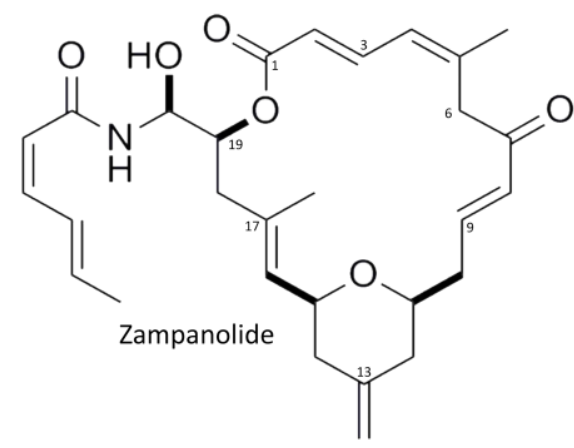

Figure 1.5 Structures of selected compounds that bind and stabilize the MT 


\subsubsection{Binding sites}

There are currently two well-known binding sites for MSAs on the MT - the well characterized taxoid site and the LAU/PEL site. Thus, MSAs can be divided into two groups depending on the site on the MT where they bind. The larger group includes PTX and its biomimetics. These compounds bind to a site in the lumen of $\beta$-tubulin in the MT, termed the taxoid binding site (Nogales et al., 1998; 1999). There is also evidence that some of these compounds target a less characterized external site that is located at pore type I of the MT (Díaz et al., 2003). The pore type I site is now considered a third binding site on the MT for MSAs, since binding to this site on its own can cause MT stabilization (Barasoain et al., 2010). Currently, all drugs that bind the taxoid site bind with 1:1 stoichiometry (one MSA molecule per heterodimer) (Díaz \& Andreu, 1993; Nogales et al., 1995); thus, binding at the luminal site and the pore type I site must be mutually exclusive. The second group of binding agents consists of LAU and PEL, which target a not yet fully characterized binding site. This site is known, however, to be biochemically distinct from the taxoid site (Pryor et al., 2002; Gaitanos et al., 2004). Experimental data supports the location of this site on both the $\alpha$ - and $\beta$-tubulin subunits, but most recent studies support a $\beta$-tubulin site. See Figure 1.6 for a schematic representation of these sites. These binding sites will be discussed in Chapter 4.1.

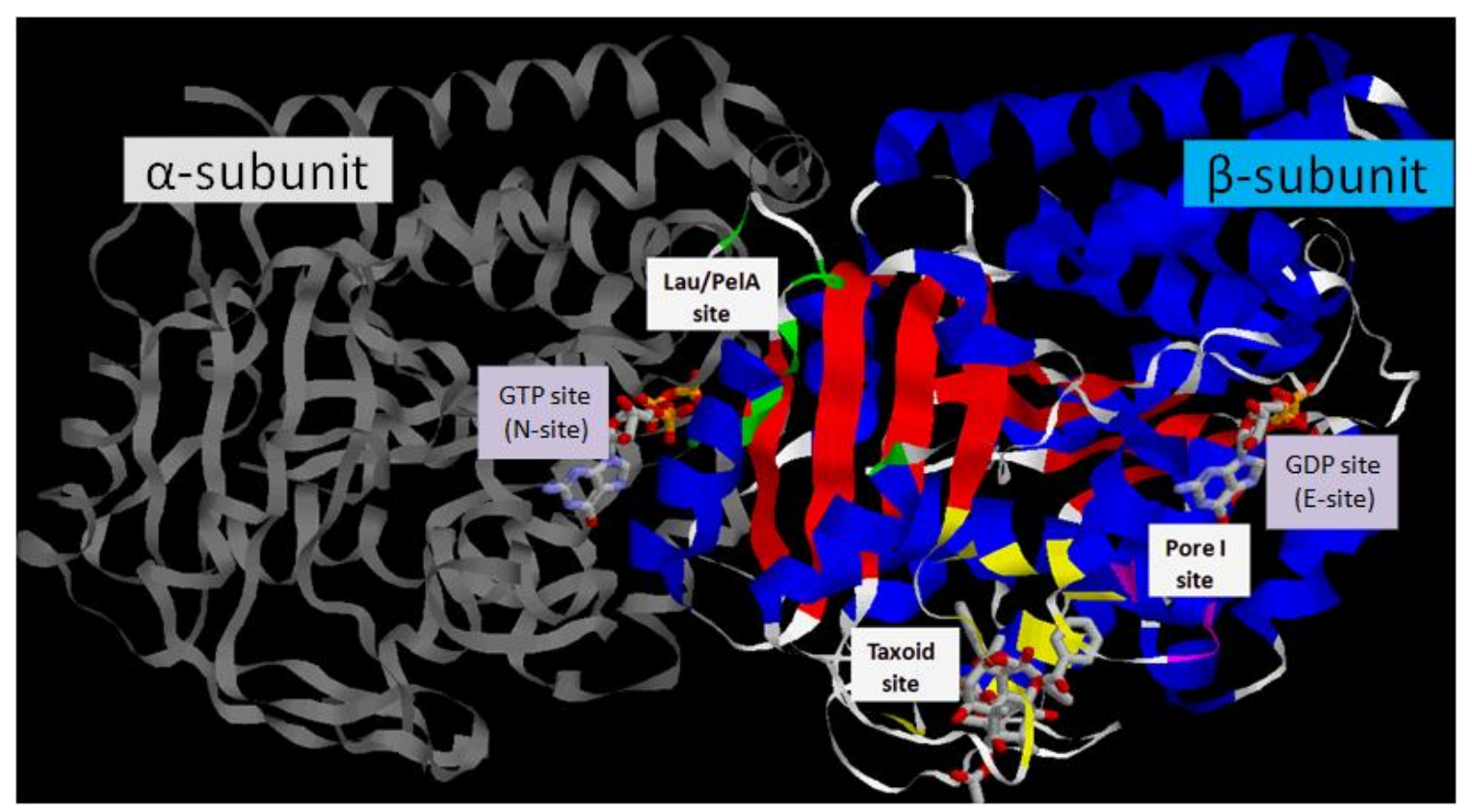

Figure 1.6 Microtubule-stabilizing agent binding sites

Tubulin heterodimer ( $\alpha$-subunit (grey), $\beta$-subunit (blue)) showing the luminal taxoid site (yellow) with bound PTX, the proposed $\beta$-tubulin LAU/PEL binding site (green) and the pore type I site (magenta). The nucleotide binding sites are shown with bound nucleotide in standard CPK coloring. 


\subsubsection{Clinical limitations of the taxanes}

Given the success of the taxanes and other MTAs in the clinic, MTs present an excellent target for anti-cancer drugs (Jordan \& Wilson 2004). Cancer cells are highly sensitive to these drugs compared to normal cells as they more frequently pass through stages of vulnerability to mitotic poisons (Jackson et al., 2007). Many tumors respond favorably to the taxanes in the first stages of treatment; however, their effectiveness can often become limited due to a number of different factors (Morris \& Fornier 2008; Dumontet \& Jordan 2010). Resistance can occur in the cancer cell due to genetic and epigenetic alterations affecting drug sensitivity. In addition, impairment in the delivery of the drug to tumor cells due to poor oral absorption or increased drug metabolism can contribute to resistance. Mechanisms of resistance include acquired expression of MDR proteins, upregulation of alternative $\beta$-tubulin isotypes, and mutations in $\beta \mathrm{I}$-tubulin (Dumontet $\&$ Jordan 2010). Other clinical implications include hypersensitivity reactions, doselimiting anti-hematopoietic activity and cumulative neurotoxicity (Morris \& Fornier, 2008). Therefore, there is a need for new agents that target MTs, avoid these mechanisms of resistance and are more soluble, for not only targeting taxane resistant tumors but to be used as frontline drugs for solid tumors. Further development of already recognized compounds could prove beneficial as well (Singh et al., 2008). The specific changes that make cells resistant or sensitive to MTAs are not well understood, and this is an active area of study. Understanding the mechanisms that cause resistance may help development of more effective drugs.

Acquired and intrinsic resistance is a huge problem for tumor cell treatment with the taxanes (Dumontet \& Jordan 2010). The most significant limitation to effective targeting of the MTs is development of resistance by the tissue. The most clinically significant mechanism is overexpression of the MDR efflux pumps. Overexpression of the ATP-binding cassette membrane transporter proteins, specifically MDR1 (P-gp) and MRP-1 in particular, confer MDR (Gottesman et al., 2002). Up-regulation of these pumps causes cancer cells to become simultaneously resistant to a range of different drugs, as these pumps have broad substrate specificity. The taxanes and the Vinca alkaloids are good substrates of the MDR pumps, including the P-gp pump (MDR-1), MRP-1, MRP-7 and BSEP (Gottesman et al., 2002). Hyper-expression of the P-gp drug efflux pump is known to correlate well with resistance found in human tumors (Tan el al., 2000), and expression of drug efflux pumps correlates well with a poor response to 
therapy and an unfavorable survival rate (Gottesman et al., 2002). These pumps are expressed in different human cancers and decrease the intracellular concentration of drugs. While the cells remain sensitive to the taxanes, the concentration of drug required to have the desired biological effect is greatly increased. As drug concentration increases to keep pace with MDR, so does the toxicity profile of the drug, not only because of the increased concentrations of potentially toxic vehicle but also due to the effect of high concentrations of anti-proliferative agents on normal, noncancerous tissues. Both of these processes lead to serious side effects. Inhibitors of MDR efflux pumps have been used in clinical trials with anticancer drugs and have been shown to be beneficial; however, these inhibitors affect the pharmacokinetics of the anticancer drugs. Thus, providing cancer drugs that are not substrates for these pumps, or are not susceptible to these pumps, would avoid this mechanism of resistance (Gottesman et al., 2002).

Overexpression of certain tubulin isotypes, which have lower sensitivity to the taxane drugs, such as $\beta$ III-tubulin, interfere with drug binding and cause resistance. $\beta$ tubulin is encoded for by a large multigene family, and there are 6 isotypes of $\beta$-tubulin in humans. The tubulin structure has remained highly conserved through evolution. Although these isotypes share significant amino acid homology, they differ in their Cterminal ends by up to 40 or more amino acids (Luduena, 1998). Additionally, different isotypes undergo different posttranslational modifications that alter their interaction with MAPs (Hammond et al., 2008). Tubulin isotypes I and IVb are expressed in all tissues; whereas, III, IVa and II are expressed mainly in brain tissue. Class I tubulin is the major isotype in mammalian cells and accounts for $70 \%$ of total $\beta$-tubulin in all tissues (Mozzetti et al., 2005). Overexpression of class III $\beta$-tubulin has been shown to inhibit PTX-induced assembly of $\beta$-tubulin by a number of different groups ( $\mathrm{Lu} \&$ Luduena, 1993). This $\beta$ III isotype is not normally found in cells, although it is found to be unregulated in taxane-resistant cells (Kavallaris et al., 1999) and in patients resistant to taxane treatment (Mozzetti et al., 2005).

Point mutations in the taxoid binding site in $\beta \mathrm{I}$-tubulin result in decreased drug affinity and decreased binding since the interaction between the drug and its target has been altered. A number of different $\beta$-tubulin mutations that cause resistance to MTAs have been shown in vitro. Such mutations were first described by Giannakakou et al., $(1997 ; 2000)$ and were later found to correlate with resistance to the drugs in non-small cell lung cancer patients (Monzo et al., 1999). This study linked mutations in tubulin 
genes to taxane resistance, but its conclusion was later discovered to be incorrect due to the sequencing of tubulin pseudogenes rather than the actual genes themselves (Sale et al., 2002; Dumontet \& Jordan, 2010). Since then, however, there have been a number of studies in patients showing that there is little evidence to support spontaneous amino acid mutations playing a clinical role in resistance (Sale et al., 2002; Tsurutani et al., 2002; Urano et al., 2003). It was concluded that $\beta$-tubulin mutations in clinical samples are rare and are therefore not thought to contribute significantly to clinical resistance (Berrieman et al., 2004). However, a problem with some of these studies is that the tumors were isolated from patients who had not been treated with MTAs and thus provided no information on resistance toward these drugs in the clinic. It has now been shown that human tumor cells can acquire resistance toward the taxanes via spontaneous mutations in $\beta$ I-tubulin, since polymorphisms can cause resistance to PTX. This suggests that human polymorphisms in $\beta \mathrm{I}$-tubulin may lead to the need for higher drug concentrations to get the same desired effect as in patients without these mutations (Yin et al., 2010). Interestingly, the most common polymorphism in $\beta I$-tubulin appears to occur at residue L217 which is a critical residue in the taxane binding site (Yin et al., 2010).

MAPs and MT-interacting proteins are also thought to contribute to resistance, although their exact role in resistance is still uncertain. For example, increased expression of MAPs, such as MAP4, which stabilizes MTs, has been shown to be associated with resistance (Kavallaris, 2010). A number of studies on MAP involvement in resistance have been reported but have yielded inconsistent results; however, there are many proteins that have not yet been examined (Cabral, 2008). There is also evidence now that a large number of other mechanisms may contribute to taxoid resistance from cell culture studies, including for example, the overexpression of stathmin (Alli et al., 2002), although its role is debatable since it is known to increase the affinity of tubulin for VBL (Devred et al., 2008). Other reported mechanisms of resistance include overexpression of MT-bound septin GTPase proteins in conjunction with increased levels of tyrosinated tubulin and tubulin polyglutamylation (Froidevaux-Klipfel et al., 2011). Drug resistance is clearly a multifactorial clinical issue for the use of MSAs, thus identification of novel MSAs that can circumvent these mechanisms of resistance would be extremely beneficial given the success of the MT as a chemotherapeutic target.

Hypersensitivity reactions (due to immune response), dose-limiting antihematopoietic activity (such as neutropenia) and cumulative neurotoxicity (due to the 
involvement of MTs in neuronal transport) are other major concerns in using the taxanes as chemotherapeutics. The risks of hypersensitivity are not due to taxanes themselves, but their insolubility or hydrophobicity, requiring their delivery using a vehicle. The surfactants used in the delivery of taxanes are polyoxyethylated castor oil (cremophor), polysorbate or ethanol. Administration of the taxanes is currently limited to intravenous routes. Although the risks from these vehicles have been reduced by premedication, it is still a significant clinical issue (Morris \& Fornier, 2008; Dumontet \& Jordan, 2010). It is thought that the castor oil may trap PTX and other chemotherapeutics in micelles inhibiting their transport to the cell interior, thus, decreasing the concentration being delivered to target cells. There are a number of different PTX formulations that have been produced in order to decrease toxic side effects seen upon administration, and many of these are used in the clinic. For example, Abraxane ${ }^{\circledR}$ has been developed to avoid the use of cremophor and thus decrease the incidence of hypersensitivity reactions. This taxane formulation was FDA approved in 2005 for metastatic breast cancer and has since been approved for use in other settings. Preclinical studies indicated that it can be administered without premedication, and a greater amount of PTX was found in the target cells (www.fda.gov/; Morris \& Fornier, 2008). This drug formulation decreased neutropenia; however, it has been associated with increased incidence of peripheral neuropathy (Gradishar et al., 2005). Thus, identifying and developing novel MTAs that have higher aqueous solubility, or improving the means by which the taxanes can be delivered, would be highly beneficial. Currently much work is going into formulations of taxanes to remove the requirement for a surfactant for administration with the goal of eliminating its hypersensitivity reactions.

\subsubsection{Synergy}

Another way to overcome some of the clinical limitations of MSAs is potentially through combination therapy. Combination therapy involves the administration of two drugs that act synergistically with each other, and this approach has become a common mechanism used clinically to improve the action profiles of therapeutic drugs (Jackson et al., 2007). Synergistic interactions can occur between two or more drugs with similar (Clark et al., 2006; Wilmes et al., 2007) or different (Amadori et al., 1996) mechanisms of action. Combinations of drugs that have a greater effect than the sum of their individual effects are considered to have a 'synergistic interaction'. Treatment of tumors with single agents 
is often ineffective, as a single drug may effectively kill the most sensitive population of cells in a tumor but leave behind a resistant population that is still capable of dividing. Therefore, each time the agent is administered, the tumor becomes more resistant and the drug less effective (Thurston, 2006). The use of a "cocktail" of drugs was first tried in the 1960s for treatment of testicular tumors and is now commonly used in the clinic. An example is the use of gemcitabine plus PTX for the treatment of metastatic breast cancer (Colomer, 2008). Combination therapy can also facilitate the simultaneous attack on different biological targets, leading to enhanced effectiveness of the treatment (Thurston, 2006). The aim of using multiple drug therapy is to improve the efficacy but at the same time maintain adequate overall toxicity against the cancer cell. Synergism is an important concept, given that the side effects associated with many current anticancer drugs are high, limiting their therapeutic effect since lower doses have to be administered to prevent toxicity. If a synergistic interaction can occur between two drugs, lower doses of each drug can then be administered, thus enhancing antitumor activity without the serious side effects associated with the use of high concentrations of a single agent. It is also very difficult for a cancer cell to develop resistance to two drugs at the same time, especially if the two drugs target different cellular pathways. It is possible that MSAs have potential secondary binding sites on tubulin or interact with other proteins in the cell, and these complex interactions may result in the observed synergistic interactions. It is also possible that two drugs may interact synergistically at one target, but act antagonistically at another target. Therefore the mechanisms underlying synergy need to be further investigated (Wilmes et al., 2011). Despite the complexity of this phenomenon, there are a number of combinations of MSAs in several clinical trials, some in phase III (Jordan \& Wilson, 2004).

\subsubsection{Microtubule-stabilizing agents in other diseases}

MSAs have also shown promise as potential therapeutics in the treatment of multiple sclerosis, and other neurodegenerative diseases, particularly Alzheimer's disease. Multiple sclerosis is an autoimmune disease that affects the central nervous system, resulting in inflammation that leads to nerve damage. The use of MSAs to treat multiple sclerosis is a new idea and has only been tested in a mouse model of the disease. Inhibition of T-cell proliferation is effective at reducing the disease. This has been shown previously but the anti-mitotic drugs used had significant toxicity effects. It has now been 
shown that PTX and PEL are effective at reducing the disease in mice, if prolonged exposure is given in an appropriate drug administration schedule (Crume et al., 2009). In neurodegenerative diseases MSAs also have a protective effect. The MAP tau is a MTstabilizer and is involved in axonal growth and neuronal transport. Tauopathies contribute to a number of different neurological disorders, the most prevalent being Alzheimer's disease, which is characterized by the accumulation of $\beta$-amyloid plaques. Tau can be hyper-phosphorylated, causing it to self-assemble into polymers, and in this state it cannot bind to and stabilize MTs, and thus loss of tau function occurs. The $\beta$ amyloid plaques in Alzheimer's are thought to enhance this phosphorylation process (Brunden et al., 2009). PTX and tau share a similar binding site on the MT (Kar et al., 2003), thus PTX can induce MT polymerization, protecting the MT network from degradation (Brunden et al., 2009). In addition, both PTX and EPOD have demonstrated good potential for treatment of Alzheimer's disease in a transgenic mouse model of the disease. However, given PTX lack of ability to cross the BBB it would not be a suitable candidate for further development. EPOD can readily penetrate the $\mathrm{BBB}$, and given its promising results is a potential candidate in treating neurological diseases (Zhang et al., 2005; Brunden et al., 2010). More recently in our laboratory, PEL has shown therapeutic potential in a primary neuronal culture model of the disease (Das \& Miller, 2012).

A major difficulty in the clinical use of MTAs for neurodegenerative diseases is access to the BBB. The $\mathrm{BBB}$ is rich in P-gp drug efflux pumps, and this prevents susceptible compounds from entering the brain (Cordon-Cardo et al., 1989). Novel MSAs that are not susceptible to MDR may be more permeable to this barrier, as already seen for the EPOs. Sagopilone was strategically designed to combine high efficacy with reduced side-effects. Sagopilone crosses the BBB in both rat and mouse models, reaching therapeutically relevant concentrations in the brain, and having a long half-life (Hoffmann et al., 2009), and is currently in clinical trials for a number of different cancer types. Most excitingly it has the potential to treat glioblastoma and CNS metastasis (Zhao et al., 2009). Given the positive therapeutic results above with the use of MSAs in both MS and AD, and the fact that novel MSAs can cross the BBB, MSAs have good potential in treating not only cancers of the brain but also other neurological disorders. 


\subsection{OTHER TARGETED CANCER APPROACHES}

Greater understanding of the molecular basis of cancer has opened a new window for targeted cancer therapeutics, development of targeted strategies that inhibit specific molecules and target specific cancer pathways (DeVita \& Rosenberg, 2012; Yap \& Workman, 2012). An example of this is the use of monoclonal antibodies to target overexpressed tumor oncogenes. Tumorigenesis is associated with genomic instability, through up-regulation and down-regulation of tumor oncogenes and suppressor genes, respectively (Lengauer et al., 1998). Numerous regulators of mitosis are not correctly expressed in tumor cells. This makes these deregulated proteins good anticancer targets. Recently, mitotic kinases have become an attractive target for anticancer therapy given their essential role in mitosis and the fact that MTAs have been so successful and have validated the mitotic spindle as an excellent target in cancer chemotherapy. For example, the Aurora kinases are important regulators of many different steps in mitosis and are overexpressed in many human tumor cells (Keen \& Taylor, 2004). However, targeting protein-protein interactions is challenging, and clinical data for inhibitors of these proteins has not been promising, with many activities only characterized as 'stable disease' (Komlodi-Pasztor et al., 2011). The main problem with kinases is that they are involved in different cellular pathways; thus, inhibiting one of these proteins may work for a period of time but in due course another kinase will up-regulate or an alternative pathway will take over and the tumor is likely to return. Causing defects in one signaltransduction pathway is likely to cause impairment of other signaling pathways, and this could lead to resistance against kinase targeting drugs (Melnikova \& Golden, 2004). Thus, blocking a single kinase may not be an efficient approach for treating cancer, and therefore the attraction of kinases as anticancer targets might not be as promising as first thought. With MTs, however, there are no other cellular structures that can segregate chromosomes. Therefore resistance to MTAs only arises from over expression of P-gp pumps, up-regulation of the $\beta$ III isoform of tubulin, or mutations in the MTA binding sites; thus, MTs are still the most successful anticancer targets. Many new MSAs are not substrates of the P-gp pump, and interestingly, there are a number of covalent MTAs, that, due to the nature of their binding, can overcome MDR by drug efflux pumps and should not affected, to the same degree, by isotype changes in tubulin expression (Singh et al., 2011), effectively eradicating these mechanisms of resistance towards MTAs Although a number of specific antimitotic drugs have not been successful in clinical 
trials, drugs that target the MT have been highly successful in the clinic for many years and will continue to be at the forefront for years to come (Komlodi-Pasztor et al., 2011).

\section{$1.7 \quad$ ZAMPANOLIDE}

The MSA ZMP (Figure 1.5), was reported by Tanaka and Higa in 1996 (Tanaka \& Higa, 1996). The compound has a 20 -membered macrolide ring that is largely unsaturated with an embedded 4-methylenetetrahydropyran ring and an unusual $\mathrm{N}$-acyl hemiaminal sidechain. In preliminary screening, ZMP demonstrated potent cytotoxicity against four tumor cell lines; P388 (mouse leukemia), A549 (human lung carcinoma), HT29 (human colon carcinoma) and MEL28 (human melanoma), all with $\mathrm{IC}_{50}$ values in the range of 2 $10 \mathrm{nM}$ (Tanaka \& Higa, 1996). A number of complete synthetic preparations of ZMP in both its natural and unnatural enantiomeric states have been reported (Smith et al., 2001; 2002; Hoye \& Hu, 2003; Uenishi et al., 2009; Ghosh \& Cheng, 2011). Formal synthesis and studies toward total synthesis have also been reported by a number of different labs. Total synthesis of ZMP and other marine macrolides has been important in determining their relative stereochemistry and also provides a means of producing sufficient material if a high demand for the natural product exists. Due to scarcity of the natural product, the mechanism of action of ZMP has only recently been elucidated, when ZMP was reisolated in 2008 by the Northcote laboratory at Victoria University of Wellington (VUW) (Field et al., 2009). It was shown to be a potent MSA with properties similar to the clinically available drugs PTX, DTX and IXA. It arrests cells in $\mathrm{G}_{2} / \mathrm{M}$ of the cell cycle, induces multiple asters in mitotic cells and MT bundles in interphase cells, and causes a concentration-dependent shift of tubulin from its soluble dimeric form to its polymerized form, both in cells and in vitro. Cells treated with ZMP increase in volume over 2.5-fold, develop multiple micronuclei and show membrane blebbing, all characteristics of mitotic slippage and induction of apoptosis. ZMP is also active in cells that overexpress the P-gp MDR pump, indicating that ZMP is not susceptible to this pump and may not be a substrate. Therefore, ZMP is a good candidate as a chemotherapeutic replacement for treating tumors that have developed resistance to the frontline taxanes via up-regulation of these pumps. In addition to this, ZMP is a good candidate for a potential anti-cancer agent as it has a low number of stereogenic centers and therefore is an ideal compound for large-scale synthesis, should clinical demand occur (Field et al., 2009). 


\subsubsection{Dactylolide}

DAC (Figure 1.5), an enantiomer of ZMP, was originally isolated from the marine sponge, Dactylospongia, collected from the Vanuatu Islands (Cutignano et al., 2001). DAC is a 20-membered macrolide lactone with an embedded 4methylenetetrahydropyran ring, the same core structure as ZMP; however, the side chain has been replaced with an aldehyde. ZMP and DAC have opposite absolute configurations (Ding \& Jennings, 2008), although this stereochemistry assigned in the literature may not be correct and needs to be further investigated (Dr. Peter Northcote, personal communication). Given that both ZMP and DAC are natural products, it seems unlikely they would have two different biosynthetic routes; therefore, it is likely that the stereochemistry of these natural compounds is the same. DAC exerted a cytotoxicity of $8.3 \mu \mathrm{M}$ against the tumor cell lines L1210 (mouse leukemia cells) and SK-0V-3 (human ovarian carcinoma cells), a concentration that corresponds to their $\mathrm{IC}_{63}$ and $\mathrm{IC}_{40}$ values, respectively. This is high compared to that of ZMP, which has nanomolar potency (Cutignano et al., 2001; Ding \& Jennings, 2008). This lower cytotoxicity is thought to be due to the loss of the reactive $\mathrm{C} 19 \mathrm{~N}-a c y l$ hemiaminal side chain in DAC, highlighting its importance in the cytotoxicity profile of ZMP. The lower cytotoxicity of DAC, however, may also be a result of the enantiomeric relationship between the two compounds if their opposite stereochemistry holds true. In studies by Ding and Jennings (2008), the unnatural enantiomer (-)-DAC, was tested against the same cell lines that (-)-ZMP was tested against in 1996 by Tanaka and Higa. A comparison of the results demonstrated that the side chain of ZMP does in fact play an important role, with (-)-DAC 10- to 1000fold less active than that of (-)-ZMP. It was also shown that (-)-DAC is approximately 2to 3-fold more active than its enantiomer (+)-DAC (Ding \& Jennings, 2008). Thus, although the absolute configuration is important, it does not affect the bioactivity to the extent of the C19 side chain moiety found in ZMP. 


\subsection{AIMS OF THIS THESIS}

There were three main aims of this research, all of which fit into the main goal of further characterizing ZMP as an MSA to help determine its potential for development as an anticancer agent.

1. To further investigate cellular effects of ZMP in taxane-resistant cells, to note any possible synergistic action with other MT-stabilizers, and to examine whether ZMP can inhibit cell migration, since a number of MTAs are successful at inhibiting cell migration.

2. To determine the location of the ZMP binding site on the MT and to investigate how it interacts with this binding site while also investigating the global effect on tubulin of ZMP binding.

3. To characterize the novel ZMP chemotype as a class of MSAs, investigating important SARs and to further investigate the effect of the ZMP side chain on its activity.

This thesis research was mainly carried out at Victoria University of Wellington under the supervision of my primary (Prof. John Miller, SBS) and secondary (Assoc. Prof. Peter Northcote) supervisors, however, aspects concerning the binding site and binding mechanisms were carried out at the Centro de Investigaciones Biológicas (CIB), Consejo Superior de Investigaciones Científicas (CSIC), Madrid, Spain under the supervision of Dr. J. Fernando Díaz. 
This chapter describes the general methods used in this thesis. More specialized methods, variations of the general methods and specific concentrations of buffers, proteins and ligands will be described in their respective chapters.

Recipes for reagents and buffers are provided in the Appendix.

Solvent concentrations were kept below $2 \%$ in all experiments.

\section{$\underline{2.1 \quad \text { CoMpounds }}$}

Compounds used in cell biology experiments

ZMP and LAU were isolated and purified from the marine sponge Cacospongia mycofijiensis collected from 'Eua and Vava'u, Tonga (Field et al., 2009). Synthetic ZMP was prepared as below. PEL was isolated and purified from the marine sponge Mycale hentscheli collected from Pelorus Sound, New Zealand (West et al., 2000). These natural products were provided by Assoc. Prof. Peter Northcote and Dr. Jonathan Singh of the School of Chemical and Physical Sciences at VUW, Wellington, New Zealand. PTX $\left(\right.$ Taxol $\left.^{\circledR}\right)$ was purchased from Sigma Chemical Co. (St. Louis, MO, USA) and IXA from Bristol-Myers Squibb (USA) as Ixempra ${ }^{\circledR}$. DTX was purchased from LC Laboratories ${ }^{\circledR}$ Woburn, MA. DSC was a kind gift from Prof. Ian Patterson, Department of Chemistry, University of Cambridge, UK. Flutax-2 (7-O-[N-(2,7-difluoro-4'-fluoresceincarbonyl)L-alanyl]-paclitaxel) (FTX-2) was kindly donated by Dr. Fernando Díaz, CIB, CSIC, Madrid, Spain. Compounds were stored in absolute ethanol or DMSO as $1 \mathrm{mM}$ or 10 $\mathrm{mM}$ stocks at $-80^{\circ} \mathrm{C}$, and working stocks of $10 \mu \mathrm{M}$ were made up in RPMI-1640 medium (Invitrogen) and stored at $-20^{\circ} \mathrm{C}$.

\section{Compounds used in biochemistry experiments}

Synthetic ZMP, DAC and analogs were synthesized by Prof. Karl-Heinz Altmann, Swiss Federal Institute of Technology (ETH) Zürich, Switzerland, as described in Zurwerra et al., (2010). The synthetic conversion of (-)-DAC into (-)-ZMP followed the strategy of Hoye and $\mathrm{Hu}$ (2003), with subsequent separation of the resulting C20 epimers by preparative reverse phase-HPLC (Zurwerra et al., 2012). PTX (Taxol ${ }^{\circledR}$ ) was supplied by Dr. Sufness, National Cancer Institute (Bethesda, USA). DTX (Taxotere ${ }^{\circledR}$ ) was kindly 
provided by Rhône Poulenc Rorer, Aventis (Schiltigheim, France). PEL was isolated as above by Drs Northcote and Singh (VUW) and supplied to Dr. Fernando Díaz as a gift. FTX-2 was synthesized in the laboratory of Dr. Wei Shuo Fan (Chinese Academy of Medical Sciences, Beijing, China) and kindly provided as a gift. Hexaflutax (7-O-[N-(6(fluorescein-4'-carboxamido)- $n$-hexanoyl)-L-alanyl]-paclitaxel (HXF) was a kind gift from Dr. Francisco Amat-Guerri (Instituto de Química Orgaánica, CSIC, Juan de la Cierva 3, Madrid 28006, Spain). MTC (2-methoxy5-(2, 3, 4-trimethoxyphenyl)-2,4,6cyclo heptatrien-1-one) was a kind gift from Dr. Thomas Fitzgerald (Florida A\&M University, Florida, USA). EPO A was purchased from Novartris. Guanine nucleotides, PDP and VBL were purchased from Sigma. All compounds were dissolved in $99.8 \% \mathrm{D}_{6}$ DMSO (Merck, Darmstad, Germany) and stored at $-80^{\circ} \mathrm{C}$, working stocks were made up in DMSO (Merck, Darmstad, Germany) and stored at $-20^{\circ} \mathrm{C}$. VBL was dissolved in milliQ water as a $0.1 \mathrm{mM}$ stock.

\subsection{Cell CUlTURE}

Various different cell lines were used in this project. All cell work was performed in a sterile Email Air Handling Class II Biological Safety Cabinet (AES Environmental Pty LTD, Australia) or in a sterile Logic Labconco ${ }^{\circledR}$ Purifier $^{\circledR}$ Biological Safety Cabinet (Total Lab Systems Ltd, New Zealand). Unless otherwise described cells were cultured in RPMI-1640 medium (Invitrogen) supplemented with 10\% fetal calf serum (FCS) (Invitrogen), 100 units/mL penicillin/streptomycin (Invitrogen) and 0.25 units/mL insulin (Sigma Chemical Co. St. Louis, MO, USA). Cells were grown at $37^{\circ} \mathrm{C}$ in a $5 \% \mathrm{CO}_{2}$ in air atmosphere using standard techniques in a SANYO Electric Co., Ltd incubator. All cell lines were stored in liquid nitrogen using $2 \mathrm{~mL}$ cryotubes (Greiner Bio One, Frickenhausen, Germany) at $1 \times 10^{6}$ cells per $\mathrm{mL}$ culture medium containing $10 \%$ DMSO. To passage cells, adherent cells were detached from the flask using trypsinEDTA (Invitrogen) and centrifuged for $5 \mathrm{~min}$ at $300 \mathrm{~g}$ to pellet cells and remove trypsinEDTA before re-suspending in fresh culture medium.

\section{$\underline{2.3 \quad \text { MTT CELLULAR PROLIFERATION ASSAY }}$}

The colorimetric MTT (3-(4, 5-dimethylthiazol-2-yl)-2, 5-diphenoyltetrazolium bromide) cell proliferation assay, first described by Mosmann (1983), was used to determine the number of viable cells after drug treatment. This assay involves the metabolic reduction 
by viable cells of a yellow tetrazolium salt into purple formazan crystals. Reduction only occurs in metabolically active cells due to the presence of NADH or NADPH. In theory, the amount of MTT reduced is relative to the number of live cells; however, only metabolizing cells produce formazan crystals; therefore, cells that are still alive but not metabolizing are not counted in this assay. 1A9 cells were seeded into 96-well plates (Microtest $^{\mathrm{TM}}$ Tissue culture plate, Falcon, Becton Dickson) at a concentration of $1 \times 10^{5}$ cells/well and left to attach overnight. Cells were then treated with varying concentrations of drug (2-fold serial dilution down a 96-well plate) and incubated for 72 $\mathrm{h}$ at $37^{\circ} \mathrm{C}$. A $20 \mu \mathrm{L}$ aliquot of MTT (5 mg/mL in PBS, see Appendix) was added to each well, followed by incubation for $2 \mathrm{~h}$ at $37^{\circ} \mathrm{C}$. The purple formazan crystals were solubilized in $100 \mu \mathrm{L}$ MTT solubilizer (see Appendix) overnight at $37^{\circ} \mathrm{C}$. The effect of various drug concentrations on cell growth was quantified spectrophotometrically at 570 $\mathrm{nm}$ in an automated multiwell plate reader (Versamax, Molecular Devices, Sunnyvale, CA) and the cell number calculated as a percentage of control (no drug):

\section{$\%$ of control $=($ sample absorbance-blank/average control-blank $) \times 100$}

Using SigmaPlot software (Systat Software Inc.), four-parameter logistic curves were fitted to the concentration-response data obtained for each drug concentration. $\mathrm{IC}_{50}$ values (concentration at which the number of viable cells is $50 \%$ that of the control wells) were calculated from the individual concentration-response curves using the equation

$$
y=\min +\frac{\max -\min }{1+\left(\mathrm{x} / \mathrm{IC}_{50}\right)^{\text {Hillslope }}}
$$

in which $x$ (concentration) and $y$ (\% of control) represent their representative axes. Max is the maximum value measured, and min the minimum value measured. Each experiment consisted of at least two technical replicates, and the $\mathrm{IC}_{50}$ value and SEM are based on at least three independent biological replicates. 


\section{$\underline{2.4}$ PURIFICATION AND PREPARATION OF TUBULIN}

Purified bovine brain tubulin is an excellent tool for in vitro experiments of MTAs. The procedure below was used in all biochemistry experiments to purify and prepare tubulin.

Bovine brain tubulin was collected, purified using a modified version of the Weisenberg procedure as described by Andreu (2007) and stored in sucrose in liquid nitrogen in $5 \mathrm{mg}-20 \mathrm{mg}$ aliquots.

On the day of the experiment tubulin was prepared by equilibration (to remove the sucrose (cryopreservant) from the storage buffer) through a Sephadex G-25 medium column (25 x $0.9 \mathrm{~cm})$ (GE Healthcare Bioscience, Upsala, Sweden) that had been preequilibrated with two volumes of cold GAB or PEDTA buffer, depending on the required conditions (Appendix). Tubulin was collected from the column in $1.5 \mathrm{~mL}$ plastic Eppendorf centrifuge tubes, and the absorbance measured at $295 \mathrm{~nm}$ using a Precision $U V$-vis spectrophotometer Cary 4000 (Varian), CA, USA. Fractions with an absorbance greater than 1.0 were pooled. To remove tubulin aggregates the pooled samples were clarified at $4^{\circ} \mathrm{C}$ by centrifugation at $50,000 \mathrm{rpm}$, for $10 \mathrm{~min}$ in a TLA 120.2 rotor in a Beckman Optima TLX ultracentrifuge (Beckman Coulter, Inc. Fullerton, CA, USA). The supernatant containing the tubulin was collected and a $50 \mu \mathrm{L}$ sample diluted 20 -fold in $10 \mathrm{mM}$ sodium phosphate (NaPi) buffer containing 1\% SDS (Sigma-Aldrich), pH 7.0, to measure the concentration of tubulin spectrophotometrically at $275 \mathrm{~nm}$ (extinction coefficient 107,000 $\mathrm{M}^{-1} \mathrm{~cm}^{-1}$ (Andreu et al., 1984)), against a buffer blank prepared in the same way. The spectrum between $240 \mathrm{nM}$ and $340 \mathrm{nM}$ was measured and the concentration of tubulin calculated from the absorbance reading at $275 \mathrm{nM}$ using the Beer-Lambert Law equation:

$$
\mathbf{A b s}=\varepsilon l c
$$

in which $A b s$ is the absorbance value obtained at $275 \mathrm{nM}, \varepsilon$ is the extinction coefficient or molar absorptivity of tubulin in this case $\left(107,000 \mathrm{M}^{-1} \mathrm{~cm}^{-1}\right), l$ is the path length in $\mathrm{cm}$ (in this case, the value is always 1 and can therefore be ignored) and $c$ is the concentration. The dilution factor (20) also needs to be taken into consideration:

$$
c=(\mathrm{Abs} / \varepsilon) * 20
$$

For tubulin assembly experiments using $\mathrm{GAB}$ buffer, $6 \mathrm{mM} \mathrm{MgCl}_{2}$ was added and the concentration of GTP increased to $1 \mathrm{mM}, \mathrm{pH} 6.5$, after purification, otherwise the buffer remained the same. PEDTA conditions remained unchanged unless otherwise stated. 


\subsection{PREPARATION OF STABILIZED TAXOID BINDING SITES}

Glutaraldehyde-stabilized cross-linked MTs are an extremely useful tool in studying MTligand interactions and have been essential in identifying the binding site and binding affinity for a number of important MT-stabilizing compounds (Pryor et al., 2002; Gaitanos et al., 2004; Buey et al., 2007). Stabilized cross-linked MTs were prepared by mild cross-linking with glutaraldehyde. A $20 \mathrm{mg}$ sample of tubulin was prepared as in section 2.4 in GAB buffer, and the concentration determined. The concentration of tubulin was then adjusted to $70 \mu \mathrm{M}$ or less and left to polymerize at $37^{\circ} \mathrm{C}$ for $30 \mathrm{~min}$. The solution becomes turbid and viscous once polymerized. A $50 \%$ glutaraldehyde solution (EMscope, microscopy grade) at a concentration of $4 \mu \mathrm{L} / \mathrm{mL}$ of tubulin (final concentration of $20 \mathrm{mM}$ ) was added to the solution to stabilize the MTs. The solution was mixed carefully with a $1 \mathrm{~mL}$ pipette so as not to disrupt the MTs and incubated at $37^{\circ} \mathrm{C}$ for $10 \mathrm{~min}$. Excess glutaraldehyde was removed with $60 \mu \mathrm{L} / \mathrm{mL}$ of $1 \mathrm{mM} \mathrm{NaBH} 4$ (Fluka) by pouring the tubulin over the $\mathrm{NaBH}_{4}$ solution in a $250 \mathrm{~mL}$ beaker on ice. Any foam that formed was left to dissipate for $10 \mathrm{~min}$ and the solution degassed by bursts of light centrifugation to recover the MTs (bench top centrifuge). The MT solution was then transferred to a Slide-A-Lyser ${ }^{\circledR} 10 \mathrm{~K}$ dialysis cassette (Pierce, Thermo scientific, Rockford, USA) that had been pre-incubated for $20 \mathrm{~s}$ in dialysis buffer (Appendix). The solution was dialyzed in the cassette against the dialysis buffer, the buffer was later replaced after 6-8 hours with new dialysis buffer and then the sample left to dialyze overnight. The following day, the stabilized crosslinked MTs were drop-frozen in liquid nitrogen. The concentration of MTs was measured as described in section 2.4, using a 1:20 dilution in $10 \mathrm{mM} \mathrm{NaPi}$ buffer containing 1\% SDS, pH 7.0. Since GTP absorbs at a similar wavelength to tubulin, if all the GTP was not lost during the dialysis (determined by the absorbance peak at $254 \mathrm{~nm}$ ), the contribution to the absorbance at $275 \mathrm{~nm}$ from residual GTP needs to be taken into account and a corrected value calculated (Dr. Fernando Díaz personal communication). This is done by solving the algebraic equations below, in which $\mathrm{A}_{254}$ and $\mathrm{A}_{275}$ are the values obtain from the spectrophotomer and [GTP] and $\mathrm{A}_{\text {tubulin }}$ are the unknowns.

$$
\begin{aligned}
& \mathbf{A}_{\mathbf{2 5 4}}=13400^{*}[G T P]+0.6^{*} A_{\text {tubulin }} \\
& \mathbf{A}_{\mathbf{2 7 5}}=8100^{*}[G T P]+A_{\text {tubulin }}
\end{aligned}
$$


The corrected value for the absorbance of tubulin at $275 \mathrm{~nm}$ is the measured absorbance

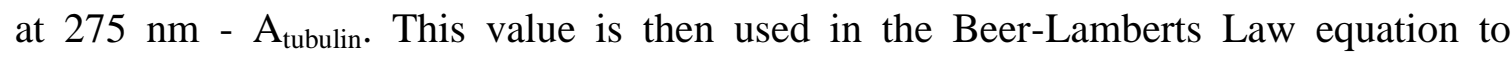
determine the concentration of MTs.

Using this concentration of tubulin, solutions of crosslinked MTs at 0.2, 0.5, 1.0, 2.0 and $5.0 \mu \mathrm{M}$ (tubulin concentration) were prepared in $10 \mathrm{mM} \mathrm{NaPi}$ buffer containing $1 \% \mathrm{SDS}$ (Sigma-Aldrich) pH 7.0. These solutions were then diluted (50 $\mu \mathrm{L}: 200 \mu \mathrm{L})$ in $4: 110$ $\mathrm{mM}$ NaPi buffer containing 1\% SDS (Sigma-Aldrich) $\mathrm{pH} 7.0$ and GAB and their fluorescence measured at $\lambda_{\text {exc }} 280 \mathrm{~nm}, \lambda_{\text {ems }} 323 \mathrm{~nm}$ using a Thermo Scientific Varioskan ${ }^{\circledR}$ Flash fluorometer. A fluorometric tubulin concentration calibration curve was constructed. The above procedure was then repeated with solutions of FTX-2 at the same concentration and in the same conditions and their fluorescence measured at $\lambda_{\text {exc }} 495 \mathrm{~nm}$, $\lambda_{\text {ems }} 520 \mathrm{~nm}$ to obtain a fluorometric FTX-2 calibration curve. The following samples were then prepared and centrifuged at $50,000 \mathrm{rpm}$ for $20 \mathrm{~min}$ at $25^{\circ} \mathrm{C}$ using a TLA100 rotor and a Beckman Optima TLX ultracentrifuge.

1. $2,5,10,20 \mu \mathrm{M}$ crosslinked MTs

2. $2 \mu \mathrm{M}$ crosslinked MTs, $5 \mu \mathrm{M}$ FTX-2

3. $2 \mu \mathrm{M}$ crosslinked MTs, $5 \mu \mathrm{M}$ FTX-2, $100 \mu \mathrm{M}$ DTX

The supernatants were collected and diluted 1:4 in $10 \mathrm{mM} \mathrm{NaPi}$ buffer containing 1\% SDS pH 7.0. The pellets were re-suspended in $200 \mu \mathrm{L} 10 \mathrm{mM} \mathrm{NaPi}$, and the samples were then diluted 1:4 in $10 \mathrm{mM} \mathrm{NaPi}$ buffer containing 1\% SDS $\mathrm{pH}$ 7.0. The concentrations of tubulin in the supernatant (soluble) and pellet (polymerized) were determined as above using the calibration curve. The concentration of tubulin in the pellet should be greater than $80 \%$, indicating that the majority of the tubulin is polymerized into MTs. The concentration of FTX-2 in each of the samples was measured using the calibration curve and the concentration of taxoid binding sites calculated as the difference in concentrations of FTX-2 in samples 1 and 2.

Under these conditions, $90 \%$ of the tubulin is assembled into MTs, and the taxoid binding site is fully functional. These assembled MTs are found to be stable against dilution and low temperatures (Díaz et al., 2000). The MTs were stored in liquid nitrogen 
until required, when they were diluted to the required concentration in GAB buffer left over from the second dialysis of the tubulin preparation procedure.

\subsection{TUBULIN POLYMERIZATION}

Tubulin polymerization can easily be monitored by quantifying the amount of pelleted polymers using centrifugation techniques. Tubulin assembles into MTs from a critical concentration $\left(\mathrm{C}_{\mathrm{r}}\right)$ (Oosawa \& Asakura, 1975). Below this concentration most of the tubulin is in the dimeric form; above this concentration, the majority of tubulin shifts into the polymerized state (Lee \& Timasheff, 1975). $C_{r}$ refers to the concentration of tubulin at which polymerization overcomes depolymerization and thus promotes assembly of MTs via non-covalent nucleation and elongation. The $\mathrm{C}_{\mathrm{r}}$ considered to be equal to the concentration of active protein in the supernatant, given essentially all the added tubulin is active the average tubulin concentration in the supernatant therefore gives an accurate measurement of the $\mathrm{C}_{\mathrm{r}}$ (Díaz et al., 1993).

Tubulin self-polymerizes at a critical concentration of $3.3 \mu \mathrm{M}$ when in the presence of $10 \mathrm{mM} \mathrm{NaPi}, 1 \mathrm{mM}$ EGTA, $1 \mathrm{mM} \mathrm{GTP}, 6 \mathrm{mM} \mathrm{MgCl}_{2}$ and $30 \%$ (3.4 M) glycerol, the so called 'favorable conditions'. This $\mathrm{C}_{\mathrm{r}}$ decreases upon MSA addition, and this decrease can be used to measure the MT assembly induction power of an MSA. Known MSAs can therefore be used as positive controls and MDAs used as negative controls, the latter significantly increasing the $C_{r}$. In conditions that are unfavorable for tubulin assembly, such as in $10 \mathrm{mM} \mathrm{NaPi}, 1 \mathrm{mM}$ EDTA, $1 \mathrm{mM}$ GTP, $6 \mathrm{mM} \mathrm{\textrm {MgCl } _ { 2 }}$ buffer, the $\mathrm{C}_{\mathrm{r}}$ of tubulin is $>200 \mu \mathrm{M}$ (Díaz et al., 1993). The $\mathrm{C}_{\mathrm{r}}$ of ZMP in both of these conditions was determined from the following methods.

\subsubsection{Microtubule assembly in glycerol buffer}

Tubulin (20 mg) was prepared in GAB buffer as described in section 2.4. For assembly conditions, $6 \mathrm{mM} \mathrm{MgCl} 2$ was added to the buffer and the concentration of GTP increased to $1 \mathrm{mM}$. Tubulin polymerization was carried out at $37^{\circ} \mathrm{C}$ for $30 \mathrm{~min}$ in $200 \mu \mathrm{L}$ polycarbonate ultracentrifuge tubes (Beckman Coulter, Inc., Fullerton, CA) at a concentration of $15 \mu \mathrm{M}$ and $20 \mu \mathrm{M}$ tubulin matched with $10 \%$ stoichiometric excess ligand concentration or the equivalent volume of DMSO. MTs were collected via ultracentrifugation at 50,000 rpm for 20 minutes at $37{ }^{\circ} \mathrm{C}$ in a TLA 100 rotor in a Beckman Optima TLX ultracentrifuge. The supernatants were collected by pipette and 
diluted 1:4 in $10 \mathrm{mM}$ NaPi buffer containing 1\% SDS pH 7.0. A $200 \mu \mathrm{L}$ aliquot of 10 $\mathrm{mM} \mathrm{NaPi}$ buffer containing $1 \%$ SDS pH 7.0 was added to the pellets to re-suspend them, and the pellets were then diluted 1:4 in a buffer containing GAB and $10 \mathrm{mM}$ NaPi buffer containing 1\% SDS pH 7.0. Tubulin concentrations of both the supernatant and the pellet were measured fluorometrically $\left(\lambda_{\mathrm{exc}}=280 \mathrm{~nm}\right.$ and $\left.\lambda_{\mathrm{em}}=323 \mathrm{~nm}\right)$ using a Modular spectrofluorometer, Fluorolog ISA ${ }^{\circledR}$ (Jobin Yvon - Spex). A standard curve was prepared with known concentrations of tubulin and measured as above. Results were analyzed using SigmaPlot software (Systat Software Inc.) and GraphPad Prism (Software version 5; San Diego, CA, USA).

\subsubsection{Induction of microtubule assembly in unfavorable conditions}

Strong MSAs that bind to the taxoid site or the LAU/PEL site stoichiometrically promote MT assembly in conditions that are normally hostile to or that inhibit tubulin assembly (i.e. in conditions where no glycerol or GTP is present) (Parness \& Horwitz, 1981; Mooberry et al., 1999; Pera et al., 2010). The ability of ZMP to promote MT assembly was tested in these inhibitory conditions using PEDTA buffer (Appendix). The key factor in this assay is the absence of glycerol. In this buffer, tubulin is unable to assemble unless a strong MSA is present, since in these conditions tubulin is dimeric and must be at concentrations $>200 \mu \mathrm{M}$ to self-assemble. This assay involves a fixed concentration of tubulin, and as the concentration of the MSA increases, the concentration of tubulin in the supernatant decreases as tubulin polymerizes. This assay measures the induction power of an MSA to cause tubulin polymerization.

Tubulin (20 mg) was equilibrated in PEDTA buffer as described in section 2.4. $\mathrm{MgCl}_{2}(6 \mathrm{mM})$ was added to the buffer and the concentration of GTP increased to $1 \mathrm{mM}$. The ZMP concentration was increased and the samples incubated at $37^{\circ} \mathrm{C}$ for $30-40 \mathrm{~min}$ in polycarbonate $200 \mu \mathrm{L}$ centrifuge tubes. The unassembled tubulin was then separated from polymerized tubulin by ultracentrifugation at $50,000 \mathrm{rpm}$ for $20 \mathrm{~min}$ at $37^{\circ} \mathrm{C}$ in a TLA 100 rotor in an Optima TLX ultracentrifuge. The supernatant was carefully collected by pipetting and the pellets re-suspended in $10 \mathrm{mM} \mathrm{NaPi} 1 \%$ SDS (SigmaAldrich) pH 7.0 buffer. A $100 \mu \mathrm{L}$ aliquot of each of the supernatant and pellet samples was diluted 1:4 in 1\% SDS pH 7.0 for fluorescence measurements. A standard curve of tubulin was prepared in the same buffer and the fluorescence of the tubulin in both supernatant and pellet measured in a Modular spectrofluorometer, Fluorolog ISA ${ }^{\circledR}$ (Jobin 
Yvon - Spex) $\left(\lambda_{\mathrm{exc}}=280 \mathrm{~nm}\right.$ and $\left.\lambda_{\mathrm{em}}=323 \mathrm{~nm}\right)$, with tubulin standards used to determine the concentration of tubulin in each of the samples. Results were analyzed using SigmaPlot software (Systat Software Inc.).

\subsection{FLUTAX-2 DISPLACEMENT ASSAY}

Flutax-1 and FTX-2 are fluorescent analogs of PTX and their fluorescence can be measured spectrophotometrically. These ligands can therefore be used as reference ligands in a competition assay for studying binding at the taxoid binding site and to determine the binding affinity of novel ligands that bind the taxoid site on MTs. This assay also allows one to distinguish between compounds which have the same mechanism of action, stabilize MTs, but which do not bind the taxoid binding site, such as LAU (Pryor et al., 2002) or PEL (Gaitanos et al., 2004). This assay will be further discussed in Chapter 4.2.

A binding competition assay using glutaraldehyde-stabilized taxoid binding sites and FTX-2 was performed to determine if ZMP binds to the taxoid site on the MT. Because these MTs are glutaraldehyde-stabilized, they do not assemble or disassemble in response to ligand addition nor do they respond to temperature changes. Thus, this assay measures the binding affinity and kinetic binding constant of a ligand in reference to their ability to displace FTX-2 from the MT. Pre-formed MTs stabilized via cross-linking with glutaraldehyde as described above were used to bind the ligand (Díaz et al., 2000). DTX and EPOA were used as controls, since they are known to compete with FTX-2 for the taxoid binding site. The displacement isotherm of each ligand was measured at least four times in different multiwell plates $\left(\mathrm{Nunc}^{\circledR}{ }^{\circledR}\right.$ FluoroNunc ${ }^{\mathrm{TM}}$, Sigma-Aldrich) using a fluorescence polarization microplate reader (Andreu \& Barasoain, 2001).

For the competition assay each well contained $200 \mu \mathrm{L}$ of GAB buffer (Appendix) with $50 \mathrm{nM}$ FTX-2, $50 \mathrm{nM}$ stabilized MTs and increasing concentrations of competitor ligand along the row of wells. Positive controls were set up for each of the ligands with no competitor. The standard contained $200 \mu \mathrm{L}$ GAB buffer and $50 \mathrm{nM}$ FTX-2, and the blank $200 \mu \mathrm{L}$ buffer with $50 \mathrm{nM}$ stabilized MTs. The competing ligands were added last and the contents of the well mixed, allowing time for binding to the MTs. The binding of these ligands in relation to FTX-2 binding and dissociation were measured by monitoring the change in ligand anisotropy at increasing temperatures. At each temperature, the plate was incubated for $20 \mathrm{~min}$ and then the reading taken. The FTX-2 anisotropy was 
measured using a Fluorolog-3-221 fluorometric spectrophotometer (Jobin Yvon-Spex, Longiumeau, France) using an excitation wavelength of $495 \mathrm{~nm}(0.1 \mathrm{~nm}$ band pass to prevent photolysis) and an emission wavelength of $525 \mathrm{~nm}(5 \mathrm{~nm}$ band pass). The binding of FTX-2 in the presence of the desired competitor ligand was then calculated (Buey et al., 2005). Anisotropy is an additive value and therefore the anisotropy of a particular mixture is the anisotropy of all its components. FTX-2 has two reference states, $r_{o}$ (bound) and $r_{\min }$ (fully displaced), and these values are known at specific temperatures (Table 2.1). The binding constants of FTX-2 at different temperatures are known (Table 2.2) (Díaz et al., 2000). The binding of FTX-2 in the absence of competitor $\left(v_{o}\right)$ was calculated using the known parameters, concentration of FTX-2 binding sites and the binding constant of FTX-2. The measured anisotropy values $\left(\mathrm{r}_{x}\right)$ were transformed into fractional saturation values $\left(v_{x}\right)$ employing the following equation:

$$
v_{x}=\frac{v o *\left(r_{x}-r_{\text {min }}\right)}{\left(r_{o}-r_{\min }\right)}
$$

These fractional saturation values of FTX-2 at different ligand concentrations were fitted to the equilibrium binding constant of the competitor ligand assuming a $1: 1$ stoichiometry and the known binding constants of FTX-2 using a PC program created by Dr. Fernando Díaz (Equigra v5, unpublished program). This program fits the experimental data by least-squares to the equilibrium binding constant of the ligand investigated, employing the known values of the reference ligand FTX-2. The binding constant of the test ligand, $\mathrm{K}(1)$, can be calculated from the known values of the binding constant of FTX-2, K(r), and the concentration of binding sites, FTX-2 and the test ligand by solving the simultaneous mass action equations (Díaz and Buey 2007):

$$
\begin{aligned}
& \mathrm{K}(1)=[\text { Ligand }]_{\text {bound }} /[\text { Sites }]_{\text {free }} *[\text { Ligand }]_{\text {free }} \\
& \mathrm{K}(\mathrm{r})=[\text { Flutax }]_{\text {bound }} /[\text { Sites }]_{\text {free }} *[\text { Flutax }]_{\text {free }} \\
& {[\text { Flutax }]_{\text {free }}=[\text { Flutax }]_{\text {total }}-[\text { Flutax }]_{\text {bound }}} \\
& {[\text { Ligand }]_{\text {free }}=[\text { Ligand }]_{\text {total }}-[\text { Ligand }]_{\text {bound }}} \\
& {[\text { Sites }]_{\text {free }}=[\text { Sites }]_{\text {total }}-[\text { Ligand }]_{\text {bound }}-[\text { Flutax }]_{\text {bound }}}
\end{aligned}
$$


Table 2.1 Fluorescence emission anisotropy of Flutax-2 $(1 \mu \mathrm{M})$ in GAB at $37^{\circ} \mathrm{C}$ $\left(\lambda_{\mathrm{ex}}=470 \mathrm{~nm}, \lambda_{\mathrm{ems}}=560 \mathrm{~nm}\right)$

\begin{tabular}{ccc}
\hline Free & Bound & Displaced \\
\hline $0.04 \pm 0.01$ & $0.26 \pm 0.02$ & $0.08 \pm 0.01$ \\
\hline
\end{tabular}

Bound: Flutax bound to MTs assembled from $20 \mu \mathrm{M}$ tubulin in GAB buffer. Displaced: Flutax displaced by the addition of $50 \mu$ M DTX (Díaz et al., 2000).

Table 2.2 Equilibrium constants of Flutax-2 binding to MTs

\begin{tabular}{ccccccccc}
\hline & $26^{\circ} \mathrm{C}$ & $27^{\circ} \mathrm{C}$ & $30^{\circ} \mathrm{C}$ & $32^{\circ} \mathrm{C}$ & $35^{\circ} \mathrm{C}$ & $37^{\circ} \mathrm{C}$ & $40^{\circ} \mathrm{C}$ & $42^{\circ} \mathrm{C}$ \\
\hline $\mathrm{Ka}\left(10^{7} \mathrm{M}^{-1}\right)$ & 6.5 & 5.9 & 4.6 & 4.2 & 3.0 & 2.2 & 2.0 & 1.8 \\
$v_{\mathrm{O}}$ (Flutax-2 bound $_{\text {Flutax-2 }}$ total & 0.378 & 0.563 & 0.522 & 0.508 & 0.451 & 0.398 & 0.382 & 0.364 \\
\hline
\end{tabular}

The parameter $v_{O}\left(\right.$ Flutax $-2_{\text {bound }} /$ Flutax- $\left.2_{\text {total }}\right)$ is calculated from the concentration of FTX-2 $(50 \mathrm{nM})$, the concentration of taxoid binding sites $(50 \mathrm{nM})$ and the binding constant of FTX-2 at the different temperatures (Díaz \& Buey, 2007), Dr. Fernando Díaz - Personal communication.

\subsection{BINDING KINETICS}

The kinetics of the reaction of compounds with cross-linked MTs was measured by determining the inhibition of FTX-2 binding to MTs. Stabilized taxoid binding sites (5 $\mu \mathrm{M}$ ) in $\mathrm{GAB}$ buffer were incubated with $6 \mu \mathrm{M}$ test compound (or an equivalent volume of DMSO) at $25^{\circ} \mathrm{C}$. After the desired time (30 min, $1 \mathrm{~h}, 2 \mathrm{~h}, 4 \mathrm{~h}, 6 \mathrm{~h}$ and overnight) 10 $\mu \mathrm{M}$ FTX-2 was added, and the samples were centrifuged to separate MTs with their bound compound from unbound compound using a TLA100 rotor in a Beckman Optima TLX ultracentrifuge at $50,000 \mathrm{rpm}$, at $37^{\circ} \mathrm{C}$ for $20 \mathrm{~min}$. The supernatant was removed with a pipette and the pellet re-suspended in $10 \mathrm{mM} \mathrm{NaPi} 1 \%$ SDS pH 7.0 buffer. A 50 $\mu \mathrm{L}$ aliquot of supernatant was then diluted in $200 \mu \mathrm{L} 10 \mathrm{mM} \mathrm{NaPi} 1 \%$ SDS pH 7.0, and $50 \mu \mathrm{L}$ pellet samples were diluted in $50 \mu \mathrm{L}$ of GAB buffer with $150 \mu \mathrm{L} 10 \mathrm{mM} \mathrm{NaPi} 1 \%$ SDS pH 7.0. A standard curve (10 $\mu \mathrm{M}-0 \mu \mathrm{M}$ FTX-2) was prepared in 1:4 GAB buffer: $10 \mathrm{nM} \mathrm{NaPi} 1 \%$ SDS buffer $\mathrm{pH}$ 7.0. All samples were added to a black 96 well FluoroNunc plate, and the amounts of FTX-2 in the pellet and supernatant were measured spectrofluorometrically $\left(\lambda_{\text {exe }} 495 \mathrm{~nm} \lambda_{\text {ems }} 520 \mathrm{~nm}\right.$ ). The free (supernatant) and bound (pellet) FTX-2 concentrations were calculated from the standard curve. 


\subsection{HIGH PERFORMANCE LIQUID CHROMATOGRAPHY}

Using high performance liquid chromatography (HPLC) separation, identification and quantification of compounds can be made based on idiosyncratic polarities and interactions with the stationary phase. Compound retention time in the column varies depending on the interaction strength of the ligand with the stationary phase of the column, the ratio or composition of the solvent and the flow rate of the mobile phase.

HPLC was used to analyze ligands for a number of different experiments. The same basic procedure was used throughout and is described below. Specific details for each experiment are described in their respective sections. All samples had an internal standard of $10 \mu \mathrm{M}$ DTX added. The organic phase of the samples was extracted $3 \mathrm{x}$ by one reaction volume of $\mathrm{CH}_{2} \mathrm{Cl}_{2}$ and air-dried using a vacuum centrifuge or a desiccator. The compounds were then dissolved in $35 \mu \mathrm{L}$ of buffer solvent (experiment dependent) for analysis. All compounds were analyzed by HPLC using an Agilent 1100 Series instrument employing a Supercosil, LC18 DB, 250 x $4.6 \mathrm{~mm}, 5 \mathrm{~mm}$ bead diameter column. Each experiment was developed in its respective solvent at a flow rate of 1 $\mathrm{mL} / \mathrm{min}$, and the absorbance was followed at the respective wavelength for the compounds being analyzed (260 nm (ZMP and DAC), $230 \mathrm{~nm}$ (DTX), $205 \mathrm{~nm}$ (PEL)). Solvent blanks were run at the start of each experiment and the concentration of ligands in each of the samples was quantified by comparison of the integrated areas of the HPLC peak with those of the internal standard.

\subsection{NANO-LIQUID CHROMATOGRAPHY AND TANDEM MASS SPECTROMETRY}

Mass spectrometry (MS) plays a major role in characterization of ligand-protein binding. Specialized MS instruments allow the detailed structural analysis of covalent-modified peptides (Calvo et al., 2008). To characterize the interaction of the compounds with MTs and unassembled tubulin, targeted MS experiments using a hybrid triple-quadrupole mass analyzer were performed. First, the test compound was fragmented using standard MS techniques to determine which of the obtained ions could act as a tracer for further experiments. The ligand was then bound to the protein and the protein digested using the protease trypsin. The ligand-protein complex was then fragmented and analyzed using standard HPLC and MS techniques. The protein peptides were analyzed by HPLC and the fragments containing the tracer ion were sequenced using MS fragmentation. This allowed identification of the ligand-labeled residue(s). 
Specific ion filtering of the adducts was carried out by precursor ion scanning (PIS) experiments using $476 \mathrm{~m} / \mathrm{z}$ as the diagnostic ion for the ZMP-derived tryptic peptide. To pinpoint the reactive residues being bound, we performed high-resolution MS (HRMS) analyses in the Orbitrap Elite system. Nano-liquid chromatography and tandem MS were carried out and analyzed by Dr. Enrique Calvo (Unidad de Proteómica, Centro Nacional de Investigaciones Cardiovasculares, 28029 Madrid, Spain).

Samples of MTs, oligomeric MTs and dimeric tubulin were prepared by me and submitted to Dr. Calvo for MS analysis.

\subsubsection{Assembled microtubules}

Tubulin (20 mg) was prepared as described in section 2.4, and then $20 \mu \mathrm{M}$ tubulin was assembled in GAB buffer with $25 \mu \mathrm{M}$ ligand or an equivalent volume of DMSO for $1 \mathrm{~h}$ at $37^{\circ} \mathrm{C}$. This method was used for the MS of strong MT inducers. The MTs were then separated by centrifugation $\left(50,000 \mathrm{rpm}, 20 \mathrm{~min}, 25^{\circ} \mathrm{C}\right)$, the supernatant discarded and the pellet re-suspended in $200 \mu \mathrm{L} 50 \mathrm{mM} \mathrm{NH}_{4} \mathrm{HCO}_{3}$ (Sigma). A $20 \mu \mathrm{L}$ aliquot of this solution was then added to $1 \mu \mathrm{g} / \mu \mathrm{L}$ pre-aliquoted trypsin (sequencing grade Promega, Madison, WI lot \#319995, specific activity $18028 \mathrm{U} / \mathrm{mg}$ ). A $20 \mu \mathrm{L}$ volume of $\mathrm{NH}_{4} \mathrm{HCO}_{3}$ was added and the samples incubated at $37^{\circ} \mathrm{C}$ for $2 \mathrm{~h}$, then dried by vacuum centrifugation overnight. Samples were stored at $-20^{\circ} \mathrm{C}$ until analysis when they were dissolved in $5 \%$ acetonitrile (ACN), $0.5 \%$ acetic acid in water for MS.

\subsubsection{Stabilized cross-linked microtubules}

In contrast to the above method on unassembled tubulin, cross-linked MTs were used, for the compounds that were weak inducers of tubulin assembly. Stabilized taxoid sites (20 $\mu \mathrm{M}$ ) were incubated with $25 \mu \mathrm{M}$ ligand or an equivalent volume of DMSO in GAB buffer for $4 \mathrm{~h}$ at $25{ }^{\circ} \mathrm{C}$. The MTs were then separated by centrifugation $(50000 \mathrm{rpm}, 20$ min, $25{ }^{\circ} \mathrm{C}$ ), the supernatant discarded and the pellet re-suspended. The samples were then processed as described in section 2.10.1.

\subsubsection{Oligomeric unassembled tubulin}

Tubulin was equilibrated using a two-step chromatography procedure in PEDTA buffer using a method that differed to that described in section 2.4. A small glass column 
(custom made) was prepared with Sephadex G-25 and washed three times with cold PEDTA buffer. The column was spun dry by centrifugation, 1,200 rpm for $3 \mathrm{~min}$ at $4^{\circ} \mathrm{C}$ using a Hettich Rotina 380R refrigerated centrifuge with rubber tube adaptors. Tubulin (20 mg) was added and centrifuged through the column at 1,200 rpm for $5 \mathrm{~min}$ at $4^{\circ} \mathrm{C}$, and the tubulin was collected at the bottom of the glass tube. This tubulin was then loaded onto a small Sephadex G-25 column (6 x 1) (GE Healthcare Bioscience, Upsala, Sweden) which had been equilibrated with $20 \mathrm{~mL}$ of cold buffer, collected and clarified as previously described in section $2.4 . \mathrm{MgCl}_{2}(1.5 \mathrm{mM}$ final concentration) was then added to the PEDTA buffer. Tubulin $(20 \mu \mathrm{M})$ was then incubated with $25 \mu \mathrm{M}$ ligand at $25^{\circ} \mathrm{C}$ for $4 \mathrm{~h}$ or overnight, depending on the potency of the ligands. The tubulin supernatant was collected by centrifugation $\left(50,000 \mathrm{rpm}\right.$ for $20 \mathrm{~min}$ at $\left.25^{\circ} \mathrm{C}\right)$, and $20 \mu \mathrm{L}$ of the sample was added to $1 \mu \mathrm{g} / \mu \mathrm{L}$ trypsin, with $20 \mu \mathrm{L} \mathrm{NH}_{4} \mathrm{HCO}_{3}$ and the samples incubated and processed as described in section 2.10.1.

\subsubsection{Dimeric unassembled tubulin}

The exact same procedure was used to prepare dimeric tubulin, except no $\mathrm{MgCl}_{2}$ was added to the buffer.

The aggregation state of the tubulin for each of the experiments was checked by analytical ultracentrifugation (AUC) using a Beckman Optima-XLA centrifuge following the same methods described in section 2.11 below.

\subsubsection{Compounds}

Free, unbound compounds were prepared to allow offline characterization of the compounds as a pre-step for the PIS experiments to determine how they interact with tubulin following fragmentation. Compounds were prepared in DMSO $(10 \mu \mathrm{L}-40 \mu \mathrm{L}$ of a $1 \mathrm{mM}-20 \mathrm{mM}$ stock) and frozen at $-80^{\circ} \mathrm{C}$ for $1 \mathrm{~h}$. Samples were then lypolized overnight and submitted for analysis in the MS.

\subsection{SMALL ANGLE X-RAY SCATTERING}

Small angle X-ray scattering (SAXS) allows the study of non-crystalline systems such as proteins. Using SAXS, the shape, size and conformation of proteins in solution can be detected by examining the scattering profile at low-medium resolution. Essentially, 
SAXS experiments consist of firing monochromatic X-rays (typically at $0.15 \mathrm{~nm}$ wavelength) into a solution (within the sample cell), and recording the X-ray photons as they elastically scatter off the molecules in solution. The scattering intensity is recorded as a function of the scattering angle. The measured signal is the difference in average electron density between molecules in the solvent. The signals from all orientations of the molecules in the irradiated solution are averaged together. The recorded scattering profile from the detector shows is unremitting and radically symmetrical (Figure 6.5A, Chapter 6) and through computer simulations provides valuable information about the global structure (size and shape) and conformation of the macromolecules at low to medium resolutions. The recorded scattering signal is derived from the difference in average electron density by the equation:

$$
\Delta \rho(r)=\rho(r)-\rho(s)
$$

in which the difference in average electron density is defined by $\Delta \rho(\mathrm{r}), \rho(\mathrm{r})$ defines the molecules in the solution, and $\rho(s)$ the solution buffer. SAXS data are collected with a buffer blank that is subtracted so that the observed scattering is from the macromolecule in solution.

SAXS is a good technique to study hollow cylindrical structures such as the MT and can monitor processes such as tubulin self-assembly and MSA-induced changes, among other factors (Putnam et al., 2007; Buey et al., 2009). In this thesis, SAXS was used to monitor changes in MT structure by determining the average number of protofilaments making up the cylinder wall in the presence of different MSA compounds. Since a protein solution is being used, the scattering profile of the buffer also needs to be measured. It is assumed that the buffer scattering intensity is constant, and therefore it is subtracted from the profile of the samples containing the test proteins (Buey et al., 2009).

In order to study the effect of the lower potency MSAs the experiments were performed in the presence of glycerol, a non-specific MT stabilizer. Control experiments were performed in PEDTA in order to compare the results with those previously reported (Matesanz et al., 2011a). Tubulin was prepared as in section 2.4, and was not diluted. Ten percent stoichiometric excess ligand or the equivalent volume of DMSO was added to the samples, in proportion to the tubulin concentration. Samples prepared in GAB buffer were incubated for $30 \mathrm{~min}$ at $37^{\circ} \mathrm{C}$; whereas, those prepared in PEDTA were 
incubated over a gradient $\left(4^{\circ} \mathrm{C}-37^{\circ} \mathrm{C}\right)$ for $1 \mathrm{~h}$ and $6 \mathrm{~min}$, followed by $30 \mathrm{~min}$ incubation at $37^{\circ} \mathrm{C}$. Buffer was analyzed as a control to obtain a buffer scattering pattern that was used as the blank. Dr. Aurora Nogales (Instituto de Estructura de la Materia, CSIC, Madrid, Spain) carried out the SAXS measurements with the Bruker NANOSTAR system. The camera was set to cover ranges of the scattering vector from 0.013 to 0.22 $\mathrm{nm}^{-1}$, and the X-ray scattering profile was recorded for $2 \mathrm{~h}$ in 30 min frames with a Bruker Hi-Star multiwire area detector. Raw data were normalized for beam intensity decay and detector response. These data was then analyzed using SigmaPlot software (Systat Software Inc.) following methods in Buey et al., 2009 and Matesanz et al., 2011a. The low-angle X-ray scattering pattern of MTs in solution can be described as the Fourier transform of a hollow cylinder. The zeroth-order Bessel function is given to a first approximation, which is the intensity, by the equation:

$$
\left|\mathrm{J}_{0}(\mathrm{qR})\right|^{2}
$$

in which $\mathrm{q}$ is the scattering angle and $\mathrm{R}$ the cylinder radius.

The data obtained from the SAXS is given in q $(1 / \AA)$, which first needed to be converted to the scattering vector $(\mathrm{s})$ in $\mathrm{nm}$.

$$
\begin{aligned}
& \mathrm{q}=4 \pi \sin \theta / \lambda \\
& \mathrm{s}=2 \sin \theta / \lambda \\
& \mathrm{q}=(2 \sin \theta / \lambda) * 10=\mathrm{s}
\end{aligned}
$$

where $q$ is the momentum transfer, $s$ is the scattering vector and $2 \theta$ is the angle between the incident X-ray beam and the scattered radiation.

The scattering of the buffer (blank) was subtracted from the MT samples, and the $s$ data graphed against the intensity. The three first peaks of the graph (intensity vs. angle of disruption) correspond to the minor maxima of the $J_{0}$ Bessel function of order $n$ and represent the low resolution transform of the excess electron density of the cylindrical structures in solution with respect to the solvent. These maxima correspond to the reciprocal radial space, which is the MT diameter. Thus, from the positions of these maxima, the average protofilament number of MTs can be determined following a series 
of transformations. The second peak of the graph directly depicts MT diameter and was analyzed by fitting a hyperbola curve to give the following the equation:

$$
\begin{aligned}
& \mathrm{y}=\mathrm{y}_{0}+\mathrm{ax}+\mathrm{bx}^{2} \\
& \mathrm{x}=-\mathrm{a} / 2 \mathrm{~b} \\
& \mathrm{a}=\mathrm{J}_{01}\left(\mathrm{~nm}^{-1}\right)
\end{aligned}
$$

The diameter of the MT can then be calculated using the following equation:

$$
\mathrm{J}_{01}=1.22 / \mathrm{D}
$$

in which $J_{01}$ is calculated from the fitted hyperbola as above, $D$ is the diameter of the MT (unknown) and 1.22 is a known constant. The number of protofilaments can then be calculated from the mean helical radius and the MT lateral interprotofilament spacing using the following equation:

$$
\text { \# protofilaments }=\left(\pi^{*} \mathrm{D}\right) / 5.7
$$

in which $5.7 \pm 0.1 \mathrm{~nm}$ is a constant calculated from the center to center lateral spacing of MTs (Andreu et al., 1994). Following this analysis, the number of protofilaments in MTs could then be compared between compounds.

\subsection{ANALYTICAL ULTRACENTRIFUGATION}

The main property of proteins is their high molecular weight. This can be studied by their sedimentation profile obtained in an analytical centrifuge. This technique allows an accurate determination of the size of the protein in solution and the approximate overall shape, providing information on the degree of homogeneity of proteins and other biological macromolecules in solution. It does this by monitoring the sedimentation of macromolecules in a centrifugal field and does so without any interaction with a matrix or a surface. It is the most accurate and adaptable means for determining the molecular weight, hydrodynamics and thermodynamics of proteins as it is solely based on the protein thermodynamics and all terms of the equations used in AUC are able to be determined experimentally. Since the 1970s, AUC has been the central technique for understanding cytoskeletal structures and allows study of the self-assembly properties of tubulin and tubulin-ligand interactions (Ralston, 1993). It relies on application of a centrifugal force and real time observation of the resulting positioning of the 
macromolecules, with quantitative analysis of the data. The sedimentation coefficient $S$ (Svedburg coefficient) defines the rate at which sedimentation of a particle occurs relative to its centrifugal force. This value increases with particle mass. The migration of macromolecules through the solution is dependent on the centrifugal force applied and is therefore opposed by molecular friction. The sedimentation rate also depends on the hydrodynamic properties of the molecules which can enable low resolution information on conformational changes. Reversibly bound complexes can dissociate and re-associate during the sedimentation process, allowing the study of weak and transient interactions as well as strong, irreversible interactions (Balbo \& Schuck 2005). Sedimentation analysis is useful for studying changes in molecular weight when molecules associate to form more complex structures. This technique is advantageous over other techniques as it involves no labeling or chemical modification of the macromolecules and no interaction with any matrix or surface (Balbo et al., 2005). Macromolecules such as tubulin can exist in several states of aggregation, and this can be clearly shown using AUC. Using sedimentation we can study the changes in tubulin aggregation in the solution when ZMP is added. Tubulin can exist in many different states of aggregation and these can be distinguished using AUC.

Tubulin $(20 \mathrm{mg})$ was prepared as in section 2.4 in the appropriate buffer. The tubulin was diluted and a 10\% stoichiometric excess of ligand or DMSO (vehicle) added to the samples, in addition to the desired amount of GTP, GDP, $\mathrm{MgCl}_{2}$, DTT and RB3 depending on the experimental conditions required (discussed in respective areas). Samples were incubated at $25^{\circ} \mathrm{C}$ or $20^{\circ} \mathrm{C}$ for $30 \mathrm{~min}$ and centrifuged at $40,000 \mathrm{rpm}$ at the respective temperatures using an An50Ti eight-hole rotor with double-sector Eponcharcoal centerpieces in a Beckman Optima XL-I analytical ultracentrifuge. Differential sedimentation coefficient distributions were calculated by least-squares boundary modeling of the experimental data using the program SEDFIT (Schuck, 2000). These experiments were run with the assistance of the AUC staff at the CIB, CSIC, Madrid, Spain.

The sedimentation coefficient is influenced by not just the size and shape of the protein but also by the density and the viscosity of the solvent. Differences in these two variables need to be taken into account between different experiments in different conditions, and thus sedimentation coefficients need to be calculated in terms of a standard solvent, 
(water at $\left.20^{\circ} \mathrm{C}\left(s_{20, w}\right)\right)$. Solvent effects can be corrected for using the equation below (Ralston, 1993):

$$
s_{20, \mathrm{w}}=s_{\mathrm{obs}}\left(\frac{\eta_{\mathrm{T}, \mathrm{w}}}{\eta_{20, \mathrm{w}}}\right)\left(\frac{\eta_{\mathrm{s}}}{\eta_{\mathrm{w}}}\right)\left(\frac{1-\bar{v} \rho_{20, \mathrm{w}}}{1-\bar{v} \rho_{\mathrm{T}, \mathrm{s}}}\right)
$$

in which $s_{20, \mathrm{w}}$ is the sedimentation coefficient expressed in terms of the standard solvent water at $20^{\circ} \mathrm{C}$ and $s_{\mathrm{obs}}$ is the measured sedimentation coefficient in the experimental solvent at the experimental temperature $T ; \eta_{\mathrm{T}, \mathrm{w}}$ and $\eta_{20, \mathrm{w}}$ are the viscosities of water at the temperature of the experiment and at $20^{\circ} \mathrm{C}$, respectively, and $\eta_{\mathrm{s}}$ and $\eta_{\mathrm{w}}$ are the viscosities of the solvent and water at a common temperature, respectively. $\bar{v}$ is the partial specific volume, $\rho_{20, \mathrm{w}}$ is the density of water at $20^{\circ} \mathrm{C}$ and $\rho_{\mathrm{T}, \mathrm{s}}$ is the density of the solvent at the temperature of the experiment.

Water density at $20^{\circ} \mathrm{C}=0.998207 \mathrm{~g} / \mathrm{mL}, 25^{\circ} \mathrm{C}=0.997048 \mathrm{~g} / \mathrm{mL}$

Water viscosity at $20^{\circ} \mathrm{C}=1.002 \mathrm{mPa} . \mathrm{s}, 25^{\circ} \mathrm{C}=0.8902 \mathrm{mPa} . \mathrm{s}$

Tubulin partial specific volume $=0.735 \mathrm{~cm}^{3} / \mathrm{g}$ 


\subsection{INTRODUCTION}

\subsubsection{Resistance to microtubule-stabilizing agents}

As discussed in Chapter 1, resistance to MSAs is a clinically important issue. A number of novel MSAs are able to overcome resistance, either because they are not substrates for the P-gp drug efflux pump or they bind with relatively equal affinity to the main $\beta$ tubulin isotypes (Dumontet \& Jordan, 2010). For example, taccalonolides are able to circumvent both mechanisms of resistance by their unique mechanism of MT stabilization (Risinger et al., 2008). Point mutations in the $\beta$ I-isotype can also contribute to resistance in the clinic if they lead to decreased drug affinity and binding, even though there has been conflicting evidence for this it is now generally accepted that they contribute (Yin et al., 2012). A number of resistant, established cell lines have been identified that have mutations in $\beta \mathrm{I}$-tubulin, resulting in a resistant phenotype to respective MSAs (Table 3.1). Many of the same amino acids mutations have been identified independently, suggesting that these mutations could occur clinically. A recent random mutagenesis approach has identified a number of novel mutations but has shown considerable crossover with previously reported mutations (Yin et al., 2012). A number of these mutations have been observed in patients that have neuronal abnormalities suggesting that single amino acid changes may cause drug resistance as well as contribute to deficits in brain development, presumably as a result of the effect of the mutations on MT function in axons and dendrites.

\subsubsection{Resistant cell lines}

Before the electron crystallography structure describing the taxoid binding site was solved, mutations in resistant cell lines provided indirect evidence for the location of the taxoid binding pocket. The Giannakakou laboratory was the first to describe PTX- and EPO-resistant cell lines (Giannakakou et al., 1997; Giannakakou et al., 2000). The PTX resistant cells have single amino acid changes at residues 272 located in S7 and 374 located in the S9-S10 loop, both involved in the binding of PTX. EPO resistant cell lines, also demonstrating cross-resistance to PTX, have single amino acid mutations at residues 276 and 284, and both of these mutations are involved in the PTX binding pocket. A number of other mutant cell lines have also been described that are resistant to some but 
not all of the PTX site compounds, indicating that ligands interact in different ways with the taxoid pocket. These mutations are summarized in Table 3.1. Yin et al. (2012) provide a brief overview of the current known mutations, a number of new mutations and possible mechanisms of resistance of PTX-resistant cell lines. The mutations all tend to cluster around the proposed binding sites; however, mutations outside the binding site could still affect the ability of an MSA to bind due to allosteric changes in the protein. In addition to allosteric effects that disrupt binding, a mutation can also cause a structural change that has global effects. Tubulin mutations often cause changes in the stability of a MT, even in the absence of a drug. Resistant cell lines have also provided support for the $\beta$-subunit as the binding site for LAU and PEL. All the above studies indicate the importance of resistance cell lines in understanding drug binding to tubulin.

\section{Human ovarian carcinoma cell lines}

A2780 cells are an adherent human ovarian cancer cell line that were first studied due to their high levels of the oncogene myc (Eva et al., 1982). The 1A9 cell line is a clone of the A2780 cell line.

PTX10 and PTX22 cells are PTX-resistant and were isolated in a single series selection in high PTX concentrations as individual clones of the 1A9 cell line, after chemical mutagenesis (Giannakakou et al., 1997). Sequence analysis of $\beta$-tubulin in these cells indicated resistance was due to single amino acid mutations in the M40 isotype of $\beta$-tubulin, $\beta$ Phe272Val and $\beta$ Ala374Thr in PTX10 and PTX22, respectively. The EPO-resistant cell lines A8 and B10 are also clones of the 1A9 cell line and were created by single lethal doses of EPOA and EPOB, respectively. After this single high concentration was gradually increased, sequencing confirmed that resistance was due to single point amino acid changes, specifically $\beta$ Thr276Ile in A8 and $\beta$ Arg284Gln in B10. These four resistant cell lines are all mutated in the taxoid binding region of $\beta$-tubulin (Giannakakou et al., 1997; Giannakakou et al., 2000) (Table 3.1).

1A9-R1 and 1A9-L4 are resistant to PEL and LAU/PEL, respectively (Kanakkanthara et al., 2011). 1A9 cells were cultured in PEL at a concentration greater than the $\mathrm{IC}_{50}$ for over 6 months; then concentrations of PEL were increased over a shorter period of time and resistant cells selected and cloned. Verapamil, a P-gp pump inhibitor, was added to the cultures to prevent selection for cells that have up-regulated this drug efflux pump. R1 cells have a single point amino acid change $\beta$ Ala296Thr. L4 
cells were created by exposure to increasing concentrations of LAU over an 18-month period, and then resistant cells were selected. L4 cells have a single amino acid change in which arginine 306 is replaced by cysteine in $33 \%$ of the cases or histidine in $66 \%$ of the cases. L4 cells are 30-fold resistant to LAU and 20-fold resistant to PEL. R1 cells are 7fold resistant to PEL but only 2-fold resistant to LAU. Neither cell line showed resistance to drugs that bind to the taxoid site on $\beta$-tubulin nor were they resistant to MDAs. Additionally, L4 cells have increased mRNA and protein levels of $\beta$ II- and $\beta$ III-tubulin isotypes (Kanakkanthara et al., 2011).

Although there are no confirmed hypotheses of the mechanism of resistance to the drugs, it is believed that reduced binding of the ligand (a decrease in $\mathrm{Km}$ ) results in the decreased cytotoxicity, rather than a defect in MT function as a result of the mutation (Giannakakou et al., 1997; Giannakakou et al., 2000). This conclusion is supported by the fact that multiple MSAs are affected by the mutations and the fact that the resistant cells are stable in drug-free medium. 
Table 3.1 Mutations in $\beta$-tubulin that confer resistance to MSAs

\begin{tabular}{|c|c|c|c|c|c|}
\hline Resistance to & Cell line & WT residue & Mutated amino acid & Location & Reference \\
\hline \multirow{6}{*}{ Paclitaxel } & 1A9-PTX10 & Phe272 & Val & M-loop & \multirow{2}{*}{ Giannakakou et al., 1997} \\
\hline & 1A9-PTX22 & Ala374 & Thr & S9-S10 loop & \\
\hline & $\mathrm{CHO}$ & Leu217 & His, Arg, Phe & H6-H7 loop & Gonzalez-Garay et al., 1999 \\
\hline & $\mathrm{CHO}$ & Leu219 & Arg, Asn, Pro & H6-H7 loop & Gonzalez-Garay et al., 1999 \\
\hline & $\mathrm{CHO}$ & Leu230 & His, Phe & H7 & Gonzalez-Garay et al., 1999 \\
\hline & KB-3-1 & Asp26 & Glu & $\mathrm{H} 1$ & Hari et al., 2006 \\
\hline Docetaxel & LNDCr & Phe272 & Ile & M-loop & Hara et al., 2010 \\
\hline \multirow{2}{*}{ Epothilone A } & $1 \mathrm{~A} 9-\mathrm{A} 8$ & Thr276 & Ile & M-loop & \multirow{2}{*}{$\begin{array}{c}\text { Giannakakou et al., } 2000 \\
\text { He et al., } 2001\end{array}$} \\
\hline & HeLa & Pro175 & Ala & T5-loop & \\
\hline \multirow{5}{*}{ Epothilone B } & 1A9-B10 & Arg284 & Gln & M-loop & \multirow{3}{*}{$\begin{array}{c}\text { Giannakakou et al., } 2000 \\
\text { He et al., } 2001 \\
\text { He et al., 2001; Verrills et al., 2003; } \\
\text { Yang et al., } 2005\end{array}$} \\
\hline & HeLa & Tyr434 & Cys & $\mathrm{H} 12$ & \\
\hline & A549, CCRF-CEM, A549 & Gln294 & Glu & H9 & \\
\hline & CCRF-CEM & Ala233 & Thr & H7 & Verrills et al., 2003 \\
\hline & A549 & Val62 & Phe & $\mathrm{H} 1^{\prime}-\mathrm{S} 2$ & Yang et al., 2005 \\
\hline Laulimalide & 1A9-L4 & $\operatorname{Arg} 308$ & His $(70 \%)$; Cys $(30 \%)$ & H9' & Kanakkanthara et al., 2011 \\
\hline \multirow{5}{*}{ Peloruside } & 1A9-R1 & Ala298 & Thr & H9-H9' loop & Kanakkanthara et al., 2011 \\
\hline & 1A9 & Ala298 & Ser & H9-H9' loop & \multirow{4}{*}{ Begaye et al., 2011} \\
\hline & $1 \mathrm{~A} 9$ & Arg308 & His & H9' & \\
\hline & $1 \mathrm{~A} 9$ & Asn339 & Asp & H10-S9 loop & \\
\hline & $1 \mathrm{~A} 9$ & Tyr342 & Ser & H10-S9 loop & \\
\hline
\end{tabular}

A number of the cells show cross resistance to other agents and the first column shows the MSA with which the cells were selected. CHO cells (Chinese hamster ovary), KB-3-1 cells (epidermoid carcinoma), LNDCr cells (human prostate cancer), A549 cells (human non-small cell lung cancer), HeLa cells (human cervical adenocarcinoma), CCRF-CEM cells (leukaemia) (desoxy-EPOB not EPOB used). 


\subsubsection{Combination therapy}

As discussed in Chapter 1.5 combination therapy involves the administration of two drugs that act synergistically with each other or are additive. This approach has become a common mechanism used clinically to improve the action profiles of therapeutic drugs, and avoid development of resistance. Synergistic interactions allow shorter administration times and lower drug doses, resulting in decreased toxicity. Currently in the clinic the taxanes are used in a number of different settings in combination with other drugs. For example the use of PTX and gemcitabine (nucleoside analog incorporated into DNA) in the treatment of metastatic breast cancer (www.fda.gov/).

\section{Synergy between microtubule-targeting agents}

It is well known that taxoid site MSAs can synergize with LAU and PEL (Gapud et al., 2004; Clark et al., 2006; Hamel et al., 2006; Wilmes et al., 2007; Wilmes et al., 2011). This synergism is thought to be due to differences in the binding mode of the compounds and the means by which they cause stabilization of the MT. Thus synergistic interactions may provide information on the binding sites of novel MSAs. In addition, PTX and some of its derivatives can synergize with some MDAs, for example PTX and vinorelbine (Photiou et al., 1997), PTX and VBL when administered sequentially (Giannakakou et al., 1998) and PTX with 2-methoxyestradiol both in vivo and in vitro (Ricker et al., 2004; Han et al., 2005). Thus, given the above and the literature on synergy between MTAs, it can be generalized that if two compounds target tubulin at different sites they are likely to synergize with one another. It is unlikely that two drugs that bind to the same site would synergize with one another, as it is more likely they would have an additive effect given that they cannot simultaneously bind the same site. PTX and DSC, however, are able to synergize in both cell culture (Martello et al., 2000; Honore et al., 2004) and xenograft tumor models (Huang et al., 2006), even though these two compounds bind to the same site on tubulin (Hung et al., 1996). The mechanism of synergy between these two drugs may not be due to their MT-stabilizing activity but due to other factors, such as effects on secondary targets. For example PTX is known to cause phosphorylation of bcl-2, a protein that promotes cell survival and has a protective effect in cancer cells. Phosphorylation of this protein causes loss of this protection and consequent cell death (Haldar et al., 1996). Additionally, the synergy seen between these drugs may be due to their potential to increase apoptosis (Martello et al., 2000). However, apoptosis is the end 
point of a process that started much earlier in the cell; therefore, more complex events must be occurring to obtain synergism between the drugs. One possibility is that different tubulin isotypes have different affinities for the individual drugs and that the compounds are thus able to distinguish between different forms of MTs within the cell (Giannakakou \& Fojo, 2000; Gertsch et al., 2009). It has been shown that the stabilization of MTs by PTX and DSC complement each other (Khrapunovich-Baine et al., 2009) and although they both bind the taxoid site, they have unique poses within this site, allowing synergy to occur. Complementary interactions may occur at both the interdimer and lateral interfaces of tubulin.

\subsubsection{Mitosis and MSAs}

MTAs mainly target mitosis, given the central role of MTs in the organization and segregation of chromosomes; however, they are also able to target cells in interphase. MTs are involved in a number of different cellular processes including for example intracellular trafficking and cell migration. Interestingly, a number of cancer oncogene protein products are trafficked by MTs. Thus, interfering with the ability of these proteins to traffic using MTs would be detrimental to cancer cell survival.

Given the success of MTAs and the understanding that their success stemmed from targeting spindle MTs, there was a push for research and development of other agents that specifically target mitosis, with the idea being that these agents would achieve at least the same or even greater cytotoxicity as the MTAs (Komlodi-Pasztor et al., 2011). For example, there has been a recent surge of mitotic kinases inhibitors as discussed in Chapter 1.6. However, many specific non-tubulin mitotic inhibitor lead compounds have been unsuccessful in clinical trials (Komlodi-Pasztor et al., 2012). Although MTs are always present in the cell, both in mitosis and interphase cells, mitotic kinases are only present in the M-phase of the cell cycle and thus can only be inhibited by a drug about $10 \%$ of the time in a cycling cell (Komlodi-Pasztor et al., 2012). It has been proposed that the rationale for targeting mitosis with MTAs may be flawed and that the efficacy of MTAs is not limited to mitotic effects but also involves interphase effects (Komlodi-Pasztor et al., 2011). Human tumors that show sensitivity to MTAs often do not divide as fast as established cancer cell lines or in vivo animal tumors, and therefore mitosis may not be the only target. For example it is now well known that disruption of MTs by MTAs affects androgen-receptor trafficking (Thadani-Mulero et al., 2012). 
Interestingly, the most recently approved MSA cabazitaxel has been approved to treat hormone-refractory metastatic prostate cancer (Galsky et al., 2010). There is also growing evidence in the literature that taxane-based therapy involves inhibition of cellular trafficking (Carbonaro et al., 2012). Numerous studies have demonstrated that MTAs can inhibit cell migration, a key process in angiogenesis (Lu et al., 2006), and there are a number of MDAs in clinical trials as vasculature disrupting agents (Tozer et al., 2005). Additional support for non-mitotic effects of MTAs comes from the neurotoxicity seen in some patients treated with these drugs. This toxicity is likely to stem from inhibition of trafficking down long nerve fibers, since neuronal cells rarely enter mitosis. In contrast to the above hypothesis by Komlodi-Pasztor et al., (2011) Kitagawa (2011) argues that new anti-mitotic agents are still needed and that tumor doubling time may not directly correlate with duration of the cell-cycle.

Given the essential role of MTs in the cell it is unlikely that only mitosis is being targeted, as MTAs can also inhibit cell migration, cellular trafficking and a number of other processes. It is also possible that MSAs have secondary targets that contribute to their therapeutic properties, for example, PTX phosphorylation of bcl-2. Proteomic studies focusing on trafficking proteins with and without MTAs would provide more insight into these processes.

\subsubsection{Angiogenesis}

Tumor vasculature is another good therapeutic target for chemotherapy, since the blood supplies the tumor with oxygen and nutrients (Tozer et al., 2005). If the blood supply is limited or cut off to a tumor, the tumor becomes necrotic. The tumor vasculature can be targeted in two ways, via anti-angiogenic pathways that prevent the formation of new blood vessels and by disruption of the established tumor vasculature. There is evidence that the vessels supplying a tumor are significantly different from normal vessels, due to many factors such as the high proliferative rate of endothelial cells and increased vascular permeability (Delmonte \& Sessa 2009). This makes it possible to selectively target the tumor vasculature. The way in which this occurs is poorly understood, although it has been shown that susceptibility correlates with increased permeability, and it is most likely related to factors in the microenvironment of the tumor that affect vascular permeability (Tozer et al., 2005). 
It is well known that COL has anti-vascular properties. Combretastatin A-4 (Figure 1.3), which binds to the COL site, was discussed in Chapter 1.4. A more soluble sodium salt analog of combretastatin A-4 is currently being developed as a vascular disrupting agent pro-drug. It is cleaved in the cell to combretastatin A-4 by endogenous non-specific phosphatases. A number of other COL derivatives have shown positive antitumor vascular effects in animal models and are at various stages of development in clinical trials (Delmonte \& Sessa 2009). The vascular disrupting effect of these compounds is present well below the maximum tolerated dose, with a wide therapeutic range (Dark et al., 1997). These compounds cause a rapid reorganization of the actin cytoskeleton via disruption of the tubulin cytoskeleton, resulting in increased vascular permeability and leading to shutdown of the tumor blood supply (Tozer et al., 2005). The key signaling pathway involved in this shutdown appears to be RHO-GTPase and RHO kinase. MT dynamics can regulate the activity of this important pathway, hence, the susceptibility to MTAs. It is likely the mechanism involves activation of RHO by guanine nucleotide binding factors (Wittmann \& Waterman-Storer, 2001).

Tumor cells have the potential to metastasize, and this involves formation of new blood vessels in the metastatic tumors. This angiogenesis process involves proliferation and migration of endothelial cells, with migration mediated by focal adhesions (connections to the extracellular matrix). Vinca alkaloids, taxoid site ligands and LAU all have varying degrees of anti-angiogenic activities through inhibition of cell and interference with the formation of focal adhesions (Zhou \& Giannakakou, 2005; Lu et al., 2006). It is well known that MTs are important in the directed migration of endothelial cells, and thus it is not surprising that MTAs can inhibit this process. Thus, tumor vasculature targeting represents a new forum in which these already successful drugs may show added benefits. 


\subsubsection{Aims of this chapter}

1. To determine how ZMP interacts with cell lines resistant to MSAs,

2. To investigate potential synergistic interactions, and

3. To determine if ZMP inhibits cell migration.

4. To generate a ZMP-resistant cell line

A number of cell lines that are resistant to MSAs have been developed. Before the crystal structure of the taxoid site was solved, these cell lines provided evidence of the possible location of the taxane binding site and the residues that interact with PTX. By testing the cytotoxicity of ZMP in these cells, insight into the location of its binding site should also be gained (Aim 1). As mentioned, MSAs can synergize with each other, and more often than not, MSAs with different binding sites are likely to synergize with one another given they can simultaneously bind to the same heterodimer. Thus, synergy assays should provide some insight into the ZMP binding site and also provide information on possible synergistic interactions that may be important clinically (Aim 2). With regard to vascular targeting, a number of different MTAs have shown promise as vascular targeting agents, and a number of MDAs are in clinical development for this reason. Many MSAs inhibit cell migration, an important process in the formation of new blood vessels, which gives evidence that they may have potential as anti-angiogenic agents. We therefore investigated the ability of ZMP to inhibit cell migration, to determine if like some MTAs ZMP is worth investigating as a potential anti-vascular agent (Aim 3). Additionally, an attempt to generate a ZMP-resistant cell line was made (Aim 4). The intention was to determine which amino acid mutations in tubulin conferred resistance to $\mathrm{ZMP}$, if any, by cloning and sequencing the $\alpha$ - and $\beta$-tubulin genes, thus providing insight into its binding site. 


\subsection{METHODS}

\subsubsection{Cell culture}

All cells were cultured as in Chapter 2.2, with variations in the medium supplements for different cell lines.

In the present study, a clone of the A2780 cell line (described in section 3.1.2) was used, the 1A9 ovarian carcinoma cell line. 1A9 cells were used to study synergy between MSAs and as a control for the 1A9 sub-line mutants PTX10, PTX22, A8, B10, $\mathrm{R} 1$ and L4, all of which are clones of the 1A9 cell line with single point mutations in the M40 gene of the class I isotype of $\beta$-tubulin. The parental (1A9) and $\beta$-tubulin mutant human ovarian carcinoma cell lines were a gift from Dr. Paraskevi Giannakakou (Weill Cornell Medical College, NY, USA). R1 cells were created in our laboratory by Dr. Anja Wilmes.

Mouse neuroblastoma cells (N2A) $\left(\right.$ ATCC $^{\circledR}$ CCL-131) ${ }^{\mathrm{TM}}$ were purchased from ATCC (Manassas, VA, USA) and cultured in RPMI-1640 medium (Invitrogen) supplemented with 10\% FCS, 2 mM L-glutamine (Sigma), 100 U/mL penicillin/streptomycin. Human skin fibroblast cells (D551) were a kind gift from Dr. Darren Day (VUW) $\left(\right.$ ATCC $^{\circledR}$ CCL-110) ${ }^{\mathrm{TM}}$, and were cultured in DMEM (high glucose, no pyruvate) medium (Invitrogen) supplemented with 10\% FCS, 100 units $/ \mathrm{mL}$ penicillin/streptomycin. Human umbilical vein endothelial cells (HUVECs), kind gifts from Dr. Darren Day (VUW) and Dr. Melanie McConnell (Malagan Institute of Medical Research), were cultured in MCDB131 medium (Invitrogen) supplemented with 2\% FCS, $10 \mathrm{ng} / \mathrm{mL}$ epidermal growth factor (Invitrogen), $12 \mu \mathrm{g} / \mathrm{mL}$ endothelial cell growth supplement (Sigma), $1 \mu \mathrm{g} / \mathrm{mL}$ hydrocortisone (Sigma), $10 \mathrm{U} / \mathrm{mL}$ heparin (Sigma), $2 \mathrm{mM}$ L-glutamine (Sigma) and $100 \mathrm{U} / \mathrm{mL}$ penicillin/streptomycin. Both D551 and HUVEC cells were washed $3 x$ in PBS before tyrpsinization to detach the cells. HUVEC cells were used at passage numbers $0-4$, since they are known to lose viability after passage 6 (Baudin et al., 2007).

\subsubsection{MTT cellular proliferation assay}

General methods for the MTT assay are described in Chapter 2.3 and were used for all cells. However, D551 and HUVEC cells were plated at $2 \times 10^{4}$ cells/well and treated with drug for $72 \mathrm{~h}$ or $120 \mathrm{~h}$. Caution needs to be made when diluting drugs down a plate since it is known that taxane compounds can bind to proteins in the culture medium and 
to plastic or glassware (Song et al., 1996). This can result in inconsistencies in the actual concentrations the cells are exposed to; thus, true $\mathrm{IC}_{50 \mathrm{~s}}$ cannot be correctly obtained since the drug does not reach a maximal inhibitory response. The problem can be eliminated by using separate pipette tips for each well dilution, and this was done for these experiments.

\section{Resistant cell studies}

Using the obtained $\mathrm{IC}_{50}$ values from the MTT assays, resistance ratios were calculated to compare the cytotoxicity of the compound in the parental cell line to that in the mutated cell line:

\section{Resistance Ratio $=\mathrm{IC}_{50}$ mutant cells $/ \mathrm{IC}_{50} 1 \mathrm{~A} 9$ cells}

Resistance ratio values were compared to a value of 1.0 using a one-sample Student's $t$ test (GraphPad Prism v5.0). Compounds with an average resistance ratio greater than one, in which $\mathrm{p}<0.05$, were taken to be significantly resistant to a drug.

\section{Synergy studies}

1A9 cells were treated with individual drugs or a combination of two drugs for $48 \mathrm{~h}$. For individual drugs, a 2-fold serial dilution was used to generate a concentration-response curve, and drugs in combination concentrations were chosen with respect to individual concentration-response curves for the compounds. Generally the combination doses were below the $\mathrm{IC}_{50}$ concentrations for each drug on its own, as it has been found that combination doses above the $\mathrm{IC}_{50}$ do not synergize (Wilmes et al., 2007). A number of different combination concentrations were chosen for each drug pair, and at least four biological replicates were used. Generally, concentrations of drug were chosen in the range that led to $50 \%$ inhibition of cell proliferation when added on their own to cells. Drug combinations can have effects that are antagonistic (drugs compete with one another and reduce their respective potencies), additive (the combined effect is the sum of the singular effects of the two drugs), or synergistic (an effect greater than the sum of the drugs individual effects).

The combination index (CI) equation (Chou \& Talalay, 1984; Berenbaum, 1985) is widely used to quantitatively assess synergy.

$$
\mathrm{CI}=\frac{\mathrm{D}_{1}}{\mathrm{D} x_{1}}+\frac{\mathrm{D}_{2}}{\mathrm{D} x_{2}}
$$


in which $\mathrm{D} x_{1}$ and $\mathrm{D} x_{2}$ are those concentrations used in the combination dose, $\mathrm{D}_{1}$ and $\mathrm{D}_{2}$ are the concentration of each drug if given individually that would produce the same response as the combination dose (determined from the corresponding concentrationresponse curve). A CI value of 1 denotes additive effects; a value less than 1 denotes a synergistic interaction between the two drugs, and a value greater than 1 denotes an antagonistic relationship.

In order to calculate the concentration of drug needed for a given response on its own, the following equation was used:

$$
\operatorname{Dose}(\mathrm{x})=\operatorname{IC} 50 *(\max -\text { response } / \text { response }-\min )^{1 / \text { hillslope }}
$$

$\mathrm{IC}_{50}$ curves were calculated as described in Chapter 2.3. The values for the min, max and hillslope were obtained from Sigma plot software programs. CI values were compared to a value of 1.0 using a one-sample Student's $t$-test (GraphPad Prism v5.0). Values in which $\mathrm{p}<0.05$ were seen as significant, and only CI values of $\leq 0.8$ were considered to be effective synergy. When synergy was found between a drug pair, repeats were carried out to ensure a robust statistical CI value representing synergy for those compounds.

\subsubsection{Wound scratch healing assay}

A number of different assays exist to study cellular migration and these have been recently reviewed in Kramer et al., 2013. In this study we used a wound scratch method derived from Rodriguez et al., 2005. On the bottom of a 24-well plate, two lines were drawn down the middle of each well (approximately $5 \mathrm{~mm}$ apart), and another line was drawn through the center, perpendicular to these lines. These lines divide the center of the plate into quadrants and are used to ensure the same section of the plate is measured at each of the time points. Cells were plated at $5 \times 10^{4}$ cells/well and grown for 2 days or until confluent. Cells were treated with drug for $5 \mathrm{~h}$ in medium without growth factors (D551 cells: DMEM high glucose medium supplemented with $100 \mathrm{U} / \mathrm{mL}$ penicillin/streptomycin. HUVEC cells: MCDB131 medium supplemented with $1 \mu \mathrm{g} / \mathrm{mL}$ hydrocortisone, $10 \mathrm{U} / \mathrm{mL}$ heparin, $2 \mathrm{mM}$ L-glutamine and $100 \mathrm{U} / \mathrm{mL}$ penicillin/streptomycin) to starve the cells of pro-migratory factors in the medium. A perspex plate insert with a $200 \mu \mathrm{L}$ pipette tip holder (made by Craig Doney, VUW) was 
used as a mechanical guide to create a consistent scratches with a sterile $200 \mu \mathrm{L}$ pipette tip, placed between the previously drawn lines in each of the wells. Cells were then washed 3 times in warm 1 x PBS buffer ( $\mathrm{pH}$ 7.4) and complete medium containing the drug added to the cells. This is time point zero $(0 \mathrm{~h})$. Photos of each of the wells were taken at 0 and $18 \mathrm{~h}$ using an Olympus IX51 inverted microscope using the 4X objective lens. Both photos were taken of the same area using the marker lines as a guide to orientate the images. The area of the wound was then calculated using the open resource tool of Image J (NIH, MD, USA). The extent of wound healing or cell migration was determined by measuring the area of the open wound at both $0 \mathrm{~h}$ and $18 \mathrm{~h}$ and calculating the percentage of wound closure after $18 \mathrm{~h}$ using the following calculation:

$$
\text { Wound healing }=100-[(\text { wound area } 18 \mathrm{hr}) /(\text { wound area } 0 \mathrm{hr})]^{*} 100
$$

The total wound closure for each of the different conditions was then analyzed using a one-way ANOVA, and the drug-treated samples compared to that of the control using a Dunnett's multiple comparison post-test (GraphPad Prism v5.0).

\subsubsection{Generation of a zampanolide-resistant cell line}

1A9 cells were grown in increasing concentrations of ZMP, starting at a low concentration just below the $\mathrm{IC}_{50}$ and increasing by 2-4 $\mathrm{nM}$ with each step. Although verapamil (a P-gp pump inhibitor) is usually used in the creation of resistant cell lines so that cells with the MDR phenotype are not selected for, given the unique binding profile of ZMP (discussed in Chapter 5) and the finding that DAC is not a substrate of the P-gp drug efflux pump (Prof. Karl-Heinz Altmann, personal communication, unpublished data), verapamil was omitted in the creation of this cell line. Cells were treated with ZMP for 1-2 days, given new medium and the next day passaged and grown to confluency in drug-free medium. This passage step into drug-free medium was necessary to select for viable cells. Cell morphology was monitored as the concentration at which they recovered from was increased. To determine their resistance to ZMP, MTT assays were performed one year after the cells were first grown in ZMP. The sensitivity of the cells to other MSA was also tested to determine if there was any difference in their cytotoxicity to different drugs as this would provide insight into the mutation (if any) that may have occurred in the cells. 


\subsection{RESULTS}

\subsubsection{Cytotoxicity of ZMP in different cell lines}

The cytotoxicity of ZMP was measured in a number of different cell lines with the MTT cell proliferation assay (Table 3.2). As expected no significant difference between natural and synthetic $\mathrm{ZMP}$ was observed. The $\mathrm{IC}_{50}$ of $\mathrm{ZMP}$ in all cell lines was in the low nanomolar range, indicating that it is a powerful cytotoxic agent, as initially shown by Tanka \& Higa (1996) and Field et al. (2009). In N2A cells, ZMP had a significantly higher $\mathrm{IC}_{50}$ value compared to the other cell lines tested and also compared to PTX and DTX. PTX and DTX had $\mathrm{IC}_{50}$ values of $81.4 \pm 11.2$ and $210 \pm 41 \mathrm{nM}$, respectively. These values appeared to be high compared to those previously determined in our lab $\left(\right.$ PTX $\mathrm{IC}_{50}$ value $=24 \mathrm{nM}$, Dr. Viswanath Das personal communication $)$. These cells were particularly hard to culture and obtain a concentration-response curve from. N2A cells are larger and known to contain large quantities of tubulin protein, and this may contribute to the higher concentration of drug required to inhibit cell proliferation. Additionally, neuroblastoma cells may not divide as rapidly as the other cell lines. 1A9 cell doubling time is approximately $24 \mathrm{~h}$ (Giannakakou et al., 2000), where N2A cells have a doubling time of $26.2 \mathrm{~h}$, however depending on the expression of certain proteins in N2A cells this time can vary significantly (Wicki-Stordeur et al., 2012). Moreover, $\mathrm{N} 2 \mathrm{~A}$ cells are very easily differentiated and this results in highly variable $\mathrm{IC}_{50}$ values (Dr. Viswanath Das personal communication). Overall ZMP is a potent MSA growth inhibitory or cytotoxic agent at low nanomolar concentrations in a number of different cell lines.

Table 3.2 Cytotoxicity of ZMP in different cell lines

\begin{tabular}{cccc}
\hline Cell line & $\mathrm{IC}_{50} \pm \mathrm{SEM}(\mathrm{nM})$ & Duration $(h)$ & $n$ \\
\hline 1A9 & $9.5 \pm 0.9$ & 48 & 46 \\
1A9 & $8.2 \pm 0.1$ & 72 & 28 \\
1A9 & $4.6 \pm 1.3$ & 72 & 6 \\
1A9 (Field et al., 2009) & $14.3 \pm 2.4$ & 72 & 6 \\
HL-60 (Field et al., 2009) & $4.3 \pm 1.1$ & 48 & 5 \\
D551 & $7.3 \pm 1.2$ & 72 & 5 \\
HUVEC & $0.6 \pm 0.1$ & 72 & 3 \\
HUVEC & $1.0 \pm 0.4$ & 120 & 3 \\
N2A & $299.9 \pm 14.1$ & 72 & 6 \\
\hline
\end{tabular}

$\mathrm{IC}_{50}$ values of ZMP in different cell lines determined using the MTT cell proliferation assay. Duration is the time in which each cell line was treated with ZMP before MTT was added. $n$ is the number of independent biological replicates. Black entries were $\mathrm{IC}_{50}$ values obtained using natural $\mathrm{ZMP}$; those in blue were obtained using synthetic ZMP. No difference in cytotoxicity of natural versus synthetic ZMP was seen in this study, as has been reported previously (Field et al., 2012). 


\subsubsection{Profile of zampanolide in resistant cell lines}

The cytotoxic or growth inhibitory activity of different MSAs in resistant cell lines were tested using an MTT cell proliferation assay (Table 3.3), and the resistance ratios for each drug in the mutant cell lines was calculated (Table 3.4; Figure 3.1). The cytotoxicity of IXA in these cell lines has not been tested previously. Consistent with previous studies, PTX10, PTX22, A8 and B10 cells were all significantly resistant to PTX and also to IXA, in the present study, given these cells all have mutations in the taxoid binding site (Giannakakou et al., 1997; Giannakakou et al., 2000). Although, EPOB has been shown to not be affected by the mutations in the PTX10 and PTX22 cell lines, suggesting it binds in a different manner, these cell lines showed significant resistance to IXA in the present study. As expected all four taxoid site cell lines demonstrated no resistance to LAU and PEL. R1 and L4 cells were sensitive to taxoid site ligands, with R1 cells resistant to PEL but not LAU and L4 cells resistant to both PEL and LAU (Table 3.3, 3.4; Figure 3.1) as previously determined (Kanakkanthara et al., 2011). PTX10 and A8 cells were not resistant to ZMP, with average $\mathrm{IC}_{50}$ values less than those in 1A9 cells. ZMP appeared to be slightly less active in PTX22 cells with a resistance ratio of 2.37; however, this was not statistically significant. B10 cells were resistant to ZMP with a resistance ratio of 3.21; however, the resistance profile of ZMP in these cells was significantly less than for PTX and IXA, which had resistance ratios of 34 and 20, respectively. Although showing some resistance these cells are far more sensitive to ZMP than to traditional taxoid site ligands. R1 cells were significantly more sensitive to ZMP with a resistance ratio of 0.56 . L4 cells showed a similar sensitivity to ZMP as $1 \mathrm{~A} 9$ cells (Figure 3.1).

Table 3.3 $\mathrm{IC}_{50}$ values (nM) for MSAs in $1 \mathrm{~A} 9$ parental cells and mutant cell lines

\begin{tabular}{cccccc}
\hline & Paclitaxel & Ixabepilone & Laulimalide & Peloruside A & Zampanolide \\
\hline 1A9 & $4.2 \pm 0.3$ & $7.3 \pm 0.6$ & $8.3 \pm 0.5$ & $20.1 \pm 0.9$ & $8.2 \pm 1.0$ \\
PTX10 & $91.7 \pm 8.2$ & $54.9 \pm 9.6$ & $11.0 \pm 1.0$ & $17.5 \pm 1.2$ & $2.3 \pm 0.9$ \\
PTX22 & $100 \pm 14.1$ & $11.4 \pm 1.7$ & $10.6 \pm 2.7$ & $19.4 \pm 5.4$ & $9.2 \pm 3.9$ \\
A8 & $94.4 \pm 5.6$ & $99.8 \pm 0.6$ & $7.2 \pm 1.1$ & $14.0 \pm 2.4$ & $14.9 \pm 4.6$ \\
B10 & $17.2 \pm 4.3$ & $106 \pm 6.5$ & $10.8 \pm 1.0$ & $24.9 \pm 1.9$ & $8.6 \pm 3.2$ \\
R1 & $8.8 \pm 2.5$ & $14.7 \pm 3.3$ & $9.8 \pm 1.5$ & $90.9 \pm 8.5$ & $5.9 \pm 1.6$ \\
L4 & $4.2 \pm 0.1$ & $4.4 \pm 0.4$ & $344 \pm 150$ & $351 \pm 126$ & $4.7 \pm 1.0$ \\
\hline
\end{tabular}

The average $\mathrm{IC}_{50}$ values $(\mathrm{nM})(72 \mathrm{~h})$ of MSAs in the parental $1 \mathrm{~A} 9$ cell line and the various cloned mutant cell lines. $n=3$ or more biological replicates. Data presented as the mean $\mathrm{IC}_{50}$ values \pm SEM. 67 Refer to table 3.1 for cell line identification.

The $\mathrm{IC}_{50}$ values for LAU treatment of L4 cells were obtained by Dr. Arun Kanakkanthara. 
Table 3.4 Resistant ratios for MSAs in mutant cells compared to 1A9 parental cells

\begin{tabular}{|c|c|c|c|c|c|c|c|c|c|c|}
\hline & Paclitaxel & $\mathrm{p}$-value & Ixabepilone & $\mathrm{p}$-value & Laulimalide & $\mathrm{p}$-value & Peloruside A & p-value & Zampanolide & p-value \\
\hline PTX10 & $21.5 \pm 2.74$ & $0.0017 * *$ & $10.3 \pm 0.83$ & $0.0004 * * *$ & $1.41 \pm 0.26$ & ns & $0.98 \pm 0.02$ & ns & $0.76 \pm 0.17$ & ns \\
\hline PTX22 & $23.7 \pm 3.69$ & $0.0035 * *$ & $2.32 \pm 0.43$ & 0.0375 & $1.18 \pm 0.19$ & ns & $1.08 \pm 0.31$ & ns & $2.37 \pm 0.17$ & ns \\
\hline A8 & $4.73 \pm 0.49$ & $0.0015 * *$ & $18.8 \pm 2.83$ & $0.0033 * *$ & $1.11 \pm 0.16$ & ns & $1.13 \pm 0.07$ & ns & $0.61 \pm 0.17$ & ns \\
\hline B10 & $34.3 \pm 7.12$ & $0.0188 *$ & $19.8 \pm 2.59$ & $0.0054 * *$ & $0.87 \pm 0.16$ & ns & $0.98 \pm 0.29$ & ns & $3.21 \pm 0.64$ & $0.0187 *$ \\
\hline $\mathrm{R} 1$ & $1.81 \pm 0.54$ & ns & $1.63 \pm 0.47$ & ns & $1.05 \pm 0.10$ & ns & $4.65 \pm 0.32$ & $0.0015 * *$ & $0.56 \pm 0.11$ & $0.0032 * *$ \\
\hline L4 & $1.25 \pm 0.16$ & ns & $0.56 \pm 0.06$ & ns & $21.1 \pm 0.35$ & $0.0495 *$ & $17.1 \pm 6.52$ & $0.0487 *$ & $0.83 \pm 0.20$ & ns \\
\hline
\end{tabular}

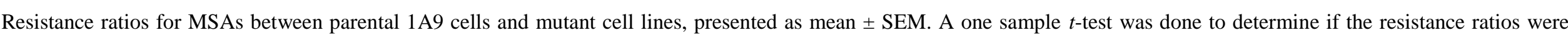

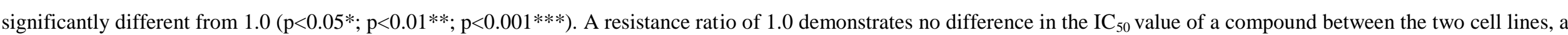

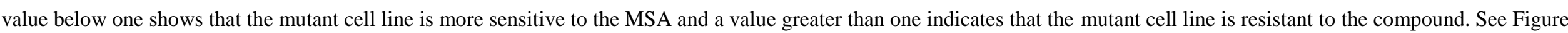
6.1 below. 
Paclitaxel-treated

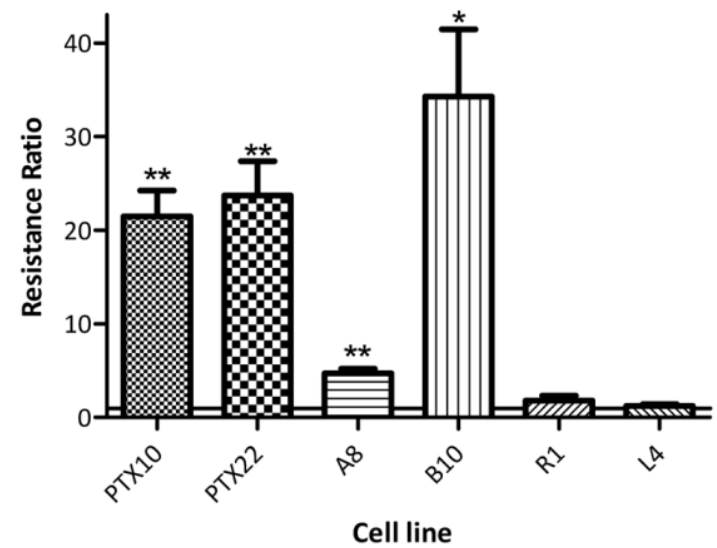

Laulimalide-treated

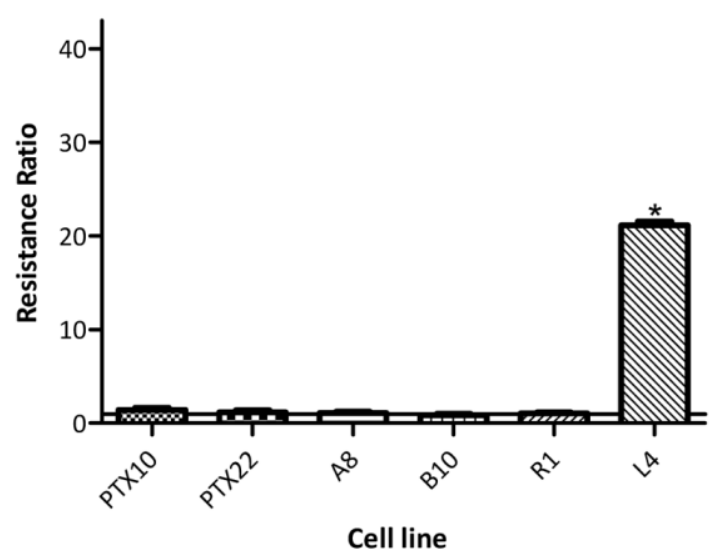

Ixabepilone-treated

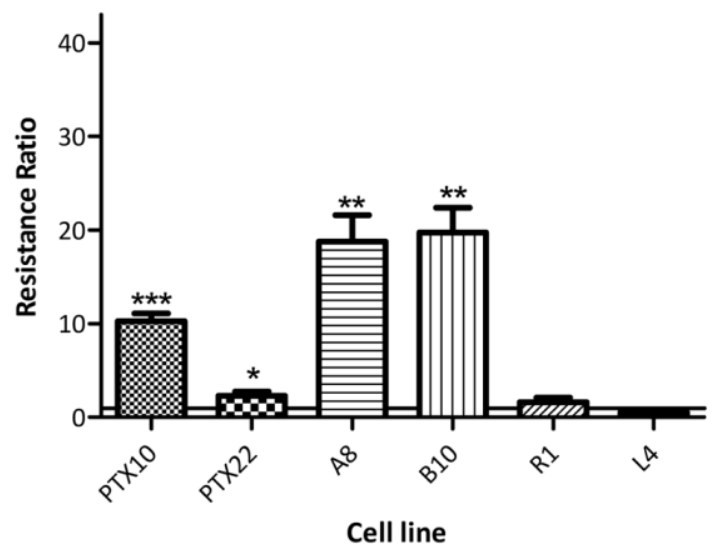

Peloruside A-treated

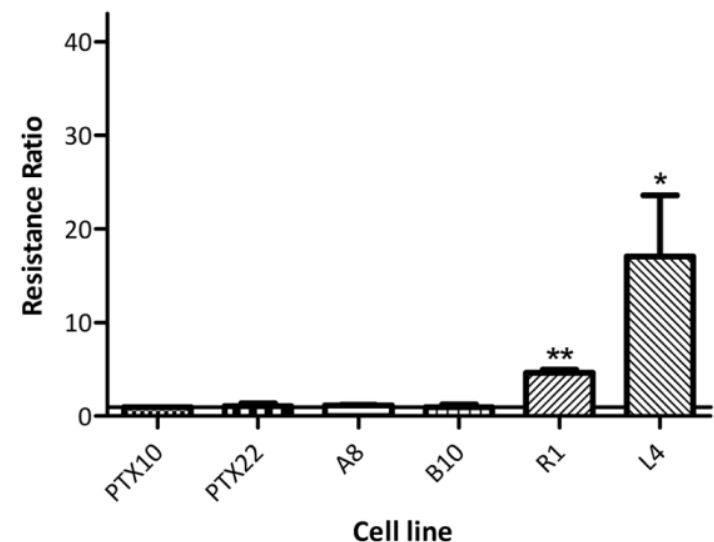

Zampanolide-treated

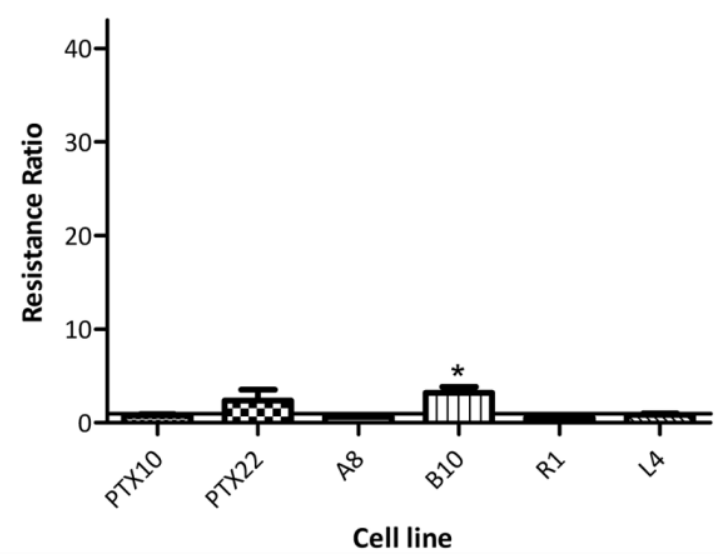

Figure 3.1 Resistance ratios of MSAs in mutant cell lines

Graphs showing the resistance ratios for MSAs between parental 1A9 cells and mutant cell lines. A one sample $t$-test was done to determine if the resistance ratios were significantly different from 1.0 $\left(\mathrm{p}<0.05^{*} ; \mathrm{p}<0.01^{* *} ; \mathrm{p}<0.001^{* * *}\right)$. Y-axis of all graphs kept the same for easy comparison between MSAs. 


\subsubsection{Zampanolide does not synergize with other MSAs}

To indirectly probe the binding site of ZMP, its ability to synergize with MSAs with known binding sites was investigated. To test for synergy between ZMP and other MSAs in their ability to inhibit cell proliferation, 1A9 cells were incubated with drugs alone or in combination for $48 \mathrm{~h}$. See Table 3.5 for $\mathrm{IC}_{50}$ values of each drug on its own in a $48 \mathrm{~h}$ MTT cell proliferation assay. Various concentrations of MSAs were chosen to look for possible synergy between ZMP and other MSAs. Control combinations of MSAs known to give synergy were used to validate the results with ZMP (Table 3.6; Figure 3.2). Combinations of PTX and DTX were used as a negative control (no synergy expected) since these drugs are analogs occupying the same binding pocket and have previously been shown to be significantly antagonistic in combination with one another (Wilmes et al., 2011). Combinations of PTX and DSC were used as a positive control since these two MSAs have been shown to synergize with each other (Martello et al., 2000; Honore et al., 2004; Huang et al., 2006; Wilmes et al., 2011). Given that synergy was only seen by Wilmes et al. (2011) at low concentrations of DSC (relative to its $\mathrm{IC}_{50}$ value), similar concentrations were used in this study. Since PEL and IXA have been shown to be synergistic in murine splenocytes and $\mathrm{CD} 4^{+} \mathrm{T}$ cells, their ability to synergize in $1 \mathrm{~A} 9$ cells was investigated in the present study. Synergy between PTX and IXA was tested since it has not been done before, although no synergy is expected. These combinations are expected to be additive or antagonistic given the overlapping binding site of these two compounds, however, synergy has been observed at some dose combinations between PTX and EPOA (Wilmes et al., 2007). The ability of ZMP to synergize with PTX, IXA, DSC, PEL and LAU was tested (Table 3.7; Figure 3.3).

As expected, PTX and DSC synergized with one another at a number of different concentrations, with CI values below 0.65 , indicating a strong synergistic relationship between the two compounds. PEL and IXA are expected to synergize with one another given their distinct binding sites, and all but three concentrations tested gave CI values below 1.0; however, only 5 of these values were statistically significant. As expected PTX and DTX did not synergize, giving high CI values for all concentrations tested. Two of these concentrations were significantly antagonistic. As expected, PTX and IXA did not synergize. ZMP showed no statistically significant synergy with PTX, IXA or DSC, with two combinations of ZMP and PTX being significantly antagonistic. Interestingly, two concentrations of ZMP and PTX and two of ZMP and DSC had CI values indicative 
of synergy; however, these were not significant using a Student's $t$-test. This suggested, along with the resistance cell data, that ZMP may be a ligand of the taxoid binding site but with a distinct pose within the site. Thus, in theory ZMP was expected to show synergy with PEL and/or LAU. However, this proved not to be the case, all concentrations of ZMP/PEL and ZMP/LAU tested showed no synergy except for one concentration of ZMP/PEL that gave a CI value of 0.79 . Although this was significantly different from $1.0(\mathrm{p}=0.0002)$, it is considered a weak synergistic relationship. The CI values obtained with ZMP and LAU were all significantly greater than 1.0, showing an antagonistic relationship between these two MSAs. CI values for ZMP and PEL combinations were additive rather than antagonistic.

Table $3.548 \mathrm{~h} \mathrm{IC}_{50}$ values for individual compounds

\begin{tabular}{cc}
\hline Compound & $\mathrm{IC}_{50} \pm$ SEM $(\mathrm{nM})$ \\
\hline Zampanolide $(n=46)$ & $9.54 \pm 0.85$ \\
Paclitaxel $(n=26)$ & $3.71 \pm 0.30$ \\
Docetaxel $(n=6)$ & $3.55 \pm 0.43$ \\
Ixabepilone $(n=28)$ & $6.65 \pm 0.33$ \\
Discodermolide $(n=18)$ & $137.93 \pm 11.58$ \\
Peloruside $_{(n=29)}$ & $23.28 \pm 1.05$ \\
Laulimalide $_{(n=7)}$ & $9.71 \pm 0.28$ \\
\hline
\end{tabular}

$\mathrm{IC}_{50}$ values (mean $\left.\pm \mathrm{SEM}\right)$ after $48 \mathrm{~h}$ of drug treatment are presented in $1 \mathrm{~A} 9$ cells $(n=$ the number of independent biological replicates) 
Table 3.6 CI values for different MSA combinations in 1A9 cells

\begin{tabular}{|c|c|c|c|c|}
\hline Paclitaxel & Discodermolide & $\mathrm{CI} \pm \mathrm{SEM}$ & $n$ & p-value \\
\hline $2 \mathrm{nM}$ & $3 \mathrm{nM}$ & $0.58 \pm 0.15$ & 6 & 0.0454 \\
\hline $2 \mathrm{nM}$ & $4 \mathrm{nM}$ & $0.37 \pm 0.06$ & 6 & 0.0002 \\
\hline $2 \mathrm{nM}$ & $7 \mathrm{nM}$ & $0.63 \pm 0.07$ & 6 & 0.0039 \\
\hline $2 \mathrm{nM}$ & $10 \mathrm{nM}$ & $0.52 \pm 0.14$ & 6 & 0.0184 \\
\hline $2 \mathrm{nM}$ & $15 \mathrm{nM}$ & $0.54 \pm 0.10$ & 6 & 0.0064 \\
\hline $3 \mathrm{nM}$ & $15 \mathrm{nM}$ & $0.92 \pm 0.16$ & 6 & ns \\
\hline Peloruside A & Ixabepilone & $\mathrm{CI} \pm \mathrm{SEM}$ & $n$ & p-value \\
\hline $5 \mathrm{nM}$ & $2.5 \mathrm{nM}$ & $0.71 \pm 0.12$ & 5 & ns \\
\hline $5 \mathrm{nM}$ & $10 \mathrm{nM}$ & $1.08 \pm 0.09$ & 4 & ns \\
\hline $6 \mathrm{nM}$ & $4 \mathrm{nM}$ & $0.75 \pm 0.13$ & 5 & ns \\
\hline $8 \mathrm{nM}$ & $2.5 \mathrm{nM}$ & $0.50 \pm 0.15$ & 5 & 0.0327 \\
\hline $10 \mathrm{nM}$ & $5 \mathrm{nM}$ & $0.85 \pm 0.12$ & 6 & ns \\
\hline $10 \mathrm{nM}$ & $6 \mathrm{nM}$ & $1.03 \pm 0.16$ & 6 & ns \\
\hline $15 \mathrm{nM}$ & $2.5 \mathrm{nM}$ & $0.46 \pm 0.11$ & 5 & 0.0082 \\
\hline $15 \mathrm{nM}$ & $3 \mathrm{nM}$ & $0.69 \pm 0.13$ & 5 & ns \\
\hline $15 \mathrm{nM}$ & $4 \mathrm{nM}$ & $0.70 \pm 0.09$ & 6 & 0.0252 \\
\hline $15 \mathrm{nM}$ & $5 \mathrm{nM}$ & $0.76 \pm 0.15$ & 5 & ns \\
\hline $15 \mathrm{nM}$ & $6 \mathrm{nM}$ & $0.86 \pm 0.12$ & 5 & ns \\
\hline $16 \mathrm{nM}$ & $4 \mathrm{nM}$ & $0.68 \pm 0.12$ & 5 & 0.485 \\
\hline $20 \mathrm{nM}$ & $6 \mathrm{nM}$ & $0.75 \pm 0.09$ & 6 & 0.0378 \\
\hline $20 \mathrm{nM}$ & $10 \mathrm{nM}$ & $1.11 \pm 0.12$ & 5 & ns \\
\hline Paclitaxel & Docetaxel & $\mathrm{CI} \pm \mathrm{SEM}$ & $n$ & p-value \\
\hline $1 \mathrm{nM}$ & $3 \mathrm{nM}$ & $1.57 \pm 0.27$ & 5 & ns \\
\hline $2 \mathrm{nM}$ & $2 \mathrm{nM}$ & $2.39 \pm 0.59$ & 5 & ns \\
\hline $2 \mathrm{nM}$ & $3 \mathrm{nM}$ & $1.40 \pm 0.05$ & 4 & 0.0035 \\
\hline $3 \mathrm{nM}$ & $1 \mathrm{nM}$ & $2.95 \pm 1.09$ & 6 & ns \\
\hline $3 \mathrm{nM}$ & $2 \mathrm{nM}$ & $2.43 \pm 0.67$ & 6 & ns \\
\hline $3 \mathrm{nM}$ & $3 \mathrm{nM}$ & $1.44 \pm 0.88$ & 6 & 0.0039 \\
\hline Paclitaxel & Ixabepilone & $\mathrm{CI} \pm \mathrm{SEM}$ & $n$ & p-value \\
\hline $2 \mathrm{nM}$ & $2.5 \mathrm{nM}$ & $1.87 \pm 0.56$ & 6 & ns \\
\hline $2 \mathrm{nM}$ & $5 \mathrm{nM}$ & $0.95 \pm 0.18$ & 5 & ns \\
\hline $3 \mathrm{nM}$ & $3 \mathrm{nM}$ & $0.70 \pm 0.13$ & 6 & ns \\
\hline $4 \mathrm{nM}$ & $3 \mathrm{nM}$ & $1.05 \pm 0.29$ & 5 & ns \\
\hline $4 \mathrm{nM}$ & $4 \mathrm{nM}$ & $1.27 \pm 0.21$ & 5 & ns \\
\hline $5 \mathrm{nM}$ & $2.5 \mathrm{nM}$ & $1.08 \pm 0.10$ & 5 & ns \\
\hline
\end{tabular}


Table 3.7 CI values for ZMP + MSA combinations in 1A9 cells

\begin{tabular}{|c|c|c|c|c|c|}
\hline Zampanolide & Paclitaxel & $\mathrm{CI} \pm \mathrm{SEM}$ & $n$ & p-value & \\
\hline $1 \mathrm{nM}$ & $1 \mathrm{nM}$ & $0.68 \pm 0.19$ & 4 & ns & \\
\hline $2 \mathrm{nM}$ & $1 \mathrm{nM}$ & $1.54 \pm 0.50$ & 4 & ns & \\
\hline $2 \mathrm{nM}$ & $2 \mathrm{nM}$ & $2.75 \pm 0.30$ & 4 & 0.0102 & \\
\hline $4 \mathrm{nM}$ & $1 \mathrm{nM}$ & $0.63 \pm 0.23$ & 4 & ns & \\
\hline $4 \mathrm{nM}$ & $2 \mathrm{nM}$ & $1.74 \pm 0.17$ & 4 & 0.0238 & \\
\hline $5 \mathrm{nM}$ & $2 \mathrm{nM}$ & $2.88 \pm 1.24$ & 4 & ns & \\
\hline Zampanolide & Ixabepilone & $\mathrm{CI} \pm \mathrm{SEM}$ & $n$ & p-value & \\
\hline $1 \mathrm{nM}$ & $2.5 \mathrm{nM}$ & $1.22 \pm 0.23$ & 4 & ns & \\
\hline $2 \mathrm{nM}$ & $2 \mathrm{nM}$ & $2.05 \pm 0.79$ & 5 & $\mathrm{~ns}$ & \\
\hline $2 \mathrm{nM}$ & $2.5 \mathrm{nM}$ & $2.01 \pm 0.58$ & 5 & ns & \\
\hline $2 \mathrm{nM}$ & $5 \mathrm{nM}$ & $1.78 \pm 0.30$ & 5 & ns & \\
\hline $4 \mathrm{nM}$ & $2.5 \mathrm{nM}$ & $1.22 \pm 0.15$ & 7 & ns & \\
\hline $4 \mathrm{nM}$ & $5 \mathrm{nM}$ & $1.56 \pm 0.27$ & 7 & ns & \\
\hline $5 \mathrm{nM}$ & $2.5 \mathrm{nM}$ & $1.07 \pm 0.18$ & 7 & ns & \\
\hline Zampanolide & Discodermolide & $\mathrm{CI} \pm \mathrm{SEM}$ & $n$ & p-value & \\
\hline $2 \mathrm{nM}$ & $7 \mathrm{nM}$ & $1.00 \pm 0.34$ & 5 & ns & \\
\hline $2 \mathrm{nM}$ & $15 \mathrm{nM}$ & $0.76 \pm 0.24$ & 5 & ns & \\
\hline $2 \mathrm{nM}$ & $50 \mathrm{nM}$ & $1.10 \pm 0.24$ & 5 & ns & \\
\hline $5 \mathrm{nM}$ & $3 \mathrm{nM}$ & $1.04 \pm 0.23$ & 5 & $\mathrm{~ns}$ & \\
\hline $5 \mathrm{nM}$ & $10 \mathrm{nM}$ & $1.03 \pm 0.27$ & 5 & ns & \\
\hline $5 \mathrm{nM}$ & $15 \mathrm{nM}$ & $0.88 \pm 0.17$ & 5 & ns & \\
\hline $5 \mathrm{nM}$ & $20 \mathrm{nM}$ & $1.09 \pm 0.19$ & 5 & ns & \\
\hline $5 \mathrm{nM}$ & $50 \mathrm{nM}$ & $0.84 \pm 0.17$ & 5 & ns & \\
\hline $10 \mathrm{nM}$ & $15 \mathrm{nM}$ & $0.90 \pm 0.17$ & 5 & ns & \\
\hline $10 \mathrm{nM}$ & $20 \mathrm{nM}$ & $0.74 \pm 0.14$ & 5 & ns & \\
\hline Zampanolide & Peloruside A & $\mathrm{CI} \pm \mathrm{SEM}$ & $n$ & p-value & \\
\hline $2 \mathrm{nM}$ & $5 \mathrm{nM}$ & $1.36 \pm 0.25$ & 4 & $\mathrm{~ns}$ & \\
\hline $2 \mathrm{nM}$ & $10 \mathrm{nM}$ & $1.15 \pm 0.12$ & 7 & ns & \\
\hline $2 \mathrm{nM}$ & $12 \mathrm{nM}$ & $0.92 \pm 0.10$ & 4 & ns & \\
\hline $5 \mathrm{nM}$ & $5 \mathrm{nM}$ & $1.04 \pm 0.12$ & 4 & ns & \\
\hline $5 \mathrm{nM}$ & $10 \mathrm{nM}$ & $1.01 \pm 0.08$ & 8 & ns & \\
\hline $5 \mathrm{nM}$ & $12 \mathrm{nM}$ & $0.88 \pm 0.07$ & 4 & ns & \\
\hline $5 \mathrm{nM}$ & $15 \mathrm{nM}$ & $0.93 \pm 0.05$ & 8 & $\mathrm{~ns}$ & \\
\hline $10 \mathrm{nM}$ & $15 \mathrm{nM}$ & $0.79 \pm 0.03$ & 7 & 0.0002 & \\
\hline Zampanolide & Laulimalide & $\mathrm{CI} \pm \mathrm{SEM}$ & $\mathbf{n}$ & p-value & \\
\hline $2 \mathrm{nM}$ & $5 \mathrm{nM}$ & $1.24 \pm 0.20$ & 4 & $\mathrm{~ns}$ & \multirow{4}{*}{73} \\
\hline $2 \mathrm{nM}$ & $10 \mathrm{nM}$ & $1.32 \pm 0.06$ & 8 & 0.001 & \\
\hline $5 \mathrm{nM}$ & $5 \mathrm{nM}$ & $1.31 \pm 0.09$ & 7 & 0.0109 & \\
\hline $5 \mathrm{nM}$ & $10 \mathrm{nM}$ & $1.30 \pm 0.09$ & 8 & 0.0143 & \\
\hline
\end{tabular}




\section{Table 3.6 Control Combination index values for MSAs}

CI values for various combinations of MSAs are presented from a $48 \mathrm{~h}$ MTT assay as controls for the ZMP data of Table 3.7. Data correspond to data in Figure 3.2. p-values were calculated from a onesample Student's t-test. ( $\mathrm{n}=$ biological replicates). CI values showing statistically significant synergy have their p-values presented in bold.

Table 3.7 Combination index values for ZMP with selected MSAs

Calculated CI values for synergy assays of specific combinations of ZMP with selected MSAs are presented. Data correspond to data in Figure 3.3. p-values were calculated from a one-sample Student's $t$-test. ( $\mathrm{n}=$ number of biological replicates). CI values showing statistically significant synergy have their $\mathrm{p}$-values presented in bold. Concentrations used were based on the $\mathrm{IC}_{50}$ values of the compounds when used individually and on previous data obtained in our laboratory (Wilmes et al., 2007, 2011).

A CI value of 1 denotes additive effects; a value less than 1 denotes a synergistic interaction between the two drugs, and a value greater than 1 denotes an antagonistic relationship. 

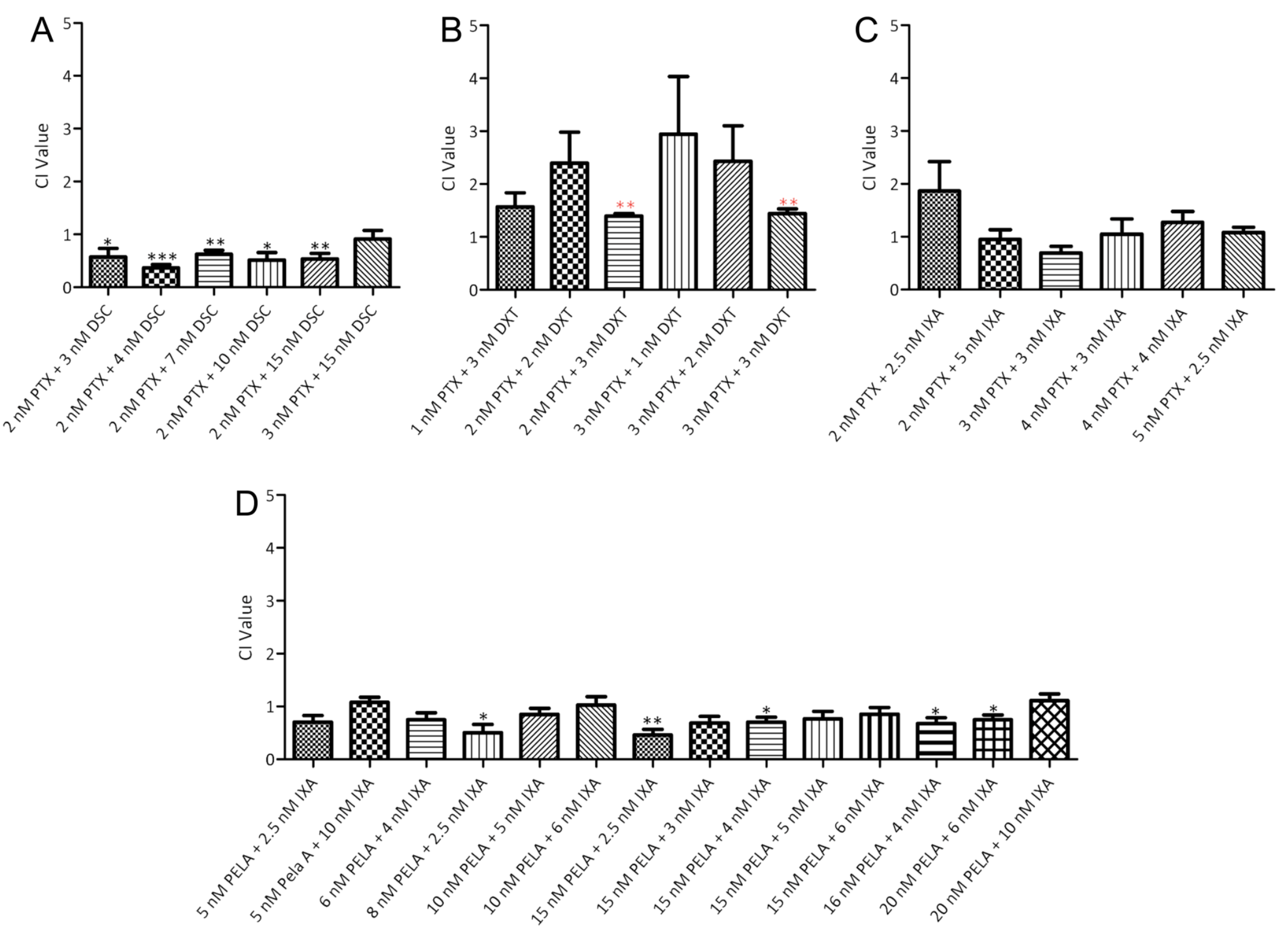

Figure 3.2 Synergy interactions of selected MSAs with one another

Graphs correspond to the data in Table 3.4. Asterisks indicate significant differences from 1.0 (one-sample Student's $t$-test). Those in red shown antagonism. * $<<0.05$; ** $\mathrm{p}<0.01$; $* * * p<0.00$. Data are the mean \pm SEM. 


\subsubsection{Zampanolide inhibits wound healing}

Angiogenesis is a viable target for treating cancer. During angiogenesis, endothelial cells proliferate and migrate in response to signals secreted from tumor cells. To determine if ZMP could inhibit cell migration, wound scratch assays were carried out in both human endothelial (HUVEC) and fibroblast (D551) cells. First the ability of both ZMP and DTX to inhibit the proliferation of theses cell lines was assessed. ZMP and DTX were shown by MTT assay to significantly inhibit the proliferation of both cell lines with low $\mathrm{IC}_{50}$ values. The $\mathrm{IC}_{50}$ values of $\mathrm{ZMP}$ in HUVEC cells were $0.60 \pm 0.13 \mathrm{nM}$ in a $72 \mathrm{~h}$ assay $(\mathrm{n}=3)$ and $1.00 \pm 0.44 \mathrm{nM}$ in a $120 \mathrm{~h}$ assay $(\mathrm{n}=3)$. DTX had an $\mathrm{IC}_{50}$ value of $1.7 \pm 0.3 \mathrm{nM}$ in HUVEC cells $(120 \mathrm{~h})(\mathrm{n}=3)\left(\mathrm{IC}_{50}\right.$ value obtained from $\mathrm{Dr}$. A. Chan). The $\mathrm{IC}_{50}$ values of ZMP and DTX in D551 cells were $7.27 \pm 1.21 \mathrm{nM}(\mathrm{n}=5)$ and $0.91 \pm 0.2 \mathrm{nM}(\mathrm{n}=3)(72$ h), respectively. The concentrations of ZMP and DTX that potentially could inhibit wound healing were chosen that were less than the $\mathrm{IC}_{50}$, as it has previously been shown that concentrations of MSAs substantially lower than those required to inhibit HUVEC proliferation are able to inhibit cell migration (Lu et al., 2006).

\section{HUVEC wound healing}

DTX was used as a positive control as it is known to significantly inhibit cell migration in wound scratch assays (Lu et al., 2006). To create the 'wound', a Perspex apparatus was used as this assay requires an accurately sized scratch wound. Before a scratch was made in the cell monolayer, the cells were cultured in drug-containing, serum-free medium for $5 \mathrm{~h}$ to minimize possible 'pro-migratory' effects of the fully supplemented medium the cells were grown in. Inhibition of wound closure was calculated by comparing the area $\left(\mu \mathrm{m}^{2}\right)$ of the wound at $0 \mathrm{~h}$ with that at $18 \mathrm{~h}$ and the percentage change in area compared to that of the control (no drug). In the controls, $76.3 \pm 1.7 \%$ of the wound closed over by $18 \mathrm{~h}$. Treatment with MSAs had a significant inhibitory effect on wound closure ( $<<0.0001$, one-way ANOVA), with all concentrations of ZMP and DTX significantly reducing wound recovery in a concentration-dependent manner $(\mathrm{p}<0.001)$ (Dunnett's multiple comparison post-test). A concentration-dependent effect was seen with both DTX and ZMP. DTX was slightly more potent at inhibiting cell migration than ZMP with both $2.5 \mathrm{nM}$ and $5 \mathrm{nM}$ DTX inhibiting migration more than $6 \mathrm{nM}$ ZMP. Both concentrations of DTX were significantly more effective at inhibiting wound closure 
compared to $2 \mathrm{nM}$ ZMP (Dunnett's multiple comparison post-test). (Figure 3.4; Table $3.8)$.

Table 3.8 \% of wound recovery after $18 \mathrm{~h}$ in HUVEC cells

\begin{tabular}{cc}
\hline Control & $76.3 \pm 1.7 * * *$ \\
$2.5 \mathrm{nM}$ DTX & $15.4 \pm 2.3 * * *$ \\
$5 \mathrm{nM}$ DTX & $10.9 \pm 1.2 * * *$ \\
$2 \mathrm{nM} \mathrm{ZMP}$ & $28.7 \pm 3.5 * * *$ \\
$4 \mathrm{nM} \mathrm{ZMP}$ & $20.3 \pm 5.2 * * *$ \\
$6 \mathrm{nM} \mathrm{ZMP}$ & $15.7 \pm 4.1 * * *$ \\
\hline
\end{tabular}

Inhibition of HUVEC cell migration is presented as percentage of wound recovery. Treatment with MSAs had a significant inhibitory effect on wound recovery ( $\mathrm{p}<0.0001$ ), with all doses of both ZMP and DTX resulting in a significant reduction in wound recovery in a concentration-dependent manner ( $<<0.001 * * *$; Dunnett's Multiple comparison post-test). Data are presented as the mean percentage \pm SEM, $\mathrm{n}=6$ independent assays run in duplicate for drug-treated and in triplicate for controls. 

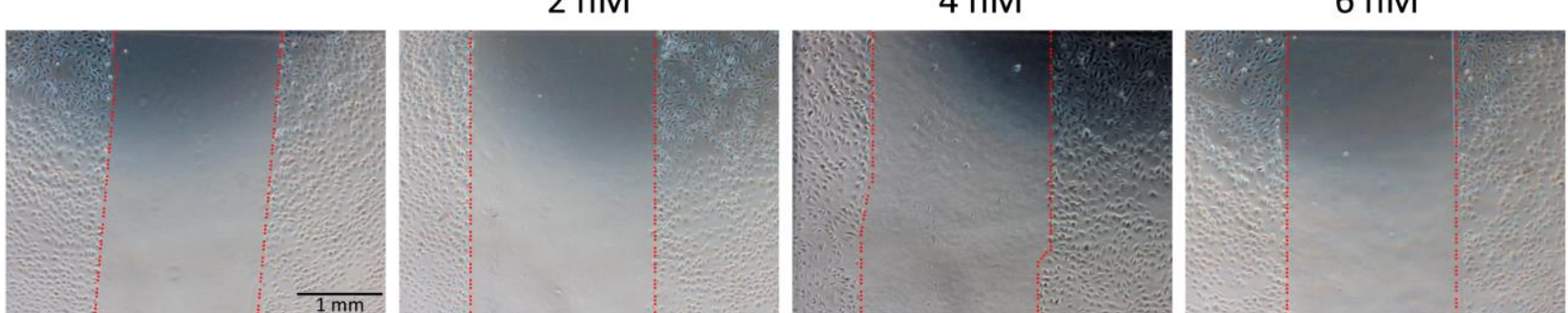

$2.5 \mathrm{nM}$

$5 \mathrm{nM}$
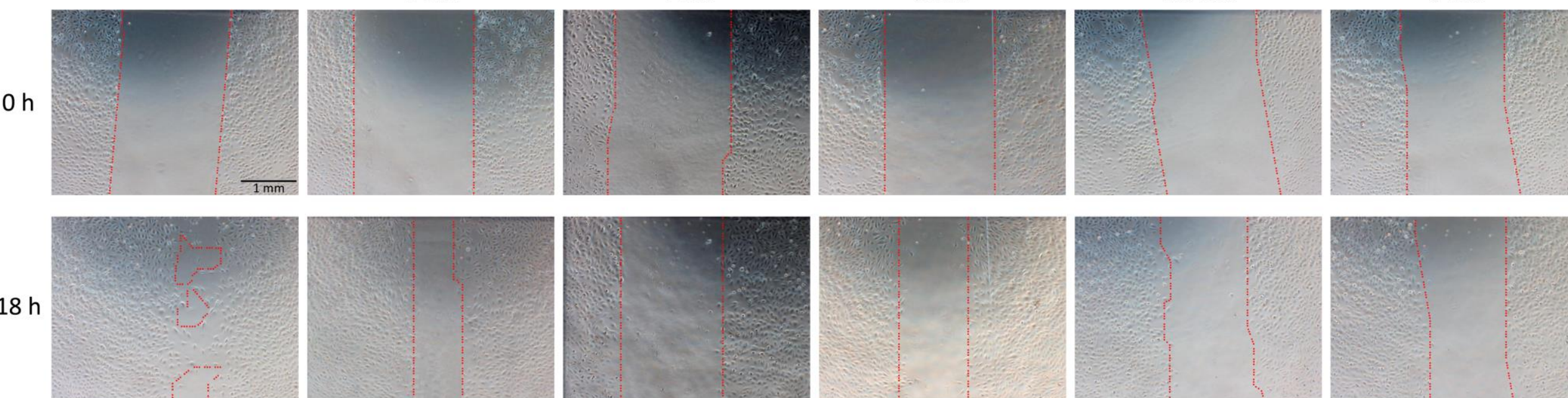

B

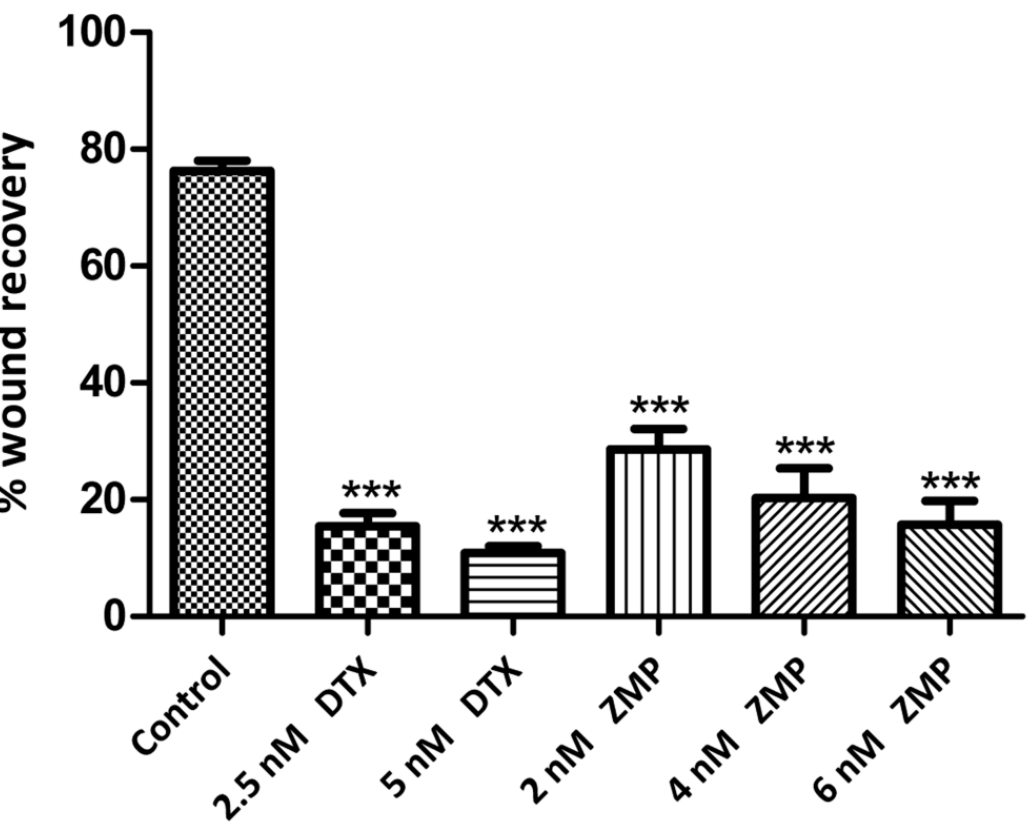

Figure 3.4 ZMP and DTX inhibition of HUVEC cell migration

Inhibition of endothelial cell migration by ZMP and DTX in a wound scratch assay. A. Representative images (4X magnification) of scratch wounds taken at time $0 \mathrm{~h}$ (upper panel) and $18 \mathrm{~h}$ (lower panel). The red dotted lines indicate the approximate location of the edges of the wound at $t=0$. Control cells showed almost complete closure of the wound by $18 \mathrm{~h}$; whereas, drug treated cells still contained a wound, indicating that migration had been inhibited or slowed down. B. Quantification of wound inhibition by ZMP and DTX compared to the control conditions. Data are presented as the mean $\% \pm$ SEM ( $n=6$ independent experiments, run in duplicate or triplicate). A Dunnett's multiple comparison post-test was used to determine significance of the drug treated samples with that of the control, and all tests were significant, indicating that ZMP and DTX significantly inhibited wound closure $(* * * p<0.001)$. See Table 3.8 for actual values. 


\section{D551 wound healing}

To determine whether ZMP inhibited fibroblast cell migration, a similar wound scratch healing assay was used as for the HUVEC cells. These assays were done to show that ZMP able to inhibit the ability of two different cell types to migrate, and to ensure the effect was not cell type specific. In the controls, full wound closure was seen in nearly all of the samples $(91.9 \pm 2.6 \%)$. Treatment with MSAs had a significant inhibitory effect on wound recovery $(\mathrm{p}<0.0001)$ (one-way ANOVA), with all doses of both ZMP and DTX resulting in a significant reduction in wound recovery in a concentration-dependent manner ( $\mathrm{p}<0.001)$ (Dunnett's multiple comparison post-test). As with the HUVEC cells, DTX inhibited wound closure to a greater extent than ZMP; however, this was only statistically significant at $5 \mathrm{nM}$ DTX versus $3 \mathrm{nM} \mathrm{ZMP}(\mathrm{p}<0.001)$ and $10 \mathrm{nM}$ DTX versus $3 \mathrm{nM}(\mathrm{p}<0.001)$ and $5 \mathrm{nM}$ ZMP $(\mathrm{p}<0.01)$ (Figure 3.5; Table 3.9).

Table 3.9 \% of wound recovery after $18 \mathrm{~h}$ in D551 cells

\begin{tabular}{cc}
\hline Control & $91.9 \pm 2.6$ \\
$5 \mathrm{nM}$ DTX & $14.7 \pm 3.0 * * *$ \\
$10 \mathrm{nM}$ DTX & $8.1 \pm 2.9 * * *$ \\
$3 \mathrm{nM} \mathrm{ZMP}$ & $47.6 \pm 7.6 * * *$ \\
$5 \mathrm{nM} \mathrm{ZMP}$ & $33.2 \pm 7.4 * * *$ \\
$10 \mathrm{nM} \mathrm{ZMP}$ & $23.6 \pm 5.4 * * *$ \\
$15 \mathrm{nM} \mathrm{ZMP}$ & $16.4 \pm 4.7 * * *$ \\
\hline
\end{tabular}

Inhibition of D551 cell migration as a percentage of wound recovery. Treatment with MSAs had a significant effect on wound recovery $(\mathrm{p}<0.0001)$, with all doses of both ZMP and DTX resulting in a significant reduction in wound recovery in a concentration dependent manner $(* * * p<0.001)$. Data are presented as the mean percentage \pm SEM, $n=6$ independent assays run in duplicate for drug treated and triplicate for controls. 
A

A

Control

$\mathrm{Oh}$

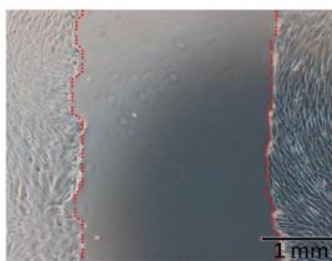

$18 \mathrm{~h}$

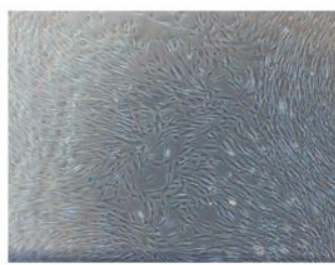

B

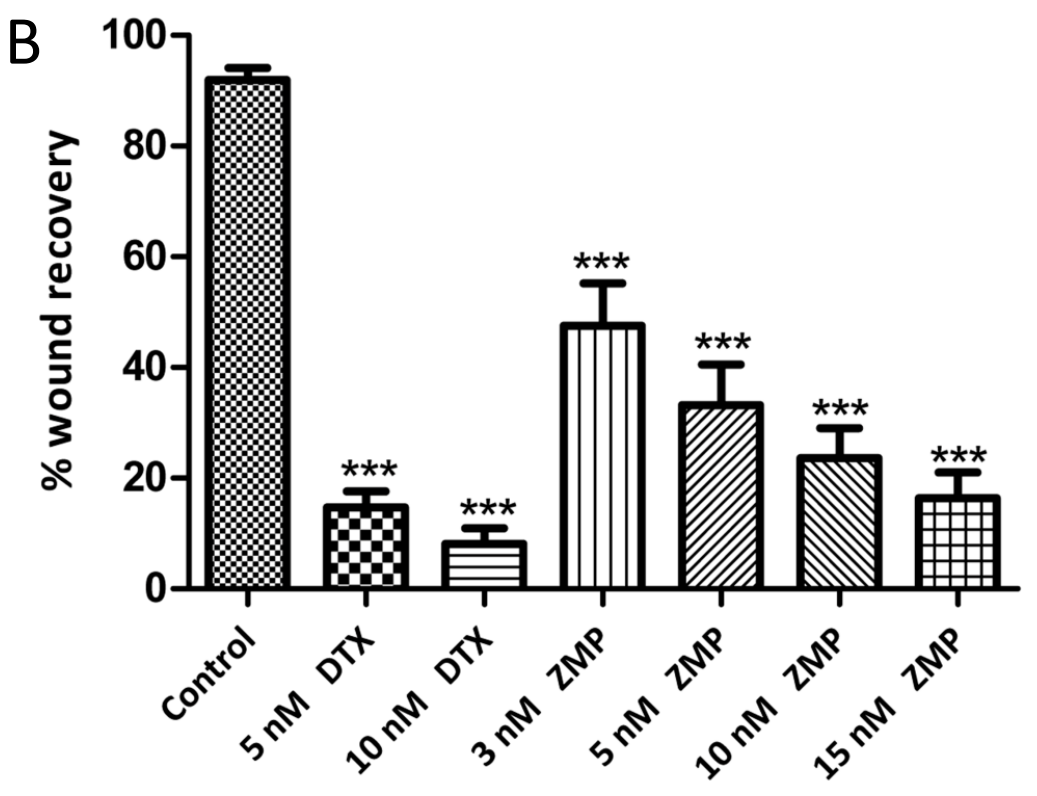

Zampanolide
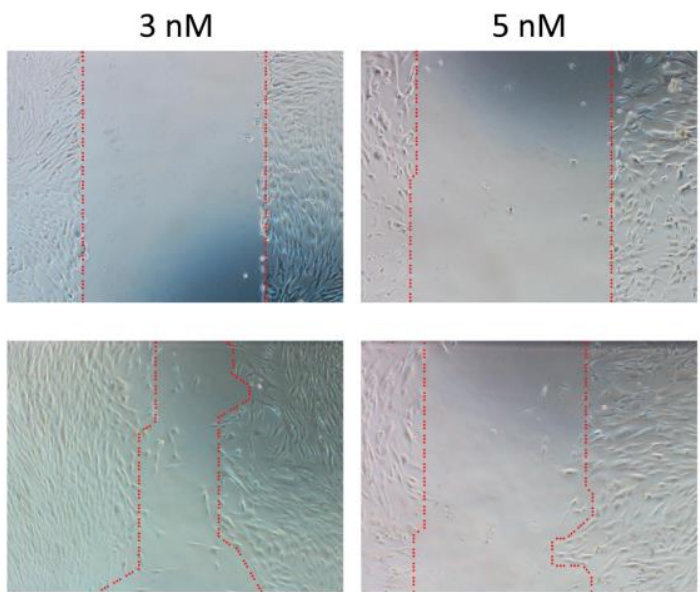
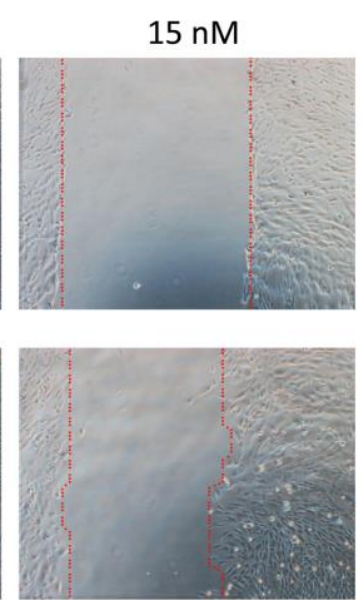

Docetaxel
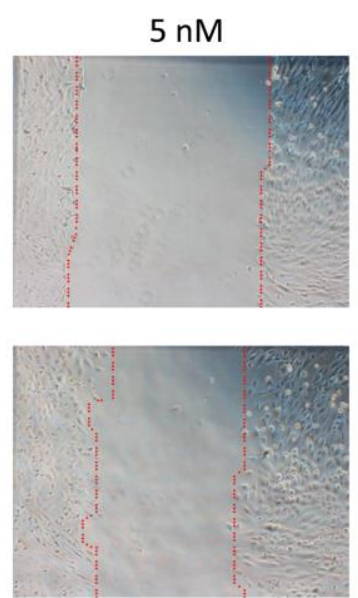

$10 \mathrm{nM}$
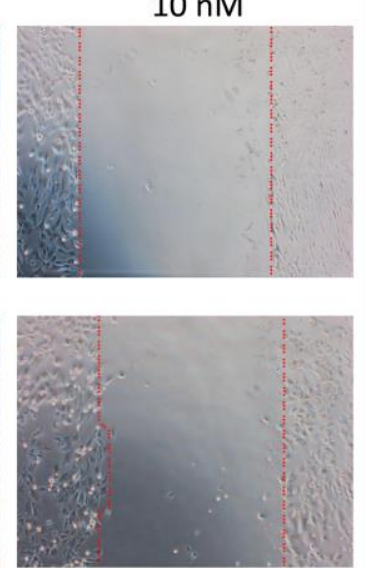

Figure 3.5 ZMP and DTX inhibition of D551 cell migration

Inhibition of fibroblast cell migration by ZMP and DTX in a wound scratch assay. A. Representative images (4X magnification) of scratch wounds taken at time $0 \mathrm{~h}$ and $18 \mathrm{~h}$ are presented as in Figure 3.4. B. Quantification of wound inhibition by ZMP and DTX compared to the control. Data are presented as the mean $\% \pm$ SEM ( $n=6$ independent experiments, run in duplicate or triplicate). One-way ANOVA gave a $\mathrm{p}$ value $<0.0001$. A Dunnett's multiple comparison post-test was used to determine significance of the drug treated samples compared to the control $(* * * \mathrm{p}<0.001)$. See Table 3.8 for values. 


\subsubsection{Generating a zampanolide-resistant cell line}

1A9 cells were grown in ZMP, starting at $2 \mathrm{nM}$, and this concentration was increased to $38 \mathrm{nM}$ over a 12-month period. Cells were treated for 1-2 days in ZMP during which a large population of the cells died. The few remaining cells were passaged after 1-2 days in drug-free medium and grown back to confluence. The cells were grown in each stepped concentration twice to ensure a resistant population was being maintained. Over time, the morphology of the cells changed significantly (Figure 3.6). The parental 1A9 cells have a spherical shape (Figure 3.6A); whereas, long-term ZMP-treated cells had a more mesenchymal phenotype with a polygonal shape (Figure 3.6C\&D), similar to the established 1A9-L4 cell line (Kanakkanthara et al., 2011) (Figure 3.6B). The cell phenotype persisted in drug-free medium for a number of months. Interestingly, however, when selected cells were grown in lower concentrations of ZMP a significant proportion of them died, even though in theory they had been selected for survival in higher concentrations of $\mathrm{ZMP}$. The $\mathrm{IC}_{50}$ values of $\mathrm{ZMP}$ and other selected MSAs were obtained in this 'resistant' cell line and compared to the $\mathrm{IC}_{50}$ values in the parental $1 \mathrm{~A} 9$ cells (Table 3.10). Unexpectedly, the cells were found to be more sensitive to ZMP with an $\mathrm{IC}_{50}$ value of $2.55 \pm 0.78 \mathrm{nM}$, compared with $1 \mathrm{~A} 9$ cells $(4.57 \pm 1.34)$. The cells were also found to be significantly resistant to PTX but more sensitive to IXA treatment compared to the parental cell line. In addition, both LAU and PEL were significantly more cytotoxic in this cell line. These results suggest that this cell line might have a mutation in the taxoid binding site involved with the binding of the taxanes but not the EPOs. It is also possible that there could be a $\beta$-tubulin isotype change that is affecting the taxoid site but not the LAU/PEL site. The $\mathrm{IC}_{50}$ for DTX was not determined due to problems with the MTT assay results and time limitations. This will be done in the future.

Table 3.10 $\mathrm{IC}_{50}$ values of MSAs in ZMP cells

\begin{tabular}{ccc}
\hline Compound & $\mathrm{IC}_{50} \pm \mathrm{SEM}(\mathrm{nM})$ & Resistance Ratio (1A9) \\
\hline Zampanolide & $2.55 \pm 0.78$ & 0.59 \\
Paclitaxel & $47.08 \pm 3.83$ & 11.21 \\
Ixabepilone & $3.57 \pm 0.25$ & 0.49 \\
Peloruside A & $13.24 \pm 0.74$ & 0.66 \\
Laulimalide & $3.35 \pm 0.26$ & 0.40 \\
\hline
\end{tabular}

$\mathrm{IC}_{50}$ values of MSAs in $1 \mathrm{~A} 9$ cells that were grown over long periods of time in the presence of stepwise increases in the concentration of ZMP. Cells were treated for $72 \mathrm{~h}, n=3-6$ independent 82 experiments. 

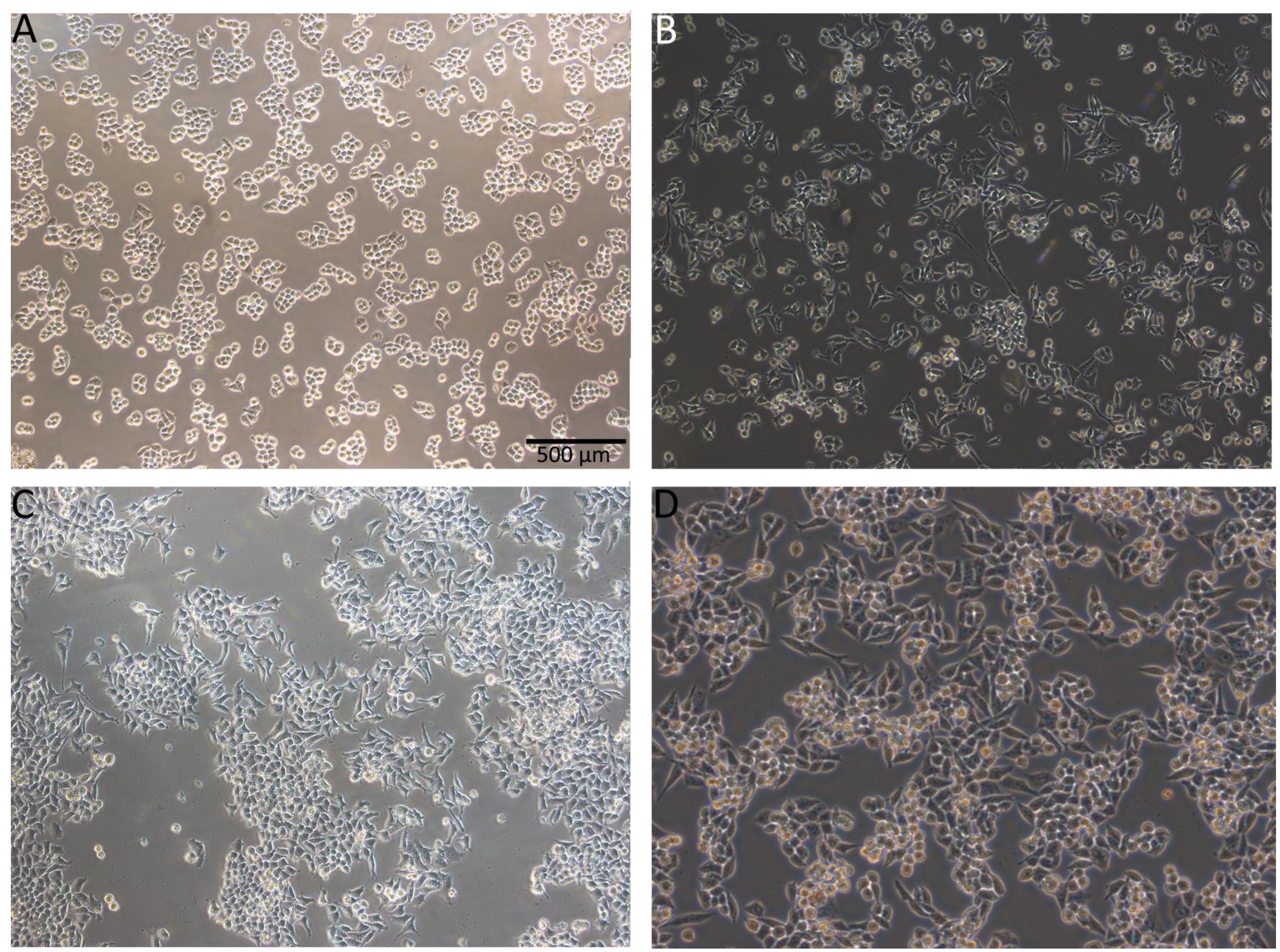
Figure 3.6 Changes in morphology of long-term ZMP-treated cells

Images of cells. A. 1A9 parental cell line. B. L4 cell line. C. ZMP cell line (taken when cells were grown at $24 \mathrm{nM}$ ). D. ZMP cell line (taken when cells were grown at $38 \mathrm{nM}$ ). ZMP cells have a significant change in morphology to a more mesenchymal phenotype with polygonal shape and regular dimensions, similar to L4 cells where 1A9 cells are spherical in shape. Scale bar $=500 \mu \mathrm{M}$. 


\subsection{DISCUSSION}

\subsubsection{Cytotoxicity of ZMP}

ZMP was found to have a potent cytotoxic/cytostatic effect in a number of different cell lines, indicating that it is a good drug candidate for further development as an MSA. This strong cytotoxicity means that low concentrations of the compound can be used to get the desired effect.

\subsubsection{Investigation into the binding site of ZMP using resistant cells}

Generation of resistant cell lines are a good approach for beginning to understand resistance in the clinic, even though the induced changes or mutations may not represent the most commonly encountered mechanisms of resistance in the clinic (Cabral, 2001). Although the most recent data show that single amino acid changes in the $\beta I$-tubulin gene are most likely involved (Yin et al., 2010). In regards to studying the binding sites of MSAs, these resistant cell lines are excellent tools. Before the taxoid site was discovered, single amino acid mutations in cell lines resulting in decreased sensitivity to taxoid site MSAs provided insight into possible binding site interactions of the taxanes and the EPOs. Through competition binding experiments, it was already known that the taxanes and the EPOs bound at the same site on tubulin (Bollag et al., 1995), and a number of different studies had located this site on the $\beta$-tubulin subunit, first described by Rao et al., 1992. The discovery and location of the taxoid binding site will be discussed in Chapter 4.

Single amino acid mutations prevent normal drug interactions with amino acid residues (such as the formation of hydrogen bonds). In addition, an allosteric effect may result from the mutation, which would cause conformational changes in the protein, bringing some amino acids closer together and moving others further apart. Each amino acid has its ideal torsion angles, and a mutation to a new amino acid may change the angle, affect the backbone of the protein and amino acid interactions. Thus although it was found that the four cell lines described by Giannakakou and colleagues (1997, 2000) had site-specific mutations, this does not always need to be the case for a cell line demonstrating resistance to an MSA. Therefore, the cell lines need to be treated with a range of MSAs with different binding sites to determine if the mutation is site-specific or if it is related to the general stability of the tubulin protein. Additionally, mutations in a specific binding site may only affect some of the compounds that bind to that site, since 
the conformation of a compound in its binding site and interacting residues are unique for each MSA. For example the two PTX-resistant cell lines, are not sensitive to EPOB; whereas, the EPO-resistant cell lines are cross-resistant to PTX (Giannakakou et al., 1997; 2000). Interestingly, in the present study all taxoid site mutated cells were resistant to IXA (Table 3.4; Figure 3.1). IXA is a semisynthetic analog of EPOB with a chemically modified lactam substitution of the naturally occurring lactone (Figure 1.5). The slight change in structure appears to have a profound effect on the manner in which the compound utilizes the taxoid binding pocket, since the mutations in PTX10 and PTX22 affect IXA but not EPOB.

\section{ZMP interaction with the taxoid site}

As described the PTX-resistant cells of Giannakakou et al. (1997) have single amino acid changes in S7 (272) (PTX10) and in the S9-S10 loop (374) (PTX22), both regions important in the taxoid binding site (Nogales et al., 1999). Based on the binding conformation of PTX proposed by Snyder et al. (2001), it is clear why mutation of either residue results in resistance to PTX binding. Residue 272, deep in the hydrophobic pocket, directly interacts with the methyl group of the C4 acetate of PTX via Van der Waals interactions and mutation of residue 374 would produce a conformational change in the pocket (Snyder et al., 2001). Substituting phenylalanine with valine at position 272 (PTX10) removes the aromatic ring from the pocket but would cause no change in polarity; substituting alanine with threonine at position 374 (PTX22) would increase the polarity within the pocket. ZMP binding does not appear to involve reside 272 as the compound was fully active in the PTX10 cell line; however, it is possible that ZMP interacts with this residue but is able to have a similar interaction with the mutated amino acid resulting in no change in cytotoxicity. Mutation of residue 374 appears to have an effect on the way in which ZMP binds to tubulin, suggesting it may bind in the taxoid site, although the resistance to ZMP was not statistically significant. This trend towards resistance could be due to the increased polarity in the taxoid pocket making binding less favorable; however, taxoid site ligands EPOB and DSC binding are not affected by this polarity change (Giannakakou et al., 1997). It has been suggested that contacts on tubulin essential for PTX binding are not the same as required by the EPOs (Nettles et al., 2004), although this model of EPO binding has recently been revised and was found to be 
fundamentally different from the earlier models based on stabilized zinc sheets (Prota $e t$ al., 2013).

In the EPO-resistant cells A8 and B10 cells, residues 276 and 284 are mutated, respectively. These mutations occur in the M-loop, an essential component MSA-induced stabilization. Mutation of residue 276 from threonine to isoleucine would make the binding pocket less polar; whereas, mutation of 284 from arginine to glutamine would decrease the side chain length and thus lengthen the distance between associating centers. Giannakakou et al (2000) proposed that in a model of unbound tubulin, solvent occupies the taxoid binding pocket, resulting in the formation of a hydrogen bonded network assisted by the polar side chains of neighboring amino acids. In this model, mutation of residues 276 and 284 would be extremely disruptive to this bonding network as they would shift the location of important water molecules and salt bridges, weakening the binding affinity and thus causing resistance in cells bearing these mutations. The main disruptions include loss of the hydroxyl group in A8 cells, causing a displacement of a water molecule. This would shift the surrounding hydrogen bonded atoms and other important molecular moieties such as the two hydrogen bonds between residue 276 and EPOA (Prota et al., 2013). These shifts are highly likely to occur with other EPO analogs, as shown by the over 18 -fold resistance to IXA in the present study. It is possible; however, these hydrogen bonds would be retained within the backbone of isoleucine. An additional hydrogen bond is formed with Gln281, and a significant structural change occurs in $\beta$-tubulin residues 278-283 when EPOA is bound; thus, it is understandable why A8 and B10 cells are strongly resistant to IXA treatment. It is known that when PTX is bound to MTs it displaces the M-loop away from H6 increasing its interactions in an adjacent subunit at the H1'-S2 loop, and this results in the formation of a stable salt bridge between Arg284 and Glu55 in a neighboring protofilament (Mitra \& Sept, 2008) (see Figure 4.1 for secondary structures of tubulin). In the absence of PTX Arg284 forms an intramonomer salt bridge with Glu290. Thus, mutation of arginine to isoleucine (B10 cells) would result in loss of the intramonomer salt bridge; this would cause an increase in the anionic charge and a flux of water. Additionally, loss of the PTX-induced salt bridge would strongly decrease the PTX-induced stability of the MT, and most likely the effect of other taxoid site MSAs. It is therefore not surprising that all taxoid site ligands so far studied are affected by this mutation. Loss of the intramonomer salt bridge (un-liganded MTs) may cause other structural problems in tubulin which may 
give reason as to why this cell line is difficult to grow compared to the other mutated 1A9 clones. ZMP was not affected by the mutation in A8 cells; however, it was significantly affected by the mutation in B10 cells, although not to the same degree as other taxoid site MSAs. ZMP had a resistance ratio of 3; whereas, PTX and IXA had significantly higher resistance ratios of 34 and 20, respectively. This suggests that the loss of the threonine is not essential for ZMP binding; whereas, loss of a positive charge and associated salt bridges due to mutation of amino acid 284 is important in the binding of ZMP as it is with all other taxoid site ligands.

Overall these results indirectly indicate that ZMP may be a ligand of the taxoid binding site but not the LAU/PEL binding site. Although the decreased resistance of the B10 cell line to ZMP compared to other taxoid site agents and the lack of ZMP resistance by the other three taxoid-resistant cell lines suggest that it may bind in a unique manner to the traditional taxoid site MSAs, possibly occupying a distinct pose within the taxoid site. These results identify residues $\beta 374$ and $\beta 284$ as being important residues in ZMP binding, these results will be further discussed in Chapter 8 .

\section{Lack of ZMP interaction with the LAU/PEL site}

Two cell lines with mutations in the LAU/PEL binding site were also studied to determine if ZMP was affected by mutations at this site in $\beta$-tubulin. Both cell lines are resistant to PEL; whereas, the L4 cell line is only resistant to LAU. Although ZMP has a similar macrolide structure to both LAU and PEL (Figure 1.5), being made up of a highly unsaturated macrolide ring (16-20 carbons) with similar length side chains, these structures are also very similar to that of the EPOs that bind to the taxoid site.

In R1 cells, alanine 298 (neutral, nonpolar) is replaced with threonine (neutral, polar, resulting in no change in charge but causing the binding pocket to increase in polarity). In L4 cells, arginine 308 is replaced with a histidine (67\% of the time) or a cysteine (33\% of the time). Arginine has the ability to form salt bridges, and loss of a salt bridge would cause a significant change in charge and water distribution. Histidine is a basic polar amino acid with a similar charge to arginine that would cause no significant change in the pocket; however, histidine has a much shorter side chain that may result in loss of potential hydrogen bonding. Replacement with cysteine would cause a loss of positive charge in the pocket and possible loss of hydrogen bonding since cysteine has a thiol side chain. This side chain thiol has the potential to react with other thiols forming 
disulphide bridges, or it may interact with other cysteine residues to form cystine. In addition, cysteine is a good nucleophile and therefore has potential to form linkages from enzymatic reactions. Using the molecular modeling program DeepView v4.1 (Guex \& Peitsch, 1997), it appears that there are no potential hydrogen bonds (in protein) available for arginine, as the side chain appears to be facing out of the MT, thus formation of intramonomer salt bridge also seems unlikely The two amino acids either side have the potential to form two hydrogen bonds (in protein) that may be disrupted by replacement of arginine with another amino acid. Residue 298 has the potential to form 3 hydrogen bonds $\left(\mathrm{NH}_{2}\right.$ of the backbone with the residue 297 side chain, backbone carbonyl with backbone residue $300 \mathrm{NH}_{2}$ and the backbone carbonyl with the 301 backbone $\mathrm{NH}_{2}$ ), although these would not occur simultaneously. Also if a drug is bound in this pocket, it would change other interactions, and therefore these hydrogen bonds may not exist in that particular conformation. To form a proper model, the wild type protein would need to be modeled with and without PEL docked to it and then the amino acids changed. Since the model was made with zinc-stabilized sheets versus 'normal' tubulin, the PTX bound to the sheets may contribute to an abnormal conformation. ZMP was found to be fully active in both R1 and L4 cells, suggesting that it is not a ligand of the LAU/PEL binding site, and this supports the hypothesis that it binds in the taxoid binding pocket as suggested by the results obtained in the taxoid mutant cells.

\subsubsection{Lack of synergistic interaction of ZMP with MSAs}

Generally, two MSAs that bind to the same site will be unable to synergize with one another (PEL and LAU), but those that bind to different sites will (PEL and PTX), with the exception of PTX and DSC. Potential synergistic interactions between ZMP and other MSAs were investigated to gain insight into the binding site of ZMP on the MT. Given the results from the mutant cell studies, it was expected that ZMP may synergize with LAU and/or PEL. It has been suggested that MSAs have secondary binding sites on

the MT that may underlie their synergy (Wilmes et al., 2011). This would only be possible if binding to both sites was mutually exclusive since all known MSAs bind in a 1:1 stoichiometry, indicating that simultaneous binding does not occur. It is possible that a drug may bind to a preferred site when present on its own, but if another MSA is present with higher affinity for the same site, then it may bind at a secondary site. Recently HDX-MS (hydrogen deuterium exchange mass spectrometry) studies have 
found evidence that LAU and PEL may also interact with the taxoid site, suggesting that when PTX is present, PEL and LAU may interact with the alternative site but when there is excess compound, they would interact with both sites. It is also possible that the HDXMS findings may be attributable to isotype differences in the purified protein used in the different experiments (Khrapunovich-Bine et al., 2011). Synergistic interactions may also occur due to interactions with other targets in the cells, or differential interactions with different $\beta$-tubulin isotypes, or differences in metabolism of the MSAs.

In the present study, ZMP was unable to synergize with the MSAs at the concentrations tested, and thus the results provided no insight into its binding site on the MT. Only one drug combination was able to synergize, ZMP and PEL $(p<0.0002)$, but the $\mathrm{CI}$ value was not convincing $(\mathrm{CI}=0.79)$. While not statistically significant, $\mathrm{ZMP}$ in combination with PTX and DSC produced CI values less than 1.0 in some combinations. This was not expected and somewhat contradicts the results seen in the resistant cell lines. However, as shown with PTX and DSC, it is possible that two taxoid site ligands can also synergize. Alternatively, perhaps ZMP has the ability to synergize with PEL and LAU, but the 'right' combinations were not tested. The one combination that did demonstrate synergy was at high ZMP (10 nM) and high PEL (15 nM), and perhaps if combinations more similar to this were tested, synergy would have been seen. However, only lower concentrations of $\mathrm{ZMP}$ were tested given its $\mathrm{IC}_{50}$ and the fact in general, synergy is more prominent at concentrations lower than the $\mathrm{IC}_{50}$.

Since some MSAs can synergize with MDAs (Photiou et al., 1997; Giannakakou et al., 1998), it would be interesting to investigate if ZMP behaves in a similar manner, especially since no synergy was seen with any MSAs.

\subsubsection{ZMP inhibits cell migration}

Angiogenesis plays an important role in tumor cell growth and metastasis. In interphase cells the MT network is a polarized dynamic structure. During cell migration the plus ends of the MT are targeted toward the newly forming focal adhesions at the cell surface (connecting the extracellular matrix with the plasma membrane and the actin cytoskeleton); whereas, the minus ends are attached to the MT-organizing center. MTAs such as COL, PTX, DTX, LAU and VBL inhibit cell migration in a number of different cell types, including endothelial and fibroblast cells (Schwartz, 2009). Thus the ability of ZMP to inhibit cell migration was also investigated in an endothelial cell line and a 
fibroblast cell line. At each ZMP concentration tested and in both cell types, cell migration was significantly inhibited, at low nanomolar concentrations. These concentrations were similar to the $\mathrm{IC}_{50}$ in HUVEC cells but significantly lower than that in D551 cells. Although ZMP was more potent than DTX at inhibiting proliferation of both HUVEC and D551 cells, it was not as effective at inhibiting cell migration. This is consistent with the literature which shows that DTX is more potent at inhibiting migration compared to other MTAs like EPOB and VBL (Bijman et al., 2006) or PEL (Chan, 2012). This may be due to different effects of the MTAs on downstream signaling pathways involved in migration. Activation of VEGF results in a number of different cellular processes, including endothelial cell migration, that are mediated by focal adhesions connecting to the extracellular matrix. The extracellular matrix acts as a ligand for cell surface integrin proteins (transmembrane proteins involved in cell attachment). DTX and LAU, for example, have differential effects on downstream targeting of VEGF receptor activation, with DTX inhibiting VEGF-induced association of a particular integrin isotype to a greater degree than LAU (Lu et al., 2006). However, this may also be due to their differences in binding site on the MT.

The exact mechanism by which MSAs inhibit cell migration is poorly understood, although disruption of tubulin dynamics would affect a number of cellular processes and not just mitosis, as discussed in Section 3.1.4. A number of downstream effects of MTAs are thought to contribute to the inhibition of cell migration, including for example impairment of the MT organizing center, interference with the formation of focal adhesions (Hotchkiss et al., 2002) or degradation of heat shock protein 90 (Murtagh et $a l ., 2006)$. The effects of MTAs on endothelial cells have been summarized in a review by Schwartz (2009). Additionally, MTs regulate the activity of a number of different proteins, such as the Rho GTPases, and thus MTAs would interfere with the processes that these proteins are involved in or have a regulatory function in. Focal adhesions are essential in cell migration and are regulated by a number of different extracellular stimuli, such as VEGF, and formation of focal adhesions require the activity of the Rho GTPases.

In the present study, ZMP was shown to inhibited cell migration in cultured cell monolayers. The mechanism by which it did this was not explored but remains a future avenue worth investigating. The next step would be to see how ZMP affects the spontaneous formation of capillary-like tubular structures that are formed by HUVEC 
cells in 3-D matrices in cell culture (Schwartz, 2009). Formation of these structures is one of the final steps in the formation of new blood vessels; thus, the ability to inhibit formation of these tube-like structures would be important to investigate when assessing a compound for its anti-angiogenic effects.

\subsubsection{Development of a ZMP-resistant cell line}

Given that patient tumor cells are frequently heterogeneous, slow growing and hard to culture, the majority of information on resistance to MSAs comes from studying established cell lines such as the 1A9 cell line. Although significantly different to the "in patient' situation, cultured cells can be easily pushed toward developing resistant phenotypes with the parental cell line acting as a good 'dependable' control. An attempt was made to develop a cell line resistant to ZMP using a variation of well-established methods that have resulted in the creation of a number of different stable mutant cell lines. Once a mutant cell line is created, it can be used to investigate the binding site and possible binding interactions of the compound with tubulin. This was the aim with development of the ZMP-resistant cell line, and knowing that PTX-resistant mutant cells and LAU/PEL-resistant mutant cells were successful in describing the potential binding sites of MSAs before definitive crystal structures became available (Giannakakou et al., 1997; Giannakakou et al., 2000; Begaye et al., 2011; Kanakkanthara et al., 2011). However, due to difficulties in generating a ZMP-resistant cell line, sequencing of the tubulin was not done and remains a future direction in this work. The highest resistance ratio attained in this study was only 11 and this was not high enough to proceed with characterization of any genetic changes in the cells. The reasons why this cell line may not have developed resistance to ZMP will be discussed in Chapter 8. Although resistance to ZMP was not obtained, the cell line did become significantly resistant to PTX (resistance ratio $=11.2$ ) and most probably to DTX, suggesting that an amino acid in the taxoid site important in taxane binding may have been mutated. This could be confirmed with $\beta$-tubulin sequencing. It would be interesting to see if the resistance of this cell line was specific for the taxoid site or if it also applied to MDAs.

As these cells were selected by multiple-step growth in increasing concentrations of ZMP, pinpointing the specific genetic or biochemical change causing the drug resistant phenotype may be difficult. Since the growth of cells between increasing drug treatments may cause the introduction of other non-tubulin-linked mutations that may 
contribute to the drug resistance. Therefore, identification of a single amino acid change may not be adequate in defining what may be a far more complex mechanism of resistance (Cabral, 2001). For example a number of different protein changes, not just in $\beta$-tubulin have been reported to cause PTX resistance, such as up-regulation of septins (GTPases involved in cytokinesis and MT skeleton organization) in addition to upregulation of $\beta$ II and $\beta$ II tubulin isotypes (Froidevaux-Klipfel et al., 2011) or downregulation of vimentin (Kanakkanthara et al., 2012). This may explain the differential results seen between different mutant cells with different MSAs binding the same site. For example, cells with increased $\beta$ III tubulin isotypes are known to be resistant to PTX (Kavallaris et al., 1999), however, this is in contrast to L4 cells that have high abundances of $\beta$ II and $\beta$ III tubulin isotypes, yet are sensitive to PTX (Kanakkanthara et $a l ., 2011)$. It can be speculated that for cells to be resistance to PTX, they may require a number of different mutations that combine to give the resistant phenotype, such as the breast cancer cells described by Froidevaux-Klipfel et al., 2011. This is not to say that other cells with increased $\beta I I$ and $\beta I$ isotype expression don't have up-regulated septins, as they have not been tested for these changes. Given that septins are involved in MT organization, it is not surprising that they are up-regulated with higher abundance of different isotypes of tubulin. Similar changes have been observed in the L4 cells used in the present study that have down-regulated vimentin (abundant type II intermediate filament protein). R1, PTX10 and A8 cells showed no down-regulation of vimentin and retained their sensitivity to LAU, even though R1 cells are mutated in the LAU binding site they show no resistance to LAU, indicating that along with the mutated $\beta$-tubulin residue, increased $\beta \mathrm{II}$ and III isotypes and vimentin also play a part in the resistance to LAU. This may help explain why some cells with mutations in the taxoid binding site retain their sensitivity to other ligands that bind this site; thus, it is possible that another mechanism of resistance is required for resistance to develop against some MSAs. The resistance towards PTX demonstrated by the ZMP-resistant cells and their lack of resistance to IXA (similar to PTX10 and PTX22 cells being resistant to PTX but not EPOB) suggest that there is a unique factor required in the development of resistance towards EPOs, and this may also be the case for ZMP.

The overall abundance of other proteins in the cells is likely to contribute to the resistance toward MSAs. By examining the proteome of resistant cells and not only the $\beta$-tubulin sequence and isotype composition, more light may be shed on the resistance 
mechanisms behind MSAs, and a proteomic study would also provide information on the interaction of MSAs with other proteins that may underpin their effectiveness in interphase cells. There is now evidence that MSAs target both mitotic and interphase cells, as discussed in section 3.1.4. First it would be interesting to investigate the effect of MSAs on non-dividing cells such as macrophages. If MSAs target primarily mitosis, their cytotoxicity would be reduced or absent in these non-cycling cells; whereas, if they target other process in which MTs are involved such as cellular trafficking they will be cytotoxic towards these cells despite the fact that they are not undergoing mitosis.

\subsubsection{Conclusions and future directions}

This study has provided insight into the binding site of ZMP and into its extended cellular effects. From the data, it can be speculated that ZMP is a ligand of the taxoid binding site, although the evidence is indirect. ZMP showed decreased potency to inhibit cell proliferation in two taxoid site mutant cell lines; although, it had significantly greater potency compared to the parental cells than the traditional taxoid site MSAs, suggesting it may interact with the pocket in a different manner or hold a distinct pose with different interacting residues. This is supported by the finding that, although not statistically significant, the CI values for ZMP demonstrated potential synergy with a number of different MSAs, including PTX and DSC. The ZMP-resistant cell line further supports a taxoid binding site for ZMP, since the cells were highly resistant, over 11-fold, to PTX, despite showing little resistance to ZMP. Overall, these results demonstrate that ZMP is a potent MSA with potent cytotoxicity in a number of different cell lines, is not affected to the same extent as other MSAs by amino acid mutations in the taxoid binding site nor is it affected by mutations in the LAU/PEL binding site. It is potent at inhibiting cell migration, giving ZMP good potential for further investigation as an anti-angiogenic compound. The synergy data did not give any clear indication to the binding site of ZMP, but suggested ZMP was not a good compound to synergize with other MSAs at the concentrations tested.

A number of directions for future research can be taken from this study. It would be interesting to further investigate synergy of ZMP with MSAs at different concentrations and to investigate its ability to synergize with MDAs or other anti-cancer compounds such as gemcitabine or doxorubicin. Second, further investigation into the mechanisms by which ZMP inhibits cell migration would be worthwhile given the 
promising preliminary results from the wound scratch assays. Effects of ZMP on tubeformation would also be of interest. If enough ZMP were available, its effect on in vivo tumors in mice would be an important set of experiments. Third, further development of a ZMP-resistant cell line could include cloning and selection for the most resistant cells to give a homogeneous population, followed by sequencing of $\alpha$ - and $\beta$-tubulin to determine if there is a gene mutation in tubulin compared to the wild type cells. 


\subsection{INTRODUCTION}

\subsubsection{The taxoid binding site}

The PTX binding site was first described on the $\beta$-tubulin subunit by direct photoaffinitylabeling (Rao et al., 1992). The binding site pocket was first modeled at $6.5 \AA$ resolution in 1995, using electron crystallography of zinc-induced sheets of antiparallel tubulin protofilaments, confirming PTX binds with a 1:1 stoichiometry and affects interprotofilament interactions (Nogales et al., 1995). These models were solved to better resolution and confirmed by later studies from the same lab (Nogales et al., 1998a; 1999; Löwe et al., 2001). Li et al. (2002) then used this information and presented the MT structure by cryo-electron microscopy ( $8 \AA$ ) of PTX-stabilized MTs in parallel, stabilized sheets. This model confirmed the important lateral interactions and highlighted the structural features that distinguish MTs from zinc-stabilized sheets (Li et al., 2002). Prior to the revelation from the electron crystallography models, a number of common pharmacophore models were proposed for taxoid site MSAs (see chapter 7.1).

Overall the crystallography models show that the PTX binding pocket consists of the S9-S10 loop and parts of helices H1, H6 and H7 (the core helix of the $\beta$-subunit), and S7 (Figure 4.1). The taxane ring sits at the N-terminal end of the MT-loop (M-loop, connecting S7 to H9), with its strongest interaction occurring at Leu275 (Figure 4.2). The M-loop protrudes out from a protofilament and is an important secondary structure for stabilization of the MT. Protofilaments are connected primarily by interprotofilament interactions between the M-loops, H1'-S2 loops, and the H2-S3 loops in adjacent protofilaments (Nogales et al., 1998a; 1999; Sui \& Downing, 2010). The most important interaction is between the M-loop and the H1'-S2 loop of an adjacent protofilament. PTX binding strengthens this contact as it can push the M-loop out towards the neighboring protofilament, resulting in more favorable contacts between adjacent protofilaments. This interaction is crucial for stabilization by PTX and its biomimetics because it increases the lateral protofilament interactions (Nogales et al., 1995; 1998a; 1999; Li et al., 2002; Sui $\&$ Downing, 2010) The luminal site in $\beta$-tubulin has PTX facing the central hole in the MT, with the equivalent area in the $\alpha$-subunit being occupied by eight extra amino acids (within the S9-S10 loop) that block the equivalent site on $\beta$-tubulin (Nogales et al., 1998a; Löwe et al., 2001) (Figure 4.1). The extra residues in $\alpha$-tubulin have a stabilizing 
effect on the M-loop, and it is thought that PTX and other taxoid site compounds may mimic this effect, but act on the $\beta$-subunit (Nogales et al., 1999; Löwe et al., 2001). This has recently been confirmed by X-ray crystallography (Prota et al., 2013).

The resolution of these earlier structures, however, were not good enough to completely characterize PTX in its bound conformation, and the fact that the information was based on zinc-stabilized sheets rather than functional MTs meant that the interactions in vivo may differ to some extent. Photoaffinity labeling showed amino acids 1-31 and 217-233 within the $\beta$-subunit were important in the binding of PTX (Rao et al., 1994; Rao et al., 1995). This same group later identified Arg284 as an important residue in the binding (Rao et al., 1999); this residue is also found to be mutated in PTX resistant cells. Thus, from these data obtained by different methods, a common binding site for PTX in tubulin was determined for zinc-stabilized sheets. In 2001, Snyder and colleagues used computer docking based on crystallography density analysis to construct a model of PTX in its bioactive conformation. This model confirmed that the taxoid binding site is located within a deep hydrophobic cleft and involves three potential hydrogen bonds and a number of hydrophobic interactions, supporting the previous research by Nogales and colleagues. A considerable number of amino acid residues are involved in direct contacts with PTX when it is bound to tubulin; these interactions are described in Table 4.1 (see Chapter 1 Figure 1.5 for carbon numbering of PTX and Figure 4.2 for the interaction maps). In summary, PTX binds the $\beta$-subunit in its second globular domain on the opposite side of the core helix from where the E-site is located and faces the central hole in the MT (Amos \& Löwe, 1999). 


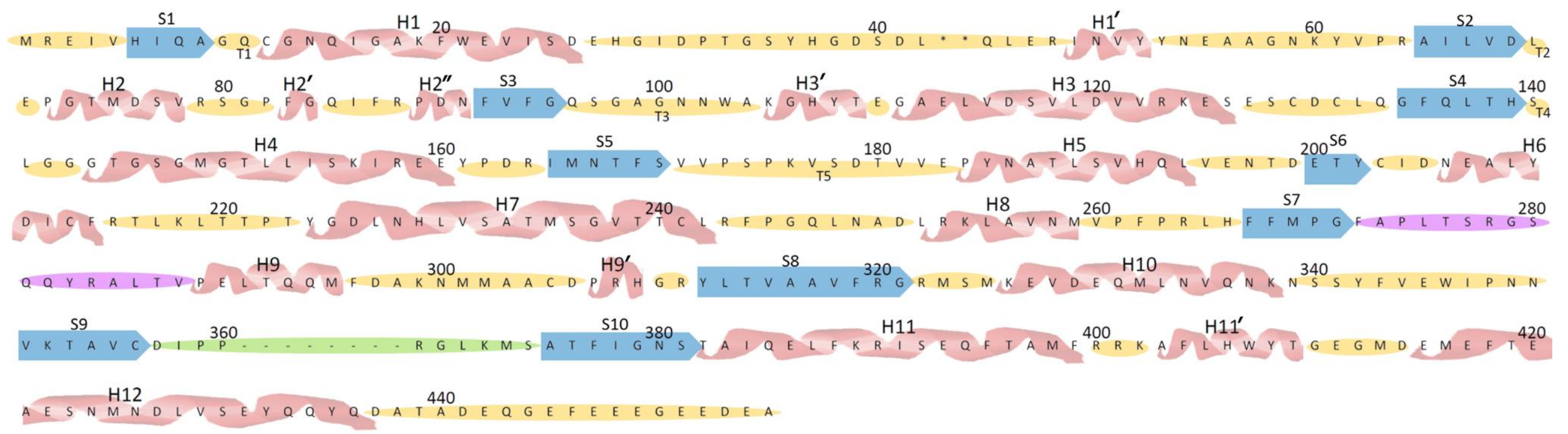

Figure 4.1 Sequence and secondary structure of $\beta$-tubulin

$\alpha$-helices are shown in pink, $\beta$-sheets in blue and joining loops in yellow. The M-loop is shown in purple and the S9-S10 loop in green. All segments are labelled and defined as in Löwe et al., 2001, protein data bank entry JFF. In the S9-S10 loop amino acids 361-368 are missing (shown by the dashes) these residues account for the extra 8 amino acids in the $\alpha$-subunit) and are included here for consistence of sequence numbering. 
Table 4.1 Tubulin regions and amino acid residues involved in MSA stabilization

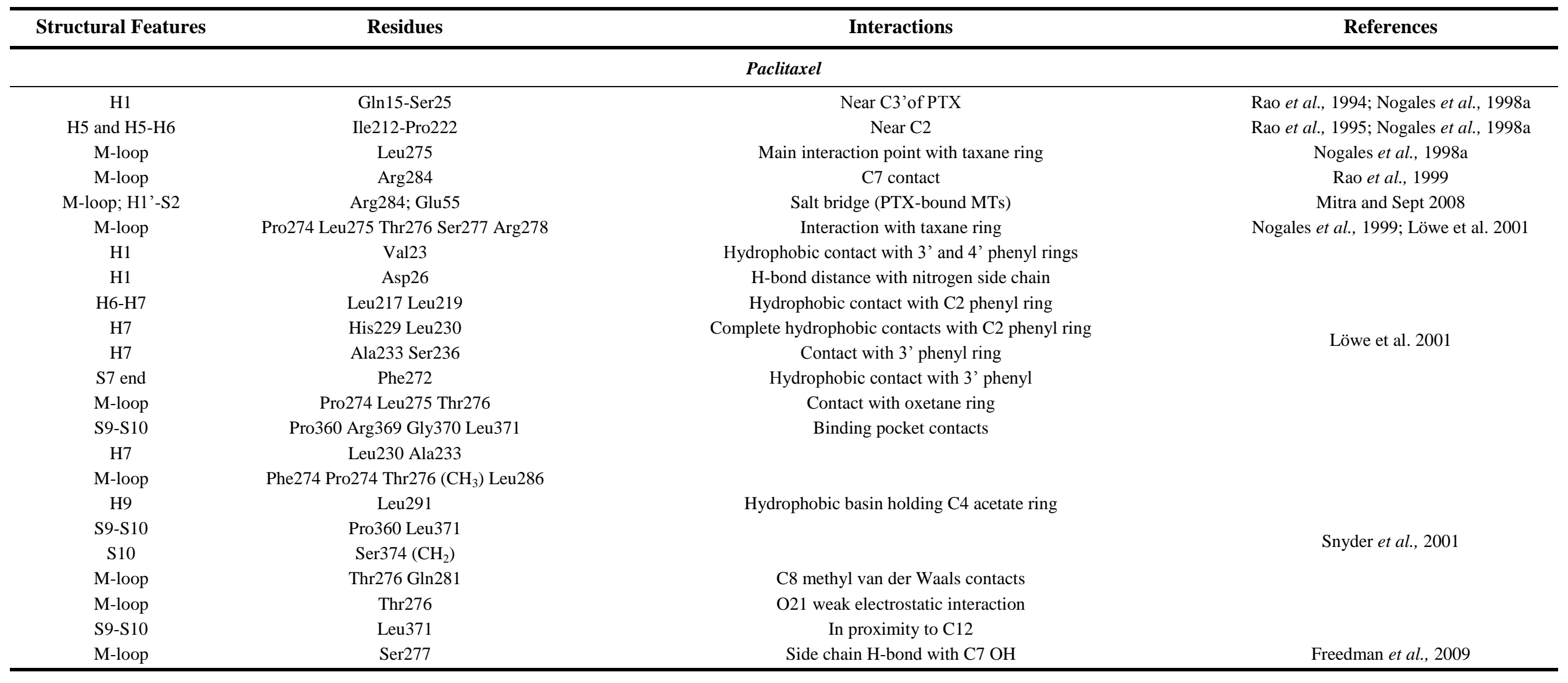




\begin{tabular}{|c|c|c|c|}
\hline \multicolumn{4}{|c|}{ Epothilone A } \\
\hline H1 & Asp226 & $\mathrm{OH} 7 \mathrm{H}-$ bond with side chain oxygen & \multirow{4}{*}{ Prota et al., 2013} \\
\hline M-loop & Thr276 & O1 H-bond back bone $\mathrm{NH} 2$ \& $\mathrm{N} 20 \mathrm{H}$-bond side chain $\mathrm{OH}$ & \\
\hline M-loop & $\mathrm{Gln} 281$ & $\mathrm{OH} 3 \mathrm{H}$-bond side chain amide nitrogen & \\
\hline M-loop & Arg278-Tyr283 & Restructured into short well-defined helix & \\
\hline \multicolumn{4}{|c|}{ Zampanolide } \\
\hline $\mathrm{H} 7$ & Asn228 & $\mathrm{C} 3$ covalent bond with side chain amide (minor) & Field et al., 2012 \\
\hline M-loop & Thr276 & $\mathrm{C} 20 \mathrm{OH} \& \mathrm{C} 1$ ' carbonyl H-bond with the main chain carbonyl \& $\mathrm{NH} 2$ & \multirow{2}{*}{ Prota et al., 2013} \\
\hline M-loop & Arg278-Tyr283 & Restructured into a short well-defined helix & \\
\hline \multicolumn{4}{|c|}{ Laulimalide ( $\beta$-subunit) } \\
\hline H10-S9 & Asn 339 & H-bond with $\mathrm{C} 15 \mathrm{OH} \&$ defines cavity entrance & \multirow{3}{*}{ Bennett et al., 2010} \\
\hline H9-H9', H10-S9 & Phe296 Arg308 Tyr342 & Side chains protrude into binding pocket $\&$ reorganize & \\
\hline H9-H9', S8, H10, H10-S9 & $\begin{array}{l}\text { Phe296 Tyr312 Val335 Asn339 Ser341 } \\
\text { Tyr342 Phe343 Ile347 }\end{array}$ & $\begin{array}{l}\text { Residues with binding interactions in pocket. O1 close to Asn339 side chain NH, O3 } \\
\text { epoxide to Tyr } 342 \text { phenolic } \mathrm{OH}, \mathrm{C} 20 \mathrm{OH} \text { to backbone } \mathrm{NH} \text { of Ser341 \& Tyr342. }\end{array}$ & \\
\hline S8 & Tyr312 & Side chain pyran O7 H-bond to Tyr312 backbone NH2 & \multirow{6}{*}{ Nguyen et al., 2010} \\
\hline H10-S9 & Phe343 aromatic ring & Side chain pyran group stacked & \\
\hline $\mathrm{H} 10-\mathrm{S} 9$ & Phe343 ring \& Ile347 side chain & C28 methyl of pyran ring wedged between residues & \\
\hline H10 & Val335 & Pyran C5-9 packed against hydrophobic side chain & \\
\hline H9-H9', S8 & Phe296 Tyr312 aromatic side chains & Favourable binding surfaces with $\mathrm{C} 30 \mathrm{CH}_{3} \& \mathrm{C} 29 \mathrm{CH}_{2}$ & \\
\hline H10-S9 & Undefined & $\begin{array}{l}\mathrm{C} 20 \mathrm{OH} \text { could } \mathrm{H} \text {-bond with this loop \& residues may form water-mediated } \mathrm{H} \text {-bonds } \\
\text { with LAU }\end{array}$ & \\
\hline
\end{tabular}




\begin{tabular}{|c|c|c|c|}
\hline \multicolumn{4}{|c|}{ Peloruside A ( $\beta$-subunit) } \\
\hline Various & $\beta 288-293,296-303,304-316,334-343$ & Important in binding pocket & Huzil et al., 2008; Chen et al., 2008 \\
\hline H9-H9', H9', H10-S9 & $\begin{array}{l}\text { Asp297, Ala298, } \text { Pro307, Arg308, Asn339, } \\
\text { Tyr342 }\end{array}$ & Pocket holding PEL macrolactone & \\
\hline H9, H9-H9', H9' H10-S9 & $\begin{array}{c}\text { Gln294, Asp297, } \operatorname{Arg} 308, \text { Asn339, Val335, } \\
\text { Try342 }\end{array}$ & Side chains involved in pocket holding macrolactone & Huzil et al., 2008 \\
\hline H9-H9' & Phe296 & C26 wedged against side chain & \multirow{7}{*}{ Nguyen et al., 2010} \\
\hline H9' & Arg308 guanidine side chain & $\begin{array}{c}\text { C9 \& C11 hydroxyl long-range electrostatic interactions or potential water-mediated } \\
\text { H-bonds or potential intermittent H-bonds between } 308 \text { \& OHs }\end{array}$ & \\
\hline H9' & Arg308 side chain & Hydrophobic binding surface for PEL. Hydrophobic interactions with C10 and C12 & \\
\hline H9' & $\operatorname{Arg} 308$ & C27 interacts with aliphatic side chain & \\
\hline $\mathrm{S} 8$ & Tyr312 & Hydrogen bond with C24 hydroxyl & \\
\hline S8 & Tyr312 & $\mathrm{C} 10 \mathrm{CH}_{3}$ groups wedged against side chain, $\mathrm{C} 23$ interacts with side chain & \\
\hline $\mathrm{H} 10$ & Val335 & C25 hydrophobic interaction & \\
\hline \multicolumn{4}{|c|}{ Cyclostreptin and analogs } \\
\hline H7 & Asn228 & Covalent bond (CYC and analogs $\mathbf{A}$ and $\mathbf{C})$ & Buey et al., 2007; Calvo et al., 2012 \\
\hline H7 & Cys241 & Analogs $\mathbf{A}$ and $\mathbf{B}$ & Calvo et al., 2012 \\
\hline H6-H7 & Thr221 & H-bond CYC C1 & \multirow{3}{*}{ Buey et al., 2007} \\
\hline M-loop & $\operatorname{Arg} 278$ & H-bond CYC C15 & \\
\hline$\alpha \mathrm{H} 3-\mathrm{S} 4$ & Glu128-Leu132 & Hydrophobic contacts (CYC) & \\
\hline M-loop & Phe272 & Hydrophobic interactions with analogs A \& B & \multirow{4}{*}{ Calvo et al., 2012} \\
\hline S8-S10 & $\operatorname{Arg} 322$ & Polar interactions with analogs A \& $\mathbf{B}$ & \\
\hline $\mathrm{H} 1-\mathrm{H} 1$ & Glu29 & H-bond with $\mathrm{C} 8 \mathrm{OH}$ in analog $\mathbf{A}$ & \\
\hline $\mathrm{H} 7$ & Ser238 & H-bond with $\mathrm{C} 6 \mathrm{OH}$ in analog $\mathbf{B}$ & \\
\hline
\end{tabular}

CYC analogs: 6-chloroacetylcyclostreptin (A), 8-chloroacetyl-cyclostreptin (B) and 8- acetyl-cyclostreptin (C) 

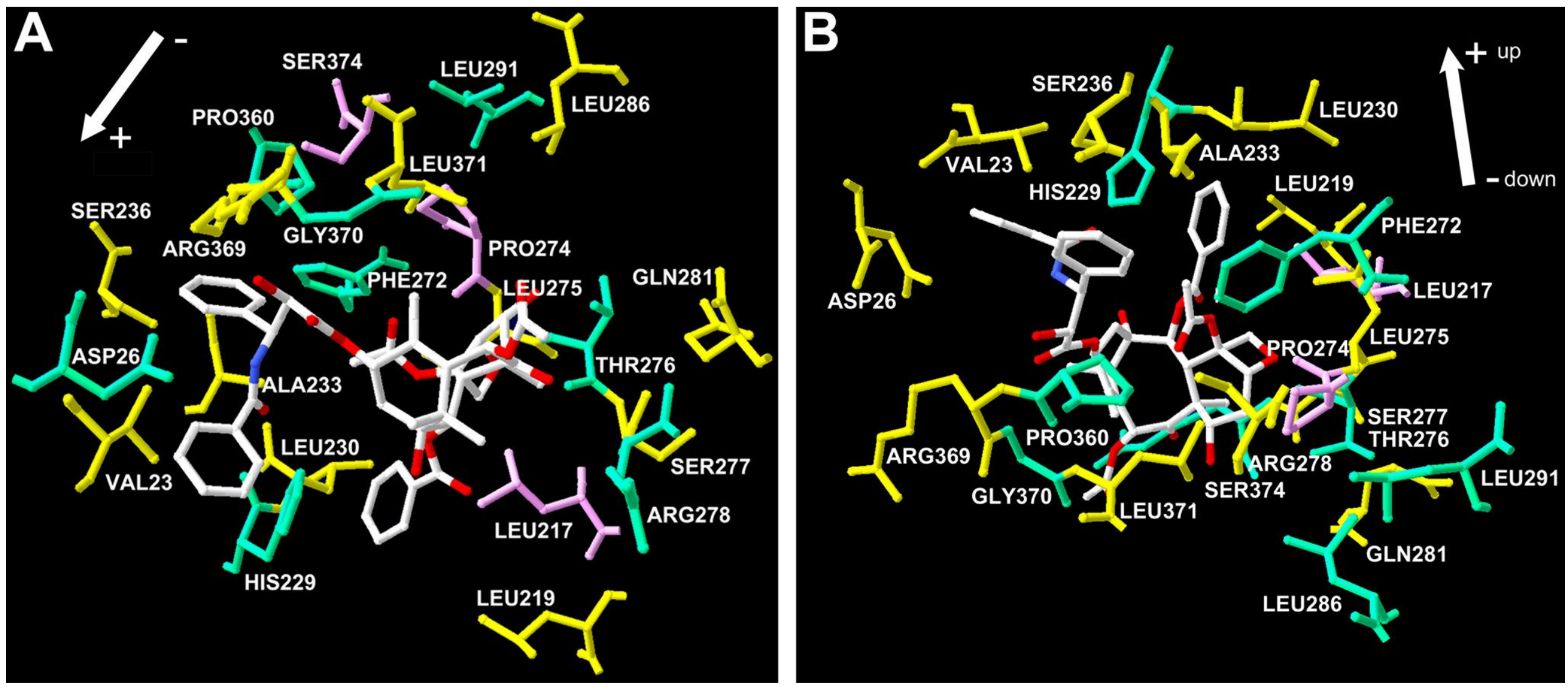

Figure 4.2 The luminal taxoid binding site with bound paclitaxel

A and B. PTX (CPK coloring) shown in its binding site with the interacting amino acids. 

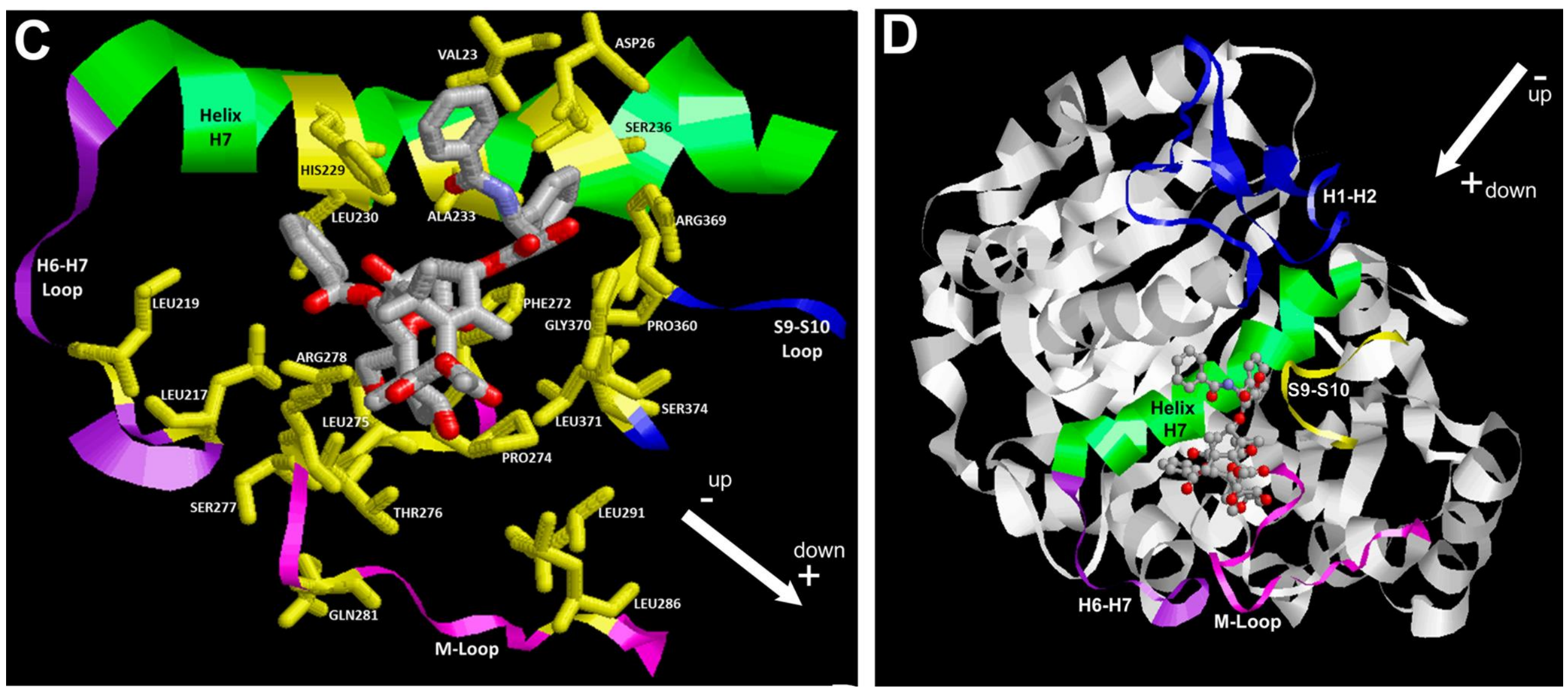

Figure 4.2 continued.

C. Close up view of PTX (CPK) shown in its binding site, interacting amino acids (yellow). The core helix, H7, is shown in green, M-loop in magenta, stabilizing S9-S10 loop in blue, and the H6-H7 loop in purple. D. View of the $\beta$-tubulin subunit with PTX (CPK) in its binding site, secondary structures coloured as above. 


\section{Biological support of the taxoid binding site}

The location of the taxoid binding pocket is also supported by mutant cell data from the Giannakakou laboratory, who described PTX- and EPO-resistant cell lines. These cells lines were discussed in Chapter 3 and have mutations in regions of the PTX binding site (Giannakakou et al., 1997; Nogales et al., 1999; Giannakakou et al., 2000). A number of other mutant cell lines have also been described that induce resistance to some but not all of the PTX-site compounds, depending on how the MSA interacts with the pocket. Table 3.1, Chapter 3 summarizes these mutations. In general, these mutations occur at specific sites within structural features that surround that taxoid binding site or mediate contacts between two tubulin subunits in the context of the protofilament. A number of these mutations have been confirmed using random mutagenesis and a number of new mutations identified, all of which showed that mutations tended to cluster around the H6H9 loop (Yin et al., 2012).

\subsubsection{The two-site binding hypothesis}

As mentioned in Chapter 1.5, the pore type I site on the $\beta$-tubulin subunit is now considered a third MSA binding site. Although this was not always the case and the progress toward determining this is discussed below.

Rapid staining of MTs with the fluorescently-labeled PTX analog FTX-2 led to speculation that PTX binds on the surface of the MT, with the C7 and C10 moieties facing towards the solvent (Evangelio et al., 1998). This is consistent with the finding that PTX rapidly induces MT flexibility in in vitro, indicating that access to the PTX binding site is relatively easy and fast (Dye et al., 1993). However, the taxoid binding site model developed by Nogales and colleagues shows that the binding site is located in the lumen of the MT. Thus, it was proposed that taxanes and other taxoid site ligands reach their luminal binding site by diffusion through openings on the MT surface (Nogales, 2001). Openings in the MT wall were first observed in 1974 (Amos \& Klug, 1974) and later modeled with high resolution by Nogales et al., (1999). In the MT structure, two different types of pores have been described, type I and type II. The type I pore has the $\beta$-subunit at its lower boundary (plus end orientated upwards), with the luminal taxoid site being in close proximity, the type II pore has the $\alpha$-subunit at its lower boundary. The main differences between the two pores are changes in the H6-H7 loop and the degree of hydrophobicity (Díaz et al., 2003). The size of one of these pores in the 
MT wall is considered far too small for passive diffusion of MSAs. Though pore size is comparable with ligand size, diffusion would be extremely slow, and larger openings are not consistent with the MT structure and kinetic data. Diffusion from the open MT ends would be incompatible, restricted and too slow. In addition, the binding of taxoids to the MT is slowed down considerably when MAPs are bound to the outer surface of the MT, indicating an effect of MAPs along the MT. Further evidence against direct diffusion of MSAs to the taxoid site is that PTX reaches its binding site far too quickly (Díaz et al., 2003), with the number of protofilaments changing within one minute of PTX addition (Díaz et al., 1998), PTX rapidly modifies MT flexibility (Dye et al., 1993) and FTX-2 rapidly stains MTs (Evangelio et al., 1998). As mentioned the taxoid binding site is also considered kinetically inaccessible as it is hidden from the exterior in MT models (Nogales et al., 1999), and this would be inconsistent with the kinetic and binding studies. To explain the observed data in view of the structure of the MT, a fast binding theory for ligands that target the taxoid site is required, and thus it has been hypothesized that an external binding site exists. Pore type I describes the space between $\alpha$ - and $\beta$ subunits from neighboring heterodimers, specifically amino acids Phe214, Thr220, Thr221 and Pro222 (Figure 4.3). In this model, binding to both sites would be mutually exclusive, and both sites may share a 'switching element' that accompanies the two different binding modes. This is supported by the 1:1 stoichiometry observed for taxoid site ligands. Díaz et al. (2005) demonstrated that fluoresceinated taxoids could be labeled by an anti-fluorescein antibody, and this would only occur if the molecule was bound at the surface, given that antibodies cannot diffuse into the luminal compartment of the MT. Additionally, doublecortin, a MAP known to bind to the MT in a position that covers the pore site, results in MT assembly (Moores et al., 2004); thus, a pore location for an assembly promoter is not a new concept (Díaz et al., 2005). In order to facilitate this movement of an MSA from the outer site to the inner site, it is proposed that there are conformational changes involving residues of the external binding site moving in toward the luminal site, resulting in loss of the external site and formation of the internal site.

The H6-H7 loop in $\beta$-tubulin, which is flexible (G225 acting as a hinge) (Figure 4.3) and highly hydrophobic, is proposed to be involved in the initial binding of PTX at the pore type I site, and this binding then assists in its translocation to the luminal site (Díaz et al., 2003). Residues Leu217 and Leu219 within this loop are known to make hydrophobic contact with PTX (Löwe et al., 2001), and mutations in this loop are 
associated with PTX resistance (Gonzalez-Garay et al., 1999; Yin et al., 2012). Using computational analysis, it has been proposed that arrangement of the H6-H7 loop causes the formation of an external binding pocket large enough to accommodate an MSA. The same computational analysis was applied to the other pore site on the dimer, pore type II; however, no binding pocket was seen, indicating that the proposed external binding site is only found at pore type I of the MT (Magnani et al., 2009). This binding model describes taxoid site drugs binding to the temporary pore type I site on the surface of the MT with the H6-H7 loop acting to transport the ligand from the pore site to the luminal PTX binding site (Díaz et al., 2003; Díaz et al., 2005; Buey et al., 2007; Magnani et al., 2009). Thus, pore type I acts as a transition station for compounds passing thought to the luminal PTX binding site. This hypothesis is supported by the fact that HXF (fluorescent taxoid derivative) is only able to bind to the pore type I site (because of its large size and the fact that it can be tagged with an antibody, but still shows binding to tubulin). HXF has similar MT-stabilizing activity to compounds that bind to the luminal site. This suggests that pore type I site can be probed as a new druggable site on MTs (Díaz et al., 2005; Barasoain et al., 2010). The existence of this external site is also supported by indirect NMR evidence for a low affinity binding site for MSAs. A fast kinetics of dissociation is required to be able to observe specific NMR signals, and these signals have been seen with the MSAs DTX and DSC (Canales et al., 2008). Therefore, it is highly plausible that a site with much lower affinity, not the luminal site that has high affinity for taxoid site MSAs, is responsible for the NMR recognition of these MSAs (Canales et al., 2011).

Consistent with this external binding site hypothesis CYC, the first MSA to show covalent binding, is able to alkylate both MTs and tubulin dimers. CYC irreversibly competes with taxoid site drugs and was reported to covalently modify two amino acids in $\beta$-tubulin, Thr220, located in pore type I, and Asn228, located near the taxoid site with its side chain facing into the E-site. In unpolymerized tubulin, only Thr220 is labeled by CYC, indicating that the pore type 1 is in fact a binding site and suggesting that pore type I may be the only binding site for MSAs in dimeric tubulin (Buey et al., 2007). Binding of CYC blocks the interaction of PTX and other taxoid site MSAs on MTs, confirming that these sites are mutually exclusive.

To summarize, binding of MSAs to the taxoid site is proposed to occur in a twostep mechanism (Díaz et al., 2000), with the first step being binding to the pore type I 
site on the outside of the MT (Buey et al., 2007) from which the MSA dissociates with fast kinetics (Díaz et al., 2000). The second step is a slow reaction that involves internalization of the MSA to the taxoid binding site. Dissociation of the MSA from the MT would see these binding events reversed, with translocation from the luminal site to the external pore site being the slow step and the second step being the fast release of the ligand from the pore site. This hypothesis explains how PTX can reach the kinetically unfavorable site in the lumen of the MT (Canales et al., 2011). Freedman et al. (2009) tested this hypothesis using computational molecular modeling and confirmed that the H6-H7 loop in the pore acted as a hinge in the first binding step of PTX. This motion was stabilized by residue Ser277 located in the M-loop by hydrogen bonding with PTX. This residue is an alanine rather than a serine in $\beta I I I$ and $\beta$ VI isotypes (Freedman et al., 2009), and it is well known that $\beta$ III tubulin is less sensitive to PTX than $\beta$ I tubulin.

In contrast, Prussia et al., (2010) debates this two-site binding model and concludes that a low affinity site on the exterior of the MT is unnecessary to explain the available data. They base their conclusion on results of molecular dynamics and modeling work that suggests that the shape and lipophilic character of pore site I make it an unlikely binding site for MSAs. It is proposed that the pore simply slows MSA diffusion into the interior of the MT, acting like a 'funnel' or channel without a distinct binding event occurring. It is further suggested that the fast binding kinetics of MSAs could be explained by a pause in the diffusion of the ligand travelling through the pore via transient interactions with specific residues, explaining the observed rapid binding. This would be consistent with CYC labeling of Thr220 since it is located in the narrowest part of the pore. It is proposed that the concept of a binding site at the pore is an artifact of the slow diffusion through the pore into the luminal site, with the pore being nothing more than an entry site. Overall Prussia et al. suggest that although a new druggable site would be welcome, structural considerations of both the MT pore and the tubulin dimer indicate that the existence of a low-affinity preliminary external site is unnecessary to explain the preliminary data on CYC.

In contrast to the model by Prussia et al., the evidence in support of the pore type I binding site has been accumulating over the last few years, and includes previously discussed HXF binding behavior and data on the binding kinetics of FTX-2 which suggest that the interaction is specific (Díaz et al., 2005; Barasoain et al., 2010). A recent study of CYC analogs has shown that two analogs also covalently modify Thr220 (Calvo 
et al., 2012), again supporting the pore site as a binding site. The current overall consensus is that MSAs may bind either the luminal or pore site in unassembled tubulin where in MTs it is likely that they first bind pore type I (fast kinetics) and move into the luminal binding site with the assistance of residues in the H6-H7 loop. This is consistent with and explains the majority of the data currently available in the field.

From the above studies, it is not yet clear whether PTX and its biomimetics bind to only the inner, only the outer, or to both sites, even though it is known that taxoids bind in a 1:1 stoichiometry (Díaz \& Andreu, 1993; Díaz et al., 2000) and that binding to either site on its own causes tubulin assembly (Barasoain et al., 2010). Both sites utilize the residues in the H6-H7 loop, indicating that when a ligand is bound at one site, binding to the other site is not possible (Magnani et al., 2009), and this has been confirmed during in the work done in this thesis. Therefore, binding to either site prevents binding to the other, and likely involves a switching element, such as the H6-H7 loop. Cryo-electron microscopy of doublecortin-stabilized MTs indicates that the taxoid binding pocket is 'open' (Fourniol et al., 2010). It is therefore likely that the pore site is a transient binding site for taxoid site compounds on their way to their final destination in the MT lumen. 

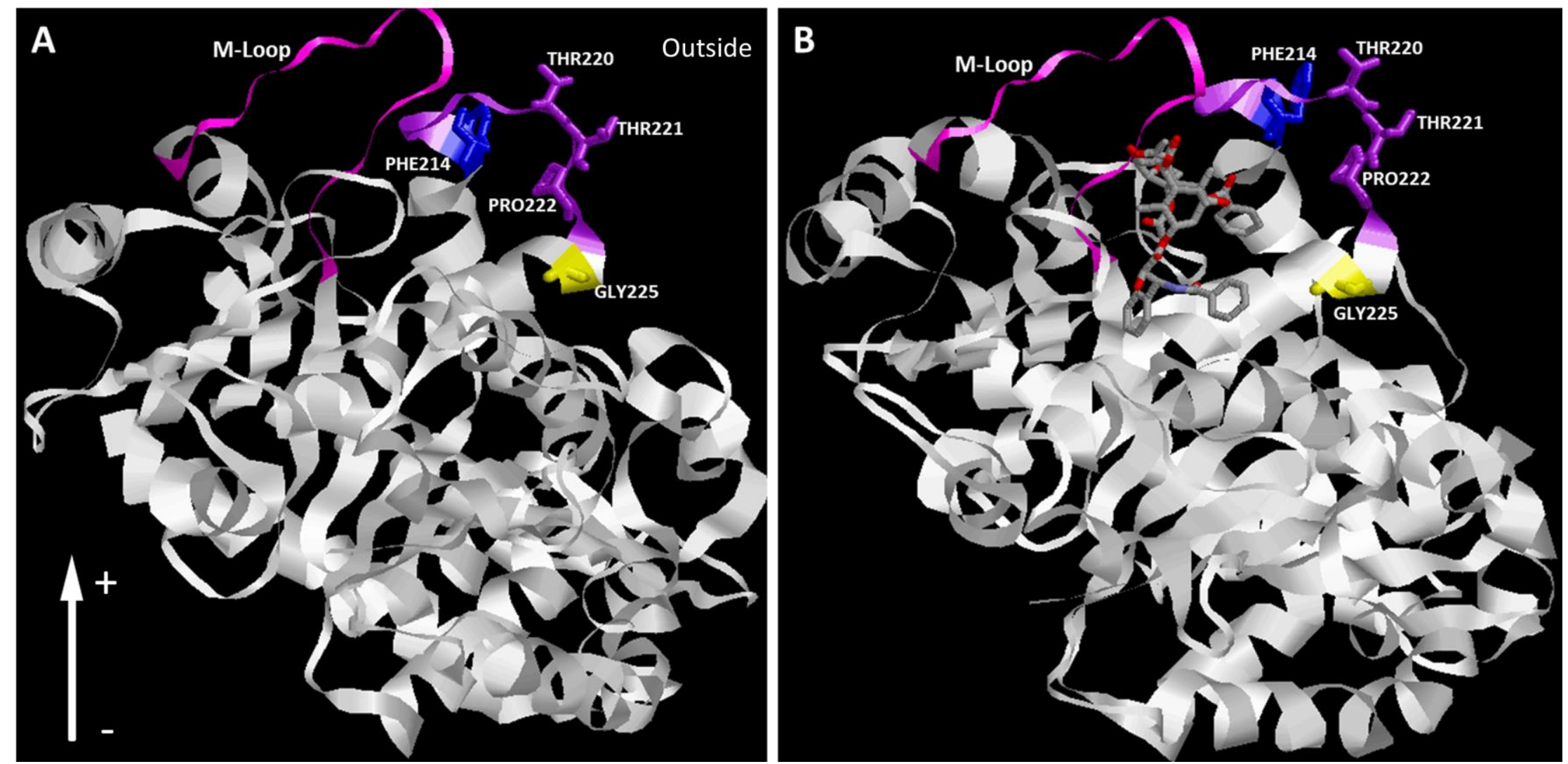

Figure 4.3 The pore type I site

A. $\beta$-subunit showing the M-loop (magenta) and the H6-H7 loop (purple). Important residues are shown in wireframe format. Gly225 (yellow) is thought to act as the hinge in the moment of this loop between the pore site and the luminal binding site. B. Same as A, with PTX (CPK colouring) bound to the luminal binding site. Note that these images have been made from zinc-stabilized sheets and therefore the H6-H7 loop is in its luminal binding site conformation rather than the pore site, thus this is not a true representative pore site image and is just proposed showing interacting components. 


\subsubsection{Other important taxoid site MSAs}

After the taxanes, the next most important MSAs are the EPOs, given that an analog (Ixempra $^{\circledR}$ ) (IXA) is currently approved for use in the clinic. The detailed binding interactions of EPOA with the MT were solved to $2.9 \AA$ resolution by electron crystallography in 2004 using zinc-stabilized sheets (Nettles et al., 2004). This gave direct evidence that the taxanes and EPOs share the same binding region of $\beta$-tubulin as previously thought. Recently, the X-ray crystallography structure of EPOA bound to unassembled tubulin has been solved to $2.6 \AA$ (Prota et al., 2013). This model contrasts with the earlier model, possibly because the X-ray crystallographic model is more defined, allowing for a clearer interpretation of the conformation and orientation of the bound ligand. In addition, the earlier model used zinc-stabilized sheets of MTs; whereas, the latest model used unassembled tubulin. In this model EPOA forms four hydrogen bonds with residues in the taxoid site (Table 4.1). When EPOA is bound, it induces restructuring of the M-loop into a short and well-defined helix that involves residues 278-283 via a number of hydrophobic and polar contacts between the side chain and these residues.

DSC is another taxoid site binding MSA with a unique profile relative to the taxanes. DSC competes with PTX for binding to MTs (Hung et al., 1996), but can also synergize with PTX, suggesting differences in binding to the site (Martello et al., 2000). Unlike other MSAs, DSC is a more flexible and linear compound (Figure 1.5). Using a photolabeled DSC analog, it was shown that residues 355-359 in $\beta$-tubulin are labeled (Xia et al., 2006), these residues are in close vicinity to the taxoid site and form part of the S9-S10 loop (stabilizing loop) (Löwe et al., 2001). NMR studies have shown that the C20-C24 side chain of DSC penetrates a hydrophobic pocket composed of Ala208, Leu209 and Ile212 in H6 and Leu230 in H7 (Sanchez-Pedregal et al., 2006). Given the synergy seen between PTX and DSC, the two compounds must bind to the taxoid site with distinct poses. Using chicken erythrocyte tubulin, PTX was shown to bind to the luminal taxoid site, orientated towards the M-loop, stabilizing the $\beta$-tubulin side of the interdimer; where DSC is orientated away from the M-loop more toward the H1-S2 loop and stabilizes the $\alpha$-tubulin side of the interdimer interface (Khrapunovich-Baine et al., 2009). In support of this distinct binding, DSC had previously been shown to be active in PTX-resistant human cells with mutations in residues 270 and 374 (Kowalski et al., 1997). 


\subsubsection{The laulimalide/peloruside binding site}

The second major MSA binding site on the MT is shared by LAU and PEL and is biochemically distinct from both the taxoid site (Pryor et al., 2002; Gaitanos et al., 2004) and the pore type I site (Buey et al., 2007). Based on competition studies, it is clear that LAU and PEL bind to a unique site; however, the actual location of the site is under debate as there is evidence to support the location of the site on either the $\alpha$ - or $\beta$-subunit. Although the PTX site was solved by electron crystallography using zinc-stabilized tubulin sheets, this technique has so far been unsuccessful with LAU, as it appears that tubulin-LAU solutions are unable to form the sheets required (Thepchatri et al., 2005). Binding to the LAU/PEL site may be more effective at causing stabilization of MTs than binding to the taxoid site since PEL is significantly more potent at inducing assembly of MTs and is highly cytotoxic relative to its binding affinity (Pera et al., 2010).

The binding site for LAU was first described to be within the S9-S10 loop region on the $\alpha$-tubulin subunit (Pineda et al., 2004). This site was supported by NMR data describing the bioactive conformation of PEL bound to the $\alpha$-subunit, with interactions centered on the M-loop (Jiménez-Barbero et al., 2006). This model proposed the involvement of $\alpha$-residues Glu22, Cys25, Thr361, and Arg320 (hydrogen bonding to the $\mathrm{OH}$ at $\mathrm{C} 24$ in PEL), and Phe244. A more recent study showed that PEL analogs with substitutions at C24 abolished polymerizing activity due to loss of this hydrogen bond (Pera et al., 2010). These studies concluded that the LAU/PEL site is located on $\alpha$ tubulin in a zone equivalent to that occupied by PTX in $\beta$-tubulin (Figure 4.4A). A limitation with this model is that the $\alpha \mathrm{S} 9-\mathrm{S} 10$ loop has eight extra amino acids (361-368). These residues are thought to occlude this site and cause stabilization of the $\alpha \mathrm{M}$-loop, giving extra strength to the lateral contacts between $\alpha$-subunits in different protofilaments (Nogales et al., 1999; Fourniol et al., 2010), thus it seems unlikely an MSA would bind here.

Using hydrogen-deuterium exchange MS it was proposed that the PEL binding site is located on the exterior surface of the $\beta$-subunit, in a cavity near the $\alpha$-, $\beta$-subunit interface (Huzil et al., 2008). Although this $\beta$-subunit site is relatively close to the taxoid site in sequence, it is structurally distant. Table 4.1 describes the binding site interactions of LAU and PEL. Using digital signal processing studies in the same lab, a technique that detects hot spots on the tubulin protein in which binding occurs, a region nearly identical to that originally proposed by Huzil et al., (2008) was identified as a likely PEL binding 
site (Chen et al., 2008). This $\beta$-subunit site location was then independently supported using modeling and biochemical studies with $\left[{ }^{3} \mathrm{H}\right]-\mathrm{PEL}$. This model provides a more reasonable structural basis for binding at the $\beta$-subunit and shows a greater array of hydrophobic and electrostatic interactions at this site rather than at the $\alpha$-site (Nguyen et al., 2010). Although binding to the same site, PEL was orientated differently and generated more hydrogen bonds to that previously proposed (Huzil et al., 2008). Specifically, an intramolecular hydrogen bond was formed that stabilized the orientation and allowed interaction of hydroxyls groups with residue 308 providing a hydrophobic platform for PEL. In the Nguyen et al. model the macrolide cores of LAU and PEL line up, and modification at $\mathrm{C} 24$ would reduce binding due to loss of a hydrogen bond with Tyr312, consistent with the findings by Pera et al. Another study by the Schriemer lab used mass shift perturbation analysis and data-directed molecular docking simulations to show LAU binds near the intradimer interface above the COL binding site in the vicinity of the type II pore (Bennett et al., 2010). Binding of a ligand in this site would not require an opening in the MT wall for access, given its external location, unlike that required for taxoid site compounds. This makes the LAU/PEL site unique by being located on the outside of the MT; whereas all other MTA sites, except for the less characterized pore type I site, are located on inner interfaces of the MT, and indicates that the LAU/PEL site would be assessable to MAPs. Binding at the site would cause reorganization of the side chains protruding into the cavity, thus promoting the stabilization of loops in this region. A more recent paper used HDX-MS to provide conformation of the $\beta$-subunit binding site and concluded that the $\alpha$-tubulin site was unlikely for LAU and PEL binding (Khrapunovich-Baine et al., 2011).

Biological support for $\beta$-tubulin subunit binding site was recently provided from mutation mapping following long-term exposure of cells to stepped concentrations of MSAs (Table 3.1). Mutations to resides important for PEL and LAU binding by the models impaired tubulin polymerization in the presence of either PEL (Ala298Thr) or both LAU and PEL (Arg308His/Cys), but not to taxoid site agents (Kanakkanthara et al., 2011). A subsequent study described four cell lines, two that were identical to those previously described and two new mutations: Tyr342Ser and Asn339Asp (Begaye et al., 2011). These four mutations all center on a cleft that is proposed to interact with the side chain of PEL (Huzil et al., 2008), and all four mutations conferred resistance to PEL and showed cross-resistance to LAU (Table 4.2). Both of these cell-based studies supported 
the $\beta$-tubulin location of the binding site rather than $\alpha$-tubulin, and both highlighted the importance of the side chain cavity. Residue Arg308 is essential in defining the entrance into the deep, narrow hydrophobic cleft in which the side chains of both compounds penetrate, and mutations in Arg308 caused the greatest resistance of the cells. LAU is thought to reorganise the side chains of amino acids that point into this cleft to cause stabilization of this region (Bennett et al., 2010) through stabilization of Arg308 via its interactions with residue 342. However, this reorganization is not thought important in PEL binding, which instead may involve interactions between residues 308 and 298 (Begaye et al., 2011) (Figure 4.4B).

The overall conclusion at this stage is that LAU and PEL most likely binds to the $\beta$-tubulin site first proposed by Huzil et al. (2008), but confirmation by X-ray crystallography is required to definitively prove this location. Although it is possible that LAU and PEL may bind at both the described $\alpha$ - and $\beta$-subunits sites, stoichiometry studies giving 1:1 ratios of binding do not support simultaneous binding (Pera et al., 2010). Thus the $\alpha$ - and $\beta$-sites, if both exist, must be mutually exclusive, similar to the taxoid site and the pore type I site as discussed. Pera et al., (2010) suggest that, like the taxoid site drugs, LAU and PEL may have a two-step binding mechanism, in which binding would involve an initial event, possibly an interaction with the $\beta$-subunit, given the proximity of this proposed site to pore type II, followed by binding at the $\alpha$-tubulin site. It is also possible that the initial binding site may be with low affinity on the $\alpha$ subunit, since this is the site that is picked up by NMR studies, and given that fast kinetics of dissociation are required to observe specific NMR signals. The final binding location would then be the $\beta$-tubulin site. This alternative mechanism of binding better explains the fact that mutations in resistant cell lines are only found in the $\beta$-subunit, much like the resistant cell lines described for PTX and EPO that have mutations in the luminal binding site rather than the pore type I site. It is not uncommon for drugs to have a two-step binding mechanism. For example, COL and a number of its analogs bind in a two-step mechanism in which the first step is slow and occurs with low affinity binding, followed by a conformational change causing high affinity binding (Skoufias \& Wilson, 1992). The difference here, however, is that there are not two distinct colchicine binding sites but two sub-sites in a single region of $\beta$-tubulin. 

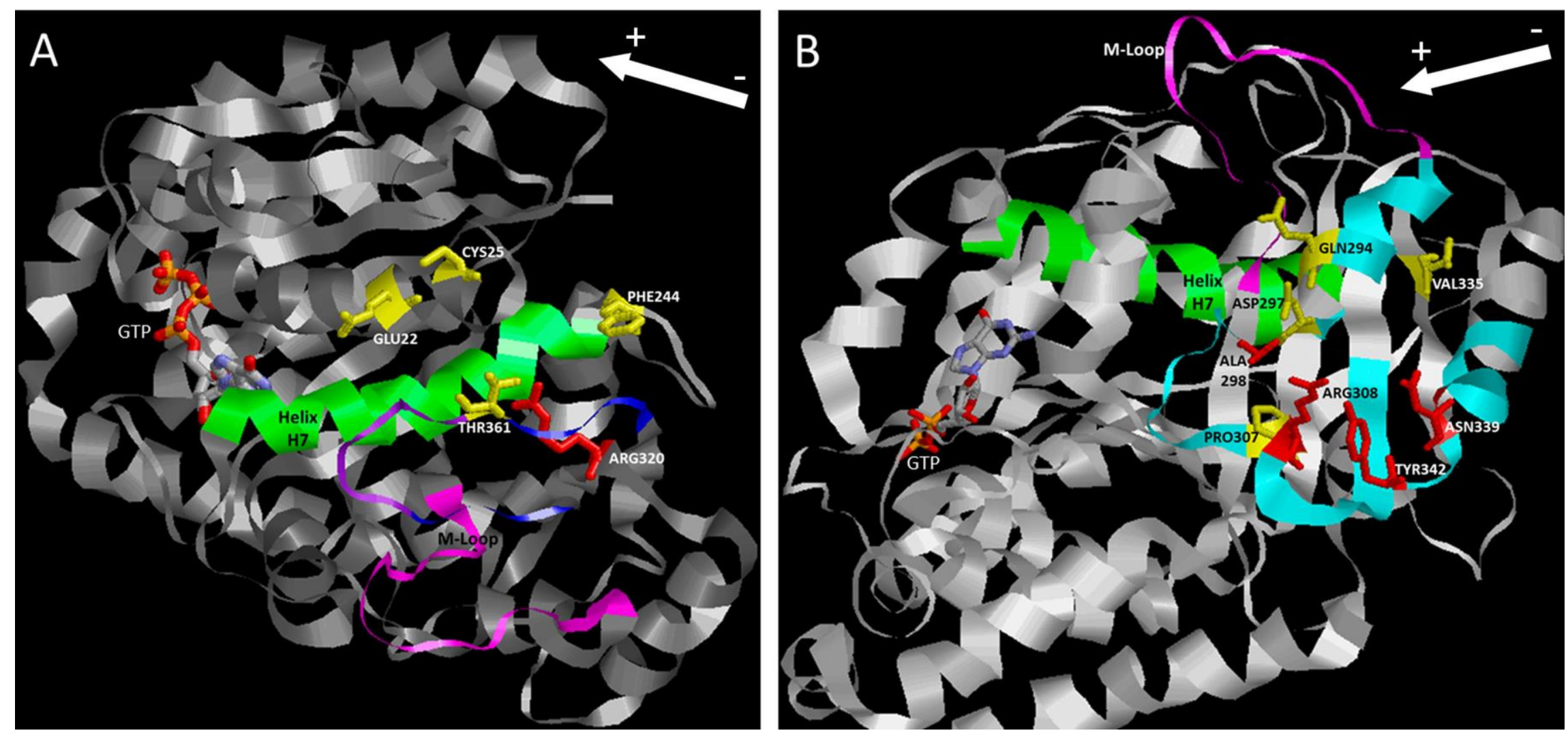

Figure 4.4 Models of the proposed LAU/PEL sites

A. Model of the proposed site in $\alpha$-tubulin. Showing the core helix (green), the M-loop (magenta), S9-S10 loop is shown in blue and the eight extra amino acids of this loop shown in purple. Amino acids important in PEL binding are coloured in yellow, Arg320 (red) hydrogen bonds with PEL. GTP is colored in CPK. B. Model of the proposed site in $\beta$ tubulin. Secondary structures coloured as above, note the missing purple residues in this subunit, the peptides shown in cyan are those identified by Huzil et al., (2008) using HDXMS. Interacting residues are shown in yellow and red. Those in red are the amino acids mutated in resistant cell lines. 


\subsubsection{Aims of this chapter}

1. To determine the activity of ZMP in vitro

2. To determine the binding site and binding stoichiometry of ZMP

3. To investigate the binding epitope of the ZMP chemotype

One of the first steps in characterizing a novel MSA is determining the site at which it binds to the MT, thus the main aim of this chapter was to determine the binding site of ZMP. Currently there are three known binding sites, the well-characterized taxoid binding site, the LAU/PEL site and the less well characterized pore type I site. Results from cellular studies presented in the previous chapter suggested that ZMP may bind at the taxoid binding site since some resistance to ZMP was seen in taxoid site mutant cell lines. To directly probe the binding site of an MSA, biochemical experiments rather than the more complex cellular experiments are needed, since isolated tubulin is easier to work with. ZMP was prepared for these biochemical experiments by determining the extinction co-efficient and the characteristic HPLC peaks, so that it could be prepared for and monitored in a number of different biochemical assays. This is particularly important when using a natural product as HPLC can be used to determine the purity of the sample.

Before the binding site was probed, it was important to determine the ability of ZMP to induce MT assembly in vitro and determine how potent it is relative to other MSAs. This would also provide information on the ligand concentration required and the assay conditions needed for further characterization of the compound. The binding site of ZMP was then directly probed using a displacement assay for the taxoid binding site, since the majority of the compounds known to stabilize MTs bind at this site. The ability of ZMP to interact with the pore type I site was also investigated, and its interaction with the LAU/PEL site was investigated by Dr. Ernest Hamel of NCI/NIH (Field et al., 2012). The second aim of the chapter was to determine the stoichiometry of ZMP binding to the tubulin heterodimer, to ensure ZMP binds with a 1:1 stoichiometry as seen with all other MSAs. The third and final aim was to investigate the binding epitope of this new MSA chemotype. At the time of this particular study, only the natural product ZMP was available and was in scarce supply, therefore DAC was used instead since DAC is a natural enantiomer of ZMP and would still provide useful information on the ZMP interaction of tubulin at the macrolide core of the compound. 


\section{$\underline{4.2 \quad \text { METHODS }}$}

\subsubsection{Preparation of zampanolide}

$H P L C$

ZMP was diluted to $5 \mu \mathrm{M}$ in ACN to a final volume of $30 \mu \mathrm{L}$. HPLC as described in Chapter 2.9 was run without an internal standard at a flow rate of $1 \mathrm{~mL} / \mathrm{min}$. The column was developed using a gradient of 10\%-100\% ACN over 10 min followed by $100 \%-10 \%$ ACN over $10 \mathrm{~min}$. This measures the absorbance of ZMP and gives its characteristic twin peaks (Tanaka and Higa, 1996). The purity of ZMP and the identity of the peaks were verified using reverse-phase HPLC-MS. ZMP was diluted to $5 \mu \mathrm{M}(\mathrm{ACN})$ and was run through HPLC-MS using electrospray ionization in a Thermo Finnigan LXQ instrument coupled to a Surveyor chromatograph and employing a Supercosil, LC18 DB, $250 \times 4.6 \mathrm{~mm}, 5 \mathrm{~mm}$ bead diameter column. HPLC was run using a gradient of $10 \%-$ $100 \%$ ACN over 10 min followed by $100 \%-10 \%$ ACN over 10 min. The peaks were then analyzed by MS to determine the molecular weight of each peak and to confirm that the two peaks were both ZMP.

\section{Spectroscopy}

ZMP was diluted to $5 \mu \mathrm{M}$ in ethanol (Spectroscopy grade Uvasol ${ }^{\circledR}$ Merek, Germany) and its absorbance measured using a Thermo Fisher Evolution 300 UV/Vis spectrometer. The sample was run against an ethanol blank. The extinction coefficient for ZMP was then calculated using Beer Lambert's Law as described in Chapter 2.4. Two extinction coefficients were determined for ZMP since it had two peaks. The same process was repeated for DAC. The spectrophotometer also picks up the DMSO diluent peak which is subtracted from the spectrum.

\subsubsection{Ligand induced microtubule assembly}

In the absence of MSAs, MT assembly is a noncovalent nucleated condensation polymerization reaction that is characterized by cooperative behavior and depends on the tubulin being above the $\mathrm{C}_{\mathrm{r}}$ for the conditions in which assembly is occurring. Below this concentration of tubulin, assembly of tubulin into MTs does not occur (Díaz et al., 1993). Adding a promoter ligand to the reaction results in a more complex reaction that may proceed via two different pathways and these will be discussed in Chapter 5.1. The apparent equilibrium constant for the MT growth reaction (addition of an assembly 
inducer) is equal to the reciprocal of the $\mathrm{C}_{\mathrm{r}}$ (with good approximation) (Díaz et al., 1993). This allows the apparent standard free energy change of assembly to be amenable to simple measurements (providing that nucleotide hydrolysis is ignored) (Díaz et al., 2009) using the equation:

$$
\Delta \mathrm{G}_{0 \mathrm{app}}=-\mathrm{RT} \ln \mathrm{K}_{\mathrm{a}}
$$

in which $\Delta \mathrm{G}$ is the standard free energy of binding, $\mathrm{R}$ is the gas constant $(8.314 \mathrm{~J} / \mathrm{mol} \mathrm{K})$, $\mathrm{T}$ is the temperature and $\mathrm{K}_{\mathrm{a}}$ is the equilibrium binding constant. The theory of linked functions is able to be employed for any ligand that is involved in protein assembly and predicts that the equilibrium of the polymerization process is displaced toward the assembled species which the ligand has higher affinity for. This model does not apply for normal MT assembly as it is not a true equilibrium due to the hydrolysis and exchange of nucleotide, which results in dynamic instability. However, MSAs can induce assembly of GDP-bound tubulin into MTs and this can be considered an equilibrium reaction of MT polymerization. Based on this, the $\mathrm{C}_{\mathrm{r}}$ is the concentration of active protein in the supernatant, and this is an accurate measurement, given that it has already been shown that essentially all the tubulin is active (Díaz et al., 1993).

The first task to characterize the mechanism of action of a suspected MSA is to confirm that it is a true MSA. To do this, it needs to be shown to induce tubulin assembly in conditions in which tubulin on its own is not able to assemble or assembles very slowly. Only strong MSAs are able to do this; whereas, weak MSAs can only assemble tubulin in conditions favorable for assembly. Glycerol is a nonspecific thermodynamic promoter of MT assembly ( $\mathrm{Na} \&$ Timasheff, 1981) and is used to create conditions in which tubulin assembles at a lower $\mathrm{C}_{\mathrm{r}}$ than without the glycerol. The difference in conditions between these two assays was described in Chapter 2.6.

The process of measuring tubulin polymerization was described in Chapter 2.6. The $\mathrm{C}_{\mathrm{r}}$ of ZMP was measured in both favorable and unfavorable assembly conditions; whereas DAC was only measured in the latter. In favorable assembly conditions, $20 \mu \mathrm{M}$ and $25 \mu \mathrm{M}$ ZMP or DAC were incubated with $15 \mu \mathrm{M}$ and $20 \mu \mathrm{M}$ tubulin for $30 \mathrm{~min}$ at $37^{\circ} \mathrm{C}$, the samples separated by centrifugation and processed as described. DTX and PDP were used as positive and negative controls, respectively, and DMSO was used as the baseline control. In unfavorable assembly conditions, increasing concentrations of ZMP (0 $\mu \mathrm{M}$ (DMSO only), $2 \mu \mathrm{M}, 5 \mu \mathrm{M}, 10 \mu \mathrm{M}, 15 \mu \mathrm{M}, 20 \mu \mathrm{M}, 25 \mu \mathrm{M}$, and $30 \mu \mathrm{M}$ ) were 
incubated with $20 \mu \mathrm{M}$ tubulin at $37^{\circ} \mathrm{C}$, separated and processed as described in section 2.6.2.

\subsubsection{Binding site determination studies}

One of the first steps in characterizing a novel MSA is determining the site at which it binds to the MT. A number of different methods exist to determine the binding site of an MSA. In principle the best strategy to probe the binding site is to perform various competition assays against compounds that bind to the known binding sites, such as PTX for one site and PEL for the other. The majority of MSAs bind to the taxoid site in the lumen of $\beta$-tubulin; therefore, this site is usually probed first. A binding competition assay using stabilized taxoid binding sites and a fluorescent derivative of PTX that binds the taxoid site can be performed to directly determine whether an MSA binds to the taxoid site on the MT. It is possible however that binding at another site on the MT may cause an allosteric change resulting in displacement of Flutax from the taxoid site; however, this is unlikely. Currently there are no fluorescently-labeled LAU or PEL ligands available; therefore, this particular assay can only distinguish between taxoid site ligands and non-taxoid site ligands. Another possible method for determining the binding site of an unknown MSA involves radioactive labeling of the positive control compound whose binding site is known, followed by competition studies and this can be done to probe either the taxoid site or the LAU/PEL site (see Edler et al., 2005 and Nguyen et al., 2010 for examples using $\left[{ }^{3} \mathrm{H}\right]-\mathrm{PTX}$ and $\left[{ }^{3} \mathrm{H}\right]$-PEL respectively). In short, this involves addition of $\left[{ }^{3} \mathrm{H}\right]$-ligand with the test ligand to pre-formed MTs, incubation and separation of the bound and free ligands using centrifugation. The samples are then dissolved in a buffer, neutralized, and the $\left[{ }^{3} \mathrm{H}\right]$-ligand content of the supernatant and pellet quantified. If the $\left[{ }^{3} \mathrm{H}\right]$-ligand is found in the supernatant then the test compound binds the same site as the labeled ligand; if it is found in the pellet then both the $\left[{ }^{3} \mathrm{H}\right]$-ligand and the test ligand are simultaneously binding the MT and therefore must interact with different binding sites. This assumes that enough ligand is added to bind most of the sites; otherwise, both ligands could bind different populations of a single site. Although the method is powerful, it requires the use of radio-labeled compound that is not always easy to obtain and/or synthesize. Alternatively, HPLC techniques can be used to quantify bound ligand using the same principle as in the above method. Basically, both ligands are added to the MTs, the ligand with the known binding site is added in excess so that all sites are 
saturated. The amount of drug in the pellet and the supernatant can be quantified against an internal standard. If both drugs are found in the pellet then both drugs are simultaneously binding to different sites, if the test ligand is only in the supernatant then it competes with the known ligand for binding to the same site.

\section{Flutax-2 competition assay}

Fluorescence anisotropy is a good technique for measuring the interaction between two molecules to determine binding constants or dissociation constants of the interaction. It is a fast, sensitive, and quantitative technique able to determine ligand-protein interactions. Using a polarizer, the sample is excited by polarized light (Bhattacharyya et al., 2010). The extent of polarization of the emission light is described by anisotropy and is the amount of the polarized light emitted from a fluorophore that has been excited by the polarized light (Lakowicz, 2006). A freely moving molecule (unbound) will scramble its emitted polarized light by radiating it in different directions from the incident light. This scrambling effect is greatest when a molecule is free in solution (freely moving with the lowest possible anisotropy) and will decrease with decreased rates of free movement. By binding to a larger, more stable molecule (i.e. a protein), a larger, more stable complex is formed that will tumble more slowly than a molecule free in solution, and this will increase the polarization of the emitted light, reducing the scrambling effect and increasing the anisotropy (LiCata \& Wowor, 2008).

PTX is not naturally fluorescent; however, it can be made fluorescent by linking a fluorescent dye to C7 using a spacer containing a free amino group (Souto et al., 1995). Flutax-1 and FTX-2 (Figure 4.5) are fluorescent derivatives of PTX. FTX-2 is more photostable than Flutax-1 and has an acidic pH that makes it fluoresce more strongly at neutral $\mathrm{pH}$ in which it is a dianion (Andreu \& Barasoain, 2001). Modifications at C7 of the PTX structure do not significantly affect the biological activity of the compound (Nicolaou et al., 1994). As mentioned in Chapter 2.7 these compounds are used as reference ligands in competition experiments to measure the affinity of ligands for the PTX site and distinguish them from ligands with the same mechanism of action that do not bind the taxoid site.

The study of the interaction between PTX and its biomimetics with their binding site is difficult due to the nature of the binding mechanism, since binding and tubulin assembly are linked reactions (Díaz et al., 1993). The majority of MSAs have high 
affinity for MTs, and it is therefore near impossible to find conditions in which the reaction is not displaced toward the end state. It is therefore difficult to obtain exact measurements of the binding affinity of these compounds. A procedure to stabilize MTs has made it possible to obtain taxoid binding sites that can be diluted to low enough concentrations to directly measure the binding affinity of reference ligands. The binding of a test ligand to stabilized MTs can be measured indirectly by measuring the change in fluorescence of the reference ligand (Díaz et al., 2000; Barasoain \& Andreu, 2001). This assay measures the change in anisotropy of bound FTX and free FTX. Upon binding, the anisotropy of FTX-2 increases. The binding constants and anisotropy values of the fluorescence emission of FTX-2 are known (Tables 2.1, 2.2) (Díaz et al., 2000). Assays performed with stabilized crosslinked MTs rather than soluble tubulin removes the requirement for overcoming the $\mathrm{C}_{\mathrm{r}}$ before MTs can form. This allows for greater competition since the starting concentration required for the compounds is much lower.

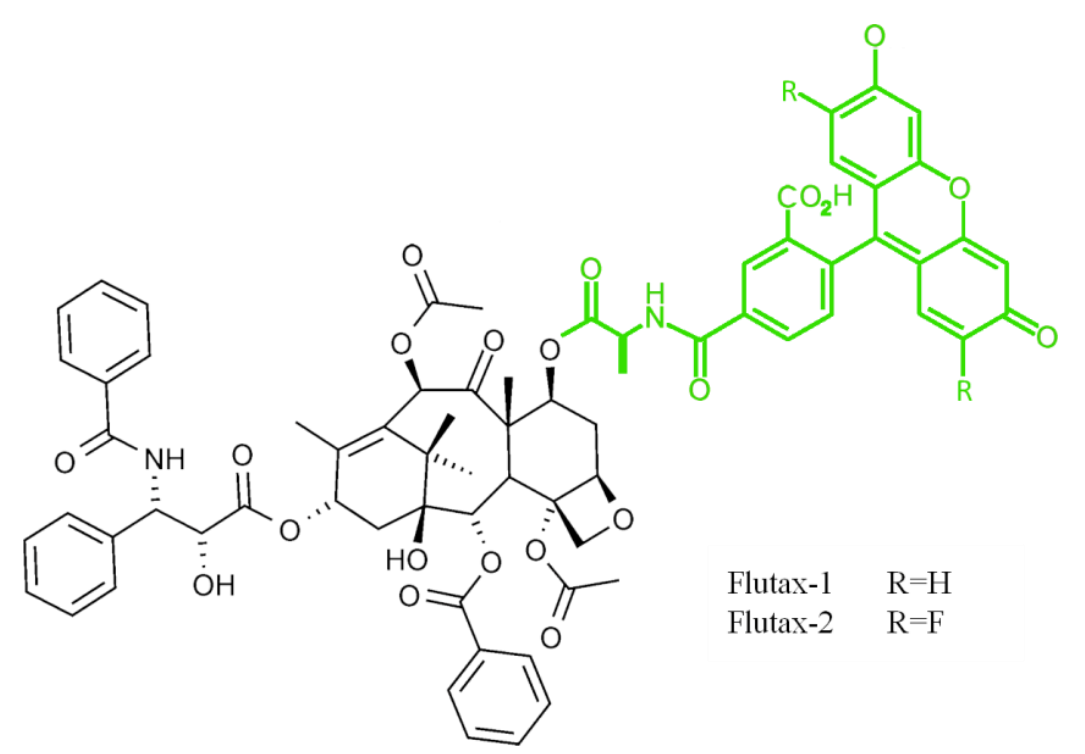

Figure 4.5 Structure of Flutax compounds

Flutax 1 and FTX-2 are fluorescent derivatives of PTX and can be used as probes of the taxoid binding site. The PTX portion of the compound is shown in black. Fluorescein (green) is attached to positon-7 of PTX in Flutax-1, and difluorofluorescein is attached to position in FTX-2. Refer to Figure 1.5 for PTX numbering. 
The FTX-2 displacement assay was carried out as described in Chapter 2.7. DTX and EPOA were used as positive controls, and VBL as a negative control. ZMP and control drugs were used at the following concentrations: $0 \mathrm{nM}, 10 \mathrm{nM}, 20 \mathrm{nM}, 50 \mathrm{nM}, 0.1 \mu \mathrm{M}$, $0.2 \mu \mathrm{M}, 0.5 \mu \mathrm{M}, 1 \mu \mathrm{M}, 5 \mu \mathrm{M}$, and $20 \mu \mathrm{M}$. DAC was used at higher concentrations (1 $\mu \mathrm{M}, 5 \mu \mathrm{M}, 10 \mu \mathrm{M}, 20 \mu \mathrm{M}, 25 \mu \mathrm{M}$ and $100 \mu \mathrm{M}$ ) because of its lower potency in inducing MT assembly.

\subsubsection{Stoichiometry}

The stoichiometry of ZMP binding to crosslinked MTs was measured by incubating stabilized taxoid binding sites $(25 \mu \mathrm{M})$ with increasing concentrations of ZMP in GAB buffer for $30 \mathrm{~min}$ at $25^{\circ} \mathrm{C}$. Samples of $10 \mu \mathrm{M} \mathrm{ZMP}$ and $0 \mu \mathrm{M}$ sites were used as a blank. MTs and bound ZMP were separated from unbound ZMP by centrifugation at 50,000 rpm using a TLA100 rotor in a Beckman Optima TLX ultracentrifuge, for 20 min at 25 ${ }^{\circ} \mathrm{C}$. The supernatant was carefully collected by pipetting and the pellet resuspended in 10 mM NaPi pH 7.0 buffer. DTX $(10 \mu \mathrm{M})$ was added to the samples as an internal standard and the samples processed as in Chapter 2.9, dissolving them in $35 \mu \mathrm{L} \mathrm{v} / \mathrm{v} 80 \%$ ACN/water and then analyzing by HPLC using a gradient of $\mathrm{ACN} / \mathrm{H}_{2} \mathrm{O}$ for $30 \mathrm{~min}(0-10$ $\min 10 \% \mathrm{ACN}, 10-20 \min 100 \% \mathrm{ACN}, 20-30 \min 90 \% \mathrm{ACN})$. The concentration of ZMP in each of the samples was quantified by comparison of the integrated areas of the HPLC peaks with those of the internal standard DTX. The above procedure was then repeated with DAC.

\subsubsection{Probing the pore type I site}

HXF (Figure 4.6) is an analog of Flutax-1 with a 6-carbon aliphatic spacer between the PTX group and the fluorescein group to separate the two moieties (Díaz et al., 2005). Antibodies against the fluorescein moiety have explicitly shown that HXF binds to the pore type I site of MTs and not to the luminal site, since large protein antibodies, unable to diffuse into the lumen, can label bound HXF. In addition, fluorescent quenching of HXF occurs very fast, indicating that the fluorescein group is solvent exposed (Díaz et al., 2005; Barasoain et al., 2010). HXF is active in cells and has similar effects to PTX, being able to polymerize tubulin in vitro. HXF has a lower binding affinity than other PTX fluorescent analogs, and is significantly less cytotoxic $(2.3 \mu \mathrm{M}$ compared to $0.3 \mu \mathrm{M}$ and $0.8 \mu \mathrm{M}$ for Flutax-1 and FTX-2, respectively) (Barasoain et al., 2010). Upon 
binding, its fluorescence intensity decreases $40 \%$ at $527 \mathrm{~nm}\left(\lambda_{\mathrm{exc}}=495\right)$. Much like FTX2, the anisotropy of HXF increases upon binding, and the anisotropy values of the fluorescence emission of HXF are known $\left(\mathrm{r}_{\text {free }}=0.06, \mathrm{r}_{\text {bound }}=0.18\right)$ (Díaz et al., 2005). $\mathrm{HXF}$ is therefore an excellent probe for the pore type I binding site.

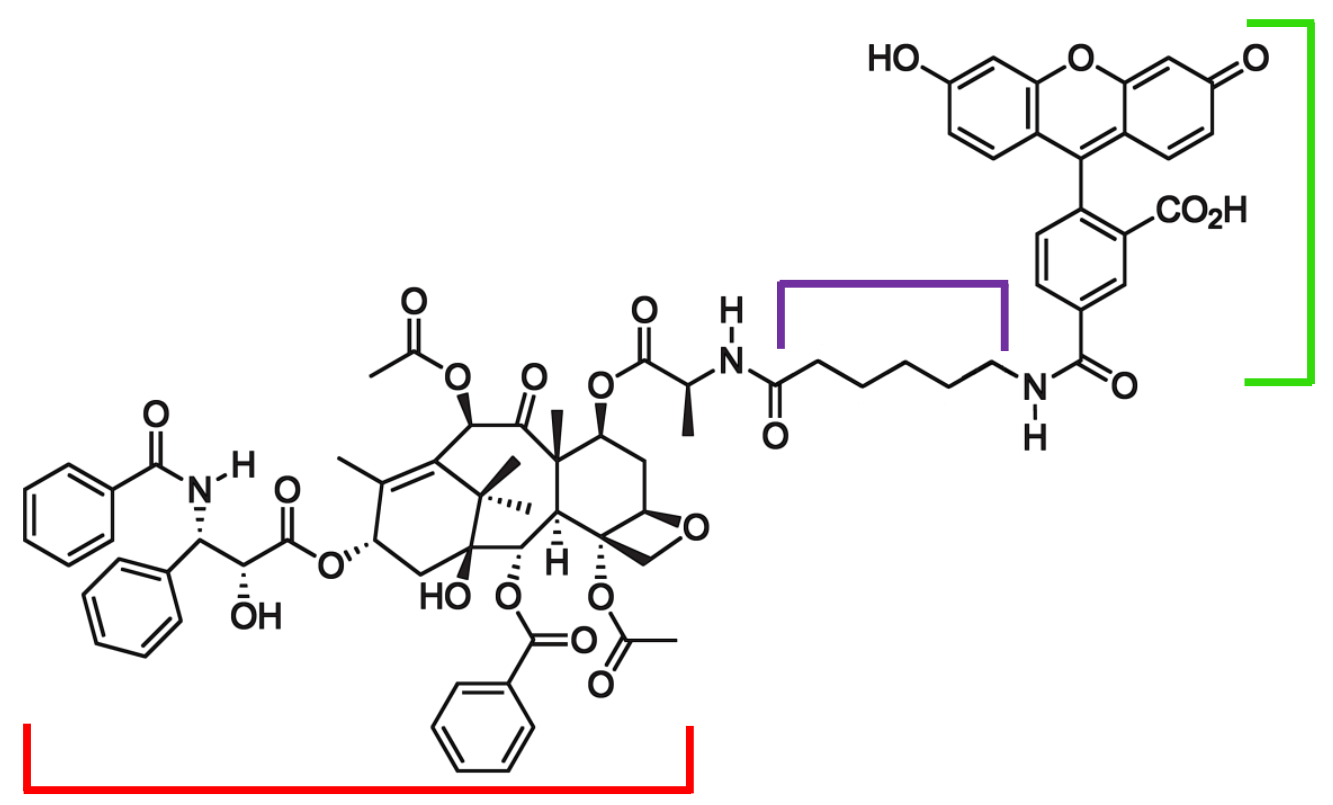

Figure 4.6 Structure of 7-Hexaflutax

HXF is a fluorescent analog of PTX. The PTX component is boxed in red, with a 6-carbon chain linker shown in purple and at position 7 of the PTX skeleton a fluorescein group is attached shown in green, similar to Flutax-1. Refer to Figure 1.5 for PTX numbering.

In order to check if ZMP binding to the taxoid site blocks binding at the pore site, and to confirm that both sites are mutually exclusive, binding at the pore site was checked using HXF. Given the low binding affinity of HXF, a concentration of $200 \mathrm{nM}$ was used. Stabilized taxoid sites $(200 \mathrm{nM})$ were incubated for $2 \mathrm{~h}$ at $25^{\circ} \mathrm{C}$ with $200 \mathrm{nM} \mathrm{HXF}$ in GAB buffer. Increasing concentrations of ZMP were then added to the samples ( $25 \mathrm{nM}-$ $1 \mu \mathrm{M})$ and incubated for $30 \mathrm{~min}$. A GAB buffer blank and a control sample (200 $\mathrm{nM}$ stabilized taxoid sites in buffer) were included. The anisotropy was then measured in a Fluorolog- 3-221 instrument (Jobin Yvon-Spex) in the T-format mode with vertically polarized excitation and correction for the sensitivity of each channel with horizontal polarized excitation. 


\subsubsection{Saturation transfer difference NMR}

The bioactive conformation of a ligand bound to a protein can be determined using NMR techniques. Proteins consist of a large system of protons that are tightly coupled by dipole-dipole interactions. These protons can be selectively saturated using magnetization pulses. The Nuclear Overhauser Effect (NOE) is the transfer of spin polarization via cross-relaxation, and NOE signals are observed between different proton pairs (Ni \& Zhu, 1994). Saturation transfer difference (STD) NMR $\left({ }^{1} \mathrm{H}\right.$ NMR) is a double resonance NOE method used to probe low affinity interactions between small molecules and proteins and is an extremely useful tool with a number of biological applications (Bhunia et al., 2012). STD-NMR can detect the bioactive conformation of small molecules bound to a macromolecule since experiments can be set up to detect saturation transfer from a given target protein to a bound ligand (Mayer \& Meyer, 1999). This provides information about how a ligand interacts with its binding site and gives a starting point for molecular modeling. This method relies on the existence of a dissociation rate that is fast on the relaxation time scale and identifies parts of the ligand that are in direct contact with the protein, otherwise the magnetic transfer would not occur (Ni \& Zhu, 1994; Mayer \& Meyer, 2001). Transferred nuclear Overhauser spectroscopy (trNOESY) allows determination of structures in solution and can be used to distinguish ligands that bind to a protein from those that do not. This technique relies on the fact that upon ligand binding to the protein, NOEs undergo huge changes resulting in the transfer of NOEs to the ligand (Ni \& Zhu, 1994). Large proteins have negative NOEs that are passed on to the bound ligand (Meyer \& Peters, 2003). Compounds that bind to the protein are identified by NOE cross-peaks that have become negative as a result of binding. Both trNOESY and STD techniques rely on a fast on/off rate of the ligand with the protein. Using these NMR techniques, the bioactive conformation of an MSA bound to MTs can be determined (Jiménez-Barbero et al., 2006; Canales et al., 2008; Canales et al., 2011).

STD requires two separate experiments to be carried out, on-resonance and offresonance, and relies on a protein passing saturation (magnetization) to a bound ligand. In the on-resonance, protein is selectively saturated with a selective pulse without saturating the ligand (an irradiation frequency is used in which only resonances of the protein protons are located in the spectra). This saturation is spread over the protein by spin diffusion (intramolecular saturation transfer). Ligands that bind the protein are then 
saturated by intermolecular saturation transfer due to their interaction with the protein. Ligands then rapidly dissociate (fast on/off rate), allowing further ligand exchange, since the ligand is in excess. The selective saturation of the ligand is then detected in solution. Ligands in the solution that do not interact with the protein are not detected. Saturation of the ligand protons reflects their proximity to the protein surface when bound. Protons that interact more with the protein show a higher degree of saturation than those that are further away. The off-resonance represents a normal ${ }^{1} \mathrm{H}$ NMR experiment. The onresonance spectrum is then subtracted from the off-resonance spectrum, and the resulting STD spectrum shows only signals from ligand protons that interacted with the protein, thus providing a useful tool to detect the bound epitope (Mayer \& Meyer, 1999; 2001) (Figure 4.7). 


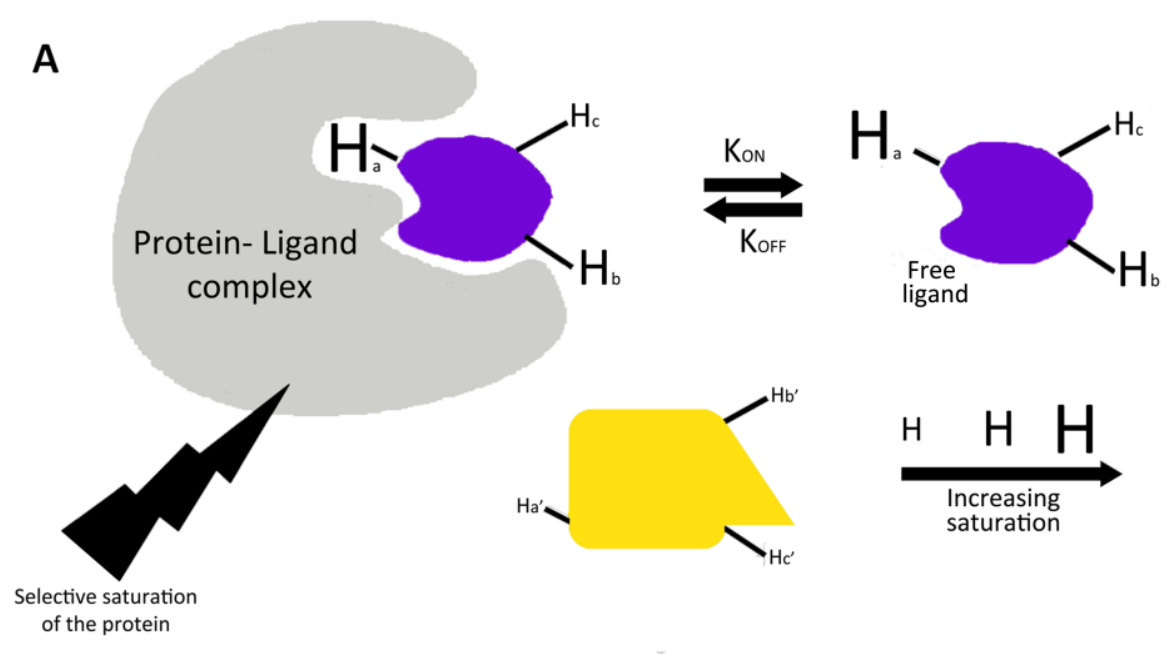

B

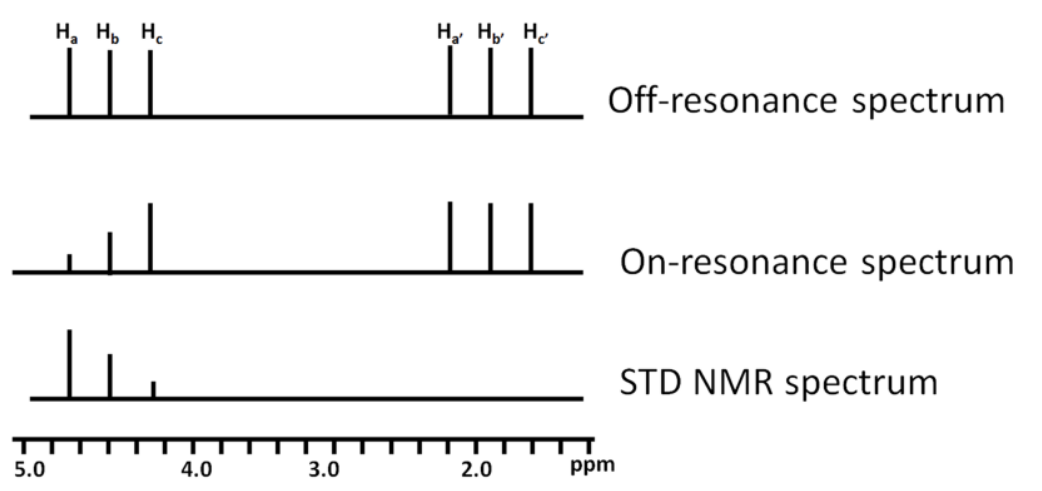

Figure 4.7 Illustration of saturation difference transfer (Modified from Mayer and Meyer 2001)

A. A selective saturation pulse is given that selectively saturates the protein. This magnetization then travels through the protein via spin diffusion and transfers onto the bound ligand. Protons that are in close contact with the protein $\left(\mathrm{H}_{\mathrm{a}}\right.$ and $\left.\mathrm{H}_{\mathrm{b}}\right)$ get saturated and this saturation is detected in the solution (free ligand). The observed STD effect depends on the distance between the protein and the ligand protons. Ligands that do not bind (yellow) do not get saturated. B. Schematic spectra showing the onand off-resonance. The STD spectrum is the off-resonance subtracted from the on-resonance. Protons $H_{a}$ and $H_{b}$ and $H_{c}$ are shown to be involved to varying extents with the protein; whereas, protons $H_{a}$, $\mathrm{H}_{\mathrm{b}}$, and $\mathrm{H}_{\mathrm{c}}$, are not detected in the STD spectrum as they are not saturated since they do not interact with the protein. 
For STD experiments, buffers were made up in deuterium oxide $\left(\mathrm{D}_{2} \mathrm{O}\right)(99.9 \%$ Merck). Buffers had their $\mathrm{pD}$ altered with $\mathrm{DCl}_{2}$ when required. It is necessary to stabilize the assembled MTs due to the long experimental time required for these experiments; however, MT assembly inductors, such as DMSO and glycerol, contain several protons, which even in trace amounts would cause large signals in the proton NMR spectrum and therefore they need to be avoided. A specific stabilizer was therefore chosen, guanylyl$(\alpha, \beta)$-methylenediphosphate (GMPCPP) a very slowly hydrolysable form of GTP. This GTP analog binds to the E-site on $\beta$-tubulin, promoting the normal polymerization of MTs, but the methylene group between the $\alpha$ - and $\beta$-phosphates makes the hydrolysis of the $\gamma$-phosphate much slower; thus, the MTs do not rapidly depolymerize. (Hyman et al., 1992). GMPCPP polymers are relatively stable and suppress MT dynamics, allowing NMR to be carried out. In $10 \mathrm{mM} \mathrm{NaPi}, 1 \mathrm{mM} \mathrm{GTP,} 6 \mathrm{mM} \mathrm{MgCl} 2$ buffer, the $\mathrm{C}_{\mathrm{r}}$ of tubulin is greater than $200 \mu \mathrm{M}$ at $37^{\circ} \mathrm{C}$. Substitution of GTP with GMPCPP decreases the $\mathrm{C}_{\mathrm{r}}$ of tubulin assembly to $5.6 \mu \mathrm{M}$ in otherwise similar conditions. Additionally, use of potassium phosphate $(\mathrm{KPi})$ rather than $\mathrm{NaPi}$ further decreases this $\mathrm{C}_{\mathrm{r}}$ to $4.6 \mu \mathrm{M}$ and slows down the hydrolysis, in otherwise similar conditions. Thus, a $10 \mathrm{mM} \mathrm{KPi}, 6 \mathrm{mM}$ $\mathrm{MgCl}_{2}, 0.1 \mathrm{mM}$ GMPCPP, $\mathrm{D}_{2} \mathrm{O}$ buffer, pD 6.4 was chosen for the NMR experiments (Jiménez-Barbero et al., 2006). Before tubulin equilibration, the Sephadex G-25 column was pre-prepared by running through $40 \mathrm{~mL}$ of $\mathrm{D}_{2} \mathrm{O}$ followed by two volumes of the desired buffer. Two samples were prepared for each of the protein conditions, DAC in buffer (sample A) and DAC bound to protein (sample B). Addition of DAC to the samples further lowers the $\mathrm{C}_{\mathrm{r}}$.

To prepare dimeric tubulin, $10 \mathrm{mg}$ of tubulin was equilibrated to remove sucrose, $\mathrm{Mg}^{2+}$ and $\mathrm{H}_{2} \mathrm{O}$ from the storage buffer and processed as in Chapter 2.4 using $10 \mathrm{mM}$ $\mathrm{NaPi}, 0.1 \mathrm{mM}$ GTP, $\mathrm{D}_{2} \mathrm{O}$, pD 7.0 buffer. Note that this buffer does not use GMPCPP or $\mathrm{KPi}$ as with polymerized tubulin. DAC $(300 \mu \mathrm{M})$ was then incubated for $30 \mathrm{~min}$ at $25^{\circ} \mathrm{C}$ with $10 \mu \mathrm{M}$ dimeric tubulin and the samples processed for NMR.

To prepare MTs, $20 \mathrm{mg}$ tubulin was equilibrated in cold $10 \mathrm{mM} \mathrm{KPi}, 6 \mathrm{mM}$ $\mathrm{MgCl}_{2}, 0.1 \mathrm{mM}$ GMPCPP buffer in $\mathrm{D}_{2} \mathrm{O}, \mathrm{pD} 6.4$ by a two-step chromatography procedure. Sucrose and GTP were removed with a drained centrifuge column of Sephadex G-25 (6 x $1 \mathrm{~cm})$ equilibrated in $10 \mathrm{mM} \mathrm{KPi}, 10 \mu \mathrm{M}$ GTP buffer in $\mathrm{D}_{2} \mathrm{O}$, pD 6.4. This was followed by another Sephadex G-25 column equilibrated in cold $10 \mathrm{mM}$ $\mathrm{KPi}, \mathrm{D}_{2} \mathrm{O}, \mathrm{pD}$ 6.7. $\mathrm{MgCl}_{2}$ and $\mathrm{GMPCPP}$ were then added to final concentrations of $6 \mathrm{mM}$ 
and $0.1 \mathrm{mM}$, respectively, $\mathrm{pD}$ 6.4. Tubulin was pelleted by centrifugation and the concentration of tubulin measured as described in Chapter 2.4. Tubulin was diluted to 20 $\mu \mathrm{M}$ and $0.1 \mathrm{mM}$ GMPCPP and $6 \mathrm{mM} \mathrm{MgCl}_{2}$ added, pD 6.7. DAC $(300 \mu \mathrm{M})$ was then added and the sample incubated at $37^{\circ} \mathrm{C}$ for $30 \mathrm{~min}$.

NMR experiments and analyses were performed by Dr. Angeles Canales (CIB, CSIC, Madrid). NMR spectra were recorded at $25^{\circ} \mathrm{C}$ (dimeric tubulin samples) or $37^{\circ} \mathrm{C}$ (MT samples) in $\mathrm{D}_{2} \mathrm{O}$ in an $\mathrm{AV}$-III $700 \mathrm{MHz}$ equipped with a triple-channel cryoprobe, an AVANCE $600 \mathrm{MHz}$ equipped with a triple-channel cryoprobe, or an AVANCE 500 $\mathrm{MHz}$ Bruker spectrometer as previously described, as were 2D-TR-NOESY experiments(Canales et al., 2008; 2011). The bioactive conformation of DAC was obtained by combining NMR data with molecular mechanics calculations. TR-NOESY experiments were acquired with different mixing times from 80 to $200 \mathrm{~ms}$, and the spectrum with the shortest mixing time was selected for analysis to minimize spin diffusion effects. 


\section{$\underline{4.3 \quad \text { RESULTS }}$}

\subsubsection{Biochemical properties of zampanolide}

When ZMP was analyzed by HPLC, it had two peaks of absorbance as expected (Figure 4.8A). These HPLC peaks were analyzed by MS to confirm that they were both ZMP. Figure $4.8 \mathrm{~B}$ shows the peaks and the absorbances that were used throughout this thesis for HPLC detection and analysis.

Using a spectrometer, the extinction coefficients of ZMP and DAC were calculated (Figure 4.8B and C). ZMP: $\varepsilon_{230}=31,000 \pm 800 \mathrm{M}^{-1} \mathrm{~cm}^{-1}, \varepsilon_{264}=42,000 \pm 1,000$ $\mathrm{M}^{-1} \mathrm{~cm}^{-1}$; DAC: $\varepsilon_{230}=15,700 \pm 300 \mathrm{M}^{-1} \mathrm{~cm}^{-1}, \varepsilon_{278}=15,500 \pm 300 \mathrm{M}^{-1} \mathrm{~cm}^{-1}$. The results for ZMP were approximately $30 \%$ higher than those originally reported by Tanaka and Higa (1996) when the compound was first isolated (ZMP: $\varepsilon_{230}=25,000 \mathrm{M}^{-1} \mathrm{~cm}^{-1}, \varepsilon_{264}=$ $30,000 \mathrm{M}^{-1} \mathrm{~cm}^{-1}$ ). The extinction coefficients of natural DAC (+ enantiomer) reported by Cutignano et al. (2001) were $\varepsilon_{266}=16,000 \mathrm{M}^{-1} \mathrm{~cm}^{-1}, \varepsilon_{222}=11,000 \mathrm{M}^{-1} \mathrm{~cm}^{-1}$ were comparable to that reported here at $\varepsilon_{278}$, but ours was $30 \%$ higher to that reported at $\varepsilon_{230}$. The synthetic DAC absorbance was shifted more toward the red end of the spectrum.
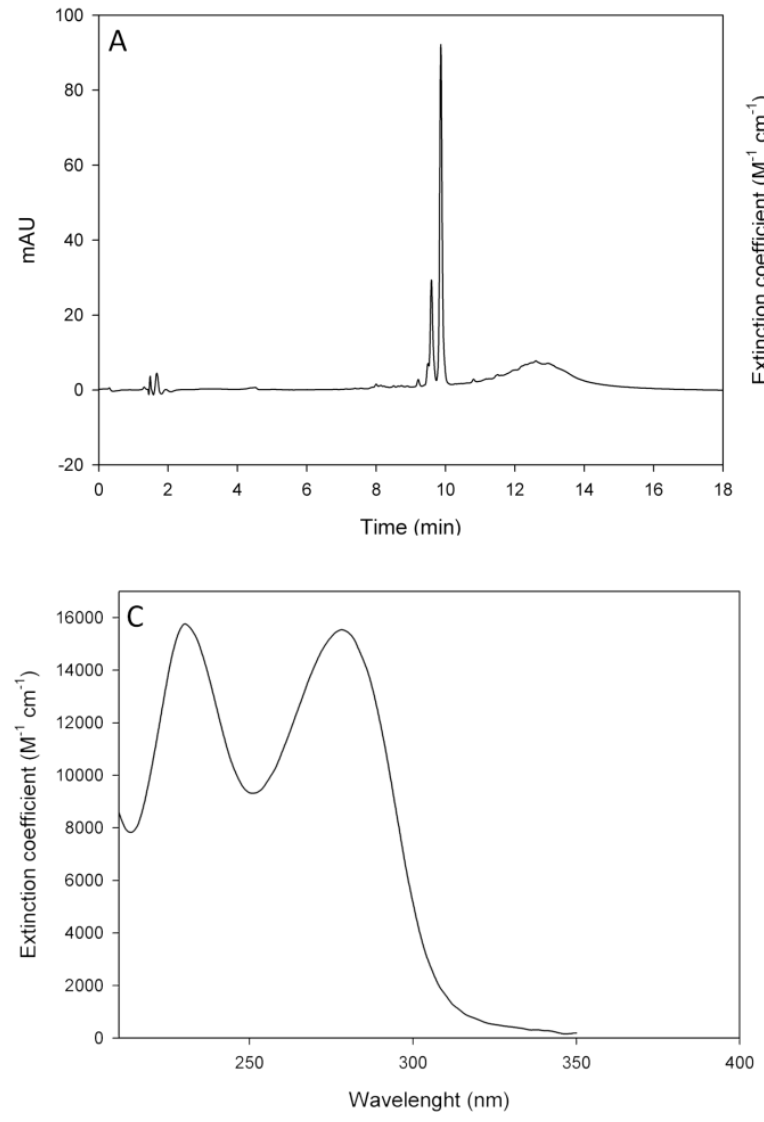

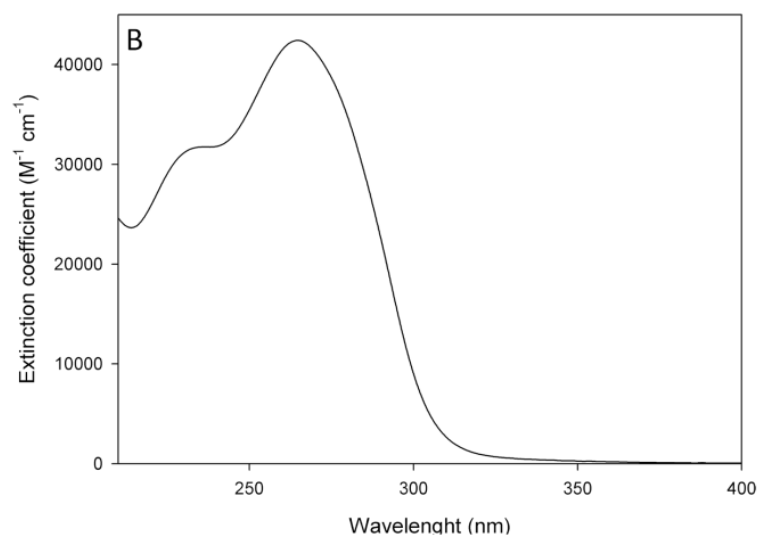

Figure 4.8 Spectra and extinction coefficients

A. Chromatogram showing the elution of ZMP at $9.59 \mathrm{~min}$ and $9.86 \mathrm{~min}$. These two peaks were analyzed by MS and were both shown to be ZMP. B. HPLC spectrum of ZMP. C. HPLC spectrum of DAC. The HPLC spectra show that both ZMP and DAC have two maximum absorbance values giving two extinction coefficients. These characteristic peaks were used throughout to detect ZMP and DAC in HPLC experiments. 


\subsubsection{Zampanolide is a potent MSA}

To confirm the MT-stabilizing activity of ZMP and DAC in vitro and to compare their potencies, a MT assembly assay was performed. In favorable conditions tubulin is able to assemble without an MSA $\left(\mathrm{C}_{\mathrm{r}}=3.3 \pm 0.30 \mu \mathrm{M}\right)$. ZMP significantly decreased the $\mathrm{C}_{\mathrm{r}}$ to $0.81 \pm 0.16 \mu \mathrm{M}(\mathrm{n}=6, \mathrm{p}<0.0001$; Student's $t$-test $)$, inducing MT assembly with a similar potency to DTX $\left(\mathrm{C}_{\mathrm{r}}=0.60 \pm 0.05 \mu \mathrm{M}, \mathrm{n}=10, \mathrm{p}<0.0001\right)$ (Figure 4.9A). DAC also enhanced tubulin assembly and did so with a $C_{r}$ of $2.10 \pm 0.15 \mu \mathrm{M}(\mathrm{n}=4, \mathrm{p}<0.004)$, reducing the $\mathrm{C}_{\mathrm{r}}$ by approximately $30 \%$. As expected, PDP significantly increased the $\mathrm{C}_{\mathrm{r}}$. The polymers formed have previously been shown to be true MTs and not just amorphous aggregates of tubulin (Field et al., 2009).

Given that ZMP significantly decreased the $\mathrm{C}_{\mathrm{r}}$ in the above conditions and was shown to be a potent stabilizer, the ability of ZMP to promote MT assembly was tested in conditions where tubulin is unable to assemble unless a strong MSA is present. In these conditions, ZMP was able to induce assembly of tubulin with a $C_{r}$ of $4.1 \mu \mathrm{M}(n=3)$, requiring little excess compound to induce maximal assembly (Figure 4.9B).

A

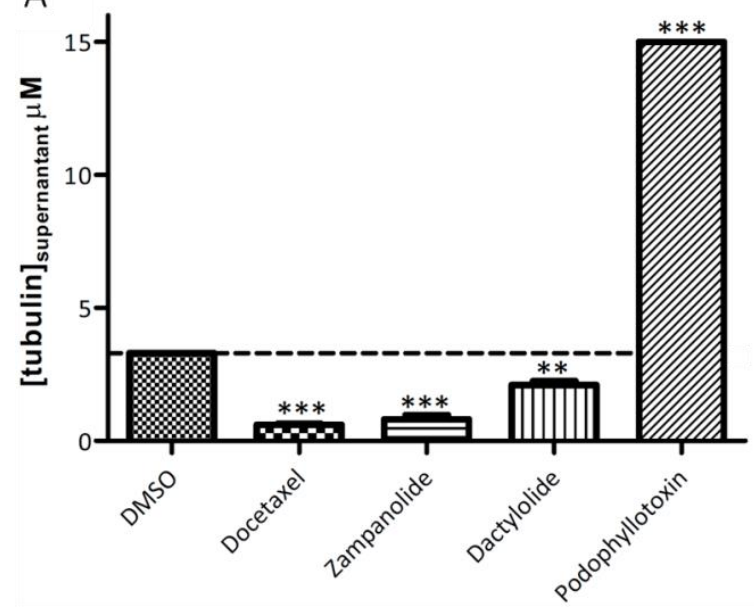

B

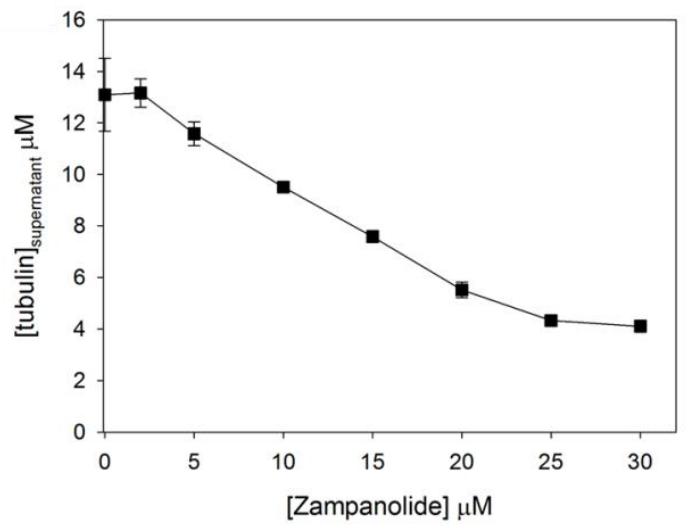

Figure 4.9 Induction of microtubule assembly

A. Induction of MT assembly by ZMP and DAC in conditions favorable for tubulin assembly. In these conditions, the $\mathrm{C}_{\mathrm{r}}$ of tubulin for self-assembly was $3.3 \mu \mathrm{M}$, shown by the DMSO bar and the dashed line. DTX and ZMP both significantly decreased this concentration to $0.60 \mu \mathrm{M}$ and $0.81 \mu \mathrm{M}$, respectively $(\mathrm{p}<0.001)$. DAC is not as potent as ZMP at causing assembly but is still able to significantly decrease the $\mathrm{C}_{\mathrm{r}}$ required for assembly to $2.10 \mu \mathrm{M}(\mathrm{p}<0.01)$. PDP was used as a negative control $(n=4-10)$. B. Induction of assembly by ZMP in conditions unfavorable for tubulin assembly. ZMP has a $\mathrm{C}_{\mathrm{r}}$ of $4.1 \mu \mathrm{M}$, requiring little excess compound to induce maximal assembly $(\mathrm{n}=3)$. All data are resented as mean \pm SEM. 


\subsubsection{Zampanolide and dactylolide are ligands of the taxoid site}

The compounds were tested for their ability to displace FTX-2 from stabilizedcrosslinked MTs. ZMP and DAC were compared with known taxoid site ligands, DTX and EPOA. All four compounds tested displaced FTX-2 from its binding site (decrease in anisotropy), with different apparent binding affinities (Figure 4.10). This directly indicates that ZMP and DAC are ligands of the taxoid binding site on $\beta$-tubulin. The apparent binding affinities of the two compounds were significantly different from one another, with the apparent binding constant of ZMP at $35^{\circ} \mathrm{C}=214 \times 10^{6} \mathrm{M}^{-1}$, over 150 times greater than that of DAC $\left(1.35 \times 10^{6} \mathrm{M}^{-1}\right)($ Table 4.2) $(\mathrm{n}=4)$.

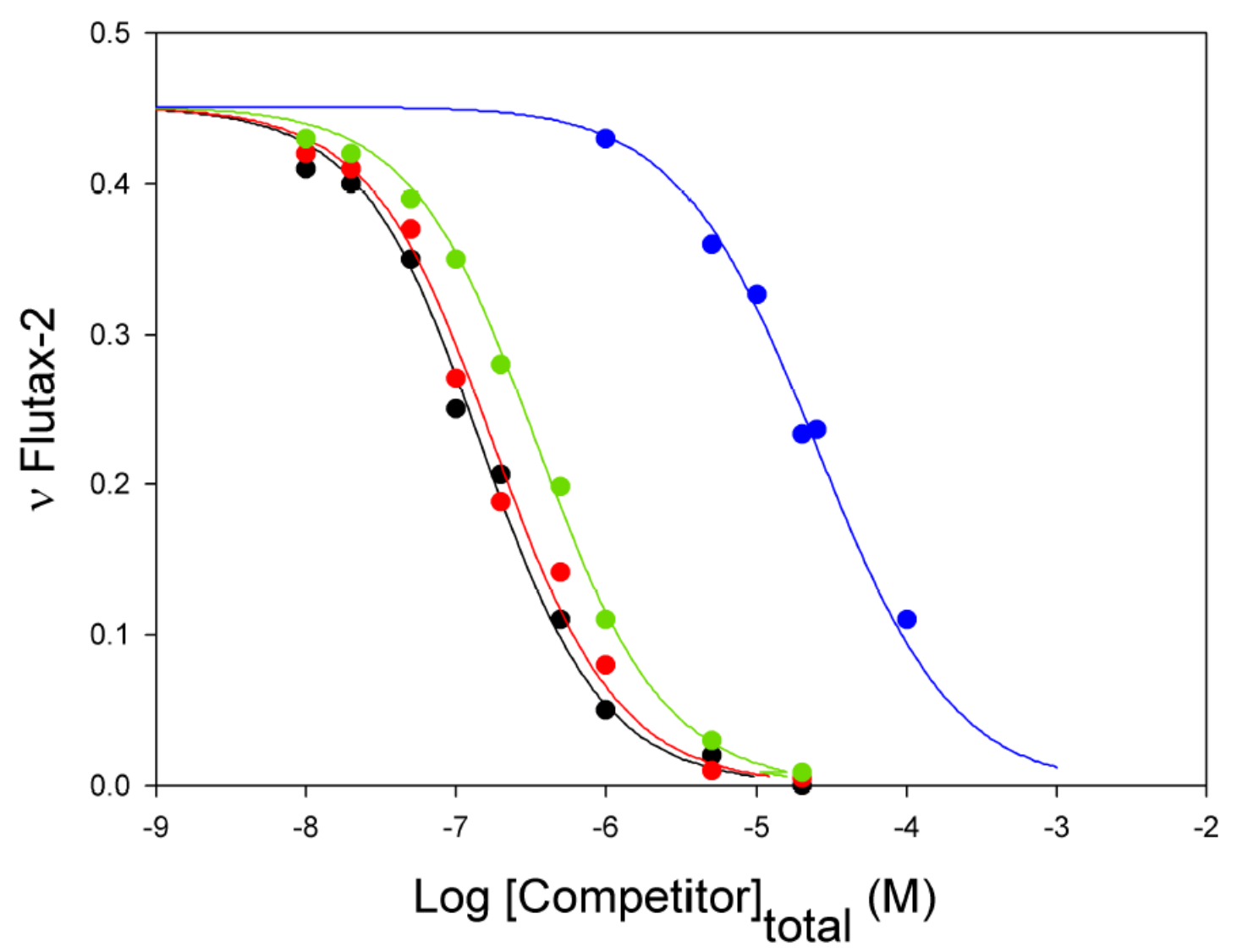

Figure 4.10 Flutax-2 displacement by zampanolide and dactylolide

Representative competition experiments between FTX-2 and MSAs for binding the taxoid site in MTs. Displacement of FTX-2 (50 nM) from MT binding sites (50 nM) by DTX (red line and symbols), EPOA (green line and symbols), ZMP (black line and symbols), and DAC (blue line and symbols). ZMP displaced FTX-2 with similar binding affinity to DTX, DAC had a significantly lower affinity for the taxoid site compared to ZMP. 
Table 4.2 Binding constants of ligands to the taxoid site $\left(\mathrm{Kb} \times 10^{\circ} \mathrm{M}^{-1}\right)$

\begin{tabular}{ccccccccc}
\hline & $26^{\circ} \mathrm{C}$ & $27^{\circ} \mathrm{C}$ & $30^{\circ} \mathrm{C}$ & $32^{\circ} \mathrm{C}$ & $35^{\circ} \mathrm{C}$ & $37^{\circ} \mathrm{C}$ & $40^{\circ} \mathrm{C}$ & $42^{\circ} \mathrm{C}$ \\
\hline Docetaxel & $69.5 \pm 4.2$ & $65.7 \pm 5.2$ & $54.2 \pm 4.2$ & $48.9 \pm 3.8$ & $39.3 \pm 2.7$ & $30.9 \pm 2.2$ & $28.9 \pm 1.7$ & $23.8 \pm 1.1$ \\
Epothilone A & $74.8 \pm 10.0$ & $69.4 \pm 10.8$ & $58.1 \pm 10.8$ & $50.0 \pm 4.9$ & $36.3 \pm 5.1$ & $29.3 \pm 4.4$ & $23.2 \pm 2.5$ & $20.8 \pm 2.1$ \\
Zampanolide & $137 \pm 29$ & $150 \pm 3.7$ & $143 \pm 48$ & $187 \pm 76$ & $214 \pm 93$ & $258 \pm 110$ & $416 \pm 173$ & $424 \pm 175$ \\
Dactylolide & $0.57 \pm 0.10$ & $0.78 \pm 0.15$ & $0.97 \pm 0.13$ & $1.20 \pm 0.14$ & $1.35 \pm 0.18$ & $1.43 \pm 0.20$ & $1.82 \pm 0.18$ & $2.14 \pm 0.25$ \\
Cyclostreptin & $10.2 \pm 1.4$ & $14.3 \pm 0.9$ & $15.9 \pm 0.7$ & $20.5 \pm 1.7$ & $20.6 \pm 1.1$ & $21.2 \pm 2.1$ & $26.7 \pm 2.4$ & $28.4 \pm 2.4$ \\
\hline
\end{tabular}

True binding constants for DXT and EPOA for the taxoid binding site, apparent binding constants for ZMP, DAC and CYC for the taxoid binding site. Both sets of measurements are derived from FTX-2 displacement studies. CYC data from Edler et al., 2005. 


\subsubsection{Zampanolide binds to the taxoid site with 1:1 stoichiometry}

The stoichiometry of ZMP and DAC binding to the taxoid site was determined using HPLC. Both ZMP and DAC induced tubulin stoichiometrically, one ligand binding per taxoid binding site (Figure 4.11). Interestingly, ZMP could not be recovered from the pellet, and the reason for this will be addressed in Chapter 5. Given this, the stoichiometry was measured by monitoring the concentration of ZMP in the supernatant alone, rather than the concentration in both fractions. ZMP in the supernatant represents ligand that is not bound to the MT. For consistency, DAC was measured in the same manner, although some DAC was able to be extracted from the pellet fraction.

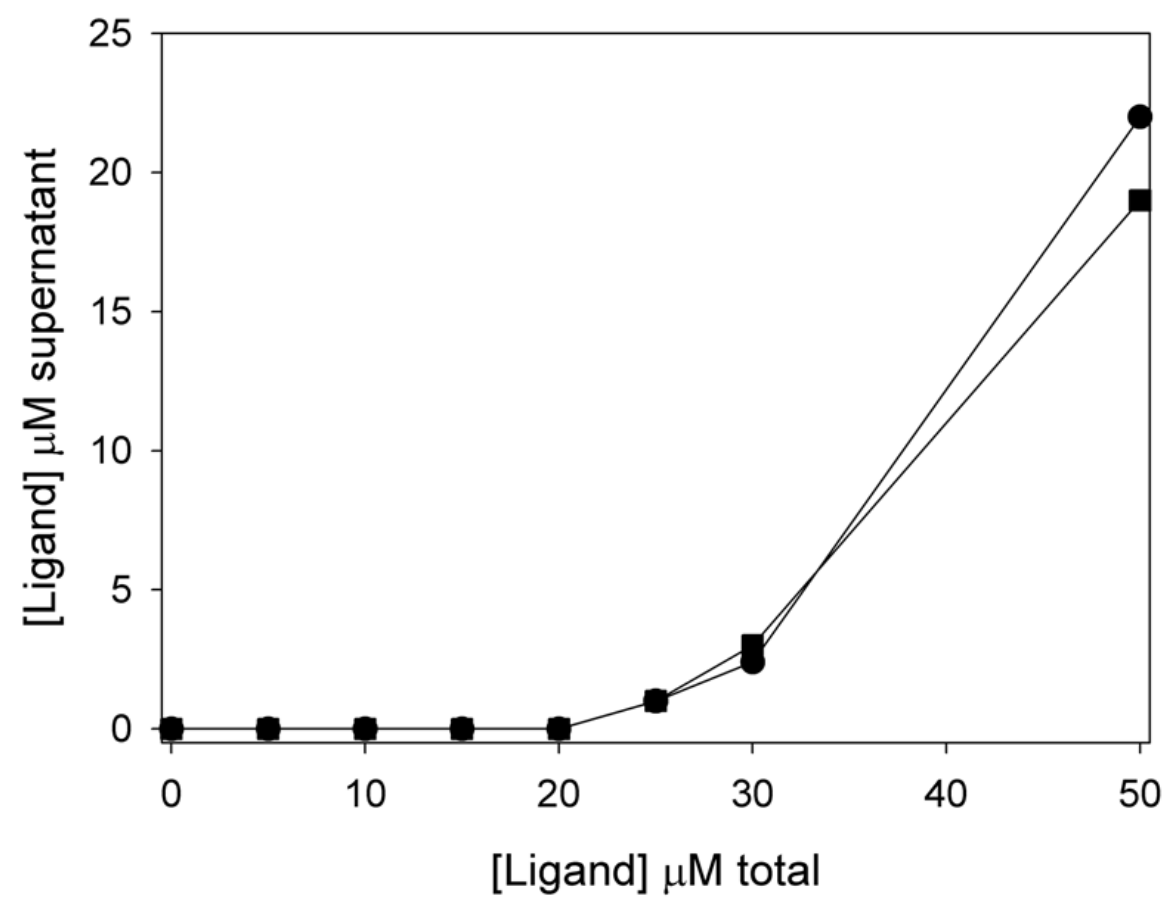

Figure 4.11 Stoichiometry of ZMP and DAC

Stoichiometric reaction of ZMP (circles) or DAC (squares) with glutaraldehyde-stabilized MTs (25 $\mu \mathrm{M})$. Ligand in the supernatant represents compound that is not bound to the taxoid sites in the glutaraldehyde-stabilized MTs. 


\subsubsection{Zampanolide binding blocks binding at the pore site}

To check if ZMP binding to the luminal site blocks binding to the pore type I site in assembled MTs, HXF and stabilized taxoid binding sites were incubated with increasing amounts of ZMP. In the absence of ZMP, $28 \pm 2 \% \mathrm{HXF}$ was bound to the pore site in MTs, (as expected from its binding constant of $2.06 \times 10^{6} \mathrm{M}^{-1}, 25^{\circ} \mathrm{C}$ (Barasoain et al., 2010) (Figure 4.12). The contribution of bound HXF to anisotropy is less than that of free HXF (bound HXF has less fluorescence than free), and this has been corrected for. No HXF was bound in the presence of ZMP at concentrations at least stoichiometric with the tubulin concentration, indicating that binding of ZMP to the MTs blocks binding at the pore site. This lack of interaction of HXF with the adduct indicates binding at the luminal site is specific and alters and/or blocks the pore site, thus preventing binding to it. This supports the previous finding that binding at either site is mutually exclusive.

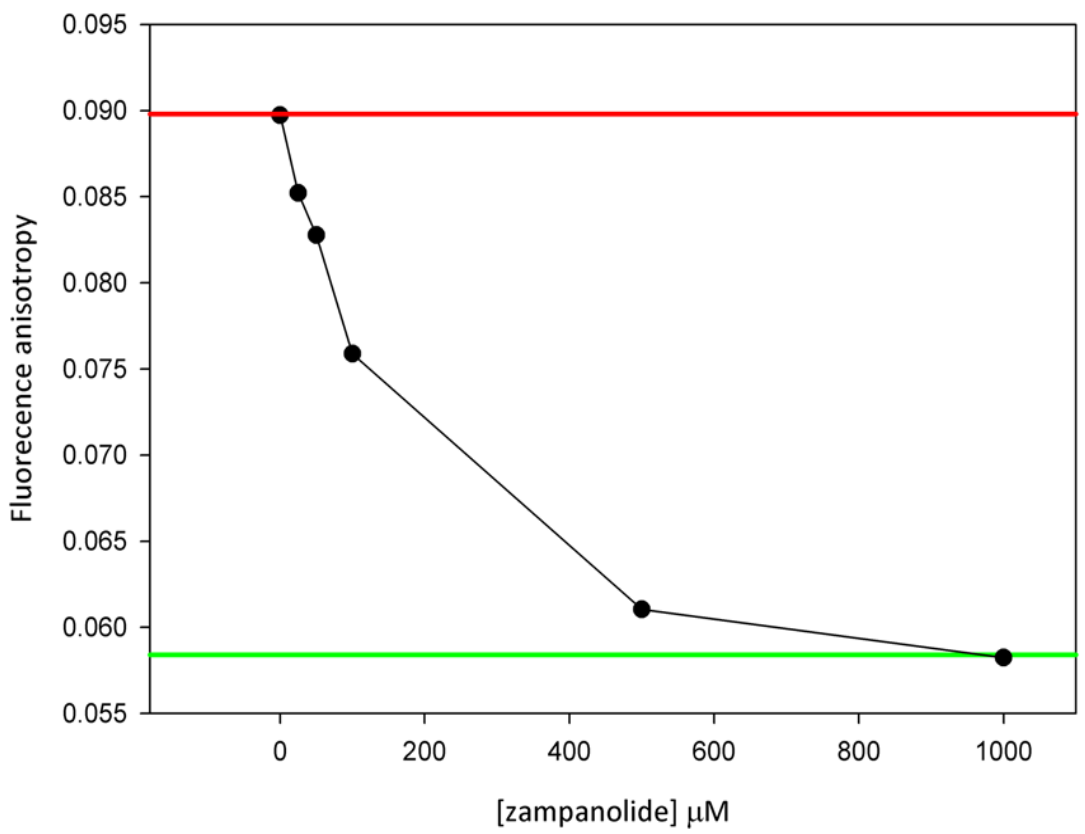

Figure 4.12 Hexaflutax binding to microtubules in the presence of ZMP

The binding of HXF to pore type I in MTs was measured by the increase in anisotropy upon binding. When HXF is bound the anisotropy value is 0.09 (red line), and unbound HXF has a lower anisotropy of 0.06 (green line). With increasing concentrations of ZMP, the fluorescence anisotropy of HXF decreases (as ZMP binding displaces HXF) to that of unbound HXF. Once ZMP reaches a concentration equal to that of the tubulin concentration (binding site saturation), no HXF binds the MT as simultaneous binding at the luminal site and the pore cannot occur. This also confirms the 1:1 stoichiometry of ZMP binding. 


\subsubsection{Bioactive conformation of DAC bound to microtubules}

DAC bound to both MTs and to unassembled tubulin produced STD signals that were easily detected (Figure 4.13A), confirming that DAC can bind to tubulin dimers and MTs. The STD effect of all the DAC protons was fairly homogeneous throughout the complete compound skeleton, thus suggesting an extensive interaction of the molecule with the binding site in both unassembled tubulin and in MTs. Interestingly, the STD profiles of DAC, bound to unassembled tubulin and to MTs, indicated that the binding epitopes of DAC in both cases were nearly identical. The same is seen with DSC but not with DTX (Canales et al., 2011). The bioactive conformation of bound DAC was deduced by analysis of the TR-NOESY crosspeaks, as shown in Figure 4.13B. Several key negative NOEs (as a result of binding) between remote protons in the molecule were detected in the presence of both protein states. This allows the determination of the bioactive conformation of DAC in its binding site. The distances obtained indicated that the bound conformations were identical between MTs and unassembled tubulin dimers. In addition, the conformation of the free compound in buffer was nearly identical, supporting the elucidated binding epitope from the STD results (data not shown). These data were analyzed by Dr. Angeles Canales (CIB, CSIC, Madrid, Spain).
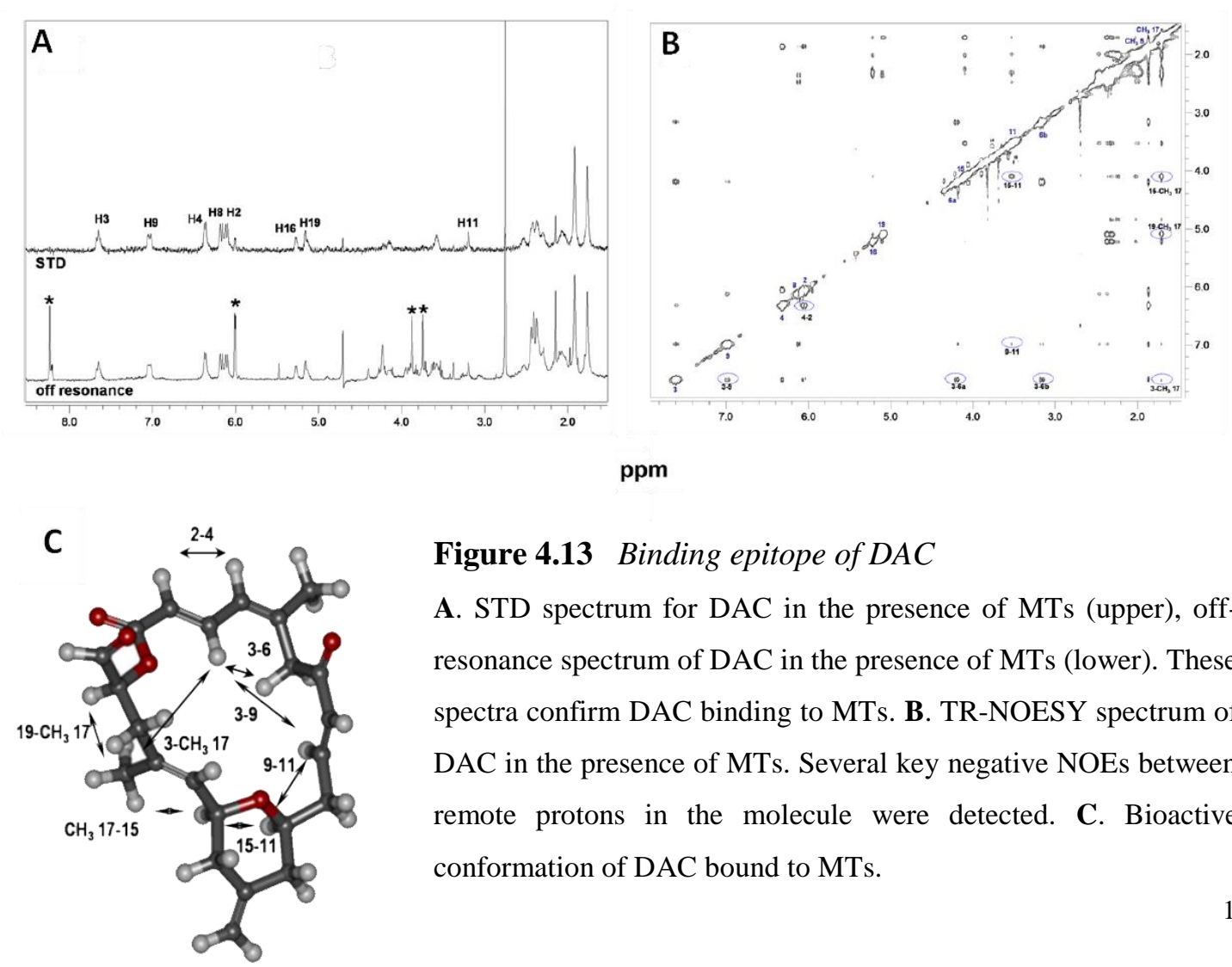

Figure 4.13 Binding epitope of DAC

A. STD spectrum for DAC in the presence of MTs (upper), offresonance spectrum of DAC in the presence of MTs (lower). These spectra confirm DAC binding to MTs. B. TR-NOESY spectrum of DAC in the presence of MTs. Several key negative NOEs between remote protons in the molecule were detected. C. Bioactive conformation of DAC bound to MTs. 


\section{$\underline{4.4 \quad \text { DISCUSSION }}$}

\subsubsection{Zampanolide is a potent inducer of MT assembly}

It is known that strong MSAs are able to bind either the taxoid site or the LAU/PEL site and promote MT assembly in a stoichiometric manner in conditions that are hostile to MT assembly (Parness \& Horwitz, 1981; Mooberry et al., 1999; Pera et al., 2010). In these conditions, ZMP was shown to be a potent MSA, since little excess of the compound was required to induce maximal assembly. This means that $10 \%$ excess ZMP is required for assays to induce maximal tubulin assembly. DAC was a poor MSA, showing minimal activity. This was further confirmed in conditions in which tubulin can self-assemble. MSAs decrease the concentration needed for this to occur. ZMP was again a strong inducer of assembly, similar to the taxanes; whereas, DAC, even though it was able to significantly enhance MT assembly, was only able to do this by $30 \%$, similar to other weak assembly promoters such as the sarcodictyins (Buey et al., 2005). These findings are consistent with the cytotoxicity results that showed that ZMP inhibited cellular proliferation in the nanomolar range; whereas, DAC was cytotoxic in the micromolar range in cancer cell lines (Cutignano et al., 2001; Ding \& Jennings, 2008). Thus, the ZMP side chain is important in ZMP's ability to induce tubulin assembly.

\subsubsection{Zampanolide is a taxoid site ligand}

The majority of MSAs bind to the taxoid site in the lumen of the MT; whereas, only two known ligand families, LAUs and PELs, bind the LAU/PEL site. The taxoid site differs from the LAU/PEL site, since many ligands with different affinities and highly diverse structures bind the taxoid site; whereas, the LAU/PEL site is smaller and less accepting of different structures, suggesting it is more defined (Bennett et al., 2010). A recent study of CYC ligands, which have diverse binding patterns, provided evidence that the S9-S10 loop, involved in the taxane pocket (Figure 4.2), is highly flexible and at least half the loop is in an open conformation, meaning that the taxoid site is larger than previously thought. This may be the reason the taxoid site can accommodate such a diverse range of compounds (Calvo et al., 2012). Taxoid site ligands are so diverse that the site lacks a defined common pharmacophore; whereas, the LAU/PEL site only binds two structurally similar ligands and is less accommodating of diverse structures, unless other LAU/PEL site binding agents exist but have not been discovered yet. The binding affinity of PEL is low, significantly weaker than most taxoid site ligands (Pera et al., 2010), and 
comparable with known low affinity MSAs such as sarcodictyin A (Buey et al., 2005). Despite this PEL is significantly more active at inducing MT-assembly and more cytotoxic to tumour cells, comparable with the taxanes. This suggests that binding to the LAU/PEL site may be more effective at causing stabilization of the MT than binding to the taxoid site, another distinguishing feature of the two sites (Pera et al., 2010). From a structural perspective, ZMP may bind to the taxoid site or the LAU/PEL site since it is structurally similar to the EPOs, LAUs and PELs. All three of these groups of compounds contain a highly unsaturated macrocyclic core with a side chain. From cellular studies in Chapter 3, it was likely that ZMP was a ligand of the taxoid binding site, and this was seen to be the case from the results in the present Chapter. ZMP displaced FTX-2 in a similar manner to DTX and EPOA both known taxoid site ligands. Additionally, ZMP has been evaluated for inhibition of binding of $\left[{ }^{3} \mathrm{H}\right]$-PTX and $\left[{ }^{3} \mathrm{H}\right]-$ PEL to preformed MTs. ZMP was able to inhibit $\left[{ }^{3} \mathrm{H}\right]-\mathrm{PTX}$ binding by $67 \%$ but had no effect on PEL binding, confirming it to be a ligand of the taxoid site (Field et al., 2012). These experiments were performed by Dr. Ernest Hamel, National Cancer Institute, NIH, Fredrick, MD, USA.

DAC is also a ligand of the taxoid site but has significantly lower affinity for binding, again indicating the importance of the C19 side chain in ZMP. The $N$-acyl hemiaminal side chain has a number of reactive centers, and these are lacking of course in DAC. Thus, DAC has decreased opportunity for interaction with the binding site compared to ZMP. The C20 hydroxyl group, the amine and the $\mathrm{C} 1$ ' carbonyl all have potential to hydrogen bond with tubulin residues, and the $\mathrm{C} 1$ ' $-\mathrm{C} 6$ ' has potential for 1,4 Michael addition. Hydrogen bonds at any of the reactive residues would increase the ligand-protein stability and may explain why ZMP is significantly more potent and a better ligand of the taxoid site than DAC.

The bioactive conformation of DAC bound to the $\alpha, \beta$-heterodimer and to the MT indicated that the conformation of DAC in both cases was essentially identical. This suggests that, unlike CYC, ZMP and DAC may bind to the same site in both dimers and MTs. CYC, on the other hand, binds the pore type I site in tubulin dimers and the luminal taxoid site in MTs (Buey et al., 2007).

Surprisingly, the apparent binding constants from the anisotropy assays for taxoid site of both ZMP and DAC increased with temperature (Table 4.2). This is the opposite of the expected results for an enthalpy-driven reaction, which is the relationship seen 
with reversibly bound MSAs like PTX and PEL (Buey et al., 2004; Gaitanos et al., 2004). This suggests that ZMP and DAC may bind to MTs in a different manner to the traditional MSAs. CYC and its analogs, although weak MSAs, covalently modify tubulin and display a similar behaviour in this assay (Edler et al., 2005; Buey et al., 2007; Calvo et al., 2012). Thus, it is possible that ZMP and DAC may covalently bind in a similar manner.

\subsubsection{Zampanolide binds with 1:1 stoichiometry}

Currently all known MSAs, regardless of where they bind, bind to the MT heterodimer with 1:1 stoichiometry. The stoichiometries of ZMP and DAC were determined by incubating MTs with increasing concentrations of compound. When the concentration of ZMP exceeded that of the tubulin, the excess ZMP began to appear in the supernatant, indicating that the reaction of ZMP proceeds in a 1:1 stoichiometry. DAC gave similar results. It is not yet clear whether PTX and its biomimetics can bind to only the inner, only the outer or to both sites in unassembled tubulin and MTs. Only one ligand can bind one heterodimer at a time since both the taxoid site and the pore site utilize residues in the H6-H7 loop (Magnani et al., 2009) and this is consistent with the observed 1:1 stoichiometry of MSAs. When ZMP is in sub-stoichiometric concentrations HFX binds at the pore site (Figure 4.12), but when ZMP is at least stoichiometric with tubulin, no HXF can bind. This confirms that binding at both sites cannot occur simultaneously, and the sites are mutually exclusive. This means that HFX is only binding to dimers that do not have bound ZMP, since when ZMP is at stoichiometric concentrations with the tubulin all the HFX is displaced.

\subsubsection{Conclusion}

Overall, this study showed that ZMP is a potent MSA representing a new chemotype that binds to the taxoid site on the MT. Differences between ZMP and DAC indicated and confirmed that the C19 side chain of ZMP is highly important in its ability to induce MT assembly, its affinity for binding the MT and its cytotoxicity as previously proposed. The correlation between binding, stabilization and cytotoxicity can be related to modifications to the compound structure as seen with EPO analogs (Buey et al., 2004), suggesting that the ZMP side chain is essential and analogs lacking this side chain will not be as potent as ZMP. Although binding with 1:1 stoichiometry, this chemotype had 
unusual binding properties suggesting it may interact with MTs in a different manner to traditional MSAs. Given this, the mechanism of binding of ZMP needs to be further validated. Increasing binding constants with increasing temperature suggests a kinetically controlled reaction. This, along with the finding that ZMP cannot be extracted from MT pellets, suggests ZMP and possibly DAC may bind irreversibly to the MT, similar to CYC (Edler et al., 2005; Buey et al., 2007). However, given the fact that STD-NMR signals were obtained with DAC binding to dimers and MTs, it is likely that DAC binds the MT in a different manner to ZMP. This is interesting since the two compounds are enantiomers of one another; suggesting that the side chain is more highly involved in the binding reaction than first thought. The actual binding mechanism of this new taxoid site chemotype is validated in the next chapter. 


\section{$5.1 \quad$ INTRODUCTION}

\subsubsection{Luminal binding site in dimeric tubulin}

MSAs promote the assembly of tubulin heterodimers into MTs, shifting the equilibrium toward the polymeric state. MSAs were not originally thought to bind, with measurable affinity, to unassembled tubulin, since it was believed the taxoid binding site only existed in assembled MTs (Parness \& Horwitz, 1981; Díaz \& Andreu, 1993). Inter-protofilament contact was therefore assumed to be required for binding to the taxoid site. It is now accepted that MSAs can bind to dimeric tubulin, because they are able to induce the formation of MTs in conditions unfavorable to MT assembly. Although ligand binding to dimeric tubulin is necessary to explain the way in which MSAs work, direct evidence of binding has only recently been provided. An MSA must have a binding affinity higher for the assembled species than the dimeric species (Díaz et al., 1993). Given this higher free energy of binding for polymerized MTs, the equilibrium would displace towards the MT polymer (Wyman \& Gill, 1990). Since MSAs can induce MT assembly in conditions in which no pre-formed MTs exist, it is reasonable to predict that a lower affinity site on dimeric tubulin is necessary to start the assembly process. NMR provided the first experimental proof of this low affinity site since TR-NOESY NMR signals can be detected from MSAs in the presence of dimeric tubulin (Canales et al., 2011). However it is not possible to know if these signals arise from low affinity binding to an uncompleted pore site or a low affinity luminal site.

\subsubsection{Mechanisms of MSA-induced assembly}

MSAs are able to promote tubulin assembly in conditions in which tubulin is unable to assemble, such as when no GTP or glycerol is present in the buffer or when GDP is bound to the $\beta$-subunit (Parness \& Horwitz, 1981; Díaz \& Andreu, 1993). Therefore, MSAs are involved in the nucleation-elongation step of MT assembly. MSAs may bind to unassembled tubulin with low affinity and induce polymerization via two possible mechanisms. Either they promote a conformational change resulting in activation of the dimer or they join two subunits together to form a high affinity site from two lower affinity sites. These two mechanisms are termed the allosteric and matchmaker processes, respectively (Díaz et al., 1993; Díaz et al., 2009) (Figure 5.1). The binding of an MSA to unassembled tubulin is the first in a series of events that lead to MSA-induced 
stabilization of MTs, and this will be further discussed in Chapter 6.1. If an MSA binds the pore site in dimeric tubulin, then the matchmaker mechanism of assembly takes place in which the full pore site is formed from two half-sites. In contrast, binding at the luminal site in dimeric tubulin, MTs would assemble by the allosteric mechanism where the luminal site is present with a low-affinity conformation that becomes high affinity after assembly. Alternatively, low-affinity binding of an MSA to the luminal site could itself induce an allosteric effect resulting in tubulin activation and consequent nucleation. Although it is known MSAs bind dimeric tubulin, currently there is only direct evidence of the matchmaker mechanism, shown by CYC labeling of the pore site and not the luminal site in dimeric tubulin (Buey et al., 2007). It is possible, of course, that both the allosteric and matchmaker mechanisms are active in tubulin MSA-induced nucleation.
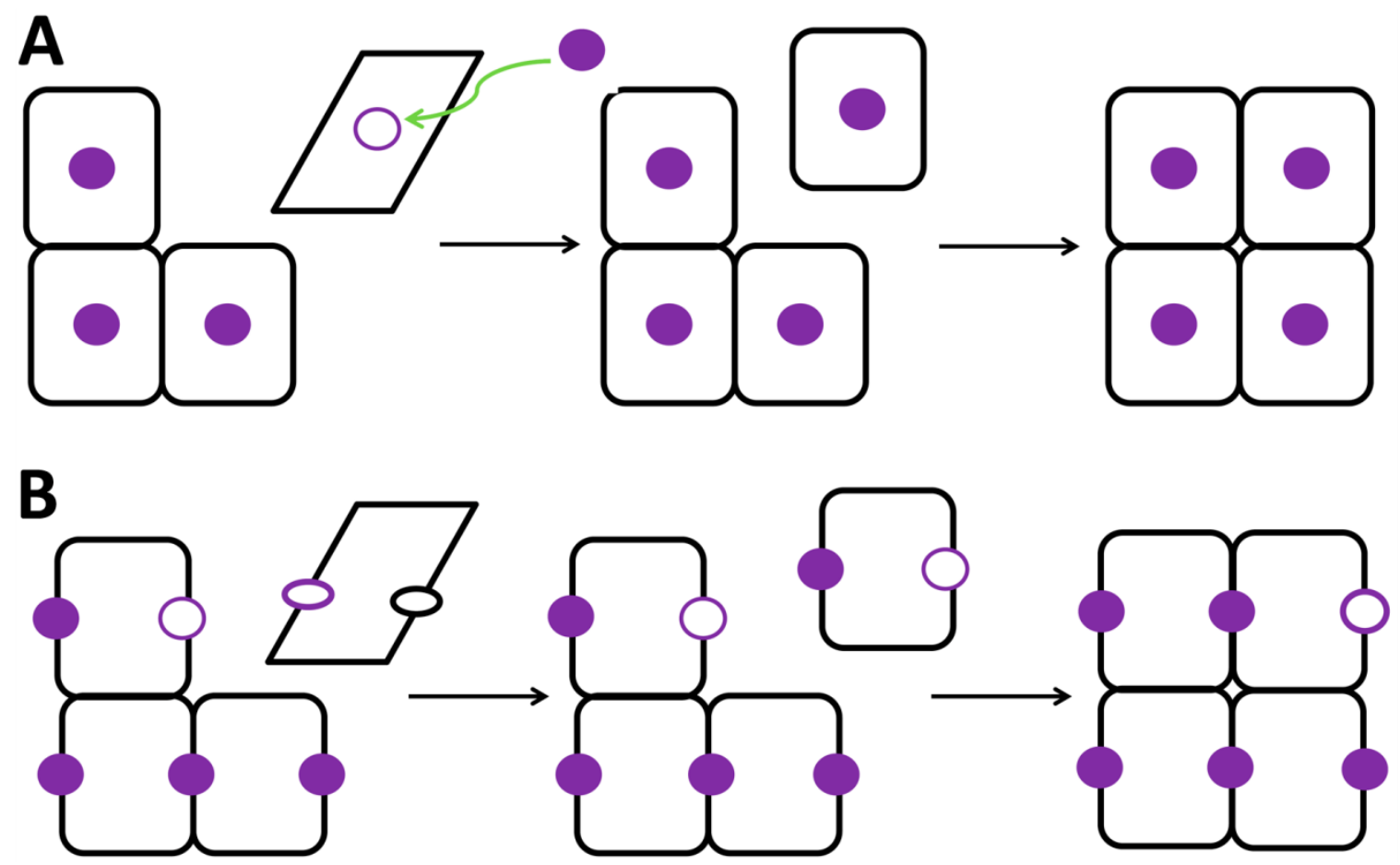

Figure 5.1 Possible mechanisms for MSA-induced elongation of MTs from dimeric tubulin (Modified from Díaz et al., 1993; 2009). A. Allosteric mechanism of assembly. Ligand binds to unassembled tubulin at the low affinity luminal site and the ligated dimer undergoes a conformational change increasing its affinity for MTs. The site then becomes a higher affinity site after assembly. Alternatively, binding could itself induce an allosteric effect resulting in tubulin activation and consequent nucleation. This model involves binding at the luminal site. B. Matchmaker mechanism of assembly. Ligand binding to unassembled tubulin joins two half sites to form a high affinity site. This model involves binding at the pore type I site. 


\subsubsection{Drug binding}

In general most drugs bind to their biological targets in a reversible (noncovalent) manner. This means that the bond does not involve the sharing of electron pairs and is not highly directional. Reversible binding involves electrostatic and hydrophobic interactions, van der Waals forces and hydrogen bonds and bonding distances may be a few $\AA$ between the interacting moieties (Matesanz et al., 2011b). This drug-target interaction is reversible, meaning the drug can bind and the drug target complex can dissociate, thus the reaction is generally in equilibrium (equation 1). Some drugs, however, are able to form covalent adducts with their targets. A covalent bond is significantly shorter and stronger than a noncovalent bond. This usually occurs through nucleophilic substitution in which a new bond is formed from an un-shared pair of electrons within the nucleophile (Zhou et al., 2005). In this scenario the drug binds to the target first in a noncovalent manner, similar to the reversible reaction which positions the nucleophile and the electrophile in the right orientation for the covalent reaction to occur. When the drug-target complex is formed it cannot dissociate back to the drug and target due to the formation of the covalent bond. This step is usually irreversible, and the dissociation constant is effectively zero (Singh et al., 2011) (equation 2). Covalent binders can be considered to have infinite affinity.

\section{Drug + Target $\leftrightarrows$ Drug-Target $(1)$}

\section{Drug + Target $\leftrightarrows$ Drug--Target $\Rightarrow$ Drug-Target (2) \\ Non-covalent \\ Reaction \\ Covalent Reaction \\ Adduct}

Equations modified from Singh et al., 2011.

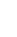


CYC analogs are also able to covalently modify dimeric tubulin and MTs. Two of the analogs bind covalently to a cysteine residue that is not labeled in the parent compound (Calvo et al., 2012). This is not surprising given that this residue is located in the vicinity of the taxoid binding site, and cysteine residues are often alkylated by tubulin binding compounds (Sackett, 2008). The CYC analogs highlighted the path CYC-like compounds, and most likely other taxoid site compounds, take to reach the taxoid binding site, confirming the previously proposed two-step binding mechanism (Díaz et al., 2003).

\subsubsection{Characteristics of covalent binding in pharmacology}

Covalent interactions proceed to completion rather than equilibrium in a time dependent manner. Covalent adduct formation permanently disrupts or stimulates the target's biological function, increasing the potency of a covalent binding drug. Activity of the target is only restored after re-synthesis of the particular target protein, essentially resulting in a long 'half-life' of the compound. Covalent binding produces a prolonged activation of the drug's target even after the residual free drug is cleared. This can lead to a more desirable clinical profile since less frequent dosing and lower drug concentrations can be used (Singh et al., 2011). Covalent binding agents, however, are also considered 'bad news', given their potential for nonspecific interactions with off-targets that may lead to systemic toxicity (Zhou et al., 2005). Despite this, a number of drugs that form covalent bonds are used in the clinic, for example, aspirin, the most widely used drug in the world, and the penicillin antibiotics. Currently there are 39 covalent drugs approved by the FDA, 20\% of which are anti-cancer compounds (Singh et al., 2011).

The pharmacodynamics of an inhibitory drug are correlated to the amount of target inhibition or occupancy of the binding site. A number of diseases, including cancer, require high target occupancy for effective treatment. This is illustrated in cancer treatment where insufficient drug-target interactions can promote development of resistant mutations in the binding site due to repeated periods of reduced exposure causing spontaneous mutations (Potashman \& Duggan, 2009). Thus in these conditions irreversible binding may be an effective treatment since formation of the covalent bond increases the degree of target inhibition and thus results in the inhibitor being more potent (Singh et al., 2011). In contrast, there are some targets that would not benefit from high target occupancy, such as the shutdown of a primary cellular pathway that is 
essential for cell survival. In this case, if the target sites were saturated, there would be on-target toxicity (Potashman \& Duggan, 2009). Additionally, random covalent modification of highly reactive proteins (for example exposed cysteine residues) would result in complications. The safety profile of a covalent agent would need to be thoroughly investigated before entering clinical trials.

\subsubsection{Covalent binding and resistance}

In oncology, drug resistance often occurs due to up-regulation of the P-gp MDR pump or the MRP-1 pump, as discussed. These pumps are over-expressed in different human cancers and cause MDR by decreasing the intracellular concentration of drugs (Gottesman et al., 2002). Resistant tumor cells presenting with up-regulated P-gp pumps are still sensitive to the action of MSAs; however, high concentrations of drug are required to gain the desired effect. At these high concentrations, the drug no longer selectively targets cancer cells and healthy cells are also targeted. Hence, often the only option once a tumor becomes resistant is to switch to a different drug.

High affinity or covalent tubulin binding compounds get trapped inside cells due to the nature of their interaction with tubulin, and they can therefore escape efflux by $\mathrm{P}$ gp pumps, avoiding this common mechanism of drug evasion by MDR cells (Yang et al., 2007; Matesanz et al., 2008). For example cryptophycin-52, a Vinca domain ligand, is a poor reversible binder, and it is this trend toward permanent, high affinity binding to the Vinca domain that is thought to be behind its reduced susceptibility to the P-gp pump (Panda et al., 1998). Taking advantage of this idea, covalent MSAs could be designed that may be able to evade MDR in cancer cells.

When cells with the MDR phenotype are treated with MSAs that are substrates for the P-gp pump, there are two mechanisms of ligand uptake, and these mechanisms oppose each other. The first is drug binding to the P-gp pump which in turn pumps the drug out of the cell. The other is binding to tubulin decreasing the intracellular levels of the drug, and the higher the affinity of the drug for tubulin, the lower the intracellular concentration of the drug. Since efflux relies on the intracellular ligand concentration, decreasing intracellular concentration results in a decrease in the concentration of ligand to below its dissociation constant from the P-gp pump, thus decreasing efflux. Therefore, by increasing the affinity of a ligand for its binding site well above the affinity for the Pgp pump (effectively decrease the efflux of the drug), MDR can be overcome (Matesanz 
et al., 2008). It is known that higher affinities of MSAs for tubulin correlate with higher cytotoxicity (Buey et al., 2004). This is most clearly demonstrated with compounds that bind to tubulin in a covalent manner, since in this case; essentially every molecule entering the cell will get covalently bound and be essentially 'trapped' by tubulin, eliminating the cells MDR phenotype. A study using derivatives of PTX has suggested that $\mathrm{P}$-gp resistance can be overcome by decreasing the concentration of unbound drug in the cell to lower to dissociation constant of the ligand for the pump (Matesanz et al., 2008).

Other forms of drug resistance, such as structural mutations in the binding site, also present a challenge in chemotherapy. Covalent drugs should theoretically overcome drug binding site mutations since mutations only effect the rate in which the adduct is formed. Therefore if adequate exposure time is given, the mutant cells that react more slowly than the wild type cell line will eventually also become inhibited to the same extent as the non-resistant cells. This is seen with CYC which is not as significantly affected by mutations in $\beta$-tubulin compared to other taxoid site MSAs (Buey et al., 2007). The increase in binding site mutations with low level exposure to MSAs should not occur with covalent binders, due to their sustained duration of inhibition (Singh et al., 2011). 


\subsubsection{Aims of this chapter}

1. To investigate the binding kinetics of ZMP and DAC

2. To confirm that the irreversible binding involved the formation of a covalent bond and to determine which residues ZMP and DAC were interacting with in the taxoid binding pocket

3. To investigate the underlying stabilization effect of this new chemotype on the MT using computer modeling

MSAs may bind in a reversible manner, like the taxanes for example, or in an irreversible manner, like CYC (Buey et al., 2007), although most drugs bind to their biological targets in a reversible manner. In the previous chapter it was found that ZMP and DAC behaved in a similar manner to CYC in that the binding constants of the compounds increased with temperature rather than decreasing like that of typical MSAs. Moreover, it was not possible to extract ZMP or DAC from the MT pellets, suggesting that these compounds bound to tubulin in an irreversible manner. Other MSAs, such as PTX or PEL, which are reversible binders, are easily recovered from MT pellets (Díaz \& Andreu, 1993; Pera et al., 2010). Using a simple pellet extraction assay (Section 5.2.1), it was shown that ZMP binds the MT in an irreversible manner; whereas, DAC, presumably because it binds more slowly to tubulin, acted like a reversible inhibitor. Aim one of this chapter was, therefore, to investigate the binding kinetics of the compounds in order to determine the differences in their binding profiles. The next aim of the chapter was to confirm that the irreversible binding involved the formation of a covalent bond and to determine which residues these compounds were interacting with in the taxoid binding pocket. At this stage of the study, it was possible that ZMP either had really strong affinity for binding similar to cryptophycin-52 (Panda et al., 1998), or it alkylated tubulin like CYC (Buey et al., 2007). MS is an important method that can be used in the characterization of ligand-protein binding. Specialized instruments allow the detailed structural analysis of covalently-modified peptides (Calvo et al., 2008). If ZMP and/or DAC proved to bind covalently, then it was essential to determine the modified amino acid residues at which alkylation occurs, providing direct MS evidence and confirming their binding site to be in the taxoid binding pocket. 


\subsection{METHODS}

\subsubsection{Detection of irreversible binding}

Given that ZMP was not being detected by HPLC in the pellets and the finding that its binding constants increased with temperature it was suspected that ZMP was binding in a covalent manner to the MT. However, STD signals were detected for DAC binding to MTs, suggesting that these two compounds interacted with tubulin in a different manner to one another. A simple assay was therefore performed to detect irreversible binding of ZMP. DAC was used as a control ligand with known reversible binding based on the STD results obtained. Stabilized MTs $(5 \mu \mathrm{M})$ were incubated with $6 \mu \mathrm{M}$ ZMP or DAC in GAB buffer for $30 \mathrm{~min}$ at $37^{\circ} \mathrm{C}$. Excess $(20 \mu \mathrm{M}) \mathrm{FTX}-2$ was then added to each sample and the samples incubated for $5 \mathrm{~min}$ at $37^{\circ} \mathrm{C}$. The MTs with bound compound were separated from unbound compound by centrifugation at $50,000 \mathrm{rpm}, 37^{\circ} \mathrm{C}, 20 \mathrm{~min}$ using a TLA100 rotor in Beckman Optima TLX ultracentrifuge. The color of the supernatant and the pellets were examined visually and then the supernatant separated by pipette, and the pellet resuspended in $10 \mathrm{mM}$ NaPi 1\% SDS pH 7.0 buffer. The amount of FTX-2 in each of the samples was measured spectrofluorometrically against a FTX-2 standard curve as described in Chapter 2.8.

\subsubsection{Ligand binding to tubulin}

Given that tubulin can exist in a number of different aggregation states; binding of the compounds to each state needed to be confirmed. These experiments are important in determining reaction time and optimal conditions of the ligands in further assays. To confirm ZMP and DAC binding to MTs and to determine the time they take to bind to MTs, tubulin oligomers and dimeric tubulin a number of different experiments were performed using HPLC as described in Chapter 2.9.

\section{Reaction times of the compounds with stabilized microtubules}

To determine the time it took for ZMP and DAC to irreversibly react with MTs, $25 \mu \mathrm{M}$ stabilized taxoid binding sites were incubated with sub-stoichiometric amounts $(20 \mu \mathrm{M})$ of ZMP, DAC or an equivalent volume of DMSO in GAB buffer, final volume $200 \mu \mathrm{L}$. Samples were incubated at $25^{\circ} \mathrm{C}$ for $30 \mathrm{~min}, 1 \mathrm{~h}, 2 \mathrm{~h}, 4 \mathrm{~h}, 6 \mathrm{~h}, 8 \mathrm{~h}$ and overnight, then centrifuged at $50,000 \mathrm{rpm}, 25^{\circ} \mathrm{C}$, for $20 \mathrm{~min}$ in a TLA100 rotor in a Beckman Optima TLX ultracentrifuge to separate MTs and bound compound from unbound compound. 
The supernatants were then collected by careful pipetting and the pellets resuspended in $200 \mu \mathrm{L} 10 \mathrm{mM}$ NaPi buffer. DTX $(10 \mu \mathrm{M})$ was added to each sample as the internal standard. The samples were extracted and processed as in Chapter 2.9. The dried samples were dissolved in $35 \mu \mathrm{L}$ v/v 70:30 methanol/water and analyzed by HPLC using an isocratic system of 70:30 methanol/water. The concentration of ligands in each of the samples was quantified by comparison of the integrated areas of the HPLC peaks with those of the internal standard. The time it took for ZMP and DAC to disappear from the pellet samples were then determined.

\section{Binding of compounds to dimeric tubulin}

Tubulin (40 mg) was prepared in PEDTA buffer as described in Chapter 2.4. The tubulin was then diluted to $20 \mu \mathrm{M}$ in PEDTA buffer and the volume split in half, with $1.5 \mathrm{mM}$ $\mathrm{MgCl}_{2}$ added to one half $\left(\mathrm{Mg}^{2+}\right.$ addition provides conditions for oligomer formation). Tubulin was then incubated at $25^{\circ} \mathrm{C}$ with $25 \mu \mathrm{M} \mathrm{ZMP}$ or DAC or an equivalent volume of DMSO in PEDTA buffer, final volume $200 \mu \mathrm{L}$, over a series of time points ( $0 \mathrm{~min}, 30$ min, $1 \mathrm{~h}, 2 \mathrm{~h}, 4 \mathrm{~h}, 8 \mathrm{~h}$, overnight). Samples were then centrifuged as above to ensure no polymerization had occurred. DTX $(10 \mu \mathrm{M})$ was added to the supernatants and samples processed as above for HPLC analysis.

\section{Compound-induced tubulin assembly into microtubules}

Tubulin (20 mg) was prepared in GAB buffer as in Chapter 2.4. The concentration was diluted, and $25 \mu \mathrm{M}$ tubulin was incubated with $20 \mu \mathrm{M}$ ligands in GAB assembly buffer for 1 hour at $37{ }^{\circ} \mathrm{C}$. Samples were centrifuged as above at $37^{\circ} \mathrm{C}$, and the supernatants were collected and the pellets resuspended in $200 \mu \mathrm{L} 10 \mathrm{mM} \mathrm{NaPi}$ buffer. DTX $(10 \mu \mathrm{M})$ was added, and the samples were processed as above for HPLC analysis and analyzed.

\section{Binding of compounds to stabilized microtubules}

Stabilized taxoid binding sites $(25 \mu \mathrm{M})$ with $20 \mu \mathrm{M}$ ZMP, DAC or an equivalent volume of DMSO in GAB buffer, final volume $200 \mu \mathrm{L}$, were incubated at $25^{\circ} \mathrm{C}$ for the desired time (DAC $4 \mathrm{~h}$ and $8 \mathrm{~h}, \mathrm{ZMP} 1 \mathrm{~h}$ ). Samples were centrifuged at 50,000 rpm at $25^{\circ} \mathrm{C}$ for $20 \mathrm{~min}$ and the supernatants collected by careful pipetting. The pellets were resuspended in $200 \mu \mathrm{L} 10 \mathrm{mM}$ NaPi buffer. DTX $(10 \mu \mathrm{M})$ was added to each sample as the internal standard and the samples processed as above for HPLC analysis. 


\subsubsection{Mass Spectrometry}

To characterize the interaction of ZMP and DAC with MTs, tubulin oligomers and tubulin dimers, MS experiments using a hybrid triple-quadruple mass analyzer were performed. MT samples were prepared and analyzed as described in Chapter 2.10.1, dimeric and oligomeric tubulin samples were prepared as described in Sections 2.10.3 and 2.10.4, respectively. All samples were run by Dr. Enrique Calvo as described in Section 2.10.

\subsubsection{Binding Kinetics}

Given that ZMP and DAC bind to tubulin irreversibly, there are no thermodynamic equilibrium parameters to characterize. The kinetic parameters of the binding can therefore be directly characterized. The binding kinetics of ZMP and DAC binding to the taxoid site were studied by two different methods. First, the kinetics of the reaction of compounds with cross-linked MTs was measured by determining the inhibition of FTX-2 binding to MTs, as described in Chapter 2.8. Second, the kinetics of the reaction of compounds with cross-linked, stabilized MTs was measured by HPLC. Crosslinked MTs in GAB $(25 \mu \mathrm{M})$ were incubated with $20 \mu \mathrm{M}$ compounds over a time course (30 min, 1 h, 2 h, 4 h, 6 h, 16 h). DTX $(10 \mu \mathrm{M})$ was added as an internal standard. Samples were processed and run by HPLC as described in Section 2.9, with samples re-suspended in v/v 70:30 methanol/water $(35 \mu \mathrm{L})$, and run using an isocratic system of 70:30 methanol/water.

\subsubsection{Immunofluorescence and confocal microscopy}

HL-60 cells were cultured using standard techniques, as described in Chapter 2.2, however the media contained no insulin. Cells were seeded in a 24 -well plate $\left(2 \times 10^{5}\right.$ cells/well, total volume of $500 \mu \mathrm{L}$ per well) and incubated for $16 \mathrm{~h}$ in the presence of varying concentrations of MSA and FTX-2. DAPI was then added at a concentration of $10 \mu \mathrm{g} / \mathrm{mL}$ and incubated for $30 \mathrm{~min}$. A $100 \mu \mathrm{L}$ aliquot from each well was then cytospun onto slides at 1,265 rpm for 5 min via a Shandon Cytospin 3, model 1302, centrifuge (Thermo Scientific). Attached cells were mounted using coverslips and $10 \mu \mathrm{L}$ glycerol buffer (0.13 M glycine/NaOH, $0.2 \mathrm{M} \mathrm{NaCl}, 70 \%$ glycerol, $\mathrm{pH}$ 8.6). Use of this buffer was determined to be an essential step in the process, and other methods of fixation did not work. The glycerol buffer preserves the taxoid binding sites, and without this buffer 
FTX-2 staining was not seen. Fluorescent staining was examined with an Olympus FluoView FV1000 confocal laser scanning microscope using a 100x oil-immersion objective. The remaining $400 \mu \mathrm{L}$ of sample was centrifuged for $5 \mathrm{~min}$ at $400 \mathrm{~g}$ onto the plate using an Eppendorf bench top centrifuge, model 5810. Fluorescence was also observed in an Olympus IX51 fluorescence microscope (U-RFET, Tokyo, Japan).

\subsubsection{Flow cytometery}

Cells treated with a reversible MSA are able to recover from MSA treatment after extensive washing of the cells and incubation in drug-free medium. A covalent MSA such as CYC, however, does not show this recovery even after extensive washing over long periods of time, and the cells remain blocked in the $G_{2} / M$ phase of the cell cycle (Buey et al., 2007). Cell cycle analysis was therefore carried out with propidium iodide (PI) staining and flow cytometry to determine if ZMP had the same effect as CYC. Optimization experiments were done to pinpoint concentrations of ZMP and PTX and time points that caused significant $\mathrm{G}_{2} \mathrm{M}$ blockage of the cells without causing too much apoptosis.

1A9 cells (cultured as described in Chapter 2.2) were plated in 12-well plates $(2 \mathrm{x}$ $10^{5}$ cells/well), allowed to attach overnight. Cells were then treated with ZMP or PTX followed by further incubation without drug. Cells were incubated for $8 \mathrm{~h}$ with ZMP at $2.5 \mathrm{nM}, 5 \mathrm{nM}$ or $10 \mathrm{nM}$ or with PTX at $5 \mathrm{nM}$ or $10 \mathrm{nM}$. At $8 \mathrm{~h}$, some wells of cells were harvested and others were extensively washed and left in culture for a further $30 \mathrm{~min}$ in drug-free medium, followed by another wash and incubation in drug-free medium for another $16 \mathrm{~h}, 24 \mathrm{~h}$ or $48 \mathrm{~h}$ before harvesting. To harvest, cells were washed once in cold PBS and collected using trypsin-EDTA and centrifugation, followed by two washes in cold PBS. The cells were then resuspended in $1.5 \mathrm{~mL}$ cold PBS and fixed in $3.5 \mathrm{~mL}$ icecold absolute ethanol, added drop by drop while vortexing the Falcon centrifuge tube. Cells were stored at $4{ }^{\circ} \mathrm{C}$ overnight or for two days in the PBS-ethanol solution. This fixes the cells so that they can be put through flow analysis at a later date, thus allowing all the samples from one experiment to be analyzed at the same time. On the day of analysis cells were centrifuged (600 g, $5 \mathrm{~min}$ ), washed once in $1 \mathrm{~mL}$ PBS and then resuspended in $500 \mu \mathrm{L}$ PBS. RNAase $(100 \mu \mathrm{g} / \mathrm{mL})$ was added and the cell solution incubated for $30 \mathrm{~min}$ at $37^{\circ} \mathrm{C}$. RNA interferes with the PI staining because PI can also stain double stranded RNA, therefore RNAase is required to be in the solution (Nunez, 2001). The cell solution 
was then centrifuged and the pellet resuspended in $250 \mu \mathrm{L}$ PI solution (Appendix) and stained for $15 \mathrm{~min}$ in the dark at room temperature. PI intercalates into DNA and can be used as a marker for the amount of DNA in a cell. DNA content was determined using a BD FacsCanto II flow cytometer. For each drug concentration and time point, 10,000 events were counted. According to the amount of DNA, cells were grouped into $G_{1}, S$, and $\mathrm{G}_{2} / \mathrm{M}$ phase using FlowJo software, version 10.0.4. The percentage of cells in the $\mathrm{G}_{2} \mathrm{M}$ phase of the cell cycle was compared between drug-treated samples and the control at each of the time points using an unpaired Student's $t$-test (GraphPad Prism v5).

\subsubsection{Molecular modeling}

The conformational search and docking calculations were carried out by Javier Rodríguez-Salarichs (a PhD student at the CIB, CSIC, Madrid, Spain); employing the bioactive conformation of DAC obtained from the NMR experiments, with consideration of all possible free torsional angles of the ZMP side chain. The best and lowest energy poses were then chosen using specialized modeling programs. The coordinates for each ligand interacting with the protein were then saved as PDB files which allows them to be manipulated in other modeling programs. Using these PDB files, I created images of the hypothetical ZMP and DAC interactions in the taxoid binding site. 


\section{$\underline{5.3 \quad \text { RESULTS }}$}

\subsubsection{Irreversible binding of zampanolide and dactylolide to tubulin}

To check ZMP and DAC binding to dimeric tubulin, oligomers, stabilized MTs and MSA-induced MTs, direct binding assays were performed using sub-stoichiometric concentrations of ZMP and DAC. The presence of compound in the supernatant or pellet was monitored using HPLC. In the absence of tubulin or stabilized MTs, the total amount of both ZMP and DAC added was recovered from the supernatant. However, in the presence of stabilized MTs or compound-induced MTs, ZMP could not be extracted from the supernatant or the pellet in any samples, indicating that ZMP was irreversibly binding to MTs within $30 \mathrm{~min}$. DAC behaved in a similar manner but took up to $4 \mathrm{~h}$ to show complete irreversible binding. This indicates that the DAC interaction is significantly slower than the ZMP interaction. These results were consistent for both compounds with

all states of tubulin studied. The binding reaction of both compounds required $\mathrm{Mg}^{2+}$ as does native MT-assembly. These results also indicated that both compounds could bind both dimeric and oligomeric tubulin. The above observations explain why DAC STD signals were obtained, as the compound was able to freely associate and dissociate from its binding site up until $4 \mathrm{~h}$ producing STD-NMR signals.

These results were confirmed using a simple FTX-2 competition assay. Even in excess, FTX-2 was unable to bind to MTs pre-incubated with ZMP but was able to bind those pre-incubated for short time periods (for example, $30 \mathrm{~min}$ ) with DAC. This was shown visually by the pellet of the ZMP sample being white, with the supernatant orange, indicating no FTX-2 was bound to MTs in the ZMP sample, even though it was in excess (note that in solution FTX-2 is green but by eye it appears orange). With DAC, the pellet was orange indicating that FTX-2 had displaced DAC and bound to the MTs when it was in excess concentration. This meant that the ZMP binding interaction is kinetically controlled, and explained why ZMP could not be extracted from pellet samples and also explained the increasing binding constants with increasing temperature in the FTX-2 assay (Figure 4.10).

\subsubsection{Mass spectrometry of ligand-modified tubulin}

Given that ZMP and DAC were both shown to bind irreversibly to tubulin and MTs, it was possible that they did so in a covalent manner. A detection method for the ligand bound to the protein was therefore required to confirm this. To characterize the 
interaction of ZMP and DAC with MTs, tubulin dimers and oligomers and to pinpoint the binding site, targeted MS experiments were performed.

To determine the modified peptides, the fragmentation spectra of ZMP and DAC were first determined by MS. This information was crucial to determine the putative diagnostic ion(s) for subsequent ion filtering experiments. Filtering ion masses of 476 $\mathrm{m} / \mathrm{z}$ and $199 \mathrm{~m} / \mathrm{z}$ were used to detect ZMP and DAC, respectively. The tryptic peptide mixtures for ion filtering experiments were then subjected to liquid chromatography coupled to hybrid MS (LC-MS). Peptides were separated by HPLC in a reversed phase C18 nanocolumn and eluted (5\% to $35 \% \mathrm{ACN}$ gradient in $80 \mathrm{~min}$ ) into a nanospray emitter for ionization and fragmentation in the mass spectrometer. The comparative study between the corresponding PIS chromatographic runs indicated the ZMP and DAC binding sites (Figures 5.2A and 5.3A, highlighted areas). Comprehensive analysis of the MS/MS spectra (carried out by Dr. Enrique Calvo) showed that both ZMP and DAC had the same reactive site in MTs, labeling the $\beta$-tubulin-derived peptide spanning the sequence 219-LTTPTYGDLNHLVSATMSGVTTCLR-243 (Figure 5.2B and 5.3B). Although some peaks appeared to be altered after ligand binding, they maintained the same mass composition.

To determine the reactive residue(s), high resolution MS analysis was performed. Residues Asn228 and His229 were determined to be the residues that reacted with both ZMP and DAC (Figure 5.2C and 5.3C). The lower expanded spectra showed that there were two possibilities for the doubly charged fragment ion $y 15$ depending on which residue was ligand bound, with the rest of the ions remaining common between both adducts. If Asn228 was the modified residue, then the value for the doubly charged $y 15$ ion would be $788.40 \mathrm{Da}$ for both ZMP and DAC (y15b). If H229 was the modified residue, then the value for this ion would be 1036.03 Da for ZMP and 980.50 Da for DAC (Figure 5.2C and 5.3C). Detection of ion y15a was significantly stronger for both ZMP and DAC, indicating that modification His229 was the major product of the reaction.

Both ZMP and DAC covalently modified all three forms of tubulin: dimeric tubulin, oligomeric tubulin and MTs in the same manner (Figure 5.4). As expected from previous results the reaction with ZMP was fast and extensive; whereas, the DAC reaction was weaker and required longer incubation time. Given that the same residues were modified in MTs as in unassembled tubulin, it was concluded that the taxoid 
binding site exists in unassembled tubulin. This provided the first direct and conclusive evidence for this site in unassembled tubulin, as previously only evidence of the pore type I site existed in the heterodimer (Buey et al., 2007). ZMP adduct formation was the same for both MTs and unassembled tubulin, indicating that the reaction mode was similar, regardless of the association state of the protein (Figure 5.4A). In contrast, this did not occur with DAC which had three main reaction products eluting at $73 \mathrm{~min}, 81$ min and $86 \mathrm{~min}$. Those eluting at $73 \mathrm{~min}$ and $81 \mathrm{~min}$ exhibited labeling of the peptide at both residues, with the peptide at $73 \mathrm{~min}$ being $1 \mathrm{Da}$ less than that at $81 \mathrm{~min}$. The third product, eluting at $86 \mathrm{~min}$, had the same molecular weight as that at $81 \mathrm{~min}$ but was only labeled at residue 229. The proportion between the three different adducts changes with the aggregation state of the protein (Figure 5.4B). 


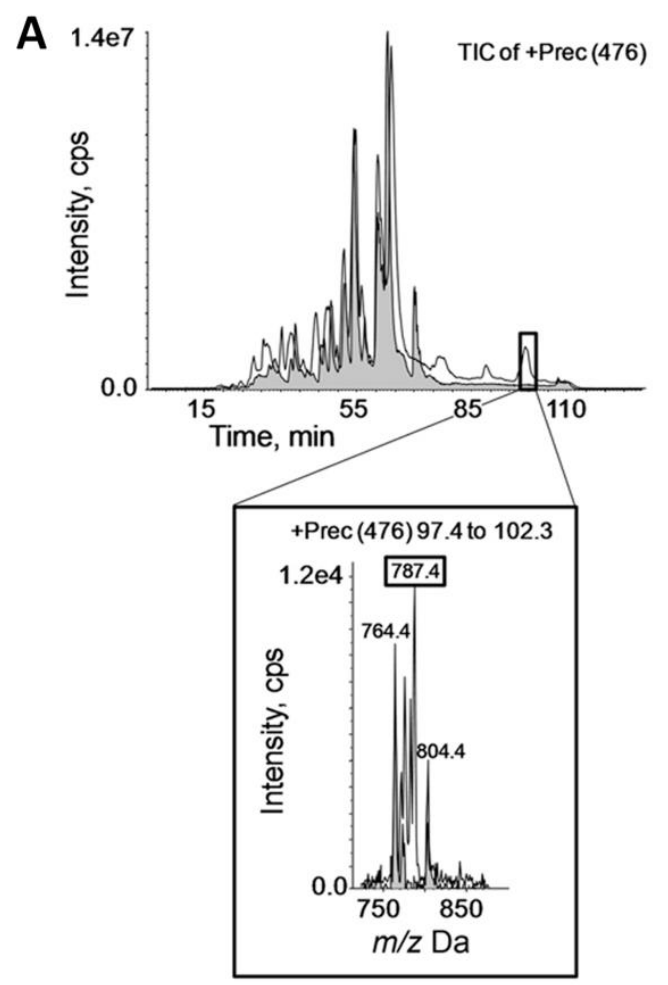

B

LTTPTYGDLNHLVSATMSGVTTCLR+Zampanolide

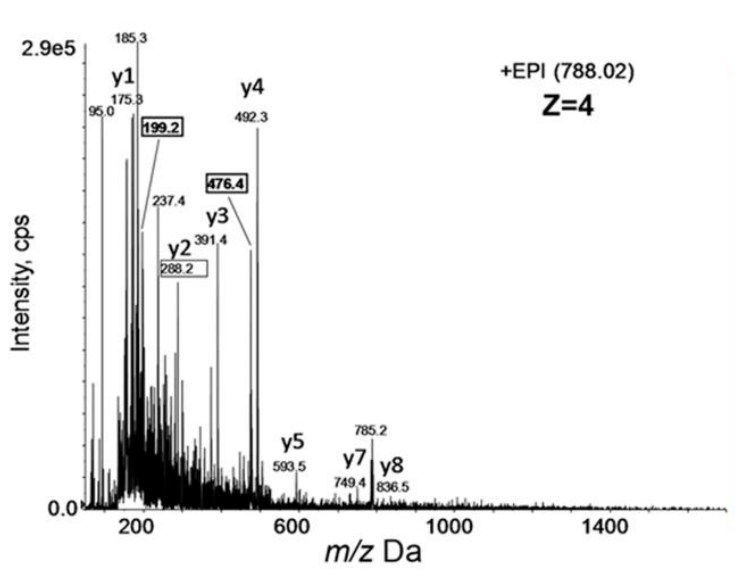

C LTTPTYGDLNHLVSATMSGVTTCLR + Zampanolide
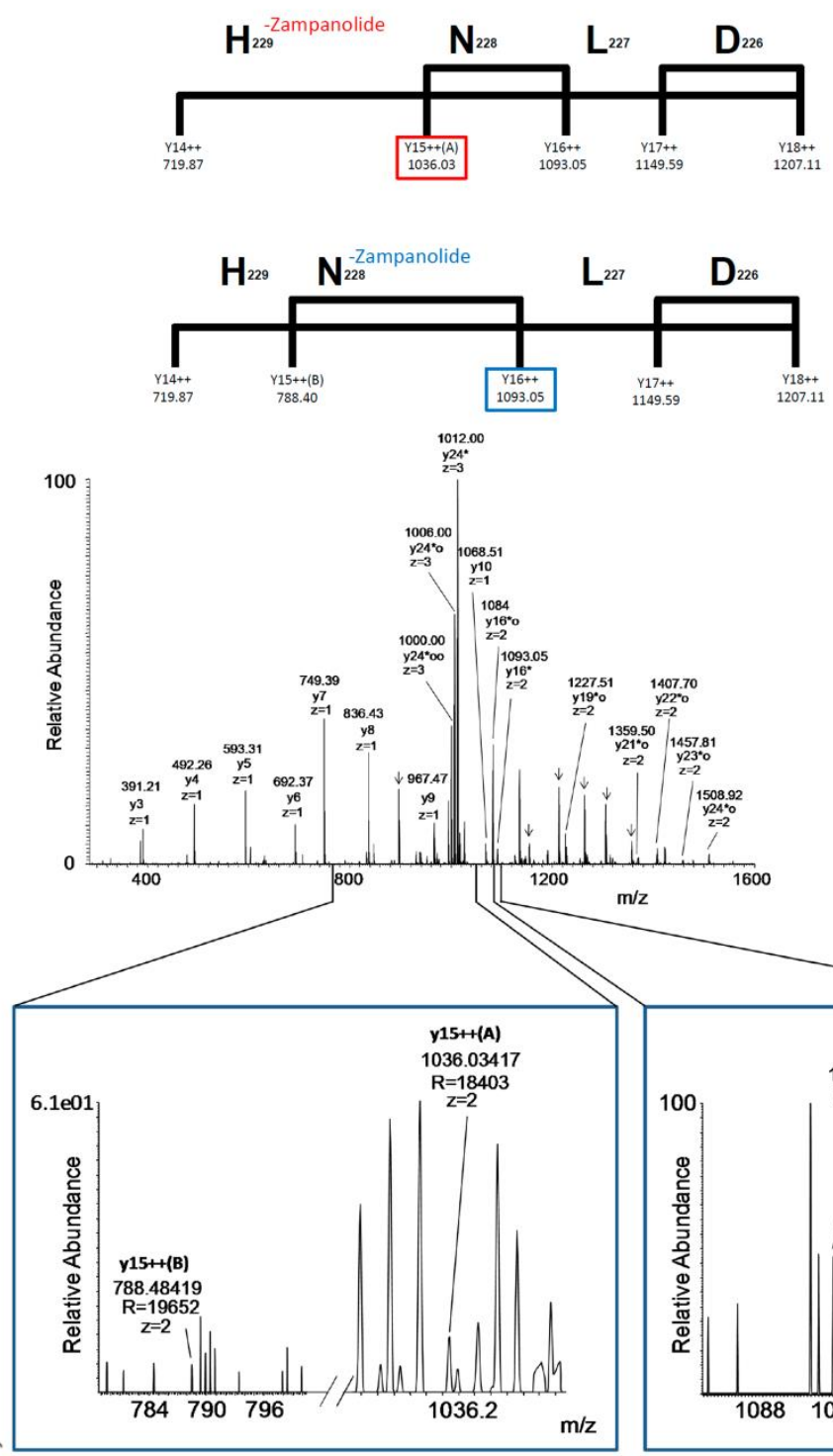

$\mathrm{m} / \mathrm{z}$
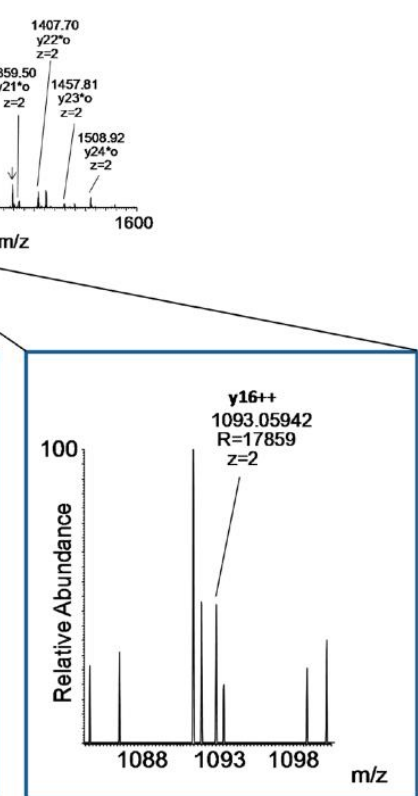

Figure 5.2 MS analysis of ZMP binding to MTs

A. Total ion chromatogram of a PIS experiment at selected $\mathrm{m} / \mathrm{z}$ values for control MTs (gray tracing) or ZMP MTs (white tracing). The chromatograms are similar except for refined differences in the black boxed region. The expanded area highlights these differences and displays some of the differential masses corresponding to the tubulin-derived peptides bound to ZMP.

B. Fragmentation MS/MS spectrum for the tubulin-derived tryptic peptides bound to ZMP. Signals correspond to peptides with four charges. Squared numbers correspond to ZMP fragments. The tryptic $\beta$-peptide

219-LTTPTYDGLNHLVSATMSGVTTCLR-243 contains attached ZMP and its interaction domain.

C. Upper: schematic indicating the ZMP peptide and the charged ions. Two different $y 15$ ion masses indicate the labeling of two different residues by ZMP. y15(A) $\mathrm{m} / \mathrm{z}=1036.03$ when His 229 is labeled, $y 15(\mathrm{~B}) \mathrm{m} / \mathrm{z}=788.40$ when Asn228 is labeled. Lower: High-resolution MS/MS spectrum for the triply-charged tryptic peptides bound to ZMP. The asterisks mark the $y$ ions bearing the bound ZMP. Water losses are labeled with the symbol "o". Some ions corresponding to the accompanying ' $a$ ' ion series are marked with an arrow. The value of $\mathrm{z}$ indicates the charge state on each fragment. Detailed information about the key fragment ions (in the y series) containing the modified residue (Asn228 or His229) is shown. 


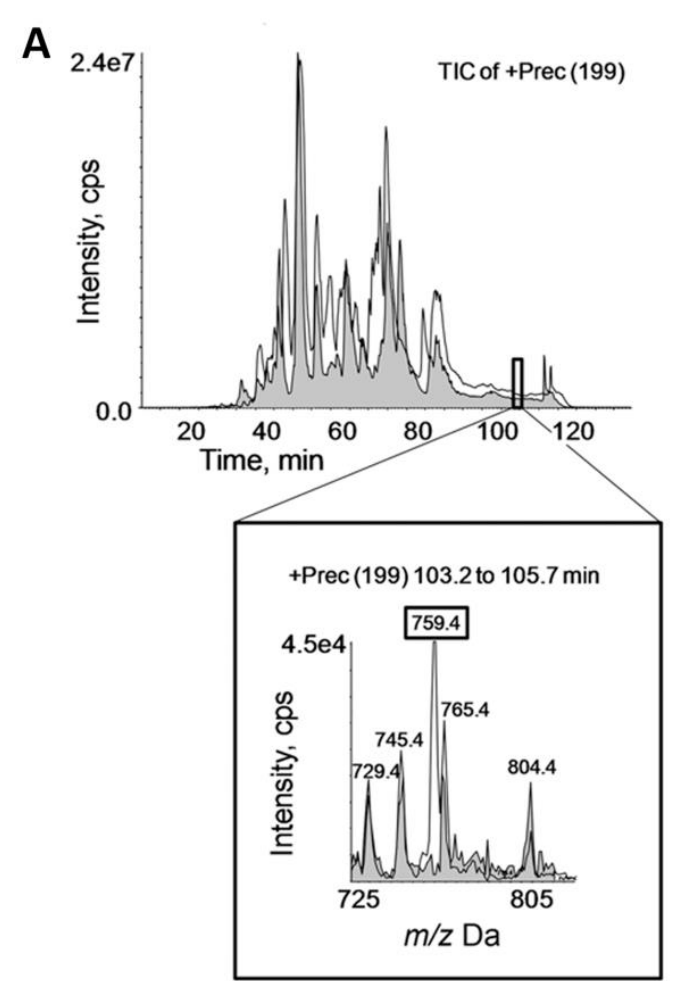

B

LTTPTYGDLNHLVSATMSGVTTCLR + Dactylolide

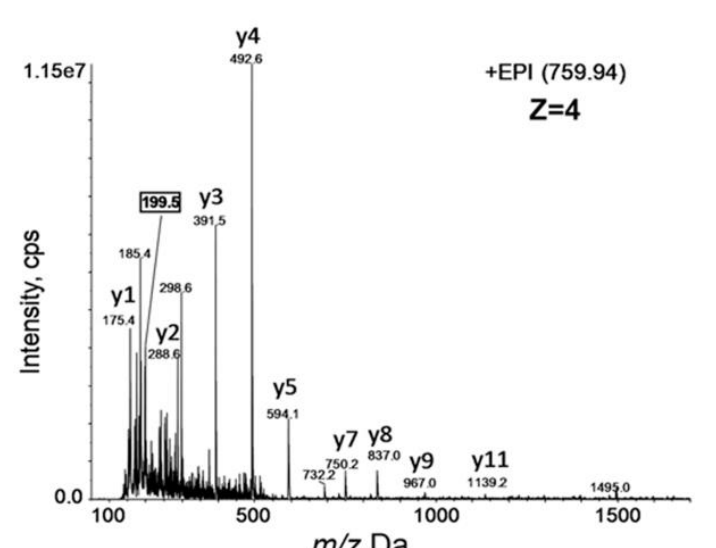

C
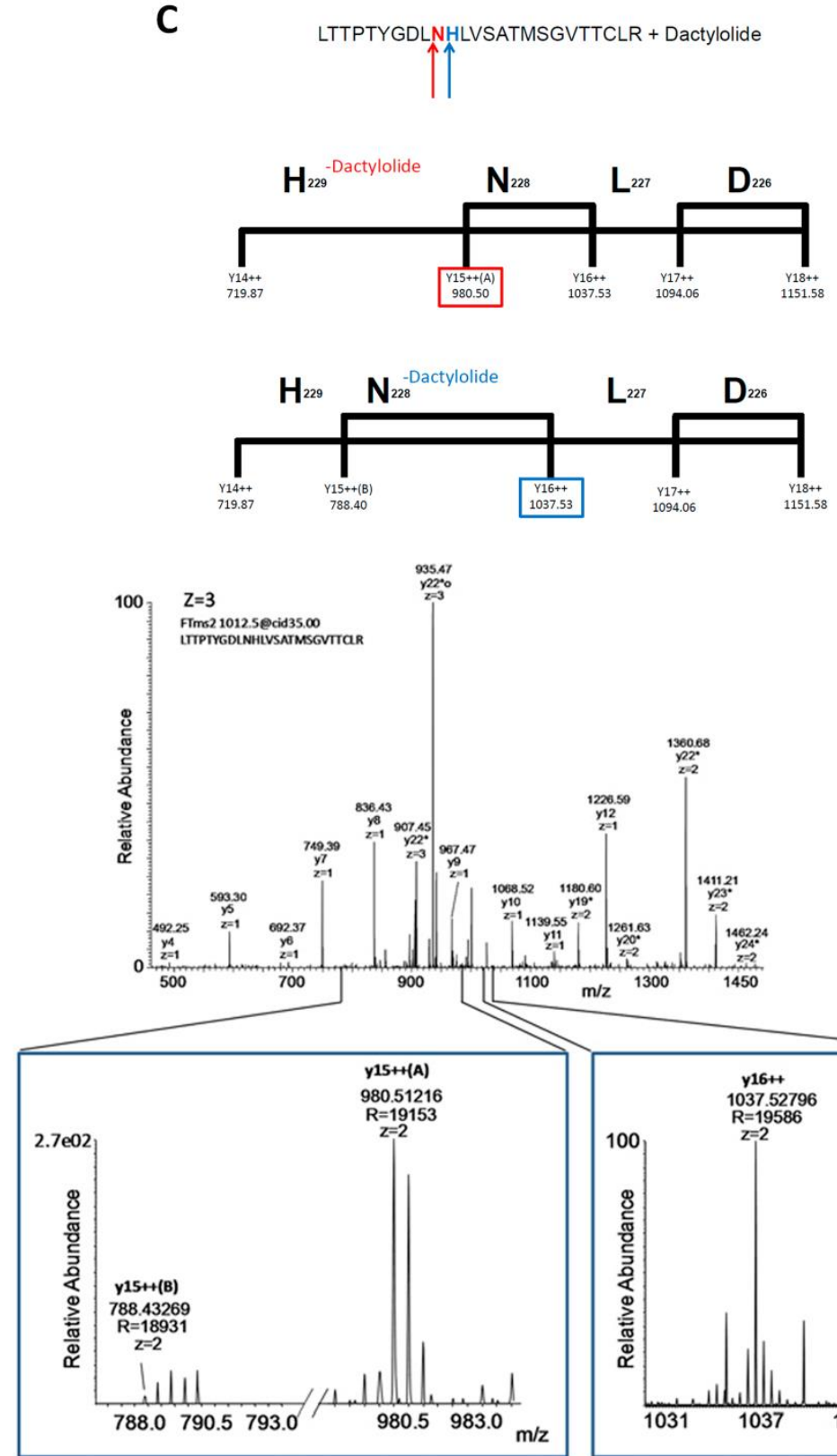

Figure 5.3 MS analysis of DAC binding to MTs

A. Total ion chromatogram of a PIS experiment at selected $\mathrm{m} / \mathrm{z}$ values for control MTs (gray tracing) or DAC MTs (white tracing). The chromatograms are similar except for refined differences in the black-boxed region. The expanded area highlights these differences and displays some of the differential masses corresponding to the tubulin-derived peptides bound to DAC.

B. Fragmentation MS/MS spectrum for the tubulin-derived tryptic peptides bound to DAC. Signals correspond to peptides with four charges. Squared numbers correspond to DAC fragments. The tryptic $\beta$-peptide 219-LTTPTYDGLNHLVSATMSGVTTCLR-243 contains attached DAC and its interaction domain.

C. Upper: Schematic indicating the DAC peptide and the charged ions. Two different $y 15$ ion masses indicate the labeling of two different residues by DAC. $y 15(\mathrm{~A})$ $\mathrm{m} / \mathrm{z}=980.50$ when His229 is labeled, $y 15(\mathrm{~B}) \mathrm{m} / \mathrm{z}=788.40$ when Asn228 is labeled. Lower: High-resolution MS/MS spectrum for the triply-charged tryptic peptides bound to DAC. The asterisks mark the y ions bearing the bound DAC. Water losses are labeled with the symbol "o". Some ions corresponding to the accompanying ' $\mathrm{a}$ ' ion series are marked with an arrow. The value of $\mathrm{z}$ indicates the charge state on each fragment. Detailed information about the key fragment ions (in the y series) containing the modified residue (Asn228 or His229) is shown. 
A Zampanolide-treated samples
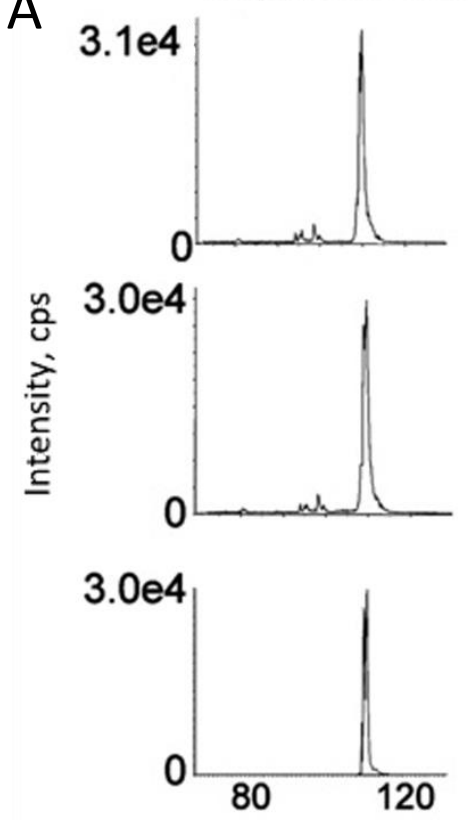

Dimeric tubulin

Oligomeric tubulin

Microtubules

Time, $\min$
B

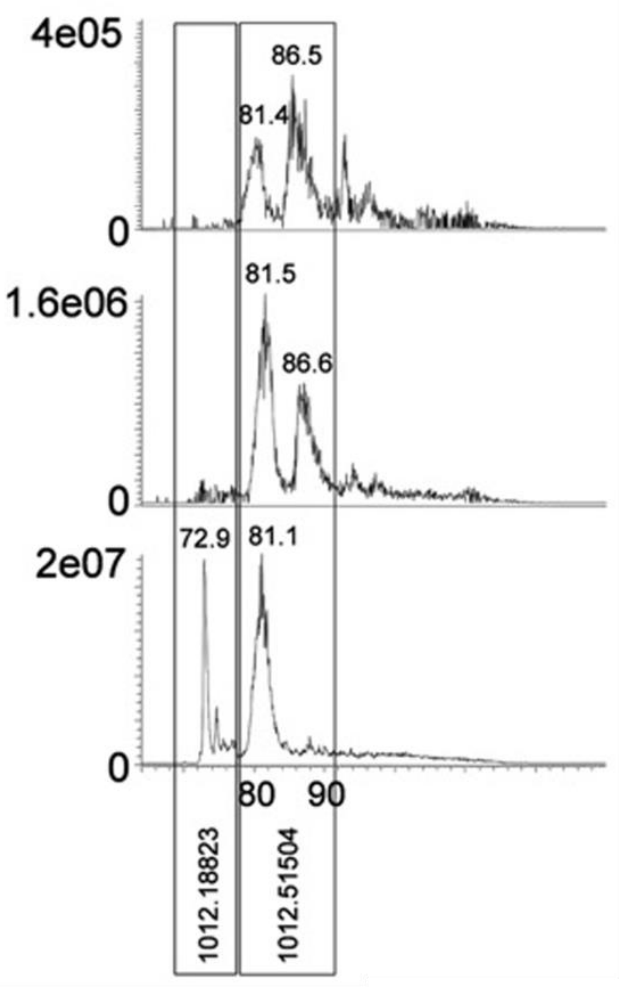

Figure 5.4 ZMP and DAC interaction with tubulin and MTs

A. ZMP interaction with dimeric tubulin, oligomeric tubulin and MTs. Selected reaction monitoring assays: the mass of the expected triply charged $\beta$-tubulin ion was monitored, and two different transitions were studied. B. DAC interaction with dimeric tubulin, oligomeric tubulin and MTs. Extracted ion chromatograms for the triply charged tubulin-derived tryptic peptide of DAC-treated samples. 


\subsubsection{Binding kinetics of zampanolide and dactylolide}

Given the fact that both ZMP and DAC can irreversibly bind MTs but do so in a different manner, the binding kinetics of ZMP and DAC were examined. Fast covalent binders will immediately bind to the MT in an irreversible manner, and no FTX-2 will be associated with the pellet (FTX-2 will be in the supernatant). With compounds that do not bind irreversibly the pellet will be FTX-2 saturated given that it is in excess concentration. Slow covalent binders such as DAC bind covalently over time, and compounds that react in this manner will show a decrease in FTX-2 associated with the pellet over time. Using two types of kinetic experiments, two different processes can be monitored to provide an estimate of the kinetics of the covalent reaction once the compound is noncovalently bound to the binding site. The first experiment measures the number of binding sites that have not yet reacted and therefore are still available for FTX-2 binding (fluorescence method). This measures the kinetics of the covalent reaction. In the second experiment, MTs are pelleted and the concentration of unreacted compound measured, such that both covalently and noncovalently reacted compound are pelleted (using an HPLC method). This measurement accounts for the kinetics of the noncovalent binding reaction. If the covalent reaction between the compound and the sites is immediate, no difference will be observed between the two experiments. In this case the covalent reaction immediately follows noncovalent binding. However, if the covalent reaction is slow and the noncovalently bound compound can exchange with unbound compound, a significant difference between measurements will be obtained.

ZMP reacted quickly with the MTs, and the covalent reaction was complete in less than $30 \mathrm{~min}$. No differences were observed between the two different measurements, indicating that the covalent reaction immediately follows the noncovalent reaction (Figure 5.3.4). In contrast, a significant difference between the two experiments was observed for DAC binding to MTs. Thus, the covalent reaction with DAC was much slower than that with ZMP, with an apparent kinetic rate constant on the order of $0.12 \mathrm{hr}^{-}$ 1 (measured by FTX-2 exchange); whereas, the noncovalent reaction as measured by HPLC was 10 times faster than the covalent reaction (apparent kinetic rate constant of the order of $1 \mathrm{hr}^{-1}$ ) (Figure 5.5). This indicates a significant delay between the noncovalent and the covalent interaction of DAC with the binding site and suggests that the covalent reaction occurs after formation of the noncovalent complex. This suggests that the noncovalently bound DAC is in fast exchange with the medium, confirming the 
STD-NMR results. Over time, the intensity of these signals decreased due to the formation of the covalent complex.

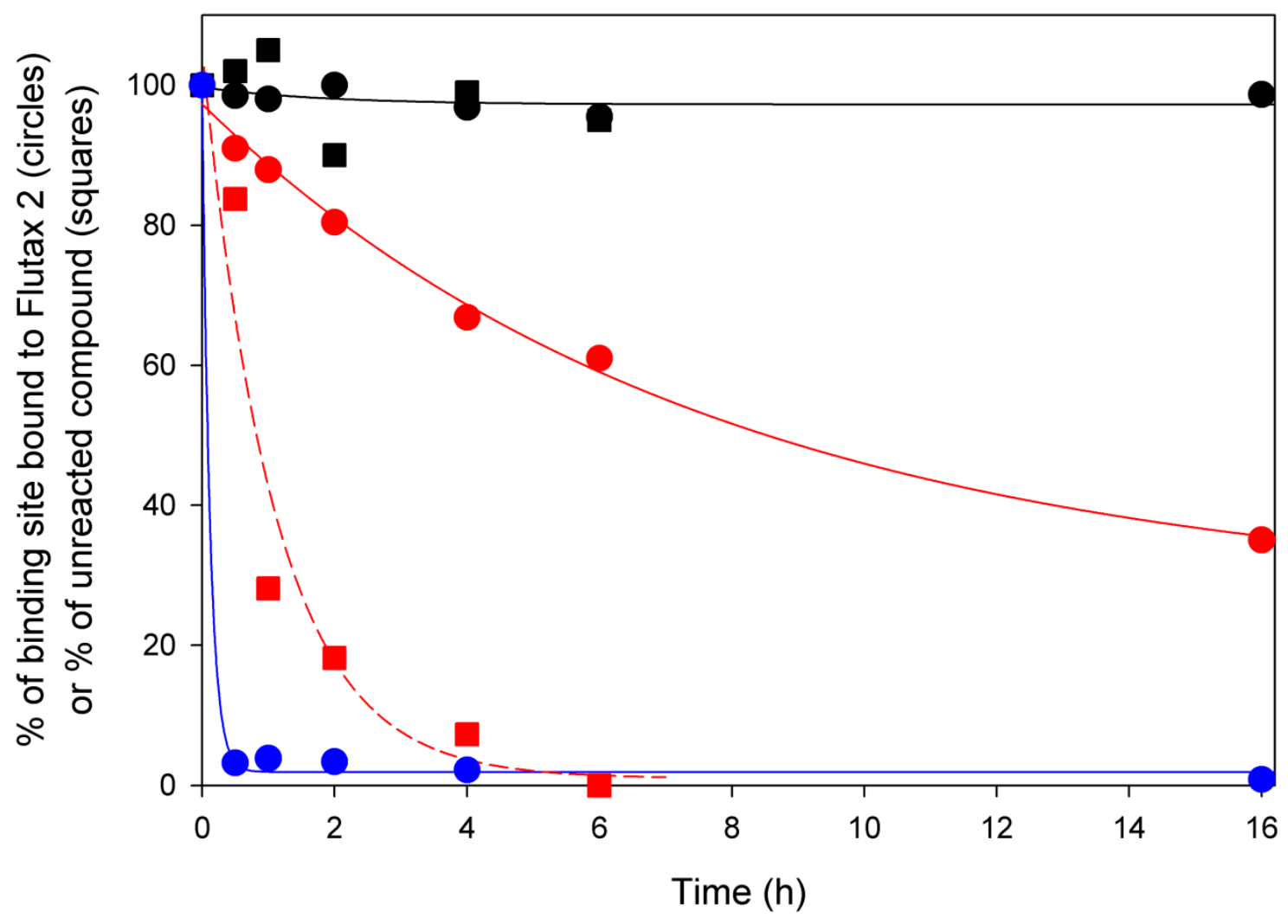

Figure 5.5 Binding kinetics of ZMP and DAC

Kinetics of the reaction of ligands with MTs studied by HPLC to measure the unreacted amount of compound, and fluorescence to measure the decrease in available binding sites. These two experiments were designed to monitor different processes and to provide an estimate of the kinetics of the covalent reaction after the compound was noncovalently bound to the binding site. The percentage of binding sites bound by FTX-2 (circles) and the percentage of unreacted compound detected by HPLC (squares): ZMP (blue circles), DAC (red circles), DMSO control (black circles), DAC (red squares) and DAC control with no MTs (black squares). Solid lines represent the loss of available binding sites, and the dashed line represents the fit for decay of unbound DAC. The difference in the two measurements for DAC indicates that the covalent reaction is slow. All FTX-2 was displaced by ZMP, and all ZMP was found bound irreversibly to the pellet within $30 \mathrm{~min}$, indicating covalent binding immediately follows the noncovalent reaction. 


\subsubsection{Covalent modification of cellular tubulin}

To confirm covalent binding in a cellular setting, FTX-2 was used to monitor compound binding to the taxoid binding site in intact cells. Cells were treated with excess FTX-2 or excess MSA and the fluorescence of FTX-2 monitored by confocal microscopy. When cells were treated with $200 \mathrm{nM}$ FTX-2 and $50 \mathrm{nM}$ taxoid site ligand (PTX, DTX, IXA or DSC), the MTs stained green with FTX-2. When cells were treated with excess taxoid ligand (200 nM) and $50 \mathrm{nM}$ FTX-2, no green MT staining was seen, since the sites were saturated with the unlabelled taxoid site MSA (Figure 5.6A). Thus, the taxoid site MSAs and FTX-2 reversibly competed with each other for binding at the taxoid site in $\beta$ tubulin. When cells were treated with LAU or PEL, even in excess, FTX-2 was still able to stain the MTs, since these two MSAs do not bind to the taxoid site on tubulin and simultaneous binding at both the taxoid site and LAU/PEL site can occur (Figure 5.6A). As shown with other taxoid site drugs, at concentrations of excess ZMP (200 nM), no FTX-2 staining of the MTs was seen, since ZMP has saturated all the binding sites. However, in contrast to other taxoid site drugs tested, even when FTX-2 was in excess over ZMP, it was unable to stain MTs as it could not displace ZMP from the binding site, indicating the irreversible binding profile of ZMP (Figure 5.6B). This visually confirmed the covalent binding of ZMP and showed that ZMP had the same mechanism of action, covalently modifying MTs, in cells as it did in vitro. 

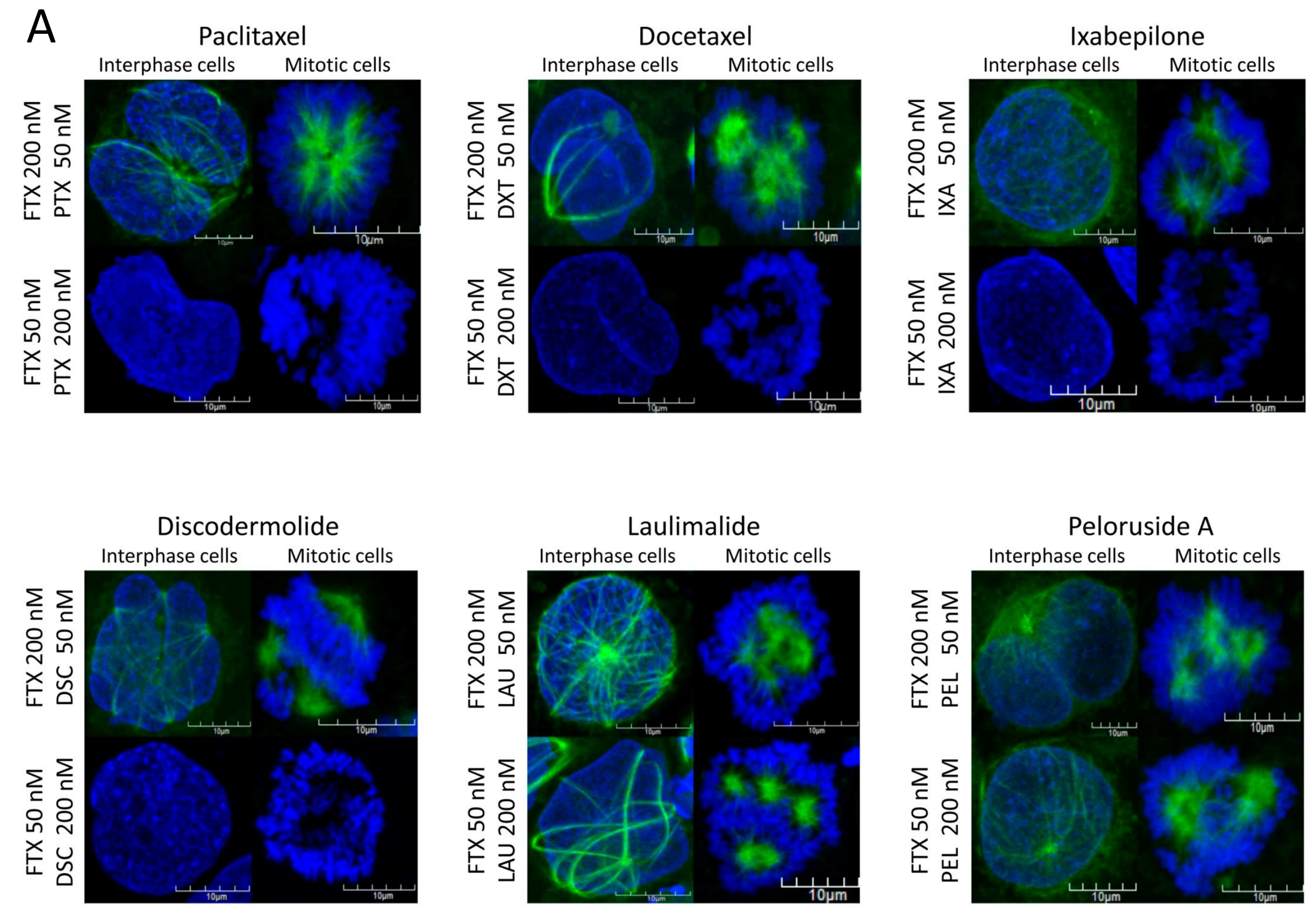

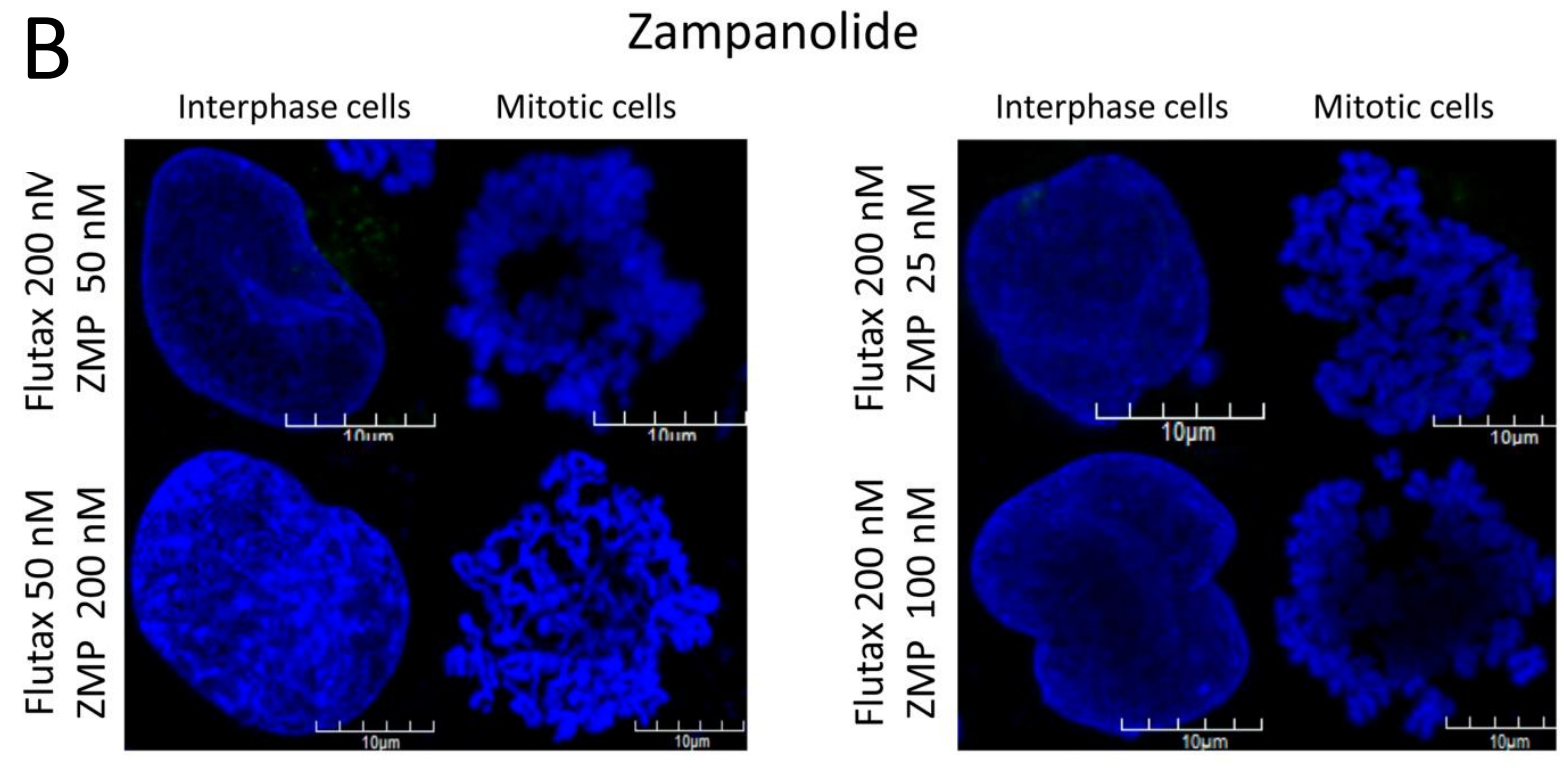

Figure 5.6 FTX-2 staining of cellular MTs with different MSAs

Cells were treated with excess MSA or FTX-2 for $16 \mathrm{~h}$, stained with DAPI and the fluorescence of the cells examined in a confocal microscope. A. FTX-2 staining of cellular MTs in the presence of different MSAs. Taxoid site ligands (PTX, DTX, IXA and DSC) when in excess inhibit FTX-2 binding since all the taxoid sites are occupied by the MSA, thus no green MTs are seen. When FTX-2 is in excess to the taxoid site ligands, the MTs stain green since FTX-2 has bound and saturated the binding sites. Regardless of the concentration of LAU or PEL, the MTs always stain green since simultaneous binding of the MSA at the LAU/PEL site and FTX-2 at the taxoid site can occur. B. When ZMP is in excess over FTX-2 no green staining of the MT is seen, as seen with the other taxoid site ligands in panel A. In contrast when FTX-2 is in excess there is no green staining of the MTs, even at low concentrations of ZMP (25 nM). This lack of green staining indicates that ZMP is covalently modifying the taxoid binding site, inhibiting FTX-2 binding and thus confirming its covalent mechanism of action in a cellular setting. 


\subsubsection{Cell recovery after MSA treatment}

Cells treated with reversible MSAs can recover from the induced $\mathrm{G}_{2} / \mathrm{M}$ cell cycle block after removal of the drug. Cells treated with CYC, a covalent MSA, cannot recover or recover extremely slowly from the $\mathrm{G}_{2} / \mathrm{M}$ cell cycle block (Buey et al., 2007). To investigate if cells could recover after ZMP treatment, the DNA content of cells was examined using PI staining and flow cytometry. In the analysis, only live cells were included. The percentage of cells in the three phases of the cell cycle is shown in Table 5.1. After $8 \mathrm{~h}$ treatment with drug, a significant proportion of the cells were blocked in the $\mathrm{G}_{2} \mathrm{M}$ phase compared to the control, and higher concentrations of drug caused more cells to be blocked; however, more drug also increased the number of dead cells. After washout and incubation in drug-free medium, PTX-treated cells recovered from the $\mathrm{G}_{2} \mathrm{M}$ block and distribute through the cell cycle similar to the control cells (Figure 5.7A; Table 5.1). With ZMP, a decrease in the $\mathrm{G}_{2} \mathrm{M}$ population was seen after washout; however, a significant proportion of the cells remained blocked in $\mathrm{G}_{2} \mathrm{M}$ (Figure 5.7B; Table 5.1), and this confirmed the covalent binding of ZMP in cells. 
Table 5.1 Cell cycle distribution (\%) of 1 A9 cells treated with MSA

\begin{tabular}{|c|c|c|c|c|c|c|c|c|c|c|c|c|}
\hline \multirow{3}{*}{ Compound } & \multicolumn{12}{|c|}{$8 \mathrm{~h}$ incubation } \\
\hline & \multicolumn{3}{|c|}{$8 \mathrm{~h}$ harvest } & \multicolumn{3}{|c|}{ Wash $+16 \mathrm{~h}$} & \multicolumn{3}{|c|}{ Wash $+24 \mathrm{~h}$} & \multicolumn{3}{|c|}{ Wash $+48 \mathrm{~h}$} \\
\hline & $G_{l}$ & $G_{2} / M$ & $S$ & $G_{1}$ & $G_{2} / M$ & $S$ & $G_{1}$ & $G_{2} / M$ & $S$ & $G_{1}$ & $G_{2} / M$ & $S$ \\
\hline Control & $55.4 \pm 1.7$ & $22.7 \pm 1.9$ & $22.0 \pm 1.6$ & $53.2 \pm 1.6$ & $25.9 \pm 0.9$ & $20.8 \pm 0.9$ & $59.0 \pm 1.2$ & $22.0 \pm 0.9$ & $19.0 \pm 0.6$ & $64.2 \pm 0.9$ & $18.7 \pm 0.5$ & $17.1 \pm 0.7$ \\
\hline ZMP $2.5 \mathrm{nM}$ & $50.0 \pm 1.9$ & $35.5 \pm 1.6$ & $14.5 \pm 1.4$ & $52.4 \pm 0.9$ & $29.8 \pm 1.1$ & $17.8 \pm 0.9$ & $56.5 \pm 1.6$ & $25.5 \pm 1.1$ & $18.1 \pm 1.2$ & $61.2 \pm 2.0$ & $25.0 \pm 1.4$ & $13.9 \pm 1.8$ \\
\hline ZMP $5 \mathrm{nM}$ & $42.8 \pm 1.3$ & $39.3 \pm 2.6$ & $17.9 \pm 2.2$ & $51.2 \pm 1.3$ & $30.1 \pm 1.6$ & $18.7 \pm 1.4$ & $58.8 \pm 0.7$ & $25.7 \pm 0.6$ & $15.5 \pm 1.0$ & $58.6 \pm 2.4$ & $23.0 \pm 2.2$ & $18.4 \pm 1.6$ \\
\hline ZMP $10 \mathrm{nM}$ & $29.7 \pm 3.7$ & $53.3 \pm 4.7$ & $16.9 \pm 1.2$ & $48.5 \pm 1.3$ & $33.0 \pm 2.1$ & $18.5 \pm 1.2$ & $55.2 \pm 1.1$ & $28.1 \pm 1.3$ & $16.6 \pm 0.8$ & $57.8 \pm 4.0$ & $26.0 \pm 3.2$ & $16.2 \pm 2.4$ \\
\hline PTX $5 \mathrm{nM}$ & $50.1 \pm 2.3$ & $31.4 \pm 2.8$ & $18.5 \pm 0.8$ & $53.6 \pm 1.6$ & $26.8 \pm 1.7$ & $19.6 \pm 1.0$ & $63.1 \pm 2.0$ & $19.2 \pm 1.6$ & $17.6 \pm 0.9$ & $63.1 \pm 1.6$ & $19.3 \pm 1.0$ & $17.6 \pm 1.3$ \\
\hline PTX $10 \mathrm{nM}$ & $28.1 \pm 2.6$ & $55.3 \pm 3.2$ & $16.7 \pm 1.6$ & $45.9 \pm 1.6$ & $30.3 \pm 2.1$ & $23.8 \pm 1.9$ & $59.3 \pm 1.9$ & $23.6 \pm 1.8$ & $17.1 \pm 0.7$ & $62.8 \pm 1.3$ & $18.6 \pm 1.0$ & $18.6 \pm 1.5$ \\
\hline
\end{tabular}

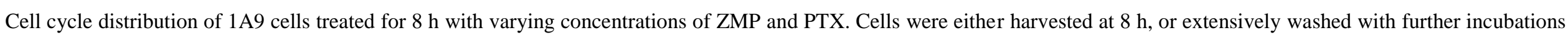

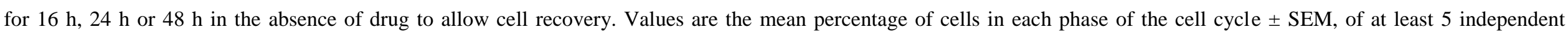

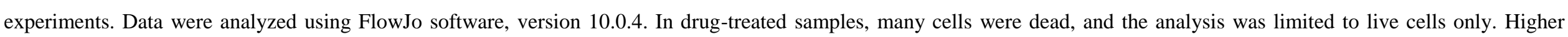
concentrations of MSA and longer incubation times produced a higher percentage of dead cells, and this is reflected by the larger SEM values for drug-treated samples. 
A

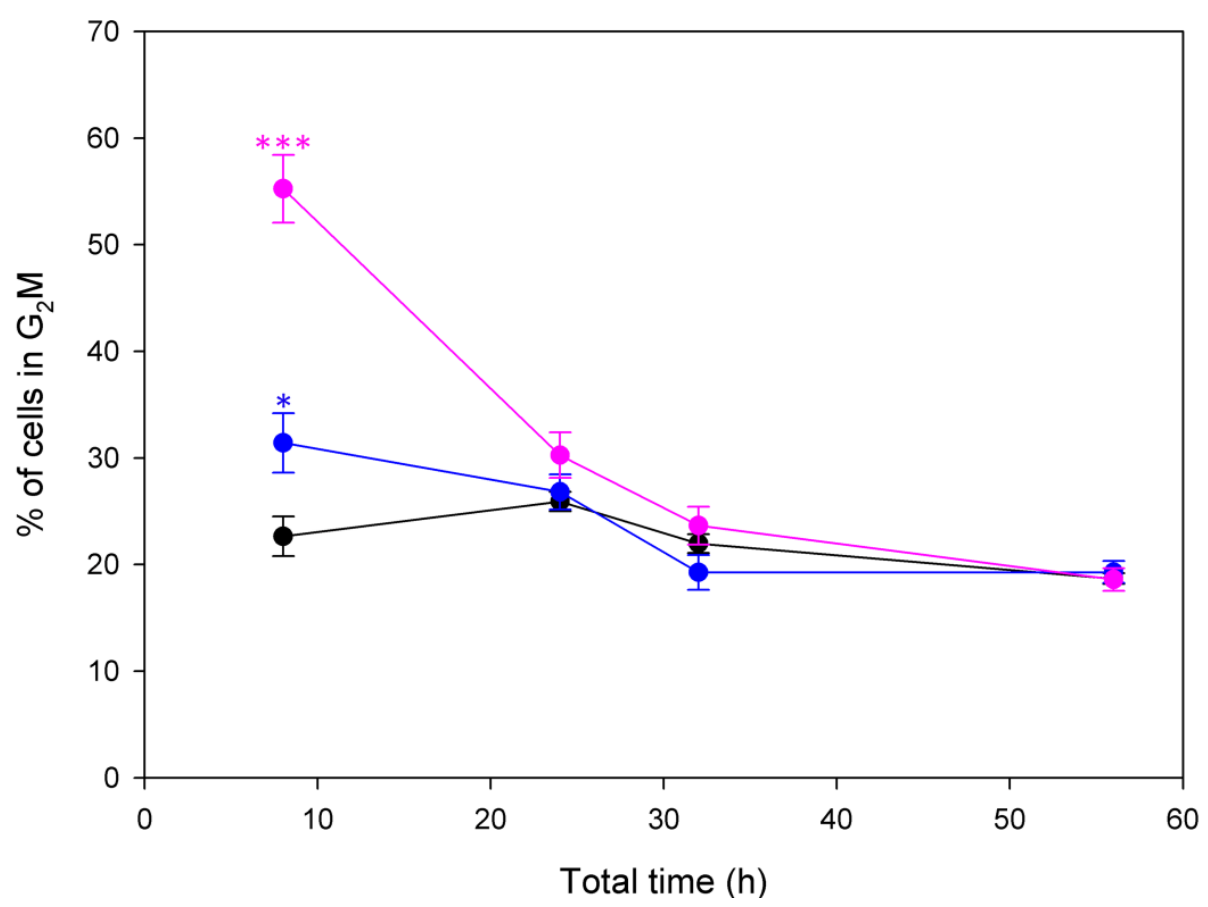

B

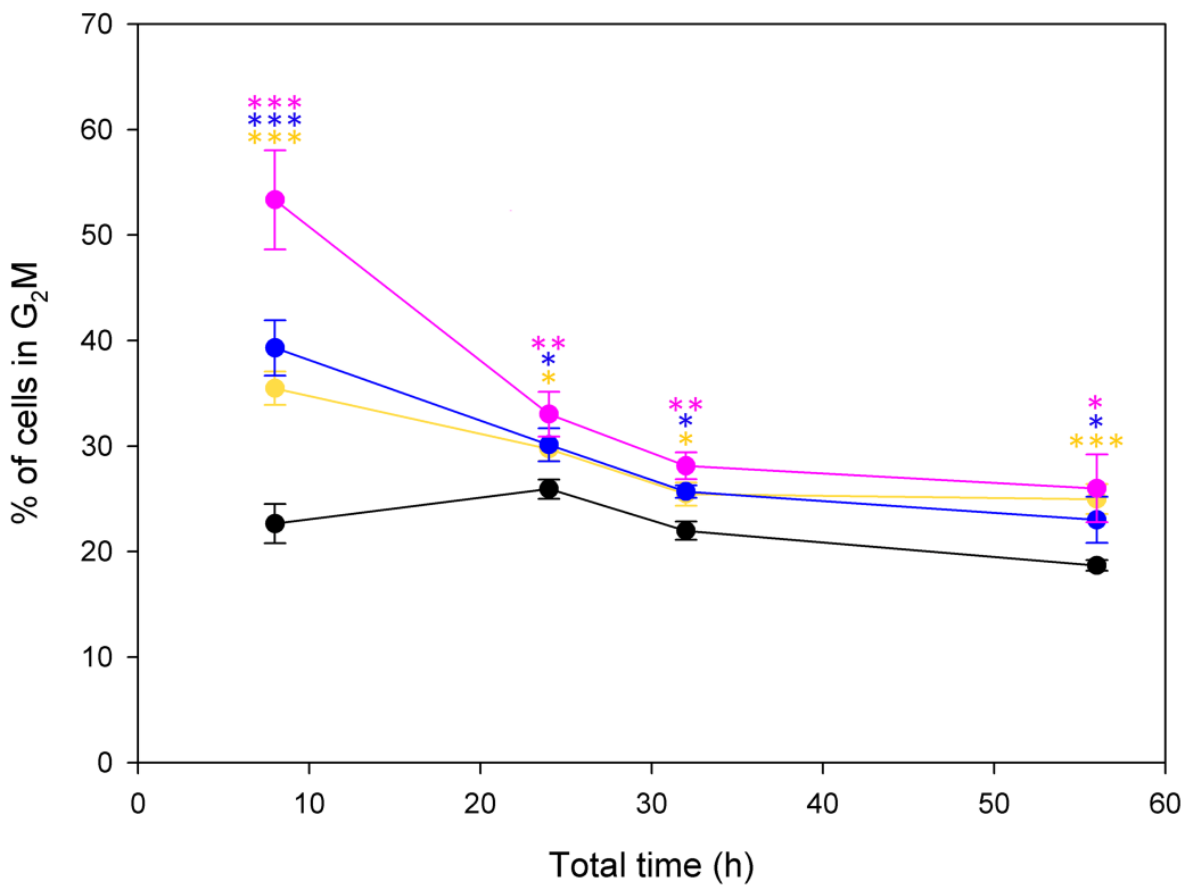

Figure 5.7 Percentage of cells in the $G_{2} M$ phase of the cell cycle

Graphs correspond to data of Table 5.1 and present the number of cells in the $\mathrm{G}_{2} \mathrm{M}$ phase of the cell cycle at the different time points examined. The time points have been presented as the total time since the drug treatment $(0 \mathrm{~h})$. Cells at the $8 \mathrm{~h}$ mark were harvested with no washing; this was used as the comparison control. At $8 \mathrm{~h}$ the other cells extensively washed, then incubated in drug-free medium for a further $16 \mathrm{~h}, 24 \mathrm{~h}$ and $48 \mathrm{~h}$, shown on the graph as $24 \mathrm{~h}, 32 \mathrm{~h}$ and $56 \mathrm{~h}$ total time, respectively. Each point represents the mean percentage \pm SEM of at least 5 independent experiments. Drug treated samples were compared to that of the control at each of the time points using an unpaired Student's $t$-test (GraphPad Prism v5). A. Control (black), $5 \mathrm{nM}$ PTX (blue), $10 \mathrm{nM}$ PTX (pink). PTX treatment of cells significantly increased the number of cells in the $\mathrm{G}_{2} \mathrm{M}$ phase of the cell cycle at the $8 \mathrm{~h}$ time point. After drug washout and incubation in drug-free medium, PTX-treated cells recovered from MSA treatment and returned to having a similar cell cycle distribution as the control. B. Control (black), $2.5 \mathrm{nM}$ ZMP (yellow), $5 \mathrm{nM}$ ZMP (blue), $10 \mathrm{nM}$ ZMP (pink). ZMP treatment caused a significant increase in the number of cells in the $\mathrm{G}_{2} \mathrm{M}$ phase of the cell cycle at $8 \mathrm{~h}$, as did PTX. After drug washout, the number of cells in the $\mathrm{G}_{2} / \mathrm{M}$ phase decreased but remained significantly greater than that in the control, indicating the cells could not recover the same due to the covalent binding of ZMP. Statistical significance of the drug treated samples compared to the control is denoted by the asterisks colored accordingly $(* \mathrm{p}<0.05, * * \mathrm{p}<0.01, * * * \mathrm{p}<0.001)$. 


\subsubsection{Molecular modeling of ZMP bound to the taxoid site}

To explain the stabilization effect of ZMP with tubulin and MTs ZMP was modeled to its binding site using the bioactive conformation of DAC obtained from the STD-NMR measurements. This built a ZMP bioactive structure and both compounds were docked to the taxoid binding pocket. The ligands were modeled in two different poses since two different reaction modes were detected by the MS. Given that the STD and TR-NOESY signals observed for DAC were those of free compound just released from its binding site and the fact that there is a slow dissociation rate for reversible MSAs from the luminal binding site, the signals could not be assumed to be due to interaction with the luminal site. Rather these signals were most likely due to interaction of DAC with the pore type I site in MTs where it would fast exchange with the medium in the absence of a covalent binding event. The interaction of MSAs with dimeric tubulin has not yet been fully characterized and most likely involves an interaction between both sites (Canales et al., 2011). Given this, it was thought that the STD-derived DAC epitope could not be soundly employed to model ZMP bound to the MT; however, the TR-NOESY signals derived for DAC were identical for binding to dimers and to MTs. This suggests that the conformation of bound DAC is stable and that this conformation could be used as a starting point to create a molecular model of these compounds in the luminal taxoid site.

The best poses of the ZMP and DAC pre-reaction complexes were selected as those with minimal interaction energy and the fact that at least one reactive moiety in the compound must be in close enough proximity for a covalent reaction to occur with His229 or Asn228. There are three reactive groups in ZMP and two in DAC. Using Auto dock 4.2 (Morris et al., 1998), acceptable dockings positioned the C1-C5 $\alpha, \beta$-unsaturated ester moiety for interaction with Asn228 and the C9 enone moiety (best Michael acceptor since there is no cross-conjugation) close to His229. Since the side chain of Asn228 faces into the E-site (interacting with the bound nucleotide via a bifurcated hydrogen bond), the 228 side chain was repositioned to face the taxoid binding pocket so that the covalent complex could be built with the $\alpha, \beta$-unsaturated ester. The covalent complex formed with His229 did not require any modification of the structure.

The molecular modeling complexes of ZMP (or DAC) in the luminal binding site indicated that both compounds bound at the bottom of the binding pocket. The shape of DAC in the pocket was concordant with the findings that the STD signals were homogenous. 


\section{Zampanolide models}

When covalently bound to Asn228 at C3, the north face of ZMP is held in place by the covalent bond. The C20 hydroxyl and C1' carbonyl group of the ZMP side chain forms two hydrogen bonds with Asn228, and Asn228 forms an additional hydrogen bond with His229. The ZMP side chain also interacts with Ser232, the latter two interactions increasing the stability of the complex. The opposite side of the side chain contacts Glu22 on the lateral side of the complex. The oxygen of the ketone moiety at C7 strongly interacts with Arg278 within the M-loop, in a similar manner to that observed with PTX binding. The western side of the molecule lays over $\mathrm{H} 7$, and the exo-cyclic methylene moiety at the bottom of the tetrahydropyran ring sits in a hydrophobic pocket between H7, the S9-S10 loop and the M-loop formed by residues Ala233, Phe272, Pro274, Thr276 and Leu231. When in complex with H229, essentially identical interactions occur except for those with the Asn228 side chain which is now in its natural configuration facing into the E-site. In addition, a new hydrogen bond is formed between the C20 side chain hydroxyl and Lys19. Figure 5.8 shows the molecular modeling of bound ZMP. This model explains the strong stabilizing activity of ZMP, given its interaction with the stabilizing M-loop that, in a similar fashion to PTX, would result in increased lateral protofilament interaction and stabilization of the MT. 


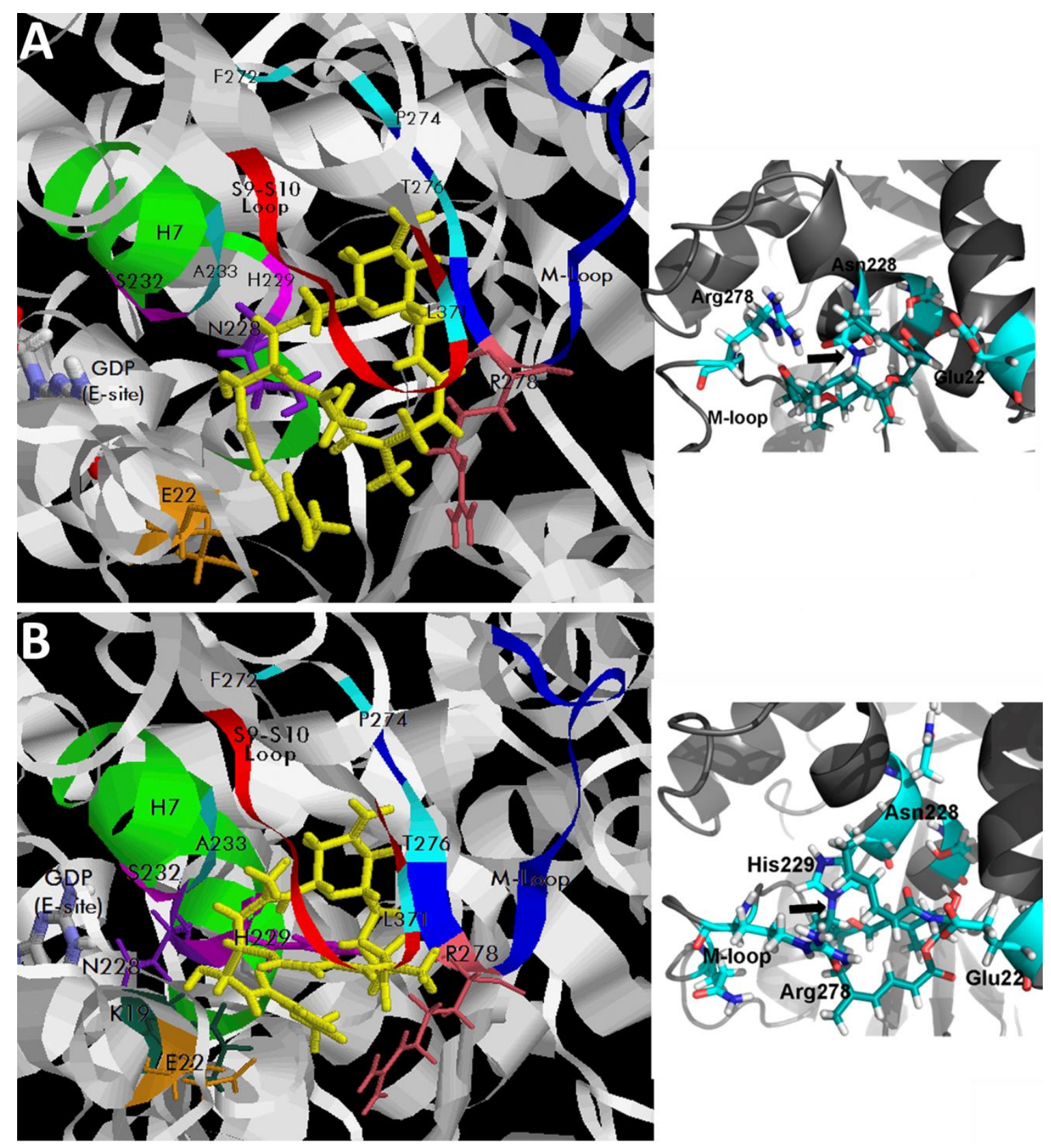

Figure 5.8 Molecular model of the ZMP-MT adduct

All interacting residues are colored differently and labeled in the left panel, the right panel images show some interacting residues and the covalent bonds are shown by the black arrows. A. ZMP (yellow left panel, CPK coloring right panel) covalently bound to Asn228 (purple) (minor product). Note in this complex the Asn228 side chain has been moved to face the taxoid binding pocket. The north face of the compound is held in place by the covalent bond, and the ZMP side chain forms two hydrogen bonds with Asn228, which forms an additional hydrogen bond with His229. The opposite side of the side chain contacts Glu22 (orange) on the lateral side of the complex. The oxygen of the ketone moiety on the eastern side of the compound strongly interacts with Arg278 (pink) within the M-loop (blue). The western side of the molecule lays over $\mathrm{H} 7$ (green), and the exo-cyclic methylene moiety at the bottom of the tetrahydropyran ring sits in a hydrophobic pocket between H7, the S9-S10 loop (red) and the M-loop formed by residues Ala233, Phe272, Pro274, Thr276 and Leu231 (cyan). B. ZMP (yellow left panel, CPK coloring right panel) covalently bound to His229 (magenta) (major product). Note, in this model the 228 side chain faces the E-site in its natural conformation. Essentially identical interactions occur as in the above model, with an additional hydrogen bond formed between the side chain and Lys19 (dark green). 


\section{Dactylolide models}

When DAC is bound to Asn228, the macrolide ring is inverted compared to that of ZMP. This is understandable since natural DAC is the enantiomer of the compound used in this study. Again the north face of the compound is held in place by the covalent interaction, and Arg278 interacts with the ester. The eastern side of the compound is in contact with the bottom of the binding site, occupying the space that the side chain occupied in the ZMP-tubulin complex. The exo-cyclic methylene moiety at C13 interacts directly with the S9-S20 loop and occupies a small pocket formed by Lys19, Glu22, Val23, Arg369 and Gly370. When covalently bound to His229, the compound is slightly displaced and closer to the M-loop, with the exocyclic methylene moiety in a hydrophobic pocket formed by Ala233, Ser236, Phe272, Pro274, Pro360 and Ser374 and resulting in an additional strong interaction with the M-loop between the C19 aldehyde and Gln281. Figure 5.9 shows the molecular modeling of bound DAC. 

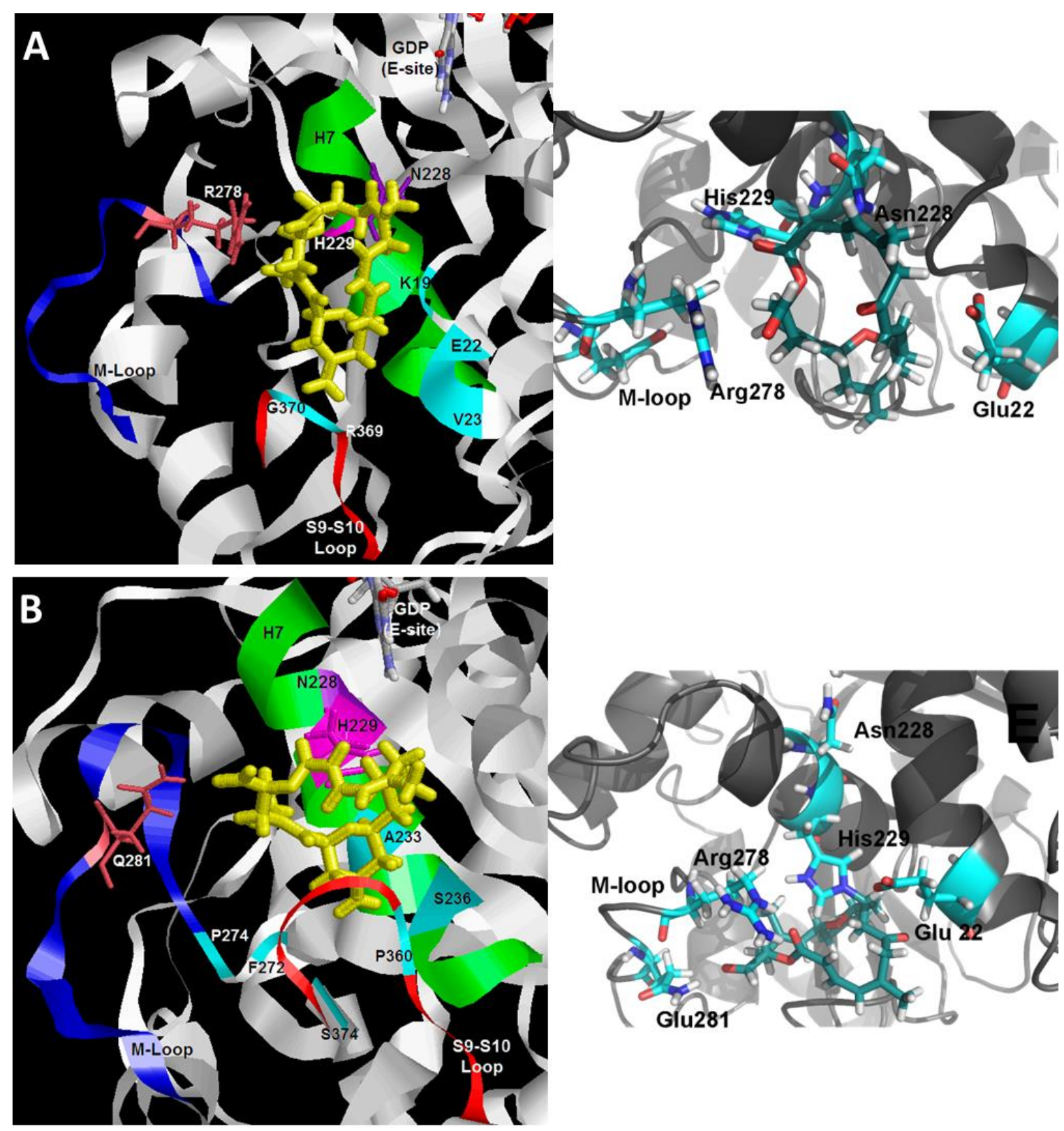

Figure 5.9 Molecular model of the DAC-MT adduct

All interacting residues are colored differently and labeled in the left panel. The right panel images show some interacting residues, and the covalent bonds are shown by the black arrows. A. DAC (yellow left panel, CPK coloring right panel) covalently bound to Asn228 (purple) (minor product). Note in this complex the Asn228 side chain has been moved to face the taxoid binding pocket, and the macrolide ring is inverted compared to that of ZMP, with the north face of the compound held in place by the covalent interaction. Arg278 (pink) interacts with the ester. The eastern side of the compound is in contact with the bottom of the binding site, occupying the space that the side chain occupied in the ZMP-tubulin complex. The exo-cyclic methylene moiety at C13 interacts directly with the S9-S20 loop (red) and occupies a small pocket formed by Lys19, Glu22, Val23, Arg369 and Gly370 (cyan). B. DAC (yellow left panel, CPK coloring right panel) covalently bound to His229 (magenta) (major product). Note, in this model the 228 side chain faces the E-site in its natural conformation. The compound is slightly displaced compared to the above complex and closer to the M-loop, with the exocyclic methylene moiety in a hydrophobic pocket formed by Ala233, Ser236, Phe272, Pro274, Pro360 and Ser374 (cyan) resulting in an additional strong interaction with the M-loop between the aldehyde and Gln281 (pink). 


\section{$5.4 \quad$ DISCUSSION}

\subsubsection{Zampanolide binds covalently to the MT}

Michael-type addition of nucleophilic tubulin residues are known to occur across $\alpha \beta$ unsaturated carbonyl systems of natural products (for example that seen in CYC; Buey et al., 2007). Given the structure of ZMP, there are three possible sites where this can occur: i) the $\alpha \beta \gamma \delta$-unsaturated lactone unit (C1-C5), ii) the C9-C11 enone moiety and iii) the $\mathrm{N}$-acyl hemiaminal side chain. In DAC, the two former sites are available for conjugation. Thus, covalent binding is not an unreasonable assumption to make.

The majority of taxoid site ligands bind endothermically and reversibly to the taxoid binding site, and their binding constants therefore decrease with temperature. However, with ZMP and DAC, the apparent binding constants increased with temperature, the opposite of what is expected for an enthalpy-driven reaction. This means that their binding reaction is not thermodynamically controlled like that of traditional MSAs, but is kinetically controlled as for any irreversible reaction. Thus, the extension of the reaction (apparent displacement) increases as temperature increases, and the reaction is limited by the rate at which the irreversible ligand binds to MTs. MS showed mass addition consistent with that of ZMP or DAC binding to a tubulin peptide involved in the taxoid binding site, confirming these compounds are ligands of this site. Both compounds alkylated $\beta$-tubulin at His229 (major product) and Asn228, showing that these compounds are covalent MSAs similar to CYC. Due to formation of the covalent bond, the ligands could not be extracted from the protein; thus, no ligand was detected by HPLC in the pellet samples. Thus, this mechanism of binding explains their biochemical properties observed in Chapter 4, and this was also confirmed in intact cells. The binding kinetics further emphasized the importance of the ZMP C19 side chain.

In the MS both the ZMP-228 adduct and the ZMP-229 adduct co-eluted, shown by a single labeled peak. However, with DAC three peaks were observed, at $73 \mathrm{~min}, 81$ min and 83 min, with that eluted at 73 min being 1 Da less and the strongest peak (Figure 5.4). This suggests three different reactions modes for DAC. The observed difference could not be due to inconsistencies in the protein sequence but is most likely due to a difference in the compound itself. This likely occurred due to the digestion process that was performed in $\mathrm{NH}_{4} \mathrm{HCO}_{3}$ buffer, as this buffer may cause the $\mathrm{C} 19$ aldehyde moiety to be partly transformed into the imine, resulting in the 1 Da mass difference in relation to the aldehyde, and accounting for the peak eluting earlier from the column. The difference 
between the two peaks eluting at 81 and 86 min may be due to interactions with different nitrogens in the His229 imidazole side chain, since it would be protonated in these conditions. These different peaks were most probably not observed for ZMP because the ZMP adduct would have a lower polarity because of its side chain, and this would increase retention time, with the adduct eluting closer to maximal ACN concentration, preventing the resolution of both peaks. Another possible explanation could be that the asparagine side chain can act as a bifunctional nucleophile that would allow two different reaction modes with DAC; however, this is the minor product and the smallest peak at 86 min only showed alkylation at residue 229. It is also feasible that in DAC, different moieties on the ligand react, either with the same site or with different sites in the protein, but with ZMP the side chain fixes the ligand in the binding site allowing only one reaction mode, and this may cause the formation of a more potent complex that gives a molecular basis to the importance of the side chain. Crystal structures of bound DAC could be used to determine where it is attaching on the protein.

Molecular modeling predicted that attachment of these residues would occur at C9 (His229) and C3 (Asn228) of the macrolide ring. The C9 enone moiety is the best Michael acceptor because of its lack of cross conjugation; thus, it is not surprising that attachment to $\mathrm{C} 9$ is the major product of the reaction. However, the actual site of attack by the protein will also depend on the most favorable binding mode of the ligand, which can lead to a large increase in the effective molarity of an alternative site with lower inherent reactivity. This is what the modeling suggests is occurring at the double bond of the conjugated lactone, which is closest to the nucleophile (Asn228) and therefore can be alkylated. This conjugated lactone is still a good acceptor for a 1,4 addition, although chemically it is not as reactive as the enone where the majority of alkylation occurs with His229. Additionally, it is possible that the C3 of the macrolide ring is not alkylated at all, and labeling of Asn228 is an artifact of the MS process. This was suggested by Prussia et al. to be the case with $\mathrm{CYC}$, and under this reasoning it cannot be ruled out here.

\subsubsection{Labeling of residue 288}

Prussia et al., (2010) argued that Asn228, with a primary amide side chain, is a poor nucleophile. However, it is also possible that the amide side chain is interacting as a nucleophile, since catalytic conjugate addition by weak $\mathrm{N}$-centered nucleophiles is not 
uncommon (Lin et al., 2007). When modeled in the taxane binding site, CYC is unlikely to interact with Asn228, given that its side chain faces away from the taxane binding pocket into the E-site where it interacts with the bound nucleotide via a bifurcated hydrogen bond. This was seen with the molecular modeling in this study, in which the side chain of Asn228 was orientated into the taxoid pocket for the modeling. Prussia et $a l$. , suggested that the labeling of Asn228 was an artifact of MS and proposed His229 as the actual site of CYC covalent attachment, in addition to Thr220. During the ionization process for MS, CYC transfers to the actual labeled residue Asn228. The shift would be driven by steric hindrance with the imidazole attachment to histidine compared to the primary amide attachment to Asn228. Rearrangement of peptides during the fragmentation process of MS is not uncommon due to the conditions of the process (Prussia et al., 2010). Binding to this amino acid in the taxoid site would influence the M-loop conformation, promoting assembly and favoring interactions between neighboring protofilaments. CYC, however, is a weak stabilizer and shows little interaction with the M-loop compared with other MSAs (Edler et al., 2005; Buey et al., 2007); therefore, it seems unlikely it would interact with residue His229 in the taxoid binding site.

Additionally, this work has shown that His229 is the major site in which ZMP alkylation occurs, if steric hindrance was an issue in attachment to this residue, then more labeling would be expected with Asn228 which is only the minor product. Prussia et al., suggested that the poor stabilizing activity of CYC stemmed from its interaction with Thr220 in dimeric tubulin, an interaction that would not lead to a polymerization effect, and suggested from molecular simulations that the M-loop blocks access to the taxoid site residues Asn228 and His229 in tubulin dimers. The present study has shown that this is unlikely since both ZMP and DAC were able to bind to the taxoid site at His229 in tubulin dimers. Taking Prussia et al. into perspective, it is possible that ZMP itself does not label residue 228 and it is in fact an artifact of the tandem MS. This seems likely since it is only a minor product of the reaction, with His 229 being the major product. As suggested by Prussia et al., it is possible that labeling at 229 may cause some steric hindrance that leads to some compound moving over to residue 228.

Additionally, the crystal structure of bound ZMP only shows labeling of His229 (Chapter 6), further supporting the conclusion that labeling of Asn228 is an artifact. However, since His229 is the major product shown by the MS it is not surprising that the 
majority of crystals formed were labeled at this residue, and thus labeling at Asn228 cannot be completely ruled out.

If available, it would be interesting to assess the cytotoxic activity of ZMP in cells with either Asn288 or His229 mutated. Residue 228 is involved in nucleotide binding in the E-site, and it is likely cells with a mutation at this site would be nonviable. A mutation in His229 would still allow a covalent bond to form with residue 228, if of course labeling of this residue is true and not an artifact. Isolation and MS of the cellular tubulin in these cells would provide information on which residue is covalently modified and would provide insight as to whether or not Asn228 can act as a suitable nucleophile for MSA attachment (Prussia et al., 2010). There are also other residues of the taxoid site that may have the ability to react as nucleophiles if His229 is not available for ZMP binding, such as Cys241 for example which is labeled by CYC analogs (Calvo et al., 2012), and it is well known that the thiols in the side chain of cysteine residues act as strong nucleophiles.

\subsubsection{Taxoid site and the pore type I site are mutually exclusive}

With the covalent mechanism of action of ZMP validated, it could now be biochemically verified that binding at the pore site and the taxoid site are mutually exclusive. It was proposed that binding at either site excludes binding at the other, and that the two sites involved a switching element (Magnani et al., 2009). ZMP covalently binds exclusively to the taxoid site, with 1:1 stoichiometry, as does HXF to the pore type I site. The absolute lack of interaction of HXF with MTs when ZMP was covalently bound, as shown in Figure 4.12, indicates that binding at the luminal taxoid site is highly specific and alters or blocks subsequent binding at the pore type I site, thus preventing simultaneous binding at both sites. This is most likely due to the fact that both sites utilize the H6-H7 loop (Díaz et al., 2003; Magnani et al., 2009). As mentioned in Chapter 4, the Prussia et al. paper argued against the pore site being a binding site for MSAs and concluded that it was nothing more than a 'funnel' for diffusion into the luminal binding site and that the observed kinetics were an artifact of a pause of the ligand in the pore. It is suggested that CYC only labels Thr220 due to the exposure and proximity of the residue in the pore, given that there are other exposed nucleophiles in tubulin. However, much of the evidence in the literature supports the pore type I site as a binding site for compounds entering the luminal site when binding MTs, and this work 
has suggested that compounds interact with the luminal site in dimeric tubulin rather than the pore site. This will be discussed in the section below.

\subsubsection{The luminal binding site exists in dimeric tubulin}

As discussed earlier in this chapter, it is evident that strong MSAs are involved in the nucleation step of MT-assembly, and this would occur by binding to unassembled tubulin with low affinity. The mechanism of nucleation would involve either inducing a conformational change that activates the tubulin dimer (allosteric model) or joining two subunits to produce a high affinity binding site from two low affinity sites (matchmaker model). Direct evidence of binding to unassembled tubulin at the pore site has been observed by MS-techniques employing CYC which only labels dimeric tubulin at pore site residue Thr220 (Buey et al., 2007). NMR and biochemical studies have confirmed that DTX and DSC bind with low affinity to unassembled tubulin (Canales et al., 2011), and other NMR studies have provided evidence of a low-affinity binding site for MSAs in a nonhomogeneous preparation of tubulin (Carlomagno et al., 2003; Sanchez-Pedregal et al., 2006). However, with these studies it was not possible to know if the binding site of MSAs in unassembled tubulin was to the pore site, indicating that the nucleation mechanism of MSAs is of the matchmaker type, or to the luminal site, indicating that the nucleation mechanism was of the allosteric type (Díaz et al., 1993; Sanchez-Pedregal \& Griesinger, 2009). The results of the present study give the first biochemical evidence that proves the luminal binding site exists in unassembled tubulin, since both ZMP and DAC label unassembled tubulin at Asn228 or His229. Thus, as previously proposed (Sanchez-Pedregal \& Griesinger, 2009; Canales et al., 2011), MSAs bind to the luminal site in unassembled tubulin and not only to the pore site, as suggested by the CYC results (Buey et al., 2007). In this way, nucleation of MTs may possibly proceed by both alternative mechanisms (matchmaking and allosteric) as previously discussed (Díaz et al., 1993). It is likely that, in general, MSAs bind to the luminal binding site in unassembled tubulin and assemble MTs via an allosteric mechanism. In the case of an MSA binding to MTs rather than dimeric tubulin, it is likely that it binds the pore site, since the luminal site would be blocked from direct access, and then moves into the taxoid binding site. The exception here is CYC which only binds the pore site in dimeric tubulin (Buey et al., 2007). 


\subsubsection{Binding kinetics}

It was evident that ZMP and DAC have different binding kinetics since in the preliminary assays, ZMP bound irreversibly in <30 min; whereas, the DAC covalent reaction took approximately $4 \mathrm{~h}$ to complete. The initial binding step with ZMP occurs much faster than for DAC, indicating that the covalent reaction immediately follows the noncovalent interaction. Since the irreversible reaction of DAC with tubulin is slow $(k=$ $\left.0.12 \mathrm{hr}^{-1}\right)$ compared with the reversible reaction $\left(\mathrm{k}=1 \mathrm{hr}^{-1}\right)$, the TRNOESY and STD signals obtained (Figure 4.13) presumably arise from DAC not yet covalently bound to the MT or dimer. The intensity of DAC signals in the NMR spectra slowly decreased with time at a kinetic rate similar to that determined for the irreversible reaction $(k=0.12$ $\mathrm{hr}^{-1}$ ), as expected when the ligand reacts with tubulin, indicating blocking of the binding site. This is indicative of covalent binding since DAC is no longer dissociating, and thus less of an STD signal is seen. This was not observed for DCX or DSC binding to MTs or tubulin dimers (Canales et al., 2011), since these MSAs bind reversibly and therefore always have an on/off dissociation rate.

The kinetic rate measured by HPLC $\left(\mathrm{k}=1 \mathrm{hr}^{-1}\right)$ is much higher than when the constant is measured by FTX-2 binding $\left(\mathrm{k}=0.12 \mathrm{hr}^{-1}\right)$, since two different parameters are being measured with the different kinetic assays, as discussed. In the FTX-2 case only sites that are covalently bound are measured since the noncovalently bound compound is rapidly displaced by the excess FTX-2, implying that it is in fast equilibrium. The existence of a difference between these two measurements implies that the noncovalently bound compound that binds tubulin is in fast exchange with the non-bound (free) compound, and this rapid exchange is essential for observing the TR-NOESY signal, in order to be able to determine the pre-reaction complex. This difference does not exist in the case ZMP since it binds rapidly in a covalent manner, thus it would not be possible to observe TR-NOESY signals from ZMP.

The difference in binding time between the two compounds may occur in three possible ways. First, placement of the electrophile in relation to the nucleophile may occur sooner with ZMP than with DAC. Second, the electrophile in ZMP may have a stronger affinity than the electrophile in DAC. It is known that ZMP covalently binds at C9 to His229 (crystal structure), and although this is mostly likely the case for DAC, binding at another electrophile cannot be ruled out. Lastly, the ZMP adduct may have 
more favorable kinetics, leading to a faster reaction that most likely involves the side chain of ZMP.

\subsubsection{Structural basis for the ZMP MT-stabilizing effect}

In order to understand the stabilization effect of ZMP and DAC, molecular models were made of the compounds bound in the taxoid binding pocket (Figure 5.8 and 5.9). The models explain the strong stabilizing activity observed with ZMP. With traditional MSAs, taxanes and EPOs, the ligand binds tighter to the MT than to unassembled tubulin (Díaz et al., 1993; Canales et al., 2011). Therefore, the higher free energy of binding to the MT over the dimer would shift the equilibrium toward the MTs; independent of the structural effect on the MT. Covalent binders are missing this property and the equilibrium is displaced through a structural allosteric effect. Thus, the modified tubulin would have higher affinity for the MT than the unmodified tubulin. With ligands that bind the taxoid site, the strong stabilization of the M-loop is the main structural MTstabilizing feature that occurs after taxane binding. A similar mechanism is proposed for the strong MSA activity observed with ZMP. This has recently been shown by crystallography which will be discussed in Chapter 6.4. With CYC, a much weaker covalent MSA, M-loop interaction does not occur to the same extent since the compound interacts with the pore in dimeric tubulin. The DAC model suggests that the compound interacts with the M-loop at residue Gln281, despite this DAC is a significantly weaker inducer of MT assembly, as evident by the biochemical assays. This suggests that the ZMP side chain should have a stronger interaction with the M-loop than the models are suggesting, or at least have a greater effect on stabilization of the M-loop (i.e. hydrogen bonding interactions) and should result in its significantly greater potency in the in vitro assays. The M-loop additionally has to be involved in the covalent reaction since ZMP binds significantly faster than DAC, but also since the labeled residues are in $\mathrm{H} 7$ and not the M-loop, thus, the loop must be involved in positioning of the two reactive centers.

The structural implications of binding will be discussed in Chapter 6.4.

\subsubsection{Zampanolide overcomes resistance}

As seen with CYC, the covalent mechanism of action of ZMP and DAC means they can avoid P-gp drug efflux pumps and likely other mechanisms of resistance. It has already been shown that ZMP is fully active in cells with the MDR phenotype (Field et al., 
2009). Although covalent binding is clearly the mechanism in which ZMP is able to evade these up-regulated pumps, the possibility that ZMP and DAC are poor substrates for the pump cannot be excluded. In fact, preliminary studies have shown that DAC is not a substrate for the P-gp drug efflux pump (Prof. Karl-Heinz Altmann, personal communication). Covalent binding would however prevent these compounds from being susceptible to any cellular pumps, not just the P-gp, and this is important since a number of efflux pumps are up-regulated in different cancers (Gottesman et al., 2002). In Chapter 3 it was shown that cell lines with taxoid site mutations have significantly decreased resistance to ZMP compared to other taxoid site MSAs. Furthermore, ZMP was fully active in L4 cells which have up-regulated $\beta$ II and $\beta$ III tubulin (Kanakkanthara et al., 2011). Since the formation of the ZMP-tubulin adduct is a kinetically controlled and irreversible process, even if ZMP has lower affinity for the modified mutant tubulin in the resistant cells, they cannot escape from the effect of ZMP, nor can they escape by upregulation of efflux pumps. Additionally, it is unlikely that cells treated with ZMP over long periods of time would develop mutations since covalent binders have a sustained duration of inhibition, since the drug is permanently attached to the target (Singh et al., 2011). Thus, design of covalent MSAs provides a means of evading cancer cell resistance.

\subsubsection{Conclusions}

In this chapter the mechanism of binding and the binding kinetics of ZMP and DAC were determined. Both compounds covalently modified the taxoid binding site at either Asn228 or His229, the later residue being the major site of modification. ZMP irreversibly reacted with tubulin in less than $30 \mathrm{~min}$; whereas, the irreversible DAC reaction took $4 \mathrm{~h}$, indicating that the STD signals for DAC binding are from the noncovalent reaction. ZMP covalently modifies cellular tubulin and permanently arrests cells in mitosis, indicating this binding mechanism holds true in cells as well as in the test tube. Covalent binding means ZMP is not affected by P-gp drug efflux pumps and is more cytotoxic in mutant cell lines compared to other MSAs. Thus, design of new generation MSAs that bind in this manner would potentially evade cancer cell resistance. Further investigation into the limitations of this binding mechanism, however, needs to be made for safety concerns involved with covalent drugs in the clinic. 


\subsection{INTRODUCTION}

\subsubsection{Microtubule formation and tubulin aggregation}

From a thermodynamic point of view, MSAs and MDAs are described as molecules capable of changing the ratio between assembled MTs and unassembled MTs. They do this by binding preferentially to either tubulin polymers or tubulin dimers. Ligands binding preferentially to dimers inhibit assembly into MTs, ligands binding preferentially to MTs prevent disassembly (Díaz et al., 2009). This is due to thermodynamic linkage and is independent of the binding site and changes in structural dynamics of both the ligand and the protein upon binding. New information gained in the course of this thesis has provided more insight into these mechanisms, and this will be reviewed in the discussion of this chapter. A number of models have been proposed to describe the structural mechanisms of assembly of tubulin into MTs. The most simple is that of oneby-one addition of dimers to the growing end of a MT or a MT nucleus in a head-to-tail fashion (VanBuren et al., 2005), described in Figure 6.1. $\mathrm{Mg}^{2+}$ is required for the selfassembly of MTs and for the stability of the protein. $\mathrm{Mg}^{2+}$ is also tightly linked to the binding of GTP at the E-site, having a significant influence on the interaction of the nucleotide with tubulin (Correia et al., 1987). Occupation of the E-site is also an important factor in the aggregation of tubulin. A number of different structural intermediates can form in the process of tubulin aggregation and MT assembly (Figure 6.1). A number of these intermediates also form when an MSA is present (Díaz et al., 1996). In low concentrations of $\mathrm{Mg}^{2+}$, GDP-bound dimers remain as dimers, even in the presence and absence of taxoid ligand. When the concentration of $\mathrm{Mg}^{2+}$ is increased, GDP-bound dimers can arrange into double rings (Howard \& Timasheff, 1986), or in the presence of taxoid ligands into ligand-induced MTs (Díaz et al., 1996) (Figure 6.1). The formation of double rings can block the formation of MTs (Díaz et al., 1996) and depolymerizing MTs result in curling protofilaments that can also form double rings. The most common ring structure comprises an inner ring of 12 tubulin dimers, surrounded by a 16 tubulin dimer outer ring (Nogales et al., 2003) (Figure 6.1).

In the presence of the MT regulatory protein stathmin, tubulin forms a tight ternary complex with an elongated shape, termed $T_{2} R$. Proteins with stathmin-like domains (SLDs) are able to sequester two tubulin heterodimers that arrange head-to-tail 
(Figure 6.2). This complex cannot polymerize into MTs, nor form double rings, and is formed under a number of conditions (Jourdain et al., 1997). Many vertebrate proteins contain highly conserved SLDs. For example, the neural protein RB3 is a stathmin family protein, with an SLD behaving in the same manner as stathmin to form the $T_{2} R$ complex. This complex has been crystallized (Gigant et al., 2000) a number of times and is an excellent tool for studying ligand-protein interactions. It has been used to determine the structure of tubulin dimers (Barbier et al., 2010), the location of both the colchicine binding site (Ravelli et al., 2004) and the Vinca domain (Gigant et al., 2005). Tubulin dimers, aggregates and MTs are often modified upon binding of different MTAs. Currently there are many different binding sites on the tubulin dimer or MT where MAPs, MSAs, MDAs, and nucleotides bind. Tubulin also has intrinsic GTPase activity. The effect of a ligand binding at any one of these sites can often affect the properties of the protein. Aspects of this will be discussed in this Chapter. 

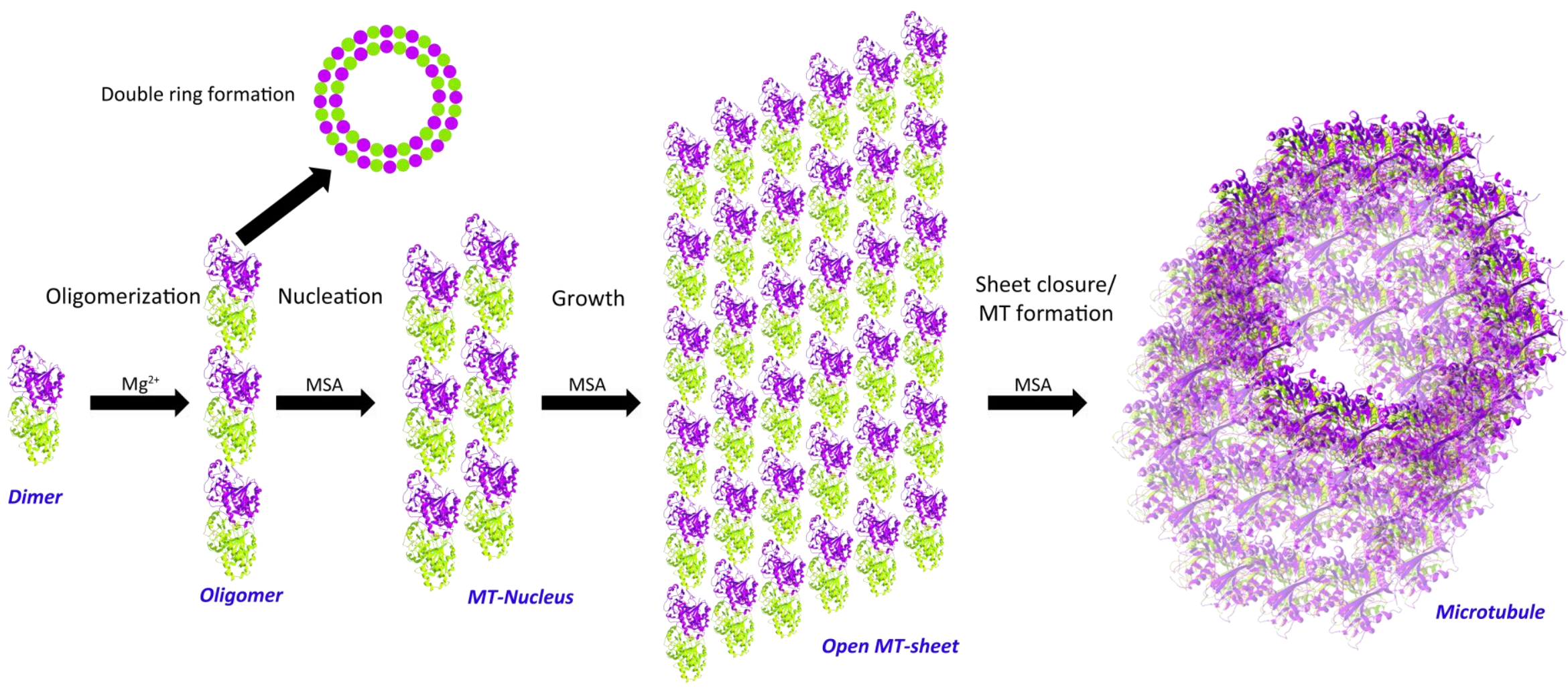

Figure 6.1 MSA induced MT-assembly from GDP bound tubulin. (Modified from Matesanz et al., 2011a)

In the presence of $\mathrm{Mg}^{2+}$, tubulin GDP-bound dimers can self-associate into double rings that consist of 16 subunits in the outer ring and 12 subunits in the inner ring. GDP-bound tubulin cannot assemble into MTs unless an MSA is present. The first structural step of MSA-induced MT assembly involves oligomerization of tubulin dimers in a "head-to-tail" fashion to form protofilaments. These protofilaments then organize into a sheet structure due to lateral contact association between subunits, and this process is assisted by the MSA. This sheet structure then takes on cylindrical morphology and forms a MT. The interprotofilament angle and number of protofilaments varies depending on the MSA the MT was assembled with. 


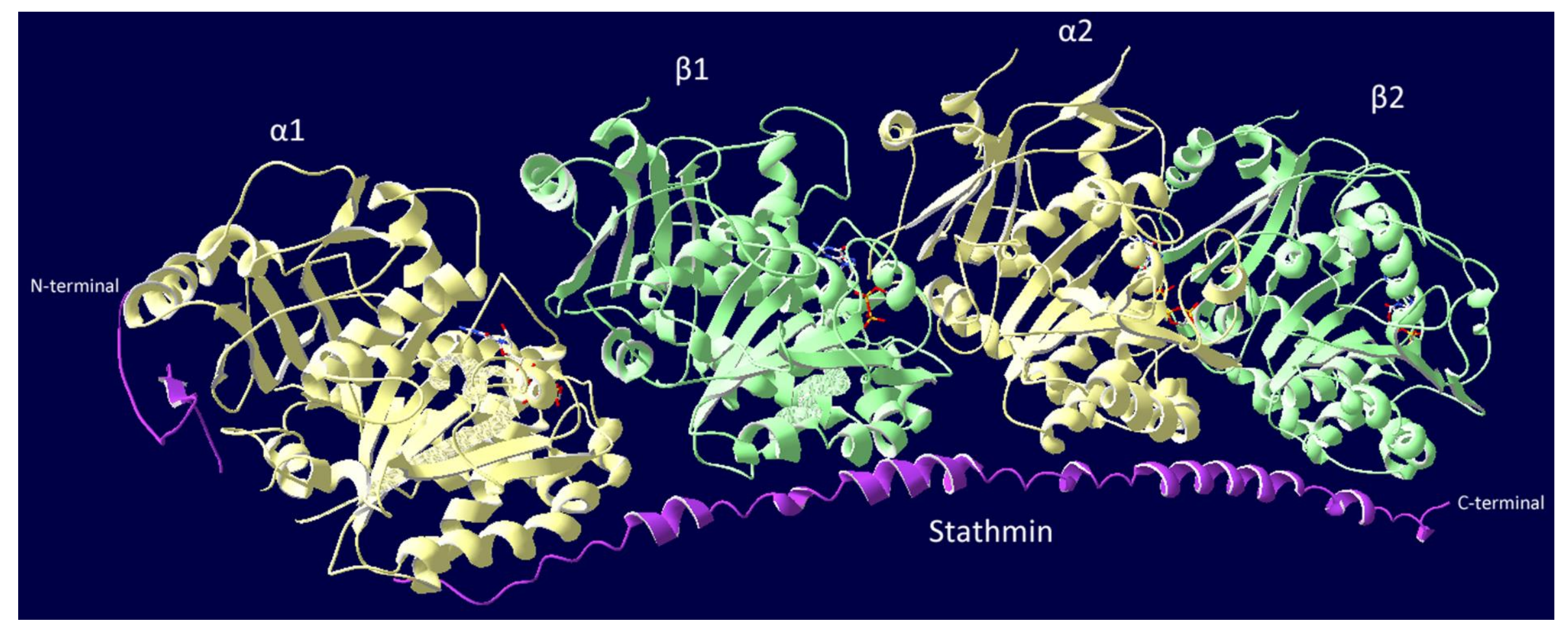

Figure 6.2 Structure of the $T_{2} R$ complex

Ternary $\mathrm{T}_{2} \mathrm{R}$ complex formed by the MT regulatory protein stathmin (magenta) and two $\alpha \beta$ tubulin heterodimers. Stathmin takes on a 'hook-like' shape incasing the two heterodimers with its $\mathrm{N}$-terminal end wrapping around the $\mathrm{C}$-terminal of the $\alpha 1$ subunit. This complex is highly stable and does not further polymerize nor aggregate into tubulin rings. Protein data bank coordinates used: 1SA0 (Ravelli et al., 2004). 


\subsubsection{GTP-bound dimers versus GDP-bound dimers}

GTP-bound dimers are able to form protofilaments; whereas, GDP dimers are inactive. In a tubulin dimer the $\alpha$-subunit $\mathrm{N}$-terminal is in contact with the $\beta$-subunit $\mathrm{C}$-terminal, and this constantly blocks the N-site, leaving the nucleotide non-hydrolysable and nonexchangeable. The $\beta$-tubulin E-site, however, is partially exposed on the dimer and free to exchange nucleotide; whereas, in MTs the GTP is hydrolysable but not exchangeable (Nogales et al., 1998b). The site of GTP hydrolysis is located in the $\beta$-subunit and is completed by head to tail contact with the residues in an $\alpha$-subunit of a neighboring heterodimer (Nogales et al., 1998b). When tubulin dimers form longitudinal contacts, the $\mathrm{N}$-terminal of a $\beta$-subunit contacts the $\mathrm{C}$-terminus of an $\alpha$-subunit in another heterodimer (Kirchner \& Mandelkow, 1985), resulting in blockage of the E-site. The nucleotide can no longer exchange even though GDP occupation at this site in the lattice is thermodynamically unstable. Hydrolysis of GTP to GDP is not required for the assembly of the MT but is required for the MT's dynamic instability as it controls the disassembly rate of the MT. Hydrolysis results in the MT being energetically unstable (Hyman et al., 1992).

\subsubsection{Allosteric or lattice model of tubulin straightening}

Until recently there were only two atomic structures of $\alpha, \beta$-tubulin known. That of straight antiparallel protofilaments of zinc sheets stabilized by PTX, with GTP bound in the E-site (Nogales et al., 1998a; Löwe et al., 2001). This structure was used to build a model of parallel protofilaments from electron density maps (Li et al., 2002). Secondly, that of the $\mathrm{T}_{2} \mathrm{R}$ complex which is curved (Gigant et al., 2000; Ravelli et al., 2004; Dorléans et al., 2009). The structure of MTs is more closely related to the straight structure modeled by the zinc sheets, although there would be discrepancies in lateral contacts compared to tubular MT structures. Structural differences between GDP-bound versus GTP-bound dimers were unknown until recently (Barbier et al., 2010).

It was originally hypothesized that GDP-dimers had a curved conformation; whereas, GTP-dimers had a straight conformation. This straight conformation was thought to be the factor that allowed GTP-dimers to assemble into MTs, and why GDPdimers in a curved conformation are prone to disassembly. This mechanism was termed the "allosteric mechanism" and involved GTP binding causing a conformational change 
resulting in straight dimers that allow lateral contacts to occur, followed by sheet closure into a MT structure (Wang \& Nogales, 2005; Nogales \& Wang, 2006).

However growing evidence has challenged this model. For example, it is known that GTP-dimers take on a GDP-dimer-like curved conformation in the absence of $\mathrm{Mg}^{2+}$ (Shearwin et al., 1994). This led to a new model proposed by Buey et al., (2006) called the lattice model which describes GTP-tubulin as being curved when free, similar to GDP-dimers, and driven to a straight conformation when lateral contacts form in the MT lattice. The $\gamma$-phosphate only lowers the free energy between the two different nucleotide dimer states (Buey et al., 2006), meaning GTP bound dimers have enhanced tendency to elongate protofilaments (Nawrotek et al., 2011). All dimers, irrespective of nucleotide bound, are in a 'MT incompatible' state, or inactive curved state, and are driven to the straight conformation as a consequence of lateral contacts made during assembly rather than a straight conformation being the cause of MT assembly (Buey et al., 2006). Further support for this model came from a number of independent findings, for example, that GTP-dimers have less curvature than GDP-dimers (Gebremichael et al., 2008).

Recently, it was found that COL analogs bind equally well to tubulin dimers as they do to $T_{2} R$ complexes (Barbier et al., 2010). These results favor the lattice model that unassembled tubulin is in a curved conformation, which switches to the straight form upon MT assembly. More recently the first atomic model of GTP-bound to the $T_{2} R$ complex was solved (Nawrotek et al., 2011). In this model the C-terminal tails of each of the subunits were cleaved by subtilisin $\left(\mathrm{sT}_{2} \mathrm{R}\right)$ but had extremely similar structure to the $\mathrm{T}_{2} \mathrm{R}$ complex. This structure demonstrated that GTP-dimers and GDP-dimers differ only in the zone of the E-site domain. In the case of GTP-bound tubulin, the $\gamma$-phosphate hydrogen bonds with Asn101 in the T3 loop (Figure 4.1); whereas, in GDP-dimers the $\beta$ phosphate interacts with the T3 loop via water-mediated interactions. In GTP-tubulin the hydrogen bond results in displacement of Asn101, resulting in close contact with Thr180 in the T5 loop, if GTP-tubulin was to take on the same conformation as GDP-tubulin. To avoid this, T5 moves outward in GTP-bound tubulin as a consequence of the T3 movement. Thus, changes in the structures are limited to the Asn101 (T3) and T5 loops at the nucleotide binding site. In addition, given that the E-site is a major contributor to longitudinal contacts in the MT, and the observation that changes in structure are restricted to this site, it can be suggested that GTP enhances the tendency of tubulin to form elongated protofilaments. This is confirmed by the T5 loop movement as it allows 
for more interactions between other tubulin heterodimers and favors dimer recruitment to MT ends and facilitates the curved-to-straight change (Nawrotek et al., 2011).

Taken together, all the structural and biochemical data support the lattice model. The dimers only differ in their flexibility to adopt a straight conformation, and this is driven by the lateral and longitudinal contacts occurring upon polymer assembly (Buey et al., 2006). The curved structure of the $T_{2} R$ complex is therefore a representative conformation of unassembled free tubulin (Ayaz et al., 2012; Pecqueur et al., 2012).

\subsubsection{Colchicine site}

Interestingly, the tubulin protein was first identified as the "colchicine binding protein" and was isolated from mammalian brain using a COL affinity assay (Weisenberg et al., 1968). COL binds to tubulin in a two-step mechanism, as discussed. Although this mechanism of binding is non-covalent, it is considered "essentially irreversible", and the COL-protein complex is highly stable (Hastie, 1991). COL binds to tubulin at a location where it is able to inhibit curved tubulin taking on a straight structure and therefore inhibits assembly of MTs (Dorléans et al., 2009). Using the $\mathrm{T}_{2} \mathrm{R}$ complex, the COL binding site was determined to be in a deep pocket in the $\beta$-tubulin subunit at the intradimer longitudinal interface with the $\alpha$-subunit in the heterodimer (Ravelli et al., 2004) (Figure 6.3). Overlay of tubulin structures (non-bound versus COL-bound) indicated that there was a significant structural change in the COL-structure. Movement of the T7 loop away from the S8-S9 loop, opens a pocket in which COL binds (Massarotti et al., 2012). The COL binding site is considered 'inherently flexible', adjusting in shape upon the binding of different ligands, thus accommodating the binding of many diverse structures (Chakraborti et al., 2012). Additionally, COL is able to increase GTP hydrolysis, (David-Pfeuty et al., 1979).

COL and a number of its analogs are non-fluorescent when free in solution but emit strong fluorescent signals upon binding to tubulin (Bhattacharyya \& Wolff, 1984; Bhattacharyya et al., 2008). The fluorescent moiety of COL is the tropolone (C) ring, and the compound is excited at $350 \mathrm{~nm}$ with emission fluorescence observed at $425 \mathrm{~nm}$ (Bhattacharyya et al., 2010). One such analog is MTC. MTC is a synthetic bicyclic derivative of $\mathrm{COL}$ that lacks the $\mathrm{B}$ ring, with the $\mathrm{A}$ and $\mathrm{C}$ rings joined by a carboncarbon single bond (Figure 1.3) (Fitzgerald, 1976). MTC binds fast and reversibly to the COL site (Andreu et al., 1984), in contrast to COL which binds in an essentially 
'irreversible' manner (Hastie, 1991). Upon binding to tubulin, MTC undergoes a large change in fluorescence, emitting a strong fluorescence signal. MTC rings are more coplanar when bound than when the compound is free in solution, and this is what causes the increase in fluorescence upon binding. Fluorescence is an excellent probe because even extremely small changes in binding can cause significant changes in fluorescence intensity. Since the COL-tubulin complex is stable and dissociates only very slowly, the measurement of COL binding site equilibrium interactions is difficult, making MTC an excellent reference ligand for studying the COL binding site (Medrano et al., 1991; Andreu et al., 1998; La Regina et al., 2007; Barbier et al., 2010).

\subsubsection{Vinca Domain}

The Vinca domain is located at the $\alpha \beta$ interdimer interface (between two heterodimers). Binding at this site inhibits the assembly of tubulin as it causes a so called 'wedge' between the two heterodimers. Vinca agents are "interfacial" inhibitors that longitudinally crosslink two heterodimers at the interdimer interface, essentially acting to blockade the formation of the interface required for polymerization. This site was originally identified using the $\mathrm{T}_{2} \mathrm{R}$ complex, similar to the way in which the COL site was determined. Figure 6.4 shows the Vinca domain. The site is made up of the T7 loop, H10 and S9 in the $\alpha$-subunit and H6, loops T5 and H6-H7 in the $\beta$-subunit (Gigant et al., 2005). Vinca site compounds inhibit the intrinsic GTPase activity of tubulin along with nucleotide exchange, and are even able to inhibit COL-induced GTPase activity (Hamel, 1992; Cormier et al., 2010). Since the Vinca domain is only complete when two heterodimers are associated, binding at this site promotes tubulin aggregation and causes stabilization of tubulin interactions to form curved or spiral assemblies, thus, preventing the straight conformation of tubulin (Gigant et al., 2005; Lobert et al., 2007; Gigant et $a l ., 2009)$. This means that the study of Vinca domain compounds and their interaction with tubulin is relatively more difficult than for other MTAs. Although now there are a number of methods to study these agents (Lobert \& Correia, 2007), using the $T_{2} R$ complex appears to be one of the most informative (Cormier et al., 2010).

An interesting compound of the Vinca domain is cryptophycin-52 (Figure 1.2), a

totally synthetic depsipeptide from the cryptophycin family, and an extremely potent MDA (Panda et al., 1998). It is known to bind rapidly to a high affinity binding site in an entropy-driven reaction, and this binding site was later shown to be the Vinca domain by 
competition experiments. Cryptophycin-52 is a poorly reversible binder of tubulin; however, the binding is not covalent as it can be dissociated from the dimer by protein denaturing. It inhibits COL-induced GTPase activity, possibly by inducing a conformational change in the protein. As discussed the poor susceptibility of cyrptophycin-52 for the P-gp drug efflux pump is most likely due to its nearly irreversibly binding profile, meaning the cells would retain the compound (Panda et al., 2000). Although cryptophycin-52 has been deemed too toxic for clinical use, its mechanism of action has offered insight into how an MDA interacts with tubulin and has provided the first evidence of covalent-like MTAs as tools for evasion of resistance due to P-gp drug efflux. 


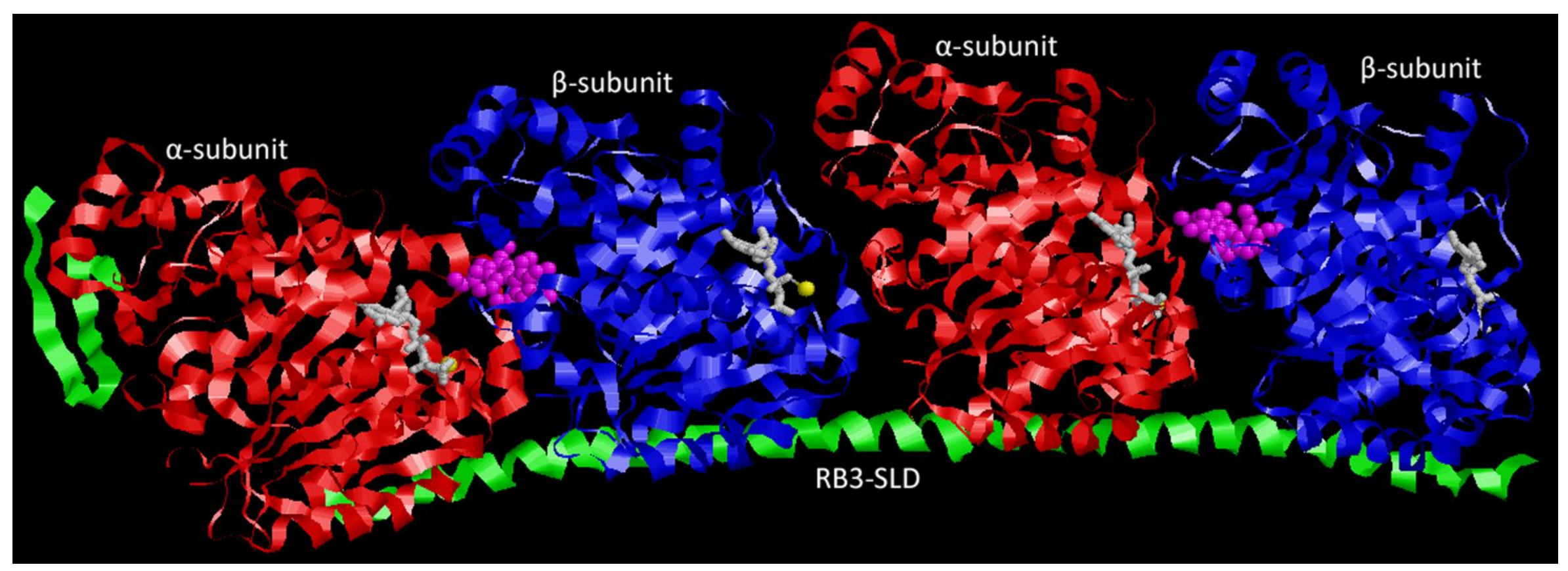

Figure 6.3 Colchicine binding site

$\mathrm{T}_{2} \mathrm{R}$ complex showing the COL binding site located on the $\beta$-tubulin subunit at the intradimer interface. COL is shown in magenta, RB3-SLD in green and the tubulin heterodimers in red and blue, $\alpha$ and $\beta$, respectively. Nucleotides are shown in grey, with the $\mathrm{Mg}^{2+}$ ion in yellow. The site is in the intermediate domain and involves S8, S9, loop T7 and H7 and H8. The compound also interacts with the T5 loop of the $\alpha$-monomer in the same heterodimer. Protein data bank coordinates used: 1SA0 (Ravelli et al., 2004). 


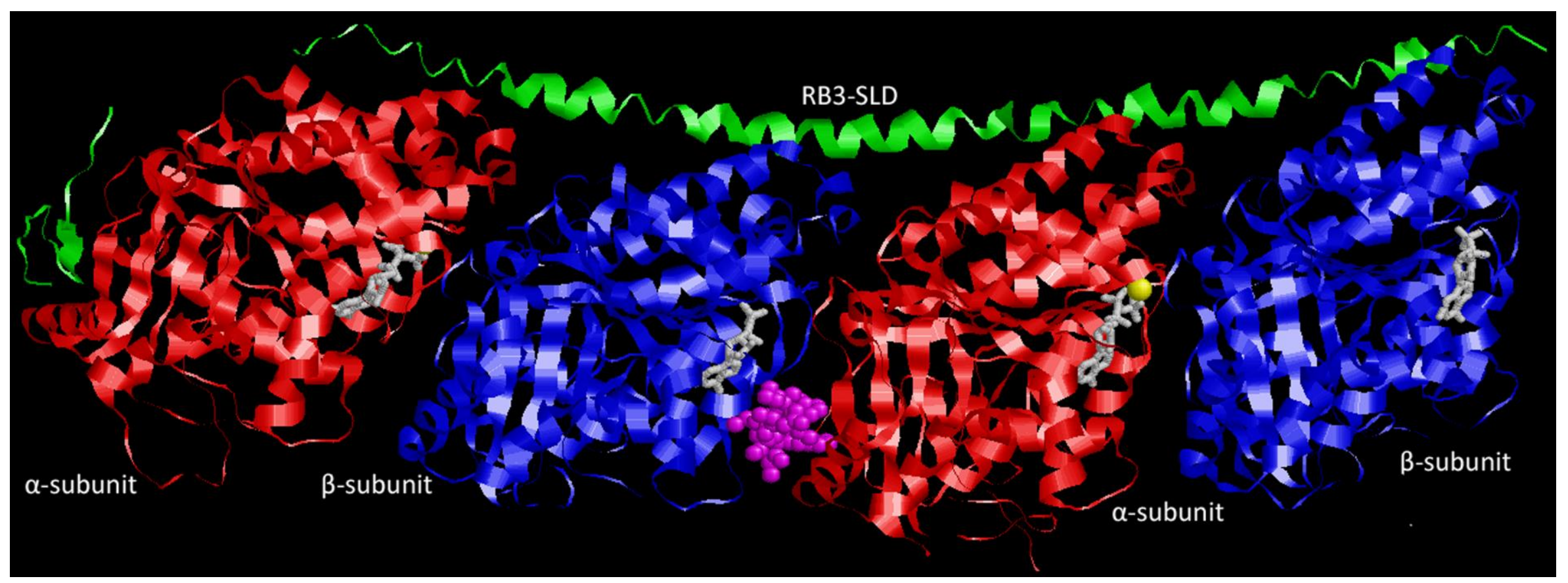

Figure 6.4 Vinca binding domain.

$\mathrm{T}_{2} \mathrm{R}$ complex showing the Vinca domain located at the interdimer interface between neighboring heterodimers. VBL is shown in magenta, RB3-SLD in green and the tubulin heterodimers in red and blue, $\alpha$ and $\beta$, respectively. Nucleotides are shown in grey, with the $\mathrm{Mg}^{2+}$ ion in yellow. The location of the Vinca domain givens visual evidence as to how VBL-induced tubulin aggregation and spiral aggregates form and how binding at this site inhibits MT assembly. Protein data bank coordinates used: 1Z2B (Gigant et al., 2005) 


\subsubsection{MSA binding determines MT size}

SAXS (Chapter 2.11) is used to study MSA effects on the diameter and number of protofilaments making up a MT lattice. In vivo MTs generally consist of 13 protofilaments, but this number can vary depending on the experimental conditions in which the MTs are formed (Chrétien et al., 1992), and the presence or absence of different MSAs. PTX reduces the mean protofilament number and MT lattice diameter (Andreu et al., 1992); whereas, DTX has no effect on the number of protofilaments (Andreu et al., 1994). Both FTX-1 and FTX-2 increase the MT diameter (Díaz et al., 2000; Matesanz et al., 2011a). MSA interactions at the taxoid site occur close to the interprotofilament region of the MT and thus dictate the number of protofilaments making up the MT. These fluctuations in protofilament number suggest differences in the lateral association between heterodimers in the MT wall. This suggests that different compounds can alter the way in which protofilaments laterally interact, changing the number of protofilaments in a MT and the interprotofilament angle (Matesanz et al., 2011a). Modification of the PTX core at C7 and C10 results in conformational changes in key secondary structures of the MT involved in lateral interactions, specifically the Mloop, S3, and H3 (Figure 4.1). Additionally, C2 modification of PTX causes changes in the interaction of C7 with the M-loop (Matesanz et al., 2011a). These structures are known to be involved in regulating contacts between neighbouring protofilaments (Nogales et al., 1999; Sui \& Downing, 2010). Since changes in protofilament number occur within a time scale of less than one minute, it is postulated that MSA-induced assembly modifies the interprotofilament angle, thus changing the number of protofilaments (Matesanz et al., 2011a). The interprotofilament angle is defined as the angle between protofilaments in the cylindrical cross-section) (Figure 6.5B). Thus, depending on the MSA bound or the conditions in which the MT is assembled, the MT will have a specific interprotofilament angle. Using SAXS, insight into structural data of ligand-induced MTs can be obtained.

\subsubsection{X-ray crystallography}

X-ray crystallography requires very pure preparations of macromolecules to be formed into crystals, which are usually grown in conditions in which molecules are driven out of the solution in a reversible manner. Care needs to be taken to ensure that these conditions do not denature the protein or cause dissociation of complexes. Similar to SAXS, X-ray 
crystallography consists of firing a monochromatic X-ray beam into the sample, with an $\mathrm{X}$-ray detector measuring the diffracted X-rays. The diffracted X-rays can be mathematically treated as if they were reflected from a plane of angle $\theta$ to the fired Xrays, and they can therefore also be considered "reflections". The main difference between X-ray crystallography and SAXS is the way in which the data collection organizes the target molecules. X-ray crystallography is not a contrast method like SAXS, and the collected data from X-ray scattering are highly organized because they are arranged in a crystal lattice, resulting in distinct diffraction maxima. Additionally, Xray crystallography provides far more information compared to SAXS, allowing the determination of atomic structures in the 3D-space of the crystal (Putnam et al., 2007).
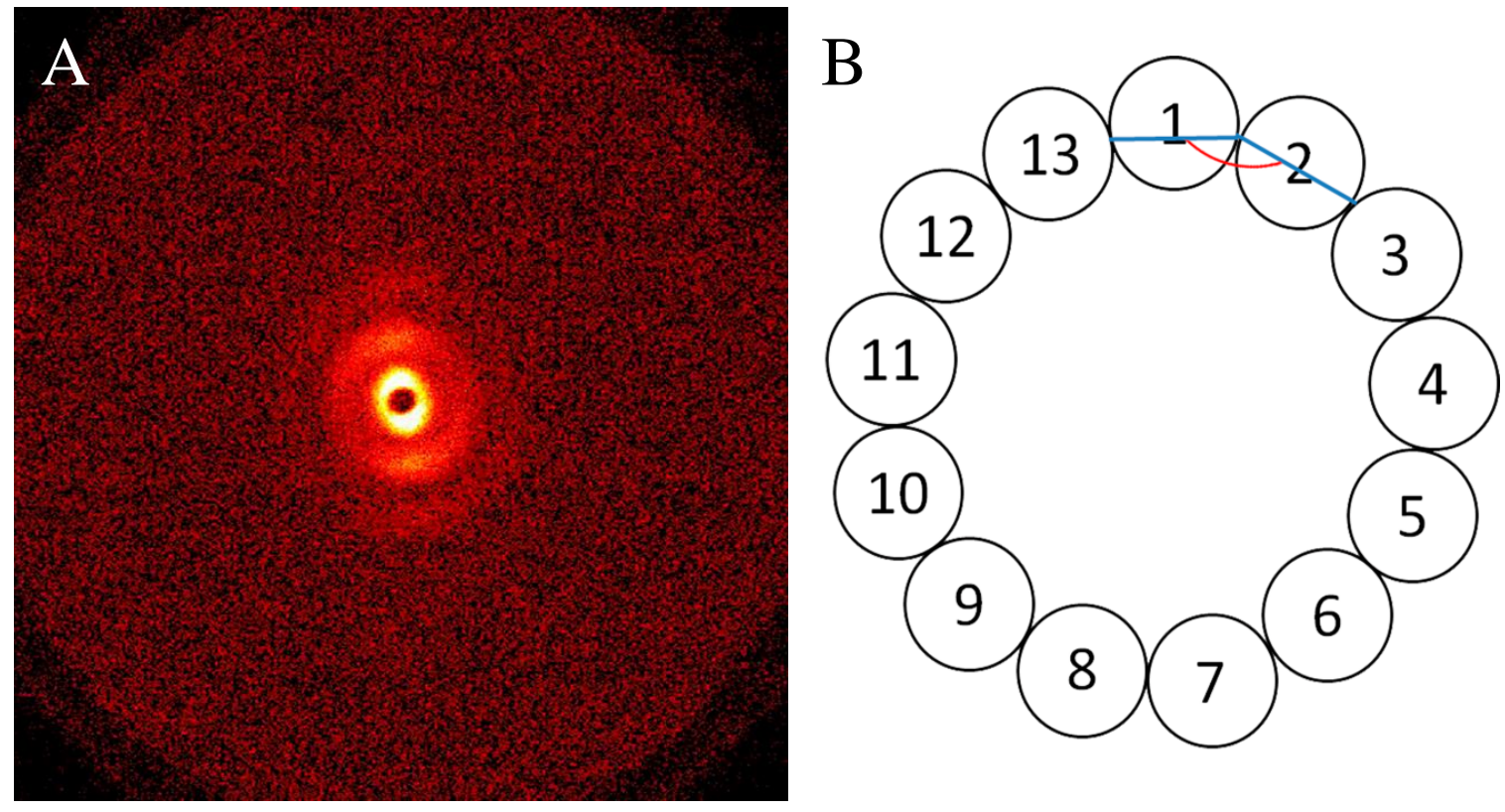

Figure 6.5 Small angle X-ray scattering

A. Typical scattering pattern of a MT by SAXS. This profile provides information about the global structure and conformation of the MTs. Image courtesy of Dr. Aurora Nogales (Instituto de Estructura de la Materia, CSIC, Madrid, Spain). B. Schematic diagram showing the interprotofilament angle between protofilaments (measured from the cylindrical cross-section). Larger MTs (greater diameter and more protofilaments) have a larger interprotofilament angle, for example those assembled in the presence of PTX, where smaller MTs have smaller angles such as those assembled in the presence of DTX. Adapted from Matesanz et al., 2011a. 


\subsubsection{Aims of this chapter}

If a drug covalently modifies tubulin, it forms a so called 'adduct', and this provides a scaffold by which the activated form of dimeric tubulin can be studied. It is likely that the ZMP-adduct would have different properties to unligated-tubulin, and therefore it is likely that ZMP-modified dimeric tubulin would aggregate differently. It is also possible that other MTA binding sites on the MT are affected by ZMP alkylation of tubulin, and it is therefore important to investigate whether ZMP affects the stoichiometry and or affinity for these sites. This will provide information not only on the global effect of ZMP on the MT but also give insight into possible synergistic interactions. Given that ZMP covalently modifies two amino acids, both of which may affect the nucleotide Esite, it is likely that ZMP modifies the properties of the E- site on $\beta$-tubulin.

Thus, the aims of this chapter are to investigate the global effect of ZMP on the MT and the properties of tubulin.

Aim 1. To determine if the tubulin aggregation properties of ZMP-ligated dimeric tubulin differ compared to unligated tubulin. This was studied using AUC (sedimentation) and complemented with X-ray crystallography.

Aim 2. To determine if ZMP modification of the protein affects ligand stoichiometry and site affinity of the LAU/PEL binding site, COL binding site and the Vinca domain.

Aim 3. To investigate the effect of ZMP binding on the E-site by determining if nucleotide binding is affected by covalent modification of residues 228 and 229 .

Aim 4. To develop a method of adduct isolation in order to provide material for crystallization of ZMP bound to tubulin and to have the structure of the adduct analyzed by X-ray crystallography. 


\subsection{METHODS}

\subsubsection{Small angle $X$-ray scattering}

SAXS was carried out as in Chapter 2.11. I prepared the samples, and Dr. Aurora Nogales (Instituto de Estructura de la Materia, CSIC, Madrid) used a Bruker NANOSTAR system to obtain the measurements. SAXS experiments were run with the assistance of Dr. Mariano Redondo-Horcajo, CIB, CSIC, Madrid. The SAXS measurements for ZMP and DAC were carried out in both PEDTA and GAB buffers and compared to control DMSO and DTX conditions.

\subsubsection{Analytical ultracentrifugation}

AUC was performed as described in Chapter 2.12 with the assistance of AUC technical staff at CIB, CSIC, Madrid, who ran the samples. Some experiments included in this section were run with the assistance of Dr. Benet Pera Gresley CIB, CSIC, Madrid. Specific experiments required specialized conditions. The aggregation properties of ZMP-modified tubulin were compared to those of un-ligated tubulin in different conditions. For all AUC experiments, tubulin was prepared as described in Chapter 2.4 in the respective buffers described below.

\section{Nucleotide effect on aggregation}

The effect of GTP versus GDP on tubulin aggregation was investigated for unligated tubulin and ZMP-ligated tubulin at different tubulin concentrations. Tubulin $(5 \mu \mathrm{M}, 20$ $\mu \mathrm{M}, 50 \mu \mathrm{M}$ and $90 \mu \mathrm{M}$ ) was prepared in PEDTA buffer containing either $1 \mathrm{mM}$ GTP or $1 \mathrm{mM} \mathrm{GDP}$ and $1 \mathrm{mM} \mathrm{MgCl}$ (or $12 \mathrm{mM}$ in the case of $90 \mu \mathrm{M}$ tubulin). All samples were treated with a $10 \%$ stoichiometric excess of ZMP or an equivalent volume of DMSO. Samples were incubated for $30 \mathrm{~min}$ at $25^{\circ} \mathrm{C}$ (or $20^{\circ} \mathrm{C}$ for the $90 \mu \mathrm{M}$ tubulin $-12 \mathrm{mM}$ $\mathrm{MgCl}_{2}$ sample) and AUC performed as described in Chapter 2.12.

\section{Effect of magnesium ions on aggregation}

Exogenous $\mathrm{Mg}^{2+}$ ions are essential in tubulin assembly, and varying the concentration of $\mathrm{Mg}^{2+}$ has variable effects on assembly. $\mathrm{Mg}^{2+}$ ions also have a significant effect on the interactions of tubulin with nucleotide. When GTP is bound to the E-site, $\mathrm{Mg}^{2+}$ enhances tubulin self-assembly and MT assembly (Grover \& Hamel, 1994; Menéndez et al., 1998). Tubulin prepared in PEDTA was diluted to $15 \mu \mathrm{M}$ and incubated with $16.5 \mu \mathrm{M}$ ZMP or 
$100 \mu \mathrm{M}$ EPOB or an equivalent volume of DMSO in different concentrations of $\mathrm{MgCl}_{2}$ for $30 \mathrm{~min}$ at $20^{\circ} \mathrm{C}$ and AUC performed as described in Chapter 2.12.

\section{Effect of zampanolide on $T_{2} R$ complex formation}

AUC is an excellent tool for studying the formation of the $T_{2} R$ complex. To see if $Z M P$ affects the formation of this complex, $10 \mu \mathrm{M}$ tubulin with $11 \mu \mathrm{M} \mathrm{ZMP}$ or the equivalent volume of DMSO was incubated with $6 \mu \mathrm{M}$ RB3 in PEDTA buffer containing $0.1 \mathrm{mM}$ DTT.

\subsubsection{Effect of zampanolide on laulimalide/peloruside binding site}

The effect of adduct formation on the affinity of the LAU/PEL site was measured using HPLC analysis. The binding affinity of PEL at $25^{\circ} \mathrm{C}$ is $3.2 \pm 0.8 \times 10^{6} \mathrm{M}^{-1}$ (Pera et al., 2010); thus, concentrations of $0.6 \mu \mathrm{M}$ PEL and $0.6 \mu \mathrm{M}$ taxoid binding sites were used in order to obtain equal concentrations of free and bound PEL. Stabilized taxoid binding sites $(0.6 \mu \mathrm{M})$ were incubated with ZMP $(0.6 \mu \mathrm{M})$ or an equivalent volume of DMSO in GAB buffer for $30 \mathrm{~min}$ at $25^{\circ} \mathrm{C}$. PEL $(0.6 \mu \mathrm{M})$ or an equivalent volume of DMSO was then added to the samples followed by a further incubation for $30 \mathrm{~min}$ at $25^{\circ} \mathrm{C}$. The samples were then centrifuged for $20 \mathrm{~min}, 25^{\circ} \mathrm{C}$ at $50,000 \mathrm{rpm}$ in a TLA 120.2 rotor in a Beckman Optima TLX centrifuge to separate out the supernatants and pellets. The pellets were resuspended in $10 \mathrm{mM} \mathrm{NaPi}$. DTX $(10 \mu \mathrm{M})$ was added to all samples as an internal standard and samples processed and analyzed by HPLC as described in Chapter 2.9. Samples were resuspended in $35 \mu \mathrm{L}$ v/v $55 \%$ methanol/water and the column developed with a gradient of $13 \mathrm{~min} 55 \%$ methanol in water, $10 \mathrm{~min} 70 \%$ methanol in water, $10 \mathrm{~min}$ $55 \%$ methanol in water. The binding constant of PEL was then calculated from these data and compared with the control (unmodified tubulin) and ZMP-modified tubulin

\subsubsection{Effect of zampanolide on colchicine binding site}

The effect of adduct formation on the COL binding site was studied using MTC. Tubulin (20 mg) was prepared in PEDTA buffer as described in Chapter 2.4. The volume of tubulin was split in half and one half incubated for $1.5 \mathrm{~h}$ at $4{ }^{\circ} \mathrm{C}$ with $10 \%$ stoichiometric excess of ZMP and the other half with the equivalent volume of DMSO. A standard MTC concentration curve was prepared in PEDTA buffer $(0 \mu \mathrm{M}-50 \mu \mathrm{M})$ and the fluorescence spectra of MTC was measured using a FluoroMax $-2^{\circledR}$ ISA ${ }^{\circledR}$ Jobin Yvon- 
Spex spectrophotometer, using the following specifications: $350 \mathrm{~nm}$ excitation and 425 $\mathrm{nm}$ emission (collecting data over the $360-500 \mathrm{~nm}$ spectrum range), using an integration of $1 \mathrm{sec}$. The fluorescence spectra of $10 \mu \mathrm{M}$ tubulin or $10 \mu \mathrm{M}$ ZMP-ligated tubulin in PEDTA buffer with increasing concentrations of MTC $(0 \mu \mathrm{M}-50 \mu \mathrm{M})$ was measured as above. Tubulin $(50 \mu \mathrm{M})$ or ZMP-ligated tubulin was prepared in a buffer containing 10 $\mu \mathrm{M}$ MTC and the florescence spectra measured as above, the sample was then diluted to $30 \mu \mathrm{M}$ tubulin in PEDTA buffer containing $10 \mu \mathrm{M}$ MTC, and the fluorescence spectra measured. This was continued until the tubulin concentration reached $0.5 \mu \mathrm{M}$. PEDTA buffer was used as the blank and the baseline fluorescence subtracted from all the samples. The free MTC baseline fluorescence was determined and subtracted from all MTC containing samples. The measurements of each set of samples were altered between experiments so that the samples were not being measured in the same order for each replicate.

\subsubsection{Effect of zampanolide on the Vinca domain}

The $\mathrm{T}_{2} \mathrm{R}$ complex was used to study the effect of ZMP on the affinity of the Vinca domain. When similar concentrations of each component of the $\mathrm{T}_{2} \mathrm{R}$ complex are present, dimers and $\mathrm{T}_{2} \mathrm{R}$ are in a $50 \%$ equilibrium (Barbier et al., 2010). When VBL is present the relative equilibrium of the $\mathrm{T}_{2} \mathrm{R}$ complex changes to $30 \%$ dimers: $70 \% \mathrm{~T}_{2} \mathrm{R}$ (F. Díaz unpublished observations), since VBL binds between heterodimers in the $T_{2} R$ complex (Gigant et al., 2005).The $\mathrm{T}_{2} \mathrm{R}$ complex and differences in equilibrium can then be used as a tool to study the influence of ZMP on the Vinca domain, since it has previously been shown that ZMP does not affect the equilibrium of tubulin- $\mathrm{T}_{2} \mathrm{R}$ complex (Prota et al., 2013; section 6.3.2 below). If ZMP does not affect the Vinca domain, we would expect to see no change in the equilibrium observed between free tubulin and $T_{2} R$ when in the presence of VBL. However, if this site is altered, a change in this equilibrium will be observed.

The $\mathrm{T}_{2} \mathrm{R}$ complex was formed in a $50 \%$ equilibrium with tubulin dimers by incubating $10 \mu \mathrm{M}$ tubulin with $7.3 \mu \mathrm{M}$ RB3-SDL, and the effect of VBL on the formation of the $\mathrm{T}_{2} \mathrm{R}$ complex was studied for the ZMP-modified and the unmodified protein using AUC (Gigant et al., 2005; Barbier et al., 2010).

Tubulin (10 mg) was prepared in buffer containing $10 \mathrm{mM} \mathrm{NaPi}, 0.1 \mathrm{mM}$ DTT, $0.1 \mathrm{mM}$ GTP, $1.5 \mathrm{mM} \mathrm{MgCl}_{2}, \mathrm{pH}$ 6.5, as in Chapter 2.4. DTT inhibits the formation of 
disulfide bond in RB3. Tubulin $(10 \mu \mathrm{M})$ was incubated for 20 min at $20^{\circ} \mathrm{C}$ in buffer (final volume of $400 \mu \mathrm{L}$ ) containing 7.3 $\mu \mathrm{M}$ RB3 + DMSO, $7.5 \mu \mathrm{M}$ RB3 + 12 $\mu \mathrm{M}$ ZMP, $7.3 \mu \mathrm{M}$ RB3 + $12 \mu \mathrm{M}$ VBL, $7.3 \mu \mathrm{M}$ RB3 + $12 \mu \mathrm{M}$ ZMP + $12 \mu \mathrm{M}$ VBL. Samples were then analyzed by AUC as described in Chapter 2.12.

\subsubsection{Effect of zampanolide on the E-site}

The concentration of the bound nucleotide was measured using two different methods in three different conditions.

To measure the nucleotide in dimeric tubulin, 20-40 $\mathrm{mg}$ of tubulin was equilibrated in PEDTA buffer as described in Chapter 2.4. The concentration of tubulin was adjusted to $40 \mu \mathrm{M}$ and incubated for $1.5-2 \mathrm{~h}$ at $25^{\circ} \mathrm{C}$ with $44 \mu \mathrm{M}$ ligand or an equivalent volume of DMSO in PEDTA buffer $(1 \mathrm{~mL})$. The samples were purified by removing excess nucleotide in a Sephadex G-25 medium column $(25$ x $0.9 \mathrm{~cm})(\mathrm{GE}$ Healthcare Bioscience, Upsala, Sweden), pre-equilibrated in PEDTA buffer (10 $\mu \mathrm{M}$ GTP, $1.5 \mu \mathrm{M} \mathrm{MgCl}_{2}$ ). Up to 16 fractions were collected, and $50 \mu \mathrm{L}$ of each sample was diluted (20x) in $10 \mathrm{mM} \mathrm{NaPi}, 1 \%$ SDS pH 7.0 and the concentration of tubulin in each sample was measured using a spectrophotometer at $275 \mathrm{~nm}$.

To measure the nucleotide in MTs, tubulin was prepared and assembled in GAB buffer as described in Chapter 2.4. The concentration of tubulin was adjusted to $40 \mu \mathrm{M}$ and incubated with $44 \mu \mathrm{M}$ ligand in GAB assembly buffer and MTs assembled at $37^{\circ} \mathrm{C}$ for $30 \mathrm{~min}(1 \mathrm{~mL})$. The samples were then centrifuged at 50,000 rpm, $37^{\circ} \mathrm{C}, 20 \mathrm{~min}$ in a Beckman Optima TLX centrifuge. The supernatant was discarded and the pellets washed in three volumes of $500 \mu \mathrm{L}$ warm GAB buffer $(0 \mu \mathrm{M}$ GTP). The pellets were then dissolved in $10 \mathrm{mM} \mathrm{NaPi}$ and the concentration of tubulin and nucleotide measured as above after a 20x dilution of each sample.

To measure the nucleotide concentration in stabilized MTs, stabilized taxoid binding sites $(20-30 \mu \mathrm{M})$ were incubated with $10 \%$ excess ligand or an equivalent volume of DMSO in GAB buffer for $30 \mathrm{~min}$ at $37^{\circ} \mathrm{C}$. Samples were then centrifuged as above and the supernatant discarded. Pellets were washed in warm GAB buffer $(0 \mu \mathrm{M}$ GTP) and resuspended in $10 \mathrm{mM} \mathrm{NaPi}$ and the concentration of tubulin and nucleotide measured as above.

Nucleotide extraction and HPLC were then carried out the same for all the above samples, similar to that described by Seckler et al. (1990). The protein was removed 
from the solution by precipitation on ice for 10 min with $1 \mathrm{M}$ ice-cold $\mathrm{HClO}_{4}$ followed by centrifugation at $4^{\circ} \mathrm{C}$ at $50,000 \mathrm{rpm}$ for $10 \mathrm{~min}$ in a TLA 120.1 rotor in a Beckman Optima TLX centrifuge to remove denatured protein. The nucleotide concentration of the supernatant was measured spectrophotometrically at $254 \mathrm{~nm}$. The supernatant was then neutralized and buffered by the addition of $1 / 6$ volume of both $1 \mathrm{M} \mathrm{K}_{2} \mathrm{HPO}_{4}$ and $3 \mathrm{M}$ $\mathrm{KOH}$, and adjusted with $0.5 \mathrm{M}$ acetic acid. Guanosine $(10 \mu \mathrm{M})$ was added as the internal standard. Samples were vortexed and frozen overnight to precipitate $\mathrm{KClO}_{4}$ and stored at $-20^{\circ} \mathrm{C}$ until HPLC analysis. Samples were thawed and precipitated $\mathrm{KClO}_{4}$ was removed by centrifugation at $12,000 \mathrm{rpm}$ for $10 \mathrm{~min}$ at $4^{\circ} \mathrm{C}$ in a bench top centrifuge. Supernatants were then cleared by membrane filtration using Minisart single-use filter units $(0.20 \mu \mathrm{m}$ pore size). Nucleotides were quantified at a wavelength of $254 \mathrm{~nm}$ and separated using tetrabutylammonium ion-pair HPLC. Tetrabutylammonium changes the properties of the HPLC column because its three methyl groups stick to the silica and the basic methylene amino group $\left(\mathrm{CH}_{2} \mathrm{NH}_{2}\right)$ faces into the column and interacts with nucleotides passing through. Nucleotides react more strongly if they have more phosphate groups; thus, GTP is retained longer in the column (Seckler et al., 1990; Díaz \& Andreu, 1993). Nucleotides $(20 \mu \mathrm{L})$ were injected into the HPLC system and separated by isocratic reversed phase on an LC18 reverse phase column Supercosil ${ }^{\mathrm{TM}} 25 \mathrm{~cm} \times 4.6 \mathrm{~mm} \times 5 \mu \mathrm{M}$. The mobile phase buffer contained $0.2 \mathrm{M} \mathrm{K}_{2} \mathrm{HPO}_{4} \cdot 3 \mathrm{H}_{2} \mathrm{O}, 0.1 \mathrm{M}$ acetic acid, $4 \mathrm{mM}$ tetrabutylammonium phosphate, $\mathrm{pH} 6.67$ and separates the three nucleotides at a flow rate of $1 \mathrm{~mL} / \mathrm{min}$. The column was prepared by thorough washing with $\mathrm{H}_{2} \mathrm{O}$ at a flow rate of $1 \mathrm{~mL} / \mathrm{min}$, then equilibration overnight in mobile phase buffer at a flow rate of $0.2 \mathrm{~mL} / \mathrm{min}(16 \mathrm{~h})$. The flow rate was then set to $1 \mathrm{~mL} / \mathrm{min}$ and the column equilibrated with the same buffer before samples were run. This overnight slow buffer run allows attachment of tetrabutylammonium groups to the silica surface of the column. Standards of $10 \mu \mathrm{M}$ guanosine, $10 \mu \mathrm{M}$ GDP and $10 \mu \mathrm{M}$ GTP were run at the start of each experiment. The area of GDP and/or GTP in each of the samples was recorded and normalized again to the $10 \mu \mathrm{M}$ guanosine internal standard to determine the concentration of each of the nucleotides in the samples.

\subsubsection{Preparation of the zampanolide-tubulin adduct for crystallization}

Fast protein liquid chromatography (FPLC) is high performance chromatography which allows the analysis and separation of mixtures of proteins and ligands. It works on the 
same basis as other forms of chromatography, taking advantage of the different affinities of components within an injected mixture for the stationary phase and the mobile phase. A fast protein liquid chromatography gel filtration system on an ÄKTA FPLC Explorer Purifier employing a HiTrap ${ }^{\mathrm{TM}} 5 \mathrm{~mL}$ desalting column (GE healthcare, Amersham Biosciences) was used to isolate the ZMP-tubulin adduct. This system was optimized for isolation of the adduct and then applied to produce large amounts of the ZMP-tubulin adduct for crystallization.

Tubulin was prepared as in Chapter 2.4 in degassed PEDTA buffer. The column was first equilibrated for $30 \mathrm{~min}$ in degassed PEDTA buffer and samples (600 $\mu \mathrm{L})$ were injected and run for $12 \mathrm{~min}$ and the absorbance monitored at 225, 260 and $280 \mathrm{~nm}$. Tubulin was used as the internal standard since it was always at the same concentration $(10 \mu \mathrm{M})$.

For crystallization, the ZMP-tubulin adduct was prepared by $1 \mathrm{~h}$ incubation of tubulin $(3 \mathrm{mg} / \mathrm{mL})$ with a slight molar excess of $\mathrm{ZMP}$ at $4{ }^{\circ} \mathrm{C}$. Using this adduct, the $\mathrm{T}_{2} \mathrm{R}-$ TTL-ZMP complex was formed. Formation of the complex, crystallization and crystallography were performed by Prof. Michel O. Steinmetz and colleagues at the Paul Scherrer Institut, CH-5232 Villigen, Switzerland. Crystallization was carried out at $20^{\circ} \mathrm{C}$, with crystals appearing overnight and reaching maximum dimensions within one week. The adduct structure was then determined by X-ray crystallography. 


\subsection{RESULTS}

\subsubsection{Microtubule diameter and number of protofilaments}

MTs assembled in GAB buffer in the absence of ligand have an average number of protofilaments of 13.3, as does DXT, similar to that previously reported (Andreu et al., 1992; Andreu et al., 1994). MTs assembled in the presence of DTX in PEDTA buffer have an average number of protofilaments of 14.0, as previously reported (Matesanz et al., 2011a). When MTs are assembled in the presence of ZMP, their diameter increases, and one additional protofilament makes up the MT lattice, most likely due to increased lateral contacts. When MTs are assembled in the presence of DAC, the lattice is made up of 15 protofilaments, two additional compared to those assembled in control conditions (Figure 6.6; Table 6.1).

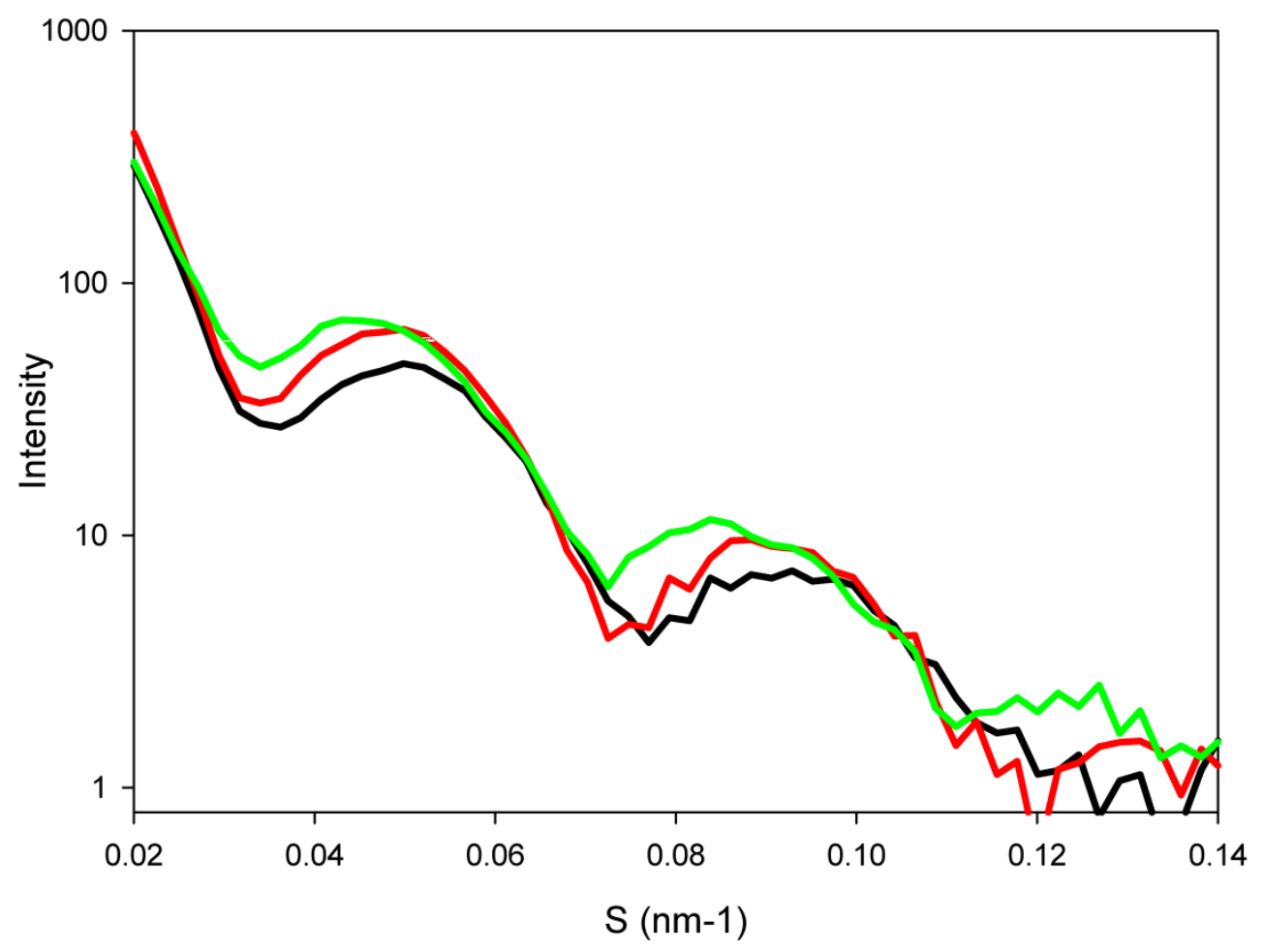

Figure 6.6 SAXS profile of microtubules

Graph showing the comparison of SAXS profiles of MTs assembled GAB buffer in the presence of DMSO control (black), ZMP (red) and DAC (green). When comparing ligand-induced MTs to control MTs, the $\mathrm{J}_{01}$ maximum increased. From these data the average MT diameter and number of protofilaments making up the lattice were calculated (Table 6.1). 
Table 6.1 Structural data of ligand induced MTs

Ligand-induced microtubules (GAB)

\begin{tabular}{cccc}
\hline Ligand & $J_{01}\left(\mathrm{~nm}^{-1}\right)$ & $\begin{array}{c}\text { Mean helical radius } \\
(\mathrm{nm})\end{array}$ & $\begin{array}{c}\text { Average number } \\
\text { protofilaments }\end{array}$ \\
\hline DMSO (vehicle) & 0.0505 & 12.07 & 13.3 \\
Zampanolide & 0.0475 & 12.84 & 14.2 \\
Dactylolide & 0.0448 & 13.61 & 15.0 \\
Docetaxel & 0.0505 & 12.07 & 13.3 \\
\hline
\end{tabular}

Ligand-induced microtubules (PEDTA)

\begin{tabular}{cccc}
\hline Zampanolide & 0.0443 & 13.76 & 15.2 \\
Dactylolide & 0.0413 & 14.77 & 16.3 \\
Docetaxel & 0.0480 & 12.71 & 14.0 \\
\hline
\end{tabular}

Values for $\mathrm{J}_{01}$ indicate the position of the maxima in the scattering profiles obtained from one measurement. The data were analyzed using SigmaPlot curve fitting software. Hyperbola curves were fitted to the second maxima peaks (first peak shown in the graph in Figure 6.6) (second peak directly relates to MT diameter). Nonlinear regression quadratic equations were used to calculate the angle, using $x=-a / 2 b$ which is equal to the value of $J_{01}\left(\mathrm{~nm}^{-1}\right)$. The diameter and number of protofilaments making up the MT lattice were then calculated as described in Chapter 2.11, with the helical radius value estimated from $\mathrm{J}_{01}=1.22 / \mathrm{D}$, and the average protofilament number from the mean helical radius and the known MT interprotofilament spacing value of $5.7 \mathrm{~nm}$ as determined by Andreu et al., 1994 . 


\subsubsection{Tubulin aggregation}

AUC was used so that the sedimentation profiles of unmodified and ZMP-modified tubulin in different conditions could be monitored. The tubulin dimer sediments at approximately $5.8 \mathrm{~S}$, and tubulin oligomers and larger aggregates including MTs sediment at higher $S$ values (Díaz et al., 1996). The $T_{2} R$ complex sediments at approximately 7.7 S (Jourdain et al., 1997). The AUC results indicated that the ZMPadduct required $\mathrm{Mg}^{2+}$ for assembly, as did unmodified protein and behaved in the same manner regardless of which nucleotide was present (Figure 6.7A\&B). At low concentrations of free $\mathrm{Mg}^{+2}(0.14 \mathrm{mM})$, no significant differences were observed between the unmodified protein and the adduct (Figure. 6.7C), thus, indicating that the binding of ZMP had no notable effect on the longitudinal tubulin-tubulin interactions responsible for the early aggregation events. In conditions with moderate concentrations of free $\mathrm{Mg}^{2+}(0.77 \mathrm{mM})$ and in the absence of $\mathrm{ZMP}$, tubulin dimers and the formation of a small proportion of tetramers and some bigger oligomers were observed. With the ZMP-adduct, however, the amount of free tubulin dramatically decreased, and the small oligomers disappeared, indicating that the adduct was assembling into MTs or large aggregates in these conditions (Figure 6.7D). In the presence of excess EPOB, the same effect occurred as expected (Figure 6.7G). This means that the effect of ZMP on the ligation was to stabilize the lateral interdimer contacts responsible for MT formation from oligomers. This indicates that the adduct is more prone to oligomerization compared to unligated-tubulin. In addition it appears that for the adduct the first step of nuclei formation from the tetramer to oligomer state of tubulin (Figure 6.1) is more straightforward than for unmodified tubulin, and a larger percentage of bigger oligomers are formed with the adduct compared to unmodified tubulin. These results were confirmed in GDP buffer (Figure 6.1E\&F). As expected, in GDP buffer and high free $\mathrm{Mg}^{+2}(6.78 \mathrm{mM})$, the adduct was more prone to assembly compared to unmodified tubulin which forms double rings (Figure 6.7H). The formation of the ZMP-adduct had no influence on the formation of the $\mathrm{T}_{2} \mathrm{R}$ complex with the $50 \%$ equilibrium between tubulin dimers and $\mathrm{T}_{2} \mathrm{R}$ remaining unchanged (data not shown). Similar experiments were carried out with DAC and no major differences were seen between unligateddimers and DAC-ligated dimers. 

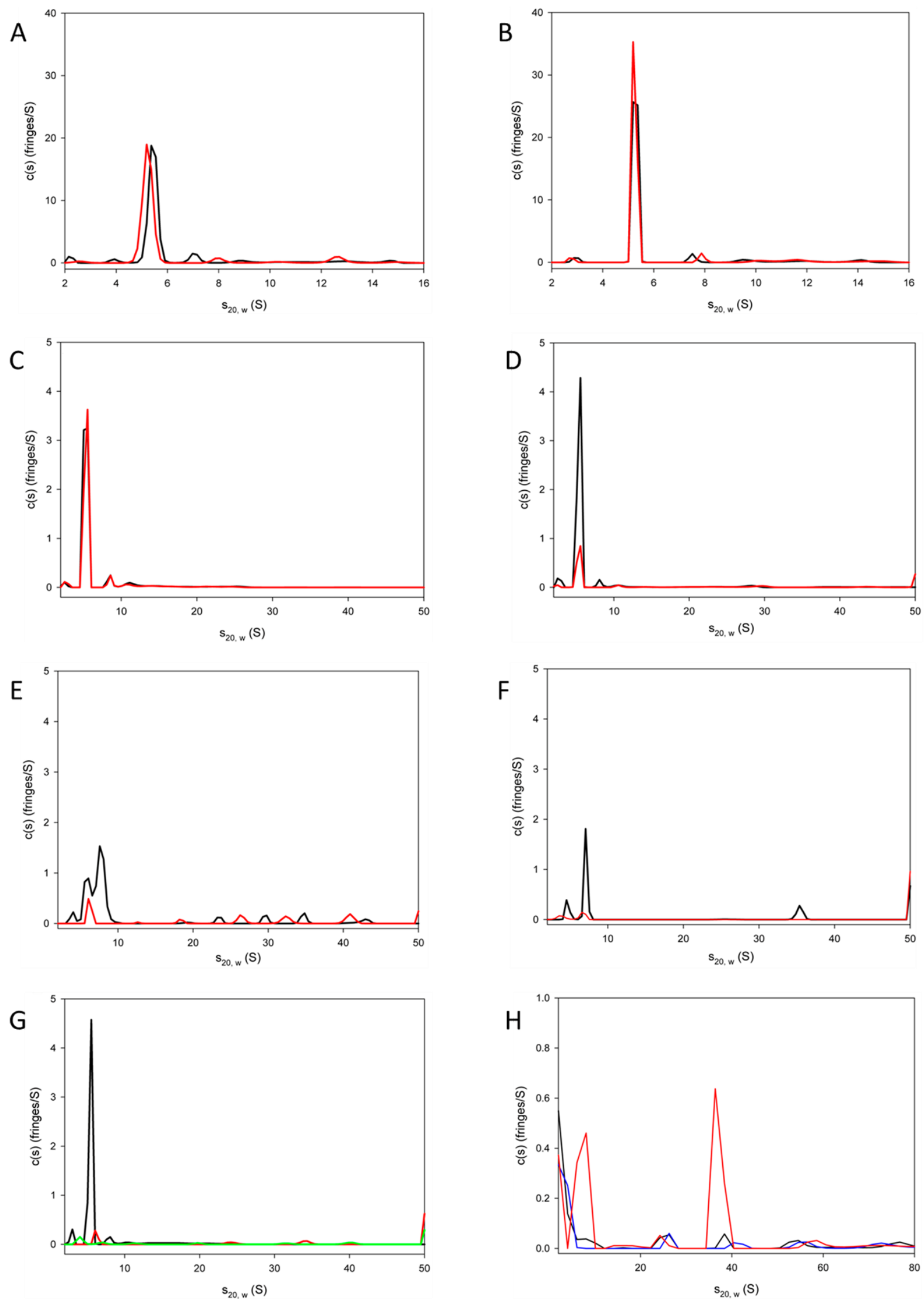

204 
Figure 6.7 Tubulin aggregation of unligated tubulin and the ZMP-tubulin adduct

Unless otherwise stated black lines represent unmodified tubulin and red lines represent ZMP-adduct.

Graphs A and B show the effect of nucleotide on tubulin aggregation. A. $50 \mu \mathrm{M}$ tubulin with $55 \mu \mathrm{M}$ ZMP or DMSO with GTP and low free $\mathrm{Mg}^{2+}(0.028 \mathrm{mM})$. B. $50 \mu \mathrm{M}$ tubulin with $55 \mu \mathrm{M}$ ZMP or DMSO with GDP and low free $\mathrm{Mg}^{2+}(0.039 \mathrm{mM})$

Graphs $C$ - $G$ show the effect of increasing concentrations of free $\mathrm{Mg}^{2+}$ on tubulin aggregation

C. Aggregation in GTP buffer with a low concentration of free $\mathrm{Mg}^{2+}\left(1.5 \mathrm{mM}\right.$ total $\mathrm{Mg}^{2+}, 0.14 \mathrm{mM}$ free $\mathrm{Mg}^{2+}$ ), indicating that the adduct requires $\mathrm{Mg}^{2+}$ for aggregation as does unligated tubulin. D. Aggregation in GTP buffer with moderate free $\mathrm{Mg}^{2+}$ concentrations $\left(3 \mathrm{mM}\right.$ total $\mathrm{Mg}^{2+}, 0.77 \mathrm{mM}$ free $\mathrm{Mg}^{2+}$ ). Unligated tubulin is unable to aggregate, with only dimers present (peak at $5.5 \mathrm{~S}$ ); however, the adduct can aggregate, showing very few dimers. E. Aggregation in GDP buffer with $1.48 \mathrm{mM}$ free $\mathrm{Mg}^{2+}$ concentration or $\mathbf{F}$. $3.31 \mathrm{mM}$ free $\mathrm{Mg}^{2+}$. G. Effect of EPOB $(100 \mu \mathrm{M})$ at different concentrations of $\mathrm{Mg}^{2+} ; 1.5 \mathrm{mM} \mathrm{MgCl}_{2}$ (0.14 mM free) (black), $3 \mathrm{mM} \mathrm{MgCl}_{2}$ (0.77 $\mathrm{mM}$ free) (red), and $4 \mathrm{mM} \mathrm{MgCl}_{2}$ (1.31 mM free) (green).

Graph H shows ZMP inhibition of double ring formation

H. Influence of adduct formation in the presence of high free $\mathrm{Mg}^{2+}$ concentrations. $1 \mathrm{mM}$ GDP, 6.78 $\mathrm{mM}$ free $\mathrm{Mg}^{2+}$ with ZMP-tubulin (black), or with unligated-tubulin (red). In these conditions tubulin double rings usually form since the dimers are GDP-bound and in excess $\mathrm{Mg}^{2+}$ conditions; however, with ZMP-ligated tubulin, all the tubulin is assembled into MTs, and no rings are seen because the conditions are well above the critical concentration required for assembly. Blue line shows ZMPtubulin in high free $(6.68 \mathrm{mM}) \mathrm{Mg}^{2+}$ concentration with GTP, indicating that the bound nucleotide has no effect on the assembly of ZMP-ligated tubulin in conditions where the free concentration of $\mathrm{Mg}^{2+}$ is high. 


\subsubsection{Effect of zampanolide on the laulimalide/peloruside A site}

Using HPLC methods the binding affinity for the LAU/PEL binding site in unligatedtubulin compared to that in ZMP-ligated tubulin was studied. The binding affinity for PEL for unmodified tubulin at $25^{\circ} \mathrm{C}$ was found to be $3.98 \pm 0.29 \times 10^{6} \mathrm{M}^{-1}$, similar to that already determined (Pera et al., 2010). This affinity was slightly lower when binding to $\mathrm{ZMP}$-modified tubulin, $\mathrm{Kb}=3.05 \pm 0.38 \times 10^{6} \mathrm{M}^{-1}$; however, these $\mathrm{Kb}$ values were not significantly different from one another (nonparametric Mann Whitney test (GraphPad Prism v5) and are therefore within the experimental error. A nonparametric statistical test was used because of the small sample size $(n=4)$. The fact that the affinity of the LAU/PEL site did not change between the two conditions indicates that the formation of the adduct does not affect ligand binding to this site (Figure 6.8; Table 6.2).

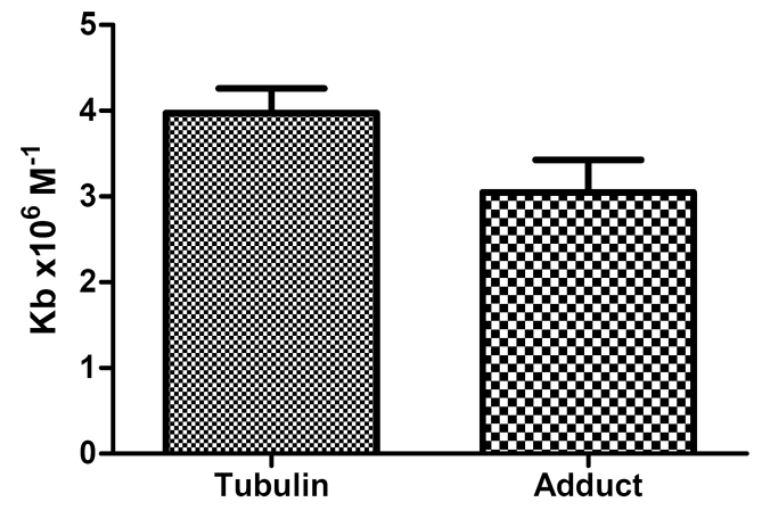

Figure 6.8 PEL binding constants

Graph showing the difference in binding constants of PEL for unligated MTs and ZMPbound MTs. Although the binding affinity for the adduct is slightly less, it is not significantly different from the affinity for unligated MTs, indicating the LAU/PEL site remains unchanged in ZMP-modified MTs.

Table 6.2 Binding affinity of PEL for unmodified and modified MTs

\begin{tabular}{lccc}
\hline & $\begin{array}{c}\text { Microtubules } \\
\text { (Pera et al., 2010) }\end{array}$ & Microtubules & Zampanolide-adduct \\
\hline Affinity $(\mathrm{Kb})\left(10^{6} \mathrm{M}^{1}\right)$ & $3.2 \pm 0.8$ & $3.98 \pm 0.29$ & $3.05 \pm 0.38$ \\
\hline
\end{tabular}

The binding constant of PEL for unligated and ZMP-modified MTs is similar to that previously determined, indicating that adduct formation has no effect of the affinity LAU/PEL binding site for binding PEL. 


\subsubsection{Effect of zampanolide on colchicine binding site}

MTC was used determine if covalent modification of tubulin by ZMP affects the stoichiometry and the affinity of ligands for the COL binding site. Increasing concentrations of MTC were added to a constant concentration of tubulin $(10 \mu \mathrm{M})$ and the fluorescence of MTC used to calculate the stoichiometry of the reaction. MTC fluorescence saturated at approximately $10 \mu \mathrm{M}$, indicating a 1:1 stoichiometry. This was repeated with ZMP-modified tubulin to determine if MTC binding to the adduct rather than unmodified tubulin changes the stoichiometry. As shown in Figure 6.9A, ZMP binding did not affect the stoichiometry of MTC for its binding site (stoichiometry remains at 1:1); however, the fluorescence of the compound was significantly higher when ZMP was bound. To measure the binding affinity of MTC for the COL binding site, $10 \mu \mathrm{M}$ MTC was added to increasing concentrations of tubulin and ZMP-bound tubulin to determine if adduct formation changes the affinity of MTC binding. As shown in Figure 6.9B and Table 6.3, the affinity of MTC for tubulin $\left(\mathrm{Kb}=4.21 \times 10^{5} \mathrm{M}^{-1}, 25^{\circ} \mathrm{C}\right)$ and ZMP-modified tubulin $\left(\mathrm{Kb}=4.51 \times 10^{5} \mathrm{M}^{-1}, 25^{\circ} \mathrm{C}\right)$ was similar, as previously been shown at $20^{\circ} \mathrm{C}$ (La Regina et al., 2007). Thus, although MTC had a higher fluorescence when bound to ZMP-modified tubulin, the stoichiometry and the affinity of the COL site did not change with ZMP binding. 

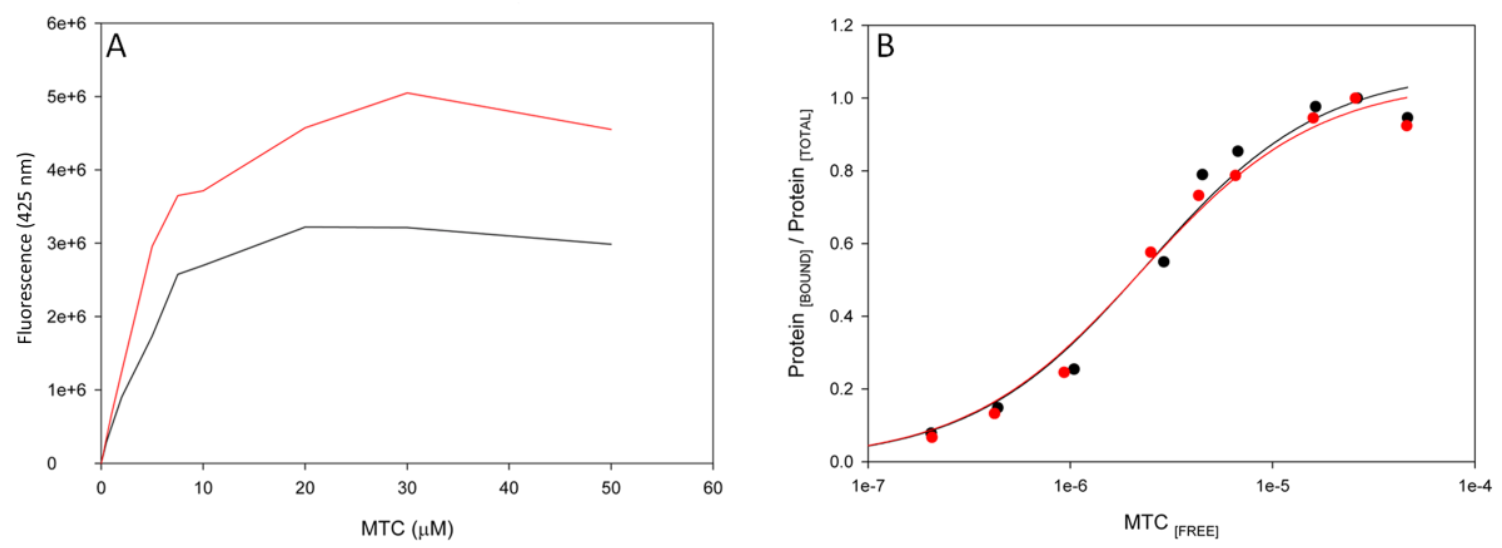

Figure 6.9 Effect of adduct formation on the affinity of the colchicine binding site

A. Fluorescence of increasing concentrations of MTC when binding the ZMP-adduct (red) or unligated tubulin (black). MTC emits higher fluorescence when binding to the adduct compared to unligated tubulin. Fluorescence saturates at $10 \mu \mathrm{M}$ MTC in both cases indicating that the stoichiometry of the COL binding site does not change upon adduct formation. B. Binding curves of MTC binding to un-ligated tubulin (black) and to the ZMP-adduct (red). ZMP binding to tubulin did not alter the affinity of the COL binding site for MTC, as the binding constants do not change between un-ligated tubulin and the ZMP adduct (Table 6.3).

Table 6.3 Binding constants of MTC binding to unmodified and modified tubulin

\begin{tabular}{cccc}
\hline & $\begin{array}{c}\text { Tubulin } \\
\text { (La Regina et al., 2007) }\end{array}$ & Tubulin & Zampanolide-adduct \\
\hline Affinity $(\mathrm{Kb})\left(\times 10^{5} \mathrm{M}^{1}\right)$ & $4.7 \pm 0.3$ & $4.21 \pm 0.16$ & $4.51 \pm 0.54$ \\
\hline
\end{tabular}

The binding constant of MTC for the COL binding site was similar to that previously determined, and there was no difference between unligated and ZMP-modified tubulin. Formation of the ZMP-adduct therefore has no effect on the affinity of the COL site for its ligands. 


\subsubsection{Effect of zampanolide on nucleotide E-site}

MTs contain 1 mole of GDP and 1 mole of GTP per tubulin heterodimer (Carlier \& Pantaloni, 1981). Since ZMP covalently reacts with His229 (majority) and N228 (minority), and the side chain of residue 228 faces the E-site, interacting with the bound nucleotide, it seemed likely that the covalent binding of ZMP would affect the binding affinity of the E-site for guanosine nucleotides. To investigate this, the nucleotide content of the ZMP-adduct and unmodified protein were compared under several different conditions. In GAB assembled MTs and dimeric tubulin, ZMP and DAC cause a significant decrease in bound nucleotide at the E-site. This effect is more apparent in dimeric tubulin than preformed MTs. ZMP displaces $100 \%$ of the E-site nucleotide bound to dimeric tubulin compared to $60 \%$ by DAC. Interestingly, cyclostreptin had no effect on the nucleotide content in assembled MTs but caused a 20\% loss of nucleotide in dimeric tubulin. These results were compared to DMSO and DTX, both of which had no effect on the nucleotide content in either of the two conditions (Table 6.4). The effect on nucleotide binding in pre-formed crosslinked MTs was also investigated; however, there was no significant loss of nucleotide as expected (data not shown). 
Table 6.4 Effect of adduct formation on the E-site $(\mu \mathrm{M})$

\begin{tabular}{cccc}
\hline \multicolumn{4}{c}{ GAB Assembled Microtubules } \\
\hline Ligand & Total Nucleotide & GDP & GTP \\
\hline DMSO & 2.00 & 1.00 & 1.00 \\
Docetaxel & $2.05 \pm 0.06$ & $1.03 \pm 0.03$ & $1.02 \pm 0.03$ \\
Zampanolide & $1.76 \pm 0.04$ & $0.84 \pm 0.03$ & $0.92 \pm 0.07$ \\
Dactylolide & $1.79 \pm 0.09$ & $0.84 \pm 0.01$ & $0.95 \pm 0.07$ \\
Cyclostreptin & $2.00 \pm 0.004$ & $0.97 \pm 0.03$ & $1.03 \pm 0.03$ \\
\hline & & & \\
\hline \multicolumn{4}{c}{ Dimeric tubulin } \\
Ligand & Total Nucleotide & GDP & GTP \\
\hline DMSO & 2.00 & 0.00 & 2.00 \\
Zampanolide & $0.93 \pm 0.13$ & 0.00 & 0.93 \\
Dactylolide & $1.40 \pm 0.16$ & 0.00 & 1.40 \\
Cyclostreptin & $1.81 \pm 0.12$ & 0.00 & 1.81 \\
\hline
\end{tabular}

Total nucleotide was measured spectrometrically and the concentration of GDP and GTP measured by HPLC. The total concentration of nucleotide should in theory be 2 moles, and the data were normalized to this value. 


\subsubsection{Crystallization of zampanolide bound to unassembled tubulin}

ZMP-tubulin adduct was isolated as described in section 6.2.7. This adduct was then further reacted to form a 2:1:1:2 tubulin-RB3-TTL-ZMP complex $\left(\mathrm{T}_{2} \mathrm{R}-\mathrm{TTL}-\mathrm{ZMP}\right)$. This complex was crystallized to a resolution of $1.8 \AA$ by Dr. Michel Steinmetz and colleagues at PSI, Switzerland (Prota et al., 2013). This is currently the highest resolution tubulin structure known. The ligand-bound structure superimposed well with the same tubulin structure in the absence of ligands, and also with zinc stabilized sheets (Löwe et al., 2001).

The crystallography confirmed that ZMP binds deep within the taxoid binding pocket in the MT lumen and covalently modifies His229, as shown by MS (Chapter 5.3). The covalent reaction involves formation of a new bond between the NE2 atom of the histidine side chain and C9 of the ZMP macrocyclic ring, likely via 1,4 Michael addition (Figure 6.10). These sites of covalent modification were predicted by the molecular modeling; however, positioning of the ligand in the taxoid site did not agree with the molecular modeling. Two hydrogen bonds are formed between the ZMP C20 hydroxyl group and main chain carbonyl oxygen of Thr276 and the O1' atom of ZMP and the NH group of Thr276 (Figure 6.11). Interestingly, no crystals formed with ZMP attached to Asn228, the second residue shown to be modified in the MS experiments. 


\subsection{DISCUSSION}

\subsubsection{Crystal structure of zampanolide bound to unassembled tubulin}

The X-ray crystal structure provided an accurate binding site description for ZMP and, as suggested by the molecular modeling, confirmed that C9 of ZMP covalently binds His229 at the NE2 atom of the side chain (Figure 6.10). Additionally, it highlighted how ZMP causes potent stabilization of MTs, confirming the biochemical results in previous chapters and also supporting the existence of the luminal binding site in unassembled tubulin. The molecular basis behind the potent MT-stabilizing activity of ZMP stems from its side chain interaction with the M-loop. ZMP induces major restructuring of the M-loop into a short and distinct helix involving M-loop residues 278-283. This helical structuring is explained by the extensive hydrophobic and polar contacts formed between the side chain of ZMP and M-loop residues Pro274, Thr276, Gly281, Arg284, and Leu286, and via an intramolecular hydrogen bonding network within the M-loop and H9. Using the same method, EPOA was also crystallized and showed similar M-loop restructuring. In the taxoid site, the side chains of ZMP and EPOA superimpose in the structures, with their macrolide cores at $90^{\circ}$ to one another, and held in the pocket by different sets of interactions. The M-loop restructuring is now considered a 'hallmark' of the ZMP and EPOA structures and explains the effect of these MSAs on MT-assembly and stabilization (Prota et al., 2013). The X-ray crystallographic structure of ZMP is consistent with the importance of the side chain of ZMP in its binding properties and potency (Figure 6.11).

Overall these results show that MSAs activate tubulin by inducing re-structuring of the M-loop in $\beta$-tubulin when they bind. This is important since the M-loop facilitates lateral tubulin interactions in adjacent protofilaments. The same structural changes would be expected for other taxoid site MSAs, including those used in the clinic. 

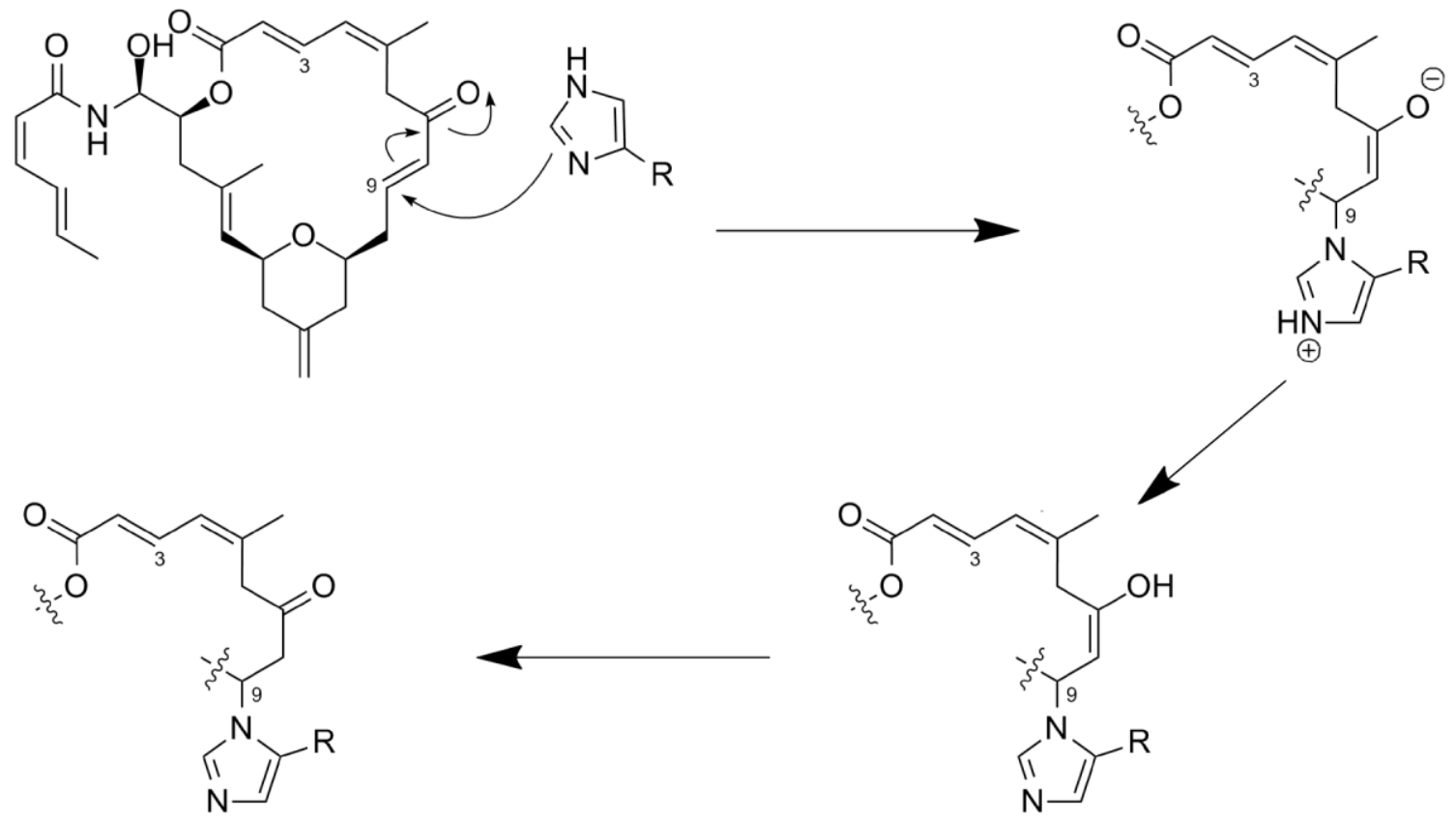

Figure 6.10 Michael addition of ZMP and histidine

ZMP covalently modifies tubulin by Michael addition to the His229 side chain which is nucleophilic and attacks the C8-C9 double bond of the enone in ZMP. ZMP attaches to His229 via a bond between C9 of the macrolide ring and NE2 of the His side chain. (See also Figure 6.11)

Figure 6.11 Crystal structure of ZMP bound to unassembled tubulin

A. Overall view of the ZMP-tubulin heterodimer complex. Tubulin subunits are labeled and shown in grey, the M-loop is shown in yellow and ZMP in green. The dotted box shows the areas depicted in B. B. Close-up view of the ZMP-tubulin interaction networks, colored as above. Interacting amino acids are shown in stick format and the hydrogen bonds are shown by the black dotted lines. The covalent bond between C9 in ZMP and the NE2 atom of His229 is shown in orange. C. Close-up view of the ZMP interaction with His229. ZMP is shown in green, the covalent bond in orange and His229 in blue. D. Close up view of the ZMP-induced restructuring of the M-loop into a short helix. Intramolecular hydrogen bonds are shown by the black dotted lines and cause stabilization of the helix. These intramolecular hydrogen bonds are formed between residues in the M-loop and H9. Individual images taken from Prota et al., 2013. Crystallography experiments were performed and images made by Dr. Michel Steinmetz and colleagues. 


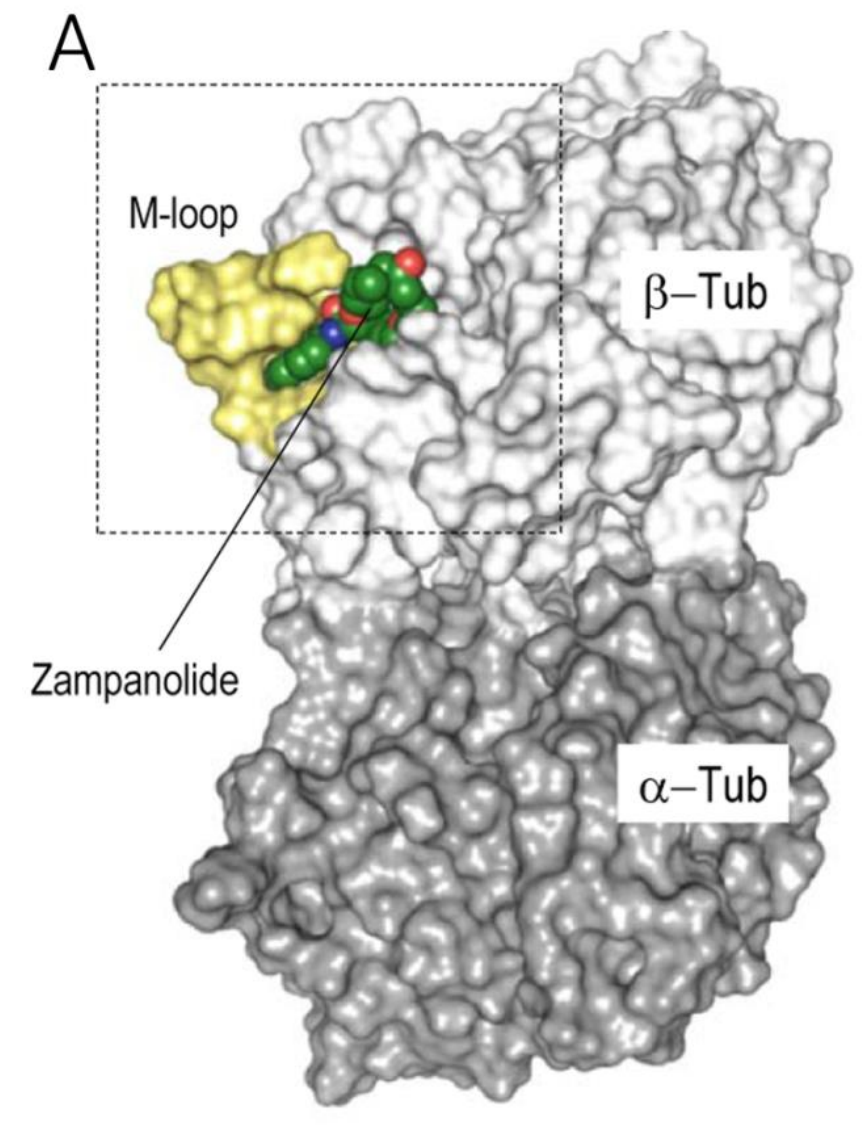

B
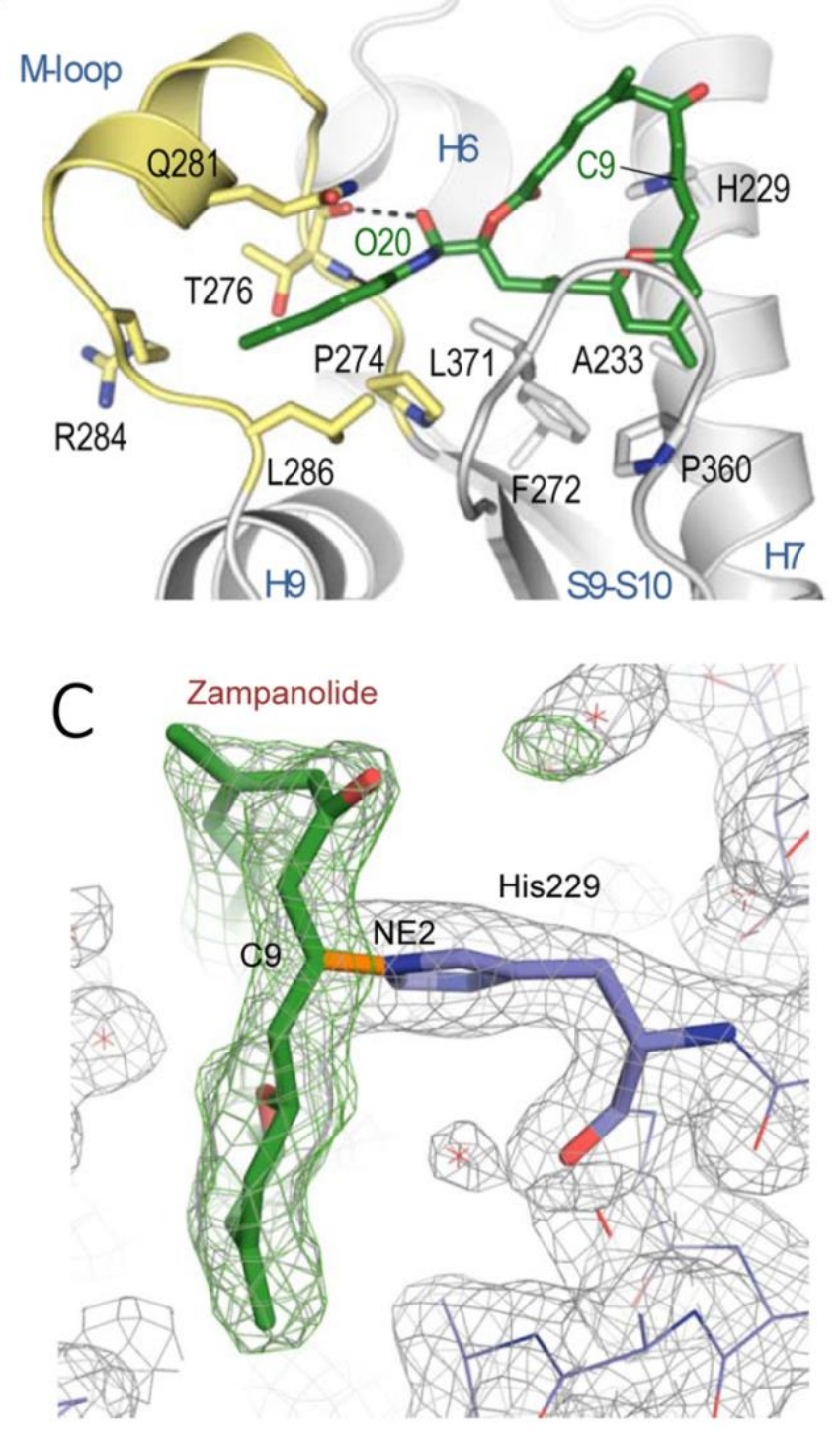

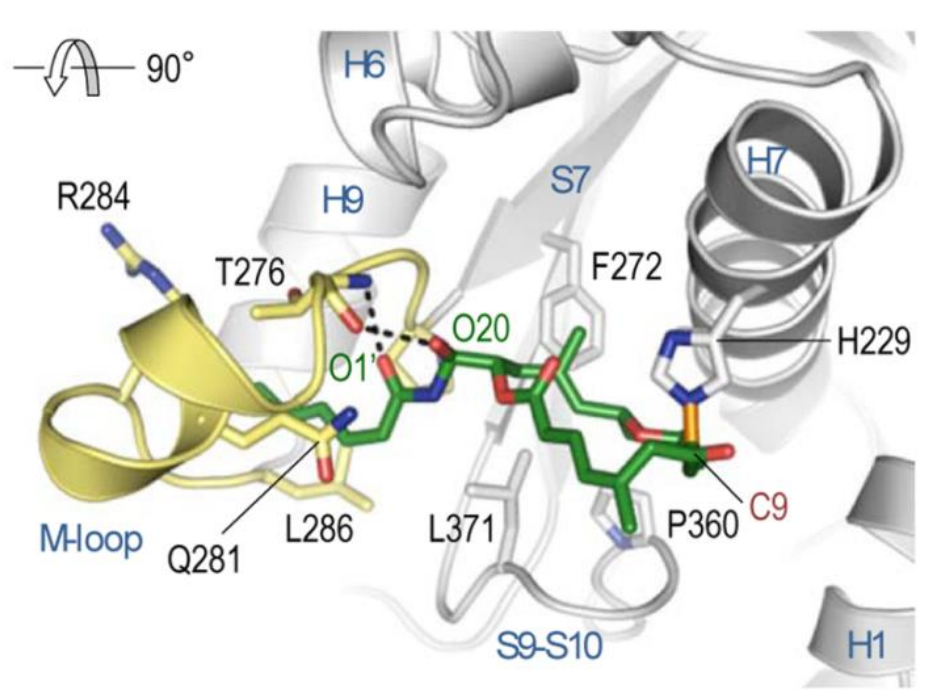

D Q282 M-loop

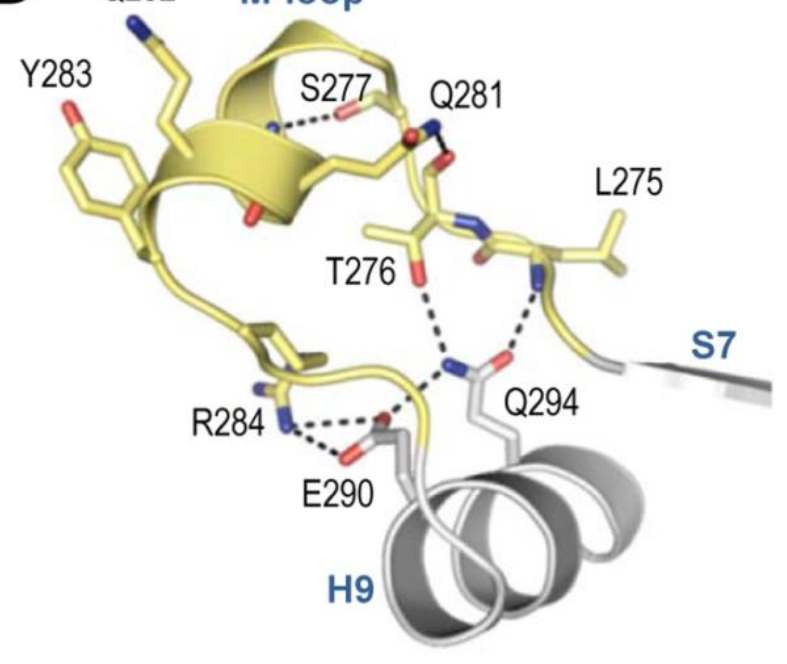




\subsubsection{Molecular mechanism of action of MSAs}

The M-loops of both tubulin subunits are now thought to have a pre-disposition to form a helical structure that establishes lateral contacts in MTs in the absence of an MSA. As described, the lattice model for tubulin assembly describes GTP-tubulin as being curved when free and driven to a straight conformation upon formation of lateral contacts in the MT lattice (Buey et al., 2006). The helical structuring of the M-loop is proposed to facilitate this curved-to-straight conformational change that occurs when tubulin is incorporated into the MT. The taxane binding site is only slightly affected by the curved to straight conformational change, with minor rearrangements of the residues shaping the pocket (Prota et al., 2013). In unassembled tubulin, this M-loop is disordered; whereas, in MTs it is helical and ordered and this explains why MSAs have higher affinity for MTs compared to unassembled tubulin (Figure 6.12). Binding of an MSA to the tubulin dimer causes structural organization of the M-loop according to the structural requirements for MT assembly (Figure 6.13). This would result in reduced entropy loss associated with the assembly of the MT in the presence of an MSA compared to assembly in the absence of the MSA. It would be expected that loss of the helical M-loop structure would trigger MT disassembly (Prota et al., 2013).

Using this crystal structure a modified, more accurate molecular mechanism for MSA-induced MT-assembly for taxoid site ligands can be proposed (Prota et al., 2013). Binding of the ligand to the dimer (un-ordered M-loop) causes it to restructure into a short and well-defined helix that is stabilized by interactions with the ligand and an intramolecular hydrogen bonding network, the latter also present in self-assembled MTs. The structured M-loop promotes polymerization by encouraging the curved-to-straight transition and the structured M-loops in both subunits stabilize the MT lattice by forming lateral contacts between protofilaments. Figure 6.13 shows a schematic diagram of the proposed molecular mechanism of MSAs. It is likely that in MTs, the MSA binds first at the pore site and then moves to the luminal taxoid binding site where it induces these structural changes. 

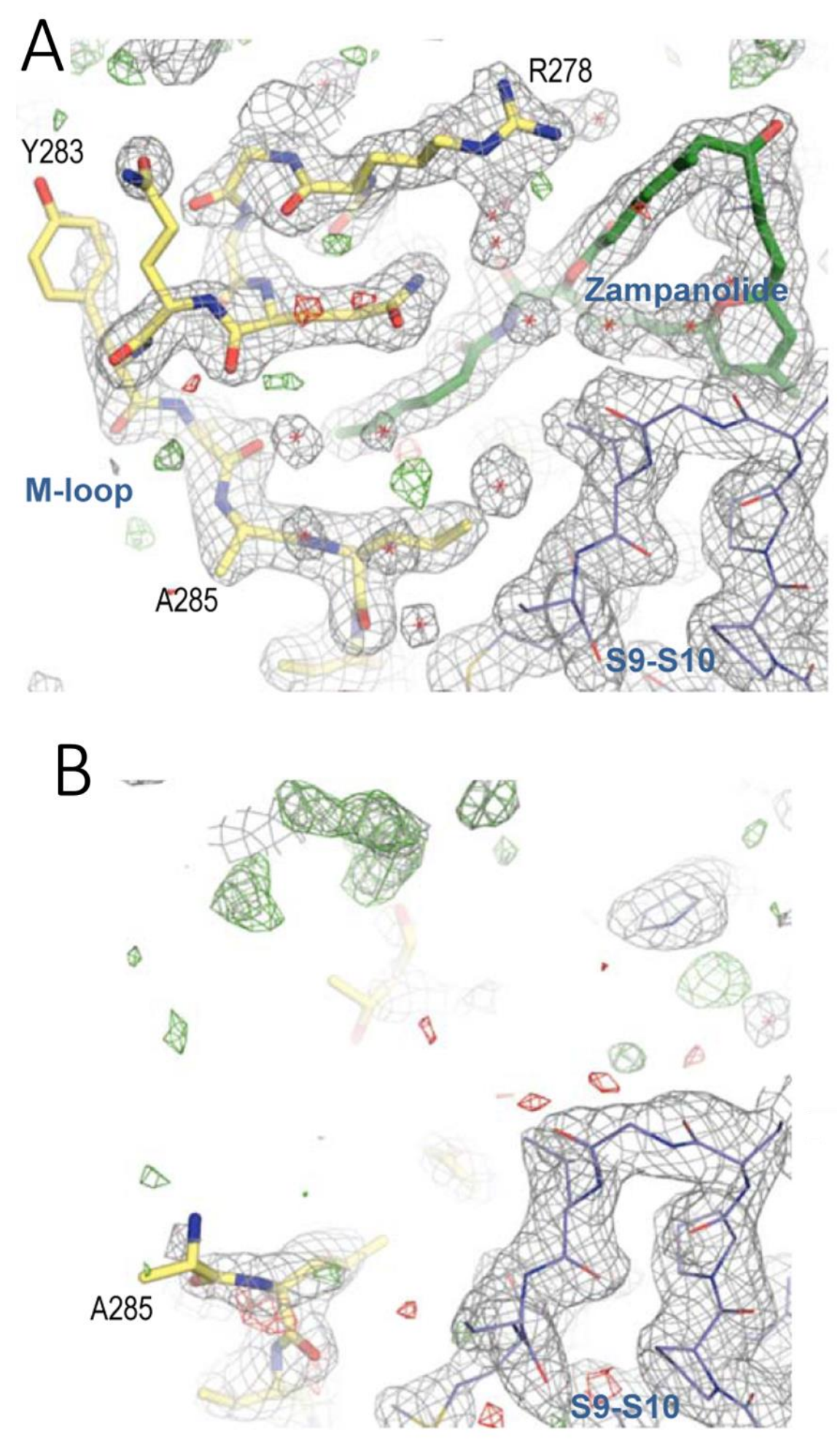

Figure 6.12 Conformation of the M-loop

Electron density maps of the region surrounding the M-loop of $\beta$-tubulin in the T2R-TTL complex. A. Complex with bound ZMP ( $\mathrm{T}_{2} \mathrm{R}$-TTL-ZMP). In this complex the M-loop is ordered since the taxoid site is occupied, and 'ordered dimers' more readily assemble since this ordering facilitates the curve-tostraight change. B. $\mathrm{T}_{2} \mathrm{R}$-TTL complex in absence of taxoid site occupation. In this complex the M-loop is unordered. Individual images taken from Prota et al., 2013. Crystallography experiments were performed and images made by Dr. Michel Steinmetz and colleagues. 


\subsubsection{Thermodynamics of MSA binding}

A common thermodynamic mechanism of assembly induction can be proposed for reversible MSAs. Reversible MSAs bind tightly, but not covalently, to the assembled form of tubulin; whereas, they do not bind with any measurable affinity to the unassembled form (Díaz et al., 1993). This is now thought to be because the M-loop is already ordered in MTs. The main thermodynamic driving force for their induced assembly is the difference in their binding constants for dimeric tubulin relative to MTs. For example, the binding constant of DSC for the dimer $\left(\mathrm{Kb}=2.0 \pm 0.7 \times 10^{4} \mathrm{M}^{-1}, 25^{\circ} \mathrm{C}\right)$ (Canales et al., 2011) is significantly lower than that for stabilized MTs (Kb $=8.7 \pm 0.8 \mathrm{x}$ $10^{9} \mathrm{M}^{-1}, 26^{\circ} \mathrm{C}$ ) (Buey et al., 2005). In structural terms, the difference in affinity and thus the mechanism of assembly induction should arise from a difference in binding site conformation. In unassembled tubulin in the absence of an MSA, the M-loop is disordered; whereas, in MSA-bound but unassembled tubulin it forms a short welldefined helix with the rest of the taxoid site binding pocket remaining unchanged (Figure 6.12) (Prota et al., 2013). In unligated dimers the M-loop has to undergo a structural change in order to accommodate the ligand, and the related free energy has to be subtracted from the overall free energy of the newly formed MSA/protein interactions. In MTs this M-loop would already be in a helical structure, and no rearrangements are needed to bind the MSA. It is evident that the linked assembly binding process should have an energetic advantage of approximately $-30 \mathrm{~kJ} / \mathrm{mol}$ over the assembly process, even if the restructuring is energetically negative. These differences in binding constants can drive the reaction towards the assembled state.

The case would be entirely different for a covalent MSA such as ZMP. In this case there is no reaction equilibrium (no off-rate of covalently bound ZMP), and the reaction is only kinetically controlled by the formation of the covalent bond and thus proceeds to completion. The energy of restructuring would be irrelevant since there is not one molecule in different states but rather there are two different molecules with different structures - tubulin and ZMP-tubulin. Once ZMP has bound, the M-loop residues have a different equilibrium conformation as a result of new bonds formed that are different from the M-loop residues in the unligated protein. This conformational change of structuring, energetically unfavorable in the case of the unligated protein, is necessary to form the lateral contacts, and the difference in the equilibrium polymerization of free energy of both processes is a reflection of the energetic cost of M-loop restructuring. In 
ligated tubulin, restructuring has already occurred prior to the lateral contacts being made. The free energy of the adduct binding to the end of a MT, which is made up of adducts has $\Delta \mathrm{G}$ of $-36.2 \mathrm{~kJ} / \mathrm{mol}\left(37^{\circ} \mathrm{C}\right.$ in $\mathrm{GAB}$ buffer, $\left.6 \mathrm{mM} \mathrm{MgCl}_{2}, \mathrm{pH} 6.7\right)$, and assembly of unligated protein has a significantly higher $\Delta \mathrm{G}$ of $-32.5 \mathrm{~kJ} / \mathrm{mol}$ (same conditions). This difference of $3.7 \mathrm{~kJ} / \mathrm{mol}$ means that the ZMP-ligated protein has lower free energy for assembly than the unligated tubulin. This is related to the fact that when the adduct binds to the end of the MT, it is already in a conformationally favorable state; whereas, when unligated tubulin dimers bind the M-loop is disordered, as discussed. Assuming the rest of the reaction is similar between the two types of dimer (ZMP-ligated versus unligated) free energy is required for addition of the unligated dimer in order to induce the required structural change in the M-loop for it to be 'organized'. 
Figure 6.13 Molecular mechanism of action of MSAs

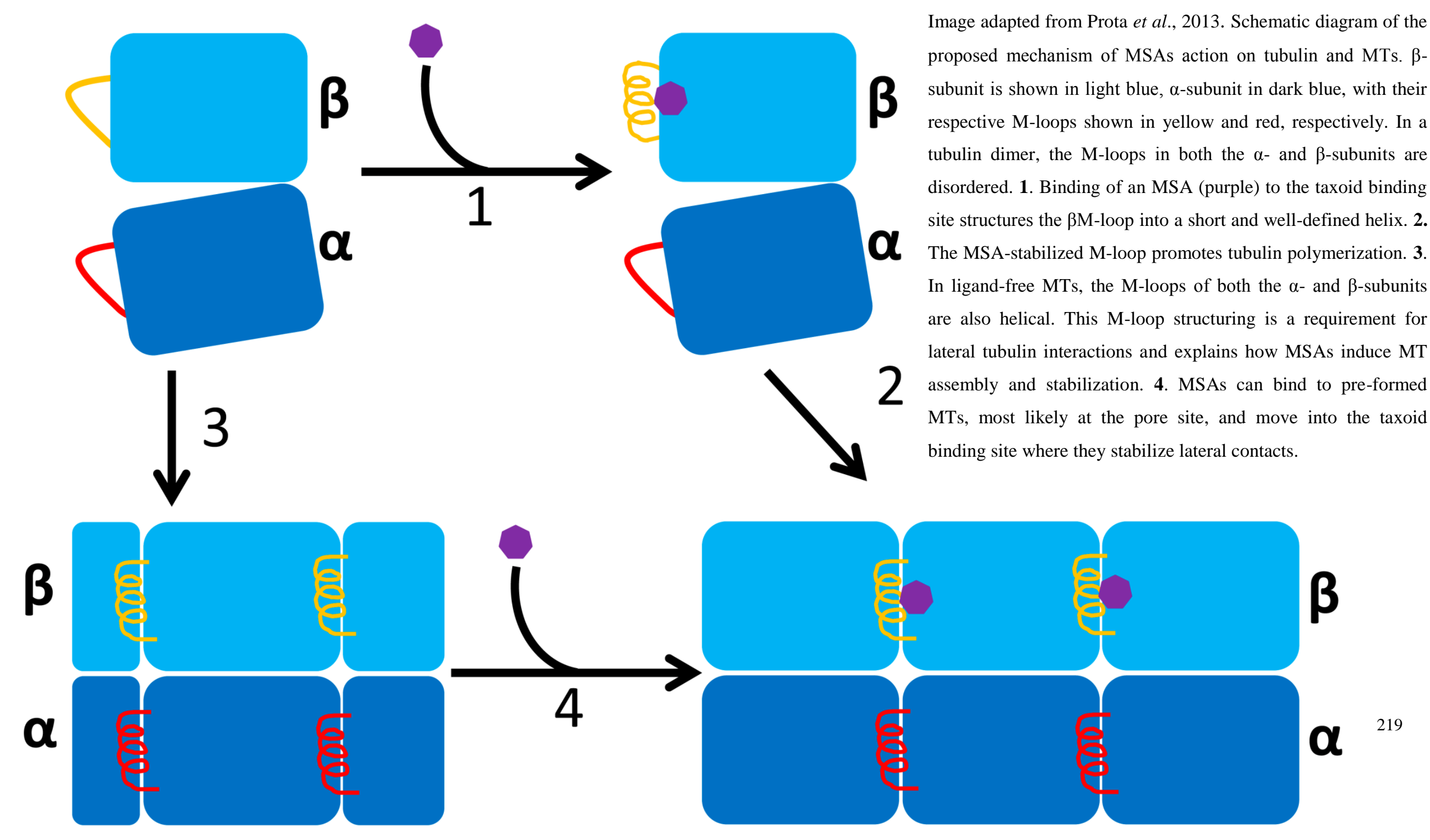




\subsubsection{Zampanolide influences MT size}

Changes in protofilament number reflect the modification of lateral interactions within the MT wall (Matesanz et al., 2011a). MTs assembled in PEDTA buffer have one additional protofilament compared to those assembled in GAB buffer. The difference in MT diameter in these two buffers is possibly related to the fact that glycerol excludes water and leads to a tighter interaction and a smaller MT. An increase in MT diameter is seen in the presence of ZMP, with the number of protofilaments increasing by approximately one. This is understandable given that ZMP is known to have extensive hydrophobic and polar interactions between its side chain and the M-loop (Figure 6.11). It is well-known that binding of PTX facilitates a greater interaction between the M-loop and loops in neighboring $\beta$-subunits, increasing the lateral protofilament interactions, and it is this that is crucial in its stabilizing activity (Nogales et al., 1999; Sui \& Downing, 2010). PTX decreases the average protofilament size by one (Andreu et al., 1992). Although both compounds interact with the M-loop it is likely that ZMP pushes the loop outward toward its respective neighboring protofilament, inducing a larger interprotofilament angle. An increased diameter by 1-2 protofilaments is expected from the perturbation of the lateral interprotofilament interactions described in the crystal structure of the adduct (Prota et al., 2013).

Surprisingly, MTs assembled with DAC have two additional protofilaments making up their lattice. Since DAC lacks the ZMP side chain and is significantly less potent it would presumably not have the same extensive interaction with the M-loop, and this is supported by the crystal structure and the biochemical data. Therefore the number of protofilaments making up a DAC-MT lattice was not expected to change. It is possible that there could be a reorganization of the binding cleft and possible movement of loop positions that result in an increase in interprotofilament angle and thus MT size. This would be feasible since MT size does not correlate with cytotoxicity (Matesanz et al., 2011a). It would be interesting to crystalize the DAC-tubulin adduct to observe if there are any significant movement in loops.

\subsubsection{Effect of adduct formation on tubulin aggregation}

Since ZMP covalent modification of tubulin essentially produces an activated tubulin dimer, it has different aggregation properties compared to unligated dimers. Like unligated tubulin, the ZMP adduct requires $\mathrm{Mg}^{2+}$ for assembly and has no notable effect 
on the longitudinal tubulin-tubulin interactions responsible for the early aggregation events. The adduct only requires a low concentration of free $\mathrm{Mg}^{2+}$ to completely assemble into MTs; therefore, ZMP must stabilize lateral interdimer contacts responsible for the MT formation from oligomers. This is consistent with the crystal structure which indicates helical structuring of the M-loop to facilitate MT-assembly. The adduct is essentially a 'pre-organized' tubulin dimer, ready for MT assembly; whereas, the unligated dimer has not yet organized its M-loop for lateral contacts. Thus, the first step in nuclei formation from the tetramer to the oligomer state of tubulin is more straightforward for the adduct than for unligated-tubulin, with a higher percentage of larger oligomers formed with the adduct. Lack of effect on the formation of the $T_{2} R-R B 3$ complex indicates the adduct is neither more curved nor more straight, and this is consistent with the crystallographic results of Prota et al. (2013). DAC was shown to be far less potent at inducing aggregation compared to ZMP, meaning that DAC-ligated dimers are less prone to assembly. This was expected from the results presented throughout this thesis due to the importance of the C19 side chain of ZMP.

Figure 6.7A\&B shows the effect of GTP versus GDP on the ZMP-adduct, although there the two peaks had a different shape and size, with the GDP peak wider and shorter. This discrepancy has been observed before with the different nucleotides, and is considered experimental error (F. Díaz personal communication). It is likely due to the fact that the tubulin dimers have a higher affinity for GDP than GTP in low free $\mathrm{Mg}^{2+}$ concentrations, and this would presumably make GDP-bound tubules more stable and compact. This may occur because when tubulin has GTP at the N-site and GDP at the E-site it reversibly binds one $\mathrm{Mg}^{2+}$ with high affinity; whereas, when tubulin has GTP bound at both sites it binds another $\mathrm{Mg}^{2+}$ ion with high affinity (Menéndez et al., 1998). This was also observed in the experiment showing that GTP-bound dimers had far less free $\mathrm{Mg}^{2+}$ (Figure 6.7A\&B legend).

\subsubsection{ZMP binding results in loss of the E-site affinity for nucleotide}

The side chain of 228 protrudes into the exchangeable nucleotide binding pocket and forms a bifurcated $\mathrm{H}$-bond with the bound nucleotide; it is likely that the ZMP interaction with both Asn229 and His228 would alter the binding affinity of this site for bound nucleotide. In the conditions of this experiment dimeric tubulin has GTP at both its nucleotide sites. GTP is then hydrolyzed during MT assembly, thus the MT has, 1 mole 
GDP and 1 mole GTP per mole of dimer. The adduct loses its binding affinity for the nucleotide, and nucleotide is lost (Table 6.4). The E-site loses binding affinity for the guanine nucleotide but not the ability to bind nucleotide. This is shown by the fact that if extra nucleotide is added to these dimers they can recover binding. In ZMP-modified dimeric tubulin, there is a greater loss of E-site nucleotide (100\% loss) compared to that lost in MTs (12\% loss), and this is thought to be due to longitudinal contacts and blockage of the E-site in the MT. Loss would be only occurring at the E-site since the Nsite is always blocked by intradimer contacts DAC displays similar nucleotide loss in GAB-assembled MTs (10.5\% loss); however, there is slightly less loss in dimeric tubulin (60\% loss), presumably because it binds less effectively to the dimer than ZMP. These results explain how ZMP and DAC can cause assembly of MTs in conditions where no nucleotide is present and additionally explain why ZMP can bind more easily to dimeric tubulin. Under these conditions, it can more easily displace the nucleotide than when the E-site is blocked by longitudinal contacts. CYC also modifies Asn228 and another amino acid in the pore site in MTs; however, it only modifies the pore residue in dimeric tubulin (Buey et al., 2007). It is interesting to note that CYC had no effect on GAB-assembled MTs, retaining $100 \%$ of its bound nucleotide, but in dimeric tubulin it displaces $20 \%$ of bound nucleotide. The lack of nucleotide loss in MTs in conjunction with the results seen with ZMP and DAC suggest that it is in fact the covalent modification of residue 229 that is causing loss of affinity for the bound nucleotide. This is consistent with 229 being the major product of covalent modification by ZMP and DAC and also consistent with the possibility that labeling of Asn228 is in fact an artifact of the MS (section 5.4).

\subsubsection{Effect of adduct formation on other drug binding sites}

To develop ZMP as a MSA, possible changes to global tubulin structure and binding at other sites on tubulin need to be investigated. This might provide insight into the potential for synergistic relationships between the compounds and also may provide information on the global effects of ZMP. The affinity of the LAU/PEL site does not change upon covalent modification of the MT by ZMP. This could be considered consistent with the lack of synergy observed between the compounds, although there is no known link between synergy and binding site affinity. Presumably if one ligand binds at a site and increases the affinity for another ligand, these compounds would likely synergize, due to the linkage of binding. For example stathmin and VBL increase the 
affinity of tubulin for one another (Barbier et al., 2010), and cells with overexpressed stathmin have increased sensitivity to the Vinca alkaloids (Devred et al., 2008).

\section{Vinca domain}

Occupation at the Vinca site prevents hydrolysis and nucleotide exchange (Cormier et al., 2010), thus ZMP may be able to act synergistically with Vinca domain agents, since it decreases affinity for the bound nucleotide, although there is no evidence for this. Using AUC, an initial experiment indicated that the $T_{2} R$ complex may form more easily with VBL when reacting with ZMP-modified tubulin compared to unmodified tubulin. This suggests that VBL reacts slightly more with the $T_{2} R$ complex that consists of ZMPmodified dimers than it does with the $\mathrm{T}_{2} \mathrm{R}$ complex composed of unligated dimers; however, no conclusions could be drawn from this experiment as there was an optical problem with the AUC machine at the time of these measurements. Although two peaks were seen (dimers and $\mathrm{T}_{2} \mathrm{R}$ ) they were not at the expected $S$ values. Generally the dimer and the tetramer have $\mathrm{S}$ values of 5.5 and 7.7, respectively. When investigating the effect of adduct formation on tubulin aggregation, similar values were obtained. However in this particular Vinca domain experiment, the values were higher for the dimer and tetramer (8.3-8.8 versus 10.4-11.8, respectively. which are far too high. A repeat for this experiment is currently underway.

\section{Colchicine binding site}

The change in fluorescence of MTC when bound to the COL site was similar to what is seen when free $\mathrm{Mg}^{2+}$ is added to MTC-bound tubulin. This change in florescence in increased $\mathrm{Mg}^{2+}$ conditions occurs due to the microenvironment of MTC binding being sensitive to the $\mathrm{Mg}^{2+}$ bound with high affinity to the $\alpha$-subunit, increasing the fluorescence of the compound (Menéndez et al., 1998). Measurement of fluorescence is an excellent tool because even extremely small changes in binding can cause significant changes in intensity that can be easily detected. MTC rings are more coplanar when bound than when free in solution, and this is what causes the increase in fluorescence upon binding to tubulin. The MTC microenvironment would only need to change by $<1$ $\AA$ to cause a measurable change in fluorescence. It is possible that when $\mathrm{Mg}^{2+}$ is added or when ZMP is pre-bound to the tubulin, the configuration of the COL binding site may change slightly, resulting in the MTC rings becoming more co-planar and thus increasing 
fluorescence. Alternatively, there may be a charge close to the MTC rings when the compound is bound that might be quenching some of the fluorescence. When $\mathrm{Mg}^{2+}$ is added or when ZMP binds, the charge may move further away from the bound MTC, reducing the quenching and thus increasing the fluorescence. From these experiments, it was clear that covalent modification at the taxoid site by ZMP does not affect the affinity of the COL binding site for MTC; however, it may cause slight changes in the site. Although no synergy has been observed between COL site agents and MSAs, this would be an interesting avenue to investigate with ZMP. It is also known that COL binding enhances GTP hydrolysis (David-Pfeuty et al., 1979) and we have now shown that ZMP affects occupation at the E-site. These relationships may have an additive or synergistic effect when both compounds are added together, depending on whether the loss of affinity for nucleotide has a positive or negative effect on hydrolysis.

\subsubsection{Conclusions}

The crystal structure of ZMP has confirmed its binding to the taxoid site and indicated specific interactions with tubulin. In addition, the elucidation of this structure, along with that of EPOA, has revealed the structural basis for MSA-induced MT assembly. This will be helpful in developing new generation MSAs by structure-guided drug engineering. Additionally, these structures have confirmed that the luminal binding site exists in unassembled tubulin as shown by the MS in Chapter 5 .

$\mathrm{Mg}^{2+}$ is still required for optimum assembly of the ZMP-tubulin adduct, which aggregates more readily in the presence of these ions. ZMP covalent modification of tubulin does not affect the affinity for the LAU/PEL site or the COL binding site; however, the E-site shows decreased affinity for its ligand but not loss of ability to bind it. Although ZMP does not alter the affinity of MDA binding sites, it would be interesting to investigate if ZMP synergizes with MDA given the link between ZMP and the E-site and the effect of targeting the Vinca domain and the COL binding sites on GTP hydrolysis. This study has provided insight into how covalent modification of the MT affects MT size, aggregation and other MTA binding sites on the MT, information important when developing a novel MSA analog. 


\subsection{INTRODUCTION}

\subsubsection{Pharmacophore models}

A pharmacophore defines a group of compounds that exhibit a similar pharmacological profile and bind to the same binding site on their target. A pharmacophore model describes functional and structural features (essential, steric and electronic features) contributing to the molecular interaction and biological activity of a set of compounds (Wermuth, 2006). It does not represent a molecular configuration but is a concept accounting for the common molecular interaction of a group of compounds with their common binding site. A SAR analysis is a traditional medicinal chemistry practice that provides information on how modification to the structure of a compound affects its activity. This enables identification of the chemical groups within a compound that interact specifically with the target.

\section{MSA pharmacophore models}

Prior to the revelation of the tubulin electron crystallography models of Nogales and colleagues (Nogales et al., 1998a; 1999, Löwe et al., 2001), a number of common pharmacophore models were proposed for taxoid site MSAs (Winkler \& Axelsen, 1996; Ojima et al., 1999; Wang et al., 1999). These models suggested motifs of the compounds that overlapped and interacted with the same sites in tubulin, using the superimposition of PTX structure with the structures of other compounds, including the EPOs, DSC, eleutherobin, and nonataxel. These models, however, did not align with each other and did not take tubulin structure into consideration. In 2000, Giannakakou et al. suggested two possible models of binding based upon single amino acid mutations in the taxoid site of $\beta$-tubulin in resistant cell lines, taking the structural data into consideration. It was proposed that the taxane ring system was mimicked by the macrolide ring of the EPOs (Giannakakou et al., 2000; He et al., 2000).

Although MSAs are a structurally diverse class of compounds, they can be categorized into three groups based on their structure: (i) the terpenoids (including PTX, DTX, and eleutherobin), (ii) the macrolides (including EPO, LAU, PEL and ZMP), (iii) the polyhydroxylated alkatetraene lactones (including DSC and dictyostatin, the latter also classed as a macrolide). The majority of these compounds have a core 14-20 
membered ring system and a substituted hydrophobic side chain (see Figure 1.5). It was originally proposed that these common features led to binding to a common site on $\beta$ tubulin that caused MT stabilization. In this model, LAU and PEL would be considered very structurally similar to the EPOs and therefore likely to bind to the same site on tubulin. It is now well known that two biochemically distinct sites exist on tubulin for MSAs (Pryor et al., 2002; Gaitanos et al., 2004) and that compounds that bind these two sites may be structurally similar (for example, EPO versus the LAU or PEL), even more similar than some compounds that bind to the same site (for example EPO and PTX are structurally very different). The overall consensus from the extensive pharmacophore modeling is that the macrolide ring system of the EPOs is analogous to and overlays the taxane ring system in the taxoid binding site. In this respect, LAU and PEL also fit this consensus; however, they clearly bind to a different site (Pryor et al., 2002; Gaitanos et al., 2004). This puts the MSA 'pharmacophore' hypothesis in doubt. Given the structural diversity of MSAs, it has been suggested that instead of fitting a common pharmacophore for binding the taxoid site, it may be better to think of the binding pocket as unrestrained, in which bound compounds can exploit many potential contacts with an ideal but distinct set of residues specific to each compound. Although this concept makes pharmacophorerationalized drug design more difficult, an unrestrained taxane binding pocket can accommodate a wide variety of MSAs with diverse chemical structures. This is evident from the findings that taxoid site mutations in cells are only resistant to some taxoid site MSAs (Giannakakou et al., 2000) and the more recent findings that suggest that the taxane binding pocket is significantly larger than first anticipated (Calvo et al., 2012).

The use of analogs that bind to the MT can provide information on what motifs in the parent compounds are important in binding and in determining potency. Extensive SAR studies have been carried out with PTX and the EPOs. In PTX, the C13 side chain, C2 benzoyl group and the oxetane ring are essential for its stabilization activity (He et al., 2000; He et al., 2001; Chen et al., 1993a); whereas, C7 and C10 are non-essential (Chen et al., 1993b). In EPO, the motifs at C12 and C13 are amenable to modification; whereas, the $\mathrm{C} 1-\mathrm{C} 8$ portion of the ring is intolerant to modification; hence, the size of the macrolide ring is essential for its biological activity, as is its side chain C16-18 (He et al., 2001). This is consistent with recent crystal structure evidence (Prota et al., 2013). Using this information, new generation MSAs can be developed on the premise that they will have improved activity and binding properties. However, these pharmacophore models 
were developed from studies in different cancer cell lines, and although these studies provided insight into a common pharmacophore for the taxoid binding pocket they prevented direct correlation of structural changes and cytotoxicity. Studying the thermodynamics of compounds with stabilized crosslinked MTs has allowed the effects of single substitutions to be studied and quantified in a systematic manner (Buey et al., 2004; Matesanz et al., 2008). In doing so, it has been shown that a gain in free binding energy also increases the cytotoxicity of taxoid site compounds (Buey et al., 2004) and that increasing the binding affinity of a compound for tubulin lowers the intracellular concentration of the compound and results in decreased P-gp drug efflux (Matesanz et al., 2008). Given these two factors, substituted taxane compounds can be studied with the aim of identifying the 'optimal' high affinity taxane compound that overcomes P-gpmediated resistance. In this manner a library of 44 substituted taxanes was synthesized and tested. These compounds confirmed the importance of $\mathrm{C} 2$ and $\mathrm{C} 13$ substituents, with modification to the latter the most effective in modulating activity. Additionally, modification to substituents at $\mathrm{C} 7$ and $\mathrm{C} 10$ has been confirmed to have little effect on binding (Matesanz et al., 2008).

\section{Current taxoid site pharmacophore}

Superimposition of the known crystal structures of MSAs binding to the taxoid site (Figure 7.1) gives insight into how these different ligands interact with the binding pocket. All ligands are known to interact with the M-loop, with ZMP and EPOA causing potent restructuring of the loop into a short helix. This is now considered the underlying molecular basis for MSA-induced stabilization, as discussed in the previous chapter, and thus PTX would therefore cause the same effect. The M-loop helix is made up of residues Arg278-Tyr283. All three compounds hydrogen bond to M-loop residue 276, and it is likely that this interaction is central in the stabilization and restructuring effect. As shown in Figure 7.1, the side chains of ZMP and EPOA superimpose well with their macrolide rings at $90^{\circ}$. The macrolide cores and the ring system of PTX, notably C2 and C13 substituents, occupy the same space in the pocket. C7 and C10 substituents of PTX appear to occupy a space in the pocket that is not involved in ZMP and EPO binding. These observations are concordant with those of Matesanz et al., (2008). 

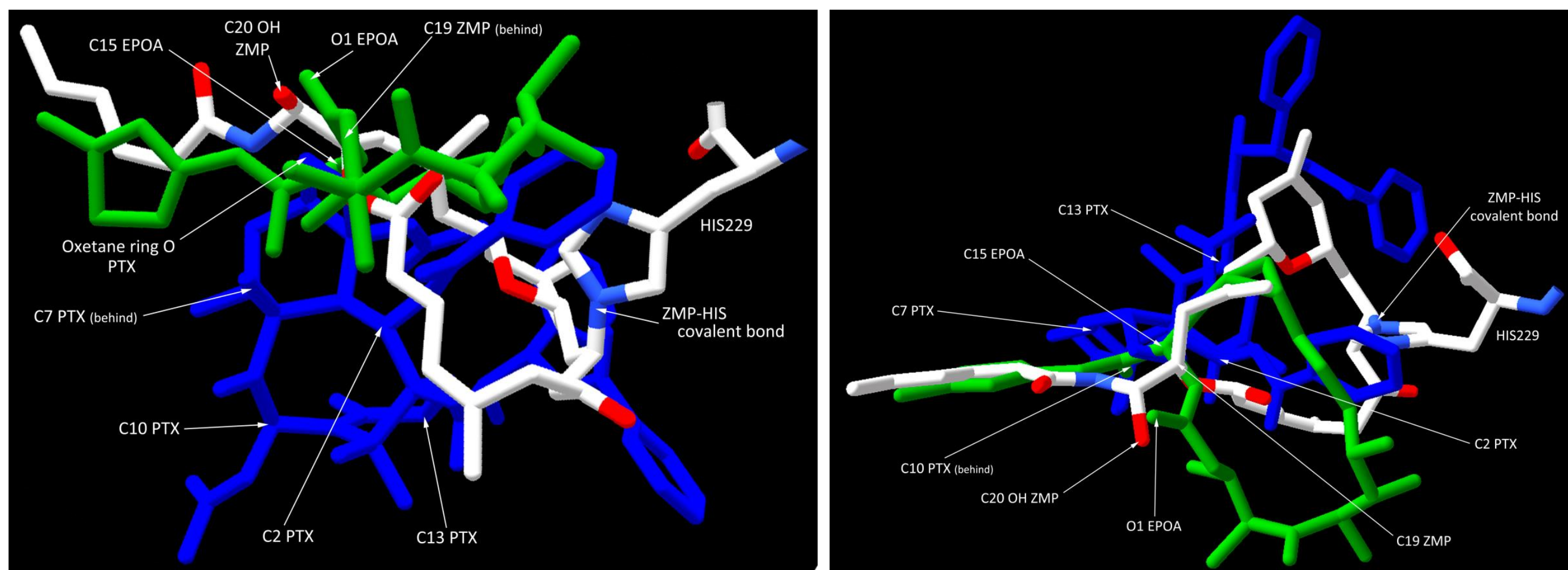

\section{Figure 7.1 ZMP, EPOA and PTX in the taxoid binding site}

Orthogonal views of three taxoid site ligands bound in the taxoid binding site, indicating a common pharmacophore for this binding site. Compounds are shown in stick representation: ZMP (CPK), EPOA (grey) and PTX (blue). His229 (CPK) is also shown covalently bound to C9 of ZMP. Important moieties are labeled. The side chains of ZMP and EPOA superimpose and have a similar interaction with the M-loop. Their macrolide cores are at $90^{\circ}$ to one another, and superimpose well with the PTX ring system. All compounds hydrogen bond to Thr276 in the M-loop (not shown for simplicity sake), and it is likely that this interaction contributes to stabilization of the M-loop. PDB files 4I4T (ZMP), 4I50 (EPOA) and JFF (PTX). 


\subsubsection{The zampanolide chemotype}

Earlier in this thesis, it was shown that DAC lacks what appears to be the essential component for stabilization, the ZMP C19 N-acyl hemiaminal side chain. This results in DAC being a weak MSA with significantly slower binding kinetics, indicating that the side chain is important but not essential for the basic interaction with tubulin; however, it is required for a strong stabilization effect. This is likely due to the lack of M-loop interaction in the absence of the side chain, as evident from the crystal structure (Prota $e t$ al., 2013).

In 2010, Altmann et al. synthesized the 13-desmethylene analog of DAC (3) and two reduced alcohols: the C19-reduced analog (2) and the reduced 13-desmethylene analog (4) (Figure 7.2) (Zurwerra et al., 2010). These compounds had similar antiproliferative activity to DAC, inhibiting human cancer cell growth with $\mathrm{IC}_{50}$ values in the sub-micromolar range. They were also shown to induce in vitro tubulin polymerization. Interestingly, the biological studies indicated that loss the exocyclic methylene group at $\mathrm{C} 13$ and the aldehyde at C20 had little effect on tubulin polymerization. In 2012, ZMP and a number of analogs were synthesized using the same strategy described by Zurwerra et al. (2010), and (-)-DAC (the unnatural enantiomer of DAC) was converted to (-)-ZMP (the natural enantiomer of ZMP) (Zurwerra et al., 2012). Two of these ZMP analogs were studied in this thesis, epi-ZMP and the monocyclic des-tetrahydropyran analog (1) (Figure 7.2). It had previously been reported that epi-ZMP was 10-fold less active than ZMP (Uenishi et al., 2009), and this was confirmed by Zurwerra et al. (2012) As expected from previous observations (Ding \& Jennings, 2008), the natural enantiomer of DAC was significantly less active than ZMP, and was additionally found to be less active that epi-ZMP. Indicating that the hemiaminal side chain in ZMP is essential for its potent cytotoxicity and this was confirmed earlier in this thesis. Thus, the absolute configuration of the macrocycle, discussed in section 1.6.1, is not as important as first thought, and the potent stabilization activity appears to be largely due to the C19 side chain. Similar to the in vitro studies, cellular proliferation studies suggested that the aldehyde functionality at C19 and the $\mathrm{C} 13$ exocyclic methylene group were not required for the anti-proliferative activity of DAC (Zurwerra et al., 2012). Compound 1 with complete loss of the tetrahydropyran ring had sub-micromolar activity 25-80 fold less potent than ZMP. This retention of some of the antiproliferative activity, however, is surprising since a large region of the parent compound is missing. 


\subsubsection{Aims of this chapter}

The aim of the research in this chapter was to determine the in vitro activity of the five ZMP analog compounds shown in Figure 7.2, and to relate these activities to SARs for this new and important MSA chemotype. It is already known that the C19 ZMP side chain is essential in its potent stabilizing activity and cytotoxicity, and is directly involved in its binding interaction; however, the significance of the remainder of the compound motifs have not been studied in great detail. Preliminary cellular proliferation studies demonstrated that reduction of the C19 DAC aldehyde functionality and loss of the exocyclic methylene group off $\mathrm{C} 13$ had no substantial effect on the activity of the compounds (Zurwerra et al., 2010; 2012) (Table 7.5). It is important to confirm these findings in vitro and to further investigate the effect of these motif changes for the development and understanding of ZMP and its mechanism of action but also for the development of new generation MSAs with improved properties. Also, since its identification in our lab as an MSA (Field et al., 2009), the synthesis of ZMP and its analogs appears to be a popular objective of a number of different laboratories (Uenishi et al., 2009; Zurwerra et al., 2010; Ghosh \& Cheng, 2011; Ghosh et al., 2012; Zurwerra et al., 2012), including that of Assoc. Professor Joanne Harvey at VUW. Additional SAR information would be beneficial for further synthesis of new, cytotoxic compounds in the ZMP chemotype. 

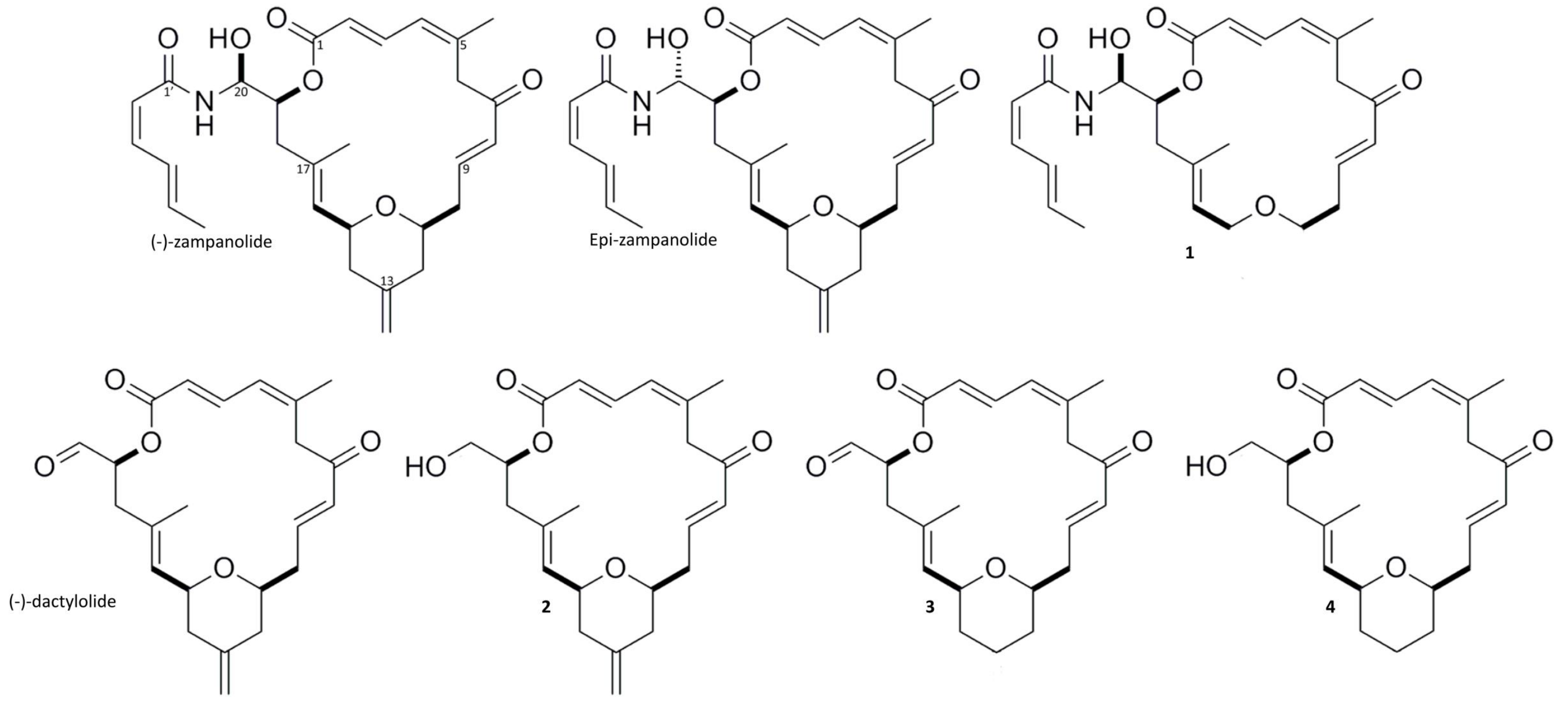

Figure 7.2 Structures of ZMP, DAC and their analogs (Zurwerra et al., 2010, 2012).

Upper row: Structure of ZMP and analogs. Lower row: Structure of DAC and analogs. ZMP has an unusual $N$-acyl hemiaminal side chain at C19, with the alcohol facing downwards. In epi-ZMP this secondary alcohol is facing upwards, with the rest of the molecule remaining the same. Compound $\mathbf{1}$ is essentially identical to ZMP but is missing the 4-methylenetetrahydropyran ring and C13 substituent at the lower part (C12-14) of the molecule, resulting in a monocyclic compound. DAC lacks the side chain of ZMP at C19 which is replaced by an aldehyde; otherwise, the compound remains the same. Similar to DAC, compound 3 (13-desmethylene DAC derivative) has an aldehyde at position C19, but it lacks the exocyclic methylene group at C13. Compounds 2 and $\mathbf{4}$ are $\mathrm{C} 19$ reduced derivatives of DAC and compound 3, respectively. The ZMP compound denotes the numbering used for these compounds throughout this chapter. Note that for consistency, compound $\mathbf{1}$ is numbered in the same manner even though the tetrahydropyran ring (C12-14) is missing. 


\subsection{METHODS}

\subsubsection{Effects on microtubule assembly}

All analog compounds were tested for their ability to induce MT assembly in conditions favorable to tubulin assembly. Their ability to induce assembly in PEDTA buffer was not evaluated, given DAC was already found to be significantly less potent that ZMP. The assays were carried out as described in Chapter 2.6.1.

\subsubsection{Binding at the taxoid binding site}

Given that both ZMP and DAC are ligands of the taxoid site, the ability of the analogs to bind this site was investigated. The FTX-2 displacement assay was carried out as described in chapter 2.7. DTX, ZMP and DAC were used as positive controls. ZMP and control drugs were used at the following concentrations: $0 \mathrm{nM}, 10 \mathrm{nM}, 20 \mathrm{nM}, 50 \mathrm{nM}$, $0.1 \mu \mathrm{M}, 0.2 \mu \mathrm{M}, 0.5 \mu \mathrm{M}, 1 \mu \mathrm{M}, 5 \mu \mathrm{M}$, and $20 \mu \mathrm{M}$. DAC and the analogs were used at 1 $\mu \mathrm{M}, 5 \mu \mathrm{M}, 10 \mu \mathrm{M}, 20 \mu \mathrm{M}, 25 \mu \mathrm{M}$ and $100 \mu \mathrm{M}$, given their lower potency.

\subsubsection{Binding kinetics}

The kinetics of the reaction of the analogs with stabilized cross-linked MTs was measured by determining the inhibition of FTX-2 binding to MTs. Stabilized taxoid binding sites $(5 \mu \mathrm{M})$ in $\mathrm{GAB}$ were incubated with $6 \mu \mathrm{M}$ test compound at different times as described in Chapter 2.8.

\subsubsection{Mass spectrometry}

To characterize the interaction and confirm the binding site of the analogs with MTs, tubulin oligomers and tubulin dimers, targeted MS experiments as described in Chapter 2.10 were performed. Given the analogs are not as potent as the parent compounds in inducing tubulin assembly; they were bound to pre-formed stabilized MTs rather than used to induce tubulin assembly from dimers.

\subsubsection{Small angle X-ray scattering}

SAXS experiments were carried out as in described in Chapter 2.12. GAB buffer was used because the analog compounds are weak MSAs. Given the lack of supply of some compounds and the limited availability of the SAXS machine, not all compounds were analyzed. The compounds used in the experiment encompass the major changes in the chemotype of these MSAs. 


\subsection{RESULTS}

\subsubsection{Effects on microtubule assembly}

To confirm that the analog compounds retained the same mechanism of action as ZMP and DAC, their ability to enhance MT assembly was investigated in conditions in which tubulin self-assembles with a $\mathrm{C}_{\mathrm{r}}$ of $3.3 \mu \mathrm{M}$ (Buey et al., 2005). ZMP is known to induce the formation of MTs with a similar potency to DTX $\left(\right.$ ZMP $C_{r}=0.81 \mu \mathrm{M}$; DTX $\mathrm{C}_{\mathrm{r}}=$ 0.60). Epi-ZMP had a significantly higher $C_{r}(1.82)$ but was more potent that DAC $\left(C_{r}=\right.$ 2.10). Compound 2 had an extremely similar $C_{r}$ value to DAC, with both compounds reducing $\mathrm{C}_{\mathrm{r}}$ by roughly $30 \%$ to the level of weak assembly inductors such as the sarcodictyins or cyclostreptin (Buey et al., 2005). Compounds 1, 3 and 4 were unable to reduce the $\mathrm{C}_{\mathrm{r}}$, with values within experimental error of the control conditions (DMSO) (Figure 7.3, Table 7.1).

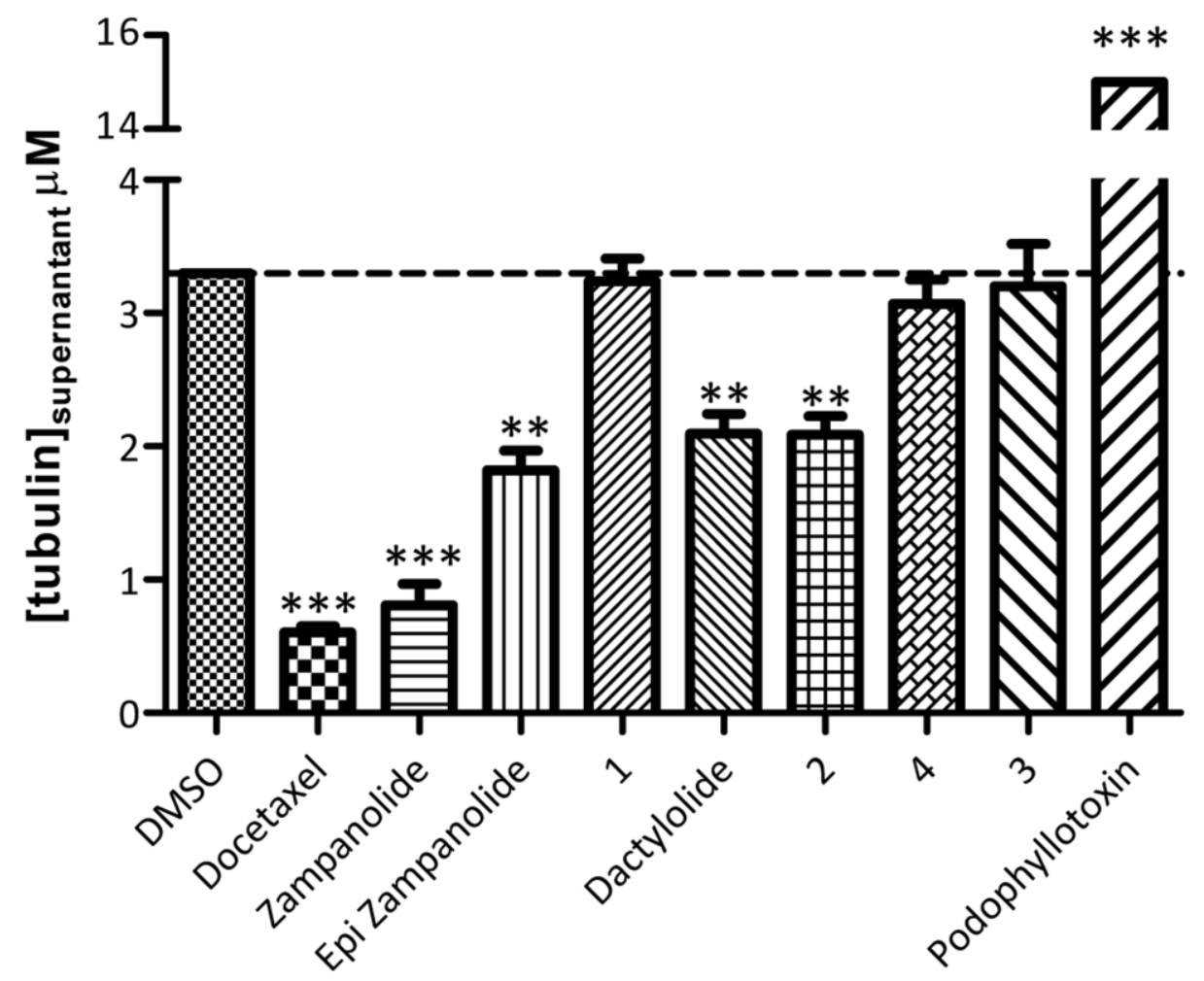

Figure 7.3 Enhancement of MT assembly by ZMP and analogs

Induction of MT assembly in conditions that are favorable for tubulin assembly. In these conditions the $\mathrm{C}_{\mathrm{r}}$ of tubulin for self-induced assembly is $3.3 \mu \mathrm{M}$, shown by the DMSO bar and the dashed line. DTX and ZMP both significantly decrease the $\mathrm{C}_{\mathrm{r}}$, DAC is not as potent as ZMP at causing assembly but is still able to significantly decrease the $\mathrm{C}_{\mathrm{r}}$ required for assembly. Epi-ZMP significantly reduced the $\mathrm{C}_{\mathrm{r}}$ to lower than that of DAC but was not as potent as ZMP. Compound $\mathbf{2}$ had a similar potency to DAC, indicating that it is a weak MSA. Compounds $\mathbf{1}, \mathbf{3}$ and $\mathbf{4}$ did not significantly decrease the $\mathrm{C}_{\mathrm{r}}$, indicating that they cannot enhance tubulin assembly in these conditions. PDP is used as a negative control. Note that the ZMP and DAC data are the same as that presented in Figure 4.9. The y-axis is broken for easy comparison of the analogs. 
Table 7.1 $\mathrm{C}_{\mathrm{r}}$ values of ligand-induced MT assembly

\begin{tabular}{cc}
\hline Ligand & $C r \pm S E M(\mu M)$ \\
\hline DMSO & 3.3 \\
Docetaxel & $0.60 \pm 0.05$ \\
Zampanolide & $0.81 \pm 0.16$ \\
Epi-zampanolide & $1.82 \pm 0.15$ \\
$\mathbf{1}$ & $3.24 \pm 0.17$ \\
Dactylolide & $2.10 \pm 0.15$ \\
$\mathbf{2}$ & $2.09 \pm 0.14$ \\
$\mathbf{4}$ & $3.07 \pm 0.18$ \\
$\mathbf{3}$ & $3.20 \pm 0.32$ \\
Podophyllotoxin & $>15$ \\
\hline
\end{tabular}

$\mathrm{C}_{\mathrm{r}}$ values for the analogs shown graphically in Figure 7.2. Values for DTX, ZMP and DAC are also presented for comparison sake. Values are the mean $\mathrm{C}_{\mathrm{r}} \pm \mathrm{SEM}$, from at least 3 independent assays.

\subsubsection{Binding at the taxoid binding site and binding kinetics}

ZMP covalently bound to MTs with an apparent binding constant of $214 \times 10^{6} \mathrm{M}^{-1}$ at $35^{\circ} \mathrm{C}$ (Table 4.2). DAC had a binding constant 150 times lower at $1.35 \times 10^{6} \mathrm{M}^{-1}$ and with a slower apparent kinetic rate constant on the order of $0.12 \mathrm{hr}^{-1}$. The ability of the analogs to displace FTX-2 was therefore investigated, first to confirm they also bind the taxoid site, and second to compare their binding kinetics, since such a large difference was seen when the side chain of ZMP is removed. The binding kinetics for each analog was measured using the FTX-2 displacement assay and by monitoring the number of sites available for FTX-2 binding over a $24 \mathrm{~h}$ period. With reversible MSAs the FTX-2 displacement assay measures the affinity of the ligand for binding to the taxoid binding site; however, with covalent MSAs the assay does not measure a true dissociation constant or the affinity but measures the kinetics of the binding reaction (the measured 
value is the ligand concentration needed to react with $50 \%$ of the available binding sites in the time of the assay). This is due to the covalent nature of their binding (Calvo et al., 2012).

All compounds displaced FTX-2 from its binding site with different apparent binding affinities (for binding affinities, see Figure 7.4, Table 7.2). Epi-ZMP had the highest apparent binding constant at $35^{\circ} \mathrm{C}\left(1.43 \times 10^{6} \mathrm{M}^{-1}\right)$ of all the analogs, followed by compound $2\left(1.04 \times 10^{6} \mathrm{M}^{-1}\right)$, which lacks the ZMP side chain. At $24 \mathrm{~h}$ the majority of compound 2 was covalently bound to the taxoid site, with only $27 \%$ of total sites available for FTX-2 binding compared to $45 \%$ with epi-ZMP (for binding kinetics, see Figure 7.5, Table 7.3). Both compounds covalently modified tubulin faster than DAC. Compounds 3 and 4 had slow kinetics (10-20\% of taxoid sites covalently bound after 24 h) and significantly lower apparent binding constants $\left(0.40\right.$ and $0.65 \times 10^{6} \mathrm{M}^{-1}$, respectively), consistent with their weak ability to induce MT assembly. Compound 1 had the lowest apparent binding constant $\left(0.090 \times 10^{6} \mathrm{M}^{-1}\right)$ and showed very slow binding kinetics, confirming its weak stabilizing activity.

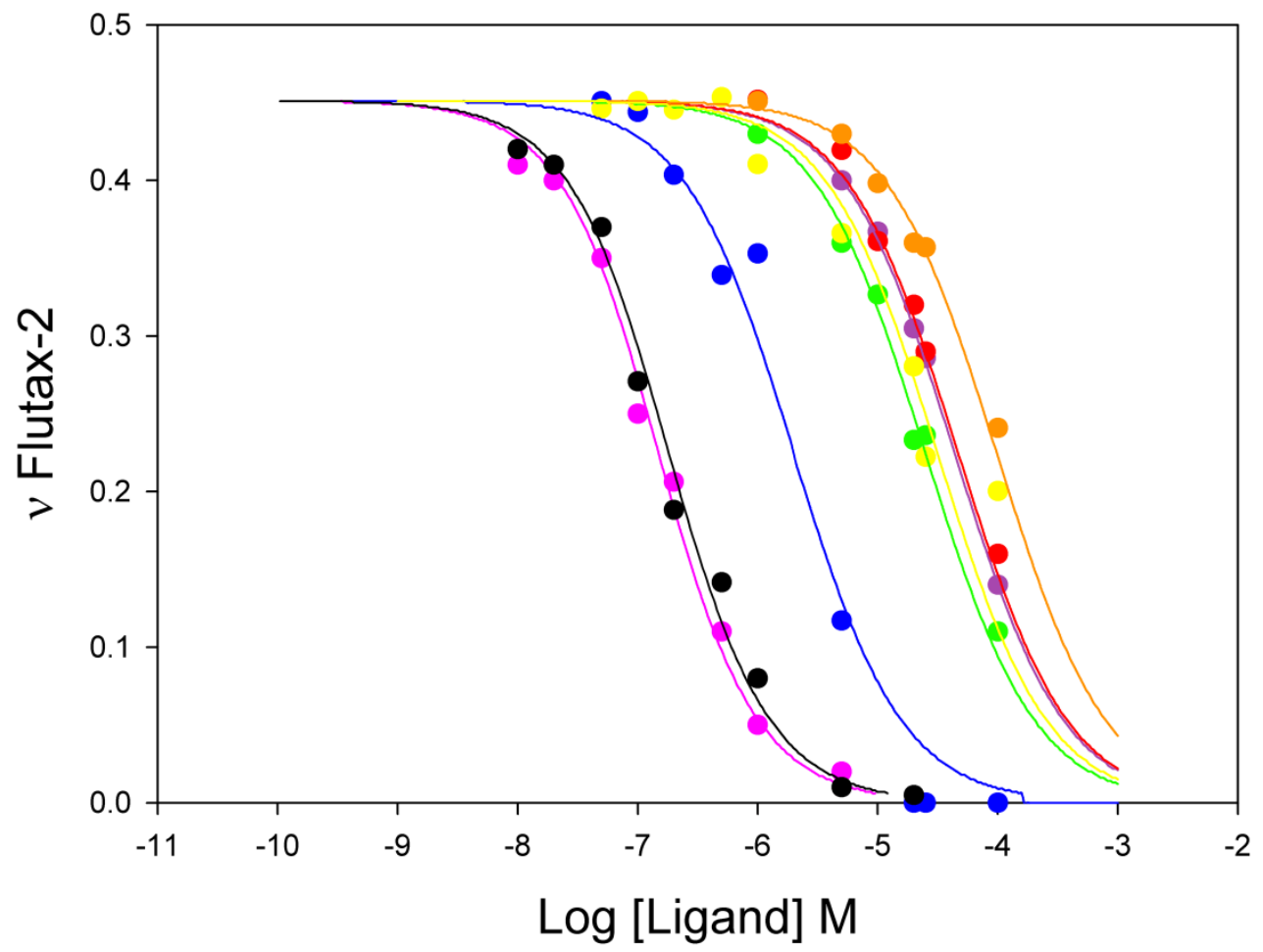

Figure 7.4 Flutax-2 displacement by the analog compounds

Representative competition experiments between FTX-2, known taxoid site MSAs and the analogs for binding to the taxoid site. Displacement of FTX-2 (50 nM) by DTX (black), ZMP (magenta), epi-ZMP (blue), 1 (yellow), DAC (green), 2 (purple), 4 (red) and $\mathbf{3}$ (orange). See also Table 7.1. ZMP, DAC, and DTX data are from Chapter 4, Figure 4.10. 
Table 7.2 Binding constants of the compounds to the taxoid site

\begin{tabular}{ccccccccc}
\hline Compound & $26^{\circ} \mathrm{C}$ & $27^{\circ} \mathrm{C}$ & $30^{\circ} \mathrm{C}$ & $32^{\circ} \mathrm{C}$ & $35^{\circ} \mathrm{C}$ & $47^{\circ} \mathrm{C}$ \\
\hline Docetaxel & $69.5 \pm 4.2$ & $65.7 \pm 5.2$ & $54.2 \pm 4.2$ & $48.9 \pm 3.8$ & $39.3 \pm 2.7$ & $30.9 \pm 2.2$ & $28.9 \pm 1.7$ & $23.8 \pm 1.1$ \\
Zampanolide & $137 \pm 29$ & $150 \pm 3.7$ & $143 \pm 48$ & $187 \pm 76$ & $214 \pm 93$ & $258 \pm 110$ & $416 \pm 173$ & $424 \pm 175$ \\
Epi-Zampa & $1.66 \pm 0.10$ & $1.84 \pm 0.09$ & $1.64 \pm 0.11$ & $1.75 \pm 0.13$ & $1.43 \pm 0.05$ & $1.31 \pm 0.10$ & $1.37 \pm 0.14$ & $1.40 \pm 0.12$ \\
Dactylolide & $0.57 \pm 0.10$ & $0.78 \pm 0.15$ & $0.97 \pm 0.13$ & $1.20 \pm 0.14$ & $1.35 \pm 0.18$ & $1.43 \pm 0.20$ & $1.82 \pm 0.18$ & $2.14 \pm 0.25$ \\
$\mathbf{2}$ & $0.45 \pm 0.13$ & $0.68 \pm 0.18$ & $0.79 \pm 0.17$ & $1.02 \pm 0.23$ & $1.04 \pm 0.25$ & $1.21 \pm 0.23$ & $1.44 \pm 0.28$ & $1.60 \pm 0.27$ \\
$\mathbf{3}$ & $0.23 \pm 0.02$ & $0.28 \pm 0.02$ & $0.35 \pm 0.04$ & $0.38 \pm 0.04$ & $0.40 \pm 0.05$ & $0.40 \pm 0.66$ & $0.44 \pm 0.05$ & $0.54 \pm 0.06$ \\
$\mathbf{4}$ & $0.35 \pm 0.06$ & $0.46 \pm 0.05$ & $0.47 \pm 0.06$ & $0.57 \pm 0.09$ & $0.65 \pm 0.11$ & $0.48 \pm 0.07$ & $0.50 \pm 0.09$ & $0.49 \pm 0.09$ \\
$\mathbf{1}$ & $0.052 \pm 0.005$ & $0.069 \pm 0.007$ & $0.071 \pm 0.005$ & $0.085 \pm 0.010$ & $0.090 \pm 0.003$ & $0.077 \pm 0.004$ & $0.102 \pm 0.004$ & $0.099 \pm 0.003$ \\
\hline
\end{tabular}

True binding constants for DXT for the taxoid binding site, and apparent binding constants for ZMP, DAC and analogs for the taxoid binding site. Both sets of measurements are derived from FTX-2 displacement studies. DTX, ZMP, and DAC results are from Chapter 4, Table 4.2. 


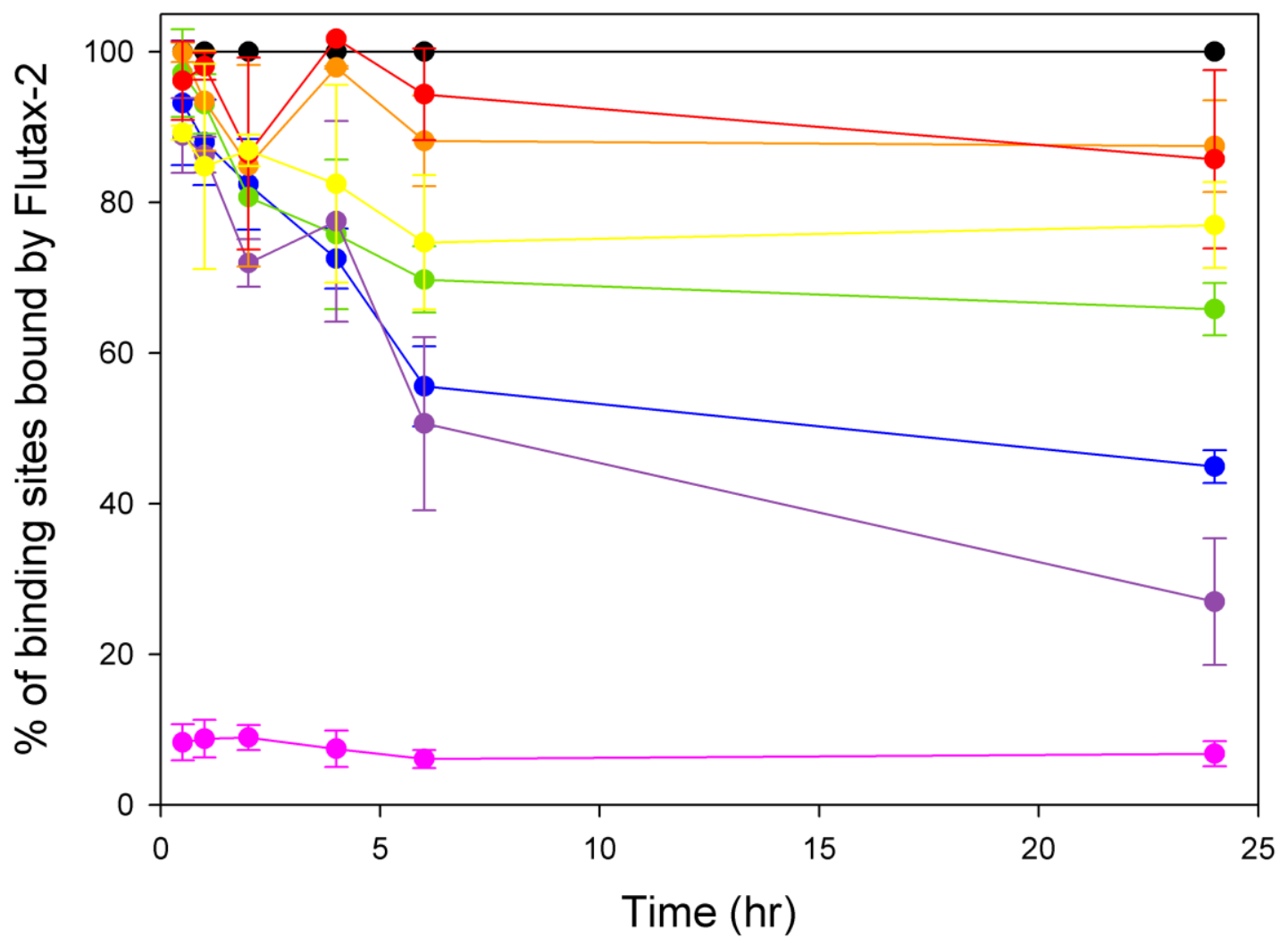

Figure 7.5 Binding Kinetics of ZMP and analogs

Binding kinetics of the covalent reactions with stabilized MTs measured as the remaining available binding sites for FTX-2 exchange after the reaction with the compounds over set periods of time. DTX (black), ZMP (magenta), epi-ZMP (blue), 1 (yellow), DAC (green), 2 (purple), 4 (red) and $\mathbf{3}$ (orange). See also Table 7.3. The lines represent the fit of decay of the FTX-2 binding sites. Error bars $=$ SEM from at least 3 independent experiments.

Table 7.3 \% of taxoid binding sites available for FTX-2 exchange

\begin{tabular}{cc}
\hline Zampanolide & $7 \%$ \\
$\mathbf{2}$ & $27 \%$ \\
Epi-zampanolide & $45 \%$ \\
Dactylolide & $66 \%$ \\
$\mathbf{1}$ & $77 \%$ \\
$\mathbf{4}$ & $86 \%$ \\
$\mathbf{3}$ & $87 \%$ \\
\hline
\end{tabular}

The percentage of free taxoid binding sites that are available for FTX-2 exchange (not covalently bound by the compound) after $24 \mathrm{~h}$ incubation. 


\subsubsection{Mass spectrometry}

The reactivity of the compounds for the $\beta$-tubulin taxoid site was confirmed using MS techniques. Tubulin-treated samples were digested with trypsin and subjected to liquid chromatography coupled to MS. All analog compounds labeled the same $\beta$-tubulin peptide as previously identified for ZMP and DAC (compare to results in Chapter 5 for ZMP and DAC), indicating that although some of them are either very weak or show no MSA activity, they still bind to the taxoid binding site (Figure 7.6). As for ZMP and DAC, all analog compounds reacted with both MTs and oligomeric-dimeric tubulin. Figure 7.6 displays the extracted ion chromatograms of the corresponding adducts. The data for Figure 7.6 were provided by Dr. Enrique Calvo.
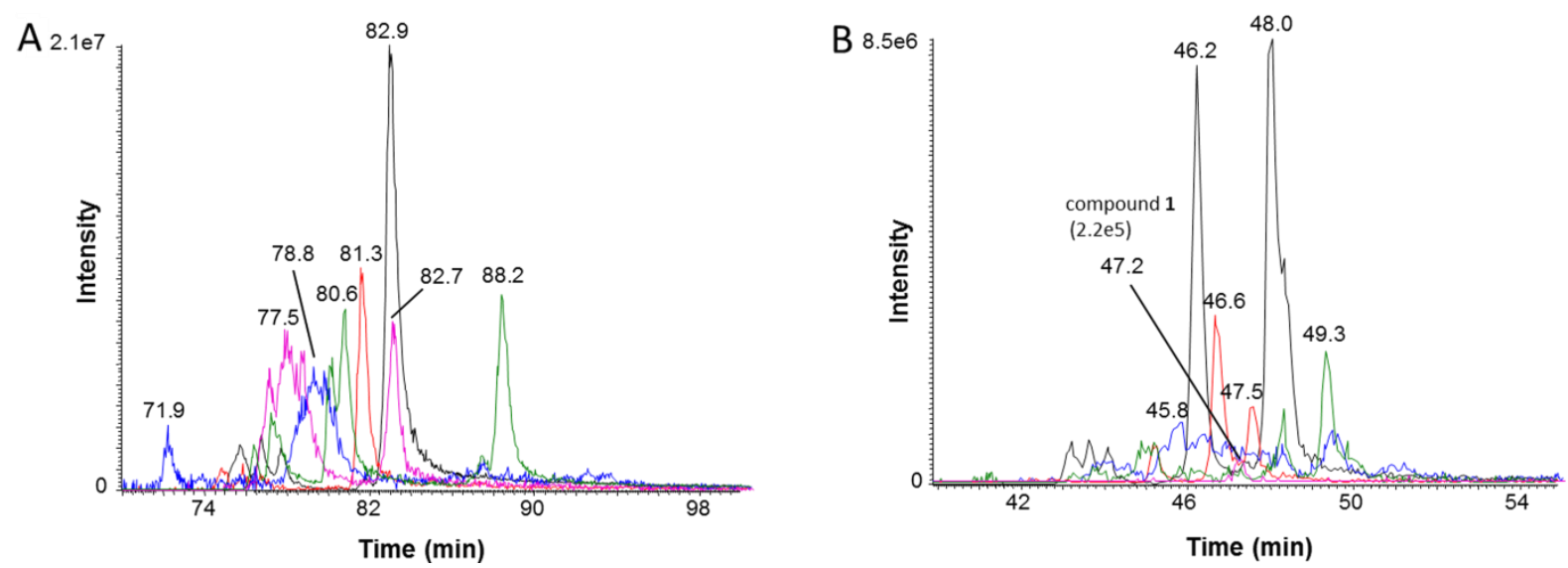

Figure 7.6 Mass spectrometry ion chromatograms of the analogs

Extracted ion chromatograms for the triply-charged $\beta$-tubulin adducts attached to the compounds at masses 1013.1835 (compound 2 (black)), 1009.1823 (compound 4 (red)), 1008.5101 (compound 3 (blue)), 1049.5307 (epi-ZMP (green)), and 1032.1913 (compound 1 (magenta)), in MTs (A) and in oligomeric/dimeric tubulin (B). Some derivatives yield different chromatographic peaks, but without any mass shift. Compound $\mathbf{3}$ yields a very wide chromatographic peak. Miss-matched retention times seen between MTs and oligomeric/dimeric tubulin are caused by the different length of the chromatographic run (130 versus $70 \mathrm{~min}$, respectively). 


\subsubsection{SAXS}

SAXS was used to measure possible changes in MT size and protofilament number induced by the analogs, as was done with ZMP and DAC in Chapter 6, Figure 6.6. Since the analog compounds are not as potent as ZMP and many of them lack the side chain, the interprotofilament angle between the analogs is not expected to change from their parent compounds. MTs assembled in GAB in the absence of ligand have an average number of protofilaments of 13.3. Interestingly, epi-ZMP increases the number of protofilaments by two, similar to DAC (Figure 7.7). MTs formed in the presence of compounds $\mathbf{3}$ and $\mathbf{1}$ have the same number of protofilaments as those assembled in the presence of ZMP, despite the side chain being absent in these compounds (Figure 7.7 and Table 7.4).

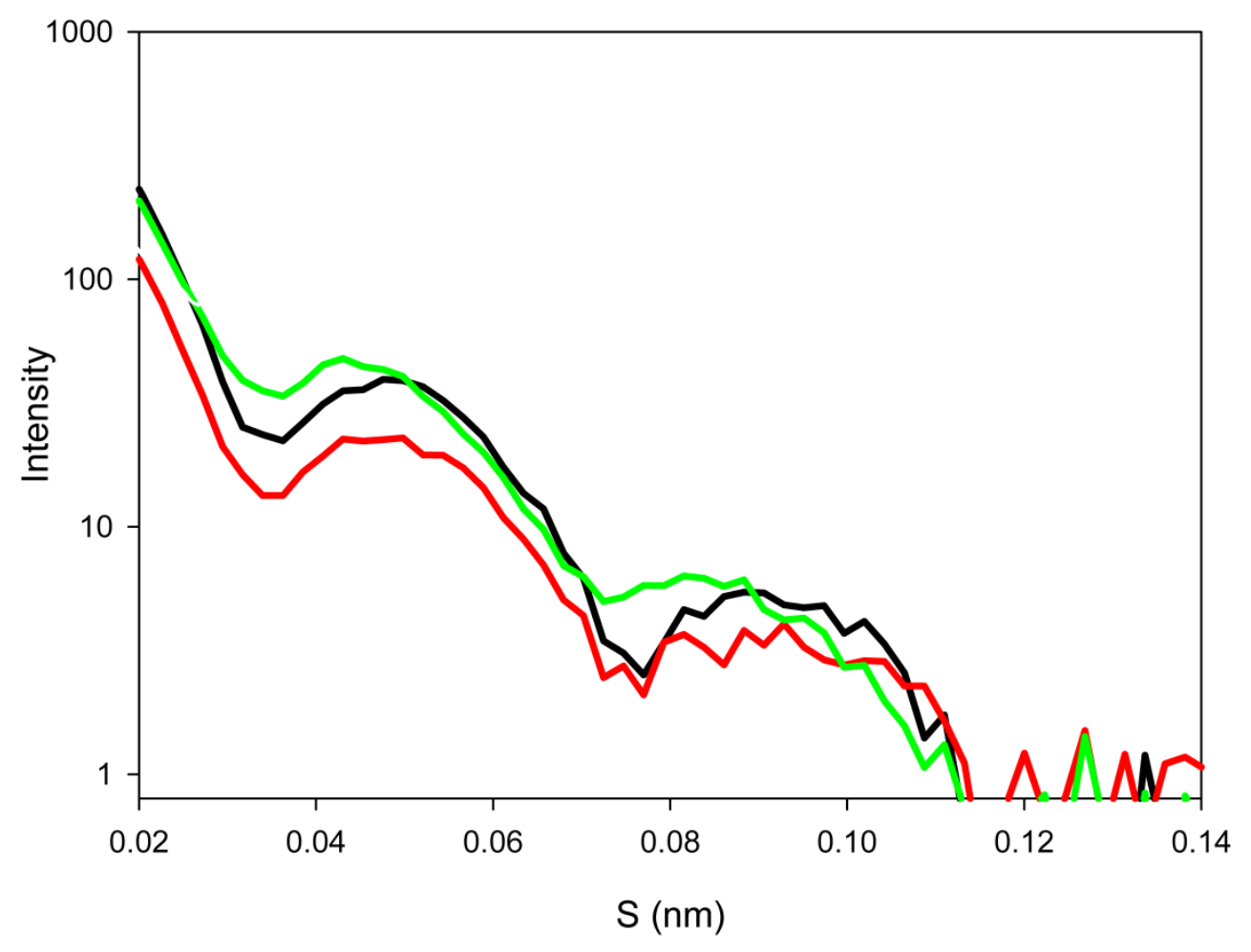

Figure 7.7 SAXS profile of MTs (GAB buffer)

Graph comparing the SAXS profiles of MTs assembled in the presence of compound 3 (black), compound 1 (red) and epi-ZMP (green). When comparing ligand-induced MTs versus control MTs, the $\mathrm{J}_{01}$ maxima were increased. From these data the average MT diameter and number of protofilaments making up the lattice were calculated and are presented in Table 7.4. 
Table 7.4 Structural data of the ligand-induced MTs

\begin{tabular}{cccc}
\hline Ligand & $J_{01}\left(\mathrm{~nm}^{-1}\right)$ & Mean helical radius $(\mathrm{nm})$ & $\begin{array}{c}\text { Average number } \\
\text { protofilaments }\end{array}$ \\
\hline DMSO (vehicle) & 0.0505 & 12.07 & 13.3 \\
Zampanolide & 0.0475 & 12.84 & 14.2 \\
Epi-zampanolide & 0.0439 & 13.89 & 15.3 \\
Dactylolide & 0.0448 & 13.61 & 15.0 \\
$\mathbf{1}$ & 0.0475 & 12.84 & 14.2 \\
$\mathbf{3}$ & 0.0475 & 12.84 & 14.2 \\
Docetaxel & 0.0505 & 12.07 & 13.3 \\
\hline
\end{tabular}

Values for $\mathrm{J}_{01}$ indicate the position of the maxima in the scattering profiles obtained from one measurement. The data were analyzed using SigmaPlot curve fitting software. Hyperbola curves were fitted to the second maxima peaks (first peak shown in the graph in Figure 7.7, this peak directly correlates to MT diameter). Nonlinear regression quadratic equations were used to calculate the angle, using $x=-a / 2 b$ which is equal to the value of $J_{01}\left(\mathrm{~nm}^{-1}\right)$. The diameter and number of protofilaments making up the MT lattice were then calculated as described in Chapter 2.11, with the helical radius value estimated from $\mathrm{J}_{01}=$ $1.22 / \mathrm{D}$, and the average protofilament number from the mean helical radius and the known MT interprotofilament spacing value of $5.7 \mathrm{~nm}$ as determined by Andreu et al., 1994. The data for DMSO, ZMP and DAC were taken from Table 6.1. 


\subsection{DISCUSSION}

\subsubsection{Structure-activity relationships of the analogs}

The antiproliferative activity of the ZMP analogs and a number of other analogs not assessed in this thesis (Figure 7.8) were previously determined by Zurwerra et al. (2010; 2012). The parent compound ZMP was the only compound with low nanomolar cytotoxicity. Interestingly, removal of the $\mathrm{C} 13$ exocyclic methylene group and reduction of the C20 aldehyde had no significant effect on the cytotoxicity, nor did removal of the tetrahydropyran ring (Zurwerra et al., 2012; Figure 7.8; Table 7.5). These results suggested that this chemotype can be modified to produce simpler chemical structures whilst retaining micromolar activity.<smiles>C=C1C[C@H](C/C=C\C)O[C@@H](/C=C(\C)C[C@H](COC)OC(=O)/C=C/C=C/C(=O)/C=C/C)C1</smiles><smiles>[2H][C@H]1CC(=C)C[C@H](C/C=C(\C)C[C@@H](OC(=O)/C=C/C=C(/C)CC(=O)/C=C/C)C(=O)O)O1</smiles><smiles>C=C1C[C@H](C)O[C@@H](/C=C(\C)C[C@H](OC(=O)/C=C/C=C(/C)CC(=O)/C=C/C)C(=O)NCCCCCC)C1</smiles>

Figure 7.8 Additional ZMP analogs

Analogs synthesized and studied by Zurwerra et al., 2012. Methylation of DAC produces compound A. Oxidation at $\mathrm{C} 19$ in DAC results in the carboxylic acid B. Both $\mathbf{A}$ and $\mathbf{B}$ are significantly less potent compared to DAC. Compound $\mathbf{C}$ is a side chain modified ZMP-analog lacking two reactive centers in the side chain.

Table 7.5 $\mathrm{IC}_{50 \mathrm{~s}}$ of the analogs

\begin{tabular}{cc} 
Compounds & $\mathbf{I C}_{\mathbf{5 0}}(\mathbf{n m})$ \\
\hline ZMP & $6.5 \pm 0.7$ \\
Epi-ZMP & $42 \pm 9.3$ \\
$\mathrm{DAC}$ & $247 \pm 2.6$ \\
$\mathbf{1}$ & $165 \pm 13$ \\
$\mathbf{2}$ & $106 \pm 3.6$ \\
$\mathbf{3}$ & $114 \pm 10.2$ \\
$\mathbf{4}$ & $68 \pm 5.6$ \\
$\mathbf{A}$ & $1489 \pm 83$ \\
$\mathbf{B}$ & $7624 \pm 303$ \\
$\mathbf{C}$ & $1138 \pm 72$ \\
\hline
\end{tabular}

Antiproliferative activity of analogs in MCF-7 cells (human breast carcinoma) determined by Zurwerra et al., 2012. Values represent the mean $\mathrm{IC}_{50}$ value from three independent experiments. Table modified from Zurwerra et al., 2012. 
In contrast to these cellular results, the biochemical assays in this study highlighted the importance of four chemical groups within this chemotype that are essential for their binding interaction and ability to assemble MTs. The importance of the $\mathrm{N}$-acylhemiaminal side chain has been further emphasized, along with the tetrahydropyran ring (C11-15), the exocyclic methylene group of $\mathrm{C} 13$, and the orientation of the $\mathrm{C} 20$ hydroxyl in the ZMP side chain. ZMP remains the most potent compound, with an apparent binding constant at least 150 times greater than any of the analogs tested. Despite their weak or complete lack of ability to enhance MT-assembly, the compounds were still shown in the present study to interact with the taxoid site by MS. All analogs were still able to covalently modify the same peptide as the parent compounds, yet this was not reflected in the binding kinetics which showed little or no covalent binding of compounds 1, 3 and $\mathbf{4}$ with tubulin. The explanation for this paradox is in the resolution of the techniques. MS can detect minimal amounts of covalently bound compound, with as little as $0.5 \%$ of ligand bound being detectable. This minimal amount of adduct formation would not be detected by the binding kinetics assay (Dr. F. Díaz, personal communication).

The differences in activity between the cellular results (Zurwerra et al., 2012) and the present in vitro results could be attributed to the duration of the experiments and formation of the covalent bond. The cellular proliferation assays were carried out over a period of $72 \mathrm{~h}$; whereas, the biochemical assays were carried out between $30 \mathrm{~min}$ and 24 h. Thus, over $72 \mathrm{~h}$, more ligand has the 'chance' to covalently interact with tubulin causing the cytotoxic effect; whereas, in the biochemical assays the ligands may not have 'enough' time to form a covalent bond. As discussed in previous chapters and stated by Singh et al., (2011), a covalent ligand will always react eventually, providing there is enough time for positioning of the reactive centers, and this may be what is occurring here. Biochemical experiments cannot be performed over longer time periods due to protein degradation at the temperatures of incubation. Additionally, the observed biochemical effect would require stoichiometric binding of one MSA ligand per tubulin heterodimer; whereas, in cells MSAs are active at much lower, sub-stoichiometric, concentrations, with only a fraction of MSA-bound cellular tubulin required for a cytotoxic effect (Díaz et al., 2009). At sub-stoichiometric concentrations, both MSAs and MDAs are able to suppress the dynamic instability of MTs, resulting in inhibition of mitosis and consequent cellular death (Jordan and Wilson 2004). Thus, depending on the 
duration it takes for the compounds to bind, a measureable effect may not be observed in vitro in the $24 \mathrm{~h}$ period; whereas, in cells even a small fractional occupancy would lead to a cytotoxic effect. It would be of interest to investigate the in vitro activity of compounds A-C.

Recently the crystal structure of ZMP bound to unassembled tubulin was elucidated (Prota et al., 2013). The structure confirmed the proposed covalent attachment of ZMP to His229 and showed that two H-bonds are formed with the $\mathrm{C} 19$ side chain at the C20 hydroxyl and the O1 oxygen atom to Thr276 (within the M-loop) at the main chain carbonyl and the $\mathrm{NH}_{2}$ group, respectively (Figure 7.9A). Based on the X-ray crystallography results, the molecular basis of the potent MT-stabilizing activity of ZMP stems from this side chain interaction with the M-loop, which induces major restructuring of this loop by numerous hydrophobic and polar contacts established between the ZMP side chain and residues in the loop (Prota et al., 2013). This confirms the importance of the ZMP side chain and explains why the analog compounds are not as potent as the parent compound.

\section{Importance of the side chain moiety}

Epi-ZMP has an apparent binding constant for the taxoid site 150 times less than that of ZMP, is significantly less potent at causing MT assembly, and is less efficient at covalently modifying MTs, since it covalently occupies only $65 \%$ of the taxoid sites after $24 \mathrm{~h}$. The only difference between these two compounds is the configuration of the hydroxyl group at C20 (Figure 7.2). The crystallization data explain why epi-ZMP is significantly less active than ZMP since the C20 hydroxyl group is orientated in the opposite direction, preventing it from forming a hydrogen bond with the same conformational constraints as seen with ZMP. Additionally, in epi-ZMP, the different orientation of the $\mathrm{C} 20$ alcohol would force the remaining side chain into a completely different conformation, and this change in orientation would severely disrupt its interaction with the M-loop, resulting in the observed loss in activity. DAC and compounds 2, 4, and $\mathbf{3}$ have either an aldehyde or an alcohol moiety at C19 and are significantly less active than both ZMP and epi-ZMP, indicating that an inappropriately orientated side chain is better than no side chain. This also suggests that the hydrogen bond with Thr276 can be disrupted without total loss of activity, and an active conformer obtained. Both these scenarios would result in variable M-loop interactions and thus 
relatively different degrees of stabilization. The bound conformation of ZMP is shown in Figure 7.9. It is known that the covalent reaction occurs mainly at $\mathrm{C} 9$ of the macrolide ring, with minimal attachment at $\mathrm{C} 3$. The side chain should therefore have no effect on the covalent reaction itself but would influence the positioning of compound, aligning it in the correct conformation for the covalent reaction to take place. A compound lacking the side chain would therefore take longer to non-covalently bind to position the analog into its correct alignment for making the covalent bond.

DAC and compound 3 have an aldehyde present at C19; whereas, compounds 2 and 4 have an alcohol. DAC and compound 2 can be compared with one another, and compounds 3 and $\mathbf{4}$ with one another, the latter two lacking the C13 exocyclic methylene moiety. It is evident that the compounds with the C19 alcohol are slightly more potent and better ligands of the taxoid site than their respective reduced analogs, apart from the binding kinetics of compound 2; however, these changes are minimal compared to complete loss of the ZMP side chain. Compound $\mathbf{2}$ is a slightly more potent MSA than DAC and is significantly better at covalently modifying its binding site, suggesting an alcohol at C19 is better at positioning the macrolide ring for the covalent reaction. Compound 4 has a higher apparent binding constant for the taxoid site and is a slightly more potent MSA than 3. When the alcohol is present on $\mathrm{C} 19$, an $\mathrm{H}$-bond can be formed by the lactone carbonyl at $\mathrm{C} 1$ of the macrolide ring. This bond may form a 6-membered ring between $\mathrm{C} 1$, the lactone carbonyl, $\mathrm{C} 19$, and position 20 of the macrocycle and the side chain alcohol oxygen (see Figure 7.2 for compound numbering), resulting in improved stability of the molecule. This ring formation may promote conjugate addition at positions $\mathrm{C} 3$ or C5; however, there is no evidence for this from molecular modeling with ZMP, but this does not mean that it would not occur with the analogs. In contrast to this, when the aldehyde is at position 19, this hydrogen bond cannot form, and the aldehyde would position itself far from the internal carbonyl, changing the conformation of the compound. Furthermore, an alcohol can act as an H-bond acceptor or a donor; whereas, an aldehyde can only be an acceptor. In the binding site, this would give the alcohol-containing compounds an advantage. There may be an hydrogen bond acceptor that the alcohol interacts with as a donor, although this would be at the expense of the internal hydrogen bond mentioned above; whereas, in the case of the aldehyde this same interaction would not be possible. It is important to note, however, that there is no proof that the 'internal' hydrogen bond actually ever forms. Previous molecular modeling has 
shown that the aldehyde of DAC interacts strongly with the M-loop at residue Q281, suggesting this same interaction occurs with the analogs and is more favorable with the alcohol than the aldehyde. Also in relation to the binding site, the relative position of the oxygen in both groups is different and would result in different binding site interactions. The $\mathrm{C} 20$ of the side chain has a $\mathrm{sp}^{2}$ orbital with the aldehyde and an $\mathrm{sp}^{3}$ orbital with the alcohol. These factors would all play a role in making the alcohol-containing compounds more efficient at binding and causing MT assembly.

Further support for the C19 alcohol being more important than the aldehyde in analogs without a side chain comes from some of the other ZMP analogs described by Zurwerra et al. (2012) in which the capacity to hydrogen bond improves the activity. When compound $\mathbf{2}$ is methylated at C19 to give compound A (Figure 7.8), there is a significant loss in antiproliferative activity (Table 7.5) (Zurwerra et al., 2012), demonstrating again that hydrogen bonding at C19 is essential for the activity of the compound. Compound A would not have the ability to hydrogen bond since it is methylated at $\mathrm{C} 19$. When DAC is oxidized at this position to form the corresponding carboxylic acid (compound B, Figure 7.8) the antiproliferative activity also decreases (Zurwerra et al., 2012), suggesting that this moiety is too large for optimal hydrogen bonding within the binding pocket. It is also possible, however; this loss in activity is due to poor cellular penetration because of the negative charge associated with the carboxyl group. It would therefore be of interest to investigate compound $\mathbf{B}$ in the in vitro assays. Additionally, with the amide-analog of ZMP ( $\mathbf{C}$, Figure 7.8) the C19 side chain is lacking the $\mathrm{C} 20$ hydroxyl which has been replaced with a carbonyl, and the $\mathrm{C} 1$ ' carbonyl is lost, along with the Z-alkene chemistry of the remaining side chain. This compound is more potent than the carboxylic acid substitution at C19 (compound B) but several hundred fold less potent than ZMP. This is presumably due to loss of the hydroxylThr276 hydrogen bond (Figure 7.9). This suggests that a hydrogen acceptor is required at C19 and explains why compounds $\mathbf{2}$ and $\mathbf{4}$ are slightly more potent than their reduced counterparts DAC and compound 3, respectively. Compound $\mathbf{C}$ is only 3-6 fold less potent than DAC with $\mu \mathrm{M}$ activity, and thus can still be considered a lead compound.

\section{Importance of the tetrahydropyran ring and exocyclic methylene group}

Compound 1 has the same $N$-acyl hemiaminal side chain as ZMP; however, it lacks the 4-methylenetetrahydropyran ring. Loss of this moiety results in it being the least active of 
the analogs with an apparent binding constant 2000 times less than that of ZMP, and significantly less than any of the other compounds studied. This indicates that the tetrahydropyran ring is even more important that the C19 side chain. Thus, this moiety needs to be retained in analogs to preserve stabilizing activity. Interestingly, however, this compound is a slighter better covalent modifier than compounds $\mathbf{4}$ and $\mathbf{3}$ which retain the tetrahydropyran ring but lack the exocyclic methylene group (23\% covalently bound vs. $13-14 \%$ ). This may be explained by the side chain that is present in 1 that would cause correct positioning of reactive centers for covalent modification, knowing that the formation of the covalent bond is faster in compounds with the side chain, as was seen when comparing ZMP to epi-ZMP. This is also supported by the fact that the DAC version of compound $\mathbf{1}$ (loss of $\mathrm{N}$-acyl hemiaminal side chain) is significantly less potent than compound 1 itself at inhibiting cellular proliferation (Zurwerra et al., 2012).

Compounds $\mathbf{4}$ and $\mathbf{3}$ lack the exocyclic methylene group, and, along with 1, are not very potent MSAs. The apparent binding constants of $\mathbf{4}$ and $\mathbf{3}$ for the taxoid site at $35^{\circ} \mathrm{C}$ are at least 500 times less than that of ZMP and two times less than DAC. In addition, they are unable to form significant covalent bonds to tubulin after $24 \mathrm{~h}$. These results indicate that the methylene group is essential for the activity of these compounds. Previously, molecular modeling indicated that this exocyclic methylene group occupies a hydrophobic pocket, which, in the case of DAC, brings it closer to the M-loop (Field et al., 2012). Loss of this moiety and the additional side chain loss may result in lack of Mloop interaction and thus lack of stabilization. The crystal structure confirmed that the exocyclic methylene group of ZMP is tucked into a cavity in tubulin that is mainly made up of hydrophobic residues (Figure 7.9). Furthermore, the tetrahydropyran ring at the bottom of the chemotype is relatively constrained, and loss of the exocyclic methylene group would result in $\mathrm{C} 13$ changing from a $\mathrm{sp}^{2}$ orbital to a $\mathrm{sp}^{3}$ orbital. When $\mathrm{C} 13$ has a $\mathrm{sp}^{2}$ orbital, it is probable that it takes on a more favorable conformation for binding than when a $\mathrm{sp}^{3}$ orbital is present at this position. Overall to maintain nanomolar activity and a potent stabilization effect the C19 side chain and tetrahydropyran ring need to be preserved. However compounds lacking the C19 side chain still have micromolar activity and can therefore still be used as lead compounds. Loss of this activity occurs with loss of the tetrahydropyran ring and thus this needs to produce active analogs. 

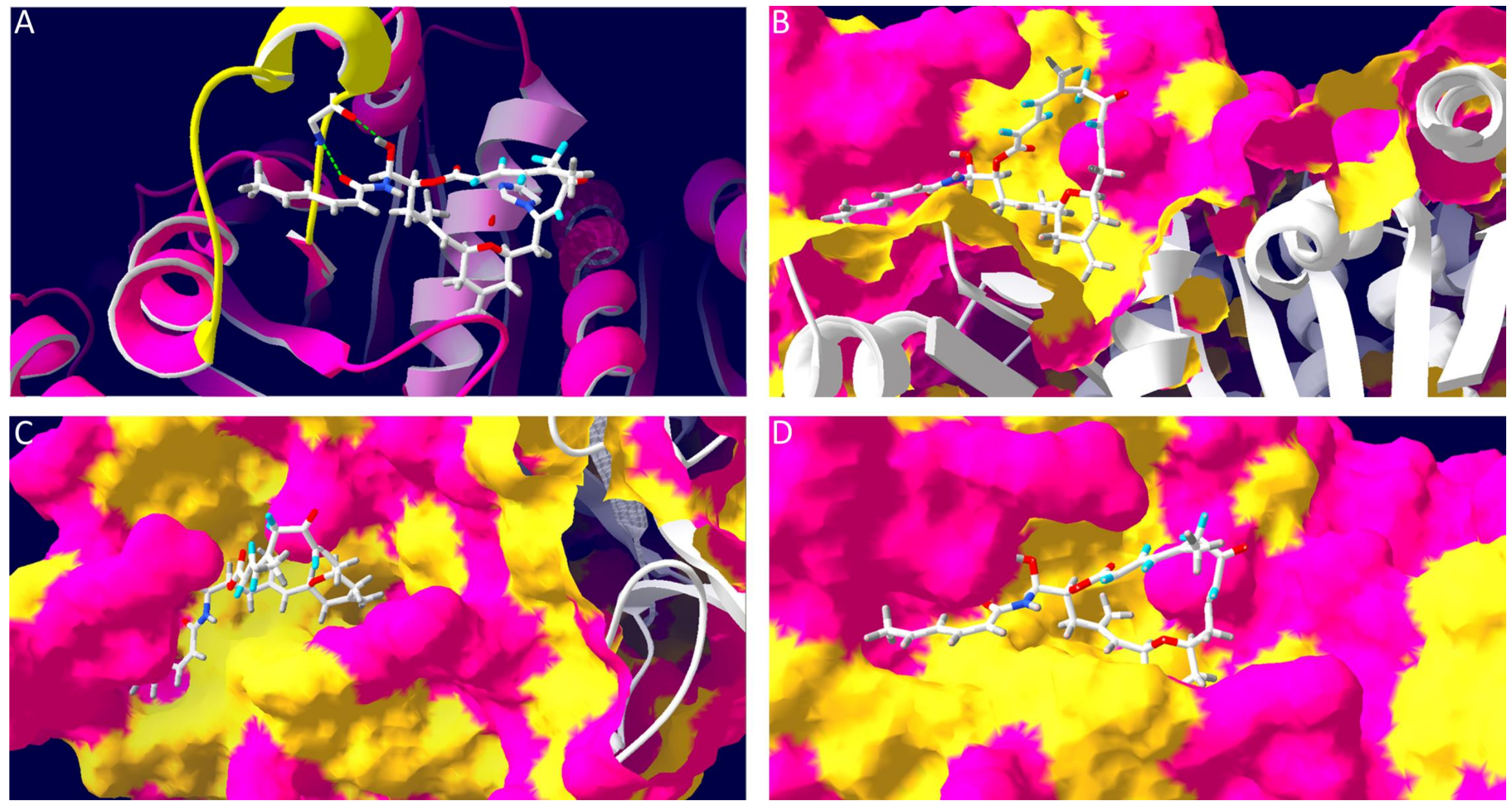

Figure 7.9 Molecular modeling of the ZMP crystal structure

A. ZMP (CPK coloring) bound in its binding site. His229 (CPK) is shown bound to C9 of the macrolide ring. The two hydrogen bonds formed between the ZMP side chain and Thr276 (CPK) are shown in green. The M-loop is shown in yellow. B-D. ZMP bound to its binding site. Hydrophobic residues are shown in yellow and polar residues in pink. The hydrophobic pocket holding the exocyclic methylene group is shown clearly in C and also evident in B and D. Loss of the side chain would result in loss of the hydrogen bonds and M-loop interaction, and further loss of the C13 moiety would result in a different conformation of the bound ligand. 


\subsubsection{Microtubule size}

Ligands binding to the MT can alter the interprotofilament angle and result in a larger or smaller MT lattice by altering the number of protofilaments making up the lattice. The ZMP chemotype gives a larger MT lattice by 1-2 protofilaments, reflecting modification of lateral interactions between protofilaments by the different chemical structures. It was expected that ZMP would cause an increase in lattice size, since the side chain has now been shown to restructure the M-loop, increasing interprotofilament contacts, and this interaction explains its potent stabilizing activity. Interestingly epi-ZMP caused the largest increase in lattice size (15.3 protofilaments). It is possible the $\mathrm{C} 20$ hydroxyl in epi-ZMP is still hydrogen bonding with the M-loop but in a different, 'less stabilizing', manner. This may occur because its $\mathrm{C} 20$ hydroxyl is oriented in the opposite direction, and this may push the M-loop further out from the protofilament to increase the interprotofilament angle and thus the lattice size. It is known that a larger MT lattice does not necessarily correlate with ligand potency. It has been shown that HXF, which only interacts with the pore type I site, causes a larger increase in protofilament number than either PTX and FTX-2 (15.1 versus 13.0 and 14.2, respectively) (Matesanz et al., 2011a). HXF is less active than FTX-2, and all fluorescent taxoids are significantly less active than PTX (Barasoain et al., 2010). The increase in MT size by DAC and compounds 1 and 3 may possibly be due to a small reorganization of the binding cleft resulting in movement of loops which gives larger interprotofilament angles and a larger lattice. It is unlikely that these compounds have significant interaction with the M-loop since they have weak or no stabilizing activity. Molecular docking of each individual analog to the binding site would provide further insight on possible interactions and loop movement. However these models would need to be taken with care since as shown with ZMP the models can be misleading, thus crystallization of the compounds would be ideal.

\subsubsection{Conclusions and future directions}

Although the east side of the compounds was not modified, we can speculate that loss of the enone moiety would significantly decrease potency since this is where covalent attachment at H229 occurs, and addition of motifs to this section of the compound may also decrease potency. Attachment of an electrophilic group in a side chain to this region may push the compound more toward the M-loop and increase the side chain interaction. According to the X-ray crystal structure, the Z-alkene in the side-chain folds it into the 
space within the M-loop (Figure 7.9), and this is likely to be important for binding. This Z-stereochemistry will therefore need to be retained in analogs with the ZMP side chain. This is evident with compound $\mathbf{C}$ which has lost the side chain double bonds and has significantly lower activity compared to the parent compound. Extension of the sidechain by addition of extra alkyl groups should be explored, although conservation of the C20 hydroxyl and the carbonyl at C1' are likely to be important for hydrogen bonding. It would be interesting to incorporate another hydrogen bond acceptor (such as an ester or a ketone) into the side chain to exploit potential hydrogen bonding interactions with the Mloop.

A new chemotype for MSAs with a covalent mechanism of action based on ZMP has now been characterized. This new chemotype competes with PTX for binding to $\beta$ tubulin. Overall the present study on analogs of ZMP and DAC has confirmed the importance of the C19 side chain, and this is further supported by the crystallography data. The tetrahydropyran ring was shown to be essential for potent cytotoxicity of the compounds, even more so that the C19 side chain. It was shown that the exocyclic methylene moiety at C13 was not just important for cytotoxicity but was also important in MT-assembly and stabilization by the compounds. Finally, it was concluded that a hydrogen bond acceptor group is required at C19 for good activity, both in cells and in vitro. All the above moieties participate in the pre-covalent binding interaction and affect the potency of the ZMP analogs. 


\subsection{OVERALL FINDINGS AND CONCLUSIONS}

\subsubsection{Zampanolide binds covalently to the taxoid site and potently stabilizes MTs}

Studies in mutant cells suggested that ZMP was a ligand of the taxoid site but that it interacted with the site in a different manner from traditional taxoid site ligands. ZMP was directly shown to be a ligand of the taxoid site by a competition assay. This was confirmed by MS which demonstrated that ZMP covalently modifies the taxoid binding site by alkylating His229 (major product) and Asn228 in both MTs and dimeric tubulin. This covalent binding profile of ZMP explains its 'unusual' biochemical properties and the results observed in the mutant tubulin cell lines. Since ZMP covalently binds exclusively to the luminal site, it has now been directly shown that binding at the taxoid binding site and the pore type I site are mutually exclusive since no pore site binding was observed in the presence of ZMP. X-ray crystallography further confirmed the binding site and binding mechanism and indicated that the covalent bond is formed between C9 of the macrolide ring and NE2 of the imidazole His229 side chain (Prota et al., 2013). Additionally, the crystal structure unveiled the mechanism of MT-assembly and stabilization of ZMP, indicating potent restructuring of the stabilizing M-loop. This information suggested how MSAs currently used in the clinic cause mitotic arrest. In accordance with the crystal structure and the proposals of the Prussia et al. (2010) paper, it is likely that labeling of residue 228 is an artifact of the MS, especially since its side chain is orientated away from the taxoid binding pocket.

\subsubsection{The luminal binding site exists in dimeric tubulin - a mechanism of nucleation}

Although it was known that MSAs must bind unassembled tubulin to account for their ability to assemble tubulin in unfavorable buffer conditions, there was only direct evidence of binding at the pore type I site (Buey et al., 2007). MS analysis of ZMP and DAC indicated that they label two luminal site amino acids in unassembled tubulin, providing direct biochemical evidence that the luminal taxoid binding site exists in dimeric tubulin (Field et al., 2012). X-ray crystallography data further confirmed the existence of this binding site since ZMP and EPOA both bound at the taxoid site in the $\mathrm{T}_{2} \mathrm{R}$ complex (Prota et al., 2013). In addition, the taxoid site in zinc-stabilized sheets is similar to that in unassembled tubulin with only small structural changes evident. 
We can now propose the mechanism for MSA-induced assembly. In dimeric tubulin, MSAs bind to the accessible luminal site, suggesting that MT nucleation induction by MSAs proceeds through an allosteric mechanism. The exception here is with CYC which binds the pore site, indicating that nucleation occurs through a matchmaker mechanism. When binding to MTs, however, MSAs most likely bind first to the pore site (this interaction is observed as the rapid Flutax staining of MTs, rapid binding kinetics and the fast on/off rate of ligands detected by STD-NMR). Once bound at the pore site, they then make their way to the luminal binding site. The evidence for this is that the MT luminal site is inaccessible and thus a pre-site is necessary to explain the available data.

\subsubsection{Zampanolide avoids the main mechanisms of resistance}

Although ZMP is a ligand of the taxoid site it is not affected by mutations in the taxoid binding site in mutant $1 \mathrm{~A} 9$ cells to the same extent that PTX and other taxoid site ligands are. The same was seen with CYC (Buey et al., 2007), indicating, as suggested by Singh et al. (2011), that irreversible inhibitors would be effective against resistant mutants as they do not affect the extent of inhibition but only the rate at which the inhibited complex forms. Therefore if sufficient exposure time were available, the reaction might be slower, but full inhibition would eventually occur due to the irreversible binding. Given this, it would be interesting to treat B10 cells (EPOB mutants) with ZMP for an extended time to see if their growth can be inhibited, since these cells showed some resistance to ZMP (resistance ratio $=3.2$; Table 3.4). This cell line is mutated at residue 284 which significantly disrupts the taxoid binding site, thus it is not surprising that these cells show some resistance to ZMP, since the covalent complex would most likely take far longer to form. In addition, ZMP is fully active in 1A9-L4 cells that have an altered tubulin isotype expression (Kanakkanthara et al., 2011), indicating that covalent binding can also overcome this form of resistance. However, since in this study 1A9-L4 cells were not resistant to neither PTX nor IXA (both taxoid site ligands), it isn't surprising that ZMP binding is not affected by tubulin isotype composition in these cells. Previously, ZMP has been shown to be fully active in cells that have the MDR phenotype, expressing upregulated P-gp pumps (Field et al., 2009). Thus, ZMP avoids all the major mechanisms of resistance presented toward MSAs in the clinic. Therefore, the rational design of new generation MSAs that bind in a covalent manner would potentially evade cancer cell 
resistance in the clinic, although possible limitations of covalent binding such as increased systemic toxicity need to be considered.

\subsubsection{Zampanolide activity in A8 EPOA-resistant mutant cells}

A8 cells are mutated at residue 276, in which threonine is replaced by isoleucine. As shown by the crystal structure, Thr276 forms two hydrogen bonds with ZMP (Prota et al., 2013). It would therefore be expected that these cells should be at least partially resistant to ZMP due to the loss of hydrogen bonding. Surprisingly, as shown in Chapter 3, A8 cells are more sensitive to ZMP compared to the parental cell line with a resistance ratio of 0.61 (Table 3.4). Thus, even though ZMP forms two hydrogen bonds with this residue, either its covalent binding mechanism overcomes the mutation, faster than it does with B10 cells for example, or other viable hydrogen bonds can be formed with the mutated isoleucine. The ZMP-amino acid hydrogen bonds involve the $\mathrm{C} 20$ hydroxyl and the $\mathrm{C} 1$ ' carbonyl oxygen of ZMP and the main chain carbonyl oxygen and the $\mathrm{NH}$ group of threonine, respectively (Figure 8.1A). These hydrogen bonds are shown by the green dotted lines labeled as A and B, respectively. Since these hydrogen bonds are forming with the amino acid backbone of threonine and not the side chain, the same bonding opportunities should exist with isoleucine, providing the torsion angles of the amino acids are not significantly different between wild type and the mutant. Modeling these interactions using DeepView (Guex \& Peitsch, 1997) (Figure 8.1B), it is evident that both hydrogen bonds remain intact with isoleucine with a third potential hydrogen bond available between the main chain carbonyl in isoleucine and the $\mathrm{C} 1$ ' oxygen atom in ZMP (labeled bond C). This can explain why ZMP is fully active in these cells. This model is further supported by the crystal structure of EPOA (Prota et al., 2013). EPOA forms two hydrogen bonds with Thr276 between O1 and N20 in EPOA with the main chain NH and the side chain hydroxyl in Thr276, respectively (Figure 8.1C, bonds labeled A and B). A8 cells were created as EPOA-resistant mutants (Giannakakou et al., 2000), and therefore it is not surprising that this is the amino acid that spontaneously mutated in high EPOA concentrations, given its extensive interactions with this MSA. Although EPOA may be able to retain one of its hydrogen bonds with the backbone of isoleucine, its $\mathrm{N} 20$ hydrogen bond to the polar threonine side chain would be lost since the side chain of isoleucine is nonpolar with no potential for hydrogen bonding. This is shown clearly in the model, where both hydrogen bonds appear to be lost (Figure 8.1D). 
Additional support for the model comes from pharmacophore studies showing that alterations in the C1-C8 portion of EPO results in reduced potency (He et al., 2001) and alterations to $\mathrm{C} 15$, especially stereochemistry of the substituent, are important, in activity (Buey et al., 2004).

Additionally, the oxetane oxygen of PTX hydrogen bonds to threonine at $\mathrm{NH}_{2}$ (Figure 7.1), and mutation to isoleucine would influence this interaction (Snyder et al., 2001). Thus, it is not surprising that these cells are also highly resistant to PTX as shown by Giannakakou et al., (2000), and in this study where they were shown to have a resistance ratio of 19 (Table 3.4).

Figure 8.1 ZMP and EPOA binding in A8 cells

Models of ZMP and EPOA interactions with residue 276 in the taxoid site. A. ZMP forms two hydrogen bonds with residue 276, between the C20 hydroxyl of ZMP and the backbone carbonyl of threonine (green dotted line labeled $A$ ), and between the $\mathrm{C} 1$ ' carbonyl of ZMP with the backbone NH2 of threonine (green dotted line labeled $B$ ). The covalent bond with His229 (pink) is also shown (black arrow). B. When reside 276 is mutated to isoleucine the two hydrogen bonds are retained, and a third potential hydrogen bond is present (labeled $C$ ). This model indicates why ZMP is fully active in A8 cells since no loss in bonding occurs. C. EPOA forms four hydrogen bonds in the taxoid site. The N20, O1, OH3 and $\mathrm{OH} 7$ groups of EPOA hydrogen bond to Thr276 at the main chain $\mathrm{NH}$ and the side chain hydroxyl, and to Gln281 at the side chain amide nitrogen and Asp226 at the side chain oxygen, respectively. These hydrogen bonds are labeled A-D (green dotted lines). A protein hydrogen bond is also present in the complex between the Gln281 side chain amide and the main chain carbonyl of Thr276 (white arrowhead). D. When threonine is mutated to isoleucine, both hydrogen bonds A and B are lost. Thus, it is not surprising that A8 cells developed this mutation in response to high concentrations of EPOA and are highly resistant to EPOA and other EPO analogs. 

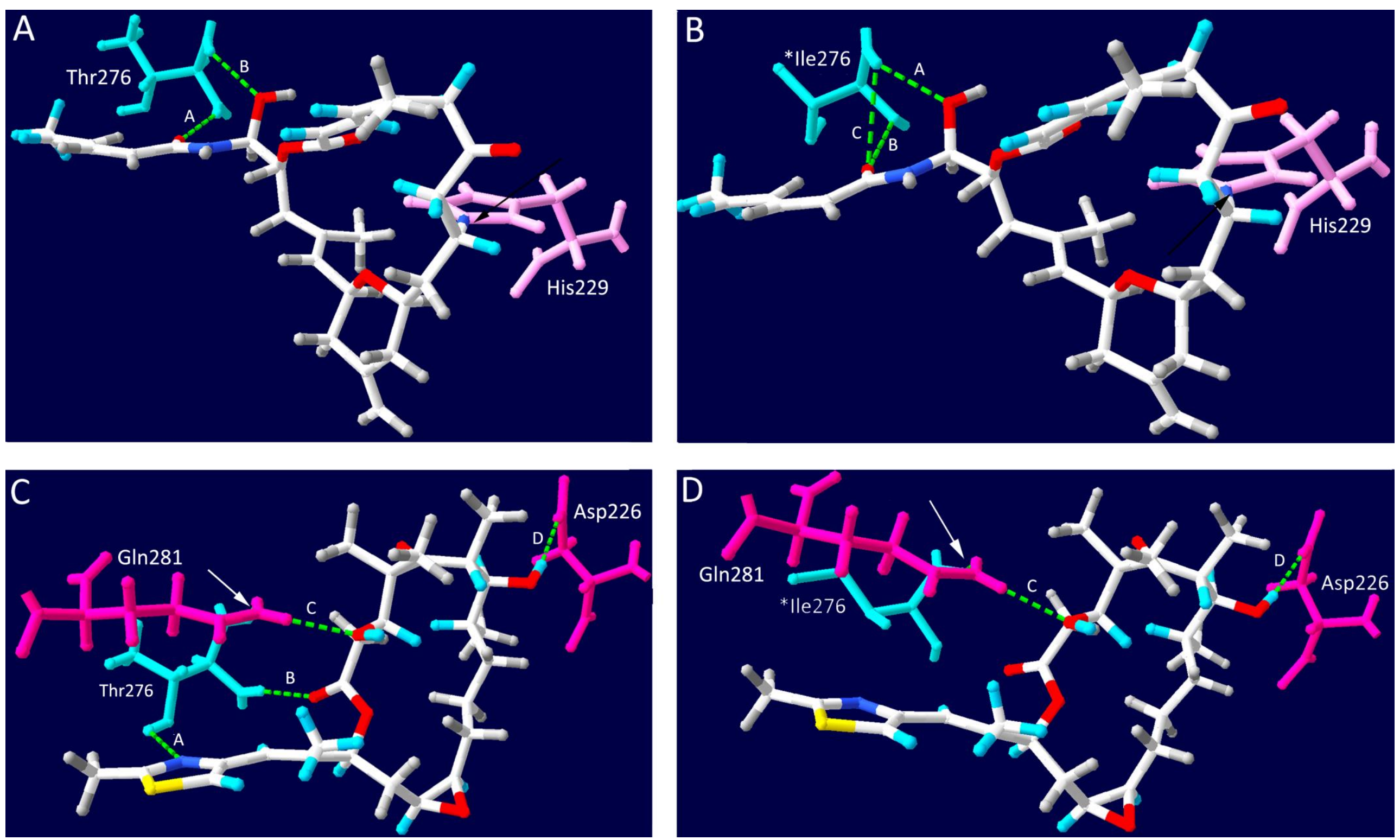


\subsubsection{Cells grown in high concentrations of zampanolide}

As described in Chapter 3, an attempt to create a ZMP-resistant cell line was made, and although no ZMP resistance was seen, the cells were resistant to PTX. The mutation in these cells, if one exists, is therefore likely to be located within the taxoid binding site. It is unlikely that it is an isotype change or a change in the stability of tubulin since IXA, PEL and LAU also remain fully active in the cells. It has been shown that mutations can occur in a binding site but not all ligands that bind that site are affected by the mutation. For example, EPOB is fully active in PTX-resistant cell lines (Giannakakou et al., 1997), and LAU in R1 cells (Kanakkanthara et al., 2011). Given the covalent binding mechanism of ZMP, it is not surprising that cells grown in high concentrations of ZMP are still sensitive to ZMP even after one year exposure. As stated above, irreversible binders will not be effected by binding site mutations since only the rate at which the covalent adduct is formed is affected (Singh et al., 2011). The cells were grown for 1-2 days in ZMP medium, allowed to recover and then the ZMP concentration further increased. If cells were treated for more than 1-2 days, then all cells died, and this can now be attributed to the covalent binding of ZMP, since a long exposure time means that all the ZMP binding sites will become occupied. Short increments of ZMP exposure still may have caused a mutation in tubulin since some PTX resistance developed (resistance ratio $=11$; Table 3.4). No resistance, however, was seen for IXA. It is possible that an MTT cell proliferation assay of shorter duration than the 72 hours tested would unmask some resistance to ZMP. It would be interesting to sequence the tubulin in this cell line to pinpoint the mutation in order to explain its resistance profile.

\subsection{SigNIFICANCE}

This study has not only further developed ZMP as an important MSA but has also shown that covalent binding presents a mechanism in which cancer cell resistance can be evaded, and this will be an important concept in the further development of known and novel MSAs. Additionally, direct evidence that the luminal binding site exists in unassembled tubulin has been provided and the mechanism of MSA-induced MTnucleation is now better understood. The analog study has provided important information relevant to further development of the ZMP chemotype. Lastly, the mechanism in which MSAs, four of which are currently used in the clinic, induce and stabilize MTs has now been shown. This is important for the understanding of this 
important class of compounds but also provides a platform for structure-guided drug engineering of new generation MSAs.

\section{$\underline{8.3 \quad \text { FUTURE DIRECTION }}$}

Synergy

It would be interesting to further investigate possible synergy of ZMP with MSAs at different concentrations than those tested, since taxoid site MSAs are well known to synergize with LAU and/or PEL. Although, given the binding mechanism of ZMP, synergy may not occur in the same manner, and this would be worth investigating on its own. Additionally, it would be of interest to investigate the ability of ZMP to synergize with MDAs or other anti-cancer compounds such as gemcitabine or doxorubicin. Although ZMP did not alter the stoichiometry or affinity of the COL site for binding MTC, there was a slight change in the site that caused MTC to have higher fluorescence. Although this is most likely due to a small shift in the positioning of bound MTC, it would be interesting to see if ZMP has the ability to synergize with COL. Testing for synergy with VBL would also be interesting since PTX is known to synergize with a number of Vinca domain agents. This would be particularly interesting if ZMP is shown to affect the affinity of binding at the Vinca domain, as discussed in Chapter 6 (this experiment is currently being carried out). In ZMP-modified tubulin, the E-site has decreased affinity for nucleotide but does not lose the ability to bind nucleotide. It would therefore be interesting to investigate if ZMP synergizes with MDAs, given the link between ZMP and E-site affinity and the effect of binding at both Vinca domain and COL site on GTP hydrolysis.

\section{Cell migration and angiogenesis}

Further investigation into the mechanisms by which ZMP inhibits cell migration would be worthwhile given the promising preliminary results from the wound scratch assays and also since MTAs are now thought to have a far bigger role at inhibiting interphase MTs than first thought. MTAs have clinically relevant anti-angiogenic and vascular disrupting properties, and a number of MTAs are currently being validated as vascular targeting agents. MTAs have been shown to inhibit endothelial cell proliferation, cell migration and the formation of 'capillary-like' structures or tubes that endothelial cells differentiate into during angiogenesis. Inhibition of these process results in inhibition of 
angiogenesis and this often occurs at low drug concentrations (Schwartz, 2009). Since ZMP has been shown to inhibit both endothelial and fibroblast cell proliferation and migration at very low nanomolar concentrations, the next step, therefore, would be to see the effect of ZMP on tube-formation. This can be studied easily in tissue culture by plating endothelial cells on a 3-D extracellular matrix where they form structures that are 'capillary-like' (Schwartz, 2009). This assay has been successfully performed in our lab, by Dr. Ariane Chan. Cells are treated with MSA and the tubule formation monitored by staining with calcien. This allows observation of the cellular morphology under the confocal microscope (Chan, 2012).

\section{Development of the zampanolide cell line}

Another future direction would be to continue developing the ZMP-resistant cell line and increase the fold-resistance to PTX. It would be interesting to see if the cells are still sensitive to MDAs such as COL and VBL, although it is expected they would be. Further development of the ZMP-resistant cell line could include cloning and selection for the most resistant cells to give a homogeneous population, followed by sequencing of $\alpha$ - and $\beta$-tubulin to determine if there is a gene mutation in tubulin compared to the wild type cells.

A different approach could have been taken to investigate possible ZMP-resistant cell lines. Yin et al., (2012) used random mutagenesis of $\beta I$-tubulin cDNA followed by transfection and selection of cells for resistance. This approach is efficient and fast at identifying mutations that alter sensitivity of the cells to MSAs. It also avoids a number of issues that limit the development of mutations in cell lines. For example, it eliminates the up-regulation of different tubulin isotypes and the MDR phenotype, although the latter may not be an issue in developing a cell line resistant to ZMP given its binding mechanism. This approach could be taken to directly mutate amino acids 228 and 229 and investigate the effect of ZMP in these cells. It is likely, however, that cells mutated at these residues may not be viable given the essential role of Asn228 in the E-site and the likelihood that His229 is involved in the positioning of 228 for this interaction. It is possible; however, that another amino acid may be able to play a similar role in the Esite. 


\section{Proteomic and animal studies}

Firstly, it would be worth investigating possible secondary protein targets of ZMP using proteomics (2-D DIGE), especially since it binds covalently and thus is likely to be attacked by nucleophile residues in other proteins. Then, if enough ZMP were to become available, which is likely since a number of complete syntheses have been completed, its effect on in vivo tumors in mice would be an important set of experiments for the development of ZMP toward the clinic and would provide understanding at a different level of its effects and pharmacological properties. This evaluation is important since in vitro activity is not necessarily retained in vivo due to pharmacokinetic properties and drug metabolism. This would be of great interest in not only further developing ZMP as a potential drug candidate but in development of future covalent MSAs, since this has never been studied before.

\section{Further development of the zampanolide chemotype}

Differences between ZMP and DAC indicated and confirmed that the N-acyl-hemiaminal C19 side chain of ZMP is very important in its ability to induce MT assembly, its affinity for binding the MT and its cytotoxicity. The correlation between binding, stabilization and cytotoxicity can be related to modifications in the compound structure as seen with EPO analogs (Buey et al., 2004). The ZMP side chain is essential, and analogs lacking this side chain will not be as potent as ZMP. However, compounds lacking this side chain moiety still have low micro-molar activity (Zurwerra et al 2010, 2012) and can therefore still be used as lead compounds. Three key motifs in the ZMP structure have been identified that dictate its potency, in addition to the presence of the C19 side chain. These moieties participate in the pre-covalent binding interaction, and it would be interesting to create analogs with these motifs or variations of these motifs with other changes to the core structure. For example, modification of $\mathrm{C} 1-\mathrm{C} 11$ has not yet been investigated. Possible structural alterations are discussed in Section 7.4.3. From this study it can be concluded that future synthetic studies should include a hydrogen bond acceptor moiety at C19 and include the tetrahydropyran ring for increased activity in compounds lacking the ZMP side chain. 
Structure-guided drug engineering

The crystal structure of ZMP has confirmed its binding to the taxoid site and indicated its specific interactions with tubulin. In addition, the elucidation of this structure, along with that of EPOA has revealed the structural basis for MSA-induced MT assembly and has indicated the mechanism in which currently used drugs cause MT stabilization (Prota et $a l ., 2013)$. This mechanism of action is key information that was lacking in this field and will provide insight into the future development of currently known and novel MSAs. Developing new generation MSAs can now be done by structure-guided drug engineering, using this information to get maximum M-loop stabilization. This could be examined in conjunction with covalent binding and will aid in the development of new MSAs that will have improved properties, including higher potency at lower concentrations and evasion of cancer cell resistance. 


\section{References}

Abrey, L., Johri, A., Wen, P. Y., Govindan, R., Reimers, H. J., Robins, H. I., de Bedout, S., Hennan, L., Ko, J. L. \& Rigas, J. R. (2007). Treatment of recurrent or progressive brain metastases with patupilone in patients with non-small cell lung cancer (NSCLC): results of a multicenter, openlabel phase II study. EJC Suppl. 5, 372-372.

Akhmanova, A. \& Steinmetz, M. O. (2008). Tracking the ends: A dynamic protein network controls the fate of microtubule tips. Nat. Rev. Mol. Cell Biol. 9, 309-322.

Alday, P. H. \& Correia, J. J. (2009). Macromolecular interaction of halichondrin B analogues eribulin (E7389) and ER-076349 with tubulin by analytical ultracentrifugation. Biochemistry 48, 79277938.

Alli, E., Bash-Babula, J., Yang, J. M. \& Hait, W. N. (2002). Effect of stathmin on the sensitivity to antimicrotubule drugs in human breast cancer. Cancer Res. 62, 6864-6869.

Altmann, K. H. \& Gertsch, J. (2007). Anticancer drugs from nature - natural products as a unique source of new microtubule-stabilizing agents. Nat. Prod. Rep. 24, 327-357.

Amadori, D., Frassineti, G. L., Zoli, W., Milandri, C., Tienghi, A., Ravaioli, A., Gentile, A. \& Salzano, E. (1996). A phase I/II study of sequential doxorubicin and paclitaxel in the treatment of advanced breast cancer. Semin. Oncol. 23, 16-22.

Amos, L. A. (2011). What tubulin drugs tell us about microtubule structure and dynamics. Semin. Cell Dev. Biol. 22, 916-926.

Amos, L. A. \& Löwe, J. (1999). How Taxol ${ }^{\circledR}$ stabilises microtubule structure. Chem. Biol. 6, R65-R69.

Amos, L. A. \& Klug, A. (1974). Arrangement of subunits in flagellar microtubules. J. Cell Sci. 14, 523549.

Andreu, J. M. (2007). Large scale purification of brain tubulin with the modified Weisenberg procedure. In Methods in Molecular Medicine ${ }^{\mathrm{TM}}$ (J. Zhou, Ed.) Microtubule Protocols: 137, (pp 17-28), Totowa, NJ: Humana Press Inc.

Andreu, J. M. \& Barasoain, I. (2001). The interaction of baccatin III with the taxol binding site of microtubules determined by a homogeneous assay with fluorescent taxoid. Biochemistry 40, $11975-11984$.

Andreu, J. M., Perez-Ramirez, B., Gorbunoff, M. J., Ayala, D. \& Timasheff, S. N. (1998). Role of the colchicine ring a and its methoxy groups in the binding to tubulin and microtubule inhibition. Biochemistry 37, 8356-8368.

Andreu, J. M., Díaz, J. F., Gil, R., Depereda, J. M., Delacoba, M. G., Peyrot, V., Briand, C., TownsAndrews, E. \& Bordas, J. (1994). Solution structure of taxotere-induced microtubules to 3-nm resolution - The change in protofilament number is linked to the binding of the taxol side-chain. $J$. Biol. Chem. 269, 31785-31792.

Andreu, J. M., Bordas, J., Díaz, J. F., Deancos, J. G., Gil, R., Medrano, F. J., Nogales, E., Pantos, E. \& Towns-Andrews, E. (1992). Low resolution structure of microtubules in solution - synchrotron Xray-scattering and electron microscopy of taxol-induced microtubules assembled from purified tubulin in comparison with glycerol and MAP-induced microtubules. J. Mol. Biol. 226, 169-184.

Andreu, J. M., Gorbunoff, M. J., Lee, J. C. \& Timasheff, S. N. (1984). Interaction of tubulin with bifunctional colchicine analogs - An equilibrium study. Biochemistry 23, 1742-1752.

Ayaz, P., Ye, X. C., Huddleston, P., Brautigam, C. A. \& Rice, L. M. (2012). A TOG: $\alpha \beta$-tubulin complex structure reveals conformation-based mechanisms for a microtubule polymerase. Science 337, 857-860.

Bacher, G., Nickel, B., Emig, P., Vanhoefer, U., Seeber, S., Shandra, A., Klenner, T. \& Beckers, T. (2001). D-24851, a novel synthetic microtubule inhibitor, exerts curative antitumoral activity in vivo, shows efficacy toward multidrug-resistant tumor cells, and lacks neurotoxicity. Cancer Res. 61, 392-399.

Bai, R. L., Lin, C. M., Nguyen, N. Y., Liu, T. Y. \& Hamel, E. (1989). Identification of the cysteine residue of $\beta$-tubulin alkylated by the antimitotic agent 2,4-dichlorobenzyl thiocyanate, facilitated by seperation of the protein subunits of tubulin by hydrophobic column chromatography. Biochemistry 28, 5606-5612.

Balbo , A. \& Schuck, P. (2005). Analytical ultracentrifugation in the study of protein self-association and heterogeneous protein-protein interactions. In A Molecular Cloning Manual (E. A. Golemis \& P. D. Adams, Eds.) Second ed. Protein-Protein Interactions NY, USA: Cold Spring Harbor Laboratory Press. 
Balbo, A., Minor, K. H., Velikovsky, C. A., Mariuzza, R. A., Peterson, C. B. \& Schuck, P. (2005). Studying multiprotein complexes by multisignal sedimentation velocity analytical ultracentrifugation. P. Natl. Acad. Sci. USA 102, 81-86.

Barasoain, I., Garcia-Carril, A. M., Matesanz, R., Maccari, G., Trigili, C., Mori, M., Shi, J. Z., Fang, W. S., Andreu, J. M., Botta, M., et al. (2010). Probing the pore drug binding site of microtubules with fluorescent taxanes: Evidence of two binding poses. Chem. Biol. 17, 243-253.

Barbier, P., Dorleans, A., Devred, F., Sanz, L., Allegro, D., Alfonso, C., Knossow, M., Peyrot, V. \& Andreu, J. M. (2010). Stathmin and interfacial microtubule inhibitors recognize a naturally curved conformation of tubulin dimers. J. Biol. Chem. 285, 31672-31681.

Baudin, B., Bruneel, A., Bosselut, N. \& Vaubourdolle, M. (2007). A protocol for isolation and culture of human umbilical vein endothelial cells. Nat. Protoc. 2, 481-485.

Begaye, A., Trostel, S., Zhao, Z. M., Taylor, R. E., Schriemer, D. C. \& Sackett, D. L. (2011). Mutations in the $\beta$-tubulin binding site for peloruside A confer resistance by targeting a cleft significant in side chain binding. Cell Cycle 10, 3387-3396.

Belarbi, E. H., Contreras Gómez, A., Chisti, Y., García Camacho, F. \& Molina Grima, E. (2003). Producing drugs from marine sponges. Biotech. Advan. 21, 585-598.

Bennett, M. J., Barakat, K., Huzil, J. T., Tuszynski, J. \& Schriemer, D. C. (2010). Discovery and characterization of the laulimalide-microtubule binding mode by mass shift perturbation mapping. Chem. Biol. 17, 725-734.

Berenbaum, M. C. (1985). The expected effect of a combination of agents - the general-solution J. Theor. Biol. 114, 413-431.

Berlin, J. D., Venook, A., Bergsland, E., Rothenberg, M., Lockhart, A. C. \& Rosen, L. (2008). Phase II trial of T138067, a novel microtubule inhibitor, in patients with metastatic, refractory colorectal carcinoma. Clin. Colorectal Canc. 7, 44-47.

Berrieman, H. K., Lind, M. J. \& Cawkwell, L. (2004). Do $\beta$-tubulin mutations have a role in resistance to chemotherapy? Lancet Oncol. 5, 158-164.

Bhattacharyya, B. \& Wolff, J. (1984). Immobilization-dependent fluorescence of colchicine. J. Biol. Chem. 259, 1836-1843.

Bhattacharyya, B., Kapoor, S. \& Panda, D. (2010). Fluorescence spectroscopic methods to analyze drugtubulin interactions. (L. Wilson \& J. J. Correia, Eds.) Methods in Cell Biology, Microtubules, in Vitro: 95, (pp 301-329): Elsevier.

Bhattacharyya, B., Panda, D., Gupta, S. \& Banerjee, M. (2008). Anti-mitotic activity of colchicine and the structural basis for its interaction with tubulin. Med. Res. Rev. 28, 155-183.

Bhunia, A., Bhattacharjya, S. \& Chatterjee, S. (2012). Applications of saturation transfer difference NMR in biological systems. Drug Discov. Today 17, 505-513.

Bijman, M. N. A., Amerongen, G. P., Laurens, N., van Hinsbergh, V. W. M. \& Boven, E. (2006). Microtubule-targeting agents inhibit angiogenesis at subtoxic concentrations, a process associated with inhibition of Rac1 and Cdc42 activity and changes in the endothelial cytoskeleton. Mol. Cancer Ther. 5, 2348-2357.

Bollag, D. M., McQueney, P. A., Zhu, J., Hensens, O., Koupal, L., Liesch, J., Goetz, M., Lazarides, E. \& Woods, C. M. (1995). Epothilones, a new class of microtubule-stabilizing agents with a Taxollike mechanism of action. Cancer Res. 55, 2325-2333.

Brunden, K. R., Zhang, B., Carroll, J., Yao, Y., Potuzak, J. S., Hogan, A. L., Iba, M., James, M. J., Xie, S. X., Ballatore, C., et al. (2010). Epothilone D improves microtubule density, axonal iIntegrity, and cognition in a transgenic mouse model of tauopathy. J. Neurosci. 30, 13861-13866.

Brunden, K. R., Trojanowski, J. Q. \& Lee, V. M. Y. (2009). Advances in tau-focused drug discovery for Alzheimer's disease and related tauopathies. Nat. Rev. Drug Discov. 8, 783-793.

Buey, R. M., Chacón, P., Andreu, J. M. \& Díaz, J. F. (2009). Protein shape and assembly studied with XRay solution scattering: Fundaments and practice. In Lecture Notes in Physics (M. Gomez, A. Nogales, M. C. Garcia-Gutierrez \& T. A. Ezquerra, Eds.) Applications of synchrotron light to scattering and diffraction in materials and life sciences: 776, (pp 245-263): Springer Berlin Heidelberg.

Buey, R. M., Calvo, E., Barasoain, I., Pineda, O., Edler, M. C., Matesanz, R., Cerezo, G., Vanderwal, C. D., Day, B. W., Sorensen, E. J., et al. (2007). Cyclostreptin binds covalently to microtubule pores and lumenal taxoid binding sites. Nat. Chem. Biol. 3, 117-125.

Buey, R. M., Díaz, J. F. \& Andreu, J. M. (2006). The nucleotide switch of tubulin and microtubule assembly: A polymerization-driven structural change. Biochemistry 45, 5933-5938.

Buey, R. M., Barasoain, I., Jackson, E., Meyer, A., Giannakakou, P., Paterson, I., Mooberry, S., Andreu, J. M. \& Díaz, J. F. (2005). Microtubule interactions with chemically diverse stabilizing agents: 
Thermodynamics of binding to the paclitaxel site predicts cytotoxicity. Chem. Biol. 12, 12691279.

Buey, R. M., Díaz, J. F., Andreu, J. M., O'Brate, A., Giannakakou, P., Nicolaou, K. C., Sasmal, P. K., Ritzen, A. \& Namoto, K. (2004). Interaction of epothilone analogs with the paclitaxel binding site: Relationship between binding affinity, microtubule stabilization, and cytotoxicity. Chem. Biol. 11, 225-236.

Cabral, F. (2008). Mechanisms of resistance to drugs that interfere with microtubule assembly. In Cancer Drug Discovery and Development (A. T. Fojo, Ed.) The Role of Microtubules in Cell Biology, Neurobiology, and Oncology, (pp 337-357): Humana Press.

Cabral, F. (2001). Factors determining cellular mechanisms of resistance to antimitotic drugs. Drug Resist. Update. 4, 3-8.

Calvo, E., Barasoain, I., Matesanz, R., Pera, B., Camafeita, E., Pineda, O., Hamel, E., Vanderwal, C. D., Andreu, J. M., Lopez, J. A., et al. (2012). Cyclostreptin derivatives specifically target cellular tubulin and further map the paclitaxel Site. Biochemistry 51, 329-341.

Calvo, E., Camafeita, E., Díaz, J. F. \& Lopez, J. A. (2008). Mass spectrometry for studying the interaction between small molecules and proteins. Curr. Proteomics 5, 20-34.

Canales, A., Rodríguez-Salarichs, J., Trigili, C., Nieto, L., Coderch, C., Andreu, J. M., Paterson, I., Jímenez-Barbero, J. \& Díaz, J. F. (2011). Insights into the interaction of discodermolide and docetaxel with tubulin. Mapping the binding sites of microtubule-stabilizing agents by using an integrated NMR and computational approach. ACS Chem. Biol. 6, 789-799.

Canales, A., Matesanz, R., Gardner, N. M., Andreu, J. M., Paterson, I., Díaz, J. F. \& Jímenez-Barbero, J. (2008). The bound conformation of microtubule-stabilizing agents: NMR insights into the bioactive 3D structure of discodermolide and dictyostatin. Chem-Eur. J. 14, 7557-7569.

Carbonaro, M., Escuin, D., O'Brate, A., Thadani-Mulero, M. \& Giannakakou, P. (2012). Microtubules regulate hypoxia-inducible factor- $1 \alpha$ protein trafficking and activity. Implications for taxane therapy. J. Biol. Chem. 287, 11859-11869.

Carlier, M. F. \& Pantaloni, D. (1981). Kinetic-analysis of guanosine 5'-triphosphate hydrolysis associated with tubulin polymerization. Biochemistry 20, 1918-1924.

Carlomagno, T., Blommers, M. J. J., Meiler, J., Jahnke, W., Schupp, T., Petersen, F., Schinzer, D., Altmann, K. H. \& Griesinger, C. (2003). The high-resolution solution structure of epothilone A bound to tubulin: An understanding of the structure-activity relationships for a powerful class of antitumor agents. Angew. Chem. Int. Ed. 42, 2511-2515.

Chakraborti, S., Chakravarty, D., Gupta, S., Chatterji, B. P., Dhar, G., Poddar, A., Panda, D., Chakrabarti, P., Dastidar, S. G. \& Bhattacharyya, B. (2012). Discrimination of ligands with different flexibilities resulting from the plasticity of the binding site in tubulin. Biochemistry 51, 71387148.

Chan, A. (2012). Cellular targets and tumor suppressive mechanisms of peloruside A. Doctor of Philosopy Biomedical Science, Victoria University of Wellington, New Zealand.

Chen, K., Huzil, J. T., Freedman, H., Ramachandran, P., Antoniou, A., Tuszynski, J. A. \& Kurgan, L. (2008). Identification of tubulin drug binding sites and prediction of relative differences in binding affinities to tubulin isotypes using digital signal processing. J Mol. Graph. Model. 27, 497-505.

Chen, S. H., Wei, J. M. \& Farina, V. (1993a). Taxol structure-activity relationships: Synthesis and biological evaluation of 2-deoxytaxol. Tetrahedron Lett. 34, 3205-3206.

Chen, S. H., Huang, S., Kant, J., Fairchild, C., Wei, J. M. \& Farina, V. (1993b). Synthesis of 7-deoxytaxol and 7,10-dideoxytaxol via radical intermediates. J. Org. Chem. 58, 5028-5029.

Chou, T. C. \& Talalay, P. (1984). Quantitative-analysis of dose-effect relationships - The combinded effects of multiple drugs or enzyme inhibitors. Adv. Enzyme. Regul. 22, 27-55.

Chou, T. C., Zhang, X., Zhong, Z.-Y., Li, Y., Feng, L., Eng, S., Myles, D. R., Johnson, R., Jr., Wu, N., Yin, Y. I., et al. (2008). Therapeutic effect against human xenograft tumors in nude mice by the third generation microtubule stabilizing epothilones. P. Natl. Acad. Sci. USA 105, 13157-13162.

Chrétien, D., Metoz, F., Verde, F., Karsenti, E. \& Wade, R. H. (1992). Lattice-defects in microtubules protofilament numbers vary within individual microtubule. J. Cell Biol. 117, 1031-1040.

Ciomei, M., Albanese, C., Pastori, W., Grandi, M., Pietra, F., D'Ambrosio, M., Guerriero, A. \& Battistini, C. (1997). Sarcodictyins: A new class of marine derivatives with mode of action similar to taxol. P. Am. Assoc. Canc. Res. 38, 5.

Clark, E. A., Hills, P. M., Davidson, B. S., Wender, P. A. \& Mooberry, S. L. (2006). Laulimalide and synthetic laulimalide analogues are synergistic with paclitaxel and 2-methoxyestradiol. Mol. Pharmaceut. 3, 457-467. 
Coleman, R. S., Campbell, E. L. \& Carper, D. J. (2009). A direct and efficient total synthesis of the tubulin-binding agents ceratamine $\mathrm{A}$ and $\mathrm{B}$; Use of IBX for a remarkable heterocycle dehydrogenation. Org. Lett. 11, 2133-2136.

Colomer, R. (2008). Review of gemcitabine plus taxane combination therapy in the first-line treatment of metastatic breast cancer. EJC Suppl. 6, 9-12.

Cordon-Cardo, C., Obrien, J. P., Casals, D., Rittmangrauer, L., Biedler, J. L., Melamed, M. R. \& Bertino, J. R. (1989). Multidrug-resistance gene (P-Glycoprotein) is expressed by endothelial-cells at bloodbrain barrier sites. P. Natl. Acad. Sci. USA 86, 695-698.

Cormier, A., Knossow, M., Wang, C. G. \& Gigant, B. (2010). The Binding of Vinca domain agents to tubulin: Structural and biochemical studies. In Methods in Cell Biology (L. Wilson \& J. J. Correia, Eds.) Methods in Cell Biology, Vol 95: Microtubules, in Vitro: 95, (pp 373-390).

Correia, J. J., Baty, L. T. \& Williams, R. C. (1987). $\mathrm{Mg}^{2+}$ dependence of guanine-nucleotide binding to tubulin. J. Biol. Chem. 262, 17278-17284.

Crume, K. P., O'Sullivan, D., Miller, J. H., Northcote, P. T. \& La Flamme, A. C. (2009). Delaying the onset of experimental autoimmune encephalomyelitis with the microtubule-stabilizing compounds, paclitaxel and peloruside A. J. Leukocyte Biol. 86, 949-958.

Cutignano, A., Bruno, I., Bifulco, G., Casapullo, A., Debitus, C., Gomez-Paloma, L. \& Riccio, R. (2001). Dactylolide, a new cytotoxic macrolide from the Vanuatu sponge Dactylospongia sp. Eur. J. Org. Chem. 775-778.

Dark, G. G., Hill, S. A., Prise, V. E., Tozer, G. M., Pettit, G. R. \& Chaplin, D. J. (1997). Combretastatin A4, an agent that displays potent and selective toxicity toward tumor vasculature. Cancer Res. 57, 1829-1834.

Das, V. \& Miller, J. H. (2012). Microtubule stabilization by peloruside A and paclitaxel rescues degenerating neurons from okadaic acid-induced tau phosphorylation. Eur. J. Neurosci. 35, 17051717.

David-Pfeuty, T., Simon, C. \& Pantaloni, D. (1979). Effect of anti-mitotic drugs on tuublin GTPase activity and self-assembly. J. Biol Chem 254, 1696-1702.

Delmonte, A. \& Sessa, C. (2009). AVE8062: a new combretastatin derivative vascular disrupting agent. Expert Opin. Inv. Drugs 18, 1541-1548.

Detrich, H. W., Williams, R. C. \& Wilson, L. (1982). Effect of colchicine binding on the reversible dissociation of the tubulin dimer. Biochemistry 21, 2392-2400.

DeVita, V. T. \& Rosenberg, S. A. (2012). Two hundred years of cancer research. New Engl. J. Med. 366, 2207-2214.

DeVita, V. T., Jr. \& Chu, E. (2008). A History of Cancer Chemotherapy. Cancer Res. 68, 8643-8653.

Devred, F., Tsvetkov, P. O., Barbier, P., Allegro, D., Horwitz, S. B., Makarov, A. A. \& Peyrot, V. (2008). Stathmin/Op18 is a novel mediator of vinblastine activity. FEBS Lett. 582, 2484-2488.

Díaz, J. F., Andreu, J. M. \& Jiménez-Barbero, J. (2009). The interaction of microtubules with stabilizers characterized at biochemical and structural levels. (T. Carlomagno, Ed.) Tubulin-Binding Agents: Synthetic, Structural and Mechanistic Insights: 286, (pp 121-149).

Díaz, J. F. \& Buey, R. M. (2007). Characterizing ligand-microtubule binding by competition methods. (J. Zhou, Ed.) Methods in Molecular Medicine, Vol 137: Microtubule Protocols: Humana Press Inc., Totowa, NJ.

Díaz, J. F., Barasoain, I., Souto, A. A., Amat-Guerri, F. \& Andreu, J. M. (2005). Macromolecular accessibility of fluorescent taxoids bound at a paclitaxel binding site in the microtubule surface. $J$ Biol. Chem. 280, 3928-3937.

Díaz, J. F., Barasoain, I. \& Andreu, J. M. (2003). Fast kinetics of taxol binding to microtubules - Effects of solution variables and microtubule-associated proteins. J Biol. Chem. 278, 8407-8419.

Díaz, J. F., Strobe, R., Engelborghs, Y., Souto, A. A. \& Andreu, J. M. (2000). Molecular recognition of Taxol by microtubules - Kinetics and thermodynamics of binding of fluorescent Taxol derivatives to an exposed site. J Biol. Chem. 275, 26265-26276.

Díaz, J. F., Valpuesta, J. M., Chacon, P., Diakun, G. \& Andreu, J. M. (1998). Changes in microtubule protofilament number induced by taxol binding to an easily accessible site - Internal microtubule dynamics. J Biol. Chem. 273, 33803-33810.

Díaz, J. F., Andreu, J. M., Diakun, G., Towns-Andrews, E. \& Bordas, J. (1996). Structural intermediates in the assembly of taxoid-induced microtubules and GDP-tubulin double rings: Time-resolved X-ray scattering. Biophys. J. 70, 2408-2420.

Díaz, J. F. \& Andreu, J. M. (1993). Assembly of purified GDP tubulin into microtubules induced by Taxol and Taxotere - Reversibility, ligand stoichiometry, and competition. Biochemistry 32, 2747-2755.

Díaz, J. F., Menendez, M. \& Andreu, J. M. (1993). Thermodynamics of ligand-induced assembly of tubulin. Biochemistry 32, 10067-10077. 
Ding, F. \& Jennings, M. P. (2008). Total synthesis of (-)-dactylolide and formal synthesis of (-)zampanolide via target oriented $\beta$-C-glycoside formation. J. Org. Chem. 73, 5965-5976.

Dorléans, A., Gigant, B., Ravelli, R. B. G., Mailliet, P., Mikol, V. \& Knossow, M. (2009). Variations in the colchicine-binding domain provide insight into the structural switch of tubulin. P. Natl. Acad. Sci. USA 106, 13775-13779.

Dumontet, C. \& Jordan, M. A. (2010). Microtubule-binding agents: a dynamic field of cancer therapeutics. Nat. Rev. Drug Discov. 9, 790-803.

Dye, R. B., Fink, S. P. \& Williams, R. C. (1993). Taxol-induced flexibility of microtubules and its reversal by MAP-2 and Tau. J. Biol. Chem. 268, 6847-6850.

Edler, M. C., Buey, R. M., Gussio, R., Marcus, A. I., Vanderwal, C. D., Sorensen, E. J., Díaz, J. F., Giannakakou, P. \& Hamel, E. (2005). Cyclostreptin (FR182877), an antitumor tubulinpolymerizing agent deficient in enhancing tubulin assembly despite its high affinity for the taxoid site. Biochemistry 45, 5932-5932.

Engelborghs, Y. (1998). General features of the recognition by tubulin of colchicine and related compounds. Eur. Biophys. J. Biophy. 27, 437-445.

Eva, A., Robbins, K. C., Andersen, P. R., Srinivasan, A., Tronick, S. R., Reddy, E. P., Ellmore, N. W., Galen, A. T., Lautenberger, J. A., Papas, T. S., et al. (1982). Cellular genes analogous to retroviral ONC genes are transcribed in human-tumor cells. Nature 295, 116-119.

Evangelio, J. A., Abal, M., Barasoain, I., Souto, A. A., Lillo, M. P., Acuna, A. U., Amat-Guerri, F. \& Andreu, J. M. (1998). Fluorescent taxoids as probes of the microtubule cytoskeleton. Cell Motil. Cytoskel. 39, 73-90.

Field, J. J., Pera, B., Calvo, E., Canales, A., Zurwerra, D., Trigili, C., Rodríguez-Salarichs, J., Matesanz, R., Kanakkanthara, A., Wakefield, S., et al. (2012). Zampanolide, a potent new microtubulestabilizing agent, covalently reacts with the taxane luminal site in tubulin $\alpha, \beta$-heterodimers and microtubules. Chem. Biol. 19, 686-698.

Field, J. J., Singh, A. J., Kanakkanthara, A., Halafihi, T., Northcote, P. T. \& Miller, J. H. (2009). Microtubule-stabilizing activity of zampanolide, a potent macrolide isolated from the tongan marine sponge Cacospongia mycofijiensis. J. Med. Chem. 52, 7328-7332.

Fitzgerald, T. J. (1976). Molecular features of colchicine associated with antimitotic activity and inhibition of tubulin. Biochem. Pharmacol. 25, 1383-1387.

Fourniol, F. J., Sindelar, C. V., Amigues, B., Clare, D. K., Thomas, G., Perderiset, M., Francis, F., Houdusse, A. \& Moores, C. A. (2010). Template-free 13-protofilament microtubule-MAP assembly visualized at 8 angstrom resolution. J.Cell Biol. 191, 463-470.

Freedman, H., Huzil, J. T., Luchko, T., Luduena, R. F. \& Tuszynski, J. A. (2009). Identification and characterization of an intermediate Taxol binding site within microtubule nanopores and a mechanism for tubulin isotype binding selectivity. J. Chem. Inf. Model. 49, 424-436.

Froidevaux-Klipfel, L., Poirier, F., Boursier, C., Crepin, R., Pous, C., Baudin, B. \& Baillet, A. (2011). Modulation of septin and molecular motor recruitment in the microtubule environment of the Taxol-resistant human breast cancer cell line MDA-MB-231. Proteomics 11, 3877-3886.

Gaitanos, T. N., Buey, R. M., Díaz, J. F., Northcote, P. T., Teesdale-Spittle, P., Andreu, J. M. \& Miller, J. H. (2004). Peloruside A does not bind to the taxoid site on $\beta$-tubulin and retains its activity in multidrug-resistant cell lines. Cancer Res. 64, 5063-5067.

Galsky, M. D., Dritselis, A., Kirkpatrick, P. \& Oh, W. K. (2010). Cabazitaxel. Nat. Rev. Drug Discov. 9, 677-678.

Gapud, E. J., Bai, R. L., Ghosh, A. K. \& Hamel, E. (2004). Laulimalide and paclitaxel: A comparison of their effects on tubulin assembly and their synergistic action when present simultaneously. Mol. Pharmacol. 66, 113-121.

Gebremichael, Y., Chu, J. W. \& Voth, G. A. (2008). Intrinsic bending and structural rearrangement of tubulin dimer: Molecular dynamics simulations and coarse-grained analysis. Biophys. J. 95, 24872499.

Gertsch, J., Meier, S., Muller, M. \& Altmann, K. H. (2009). Differential Effects of Natural Product Microtubule Stabilizers on Microtubule Assembly: Single Agent and Combination Studies with Taxol, Epothilone B, and Discodermolide. Chembiochem 10, 166-175.

Ghosh, A. K., Cheng, X., Bai, R. L. \& Hamel, E. (2012). Total synthesis of potent antitumor macrolide (-)zampanolide: An oxidative intramolecular cyclization-based strategy. Eur. J. Org. Chem. 41304139.

Ghosh, A. K. \& Cheng, X. (2011). Enantioselective Total Synthesis of (-)-Zampanolide, a Potent Microtubule-Stabilizing Agent. Org. Lett. 13, 4108-4111.

Giannakakou, P. \& Fojo, T. (2000). Discodermolide: Just another microtubule-stabilizing agent? No! A lesson in synergy. Clin. Cancer Res. 6, 1613-1615. 
Giannakakou, P., Gussio, R., Nogales, E., Downing, K. H., Zaharevitz, D., Bollbuck, B., Poy, G., Sackett, D., Nicolaou, K. C. \& Fojo, T. (2000). A common pharmacophore for epothilone and taxanes: Molecular basis for drug resistance conferred by tubulin mutations in human cancer cells. P. Natl. Acad. Sci. USA 97, 2904-2909.

Giannakakou, P., Villalba, L., Li, H., Poruchynsky, M. \& Fojo, T. (1998). Combinations of paclitaxel and vinblastine and their effects on tubulin polymerization and cellular cytotoxicity: Characterization of a synergistic schedule. Int. J. Cancer 75, 57-63.

Giannakakou, P., Sackett, D. L., Kang, Y. K., Zhan, Z. R., Buters, J. T. M., Fojo, T. \& Poruchynsky, M. S. (1997). Paclitaxel-resistant human ovarian cancer cells have mutant $\beta$-tubulins that exhibit impaired paclitaxel-driven polymerization. J Biol. Chem. 272, 17118-17125.

Gigant, B., Cormier, A., Dorleans, A., Ravelli, R. B. G. \& Knossow, M. (2009). Microtubule-destabilizing agents: Structural and mechanistic insights from the interaction of colchicine and vinblastine with tubulin. In Topics in Current Chemistry (T. Carlomagno, Ed.) Tubulin-Binding Agents: Synthetic, Structural and Mechanistic Insights: 286, (pp 259-278).

Gigant, B., Wang, C. G., Ravelli, R. B. G., Roussi, F., Steinmetz, M. O., Curmi, P. A., Sobel, A. \& Knossow, M. (2005). Structural basis for the regulation of tubulin by vinblastine. Nature $\mathbf{4 3 5}$, 519-522.

Gigant, B., Curmi, P. A., Martin-Barbey, C., Charbaut, E., Lachkar, S., Lebeau, L., Siavoshian, S., Sobel, A. \& Knossow, M. (2000). The 4 angstrom X-ray structure of a tubulin : stathmin-like domain complex. Cell 102, 809-816.

Gonzalez-Garay, M. L., Chang, L., Blade, K., Menick, D. R. \& Cabral, F. (1999). A $\beta$-tubulin leucine cluster involved in microtubule assembly and paclitaxel resistance. J. Biol. Chem. 274, 2387523882.

Goodin, S., Kane, M. P. \& Rubin, E. H. (2004). Epothilones: Mechanism of action and biologic activity. Journal of Clinical Oncology 22, 2015-2025.

Gottesman, M. M., Fojo, T. \& Bates, S. E. (2002). Multidrug resistance in cancer: Role of ATP-dependent transporters. Nat. Rev. Cancer 2, 48-58.

Gradishar, W. J., Tjulandin, S., Davidson, N., Shaw, H., Desai, N., Bhar, P., Hawkins, M. \& O'Shaughnessy, J. (2005). Phase III trial of nanoparticle albumin-bound paclitaxel compared with polyethylated castor oil-based paclitaxel in women with breast cancer. J. Clin. Oncol. 23, 77947803.

Grover, S. \& Hamel, E. (1994). The magnesium-GTP, interaction in microtubule assembly. Eur. J. Biochem. 222, 163-172.

Guex, N. \& Peitsch, M. C. (1997). SWISS-MODEL and the Swiss-PdbViewer: An environment for comparative protein modeling. Electrophoresis 18, 2714-2723.

Haldar, S., Chintapalli, J. \& Croce, C. M. (1996). Taxol induces bcl-2 phosphorylation and death of prostate cancer cells. Cancer Res. 56, 1253-1255.

Hamel, E. (2008). An overview of compounds that interact with tubulin and their effects of microtubule assembly. In Cancer Drug Discovery and Development (A. T. Fojo, Ed.) The Role of Microtubules in Cell Biology, Neurobiology, and Oncology, (pp 1-20): Humana Press.

Hamel, E., Day, B. W., Miller, J. H., Jung, M. K., Northcote, P. T., Ghosh, A. K., Curran, D. P., Cushman, M., Nicolaou, K. C., Paterson, I., et al. (2006). Synergistic effects of peloruside a and laulimalide with taxoid site drugs, but not with each other, on tubulin assembly. Mol. Pharmacol. 70, 1555 1564.

Hamel, E., Sackett, D. L., Vourloumis, D. \& Nicolaou, K. C. (1999). The coral-derived natural products eleutherobin and sarcodictyins A and B: Effects on the assembly of purified tubulin with and without microtubule-associated proteins and binding at the polymer taxoid site. Biochemistry 38, 5490-5498.

Hamel, E. (1992). Natural products which interact with tublulin in the vinca domain: Maythansine, rhizoxin, phomipsin-A, dolastatins 10 and 15 andhalichondrin B. Pharmacol. Therapeut. 55, 3151.

Hammond, J. W., Cai, D. W. \& Verhey, K. J. (2008). Tubulin modifications and their cellular functions. Curr. Opin. Cell Biol. 20, 71-76.

Han, G. Z., Liu, Z. J., Shimoi, K. \& Zhu, B. T. (2005). Synergism between the anticancer actions of 2 methoxyestradiol and microtubule-disrupting agents in human breast cancer. Cancer Res. 65, 387 393.

Hara, T., Ushio, K., Nishiwaki, M., Kouno, J., Araki, H., Hikichi, Y., Hattori, M., Imai, Y. \& Yamaoka, M. (2010). A mutation in $\beta$-tubulin and a sustained dependence on androgen receptor signalling in a newly established docetaxel-resistant prostate cancer cell line. Cell Biol. Int. 34, 177-184. 
Hari, M., Loganzo, F., Annable, T., Tan, X. Z., Musto, S., Morilla, D. B., Nettles, J. H., Snyder, J. P. \& Greenberger, L. M. (2006). Paclitaxel-resistant cells have a mutation in the paclitaxel-binding region of $\beta$-tubulin $\left(\mathrm{Asp}^{26} \mathrm{Glu}\right)$ and less stable microtubules. Mol. Cancer Ther. 5, 270-278.

Harvey, A. L., Clark, R. L., Mackay, S. P. \& Johnston, B. F. (2010). Current strategies for drug discovery through natural products. Expert Opin. Drug Dis. 5, 559-568.

Hastie, S. B. (1991). Interactions of colchicine with tubulin. Pharmacol. Therapeut. 51, 377-401.

He, L. F., Orr, G. A. \& Horwitz, S. B. (2001). Novel molecules that interact with microtubules and have functional activity similar to Taxol ${ }^{\mathrm{TM}}$. Drug Discov. Today 6, 1153-1164.

He, L. F., Jagtap, P. G. F., Kingston, D. G. I., Shen, H. J., Orr, G. A. \& Horwitz, S. B. (2000). A common pharmacophore for Taxol and the epothilones based on the biological activity of a taxane molecule lacking a C-13 side chain. Biochemistry 39, 3972-3978.

Hoffmann, J., Fichtner, I., Lemm, M., Lienau, P., Hess-Stumpp, H., Rotgeri, A., Hofmann, B. \& Klar, U. (2009). Sagopilone crosses the blood-brain barrier in vivo to inhibit brain tumor growth and metastases. Neuro-Oncol 11, 158-166.

Honore, S., Kamath, K., Braguer, D., Horwitz, S. B., Wilson, L., Briand, C. \& Jordan, M. A. (2004). Synergistic suppression of microtubule dynamics by discodermolide and paclitaxel in non-small cell lung carcinoma cells. Cancer Res. 64, 4957-4964.

Hood, K. A., West, L. M., Rouwé, B., Northcote, P. T., Berridge, M. V., Wakefield, S. J. \& Miller, J. H. (2002). Peloruside A, a novel antimitotic agent with paclitaxel-like microtubule-stabilizing activity. Cancer Res. 62, 3356-3360.

Hotchkiss, K. A., Ashton, A. W., Mahmood, R., Russell, R. G., Sparano, J. A. \& Schwartz, E. L. (2002). Inhibition of endothelial cell function in vitro and angiogenesis in vivo by docetaxel (Taxotere): Association with impaired repositioning of the microtubule organizing center. Mol. Cancer Ther. 1, 1191-1200.

Howard, W. D. \& Timasheff, S. N. (1986). GDP state of tubulin-stabilization of double rings. Biochemistry 25, 8292-8300.

Hoye, T. R. \& Hu, M. (2003). Macrolactonization via Ti(IV)-mediated epoxy-acid coupling: A total synthesis of (-)-dactylolide [and zampanolide]. J. Am. Chem. Soc. 125, 9576-9577.

Huang, G. S., Lopez-Barcons, L., Freeze, B. S., Smith, A. B., Goldberg, G. L., Horwitz, S. B. \& McDaid, H. M. (2006). Potentiation of taxol efficacy and by discodermolide in ovarian carcinoma xenograft-bearing mice. Clin. Cancer Res. 12, 298-304.

Hung, D. T., Chen, J. \& Schreiber, S. L. (1996). (+)-Discodermolide binds to microtubules in stoichiometric ratio to tubulin dimers, blocks Taxol binding and results in mitotic arrest. Chem. Biol. 3, 287-293.

Huzil, J. T., Chik, J. K., Slysz, G. W., Freedman, H., Tuszynski, J., Taylor, R. E., Sackett, D. L. \& Schriemer, D. C. (2008). A unique mode of microtubule stabilization induced by peloruside A. $J$. Mol. Biol. 378, 1016-1030.

Hyman, A. A., Salser, S., Drechsel, D. N., Unwin, N. \& Mitchison, T. J. (1992). Role of GTP hydrolysis in microtubule dynamics: Information from a slowly hydrolyzable analog, GMPCPP. Mol. Biol. Cell 3, 1155-1167.

Isbrucker, R. A., Cummins, J., Pomponi, S. A., Longley, R. E. \& Wright, A. E. (2003). Tubulin polymerizing activity of dictyostatin-1, a polyketide of marine sponge origin. Biochem. Pharmacol. 66, 75-82.

Jackson, J. R., Patrick, D. R., Dar, M. M. \& Huang, P. S. (2007). Targeted anti-mitotic therapies: can we improve on tubulin agents? Nat. Rev. Cancer 7, 107-117.

Jemal, A., Bray, F., Center, M. M., Ferlay, J., Ward, E. \& Forman, D. (2011). Global Cancer Statistics. CACancer J. Clin. 61, 69-90.

Jiménez-Barbero, J., Canales, A., Northcote, P. T., Buey, R. M., Andreu, J. M. \& Díaz, J. F. (2006). NMR determination of the bioactive conformation of peloruside a bound to microtubules. J. Am. Chem. Soc. 128, 8757-8765.

Johnson, I. S., Wright, H. F. \& Svoboda, G. H. (1959). Experimental basis for clinical evaluation of anti tumor principles derived from Vinca-Rosea Linn. J. Lab. Clin. Med. 54, 830-830.

Jordan, M. A. \& Wilson, L. (2004). Microtubules as a target for anticancer drugs. Nat. Rev. Cancer 4, $253-$ 265.

Jordan, A., Hadfield, J. A., Lawrence, N. J. \& McGown, A. T. (1998). Tubulin as a target for anticancer drugs: Agents which interact with the mitotic spindle. Med. Res. Rev. 18, 259-296.

Jordan, M. A., Toso, R. J., Thrower, D. \& Wilson, L. (1993). Mechanism of mitotic block and inhibition of cell-proliferation by taxol at low concentrations. P. Natl. Acad. Sci. USA 90, 9552-9556. 
Jourdain, L., Curmi, P., Sobel, A., Pantaloni, D. \& Carlier, M. F. (1997). Stathmin: A tubulin-sequestering protein which forms a ternary $\mathrm{T}_{2} \mathrm{~S}$ complex with two tubulin molecules. Biochemistry 36, 1081710821.

Kanakkanthara, A., Rawson, P., Northcote, P. T. \& Miller, J. H. (2012). Acquired resistance to peloruside A and laulimalide is associated with downregulation of vimentin in human ovarian carcinoma cells. Pharm. Res. 29, 3022-3032.

Kanakkanthara, A., Wilmes, A., O'Brate, A., Escuin, D., Chan, A., Gjyrezi, A., Crawford, J., Rawson, P., Kivell, B., Northcote, P. T., et al. (2011). Peloruside- and laulimalide-resistant human ovarian carcinoma cells have $\beta I$-tubulin mutations and altered expression of $\beta$ II- and $\beta$ III-tubulin isotypes. Mol. Cancer Ther. 10, 1419-1429.

Kar, S., Florence, G. J., Paterson, I. \& Amos, L. A. (2003). Discodermolide interferes with the binding of tau protein to microtubules. FEBS Lett. 539, 34-36.

Karjala, G., Chan, Q., Manzo, E., Andersen, R. J. \& Roberge, N. (2005). Ceratamines, structurally simple microtubule-stabilizing antimitotic agents with unusual cellular effects. Cancer Res. 65, 30403043.

Kavallaris, M., Burkhart, C. A. \& Horwitz, S. B. (1999). Antisense oligonucleotides to class III $\beta$-tubulin sensitize drug-resistant cells to Taxol. Brit. J Cancer 80, 1020-1025.

Kavallaris, M. (2010). Microtubules and resistance to tubulin-binding agents. Nat. Rev. Cancer 10, 194204.

Keen, N. \& Taylor, S. (2004). Aurora-kinase inhibitors as anticancer agents. Nat. Rev. Cancer 4, 927-936.

Khrapunovich-Baine, M., Menon, V., Yang, C. P. H., Northcote, P. T., Miller, J. H., Angeletti, R. H., Fiser, A., Horwitz, S. B. \& Xiao, H. (2011). Hallmarks of molecular action of microtubule stabilizing agents. Effects of epothilone B, ixabepilone, peloruside $\mathrm{A}$ and laulimalide on microtubule conformation. J. Biol. Chem. 286, 11765-11778.

Khrapunovich-Baine, M., Menon, V., Verdier-Pinard, P., Smith, A. B., Angeletti, R. H., Fiser, A., Horwitz, S. B. \& Xiao, H. (2009). Distinct pose of discodermolide in taxol binding pocket drives a complementary mode of microtubule stabilization. Biochemistry 48, 11664-11677.

Kirchner, K. \& Mandelkow, E. M. (1985). Tubulin domains responsible for assembly of dimers and protofilaments. EMBO J. 4, 2397-2402.

Komlodi-Pasztor, E., Sackett, D. L. \& Fojo, A. T. (2012). Inhibitors targeting mitosis: Tales of how great drugs against a promising target were brought down by a flawed rationale. Clin. Cancer Res. 18, 51-63.

Komlodi-Pasztor, E., Sackett, D., Wilkerson, J. \& Fojo, T. (2011). OPINION. Mitosis is not a key target of microtubule agents in patient tumors. Nat. Rev. Clin. Oncol. 8, 244-250.

Kowalski, R. J., Giannakakou, P., Gunasekera, S. P., Longley, R. E., Day, B. W. \& Hamel, E. (1997). The microtubule-stabilizing agent discodermolide competitively inhibits the binding of paclitaxel (Taxol) to tubulin polymers, enhances tubulin nucleation reactions more potently than paclitaxel, and inhibits the growth of paclitaxel-resistant cells. Mol. Pharmacol. 52, 613-622.

Kramer, N., Walzl, A., Unger, C., Rosner, M., Krupitza, G., Hengstschlager, M. \& Dolznig, H. (2013). In vitro cell migration and invasion assays. Mutat. Res-Rev Mutat. 752, 10-24.

La Regina, G., Edler, M. C., Brancale, A., Kandil, S., Coluccia, A., Piscitelli, F., Hamel, E., De Martino, G., Matesanz, R., Díaz, J. F., et al. (2007). Arylthioindole inhibitors of tubulin polymerization. 3. Biological evaluation, structure-activity relationships and molecular modeling studies. J. Med. Chem. 50, 2865-2874.

Lakowicz, J. R. (2006). Fluorescence Anisotropy. (J. R. Lakowicz, Ed.) Principles of Fluorescence Spectroscopy, (pp 353-382): Springer US.

Lechleider, R. J., Kaminskas, E., Jiang, X. P., Aziz, R., Bullock, J., Kasliwal, R., Harapanhalli, R., Pope, S., Sridhara, R., Leighton, J., et al. (2008). Ixabepilone in combination with capecitabine and as monotherapy for treatment of advanced breast cancer refractory to previous chemotherapies. Clin. Cancer Res. 14, 4378-4384.

Lee, J. C. \& Timasheff, S. N. (1975). Reconstitution of microtubules from purified calf brain tubulin. Biochemistry 14, 5183-5187.

Lee, V. M. Y., Daughenbaugh, R. \& Trojanowski, J. Q. (1994). Microtubule stabilizing drugs for the treatment of alzheimers-disease. Neurobiol. Aging 15, S87-S89.

Lengauer, C., Kinzler, K. W. \& Vogelstein, B. (1998). Genetic instabilities in human cancers. Nature 396, 643-649.

Li, H. L., DeRosier, D. J., Nicholson, W. V., Nogales, E. \& Downing, K. H. (2002). Microtubule structure at 8 angstrom Resolution. Structure 10, 1317-1328.

Li, J. W. H. \& Vederas, J. C. (2009). Drug discovery and natural products: End of an era or an endless frontier? Science 325, 161-165. 
LiCata, V. J. \& Wowor, A. J. (2008). Applications of Fluorescence Anisotropy to the Study of ProteinDNA Interactions. (J. C. Dr. John \& Dr. H. William Detrich, III, Eds.) Methods in Cell Biology: Volume 84, (pp 243-262): Elsevier Inc. Academic Press.

Lin, Y. D., Kao, J. Q. \& Chen, C. T. (2007). Catalytic conjugate additions of nitrogen-, phosphorus-, and carbon-containing nucleophiles by amphoteric vanadyl triflate. Org. Lett. 9, 5195-5198.

Liu, J. K., Towle, M. J., Cheng, H. S., Saxton, P., Reardon, C., Wu, J. Y., Murphy, E. A., Kuznetsov, G., Johannes, C. W., Tremblay, M. R., et al. (2007). In vitro and in vivo anticancer activities of synthetic (-)-laulimalide, a marine natural product microtubule stabilizing agent. Anticancer Res. 27, 1509-1518.

Lobert, S. \& Correia, J. (2007). Methods for studying Vinca alkaloid interactions with tubulin. In Methods in Molecular Medicine ${ }^{\mathrm{TM}}$ (J. Zhou, Ed.) Methods in Molecular Medicine, Vol 137: Microtubule Protocols, New Jersey, USA: Humana Press Inc.

Lobert, S., Ingram, J. W. \& Correia, J. J. (2007). The thermodynamics of vinca alkaloid-induced tubulin spirals formation. Biophys. Chem. 126, 50-58.

Long, B. H., Carboni, J. M., Wasserman, A. J., Cornell, L. A., Casazza, A. M., Jensen, P. R., Lindel, T., Fenical, W. \& Fairchild, C. R. (1998). Eleutherobin, a novel cytotoxic agent that induces tubulin polymerization, is similar to paclitaxel $\left(\right.$ Taxol $\left.^{\circledR}\right)$. Cancer Res. 58, 1111-1115.

Löwe, J., Li, H., Downing, K. H. \& Nogales, E. (2001). Refined structure of $\alpha \beta$-tubulin at $3.5 \AA$ resolution. J. Mol. Biol. 313, 1045-1057.

Lu, H. Y., Murtagh, J. \& Schwartz, E. L. (2006). The microtubule binding drug laulimalide inhibits vascular endothelial growth factor-induced human endothelial cell migration and is synergistic when combined with docetaxel taxotere). Mol. Pharmacol. 69, 1207-1215.

Lu, Q. \& Luduena, R. F. (1993). Removal of $\beta$ III-isotype enhances taxol-induced microtubule assembly. Cell Struct. Funct. 18, 173-182.

Luduena, R. F. (1998). Multiple forms of tubulin: Different gene products and covalent modifications. In International Review of Cytology-a Survey of Cell Biology (K. W. Jeon, Ed.) Int.Rev. Cytol.: 178, (pp 207-275).

Ma, X. \& Yu, H. (2006). Global Burden of Cancer. Yale J. Biol. Med. 79, 85-94.

Madiraju, C., Edler, M. C., Hamel, E., Raccor, B. S., Balachandran, R., Zhu, G. Y., Giuliano, K. A., Vogt, A., Shin, Y. S., Fournier, J. H., et al. (2005). Tubulin assembly, taxoid site binding, and cellular effects of the microtubule-stabilizing agent dictyostatin. Biochemistry 44, 15053-15063.

Magnani, M., Maccari, G., Andreu, J. M., Díaz, J. F. \& Botta, M. (2009). Possible binding site for paclitaxel at microtubule pores. FEBS J. 276, 2701-2712.

Martello, L. A., McDaid, H. M., Regl, D. L., Yang, C. P. H., Meng, D. F., Pettus, T. R. R., Kaufman, M. D., Arimoto, H., Danishefsky, S. J., Smith, A. B., et al. (2000). Taxol and discodermolide represent a synergistic drug combination in human carcinoma cell lines. Clin. Cancer Res. 6, 1978-1987.

Massarotti, A., Coluccia, A., Silvestri, R., Sorba, G. \& Brancale, A. (2012). The tubulin colchicine domain: A molecular modeling perspective. Chemmedchem 7, 33-42.

Matesanz, R., Rodríguez-Salarichs, J., Pera, B., Canales, A., Andreu, J. M., Jiménez-Barbero, J., Bras, W., Nogales, A., Fang, W. S. \& Díaz, J. F. (2011a). Modulation of microtubule interprotofilament interactions by modified taxanes. Biophys. J. 101, 2970-2980.

Matesanz, R., Benet, P. \& Díaz, J. F. (2011b). Chapter 28: Thermodynamics as a Tool for the Optimization of Drug Binding. (J. C. Moreno-Pirajan, Ed.) Thermodynamics - Interaction Studies - Solids, Liquids and Gases: .

Matesanz, R., Barasoain, I., Yang, C. G., Wang, L., Li, X., De Ines, C., Coderch, C., Gago, F., Barbero, J. J., Andreu, J. M., et al. (2008). Optimization of taxane binding to microtubules: Binding affinity dissection and incremental construction of a high-affinity analog of paclitaxel. Chem. Biol. 15, 573-585.

Mayer, A. M. S. \& Gustafson, K. R. (2008). Marine pharmacology in 2005-2006: Antitumour and cytotoxic compounds. Eur. J. Cancer 44, 2357-2387.

Mayer, M. \& Meyer, B. (2001). Group epitope mapping by saturation transfer difference NMR to identify segments of a ligand in direct contact with a protein receptor. J. Am. Chem. Soc. 123, 6108-6117.

Mayer, M. \& Meyer, B. (1999). Characterization of ligand binding by saturation transfer difference NMR spectroscopy. Angew. Chem. Int. Ed. 38, 1784-1788.

Medrano, F. J., Andreu, J. M., Gorbunoff, M. J. \& Timasheff, S. N. (1991). Roles of ring-C oxygens in the binding if colchicine to tubulin. Biochemistry 30, 3770-3777.

Melnikova, I. \& Golden, J. (2004). Targeting protein kinases. Nat. Rev. Drug Discov. 3, 993-994. 
Menéndez, M., Rivas, G., Díaz, J. F. \& Andreu, J. M. (1998). Control of the structural stability of the tubulin dimer by one high affinity bound magnesium ion at nucleotide $\mathrm{N}$-site. J Biol. Chem. 273, 167-176.

Meyer, B. \& Peters, T. (2003). NMR Spectroscopy techniques for screening and identifying ligand binding to protein receptors. Angew. Chem. Int. Ed. 42, 864-890.

Meyer, C., Ferguson, D., Krauth, M., Wick, M. \& Northcote, P. (2006). RTA 301 (peloruside): a novel microtubule stabilizer with potent vivo activity against lung cancer and resistant breast cancer. EJC Suppl. 4, 192-193.

Mitchison, T. \& Kirschner, M. (1984). Dynamic instability of microtubule growth. Nature 312, $237-242$.

Mitra, A. \& Sept, D. (2008). Taxol allosterically alters the dynamics of the tubulin dimer and increases the flexibility of microtubules. Biophys. J. 95, 3252-3258.

Monzo, M., Rosell, R., Sanchez, J. J., Lee, J. S., O'Brate, A., Gonzalez-Larriba, J. L., Alberola, V., Lorenzo, J. C., Nunez, L., Ro, J. Y., et al. (1999). Paclitaxel resistance in non-small-cell lung cancer associated with beta-tubulin gene mutations. J. Clin. Onco. 17, 1786-1793.

Mooberry, S. L., Tien, G., Hernandez, A. H., Plubrukarn, A. \& Davidson, B. S. (1999). Laulimalide and isolaulimalide, new paclitaxel-like microtubule stabilizing agents. Cancer Res. 59, 653-660.

Mooney, C. J., Nagaiah, G., Fu, P., Wasman, J. K., Cooney, M. M., Savvides, P. S., Bokar, J. A., Dowlati, A., Wang, D., Agarwala, S. S., et al. (2009). A phase II trial of fosbretabulin in advanced anaplastic thyroid carcinoma and correlation of baseline serum-soluble intracellular adhesion molecule-1 with outcome. Thyroid 19, 233-240.

Moores, C. A., Perderiset, M., Francis, F., Chelly, J., Houdusse, A. \& Milligan, R. A. (2004). Mechanism of microtubule stabilization by doublecortin. Mol. Cell 14, 833-839.

Morris, G. M., Goodsell, D. S., Halliday, R. S., Huey, R., Hart, W. E., Belew, R. K. \& Olson, A. J. (1998). Automated docking using a Lamarckian genetic algorithm and an empirical binding free energy function. J.Comput. Chem. 19, 1639-1662.

Morris, P. G. \& Fornier, M. N. (2008). Microtubule active agents: Beyond the taxane frontier. Clin. Cancer Res. 14, 7167-7172.

Mosmann, T. (1983). Rapid colourmetric assay for cellular growth and survival - application to proliferation and cyto-toxicity assays. J. Immuno. Meth. 65, 55-63.

Mozzetti, S., Ferlini, C., Concolino, P., Filippetti, F., Raspaglio, G., Prislei, S., Gallo, D., Martinelli, E., Ranelletti, F. O., Ferrandina, G., et al. (2005). Class III $\beta$-tubulin overexpression is a prominent mechanism of paclitaxel resistance in ovarian cancer patients. Clin. Cancer Res. 11, 298-305.

Murtagh, J., Lu, H. Y. \& Schwartz, E. L. (2006). Taxotere-induced inhibition of human endothelial cell migration is a result of heat shock protein 90 degradation. Cancer Res. 66, 8192-8199.

Na, G. C. \& Timasheff, S. N. (1981). Interaction of calf tubulin with glycerol. J. Mol. Biol. 151, 165-178.

Nawrotek, A., Knossow, M. \& Gigant, B. (2011). The determinants that govern microtubule assembly from the atomic structure of GTP-tubulin. J. Mol. Biol. 412, 35-42.

Nettles, J. H., Li, H. L., Cornett, B., Krahn, J. M., Snyder, J. P. \& Downing, K. H. (2004). The binding mode of epothilone A on $\alpha, \beta$-tubulin by electron crystallography. Science $\mathbf{3 0 5}, 866-869$.

Newman, D. J. \& Cragg, G. M. (2012). Natural products as sources of new drugs over the 30 years from 1981 to 2010. J. Nat. Prod. 75, 311-335.

Newman, D. J. \& Cragg, G. M. (2004). The discovery of anticancer drugs from natural sources. (L. Zhang \& A. L. Demain, Eds.) Natural Products: Drug Discovery and Therapeutic Medicine: Humana Press Inc., Totowa, NJ.

Nguyen, T. L., Xu, X. M., Gussio, R., Ghosh, A. K. \& Hamel, E. (2010). The assembly-inducing laulimalide/peloruside A binding site on tubulin: Molecular modeling and biochemical studies with $\left[{ }^{3} \mathrm{H}\right]$-peloruside A. J. Chem. Inf. Model. 50, 2019-2028.

Ni, F. \& Zhu, Y. (1994). Accounting for Ligand-Protein interactions in the relaxation-matrix analysis of transferred nuclear overhauser effects. J. Magn. Reson. Ser. B 103, 180-184.

Nicolaou, K. C., Dai, W. M. \& Guy, R. K. (1994). Chemistry and biology of taxol. Angew. Chem. Int. Ed. 33, $15-44$.

Noble, R. L., Beer, C. T. \& Cutts, J. H. (1958). Further biological activities of vincaleukoblastine - an alkaloid isolated from Vinca-Rosea-(L). Biochem. Pharmacol. 1, 347-348.

Nogales, E. \& Wang, H. W. (2006). Structural mechanisms underlying nucleotide-dependent self-assembly of tubulin and its relatives. Curr. Opin. Struc. Biol. 16, 221-229.

Nogales, E., Wang, H. W. \& Niederstrasser, H. (2003). Tubulin rings: which way do they curve? Curr. Opin. Struc. Biol. 13, 256-261.

Nogales, E. (2001). Structural insights into microtubule function. Annu. Rev. Bioph. Biom. 30, 397-420.

Nogales, E., Whittaker, M., Milligan, R. A. \& Downing, K. H. (1999). High-resolution model of the microtubule. Cell 96, 79-88. 
Nogales, E., Wolf, S. G. \& Downing, K. H. (1998a). Structure of the $\alpha \beta$ tubulin dimer by electron crystallography. Nature 391, 199-203.

Nogales, E., Downing, K. H., Amos, L. A. \& Löwe, J. (1998b). Tubulin and FtsZ form a distinct family of GTPases. Nat. Struct. Biol. 5, 451-458.

Nogales, E., Wolf, S. G., Khan, I. A., Luduena, R. F. \& Downing, K. H. (1995). Structure of tubulin at 6.5 $\AA$ and location of the taxol-binding site. Nature 375, 424-427.

Nunez, R. (2001). DNA measurement and cell cycle analysis by flow cytometry. Curr. Issues Mol. Biol. 3, 67-70.

Ojima, I., Chakravarty, S., Inoue, T., Lin, S. N., He, L. F., Horwitz, S. B., Kuduk, S. D. \& Danishefsky, S. J. (1999). A common pharmacophore for cytotoxic natural products that stabilize microtubules. $P$. Natl. Acad. Sci. USA 96, 4256-4261.

Oosawa, F. \& Asakura, S. (1975). Thermodynamics of the polymerization of protein: London, Academic Press.

Panda, D., Chakrabarti, G., Hudson, J., Pigg, K., Miller, H. P., Wilson, L. \& Himes, R. H. (2000). Suppression of microtubule dynamic instability and treadmilling by deuterium oxide. Biochemistry 39, 5075-5081.

Panda, D., DeLuca, K., Williams, D., Jordan, M. A. \& Wilson, L. (1998). Antiproliferative mechanism of action of cryptophycin-52: Kinetic stabilization of microtubule dynamics by high-affinity binding to microtubule ends. P. Natl. Acad. Sci. USA 95, 9313-9318.

Parness, J. \& Horwitz, S. B. (1981). Taxol binds to polymerized tubulin invitro. J. Cell Biol. 91, 479-487.

Pecqueur, L., Duellberg, C., Dreier, B., Jiang, Q. Y., Wang, C. G., Pluckthun, A., Surrey, T., Gigant, B. \& Knossow, M. (2012). A designed ankyrin repeat protein selected to bind to tubulin caps the microtubule plus end. P. Natl. Acad. Sci. USA 109, 12011-12016.

Pera, B., Razzak, M., Trigili, C., Pineda, O., Canales, A., Buey, R. M., Jiménez-Barbero, J., Northcote, P. T., Paterson, I., Barasoain, I., et al. (2010). Molecular recognition of peloruside A by microtubules. The C24 primary alcohol is essential for biological activity. Chembiochem 11, 1669-1678.

Photiou, A., Shah, P., Leong, L. K., Moss, J. \& Retsas, S. (1997). In vitro synergy of paclitaxel (Taxol) and vinorelbine (Navelbine) against human melanoma cell lines. Eur. J. Cancer 33, 463-470.

Pineda, O., Farras, J., Maccari, L., Manetti, F., Botta, M. \& Vilarrasa, J. (2004). Computational comparison of microtubule-stabilising agents laulimalide and peloruside with taxol and colchicine. Bioorg. Med. Chem. Lett. 14, 4825-4829.

Potashman, M. H. \& Duggan, M. E. (2009). Covalent modifiers: An orthogonal approach to drug design. $J$. Med. Chem. 52, 1231-1246.

Prota, A. E., Bargsten, K., Zurwerra, D., Field, J. J., Diaz, J. F., Altmann, K. H. \& Steinmetz, M. O. (2013). Molecular mechanism of action of microtubule-stabilizing anticancer agents. Science 339, 587590.

Prussia, A. J., Yang, Y. T., Geballe, M. T. \& Snyder, J. P. (2010). Cyclostreptin and microtubules: Is a low-affinity binding site required? Chembiochem 11, 101-109.

Pryor, D. E., O'Brate, A., Bilcer, G., Díaz, J. F., Wang, Y. F., Wang, Y., Kabaki, M., Jung, M. K., Andreu, J. M., Ghosh, A. K., et al. (2002). The microtubule stabilizing agent laulimalide does not bind in the taxoid site, kills cells resistant to paclitaxel and epothilones, and may not require its epoxide moiety for activity. Biochemistry 41, 9109-9115.

Putnam, C. D., Hammel, M., Hura, G. L. \& Tainer, J. A. (2007). X-ray solution scattering (SAXS) combined with crystallography and computation: defining accurate macromolecular structures, conformations and assemblies in solution. Q. Rev. Biophys. 40, 191-285.

Qi, Y. K. \& Ma, S. T. (2011). The medicinal potential of promising marine macrolides with anticancer activity. Chemmedchem 6, 399-409.

Ralston, G. (1993). Introduction to analytical ultracentrifugation. Beckman handbook. Department of Biochemistry, The University of Sydney, Australia: Beckman Intruments Inc. CA USA.

Rao, S., He, L. F., Chakravarty, S., Ojima, I., Orr, G. A. \& Horwitz, S. B. (1999). Characterization of the taxol binding site on the microtubule: Identification of $\mathrm{Arg}^{282}$ in $\beta$-tubulin as the site of photoincorporation of a 7-benzophenone analogue of taxol. J. Biol. Chem. 274, 37990-37994.

Rao, S., Orr, G. A., Chaudhary, A. G., Kingston, D. G. I. \& Horwitz, S. B. (1995). Characterization of the taxol binding-site on the microtubule -2-( $m$-Azidobenzoyl $)$ taxol photolabels a peptide (amino acids 271-233) of $\beta$-tubulin. J. Biol. Chem. 270, 20235-20238.

Rao, S., Krauss, N. E., Heerding, J. M., Swindell, C. S., Ringel, I., Orr, G. A. \& Horwitz, S. B. (1994). 3'( $p$-Azidobenzamido)taxol photolabels the N-terminal 31 amino-acids of $\beta$-tubulin. J. Biol. Chem. 269, 3132-3134. 
Rao, S., Horwitz, S. B. \& Ringel, I. (1992). Direct photoaffinity-labeling of tubulin with taxol. J. Nat. Cancer Inst. 84, 785-788.

Ravelli, R. B. G., Gigant, B., Curmi, P. A., Jourdain, I., Lachkar, S., Sobel, A. \& Knossow, M. (2004). Insight into tubulin regulation from a complex with colchicine and a stathmin-like domain. Nature 428, 198-202.

Ricker, J. L., Chen, Z., Yang, X. P., Pribluda, V. S., Swartz, G. M. \& Van Waes, C. (2004). $2-$ methoxyestradiol inhibits hypoxia-inducible factor lot, tumor growth, and angiogenesis and augments paclitaxel efficacy in head and neck squamous cell carcinoma. Clin. Cancer Res. 10, 8665-8673.

Risinger, A. L., Giles, F. J. \& Mooberry, S. L. (2009). Microtubule dynamics as a target in oncology. Cancer Treat Rev. 35, 255-261.

Risinger, A. L., Jackson, E. M., Polin, L. A., Helms, G. L., LeBoeuf, D. A., Joe, P. A., Hopper-Borge, E., Luduena, R. F., Kruh, G. D. \& Mooberry, S. L. (2008). The taccalonolides; Microtubule stabilizers that circumvent clinically relevant taxane resistance mechanisms. Cancer Res. 68, 8881-8888.

Rodriguez., L. G., Wu., X. \& Guan., J.-L. (2005). Wound-healing assay. (J.-L. Guan., Ed.) Methods in Molecular Biology, Vol 294: Microtubule Protocols Cell migration - Developmental methods and protocols, Humana Press Inc., Totowa, NJ.

Sackett, D. L. (2008). Antimicrotubule agents that bind covalently to tubulin. In Cancer Drug Discovery and Development (A. T. Fojo, Ed.) The Role of Microtubules in Cell Biology, Neurobiology, and Oncology, (pp 281-306): Humana Press.

Sackett, D. L. (1993). Podophyllotoxin, steganacin and combretastatin - natural producets that bind the colchicine site of tubulin. Pharmacol. Therapeut. 59, 163-228.

Sale, S., Sung, R., Shen, P. D., Yu, K., Wang, Y., Duran, G. E., Kim, J. H., Fojo, T., Oefner, P. J. \& Sikic, B. I. (2002). Conservation of the class I $\beta$-tubulin gene in human populations and lack of mutations in lung cancers and paclitaxel-resistant ovarian cancers. Mol. Cancer Ther. 1, 215-225.

Sanchez-Pedregal, V. M. \& Griesinger, C. (2009). The tubulin binding mode of MT stabilizing and destabilizing agents studied by NMR. (T. Carlomagno, Ed.) Tubulin-Binding Agents: Synthetic, Structural \& Mechanistic Insights: 286, (pp 151-208).

Sanchez-Pedregal, V. M., Kubicek, K., Meiler, J., Lyothier, I., Paterson, I. \& Carlomagno, T. (2006). The tubulin-bound conformation of discodermolide derived by NMR studies in solution supports a common pharmacophore model for epothilone and discodermolide. Angew. Chem. Int. Ed. 45, 7388-7394.

Sato, B., Muramatsu, H., Miyauchi, M., Hori, Y., Takase, S., Hino, M., Hashimoto, S. \& Terano, H. (2000). A new antimitotic substance, FRI82877 I. Taxonomy, fermentation, isolation, physicochemical properties and biological activities. J. Antibiot. 53, 123-130.

Schiff, P. B., Fant, J. \& Horwitz, S. B. (1979). Promotion of microtubule assembly in vitro by taxol. Nature 277, 665-667.

Schuck, P. (2000). Size-distribution analysis of macromolecules by sedimentation velocity ultracentrifugation and Lamm equation modeling. Biophys. J. 78, 1606-1619.

Schwartz, E. L. (2009). Antivascular Actions of Microtubule-Binding Drugs. Clin. Cancer Res. 15, 25942601.

Seckler, R., Wu, G. M. \& Timasheff, S. N. (1990). Interactions of tubulin with guanylyl-( $\beta-\gamma$-methylene) diphosphonate - Formation and assembly of a stoichiometric complex. J. Biol. Chem. 265, 76557661.

Shan, B., Medina, J. C., Santha, E., Frankmoelle, W. P., Chou, T. C., Learned, R. M., Narbut, M. R., Stott, D., Wu, P. G., Jaen, J. C., et al. (1999). Selective, covalent modification of $\beta$-tubulin residue Cys239 by T138067, an antitumor agent with in vivo efficacy against multidrug-resistant tumors. $P$. Natl. Acad. Sci. USA 96, 5686-5691.

Shearwin, K. E., Perezramirez, B. \& Timasheff, S. N. (1994). Linkages between the dissociation of $\alpha \beta$ tubulin into subunits and ligand binding: The ground state of tubulin is the GDP conformation. Biochemistry 33, 885-893.

Singh, A. J., Xu, C. X., Xu, X. M., West, L. M., Wilmes, A., Chan, A., Hamel, E., Miller, J. H., Northcote, P. T. \& Ghosh, A. K. (2010). Peloruside B, A potent antitumor macrolide from the New Zealand marine sponge Mycale hentscheli: Isolation, structure, total synthesis, and bioactivity. J. Org. Chem. 75, 2-10.

Singh, J., Petter, R. C., Baillie, T. A. \& Whitty, A. (2011). The resurgence of covalent drugs. Nat. Rev. Drug Discov. 10, 307-317.

Singh, P., Rathinasamy, K., Mohan, R. \& Panda, D. (2008). Microtubule assembly dynamics: An attractive target for anticancer drugs. IUBMB Life 60, 368-375. 
Skoufias, D. A. \& Wilson, L. (1992). Mechanism of inhibition of microtubule polymerization by colchicine: Inhibitory potencies of unliganded colchicine and tubulin colchicine complexes. Biochemistry 31, 738-746.

Smith, A. B., Safonov, I. G. \& Corbett, R. M. (2002). Total syntheses of (+)-zampanolide and (+)dactylolide exploiting a unified strategy. J. Am. Chem. Soc. 124, 11102-11113.

Smith, A. B., Safonov, I. G. \& Corbett, R. M. (2001). Total synthesis of (+)-zampanolide. J. Am. Chem. Soc. 123, 12426-12427.

Snyder, J. P., Nettles, J. H., Cornett, B., Downing, K. H. \& Nogales, E. (2001). The binding conformation of Taxol in $\beta$-tubulin: A model based on electron crystallographic density. P. Natl. Acad. Sci. USA 98, 5312-5316.

Song, D., Hsu, L. F. \& Au, J. L. S. (1996). Binding of taxol to plastic and glass containers and protein under in vitro conditions. J. Pharm. Sci. 85, 29-31.

Souto, A. A., Acuna, A. U., Andreu, J. M., Barasoain, I., Abal, M. \& AmatGuerri, F. (1995). New fluorescent water-soluble taxol derivatives. Angew. Chem. Int. Ed. 34, 2710-2712.

Sui, H. X. \& Downing, K. H. (2010). Structural basis of interprotofilament interaction and lateral deformation of microtubules. Structure 18, 1022-1031.

Tanaka, J. \& Higa, T. (1996). Zampanolide, a new cytotoxic macrolide from a marine sponge. Tetrahedron Lett. 37, 5535-5538

Tanaka, J., Higa, T., Bernardinelli, G. \& Jefford, C. W. (1996). New cytotoxic macrolides from the sponge Fasciospongia rimosa. Chem. Lett. 255-256.

ter Haar, E., Kowalski, R. J., Hamel, E., Lin, C. M., Longley, R. E., Gunasekera, S. P., Rosenkranz, H. S. \& Day, B. W. (1996). Discodermolide, a cytotoxic marine agent that stabilizes microtubules more potently than taxol. Biochemistry 35, 243-250.

Thadani-Mulero, M., Nanus, D. M. \& Giannakakou, P. (2012). Androgen receptor on the move: Boarding the microtubule expressway to the nucleus. Cancer Res. 72, 4611-4615.

Thepchatri, P., Cicero, D. O., Monteagudo, E., Ghosh, A. K., Cornett, B., Weeks, E. R. \& Snyder, J. P. (2005). Conformations of laulimalide in DMSO- $d_{6}$. J. Am. Chem. Soc. 127, 12838-12846.

Thurston, D. E. (2006). Chemistry and pharmacology of anticancer drugs: CRC Press Taylor \& Francis group.

Tinley, T. L., Randall-Hlubek, D. A., Leal, R. M., Jackson, E. M., Cessac, J. W., Quada, J. C., Hemscheidt, T. K. \& Mooberry, S. L. (2003). Taccalonolides E and A: Plant-derived steroids with microtubule-stabilizing activity. Cancer Res. 63, 3211-3220.

Tozer, G. M., Kanthou, C. \& Baguley, B. C. (2005). Disrupting tumour blood vessels. Nat. Rev. Cancer 5, 423-435.

Tsurutani, J., Komiya, T., Uejima, H., Tada, H., Syunichi, N., Oka, M., Kohno, S., Fukuoka, M. \& Nakagawa, K. (2002). Mutational analysis of the $\beta$-tubulin gene in lung cancer. Lung Cancer 35, 11-16.

Uenishi, J. i., Iwamoto, T. \& Tanaka, J. (2009). Total Synthesis of (-)-zampanolide and questionable existence of (-)-dactylolide as the elusive biosynthetic precursor of (-)-zampanolide in an Okinawan Sponge. Org. Lett.

Urano, N., Fujiwara, Y., Hasegawa, S., Miyoshi, Y., Noguchi, S., Takiguchi, S., Yasuda, T., Yano, M. \& Monden, M. (2003). Absence of beta-tubulin gene mutation in gastric carcinoma. Gastric cancer 6, 108-112.

VanBuren, V., Cassimeris, L. \& Odde, D. J. (2005). Mechanochemical model of microtubule structure and self-assembly kinetics. Biophys. J. 89, 2911-2926.

Verrills, N. M., Flemming, C. L., Liu, M., Ivery, M. T., Cobon, G. S., Norris, M. D., Haber, M. \& Kavallaris, M. (2003). Microtubule alterations and mutations induced by desoxyepothilone B: Implications for drug-target interactions. Chem. Biol. 10, 597-607.

Wang, H. W. \& Nogales, E. (2005). Nucleotide-dependent bending flexibility of tubulin regulates microtubule assembly. Nature 435, 911-915.

Wang, M. M., Xia, X. Y., Kim, Y., Hwang, D., Jansen, J. M., Botta, M., Liotta, D. C. \& Snyder, J. P. (1999). A unified and quantitative receptor model for the microtubule binding of paclitaxel and epothilone. Org. Lett. 1, 43-46.

Wani, M. C., Taylor, H. L., Wall, M. E., Coggon, P. \& McPhail, A. T. (1971). Plant antitumor agents. isolation and structure of Taxol, a novel antileukemic and antitumor agent from Taxus-brevifolia. J. Am. Chem. Soc. 93.

Weisenberg, R., Borisy, G. G. \& Taylor, E. W. (1968). Colchicine-binding protein of mammalian brain and its relation to microtubules. Biochemistry 7, 4466-4479. 
Wermuth, C. G. (2006). Pharmacophores: Historical perspective and viewpoint from a medicinal chemist. (T. Langer \& R. D. Hoffmann, Eds.) Methods and principles in medicinal chemistry. Pharmacophores and pharmacophore searches.: 32, Germany: Wiley-Vch.

West, L. M., Northcote, P. T. \& Battershill, C. N. (2000). Peloruside A: A potent cytotoxic macrolide isolated from the New Zealand marine sponge Mycale sp. J. Org. Chem. 65, 445-449.

Wicki-Stordeur, L. E., Dzugalo, A. D., Swansburg, R. M., Suits, J. M. \& Swayne, L. A. (2012). Pannexin 1 regulates postnatal neural stem and progenitor cell proliferation. Neural Dev. 7.

Wienecke, A. \& Bacher, G. (2009). Indibulin, a novel microtubule inhibitor, discriminates between mature neuronal and nonneuronal tubulin. Cancer Res. 69, 171-177.

Wilmes, A., O’Sullivan, D., Chan, A., Chandrahasen, C., Paterson, I., Northcote, P. T., La Flamme, A. C. \& J.H., M. (2011). Synergistic interactions between peloruside A and other microtubulestabilizing and destabilizing agents in cultured human ovarian carcinoma cells and murine T cells. Cancer Chemoth. Pharm.

Wilmes, A., Bargh, K., Kelly, C., Northcote, P. T. \& Miller, J. H. (2007). Peloruside a synergizes with other microtubule stabilizing agents in cultured cancer cell lines. Mol. Pharmacol. 4, 269-280.

Winkler, J. D. \& Axelsen, P. H. (1996). A model for the taxol (paclitaxel)/epothilone pharmacophore. Bioorg. Med. Chem. Lett. 6, 2963-2966.

Wittmann, T. \& Waterman-Storer, C. M. (2001). Cell motility: can Rho GTPases and microtubules point the way? J. Cell Sci. 114, 3795-3803.

www.fda.gov/.

Wyman, J. \& Gill, S. J. (1990). Binding and Linkage: Functional Chemistry of Biological Molecules: (Mill Valley, Ca, University Science Books).

Xia, S. J., Kenesky, C. S., Rucker, P. V., Smith, A. B., Orr, G. A. \& Horwitz, S. B. (2006). A photoaffinity analogue of discodermolide specifically labels a peptide in $\beta$-tubulin. Biochemistry 45, 1176211775.

Yang, C. G., Barasoain, I., Li, X., Matesanz, R., Liu, R. H., Sharom, F. J., Yin, D. L., Diaz, J. F. \& Fang, W. S. (2007). Overcoming tumor drug resistance with high-affinity taxanes: A SAR study of C2modified 7-acyl-10-deacetyl cephalomannines. Chemmedchem 2, 691-701.

Yang, C. P. H., Verdier-Pinard, P., Wang, F., Lippaine-Horvath, E., He, L. F., Li, D. S., Hofle, G., Ojima, I., Orr, G. A. \& Horwitz, S. B. (2005). A highly epothilone B-resistant A549 cell line with mutations in tubulin that confer drug dependence. Mol. Cancer Ther. 4, 987-995.

Yap, T. A. \& Workman, P. (2012). Exploiting the cancer genome: Strategies for the discovery and clinical development of targeted molecular therapeutics. In Annual Review of Pharmacology and Toxicology (P. A. Insel, S. G. Amara \& T. F. Blaschke, Eds.) Annu. Rev. Pharmacol.: 52, (pp 549-+).

Yin, S. H., Zeng, C. Q., Hari, M. \& Cabral, F. (2012). Random mutagenesis of $\beta$-tubulin defines a set of dispersed mutations that confer paclitaxel resistance. Pharm. Res. 29, 2994-3006.

Yin, S. H., Bhattacharya, R. \& Cabral, F. (2010). Human mutations that confer paclitaxel resistance. Mol. Cancer Ther. 9, 327-335.

Yue, Q. X., Liu, X. A. \& Guo, D. A. (2010). Microtubule-binding natural products for cancer therapy. Planta Med. 76, 1037-1043.

Zhang, B., Maiti, A., Shively, S., Lakhani, F., McDonald-Jones, G., Bruce, J., Lee, E. B., Xie, S. X., Joyce, S., Li, C., et al. (2005). Microtubule-binding drugs offset tau sequestration by stabilizing microtubules and reversing fast axonal transport deficits in a tauopathy model. P. Natl. Acad. Sci. USA 102, 227-231.

Zhao, Y., Fang, W. S. \& Pors, K. (2009). Microtubule stabilising agents for cancer chemotherapy. Expert Opin. Ther. Pat. 19, 607-622.

Zhou, J. \& Giannakakou, P. (2005). Targeting microtubules for cancer chemotherapy. Curr. Med. Chem. Anti-Cancer. Agents 5, 65-71.

Zhou, S. F., Chan, E., Duan, W., Huang, M. \& Chen, Y. Z. (2005). Drug bioactivation, covalent binding to target proteins and toxicity relevance. Drug Metab. Rev. 37, 41-213.

Zurwerra, D., Glaus, F., Betschart, L., Schuster, J., Gertsch, J., Ganci, W. \& Altmann, K.-H. (2012). Total Synthesis of (-)-zampanolide and structure-activity relationship studies on (-)-dactylolide derivatives. Chem-Eur. J. doi: 10.1002/chem.201202553.

Zurwerra, D., Gertsch, J. \& Altmann, K. H. (2010). Synthesis of (-)-dactylolide and 13-desmethylene-(-)dactylolide and their effects on tubulin. Org. Lett. 12, 2302-2305. 


\section{GENERAL SOLUTIONS}

$P B S(1 L)$

$137 \mathrm{mM} \mathrm{NaCl} \quad 8 \mathrm{~g}$

$1.6 \mathrm{mM} \mathrm{KCL} \quad 0.2 \mathrm{~g}$

$10.1 \mathrm{mM} \mathrm{Na}_{2} \mathrm{HPO}_{4} \quad 1.44 \mathrm{~g}$

$1.8 \mathrm{mM} \mathrm{KH}_{2} \mathrm{PO}_{4} \quad 0.24 \mathrm{~g}$

$\mathrm{pH} 7.5$

MTT solution $(80 \mathrm{~mL})$

$5 \mathrm{mg} / \mathrm{mL}$ MTT (Invitrogen or Sigma) $400 \mathrm{mg}$

$10 \mathrm{mM}$ filtered PBS $\quad 80 \mathrm{~mL}$

MTT solubilizer $(500 \mathrm{~mL})$

$10 \%$ sodium dodecyl sulphate (SDS) $\quad 50 \mathrm{~g}$

$45 \%$ dimethylformamide (DMF) $225 \mathrm{~mL}$

$\mathrm{pH} 4.5$ adjusted with acetic acid

Diluted to $500 \mathrm{~mL}$ with $\mathrm{ddH}_{2} \mathrm{O}$

Propidium iodide staining solution

$0.05 \mathrm{mg} / \mathrm{mL}$ propidium iodide (Sigma)

$0.1 \%$ sodium citrate

$0.1 \%$ Triton $\mathrm{X}-100$ 


\section{REAGENTS FOR TUBULIN AND MICROTUBULE EXPERIMENTS}

Dialysis buffer 0.5 L (GAB6-0.1 mM GTP)

$150 \mathrm{~mL}$ glycerol

$25 \mathrm{~mL} 0.2 \mathrm{M}$ Sodium phosphate

5 mL 0.1 M EGTA

$3 \mathrm{~mL} 1 \mathrm{M} \mathrm{MgCl}_{2}$

$0.1 \mathrm{mM} \mathrm{GTP}$

Glycerol-EGTA buffer $(G A B)$ - Assembly buffer

3.4 M glycerol

$10 \mathrm{mM}$ sodium phosphate

1 mM EGTA

$6 \mathrm{mM} \mathrm{MgCl}{ }_{2}$

$1 \mathrm{mM} \mathrm{GTP}$

pH 6.5

Prepared on the day of use without $\mathrm{MgCl}_{2}$ and with only $0.1 \mathrm{mM} \mathrm{GTP,} \mathrm{pH}$ adjusted to 6.8 and kept cold on ice. After column equilibration, $6 \mathrm{mM} \mathrm{MgCl}_{2}$ was added and the concentration of GTP increased to $1 \mathrm{mM}$ for assays requiring tubulin assembly conditions, this shifts the $\mathrm{pH}$ to 6.5 .

In experiments that use stabilized MTs, the GAB buffer left over from the dialysis (recipe above) is kept in the fridge and used to dilute the stabilized MTs and act as the experimental buffer.

Phosphate-EDTA Buffer (PEDTA), $0.1 \mathrm{mM} \mathrm{GTP}$

$10 \mathrm{mM}$ sodium phosphate

1 mM EDTA

$0.1 \mathrm{mM} \mathrm{GTP}$

$\mathrm{pH} 7.0$

Prepare on the day of use and keep cold on ice. When required, increase the GTP concentration to $1 \mathrm{mM}$ and add the required amount of $\mathrm{MgCl}_{2}$. 